\title{
GROUND-WATER LEVELS IN OBSERVATION WELLS IN OKLAHOMA 1982-83 CLIMATIC YEARS
}

By Robert L. Goemaat, Lionel D. Mize, and Dannie E. Spiser

U.S. GEOLOGICAL SURVEY

OPEN-FILE REPORT 84-472

Prepared in cooperation with the OKLAHOMA WATER RESOURCES BOARD

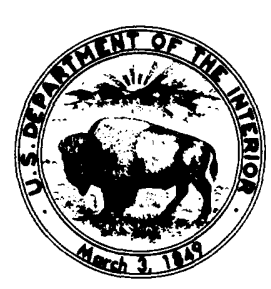

Oklahoma City, Oklahoma 1984 


\title{
UNITED STATES DEPARTMENT OF THE INTERIOR WILLIAM P. CLARK, Secretary
}

\author{
GEOLOGICAL SURVEY \\ Dallas L. Peck, Director
}

For additional information write to:

District Chief

U.S. Geological Survey

Water Resources Division

Room 621, Old Post Office Bullding

215 Dean A. McGee Avenue

Oklahoma City, Okiahoma 73102

Telephone: (405) 231-4256
Copies of this report can be purchased from:

Open-Flle Services Section Western Distribution Branch

U.S. Geological Survey Box 25425, Federal Center

Lakewood, Colorado 80225

Telephone: (303) 234-5888 


\section{CONTENTS}

Page

Abstract....................................... 1

Introduction........................................ 1

Cooperation..................................... 2

Definition of terms.............................. 2

Collection of data................................. 4

Well-numbering system............................ 5

Publications................................... 6

\section{ILLUSTRATIONS}

Figure 1. Map showing frequency of water-level measurements...... 8

Figure 2. Representative hydrographs from continuous recorders... 9

Figure 3. Representative hydrographs from annual measurements.... 10

Figure 4. Representative hydrographs from precipitation records... 11 


\section{TABLES}

Page

Table 1. List of sites at which water levels are measured

continuously, monthly, or quarterly............. 12

Counties for which water levels are published:

Alfalfa.................................. 12

Beaver.................................. 12

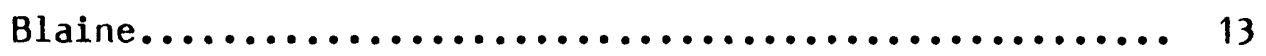

Canadian................................. 14

Cimarron.................................. 15

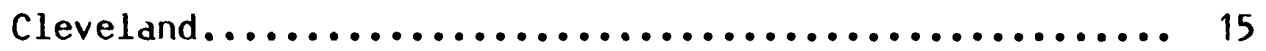

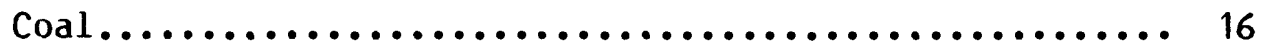

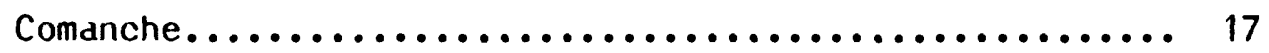

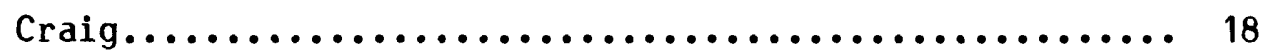

Creek................................... 19

Custer................................. 20

Ellis.................................... 21

Grady................................. 23

Haskell.................................. 24

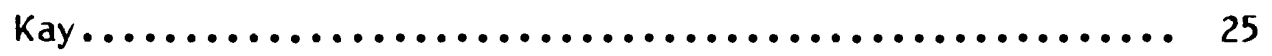

Latimer................................. 26

Le Flore................................. 27

Lincoln................................... 29

Muskogee.................................. 30

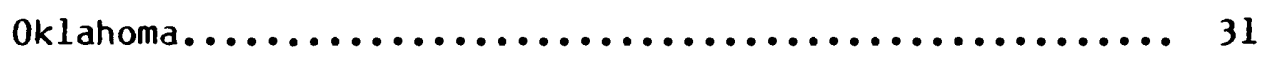


0sage....................................... 34

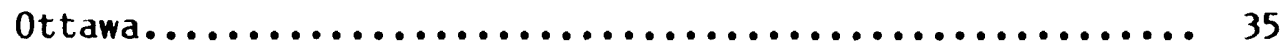

Payne.................................... 36

Pittsburg.............................. 40

Pontotoc.................................. 41

Roger Mills.............................. 42

Sequoyah................................ 43

Texas....................................... 44

Washita................................. 45

Woods.................................... 46

Woodward................................ 47

Table 2. List of wells and water levels measured annually....... 49 Counties for which water levels are published:

Adair.................................... 49

Alfalfa................................. 51

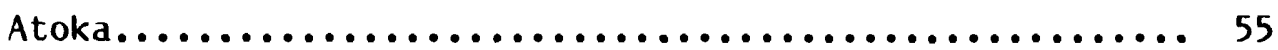

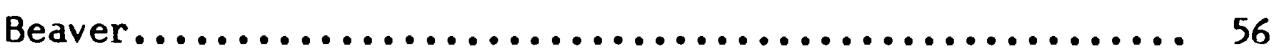

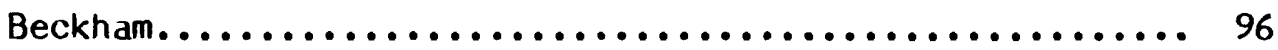

Blaine...................................... 114

Bryan...................................... 119

Caddo....................................... 120

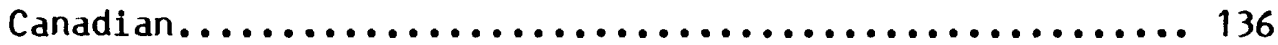

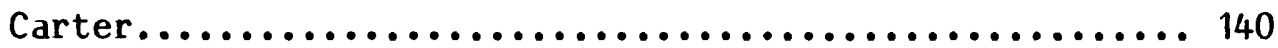

Cherokee................................... 141 
Choctaw................................ 142

Cimarron.............................. 148

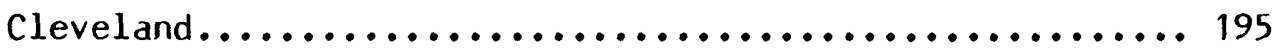

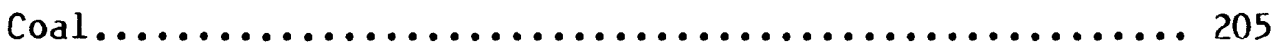

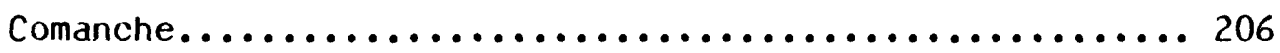

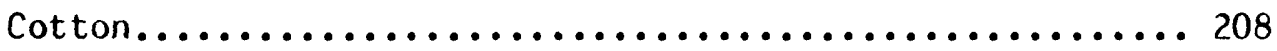

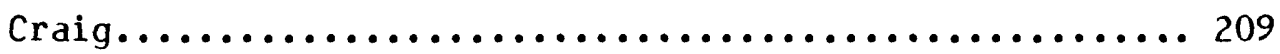

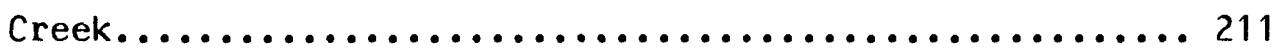

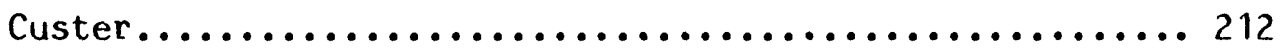

Delaware................................. 216

Dewey................................... 218

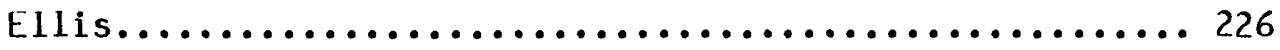

Garfield.................................. 291

Garvin................................... 298

Grady.................................... 299

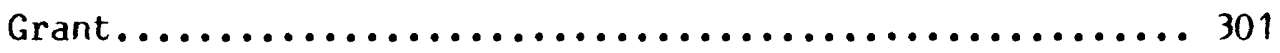

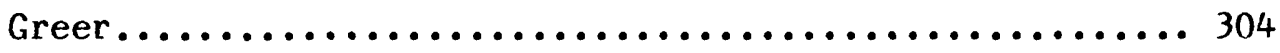

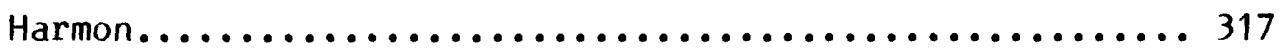

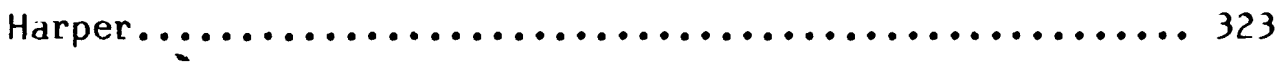

Haskell................................. 331

Hughes.................................. 332

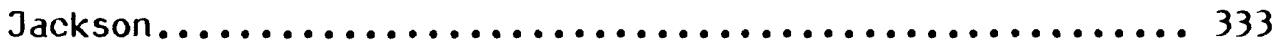

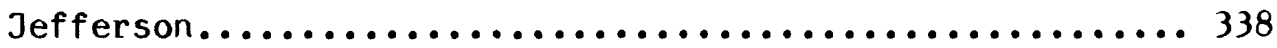

Johnston................................. 339

Kay................................... 341 


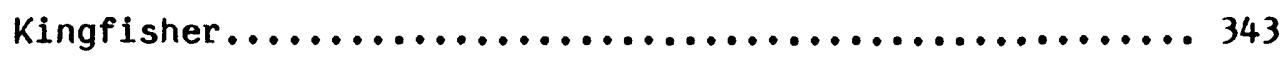

kiowa..................................... 359

Latimer ....................................... 370

Le Flore................................... 371

Lincoln...................................... 372

Logan..................................... 374

Love...................................... 378

Mcclain....................................... 379

McCurtain................................. 381

McIntosh................................ 382

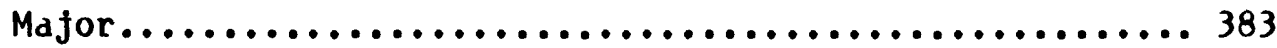

Marshall.................................. 393

Mayes....................................... 394

Murray...................................... 396

Muskogee.................................... 397

Noble...................................... 398

Nowata...................................... 399

0kfuskee................................. 401

0klahoma................................ 402

0kmulgee................................ 426

0sage.................................... 427

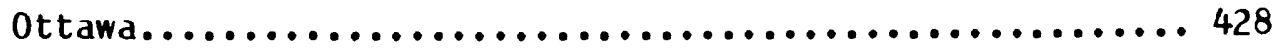

Pawnee.....................................429

Payne..................................... 430

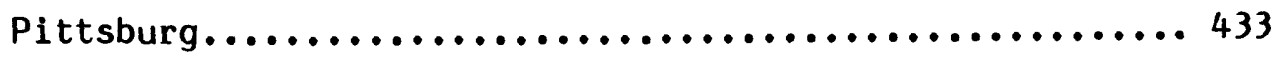


CONTENTS--Continued Page

Pontotoc..................................4434

Pottawatomie.............................4 435

Pushmataha.................................4439

Roger Mills..............................440

Rogers.................................456

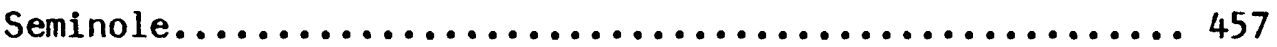

Sequoyah................................ 458

Stephens.................................. 459

Texas...................................460

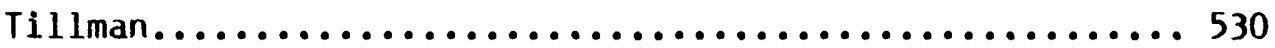

Tulsa..................................... 548

Wagoner.................................... 549

Washington............................... 550

Washita................................. 552

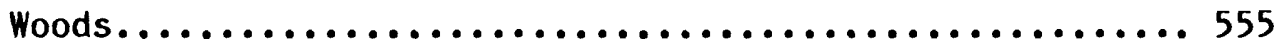

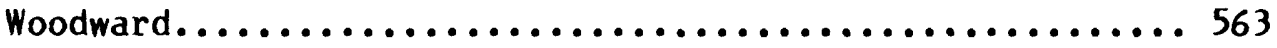

\section{CONVERSION FACTORS}

Inch-pound units used in this report may be converted to metric units by the following conversion factors.

$\begin{array}{lcc}\text { Multiply Inch-Pound Units } & \text { by } & \text { To obtain Metric Units } \\ \text { acre } & 4.047 & \mathrm{~m}^{2} \text { (square meter) } \\ \mathrm{ft} \text { (foot) } & .3048 & \mathrm{~m} \text { (meter) }\end{array}$


GROUND-WATER LEVELS IN OBSERVATION WELLS

IN OKLAHOMA 1982-83 CLIMATIC YEARS

By Robert L. Goemaat 1, Lionel D. Mize 1, and Dannie E. Spiser 2

\section{ABSTRACT}

In the 1982-83 climatic years, the U. S. Geological Survey, in cooperation with the Oklahoma Water Resources Board, collected ground-water level data in Oklahoma from 1,087 sites in 77 counties. This report presents those data points.

\section{INTRODUCTION}

A systematic program to collect water-level records in 0klahoma began in 1937. The objectives of this program are (1) to provide long-term records of water-level fluctuations in representative wells, (2) to facilitate the prediction of water-level trends and indicate future availability of ground-water supplies, and (3) to provide information for use in basic research. The stratigraphic nomenclature and age determination used in this report are those accepted by the Oklahoma Geological Survey and do not necessarily agree with those of the U.S. Geological Survey except for the Cheyenne Sandstone which is considered to be Purgatoire Sandstone by the Oklahoma Geological Survey (Robert 0. Fay, personal communication, August 9, 1979).

1 U.S. Geological Survey

2 Oklahoma Water Resources Board 
Water resources data for Oklahoma for the 1982 and 1983 climatic years are presented in this report. Data consist of records from continuous recorders, and from monthly, quarterly, and annual measurements. This report contains data for 37 wells with continuous recorders, 3 wells measured monthly, 6 quarterly, and 1041 annually. The climatic year, which begins April 1 and ends March 31, is the calendar year in which the climatic year ends.

\section{COOPERATION}

The U.S. Geological Survey and the Oklahoma Water Resources Board-James R. Barnett, Executive Director, Michael R. Melton, Assistant Director, and Duane Smith, Chief, Ground Water Division, have a cooperative agreement for the systematic collection of ground-water levels. Records have been collected in accordance with U.S. Geological Survey specifications and under Geological Survey quality control.

\section{DEF INITION OF TERMS}

AQUIFER is a geologic formation, group of formations, or part of a formation that contains sufficient saturated permeable material to yield significant quantities of water to wells. ARTESIAN means "confined" and is used to describe a well in which the water level stands above the top of the aquifer tapped by the well. A flowing artesian well is one in which the water level is above the land surface. 
CLIMATIC YEAR is the 12-month period beginning April 1 and ending March 31. The climatic year is designated by the calendar year in which it ends.

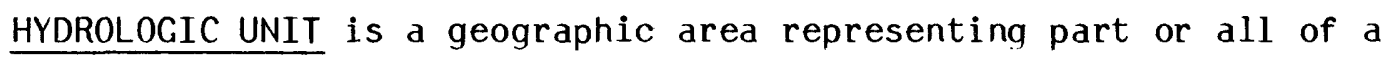
surface drainage basin of distinct hydrologic feature as delineated by the Office of Water Data Coordination on the State Hydroloqic Unit Map of Oklahoma. Each hydrologic unit is identified by an 8-digit number.

LAND-SURFACE DATUM is a datum plane that is approximately at land surface at each well.

SITE STATUS shows what occurred at the well when the measurement was made. The following are the letter codes used in the water-level tables to designate site status:
D- Dry
J- Injector site monitor
E- Flowed recently
$\mathrm{N}$ - Measurement discontinued
F- Flowing
0- Obstruction
G- Nearby well flowing
P- Well pumping
H- Nearby well recently flowed
R- Well recently pumped
I- Injector site
S- Nearby well pumped
T- Nearby well recently pumped
$X$ - Surface-water effect
V- Foreign substance
Z- Other
W- Destroyed 


\section{COLLECTION OF DATA}

Only ground-water level data from the observation well network are published herein. This network contains observation wells located so that significant data are obtained from the most important aquifers. Each well is identified by means of (1) a 15-digit number that is based on latitude and longitude and (2) a local number that is provided for local needs. Water-level measurements in this report are given in feet above or below (-) land-surface datum (LSD). Water levels in wells equipped with automatic digital recorders are published as a daily mean. Water levels in wells equipped with chart recorders are reported for every fifth day and at the end of each month (EOM) with the high of the day being reported. Water levels are reported to as many significant figures as can be justified by the local conditions. Accordingly, most measurements are reported to a hundredth of a foot, but some are given only to a tenth of a foot or a larger unit. 
The standard method of giving location by fractional section, section, township, and range is replaced by the method illustrated in the diagram below. The location of the well indicated by the dot normally would be described as SE $1 / 4 \mathrm{NE} 1 / 4 \mathrm{NE} 1 / 4$ sec. 36, T. $18 \mathrm{~N} ., \mathrm{R} .25 \mathrm{E}$. The method used in this report reverses the order and indicates quarter subdivisions of the section by letters. By this method the location of the well is given as $18 \mathrm{~N}-25 \mathrm{E}-36 \mathrm{AAD} 1$. The final digit (1) is the sequential number of a well within the smallest fractional subdivision. For some wells the location is known only to the quarter section (160-acre tract) or quarter-quarter section (40-acre tract) and is so indicated by the well number.

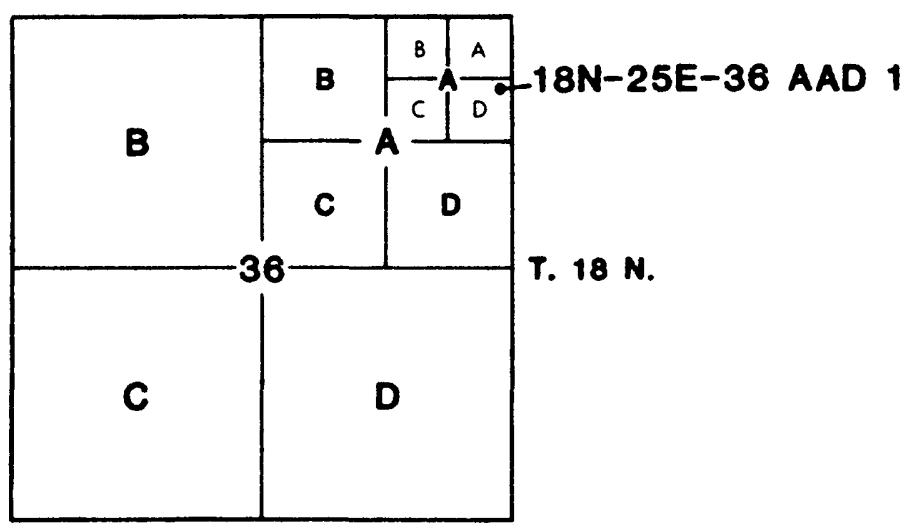

R. 25 E. 


\section{PUBLICATIONS}

Publication of ground-water level data for the United States in Water-Supply Papers was begun by the Geological Survey in 1935. From 1935 through 1939, a single Water-Supply Paper for each year covering the entire nation was issued (Water-Supply Papers 777, 817, 840, 845, and 886). From 1940 through 1974, separate Water-Supply Papers were issued for 6 sections of the United States. Water-level data for Oklahoma are in the Water-Supply Papers listed below, each report containing one or more calendar years (January-December) of data.

\begin{tabular}{lccc} 
Calendar & \multicolumn{3}{c}{ Calendar } \\
Year & Water-Supply Paper & Year & Water-Supply Paper \\
\hline 1935 & 777 & 1947 & 1099 \\
1936 & 817 & 1948 & 1129 \\
1937 & 840 & 1949 & 1159 \\
1938 & 845 & 1950 & 1168 \\
1939 & 886 & 1951 & 1194 \\
1940 & 909 & 1952 & 1244 \\
1941 & 939 & 1953 & 1268 \\
1942 & 947 & 1954 & 1324 \\
1943 & 989 & 1955 & 1407 \\
1944 & 1019 & $1956-59$ & 1549 \\
1945 & 1026 & $1960-64$ & 1824 \\
1946 & 1074 & $1965-69$ & 1979 \\
& & $1970-74$ & 2172
\end{tabular}


In addition to the Water-Supply Papers listed, the U.S. Geoloqical Survey, in cooperation with the Oklahoma Water Resources Bodrd, has published the following open-file reports on water levels in Oklahoma:

Ground-water levels in observation wells in 0klahoma, 1956-60. Ground-water levels in observation wells in Oklahoma, 1961-62. Ground-water levels in observation wells in Oklahoma, 1963-64. Ground-water levels in observation wells in 0klahoma, 1965-66. Ground-water levels in observation wells in Oklahoma, 1967-68. Ground-water levels in observation wells in 0klahoma, 1969-70. Ground-water levels in observation wells in Oklahoma, 1971-74. Ground-water levels in observation wells in 0klahoma, 1975. Water Resources Data for Oklahoma, Water Year 1976, OK-76-1. Water Resources Data for Oklahoma, Water Year 1976, OK-76-2. Records of water-level measurements in wells in the Oklahoma Panhandle, 1966-70.

Records of water-level measurements in wells in the Oklahoma Panhandle, 1971-72.

Selected water-level records for western 0klahoma, 1950-75.

Selected water-level records for western Oklahoma, 1975-76.

Selected water-level records for Oklahoma, 1975-77.

Selected water-level records for Oklahoma, 1976-78.

Selected water-level records for Oklahoma, 1979-80.

Water Resources Data for OKLahoma, OK-79-1.

Water Resources Data for OKlahoma, OK-79-2.

Water Resources Data for OKlahoma, OK-80.

Ground-water levels in observation wells in 0klahoma, 1980-82. 


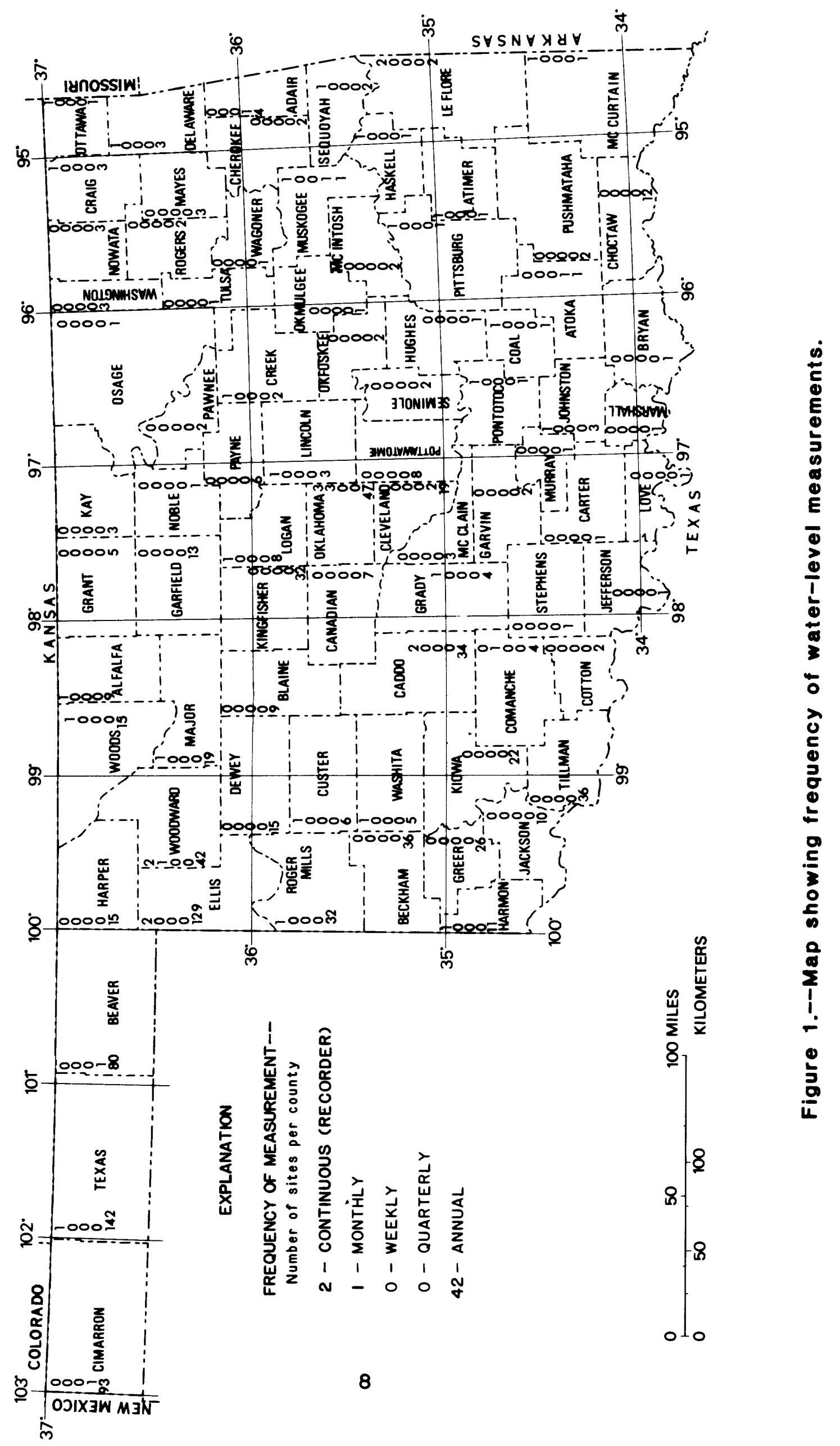




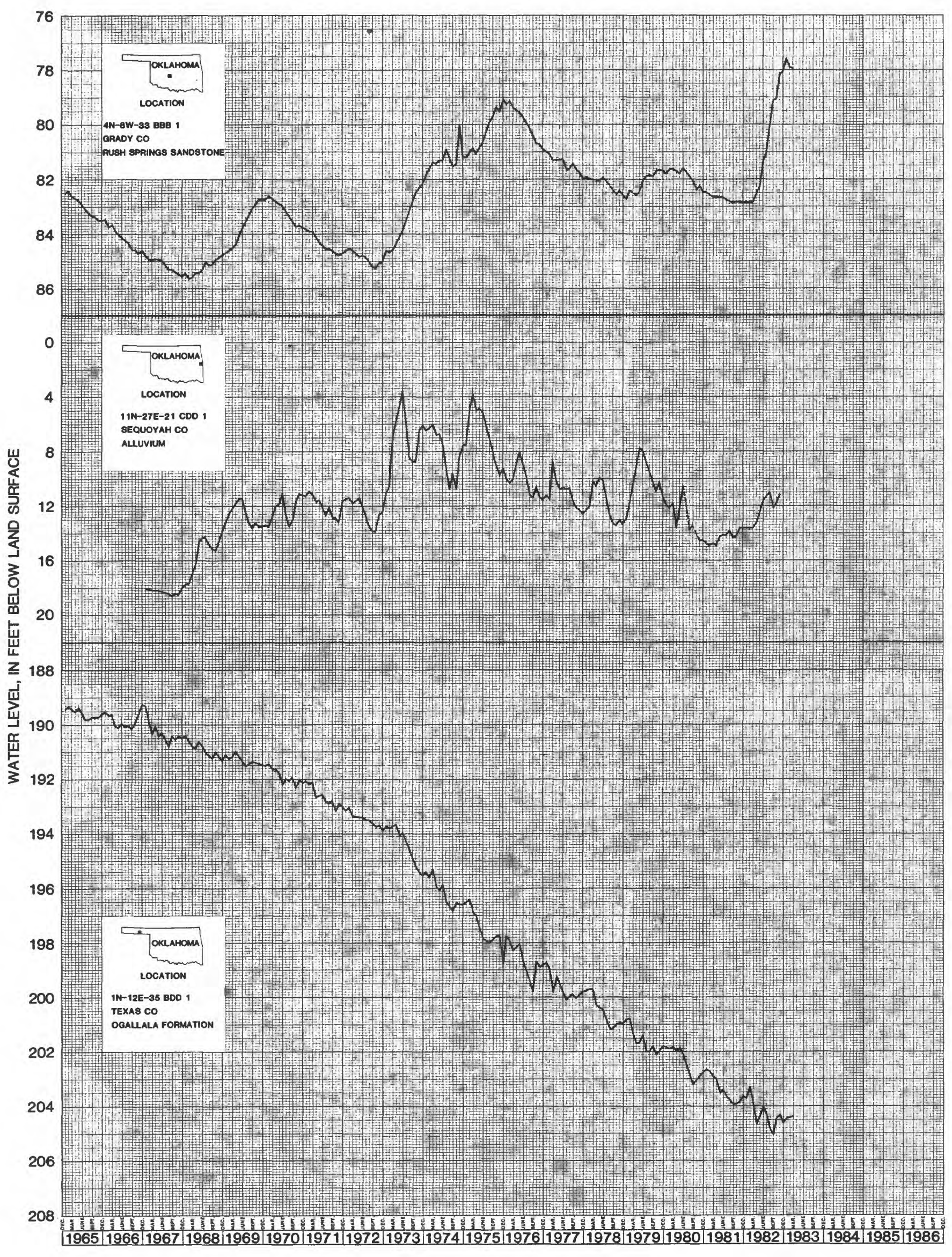

Figure 2.--Representative hydrographs from continuous recorders. 

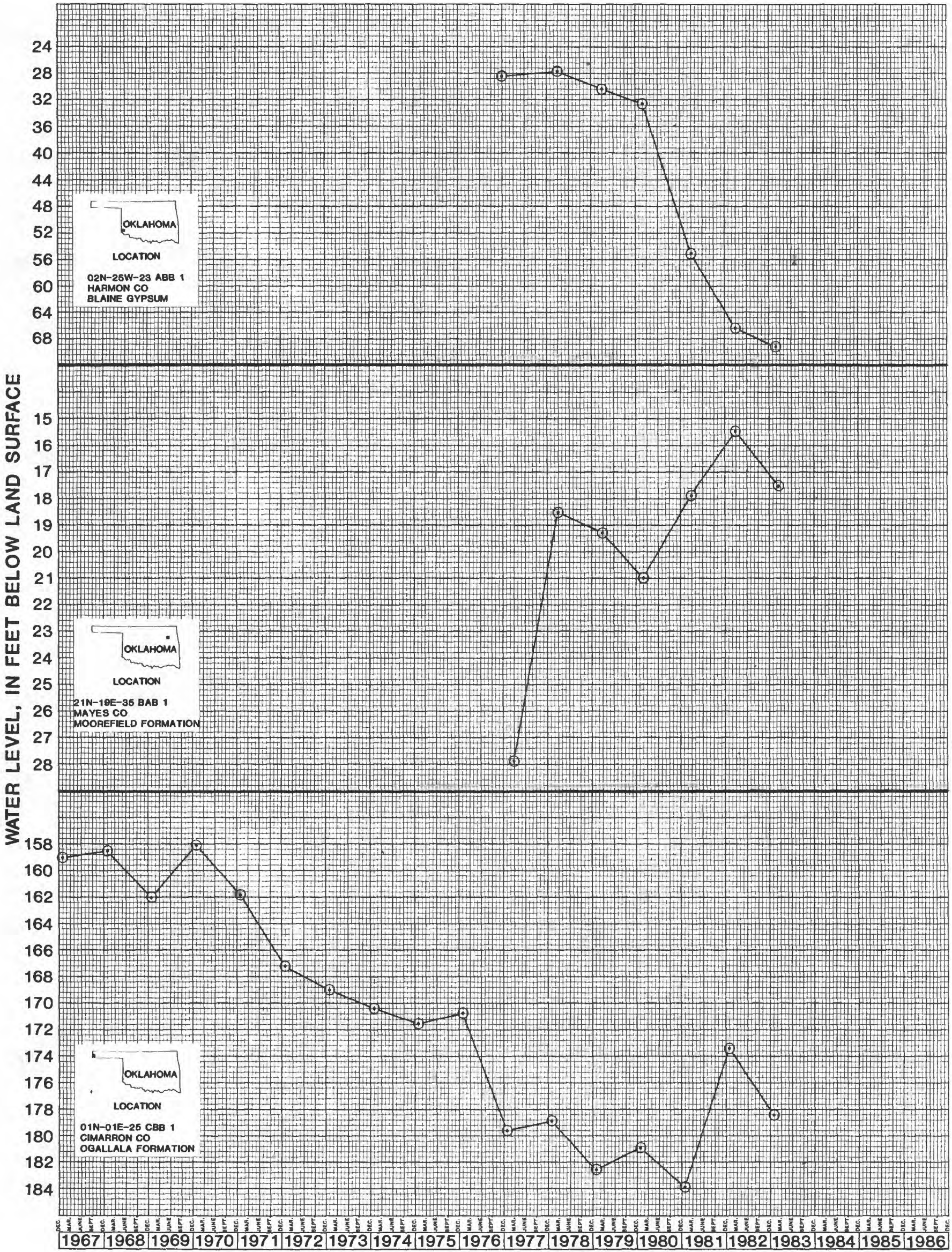

Figure 3.--Representative hydrographs from annual measurements. 


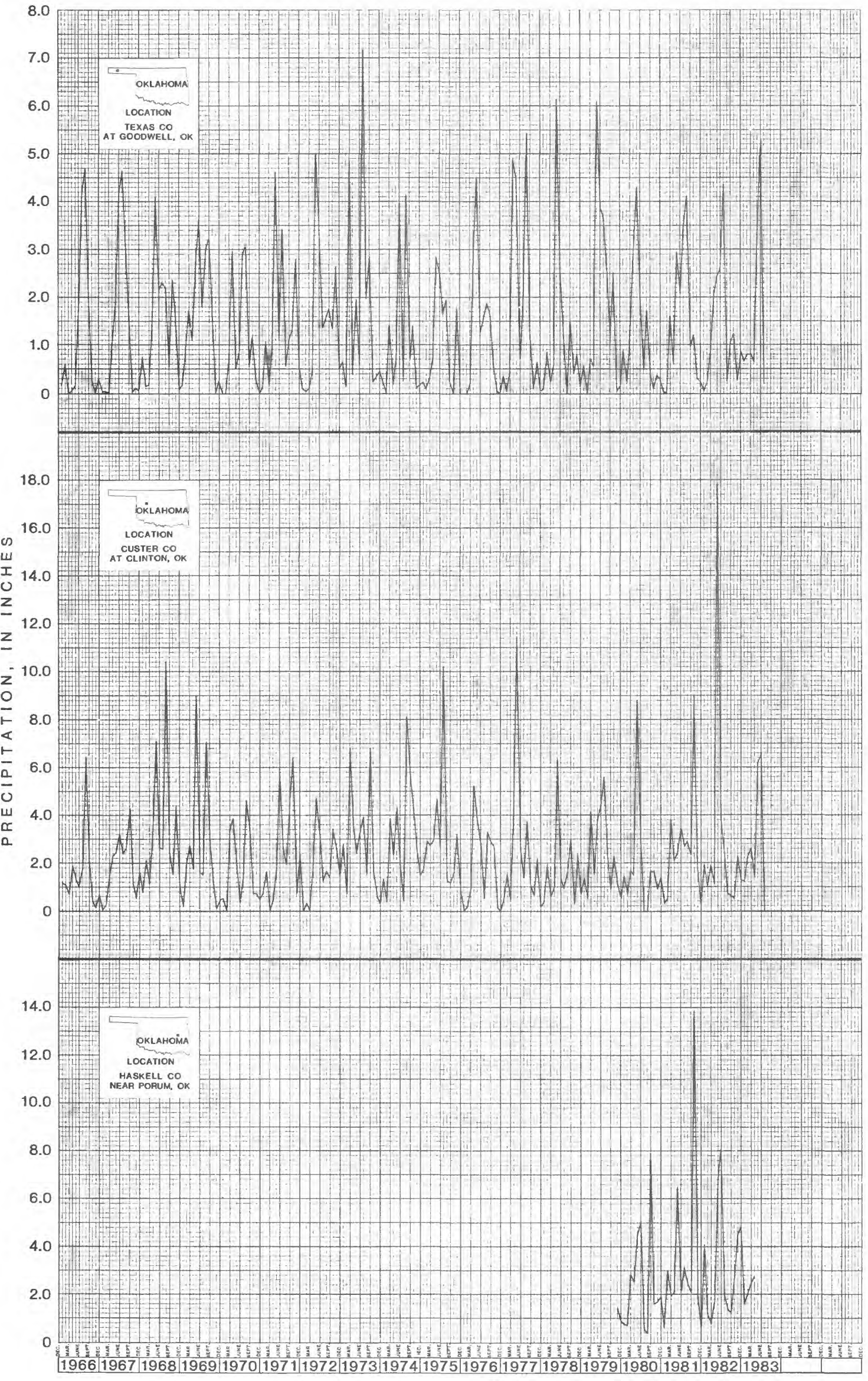

Figure 4.--Representative hydrographs from precipitation records. 
Table 1. List of sites at wich water levels are measured continuously, monthly, or quarterly. GROUND-WATER LEVELS

ALFALFA COUNTY

365342098175301 , LOCAL NUMBER(CORRECTED), 28N-11W-27 DAD 1.

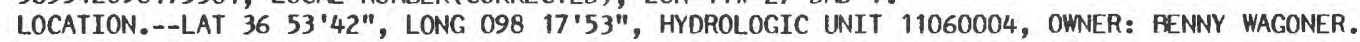
AQUIFER.--TERRACE DEPOSITS. WELL. CHARACTERISTICS._-DRILLED UNUSED STOCK WELL, DIAMETER 6 IN $(0.15 M)$, DEPTH 36 FT $(11.0 M)$. DATUM.--ALTITUDE OF LAND-SURFACE DATUM IS $1185 \mathrm{FT}(361 \mathrm{M})$. MEASURING POINT: TOP OF CASING 4.00 FT (1.22M) ABOVE LAND-SURFACE DATUM. REMARKS.--RECORDS FURNISHED BY OKLAHOMA WATER RESOURCES BOARD. PERIOD OF RECORD.--1967 TO CURRENT YEAR. EXTREMES FOR PERIOD OF RECORD.--HIGHEST WATER LEVEL, 4.80 FT (1.463M) BELOW LAND-SURFACE DATUM, MARCH 20, 1975; LOMEST, 16.95 FT (5.166M) BELOW LAND-SURFACE DATUM, JUNE 10, 1972. WATER LEVEL.S IN FEET BELOW LAND SURFACE DATUM, CLIMATIC YEAR APRIL 1982 TO MARCH 1983

$\begin{array}{ll}\text { WATER } \\ \text { DATE } & \text { LEVEL }\end{array}$

MAR 02, $1983 \quad 5.49$

GROUND-WATER LEVELS

BEAVER COUNTY

363853100311001. LOCAL NUMBER, 02N-24E-07 CCD 1.

LOCATION.--LAT 36 38'53", LONG $10031^{\prime} 10^{\prime \prime}$, HYOROLOGIC UNIT 11100201, OWNER: JAMES W. PARKER. AQUIFER.--OGALLALA FORMATION.

WELL. CHARACTERISTICS.--DRILLED STOCK WELL, DIAMETER 6 IN (0.15M), DEPTH 94 FT (28.7M).

DATUM.--ALTITUDE OF LAND-SURFACE DATUM IS $2625 \mathrm{FT}(800 \mathrm{M})$. MEASURING POINT: HIGHEST POINT ON NORTH SIDE OF CASING $0.50 \mathrm{FT}(0.15 \mathrm{M})$ ABOVE LAND-SURFACE DATUM.

PERIOD OF RECORD.--1946, 1967 TO CURRENT YEAR.

EXTREMES FOR PERIOD OF RECORD.--HIGHEST WATER LEVEL, 71.37 FT (21.754M) BELOW LAND-SURFACE DATUM, JAN. 24, 1979; LOWEST, 81.35 FT (24.795M) BELOW LAND-SURFACE DATUM, MARCH 1, 1978.

WATER LEVELS IN FEET BELOW LAND SURFACE DATUM, CLIMATIC YEAR APRIL 1982 TO MARCH 1983

\begin{tabular}{|c|c|c|c|c|c|}
\hline & WATER & & MATER & & $\begin{array}{l}\text { WATER } \\
\text { LEVEL }\end{array}$ \\
\hline DATE & LEVEL & DATE & LEVELL. & DATE & LEVEL \\
\hline $\begin{array}{l}15, \quad 1982 \\
14\end{array}$ & $\begin{array}{l}76.89 \\
76.75\end{array}$ & ОСТ 07,1982 & 76.33 & JAN 26, 1983 & 76.61 \\
\hline
\end{tabular}


GROUND-WATER LEVELS

BLAINE COUNTY

354412098172701. LOCAL NUMBER, 15N-10W-32 BAC 1.

LOCATION.--LAT 3S 44'12''. LONG 098 17'27''. HYDROLOGIC UNIT 11100301, OWNER: CLARENCE HODGES.

AQUIFER.--TERRACE, HIGH, DEPOSTIS.

WELL CHARACTERISTICS.--DRILLED WELL, DIAMETER 5 IN. $(0.15 \mathrm{M})$, DEPTH $51.5 \mathrm{FT}(15.7 \mathrm{M})$.

DATUM.--ALTITUDE OF LAND-SURFACE DATUM $1465 \mathrm{FT}(447 \mathrm{M})$.

MEASURING POINT: TOP OF CASING $1.20 \mathrm{FT}(0.366 \mathrm{M})$ ABOVE LAND-SURFACE DATUM.

PERIOD OF RECORD..- 1980 TO CURRENT YEAR.

EXTREMES FOR PERIOD OF RECORD.-- HIGHEST WATER LEVEL, 16.44 FT (5.010 M) BELOW

LAND-SURFACE DATUM, JUNE 6, 1980; LOWEST, 20.10 FT (6.130 M) BELOW LAND-SURFACE

DATUM, OCT. $28,1980$.

WATER LEVEL, IN FEET BELOW LAND SURFACE DATUM, CLIMATIC YEAR APRIL 1982 TO MARCH 1983 MEAN VALUES

\begin{tabular}{|c|c|c|c|c|c|c|c|c|c|c|c|c|}
\hline DAY & APR & MAY & JUN & JUL & AUG & SEP & OCT & NOV & $\mathrm{DEC}$ & JAN & FEB & MAR \\
\hline $\begin{array}{l}1 \\
2 \\
3 \\
4 \\
5\end{array}$ & $\begin{array}{l}19.17 \\
19.16 \\
19.23 \\
19.15 \\
19.22\end{array}$ & $\begin{array}{l}19.21 \\
19.20 \\
19.18 \\
19.17 \\
19.16\end{array}$ & $\begin{array}{l}16.84 \\
16.73 \\
16.63 \\
16.55 \\
16.47\end{array}$ & $\begin{array}{l}17.10 \\
17.11 \\
17.13 \\
17.15 \\
17.16\end{array}$ & $\begin{array}{l}17.64 \\
17.66 \\
17.70 \\
17.73 \\
17.76\end{array}$ & $\begin{array}{l}-- \\
-\cdots \\
-- \\
--\end{array}$ & $\begin{array}{l}18.68 \\
18.71 \\
18.69 \\
18.71 \\
18.73\end{array}$ & $\begin{array}{l}18.96 \\
19.02 \\
19.00 \\
18.97 \\
18.92\end{array}$ & $\begin{array}{l}18.76 \\
18.81 \\
18.81 \\
18.77 \\
18.85\end{array}$ & $\begin{array}{l}18.77 \\
18.78 \\
18.75 \\
18.73 \\
18.72\end{array}$ & $\begin{array}{l}18.61 \\
18.66 \\
18.71 \\
18.69 \\
18.64\end{array}$ & $\begin{array}{l}18.52 \\
18.51 \\
18.48 \\
18.45 \\
18.44\end{array}$ \\
\hline $\begin{array}{r}6 \\
7 \\
8 \\
9 \\
10\end{array}$ & $\begin{array}{l}19.23 \\
19.15 \\
19.19 \\
19.21 \\
19.21\end{array}$ & $\begin{array}{l}19.19 \\
19.19 \\
19.18 \\
19.18 \\
19.17\end{array}$ & $\begin{array}{l}16.44 \\
16.45 \\
16.45 \\
16.53 \\
16.58\end{array}$ & $\begin{array}{l}17.20 \\
17.25 \\
17.24 \\
17.22 \\
17.28\end{array}$ & $\begin{array}{l}17.78 \\
17.80 \\
17.81 \\
17.83 \\
17.85\end{array}$ & $\begin{array}{r}-.- \\
18.46 \\
18.47 \\
18.49\end{array}$ & $\begin{array}{l}18.72 \\
18.66 \\
18.74 \\
18.78 \\
18.78\end{array}$ & $\begin{array}{l}18.90 \\
18.92 \\
18.93 \\
18.93 \\
18.87\end{array}$ & $\begin{array}{l}18.84 \\
18.90 \\
18.93 \\
18.81 \\
18.80\end{array}$ & $\begin{array}{l}18.71 \\
18.70 \\
18.68 \\
18.68 \\
18.71\end{array}$ & $\begin{array}{l}18.67 \\
18.63 \\
18.61 \\
18.61 \\
18.61\end{array}$ & $\begin{array}{l}18.52 \\
18.56 \\
18.58 \\
18.61 \\
18.60\end{array}$ \\
\hline $\begin{array}{l}11 \\
12 \\
13 \\
14 \\
15\end{array}$ & $\begin{array}{l}19.17 \\
19.13 \\
19.18 \\
19.15 \\
19.12\end{array}$ & $\begin{array}{l}19.17 \\
19.15 \\
19.13 \\
19.12 \\
19.11\end{array}$ & $\begin{array}{l}16.58 \\
16.64 \\
16.68 \\
16.67 \\
16.74\end{array}$ & $\begin{array}{l}17.31 \\
17.32 \\
17.32 \\
17.33 \\
17.34\end{array}$ & $\begin{array}{l}17.87 \\
17.88 \\
17.89 \\
17.92 \\
17.95\end{array}$ & $\begin{array}{l}18.50 \\
18.52 \\
18.55 \\
18.58 \\
18.55\end{array}$ & $\begin{array}{l}18.80 \\
18.92 \\
18.93 \\
18.91 \\
18.95\end{array}$ & $\begin{array}{l}18.93 \\
18.99 \\
18.92 \\
18.98 \\
18.87\end{array}$ & $\begin{array}{l}18.84 \\
18.77 \\
18.71 \\
18.79 \\
18.82\end{array}$ & $\begin{array}{l}18.78 \\
18.72 \\
18.69 \\
18.77 \\
18.73\end{array}$ & $\begin{array}{l}18.66 \\
18.67 \\
18.60 \\
18.57 \\
18.61\end{array}$ & $\begin{array}{r}18.61 \\
\ldots \\
- \\
- \\
- \\
--\end{array}$ \\
\hline $\begin{array}{l}16 \\
17 \\
18 \\
19 \\
20\end{array}$ & $\begin{array}{l}19.18 \\
19.23 \\
19.18 \\
19.17 \\
19.23\end{array}$ & $\begin{array}{l}19.07 \\
18.67 \\
18.22 \\
17.84 \\
17.53\end{array}$ & $\begin{array}{l}16.81 \\
16.82 \\
16.87 \\
16.93 \\
16.92\end{array}$ & $\begin{array}{l}17.35 \\
17.38 \\
17.39 \\
17.46 \\
17.48\end{array}$ & $\begin{array}{l}18.00 \\
18.03 \\
18.05 \\
18.07 \\
18.10\end{array}$ & $\begin{array}{l}18.55 \\
18.59 \\
18.57 \\
18.61 \\
18.63\end{array}$ & $\begin{array}{l}18.90 \\
18.88 \\
18.92 \\
18.99 \\
18.96\end{array}$ & $\begin{array}{l}18.87 \\
18.85 \\
18.82 \\
18.82 \\
18.89\end{array}$ & $\begin{array}{l}18.79 \\
18.71 \\
18.78 \\
18.76 \\
18.74\end{array}$ & $\begin{array}{l}18.68 \\
18.70 \\
18.70 \\
18.68 \\
18.66\end{array}$ & $\begin{array}{l}18.60 \\
18.60 \\
18.57 \\
18.52 \\
18.59\end{array}$ & $\begin{array}{l}--- \\
--- \\
--- \\
--- \\
---\end{array}$ \\
\hline $\begin{array}{l}21 \\
22 \\
23 \\
24 \\
25\end{array}$ & $\begin{array}{l}19.24 \\
19.24 \\
19.21 \\
19.16 \\
19.15\end{array}$ & $\begin{array}{l}17.36 \\
17.25 \\
17.17 \\
17.07 \\
17.02\end{array}$ & $\begin{array}{l}16.95 \\
16.99 \\
17.01 \\
17.03 \\
17.02\end{array}$ & $\begin{array}{l}17.49 \\
17.50 \\
17.52 \\
17.53 \\
17.55\end{array}$ & $\begin{array}{r}18.11 \\
18.10 \\
18.12 \\
--- \\
---\end{array}$ & $\begin{array}{l}18.60 \\
18.57 \\
18.62 \\
18.64 \\
18.60\end{array}$ & $\begin{array}{l}18.97 \\
18.96 \\
18.96 \\
18.96 \\
18.95\end{array}$ & $\begin{array}{l}18.85 \\
18.87 \\
18.97 \\
18.92 \\
18.88\end{array}$ & $\begin{array}{l}18.70 \\
18.68 \\
18.65 \\
18.72 \\
18.83\end{array}$ & $\begin{array}{l}18.66 \\
18.66 \\
18.66 \\
18.69 \\
18.66\end{array}$ & $\begin{array}{l}18.60 \\
18.59 \\
18.56 \\
18.58 \\
18.59\end{array}$ & $\begin{array}{l}--- \\
--- \\
--- \\
--- \\
--\end{array}$ \\
\hline $\begin{array}{l}26 \\
27 \\
28 \\
29 \\
30 \\
31\end{array}$ & $\begin{array}{r}19.20 \\
19.20 \\
19.18 \\
19.19 \\
19.21 \\
-.-\end{array}$ & $\begin{array}{l}17.00 \\
17.02 \\
17.01 \\
17.03 \\
17.04 \\
17.05\end{array}$ & $\begin{array}{r}17.01 \\
17.02 \\
17.02 \\
17.04 \\
17.08 \\
\ldots\end{array}$ & $\begin{array}{l}17.57 \\
17.58 \\
17.60 \\
17.61 \\
17.64 \\
17.65\end{array}$ & $\begin{array}{l}-- \\
--- \\
-- \\
--- \\
-\cdots \\
-\ldots\end{array}$ & $\begin{array}{r}18.57 \\
18.62 \\
18.67 \\
18.69 \\
18.68 \\
\ldots . .\end{array}$ & $\begin{array}{l}18.90 \\
18.91 \\
18.97 \\
18.92 \\
18.93 \\
18.92\end{array}$ & $\begin{array}{r}18.87 \\
18.78 \\
18.77 \\
18.77 \\
18.76 \\
-\end{array}$ & $\begin{array}{l}18.74 \\
18.72 \\
18.82 \\
18.79 \\
18.77 \\
18.78\end{array}$ & $\begin{array}{l}18.71 \\
18.67 \\
18.62 \\
18.67 \\
18.65 \\
18.62\end{array}$ & $\begin{array}{r}18.58 \\
18.55 \\
18.53 \\
-.- \\
-.-\end{array}$ & $\begin{array}{l}--- \\
--- \\
--- \\
--- \\
--- \\
---\end{array}$ \\
\hline
\end{tabular}




\section{GROUND-WATER LEVELS}

CANADIAN COUNTY

353107097453701. LOCAL NUMBER, 12N-05W-18 ADA 1.

LOCATION.--LAT $3531^{\prime} 07^{\prime \prime}$. LONG $09745^{\prime} 37^{\prime \prime}$ ', HYDROLOGIN UNIT 11100301, OWNER: CITY OF YUKON.

AQUIFER.--TERRACE, LOW, DEPOSITS.

WELL CHARACTERISTICS.--DRILLED ABANDONED CITY WELL, DIAMETER 12 IN. (0.30 M), DEPTH 47.2 FT(14.4 M).

DATUM.--ALTITUDE OF LAND-SURFACE DATUM $1270 \mathrm{FT}(387 \mathrm{M})$. MEASURING POINT: TOP WEST EDGE OF CASING

1.30 FT (0.396 M) ABOVE LAND-SURFACE DATUM.

PERIOD OF RECORD.--1980 TO CURRENT YEAR.

EXTREMES FOR PERIOD OF RECORD.-- HIGHEST WATER LEVEL, 7.98 FT )2.430M) BELOW

LAND-SURFACE DATUM, MAY 30, 1980; LOWEST, $13.41 \mathrm{FT}(4.090 \mathrm{M})$ BELOW LAND-SURFACE

DATUM, DEC. $19,1980$.

WATER LEVEL, IN FEET BELOW LAND SURFACE DATUM, CLIMATIC YEAR APRIL 1982 TO MARCH 1983

MEAN VALUES

\begin{tabular}{|c|c|c|c|c|c|c|c|c|c|c|c|c|}
\hline DAY & APR & MAY & JUN & JUL & AUG & SEP & OCT & NOV & DEC & JAN & FEB & MAR \\
\hline $\begin{array}{l}1 \\
2 \\
3 \\
4 \\
3\end{array}$ & $\begin{array}{l}11.21 \\
11.18 \\
11.31 \\
11.20 \\
11.31\end{array}$ & $\begin{array}{l}11.57 \\
11.54 \\
11.53 \\
11.53 \\
11.54\end{array}$ & $\begin{array}{l}9.00 \\
8.89 \\
8.78 \\
8.77 \\
8.70\end{array}$ & $\begin{array}{l}8.51 \\
8.52 \\
8.55 \\
8.59 \\
8.62\end{array}$ & $\begin{array}{l}9.32 \\
9.36 \\
9.42 \\
9.46 \\
9.50\end{array}$ & $\begin{array}{l}10.53 \\
10.58 \\
10.63 \\
10.64 \\
10.66\end{array}$ & $\begin{array}{l}11.38 \\
11.40 \\
11.44 \\
11.44 \\
11.47\end{array}$ & $\begin{array}{l}11.88 \\
11.93 \\
11.96 \\
11.95 \\
11.94\end{array}$ & $\begin{array}{l}11.98 \\
12.03 \\
12.06 \\
12.04 \\
12.05\end{array}$ & $\begin{array}{l}--- \\
--- \\
--- \\
---\end{array}$ & $\begin{array}{l}11.85 \\
11.88 \\
11.94 \\
11.93 \\
11.84\end{array}$ & $\begin{array}{l}-- \\
-\cdots \\
--- \\
--\end{array}$ \\
\hline $\begin{array}{r}6 \\
7 \\
8 \\
9 \\
10\end{array}$ & $\begin{array}{l}11.34 \\
11.23 \\
11.30 \\
11.34 \\
11.35\end{array}$ & $\begin{array}{l}11.45 \\
11.47 \\
11.49 \\
11.51 \\
11.52\end{array}$ & $\begin{array}{l}8.68 \\
8.68 \\
8.65 \\
8.69 \\
8.72\end{array}$ & $\begin{array}{l}8.66 \\
8.58 \\
8.56 \\
8.53 \\
8.57\end{array}$ & $\begin{array}{l}9.52 \\
9.55 \\
9.58 \\
9.60 \\
9.64\end{array}$ & $\begin{array}{l}10.70 \\
10.74 \\
10.76 \\
10.78 \\
10.80\end{array}$ & $\begin{array}{l}11.51 \\
11.51 \\
11.49 \\
11.60 \\
11.63\end{array}$ & $\begin{array}{l}11.91 \\
11.94 \\
11.98 \\
11.99 \\
11.98\end{array}$ & $\begin{array}{r}12.12 \\
\ldots \\
- \\
- \\
- \\
-\end{array}$ & $\begin{array}{l}12.06 \\
12.06 \\
12.03 \\
12.08\end{array}$ & $\begin{array}{l}11.86 \\
11.82 \\
11.77 \\
11.74 \\
11.72\end{array}$ & $\begin{array}{l}--- \\
--- \\
-\cdots \\
-- \\
-\ldots\end{array}$ \\
\hline $\begin{array}{l}11 \\
12 \\
13 \\
14 \\
15\end{array}$ & $\begin{array}{l}11.30 \\
11.25 \\
11.35 \\
11.34 \\
11.33\end{array}$ & $\begin{array}{r}11.53 \\
10.99 \\
10.60 \\
10.81 \\
10.92\end{array}$ & $\begin{array}{l}8.66 \\
8.65 \\
8.65 \\
8.61 \\
8.62\end{array}$ & $\begin{array}{l}8.61 \\
8.62 \\
8.62 \\
8.63 \\
8.66\end{array}$ & $\begin{array}{l}9.68 \\
9.70 \\
9.73 \\
9.78 \\
9.84\end{array}$ & $\begin{array}{l}10.84 \\
10.88 \\
10.94 \\
10.97 \\
10.99\end{array}$ & $\begin{array}{l}11.63 \\
11.62 \\
11.64 \\
11.65 \\
11.64\end{array}$ & $\begin{array}{l}11.90 \\
12.07 \\
12.00 \\
12.05 \\
12.01\end{array}$ & $\begin{array}{l}--- \\
--- \\
--- \\
--- \\
---\end{array}$ & $\begin{array}{l}12.14 \\
12.13 \\
12.08 \\
12.15 \\
12.15\end{array}$ & $\begin{array}{l}11.73 \\
11.75 \\
11.66 \\
11.60 \\
11.64\end{array}$ & $\begin{array}{l}--- \\
--- \\
--- \\
-- \\
-\ldots\end{array}$ \\
\hline $\begin{array}{l}16 \\
17 \\
18 \\
19 \\
20\end{array}$ & $\begin{array}{l}11.43 \\
11.53 \\
11.45 \\
11.46 \\
11.58\end{array}$ & $\begin{array}{r}10.91 \\
9.68 \\
9.89 \\
10.08 \\
10.03\end{array}$ & $\begin{array}{l}8.67 \\
8.65 \\
8.67 \\
8.68 \\
8.64\end{array}$ & $\begin{array}{l}8.69 \\
8.74 \\
8.79 \\
8.83 \\
8.90\end{array}$ & $\begin{array}{r}9.91 \\
9.94 \\
9.97 \\
10.02 \\
10.07\end{array}$ & $\begin{array}{l}10.96 \\
10.97 \\
11.02 \\
11.00 \\
11.05\end{array}$ & $\begin{array}{l}11.69 \\
11.65 \\
11.65 \\
11.73 \\
11.77\end{array}$ & $\begin{array}{l}11.98 \\
11.99 \\
11.99 \\
11.98 \\
12.06\end{array}$ & $\begin{array}{l}--- \\
--- \\
--- \\
--- \\
---\end{array}$ & $\begin{array}{l}12.07 \\
12.10 \\
12.12 \\
12.09 \\
12.07\end{array}$ & $\begin{array}{r}11.61 \\
11.60 \\
11.57 \\
.-- \\
.--\end{array}$ & $\begin{array}{l}--- \\
--- \\
--- \\
--\end{array}$ \\
\hline $\begin{array}{l}21 \\
22 \\
23 \\
24 \\
23\end{array}$ & $\begin{array}{l}11.61 \\
11.61 \\
11.59 \\
11.54 \\
11.51\end{array}$ & $\begin{array}{r}10.05 \\
10.07 \\
9.97 \\
9.43 \\
9.33\end{array}$ & $\begin{array}{l}8.63 \\
8.66 \\
8.65 \\
8.64 \\
8.60\end{array}$ & $\begin{array}{l}8.96 \\
9.01 \\
9.05 \\
9.09 \\
9.13\end{array}$ & $\begin{array}{l}10.09 \\
10.10 \\
10.15 \\
10.20 \\
10.27\end{array}$ & $\begin{array}{l}11.07 \\
11.04 \\
11.04 \\
11.16 \\
11.26\end{array}$ & $\begin{array}{l}11.76 \\
11.76 \\
11.77 \\
11.78 \\
11.79\end{array}$ & $\begin{array}{l}12.10 \\
12.06 \\
12.15 \\
12.15 \\
12.09\end{array}$ & $\begin{array}{l}--- \\
--- \\
--- \\
--- \\
---\end{array}$ & $\begin{array}{l}12.06 \\
12.06 \\
12.07 \\
12.10 \\
12.08\end{array}$ & $\begin{array}{l}--- \\
-- \\
--- \\
---\end{array}$ & $\begin{array}{l}--- \\
-- \\
-- \\
--\end{array}$ \\
\hline $\begin{array}{l}26 \\
27 \\
28 \\
29 \\
30 \\
31\end{array}$ & $\begin{array}{r}11.54 \\
11.58 \\
11.55 \\
11.57 \\
11.59 \\
=-.\end{array}$ & $\begin{array}{l}9.41 \\
9.45 \\
9.31 \\
9.33 \\
9.34 \\
9.09\end{array}$ & $\begin{array}{r}8.59 \\
8.58 \\
8.46 \\
8.46 \\
8.49 \\
. .-\end{array}$ & $\begin{array}{l}9.17 \\
9.21 \\
9.24 \\
9.27 \\
9.30 \\
9.31\end{array}$ & $\begin{array}{l}10.26 \\
10.33 \\
10.41 \\
10.42 \\
10.45 \\
10.50\end{array}$ & $\begin{array}{r}11.24 \\
11.24 \\
11.30 \\
11.35 \\
11.38 \\
\ldots--\end{array}$ & $\begin{array}{l}11.79 \\
11.77 \\
11.83 \\
11.84 \\
11.84 \\
11.87\end{array}$ & $\begin{array}{r}12.08 \\
12.00 \\
11.95 \\
11.97 \\
11.99 \\
\ldots\end{array}$ & $\begin{array}{l}--- \\
--- \\
--- \\
--- \\
--- \\
---\end{array}$ & $\begin{array}{l}12.08 \\
12.09 \\
12.00 \\
12.03 \\
12.05 \\
12.00\end{array}$ & $\begin{array}{l}--- \\
--- \\
--- \\
--- \\
--- \\
-\cdots\end{array}$ & $\begin{array}{l}--- \\
--- \\
--- \\
--- \\
--\end{array}$ \\
\hline
\end{tabular}


GROUND-WATER LEVELS

\section{CIMARRON COUNTY}

364450102190001. LOCAL NUMBER, 03N-07E-09 BBB 1.

LOCATION.--LAT 36 44'50", LONG 102 19'00", HYDROLOGIC UNIT 11100101, OWNER: ELMER J. BEHRENDT. AQUIFER. --OGALLALA FORMATION.

WELL CHARACTERISTICS.--DRILLED UNUSED STOCK WELL, DIAMETER 6 IN (0.15M), DEPTH 61 FT $(18.6 \mathrm{M})$. DATUM.--ALTITUDE OF LAND-SURFACE DATUM IS $3960 \mathrm{FT}$ (1207M). MEASURING POINT: TOP OF CASING ON SOUTH SIDE $0.50 \mathrm{FT}(0.15 \mathrm{M})$ ABOVE LAND-SURFACE DATUM.

PERIOD OF RECORD.-- 1938 TO CURRENT YEAR.

EXIREMES FOR PERIOD OF RECORD.-- HIGHEST WATER LEVEL, $28.50 \mathrm{FT}$ (8.687M) BELOW LAND-SURFACE DATUM, JAN. 12, 1977; LOWEST, 32.41 FT (9.879M) BELOW LAND-SURFACE DATUM, FEB. 131969.

WATER LEVELS IN FEET BELOW LAND SURFACE DATUM, CLIMATIC YEAR APRIL 1982 TO MARCH 1983

\begin{tabular}{|c|c|c|c|c|c|c|c|}
\hline DATE & $\begin{array}{l}\text { WATER } \\
\text { LEVEL }\end{array}$ & DATE & $\begin{array}{l}\text { WATER } \\
\text { LEVEL }\end{array}$ & DATE & $\begin{array}{l}\text { WATER } \\
\text { LEVEL }\end{array}$ & DATE & $\begin{array}{l}\text { WATER } \\
\text { LEVEL }\end{array}$ \\
\hline APR 14,1982 & 31.54 & JUL 13, 1982 & 31.50 & ОСТ 06, 1982 & 29.23 & JAN 25, 1983 & 29.40 \\
\hline
\end{tabular}

GROUND-WATER LEVELS

CLEVELAND COUNTY

350816097233101. LOCAL NUMBER, 08N-02W-27 ACD 1.

LOCATION.--LAT 35 08'16", LONG 097 23'31", HYDROLOGIC UNIT 11090202,

OWNER: TOWN OF NOBLE.

AOUIFER. --GARBER SANDSTONE.

WELL CHARACTERISTICS.--DRILLED UNUSED WELL, DIAMETER 12 IN (0.30M) REDUCED TO

8 IN (0.20M), DEPTH 461 FT (141M).

DATUM.--MEASURING POINT: TOP OF 1-IN (0.03M) PIPE CEMENTED OVER CASING $1.40 \mathrm{FT}$

(0.43M) ABOVE LAND-SURFACE DATUM.

REMARKS.--PERFORATIONS 235-245 FT (71.6M-74.7M) AND 415-455 FT (126M-139M).

PERIOD OF RECORD.--1943 TO CURRENT YEAR.

EXTREMLS FOR PERIOD OF RECORD...- HIGHEST WATER LEVEL, 165.43 FT (50.423M) BELOW

LAND-SURFACE DATUM, JULY 7, 1943; LOWEST, 221.74 FT (67.586M) BELOW LAND-SURFACE

DATUM, DEC. $23,1948$.

WATER LEVELS IN FEET BELOW LAND SURFACE DATUM, CLIMATIC YEAR APRIL 1982 TO MARCH 1983

\begin{tabular}{|c|c|c|c|c|c|c|c|}
\hline DATE & $\begin{array}{l}\text { WATER } \\
\text { LEVEL }\end{array}$ & DATE & $\begin{array}{l}\text { WATER } \\
\text { LEVEL }\end{array}$ & DATE & $\begin{array}{l}\text { WATER } \\
\text { LEVEL }\end{array}$ & DATE & $\begin{array}{l}\text { WATER } \\
\text { LEVEL }\end{array}$ \\
\hline 14,1982 & 205.74 & SEP 09, 1982 & 214.05 & DEC 01,1982 & 213.20 & FEB 22,1983 & 209.35 \\
\hline
\end{tabular}




\section{GROUND-WATER LEVELS}

COAL COUNTY

342619096144201. LOCAL NUMBER, 01S-10E-27 BAB 1.

LOCATION.--LAT 34 26'19" LONG 096 14'42", HYDROLOGIC UNIT 11140103,

OWNER: HENRY MANNING.

AQUIFER.--MCALESTER SANDSTONE.

WELL CHARACTERISTICS.--DRILLED WELL, DIAMETER 8 IN $(0.20 \mathrm{M})$, DEPTH 32 FT (9.75M).

DATUM.--ALTITUDE OF LAND-SURFACE DATUM IS $630 \mathrm{FT}$ (192M). MEASURING POINT: $2.50 \mathrm{FT}$

(0.76M) ABOVE LAND-SURFACE DATUM.

PERIOD OF RECORD.--MARCH 1978 TO CURRENT YEAR.

EXTREMES FOR PERIOD OF RECORD.--HIGHEST WATER LEVEL, 5.36 FT (1.63M) BELOW LAND.

SURFACE DATUM, NOV. 1, 1981; LOWEST, 13.47 FT (4.11M) BELOW LAND-SURFACE DATUM,

SEPT. $12,1980$.

WATER LEVEL, IN FEET BELOW LAND SURFACE DATUM, CLIMATIC YEAR APRIL 1982 TO MARCH 1983

MEAN VALUES

\begin{tabular}{|c|c|c|c|c|c|c|c|c|c|c|c|c|}
\hline DAY & APR & MAY & JUN & JUL & AUG & SEP & OCT & NOV & DEC & JAN & FEB & MAR \\
\hline 1 & --- & -- & -- & --- & --- & 7.19 & --- & --- & 8.98 & --- & -- & --- \\
\hline 2 & -- & $\ldots$ & --- & --- & --- & 7.21 & -- & 9.01 & 8.93 & --- & --- & --- \\
\hline 3 & --- & --- & --- & --- & --- & 7.25 &.-- & 9.02 & 8.88 & -- & --- & --- \\
\hline 4 & -- &.- & -- & --- & --- & 7.31 & --- & 9.05 & 8.84 & -- & -- & -- \\
\hline$s$ & --- & --- & -- & --- &.-- & 7.38 & --- & 9.07 & 8.79 & -- & --- & -- \\
\hline 6 & -- & -- & -- & -- & -- & 7.46 & -- & 9.07 & 8.77 & --- & - & -- \\
\hline 7 & --- & -- & -- & --- & --- & 7.53 & --- & 9.06 & 8.76 & -- & -- & --- \\
\hline 8 & --- & -- & --- & --- & --- & 7.59 & --- & 9.06 & 8.78 & --- & $\cdots$ & -- \\
\hline 9 & --- & --- & --- & --- & -- & --- & --- & 9.07 & 8.83 & --- & -- & $\cdots$ \\
\hline 10 & --- & $-\cdots$ & --- & --- & --- & --- & --- & 9.07 & --- & -- & -- & -- \\
\hline 11 & --- & -- & --- & --- & --- & -- & --- & 9.06 & -- & -- & -- & -- \\
\hline 12 & -- & -- & --- & --- & --- & -- & --- & 9.03 & --- & -- & -- & -- \\
\hline 13 & --- & -- & -- & --- & --- & -- & --- & 9.07 & --- & -- & -- & -- \\
\hline 14 & --- & --- & --- & --- & --- & --- & --- & 9.09 & --- & --- & --- & -- \\
\hline 15 & -- & --- & $-\cdot$ & -- & --- & $-\cdots$ & -- & 9.13 & -- & -- & -- & -- \\
\hline 16 & --- & --- &.-- & -- & --- & -- & -- & 9.13 & --- & --- &..- & -- \\
\hline 17 & --- & --- & --- & --- & --- & -- & --- & 9.11 & --- & --- & --- & $-\cdot$ \\
\hline 18 & --- & $\cdots$ & -- & -- & --- & --- & --- & 9.09 & --- & -- & -- & -- \\
\hline 19 & -- & --- & --- & --- & --- & --- & --- & 9.06 & --- & --- & -- & -- \\
\hline 20 & --- & -- & --- & -- & --- & -- & --- & 9.04 & --- & -- & $\cdots$ & -- \\
\hline 21 & --- & -- & -- & -- & -- & -- & $\cdots$ & 9.04 & -- & -- & -- & --- \\
\hline 22 & -- & -- & -- & -- & --- & -- & -- & 9.06 & -- & - & -- & -- \\
\hline 23 & --- & -- & --- & --- & -- & -- & -- & 9.07 & -- & -- & $-\cdot$ & --- \\
\hline 24 & --- & -- & --- & --- & $\cdots$ & --- & --- & 9.14 & --- & -- & --- & --- \\
\hline 25 & -- & -- & --- & -- & --- & --- & -- & 9.21 & --- & -- & $\cdots$ & --- \\
\hline 26 & -- & -- & --- & -- & 6.79 & --- & -- & 9.25 & -- & -- & -.- & -- \\
\hline 27 & $\cdots$ & -- & -- & --- & 6.88 & --- & --- & 9.21 & -- & -- & -- & - \\
\hline 28 & $\cdots$ & -- & $\cdots$ & -- & 6.94 & -- & -- & 9.12 & -- & -- & -- & - \\
\hline 29 & $\cdots$ & -- & $\ldots$ & --- & 7.02 & --- & -- & 9.07 & $\cdots$ & --- & $\cdots$ & $\cdots$ \\
\hline 30 & -- & -- & --- & --- & 7.09 & --- & -- & 9.02 & -- & -- & -- & $\ldots$ \\
\hline 31 & -- & -- & --- & --- & 7.15 & -- & -- & -- & $\cdots$ & -- & -- & \\
\hline
\end{tabular}




\section{GROUND-WATER LEVELS}

COMANCHE COUNTY

343540098342001. LOCAL NUMBER, 01N-13W-04 BAA 1.

LOCATION.--LAT 34 35'40", LONG 098 34'20", HYDROLOGIC UNIT 11130203, OWNER: U.S. GEOLOGICAL SURVEY. AQUIFER.--LOWER ARBUCKLE GROUP.

WELL CHARACTERISTICS.--TEST WELL, DIAMETER 6 IN (0.15M), DEPTH 997 FT (304M).

DATUM.--ALTITUDE OF LAND-SURFACE DATUM IS $1191 \mathrm{FT}$ (363M). MEASURING POINT: TOP OF CASING $1.8 \mathrm{FT}$

(0.55M) ABOVE LAND-SURFACE DATUM.

PERIOD OF RECORD.-- 1972 TO CURRENT YEAR.

EXTREMES FOR PERIOD OF RLCORD.--HIGHEST WATER LEVEL, 71.03 FT (21.650M) BELOW LAND-SURFACE

DATUM, SEPT. 25, 1974; LOWEST, 88.62 FT (27.011M) BELOW LAND-SURFACE DATUM,

MAY $10,1972$.

WATER LEVELS IN FEET BELOW LAND SURFACE DATUM, CLIMATIC YEAR APRIL 1982 TO MARCH 1983

\begin{tabular}{|c|c|c|c|c|c|c|c|c|}
\hline DATE & $\begin{array}{l}\text { WATER } \\
\text { LEVEL }\end{array}$ & DATE & $\begin{array}{l}\text { WATER } \\
\text { LEVEL }\end{array}$ & & DATE & $\begin{array}{l}\text { WATER } \\
\text { LEVEL }\end{array}$ & DATE & $\begin{array}{l}\text { WATER } \\
\text { LEVEL }\end{array}$ \\
\hline $\begin{array}{l}03,1982 \\
03\end{array}$ & $\begin{array}{l}76.52 \\
76.47\end{array}$ & SEP 30,1982 & 77.19 & OCT & 15, 1982 & 76.64 & JAN 14, 1983 & 76.62 \\
\hline
\end{tabular}




\section{GROUND WATER LEVELS}

CRAIG COUNTY

364705095135302. LOCAL NUMBER, 27N-19E-25 CBB 2.

LOCATION.--LAT 3647 '05\%, LONG 09513 '53\%, HYDROLOGIC UNIT 11070209, OWNER: MOONEY. AQUIFER.--SENORA FORMATION.

WELL CHARACTERISTICS.--DRILLED DOMESTIC WELL, DIAMETER 6 IN. (0.15M). DEPTH 102 FR (31.1M). DATUM.--MEASURING POINT: TOP OF SHELTER FLOOR EAST SIDE $2.40 \mathrm{FT}$ (0.73M) ABOVE LAND-SURFACE

DATUM.

REMARKS.--WELL USED AS OBSERVATION WELL ONLY.

PERIOD OF RECORD.--1980 TO CURRENT YEAR.

EXTREMES FOR PERIOD OF RECORD.--HIGHEST WATER LEVEL, 2.19 FT (0.668M) BELOW LAND-SURFACE DATUM, APR. 27, 1980; LOWEST WATER LEVEL, 27.20 FT (8.290M) BELOW LAND-SURFACE DATUM, OCT. 27, 1980. WATER LEVEL, IN FEET BELOW LAND SURFACE DATUM, CLIMATIC YEAR APRIL 1982 TO MARCH 1983 MEAN VALUES

\begin{tabular}{|c|c|c|c|c|c|c|c|c|c|c|c|c|}
\hline DAY & APR & MAY & JUN & JUL & AUG & SEP & OCT & NOV & DEC & JAN & FEB & MAR \\
\hline $\begin{array}{l}1 \\
2 \\
3 \\
4 \\
5\end{array}$ & $\begin{array}{r}10.15 \\
9.74 \\
9.74 \\
9.92 \\
10.03\end{array}$ & $\begin{array}{l}10.73 \\
10.77 \\
10.78 \\
10.76 \\
10.74\end{array}$ & $\begin{array}{l}5.73 \\
6.34 \\
5.66 \\
3.80 \\
4.03\end{array}$ & $\begin{array}{l}--- \\
9.56 \\
9.73 \\
9.90 \\
9.95\end{array}$ & $\begin{array}{l}-\cdots \\
-\cdots \\
-\cdots \\
---\end{array}$ & $\begin{array}{l}11.44 \\
11.45 \\
11.52 \\
11.54 \\
11.56\end{array}$ & $\begin{array}{l}13.41 \\
13.55 \\
13.69 \\
13.91 \\
14.22\end{array}$ & $\begin{array}{l}22.96 \\
23.00 \\
23.12 \\
23.30 \\
23.46\end{array}$ & $\begin{array}{l}25.01 \\
24.89 \\
24.74 \\
24.65 \\
24.64\end{array}$ & $\begin{array}{l}9.84 \\
9.84 \\
9.84 \\
9.84 \\
9.84\end{array}$ & $\begin{array}{l}--- \\
--- \\
--- \\
--- \\
--\end{array}$ & $\begin{array}{l}--- \\
-- \\
--- \\
---\end{array}$ \\
\hline $\begin{array}{r}6 \\
7 \\
8 \\
9 \\
10\end{array}$ & $\begin{array}{l}10.47 \\
10.39 \\
10.36 \\
10.55 \\
10.64\end{array}$ & $\begin{array}{l}10.33 \\
10.21 \\
10.44 \\
10.55 \\
10.64\end{array}$ & $\begin{array}{l}4.49 \\
5.50 \\
6.59 \\
7.63 \\
8.58\end{array}$ & $\begin{array}{r}9.99 \\
10.14 \\
10.19 \\
10.14 \\
10.16\end{array}$ & $\begin{array}{l}--- \\
--- \\
--- \\
---\end{array}$ & $\begin{array}{l}11.59 \\
11.62 \\
11.63 \\
11.64 \\
11.63\end{array}$ & $\begin{array}{l}14.64 \\
15.10 \\
15.40 \\
15.71 \\
16.25\end{array}$ & $\begin{array}{l}23.58 \\
23.65 \\
23.72 \\
23.82 \\
23.92\end{array}$ & $\begin{array}{l}24.64 \\
24.64 \\
19.49 \\
13.90 \\
13.90\end{array}$ & $\begin{array}{c}9.84 \\
--- \\
--- \\
--- \\
---\end{array}$ & $\begin{array}{l}--- \\
--- \\
--- \\
--- \\
--\end{array}$ & $\begin{array}{l}--- \\
--- \\
--- \\
--- \\
--\end{array}$ \\
\hline $\begin{array}{l}11 \\
12 \\
13 \\
14 \\
15\end{array}$ & $\begin{array}{l}10.63 \\
10.46 \\
10.55 \\
10.65 \\
10.56\end{array}$ & $\begin{array}{r}10.70 \\
6.85 \\
4.77 \\
2.93 \\
3.04\end{array}$ & $\begin{array}{l}8.54 \\
8.46 \\
8.80 \\
9.03 \\
8.53\end{array}$ & $\begin{array}{r}10.34 \\
10.44 \\
10.53 \\
10.85 \\
\ldots\end{array}$ & $\begin{array}{l}--- \\
--- \\
--- \\
---\end{array}$ & $\begin{array}{l}11.64 \\
11.66 \\
11.70 \\
11.75 \\
11.83\end{array}$ & $\begin{array}{l}16.90 \\
17.50 \\
18.03 \\
18.54 \\
18.93\end{array}$ & $\begin{array}{l}23.98 \\
24.00 \\
24.13 \\
24.24 \\
24.37\end{array}$ & $\begin{array}{l}13.90 \\
13.90 \\
13.88 \\
13.85 \\
13.87\end{array}$ & $\begin{array}{l}--- \\
-- \\
-- \\
-- \\
--\end{array}$ & $\begin{array}{l}--- \\
--- \\
--- \\
---\end{array}$ & $\begin{array}{l}--- \\
--- \\
--- \\
---\end{array}$ \\
\hline $\begin{array}{l}16 \\
17 \\
18 \\
19 \\
20\end{array}$ & $\begin{array}{l}10.54 \\
10.81 \\
10.78 \\
10.67 \\
10.81\end{array}$ & $\begin{array}{l}3.71 \\
4.14 \\
4.91 \\
6.13 \\
6.98\end{array}$ & $\begin{array}{c}8.02 \\
\ldots-- \\
--. \\
---\end{array}$ & $\begin{array}{l}-- \\
-- \\
-- \\
-- \\
--\end{array}$ & $\begin{array}{r}--- \\
11.24 \\
11.21 \\
11.32 \\
11.34\end{array}$ & $\begin{array}{l}11.91 \\
11.92 \\
12.00 \\
12.07 \\
12.14\end{array}$ & $\begin{array}{l}19.31 \\
19.70 \\
20.00 \\
20.24 \\
20.55\end{array}$ & $\begin{array}{l}24.46 \\
24.51 \\
24.56 \\
24.58 \\
24.58\end{array}$ & $\begin{array}{l}13.91 \\
13.91 \\
13.92 \\
13.92 \\
13.92\end{array}$ & $\begin{array}{l}--. \\
-- \\
-- \\
--\end{array}$ & $\begin{array}{l}--- \\
--- \\
--- \\
--- \\
--\end{array}$ & $\begin{array}{l}-- \\
- \\
- \\
- \\
--\end{array}$ \\
\hline $\begin{array}{l}21 \\
22 \\
23 \\
24 \\
25\end{array}$ & $\begin{array}{l}10.92 \\
10.93 \\
10.91 \\
10.81 \\
10.74\end{array}$ & $\begin{array}{l}7.81 \\
8.58 \\
8.95 \\
8.85 \\
7.89\end{array}$ & $\begin{array}{l}--- \\
--- \\
--- \\
---\end{array}$ & $\begin{array}{l}--- \\
--- \\
--- \\
--- \\
--\end{array}$ & $\begin{array}{l}11.36 \\
11.35 \\
11.35 \\
11.36 \\
11.40\end{array}$ & $\begin{array}{l}12.27 \\
12.40 \\
12.48 \\
12.57 \\
12.70\end{array}$ & $\begin{array}{l}20.92 \\
21.27 \\
21.59 \\
21.87 \\
22.11\end{array}$ & $\begin{array}{l}24.62 \\
24.69 \\
24.74 \\
24.86 \\
24.98\end{array}$ & $\begin{array}{l}13.92 \\
13.92 \\
13.92 \\
13.85 \\
13.84\end{array}$ & $\begin{array}{l}-.- \\
-- \\
-- \\
--\end{array}$ & $\begin{array}{l}-- \\
-- \\
-- \\
-- \\
--\end{array}$ & $\begin{array}{l}--- \\
--- \\
-- \\
--\end{array}$ \\
\hline $\begin{array}{l}26 \\
27 \\
28 \\
29 \\
30 \\
31\end{array}$ & $\begin{array}{c}10.68 \\
10.75 \\
10.70 \\
10.47 \\
10.62 \\
---\end{array}$ & $\begin{array}{l}8.18 \\
8.17 \\
5.30 \\
5.95 \\
6.19 \\
4.74\end{array}$ & $\begin{array}{l}--- \\
-- \\
-- \\
--- \\
---\end{array}$ & $\begin{array}{l}--- \\
--- \\
-\ldots \\
-\ldots \\
-\ldots \\
-\ldots\end{array}$ & $\begin{array}{l}11.38 \\
11.36 \\
11.40 \\
11.42 \\
11.42 \\
11.43\end{array}$ & $\begin{array}{r}12.82 \\
12.90 \\
12.98 \\
13.11 \\
13.25 \\
.--\end{array}$ & $\begin{array}{l}22.33 \\
22.50 \\
22.61 \\
22.74 \\
22.86 \\
22.93\end{array}$ & $\begin{array}{r}25.05 \\
25.10 \\
25.10 \\
25.09 \\
25.08 \\
.\end{array}$ & $\begin{array}{r}13.98 \\
13.64 \\
10.63 \\
9.82 \\
9.84 \\
9.84\end{array}$ & $\begin{array}{l}--- \\
--- \\
--- \\
--- \\
---\end{array}$ & $\begin{array}{l}-- \\
--- \\
-- \\
-- \\
--\end{array}$ & $\begin{array}{l}-- \\
-- \\
-- \\
-- \\
-- \\
--\end{array}$ \\
\hline
\end{tabular}


GROUND WATER LEVELS

CREEK COUNTY

355510096293501 . LOCAL NUMBER, 17N-08E-30 CBB 1.

LOCATION.--LAT 35 55'10", LONG 096 29'35", HYDROLOGIC UNIT 11100303, OWNER: EVERETT MATHERLY. AQUIFER.--VAMOOSA FORMATION.

WELL CHARACTERISTICS.--DRILLED UNUSED STOCK WELL, DIAMETER 6 IN $(0.15 M)$, DEPTH 58 FT $(17.7 M)$. DATUM.--ALTITUDE OF LAND-SURFACE DATUM IS $960 \mathrm{FT}(293 \mathrm{M})$. MEASURING POINT: BASE OF RECORDER SHELTER $1.00 \mathrm{FT}(0.30 \mathrm{M})$ ABOVE LAND-SURFACE DATUM.

REMARKS.--RECORDER SITL.

PERIOD OF RECORD.-- 1969 TO CURRENT YEAR.

EXTREMES FOR PERIOD OF RECORD.--HIGHEST WATER LEVEL, 34.30 FT (10.455M) BELOW LAND-SURFACE

DATUM, JUNE 5, 1975; LOWEST, 42.77 FT (13.036M) BELOW LAND-SURFACE DATUM, MAY 12, 1970.

WATER LEVEL, IN FEET BELOW LAND SURFACE DATUM, CLIMATIC YEAR APRIL 1982 TO MARCH 1983

MEAN VALUES

\begin{tabular}{|c|c|c|c|c|c|c|c|c|c|c|c|c|}
\hline DAY & APR & MAY & JUN & JUL & AUG & SEP & OCT & NOV & DEC & JAN & FEB & MAR \\
\hline 1 & 39.38 & 39.80 & 38.77 & 38.12 & 38.24 & 38.68 & 39.10 & 39.23 & 39.33 & 40.07 & 39.60 & 39.66 \\
\hline 2 & 38.99 & 39.80 & 38.56 & 38.06 & 38.20 & 38.74 & 39.00 & 39.45 & 39.48 & 40.11 & 39.83 & 39.64 \\
\hline 3 & 39.56 & 39.80 & 38.53 & 38.02 & 38.23 & 38.90 & 39.12 & 39.77 & 39.65 & 40.16 & 40.25 & 39.51 \\
\hline 4 & 39.22 & 39.80 & 38.63 & 38.01 & 38.29 & 38.86 & 39.07 & 39.75 & 39.63 & 40.00 & 40.34 & 39.23 \\
\hline 5 & 39.42 & 39.80 & 38.57 & 37.97 & 38.35 & 38.82 & 39.08 & 39.68 & 39.61 & 39.23 & 39.99 & 39.13 \\
\hline 6 & 39.81 & 39.80 & 38.44 & 38.00 & 38.32 & 38.87 & 39.14 & 39.45 & 39.97 & 39.87 & 40.10 & 39.40 \\
\hline 7 & 39.80 & 39.80 & 38.46 & 38.14 & 38.31 & 38.90 & 39.13 & 39.43 & 39.98 & 39.88 & 40.05 & 39.70 \\
\hline 8 & 39.80 & 39.72 & 38.37 & 38.10 & 38.38 & 38.89 & 38.87 & 39.53 & 40.36 & 39.88 & 39.88 & 39.83 \\
\hline 9 & 39.80 & 39.23 & 38.39 & 37.97 & 38.41 & 38.82 & 39.10 & 39.60 & 40.16 & 39.85 & 39.51 & 39.94 \\
\hline 10 & 39.80 & 39.14 & 38.62 & 38.05 & 38.42 & 38.69 & 39.34 & 39.56 & 39.86 & 39.87 & 37.83 & 39.94 \\
\hline 11 & 39.80 & 39.12 & 38.42 & 38.17 & 38.45 & 38.66 & 39.40 & 39.20 & 39.94 & 40.04 & 40.10 & 39.95 \\
\hline 12 & 39.80 & 39.04 & 38.43 & 38.21 & 38.41 & 38.68 & 39.34 & 39.77 & 39.96 & 40.22 & 40.29 & 39.82 \\
\hline 13 & 39.60 & 38.96 & 38.46 & 38.16 & 38.31 & 38.80 & 39.34 & 39.94 & 39.33 & 39.94 & 40.04 & 39.82 \\
\hline 14 & 39.24 & 39.01 & 38.25 & 38.10 & 38.32 & 38.90 & 39.40 & 39.91 & 39.54 & 40.19 & 39.83 & 39.82 \\
\hline 15 & 39.05 & 39.13 & 38.11 & 38.08 & 38.37 & 39.10 & 39.27 & 39.93 & 39.94 & 40.27 & 39.51 & 39.82 \\
\hline 16 & 39.20 & 39.13 & 38.30 & 38.06 & 38.51 & 39.03 & 39.48 & 39.50 & 39.99 & 39.93 & 39.92 & 39.82 \\
\hline 17 & 39.69 & 39.08 & 38.25 & 38.07 & 38.58 & 38.92 & 39.30 & 39.48 & 39.75 & 39.98 & 39.96 & 39.82 \\
\hline 18 & 39.80 & 39.01 & 38.22 & 38.08 & 38.59 & 39.03 & 39.18 & 39.45 & 39.60 & 40.12 & 39.90 & 39.82 \\
\hline 19 & 39.80 & 38.93 & 38.36 & 38.05 & 38.63 & 38.97 & 39.28 & 39.30 & 39.93 & 40.01 & 39.83 & 39.82 \\
\hline 20 & 39.80 & 38.85 & 38.27 & 38.11 & 38.68 & 39.10 & 39.65 & 39.42 & 39.86 & 39.91 & 39.87 & 39.82 \\
\hline 21 & 39.80 & 38.94 & 38.19 & 38.19 & 38.65 & 39.25 & 39.60 & 39.67 & 39.85 & 39.84 & 39.91 & 39.82 \\
\hline 22 & 39.80 & 38.98 & 38.23 & 38.23 & 38.52 & 39.14 & 39.60 & 39.50 & 39.85 & 39.85 & 39.87 & 39.81 \\
\hline 23 & 39.80 & 38.90 & 38.22 & 38.24 & 38.47 & 38.96 & 39.54 & 39.85 & 39.85 & 39.92 & 39.86 & 39.68 \\
\hline 24 & 39.80 & 38.76 & 38.14 & 38.23 & 38.50 & 39.00 & 39.54 & 40.18 & 39.85 & 40.05 & 39.88 & 39.62 \\
\hline 25 & 39.80 & 38.66 & 38.08 & 38.23 & 38.67 & 39.07 & 39.49 & 39.93 & 40.05 & 40.02 & 39.99 & 39.43 \\
\hline 26 & 39.80 & 38.59 & 38.04 & 38.24 & 38.50 & 38.92 & 39.42 & 39.89 & 40.11 & 40.06 & 39.96 & 39.09 \\
\hline 27 & 39.80 & 38.63 & 38.01 & 38.22 & 38.51 & 38.78 & 39.19 & 39.88 & 39.85 & 40.15 & 39.87 & 39.60 \\
\hline 28 & 39.80 & 38.50 & 37.98 & 38.22 & 38.77 & 38.87 & 39.20 & 39.88 & 40.13 & 39.88 & 39.72 & 39.85 \\
\hline 29 & 39.80 & 38.54 & 38.02 & 38.22 & 38.74 & 39.02 & 39.34 & 39.88 & 40.24 & 39.91 & -- & 39.82 \\
\hline 30 & 39.80 & 38.54 & 38.08 & 38.30 & 38.68 & 39.14 & 39.27 & 39.86 & 40.10 & 40.02 & -- & 39.82 \\
\hline 31 & -- & 38.74 & -- & 38.31 & 38.71 & --- & 39.27 & -- & 40.11 & 39.90 & -- & 39.82 \\
\hline
\end{tabular}


GROUND WATER LEVELS

CUSTER COUNTY

354112098430601. LOCAL NUMBER, 14N-14W-17 CBD 1.

LOCATION.--LAT 35 41'12", LONG 098 43'06", HYDROLOGIC UNIT 11090201, OWNER: MELT HERRONG. AQUIFER.--RUSH SPRINGS FORMATION.

WELL CHARACTERISTICS.--DRILLED UNUSED IRRIGATION WELL, DIAMETER 16 IN (0.41M), DEPTH 320 FT (97.5M). DATUM.--ALTITUDE OF LAND -SURFACE DATUM IS $1710 \mathrm{FT}$ (521M). MEASURING POINT: TOP OF WOOD RECORDER BASE $0.40 \mathrm{FT}(0.12 \mathrm{M})$ ABOVE LAND-SURFACE DATUM.

PERIOD OF RECORD.-- 1971 TO CURRENT YEAR.

EXTREMES FOR PERIOD OF RECORD.--HIGHEST WATER LEVEL, 24.59 FT (7.495M) BELOW LAND-SURFACE

DATUM, JULY 5, 1975; LOWEST, 30.08 FT (9.168M) BELOW LAND-SURFACE DATUM SEPT. 10, 1972.

WATER LEVEL, IN FEET BELOW LAND SURFACE DATUM, CLIMATIC YEAR APRIL 1982 TO MARCH 1983

MEAN VALUES

\begin{tabular}{|c|c|c|c|c|c|c|c|c|c|c|c|c|}
\hline DAY & APR & MAY & JUN & JUL & AUG & SEP & ОСТ & NOV & DEC & JAN & FEB & MAR \\
\hline $\begin{array}{l}1 \\
2 \\
3 \\
4 \\
5\end{array}$ & $\begin{array}{l}27.41 \\
27.33 \\
27.38 \\
27.38 \\
27.33\end{array}$ & $\begin{array}{l}27.57 \\
27.60 \\
27.60 \\
27.59 \\
27.58\end{array}$ & $\begin{array}{l}26.61 \\
26.49 \\
26.45 \\
26.44 \\
26.43\end{array}$ & $\begin{array}{l}25.79 \\
25.77 \\
25.78 \\
25.76 \\
25.72\end{array}$ & $\begin{array}{l}25.35 \\
25.35 \\
25.35 \\
25.33 \\
25.33\end{array}$ & $\begin{array}{l}25.56 \\
25.58 \\
25.59 \\
25.60 \\
25.63\end{array}$ & $\begin{array}{l}26.06 \\
26.08 \\
26.04 \\
26.04 \\
26.05\end{array}$ & $\begin{array}{l}26.17 \\
26.23 \\
26.24 \\
26.24 \\
26.22\end{array}$ & $\begin{array}{l}26.15 \\
26.18 \\
26.16 \\
26.15 \\
26.19\end{array}$ & $\begin{array}{l}26.13 \\
26.12 \\
26.09 \\
26.10 \\
26.08\end{array}$ & $\begin{array}{l}26.10 \\
26.15 \\
26.15 \\
26.09 \\
26.13\end{array}$ & $\begin{array}{l}--- \\
--- \\
--- \\
---\end{array}$ \\
\hline $\begin{array}{r}6 \\
7 \\
8 \\
9 \\
10\end{array}$ & $\begin{array}{l}27.41 \\
27.38 \\
27.33 \\
27.38 \\
27.39\end{array}$ & $\begin{array}{l}27.57 \\
27.56 \\
27.58 \\
27.57 \\
27.57\end{array}$ & $\begin{array}{l}26.39 \\
26.36 \\
26.34 \\
26.30 \\
26.26\end{array}$ & $\begin{array}{l}25.70 \\
25.70 \\
25.69 \\
25.66 \\
25.63\end{array}$ & $\begin{array}{l}25.33 \\
25.32 \\
25.31 \\
25.31 \\
25.30\end{array}$ & $\begin{array}{l}25.65 \\
25.67 \\
25.69 \\
25.70 \\
25.74\end{array}$ & $\begin{array}{l}26.02 \\
25.97 \\
26.02 \\
26.04 \\
26.03\end{array}$ & $\begin{array}{l}26.23 \\
26.28 \\
26.30 \\
26.32 \\
26.26\end{array}$ & $\begin{array}{l}26.18 \\
26.24 \\
26.20 \\
26.11 \\
26.15\end{array}$ & $\begin{array}{l}26.08 \\
26.09 \\
26.05 \\
26.09 \\
26.13\end{array}$ & $\begin{array}{r}26.12 \\
-\cdots \\
-\cdots \\
--.\end{array}$ & $\begin{array}{l}--- \\
--- \\
--- \\
--\end{array}$ \\
\hline $\begin{array}{l}11 \\
12 \\
13 \\
14 \\
15\end{array}$ & $\begin{array}{l}27.39 \\
27.35 \\
27.32 \\
27.36 \\
27.35\end{array}$ & $\begin{array}{l}27.56 \\
27.55 \\
27.55 \\
27.47 \\
27.45\end{array}$ & $\begin{array}{l}26.25 \\
26.23 \\
26.20 \\
26.19 \\
26.17\end{array}$ & $\begin{array}{l}25.61 \\
25.59 \\
25.57 \\
25.56 \\
25.55\end{array}$ & $\begin{array}{l}25.29 \\
25.29 \\
25.29 \\
25.31 \\
25.31\end{array}$ & $\begin{array}{l}25.77 \\
25.82 \\
25.87 \\
25.94 \\
25.96\end{array}$ & $\begin{array}{l}26.02 \\
26.01 \\
26.01 \\
25.98 \\
26.01\end{array}$ & $\begin{array}{l}26.40 \\
26.37 \\
26.41 \\
26.40 \\
26.36\end{array}$ & $\begin{array}{l}26.15 \\
26.06 \\
26.07 \\
26.15 \\
26.14\end{array}$ & $\begin{array}{l}26.12 \\
26.09 \\
26.14 \\
26.14 \\
26.08\end{array}$ & $\begin{array}{l}--. \\
--- \\
-- \\
--\end{array}$ & $\begin{array}{l}--- \\
--- \\
--- \\
--\end{array}$ \\
\hline $\begin{array}{l}16 \\
17 \\
18 \\
19 \\
20\end{array}$ & $\begin{array}{l}27.32 \\
27.35 \\
27.42 \\
27.38 \\
27.35\end{array}$ & $\begin{array}{l}27.44 \\
27.43 \\
27.39 \\
27.32 \\
27.29\end{array}$ & $\begin{array}{l}26.14 \\
26.12 \\
26.11 \\
26.08 \\
26.05\end{array}$ & $\begin{array}{l}25.53 \\
25.52 \\
25.52 \\
25.51 \\
25.49\end{array}$ & $\begin{array}{l}25.31 \\
25.31 \\
25.31 \\
25.31 \\
25.30\end{array}$ & $\begin{array}{l}25.98 \\
26.04 \\
26.05 \\
26.11 \\
26.15\end{array}$ & $\begin{array}{l}25.96 \\
25.93 \\
25.96 \\
26.01 \\
25.99\end{array}$ & $\begin{array}{l}26.35 \\
26.33 \\
26.29 \\
26.33 \\
26.34\end{array}$ & $\begin{array}{l}26.09 \\
26.08 \\
26.13 \\
26.10 \\
25.73\end{array}$ & $\begin{array}{l}26.10 \\
26.12 \\
26.10 \\
26.09 \\
26.09\end{array}$ & $\begin{array}{l}\cdots \\
\cdots \\
\cdots \\
\cdots\end{array}$ & $\begin{array}{l}--- \\
-- \\
--- \\
---\end{array}$ \\
\hline $\begin{array}{l}21 \\
22 \\
23 \\
24 \\
25\end{array}$ & $\begin{array}{l}27.40 \\
27.43 \\
27.44 \\
27.44 \\
27.41\end{array}$ & $\begin{array}{l}27.23 \\
27.17 \\
27.12 \\
27.09 \\
27.05\end{array}$ & $\begin{array}{l}26.03 \\
26.00 \\
25.97 \\
25.94 \\
25.92\end{array}$ & $\begin{array}{l}25.47 \\
25.46 \\
25.45 \\
25.44 \\
25.43\end{array}$ & $\begin{array}{l}25.29 \\
25.29 \\
25.32 \\
25.30 \\
25.30\end{array}$ & $\begin{array}{l}26.14 \\
26.12 \\
26.15 \\
26.15 \\
26.10\end{array}$ & $\begin{array}{l}26.00 \\
26.00 \\
26.03 \\
26.05 \\
26.06\end{array}$ & $\begin{array}{l}26.29 \\
26.34 \\
26.36 \\
26.30 \\
26.28\end{array}$ & $\begin{array}{l}26.04 \\
26.02 \\
26.01 \\
26.14 \\
26.12\end{array}$ & $\begin{array}{l}26.09 \\
26.10 \\
26.12 \\
26.10 \\
26.12\end{array}$ & $\begin{array}{l}\cdots \\
\cdots \\
\cdots\end{array}$ & $\begin{array}{l}-- \\
-\cdots \\
-\cdots \\
--\end{array}$ \\
\hline $\begin{array}{l}26 \\
27 \\
28 \\
29 \\
30 \\
31\end{array}$ & $\begin{array}{r}27.39 \\
27.45 \\
27.51 \\
27.53 \\
27.55 \\
.\end{array}$ & $\begin{array}{l}26.99 \\
26.94 \\
26.90 \\
26.75 \\
26.71 \\
26.65\end{array}$ & $\begin{array}{r}25.90 \\
25.89 \\
25.86 \\
25.84 \\
25.82 \\
\end{array}$ & $\begin{array}{l}25.41 \\
25.41 \\
25.40 \\
25.38 \\
25.36 \\
25.35\end{array}$ & $\begin{array}{l}25.35 \\
25.39 \\
25.42 \\
25.46 \\
25.49 \\
25.51\end{array}$ & $\begin{array}{r}26.07 \\
26.08 \\
26.10 \\
26.10 \\
26.08 \\
.--\end{array}$ & $\begin{array}{l}26.04 \\
26.08 \\
26.10 \\
26.08 \\
26.10 \\
26.12\end{array}$ & $\begin{array}{r}26.23 \\
26.17 \\
26.17 \\
26.16 \\
26.12 \\
. .-\end{array}$ & $\begin{array}{l}26.03 \\
26.12 \\
26.13 \\
26.11 \\
26.12 \\
26.12\end{array}$ & $\begin{array}{l}26.12 \\
26.06 \\
26.09 \\
26.11 \\
26.09 \\
26.06\end{array}$ & $\begin{array}{l}-- \\
-- \\
-- \\
-- \\
--\end{array}$ & $\begin{array}{l}-- \\
-- \\
-\cdots \\
-\cdots \\
-- \\
--\end{array}$ \\
\hline
\end{tabular}


GROUND WATER LEVELS

\section{ELLIS COUNTY}

361536099464601. LOCAL NUMBER, 21N-24W-33 BBD 1.

LOCATION.--LAT 36 15'36", LONG 099 46'46", HYDROLOGIC UNIT 11100203. OWNER:

AQUIFER . - OGALLALA FORMATION.

WELL CHARACTERISTICS.--DRILLED STOCK WELL, DIAMETER 5 IN (0.13M), DEPTH 205 FT (62.5M).

DATUM.--ALTITUDE OF LAND-SURFACE DATUM IS $2280 \mathrm{FT}$ (695M). MEASURING POINT: TOP OF WOODEN RECORDER BASE $3.10 \mathrm{FT}(0.94 \mathrm{M})$ ABOVE LAND-SURFACE DATUM.

PERIOD OF RECORD.--APR. 1977 TO CURRENT YEAR.

EXTREMUS FOR PERIOD OF RECORD. -- HIGHEST WATER LEVEL, $80.53 \mathrm{FT}$ (24.546M) BELOW LAND-SURFACE

DATUM, JUNE 5, 1980; LOWEST, 84.40 FT (25.725M) BELOW LAND-SURFACE DATUM, APR. 15, 1977.

WATER LEVEL, IN FEET BELOW LAND SURFACE DATUM, CLIMATIC YEAR APRIL 1982 TO MARCH 1983 MEAN VALUES

\begin{tabular}{|c|c|c|c|c|c|c|c|c|c|c|c|c|}
\hline DAY & APR & MAY & JUN & JUL & AUG & SEP & OCT & NOV & DEC & JAN & FEB & MAR \\
\hline $\begin{array}{l}1 \\
2 \\
3 \\
4 \\
5\end{array}$ & $\begin{array}{l}81.53 \\
81.33 \\
81.45 \\
81.42 \\
81.41\end{array}$ & $\begin{array}{l}81.87 \\
81.87 \\
81.83 \\
81.76 \\
81.72\end{array}$ & $\begin{array}{l}81.22 \\
81.13 \\
81.12 \\
81.16 \\
81.12\end{array}$ & $\begin{array}{l}81.05 \\
81.04 \\
81.02 \\
81.01 \\
80.98\end{array}$ & $\begin{array}{l}81.59 \\
81.47 \\
81.38 \\
81.35 \\
81.40\end{array}$ & $\begin{array}{l}82.47 \\
82.37 \\
82.47 \\
82.52 \\
82.43\end{array}$ & $\begin{array}{l}82.42 \\
82.42 \\
82.61 \\
82.59 \\
82.36\end{array}$ & $\begin{array}{l}82.00 \\
82.11 \\
82.29 \\
82.36 \\
82.28\end{array}$ & $\begin{array}{l}81.62 \\
81.65 \\
81.78 \\
81.81 \\
81.79\end{array}$ & $\begin{array}{l}81.81 \\
81.82 \\
81.80 \\
81.71 \\
81.70\end{array}$ & $\begin{array}{l}81.35 \\
81.39 \\
81.54 \\
81.62 \\
81.52\end{array}$ & $\begin{array}{l}81.41 \\
81.41 \\
81.32 \\
81.17 \\
81.07\end{array}$ \\
\hline $\begin{array}{r}6 \\
7 \\
8 \\
9 \\
10\end{array}$ & $\begin{array}{l}81.59 \\
81.47 \\
81.45 \\
81.54 \\
81.60\end{array}$ & $\begin{array}{l}81.74 \\
81.79 \\
81.76 \\
81.74 \\
81.72\end{array}$ & $\begin{array}{l}81.05 \\
81.06 \\
81.05 \\
81.04 \\
81.16\end{array}$ & $\begin{array}{l}80.97 \\
81.06 \\
81.15 \\
81.19 \\
81.14\end{array}$ & $\begin{array}{l}81.54 \\
81.70 \\
81.69 \\
81.63 \\
81.56\end{array}$ & $\begin{array}{l}82.43 \\
82.58 \\
82.63 \\
82.67 \\
82.61\end{array}$ & $\begin{array}{l}82.29 \\
82.35 \\
82.36 \\
82.49 \\
82.58\end{array}$ & $\begin{array}{l}82.11 \\
82.10 \\
82.32 \\
82.42 \\
82.34\end{array}$ & $\begin{array}{l}81.89 \\
81.93 \\
82.13 \\
82.15 \\
81.92\end{array}$ & $\begin{array}{l}81.65 \\
81.63 \\
81.61 \\
81.50 \\
81.56\end{array}$ & $\begin{array}{l}81.56 \\
81.54 \\
81.48 \\
81.46 \\
81.46\end{array}$ & $\begin{array}{l}81.19 \\
81.38 \\
81.50 \\
81.62 \\
81.64\end{array}$ \\
\hline $\begin{array}{l}11 \\
12 \\
13 \\
14 \\
15\end{array}$ & $\begin{array}{l}81.58 \\
81.43 \\
81.46 \\
81.48 \\
81.38\end{array}$ & $\begin{array}{l}81.72 \\
81.71 \\
81.66 \\
81.68 \\
81.75\end{array}$ & $\begin{array}{l}81.15 \\
81.12 \\
81.17 \\
81.11 \\
81.02\end{array}$ & $\begin{array}{l}81.17 \\
81.17 \\
81.22 \\
81.24 \\
81.24\end{array}$ & $\begin{array}{l}81.65 \\
81.83 \\
81.92 \\
81.85 \\
81.74\end{array}$ & $\begin{array}{l}82.50 \\
82.38 \\
82.28 \\
82.24 \\
82.31\end{array}$ & $\begin{array}{l}82.67 \\
82.54 \\
82.42 \\
82.35 \\
82.25\end{array}$ & $\begin{array}{l}82.08 \\
82.15 \\
82.16 \\
82.15 \\
82.13\end{array}$ & $\begin{array}{l}81.92 \\
81.89 \\
81.68 \\
81.60 \\
81.76\end{array}$ & $\begin{array}{l}81.67 \\
81.75 \\
81.68 \\
81.73 \\
81.80\end{array}$ & $\begin{array}{l}81.55 \\
81.66 \\
81.59 \\
81.43 \\
81.50\end{array}$ & $\begin{array}{l}81.64 \\
81.52 \\
81.42 \\
81.33 \\
81.25\end{array}$ \\
\hline $\begin{array}{l}16 \\
17 \\
18 \\
19 \\
20\end{array}$ & $\begin{array}{l}81.40 \\
81.66 \\
81.68 \\
81.57 \\
81.69\end{array}$ & $\begin{array}{l}81.67 \\
81.57 \\
81.58 \\
81.50 \\
81.42\end{array}$ & $\begin{array}{l}81.08 \\
81.07 \\
81.09 \\
81.16 \\
81.14\end{array}$ & $\begin{array}{l}81.26 \\
81.34 \\
81.50 \\
81.59 \\
81.66\end{array}$ & $\begin{array}{l}81.76 \\
81.80 \\
81.87 \\
82.04 \\
82.21\end{array}$ & $\begin{array}{l}82.42 \\
82.56 \\
82.68 \\
82.65 \\
82.64\end{array}$ & $\begin{array}{l}82.32 \\
82.35 \\
82.22 \\
82.18 \\
82.38\end{array}$ & $\begin{array}{l}81.96 \\
81.90 \\
81.94 \\
82.06 \\
82.19\end{array}$ & $\begin{array}{l}81.82 \\
81.75 \\
81.66 \\
81.77 \\
81.74\end{array}$ & $\begin{array}{l}81.66 \\
81.61 \\
81.61 \\
81.55 \\
81.51\end{array}$ & $\begin{array}{l}81.52 \\
81.51 \\
81.51 \\
81.32 \\
81.37\end{array}$ & $\begin{array}{l}81.33 \\
81.43 \\
81.41 \\
81.35 \\
81.41\end{array}$ \\
\hline $\begin{array}{l}21 \\
22 \\
23 \\
24 \\
25\end{array}$ & $\begin{array}{l}81.82 \\
81.89 \\
81.88 \\
81.71 \\
81.56\end{array}$ & $\begin{array}{l}81.44 \\
81.46 \\
81.42 \\
81.35 \\
81.28\end{array}$ & $\begin{array}{l}81.09 \\
81.12 \\
81.20 \\
81.24 \\
81.19\end{array}$ & $\begin{array}{l}81.65 \\
81.60 \\
81.62 \\
81.76 \\
81.83\end{array}$ & $\begin{array}{l}82.34 \\
82.37 \\
82.28 \\
82.44 \\
82.61\end{array}$ & $\begin{array}{l}82.60 \\
82.49 \\
82.31 \\
82.31 \\
82.41\end{array}$ & $\begin{array}{l}82.35 \\
82.43 \\
82.45 \\
82.37 \\
82.30\end{array}$ & $\begin{array}{l}82.41 \\
82.30 \\
82.29 \\
82.36 \\
82.24\end{array}$ & $\begin{array}{l}81.65 \\
81.55 \\
81.47 \\
81.37 \\
81.64\end{array}$ & $\begin{array}{l}81.50 \\
81.49 \\
81.49 \\
81.54 \\
81.52\end{array}$ & $\begin{array}{l}81.50 \\
81.52 \\
81.49 \\
81.48 \\
81.53\end{array}$ & $\begin{array}{l}81.49 \\
81.49 \\
81.47 \\
81.43 \\
81.28\end{array}$ \\
\hline $\begin{array}{l}26 \\
27 \\
28 \\
29 \\
30 \\
31\end{array}$ & $\begin{array}{r}81.64 \\
81.74 \\
81.74 \\
81.75 \\
81.79 \\
\ldots\end{array}$ & $\begin{array}{l}81.21 \\
81.17 \\
81.07 \\
81.07 \\
81.08 \\
81.12\end{array}$ & $\begin{array}{r}81.14 \\
81.09 \\
81.06 \\
81.02 \\
81.02 \\
\ldots--\end{array}$ & $\begin{array}{l}81.90 \\
81.92 \\
81.97 \\
81.93 \\
81.82 \\
81.72\end{array}$ & $\begin{array}{l}82.59 \\
82.58 \\
82.73 \\
82.73 \\
82.62 \\
82.58\end{array}$ & $\begin{array}{r}82.36 \\
82.27 \\
82.26 \\
82.31 \\
82.37 \\
. .-\end{array}$ & $\begin{array}{l}82.19 \\
82.05 \\
82.03 \\
82.17 \\
82.12 \\
82.05\end{array}$ & $\begin{array}{r}82.16 \\
82.05 \\
81.82 \\
81.74 \\
81.69 \\
\ldots\end{array}$ & $\begin{array}{l}81.81 \\
81.65 \\
81.73 \\
81.84 \\
81.80 \\
81.81\end{array}$ & $\begin{array}{l}81.58 \\
81.60 \\
81.42 \\
81.44 \\
81.48 \\
81.46\end{array}$ & $\begin{array}{r}81.51 \\
81.46 \\
81.42 \\
\ldots .- \\
\ldots- \\
\ldots-\end{array}$ & $\begin{array}{l}81.17 \\
81.34 \\
81.47 \\
81.46 \\
81.48 \\
81.33\end{array}$ \\
\hline
\end{tabular}


ELLIS COUNTY -- CONTINUED

363235099592801. LOCAL NUMBER, 24N-26W-21 CAA 1.

LOCATION.--LAT 36 32'35", LONG 099 59'28", HYDROLOGIC UNIT 11100201, OWNER: HINER. AQUIFER.--OGALLALA FORMATION.

WELL CHARACTERISTICS.--DRILLED UNUSED WELL, DIAMETER 5 IN $(0.13 \mathrm{M})$, DEPTH $120 \mathrm{FT}(36.6 \mathrm{M})$. DATUM.--ALTITUDE OF LAND-SURFACE DATUM IS $2345 \mathrm{FT}$ (715M). MEASURING POINT: TOP EDGE OF PLYWOOD SHELTER BASE $1.50 \mathrm{FT}(0.46 \mathrm{M})$ ABOVE LAND-SURFACE DATUM.

PERIOD OF RECORD.--1972 TO CURRENT YEAR.

EXTREMES FOR PERIOD OF RECORD.--HICHEST WATER LEVEL, 30.11 FT (9.178M) BELOW LAND-SURFACE

DATUM, MAY 10, 1974; LOWEST, 33.25 FT (10.135M) BELOW LAND-SURFACE DATUM, OCT. 25, 1972.

WATER LEVEL, IN FEET BELOW LAND SURFACE DATUM, CLIMATIC YEAR APRIL 1982 TO MARCH 1983 MEAN VALUES

\begin{tabular}{|c|c|c|c|c|c|c|c|c|c|c|c|c|}
\hline DAY & APR & MAY & JUN & JUL & AUG & SEP & OCT & NOV & DEC & JAN & FEB & MAR \\
\hline 1 & 33.11 & 33.10 & 32.88 & 32.73 & 32.26 & 32.36 & 32.61 & 32.73 & 32.59 & 32.54 & 32.45 & 32.43 \\
\hline 2 & 33.09 & 33.09 & 32.86 & 32.72 & 32.24 & 32.39 & 32.61 & 32.74 & 32.61 & 32.54 & 32.46 & 32.43 \\
\hline 3 & 33.13 & 33.09 & 32.86 & 32.71 & 32.24 & 32.40 & 32.63 & 32.74 & 32.63 & 32.53 & 32.49 & 32.41 \\
\hline 4 & 33.10 & 33.08 & 32.86 & 32.71 & 32.23 & 32.40 & 32.62 & 32.74 & 32.61 & 32.52 & 32.48 & 32.40 \\
\hline 5 & 33.12 & 33.08 & 32.84 & 32.71 & 32.24 & 32.40 & 32.62 & 32.72 & 32.60 & 32.52 & 32.45 & 32.40 \\
\hline 6 & 33.13 & 33.09 & 32.83 & 32.71 & 32.24 & 32.43 & 32.64 & 32.70 & 32.61 & 32.52 & 32.47 & 32.43 \\
\hline 7 & 33.09 & 33.08 & 32.83 & 32.72 & 32.23 & 32.43 & 32.64 & 32.70 & 32.61 & 32.52 & 32.46 & 32.45 \\
\hline 8 & 33.11 & 33.07 & 32.82 & 32.72 & 32.23 & 32.43 & 32.63 & 32.71 & 32.65 & 32.51 & 32.45 & 32.45 \\
\hline 9 & 33.12 & 33.07 & 32.83 & 32.71 & 32.23 & 32.44 & 32.66 & 32.71 & 32.61 & 32.50 & 32.45 & 32.45 \\
\hline 10 & 33.12 & 33.07 & 32.83 & 32.73 & 32.23 & 32.44 & 32.67 & 32.71 & 32.57 & 32.52 & 32.45 & 32.44 \\
\hline 11 & 33.10 & 33.07 & 32.81 & 32.72 & 32.23 & 32.45 & 32.68 & 32.66 & 32.60 & 32.53 & 32.47 & 32.43 \\
\hline 12 & 33.09 & 33.07 & 32.81 & 32.70 & 32.23 & 32.46 & 32.68 & 32.73 & 32.58 & 32.52 & 32.47 & 32.41 \\
\hline 13 & 33.11 & 33.06 & 32.81 & 32.67 & 32.22 & 32.48 & 32.69 & 32.69 & 32.54 & 32.50 & 32.45 & 32.41 \\
\hline 14 & 33.10 & 33.07 & 32.77 & 32.64 & 32.23 & 32.50 & 32.69 & 32.71 & 32.57 & 32.53 & 32.44 & 32.40 \\
\hline 15 & 33.09 & 33.07 & 32.80 & 32.59 & 32.24 & 32.51 & 32.70 & 32.68 & 32.60 & 32.52 & 32.46 & 32.40 \\
\hline 16 & 33.11 & 33.06 & 32.81 & 32.53 & 32.26 & 32.50 & 32.70 & 32.67 & 32.59 & 32.50 & 32.46 & 32.43 \\
\hline 17 & 33.13 & 33.06 & 32.79 & 32.49 & 32.26 & 32.51 & 32.69 & 32.66 & 32.56 & 32.50 & 32.46 & 32.43 \\
\hline 18 & 33.10 & 33.05 & 32.80 & 32.45 & 32.26 & 32.53 & 32.70 & 32.65 & 32.56 & 32.51 & 32.44 & 32.41 \\
\hline 19 & 33.09 & 33.04 & 32.81 & 32.41 & 32.27 & 32.52 & 32.72 & 32.64 & 32.57 & 32.49 & 32.41 & 32.41 \\
\hline 20 & 33.12 & 33.03 & 32.79 & 32.38 & 32.28 & 32.54 & 32.73 & 32.66 & 32.56 & 32.49 & 32.46 & 32.42 \\
\hline 21 & 33.12 & 33.03 & 32.78 & 32.35 & 32.29 & 32.54 & 32.73 & 32.67 & 32.54 & 32.49 & 32.47 & 32.42 \\
\hline 22 & 33.12 & 33.01 & 32.78 & 32.34 & 32.28 & 32.54 & 32.73 & 32.65 & 32.53 & 32.49 & 32.46 & 32.41 \\
\hline 23 & 33.10 & 33.00 & 32.78 & 32.32 & 32.29 & 32.53 & 32.72 & 32.67 & 32.53 & 32.49 & 32.44 & 32.41 \\
\hline 24 & 33.08 & 32.98 & 32.77 & 32.31 & 32.30 & 32.56 & 32.73 & 32.68 & 32.52 & 32.50 & 32.44 & 32.40 \\
\hline 25 & 33.09 & 32.97 & 32.77 & 32.30 & 32.32 & 32.56 & 32.73 & 32.64 & 32.59 & 32.48 & 32.44 & 32.38 \\
\hline 26 & 33.11 & 32.95 & 32.77 & 32.29 & 32.31 & 32.55 & 32.72 & 32.65 & 32.57 & 32.50 & 32.43 & 32.39 \\
\hline 27 & 33.11 & 32.94 & 32.76 & 32.29 & 32.33 & 32.55 & 32.70 & 32.63 & 32.53 & 32.48 & 32.43 & 32.43 \\
\hline 28 & 33.09 & 32.92 & 32.75 & 32.27 & 32.35 & 32.57 & 32.73 & 32.60 & 32.56 & 32.45 & 32.43 & 32.43 \\
\hline 29 & 33.09 & 32.92 & 32.74 & 32.27 & 32.34 & 32.59 & 32.73 & 32.60 & 32.56 & 32.48 & $-\cdots$ & 32.42 \\
\hline 30 & 33.10 & 32.90 & 32.74 & 32.27 & 32.35 & 32.61 & 32.72 & 32.60 & 32.53 & 32.48 & -- & 32.41 \\
\hline 31 & -- & 32.90 & $\cdots$ & 32.27 & 32.36 & -- & 32.72 & -- & 32.54 & 32.47 & -- & 32.38 \\
\hline
\end{tabular}


GRADY COUNTY

344656098031401. LOCAL NUMBER, 04N-08W-33 BEB 1.

LOCATION.--LAT 34 46'56", LONG 098 03'14", HYDROLOGIC UNIT 11130208 , OWNER: U.S. GEOLOGICAL SURVEY.

AQUIFER.--RUSH SPRINGS FORMATION.

WELL CHARACTERISTICS.--DRILLED TEST WELL, DIAMETER 6 IN (0.15M), DEPTH 254 FT (77.4M).

DATUM.--MLASURING POINT: TOP OF CASING 4.18 FT (1.27M) ABOVE LAND-SURFACE DATUM.

PERIOD OF RECORD.-- 1948 TO CURRENT YEAR.

EXTRLMES FOR PERIOD OF RECORD.-- HIGHEST WATER LEVEL, 77.55 FT (23.6374M) BELOW

LAND-SURFACE DATUM, JAN. 28, 1983; LOWEST, $85.67 \mathrm{FT}$ (26.112M) BELOW LAND-SURFACE

DATUM, FEB. 29, 1968.

WATER LEVEL, IN FEET BELOW LAND SURFACE DATUM, CLIMATIC YEAR APRIL 1982 TO MARCH 1983

MEAN VALUES

\begin{tabular}{|c|c|c|c|c|c|c|c|c|c|c|c|c|}
\hline DAY & APR & MAY & JUN & JUL & AUG & SEP & OCT & NOV & DEC & JAN & FEB & MAR \\
\hline $\begin{array}{l}1 \\
2 \\
3 \\
4 \\
5\end{array}$ & $\begin{array}{l}82.41 \\
82.36 \\
82.54 \\
82.36 \\
82.52\end{array}$ & $\begin{array}{l}82.54 \\
82.52 \\
82.48 \\
82.45 \\
82.44\end{array}$ & $\begin{array}{l}82.19 \\
82.08 \\
82.10 \\
82.14 \\
82.04\end{array}$ & $\begin{array}{l}81.36 \\
81.31 \\
81.27 \\
81.24 \\
81.20\end{array}$ & $\begin{array}{l}--- \\
--- \\
--- \\
-- \\
--\end{array}$ & $\begin{array}{r}--- \\
79.18 \\
79.23 \\
79.17 \\
79.15\end{array}$ & $\begin{array}{l}79.09 \\
79.07 \\
79.11 \\
79.07 \\
79.06\end{array}$ & $\begin{array}{l}79.05 \\
79.16 \\
79.28 \\
79.22 \\
79.17\end{array}$ & $\begin{array}{l}78.12 \\
78.18 \\
78.24 \\
78.19 \\
78.19\end{array}$ & $\begin{array}{l}77.99 \\
78.00 \\
77.97 \\
77.87 \\
77.87\end{array}$ & $\begin{array}{l}77.62 \\
77.80 \\
77.90 \\
77.80 \\
77.76\end{array}$ & $\begin{array}{l}77.91 \\
77.87 \\
77.79 \\
77.72 \\
77.79\end{array}$ \\
\hline $\begin{array}{r}6 \\
7 \\
8 \\
9 \\
10\end{array}$ & $\begin{array}{l}82.54 \\
82.38 \\
82.49 \\
82.52 \\
82.54\end{array}$ & $\begin{array}{l}82.51 \\
82.50 \\
82.48 \\
82.47 \\
82.44\end{array}$ & $\begin{array}{l}82.01 \\
82.00 \\
81.95 \\
81.95 \\
82.00\end{array}$ & $\begin{array}{l}81.18 \\
81.21 \\
81.15 \\
81.07 \\
81.07\end{array}$ & $\begin{array}{l}--- \\
--- \\
--- \\
--- \\
---\end{array}$ & $\begin{array}{l}79.17 \\
79.17 \\
79.15 \\
79.11 \\
79.06\end{array}$ & $\begin{array}{l}79.11 \\
79.06 \\
78.96 \\
79.15 \\
79.20\end{array}$ & $\begin{array}{l}79.09 \\
79.09 \\
79.15 \\
79.16 \\
79.14\end{array}$ & $\begin{array}{l}78.31 \\
78.31 \\
78.43 \\
78.32 \\
78.25\end{array}$ & $\begin{array}{l}77.78 \\
77.75 \\
77.72 \\
77.68 \\
77.74\end{array}$ & $\begin{array}{l}77.85 \\
77.74 \\
77.73 \\
77.75 \\
77.80\end{array}$ & $\begin{array}{l}78.01 \\
78.05 \\
78.14 \\
78.15 \\
78.17\end{array}$ \\
\hline $\begin{array}{l}11 \\
12 \\
13 \\
14 \\
15\end{array}$ & $\begin{array}{l}82.45 \\
82.34 \\
82.47 \\
82.42 \\
82.37\end{array}$ & $\begin{array}{l}82.44 \\
82.40 \\
82.38 \\
82.44 \\
82.47\end{array}$ & $\begin{array}{l}81.88 \\
81.88 \\
81.87 \\
81.76 \\
81.72\end{array}$ & $\begin{array}{l}--- \\
--. \\
-- \\
--- \\
--\end{array}$ & $\begin{array}{l}--- \\
--- \\
--- \\
--- \\
---\end{array}$ & $\begin{array}{l}79.06 \\
79.08 \\
79.12 \\
79.15 \\
79.21\end{array}$ & $\begin{array}{l}79.17 \\
79.14 \\
79.15 \\
79.14 \\
79.09\end{array}$ & $\begin{array}{l}79.00 \\
79.37 \\
79.21 \\
79.31 \\
79.22\end{array}$ & $\begin{array}{l}78.35 \\
78.34 \\
78.22 \\
78.21 \\
78.36\end{array}$ & $\begin{array}{l}77.88 \\
77.73 \\
77.66 \\
77.82 \\
77.67\end{array}$ & $\begin{array}{l}77.96 \\
77.94 \\
77.74 \\
77.77 \\
77.87\end{array}$ & $\begin{array}{l}78.12 \\
77.99 \\
77.98 \\
77.93 \\
77.97\end{array}$ \\
\hline $\begin{array}{l}16 \\
17 \\
18 \\
19 \\
20\end{array}$ & $\begin{array}{l}82.48 \\
82.63 \\
82.48 \\
82.44 \\
82.60\end{array}$ & $\begin{array}{l}82.45 \\
82.46 \\
82.42 \\
82.38 \\
82.36\end{array}$ & $\begin{array}{l}81.80 \\
81.71 \\
81.70 \\
81.72 \\
81.64\end{array}$ & $\begin{array}{l}-- \\
-- \\
-- \\
-\cdots \\
-\end{array}$ & $\begin{array}{l}-\cdots \\
--- \\
--- \\
--- \\
\cdots\end{array}$ & $\begin{array}{l}79.12 \\
79.09 \\
79.13 \\
79.08 \\
79.16\end{array}$ & $\begin{array}{l}79.15 \\
79.04 \\
79.03 \\
79.13 \\
79.23\end{array}$ & $\begin{array}{l}79.10 \\
79.11 \\
78.21 \\
78.15 \\
78.26\end{array}$ & $\begin{array}{l}78.31 \\
78.14 \\
78.14 \\
78.25 \\
78.14\end{array}$ & $\begin{array}{l}77.58 \\
77.64 \\
77.65 \\
77.61 \\
77.57\end{array}$ & $\begin{array}{l}77.85 \\
77.89 \\
77.77 \\
77.73 \\
77.91\end{array}$ & $\begin{array}{l}78.08 \\
78.08 \\
78.07 \\
78.08 \\
78.19\end{array}$ \\
\hline $\begin{array}{l}21 \\
22 \\
23 \\
24 \\
25\end{array}$ & $\begin{array}{l}82.62 \\
82.60 \\
82.53 \\
82.40 \\
82.39\end{array}$ & $\begin{array}{l}82.41 \\
82.40 \\
82.35 \\
82.30 \\
82.29\end{array}$ & $\begin{array}{l}81.60 \\
81.61 \\
81.57 \\
81.53 \\
81.50\end{array}$ & $\begin{array}{l}--- \\
-- \\
-- \\
--\end{array}$ & $\begin{array}{l}--- \\
--- \\
--- \\
--- \\
--\end{array}$ & $\begin{array}{l}79.19 \\
79.10 \\
79.04 \\
79.10 \\
79.09\end{array}$ & $\begin{array}{l}79.19 \\
79.18 \\
79.14 \\
79.14 \\
79.12\end{array}$ & $\begin{array}{l}78.32 \\
78.26 \\
78.46 \\
78.51 \\
78.34\end{array}$ & $\begin{array}{l}78.03 \\
77.98 \\
77.93 \\
77.95 \\
78.25\end{array}$ & $\begin{array}{l}77.59 \\
77.64 \\
77.64 \\
77.73 \\
77.63\end{array}$ & $\begin{array}{l}77.91 \\
77.92 \\
77.86 \\
77.97 \\
77.97\end{array}$ & $\begin{array}{l}78.22 \\
78.18 \\
78.15 \\
78.13 \\
77.99\end{array}$ \\
\hline $\begin{array}{l}26 \\
27 \\
28 \\
29 \\
30 \\
31\end{array}$ & $\begin{array}{r}82.52 \\
82.54 \\
82.47 \\
82.50 \\
82.55 \\
.--\end{array}$ & $\begin{array}{l}82.24 \\
82.25 \\
82.18 \\
82.21 \\
82.17 \\
82.26\end{array}$ & $\begin{array}{r}81.46 \\
81.44 \\
81.40 \\
81.37 \\
81.37 \\
\ldots--\end{array}$ & $\begin{array}{l}--. \\
-- \\
-- \\
-- \\
--\end{array}$ & $\begin{array}{l}-- \\
-\cdots \\
--- \\
-- \\
--- \\
--\end{array}$ & $\begin{array}{r}79.00 \\
78.98 \\
79.04 \\
79.09 \\
79.10 \\
.\end{array}$ & $\begin{array}{l}79.07 \\
79.00 \\
79.09 \\
79.11 \\
79.04 \\
79.06\end{array}$ & $\begin{array}{r}78.29 \\
78.20 \\
78.17 \\
78.18 \\
78.17 \\
. . .\end{array}$ & $\begin{array}{l}78.12 \\
77.92 \\
78.16 \\
78.12 \\
78.02 \\
78.03\end{array}$ & $\begin{array}{l}77.78 \\
77.66 \\
77.55 \\
77.73 \\
77.66 \\
77.56\end{array}$ & $\begin{array}{r}77.96 \\
77.91 \\
77.86 \\
--- \\
--- \\
--\end{array}$ & $\begin{array}{l}78.09 \\
78.32 \\
78.24 \\
78.23 \\
78.20 \\
77.95\end{array}$ \\
\hline
\end{tabular}


HASKELL COUNTY

352006095080101. LOCAL NUMBER; 10N-20E-13 DDD 1.

LOCATION.--LAT $3520^{\prime} 06^{\prime \prime}$, LONG 095 08'01", HYDROLOGIC UNIT 11090204, OWNER: BUREAU OF LAND MANAGEMENT .

AQUIFER.- - HARTSHORNE SANDSTONE.

WELL CHARACTERISTICS.--DRILLED TEST HOLE, DIAMETER 6 IN $(0.15 \mathrm{M})$, DEPTH $148 \mathrm{FT}(45.1 \mathrm{M})$.

DATUM.---MEASURING POINT: TOP OF CASING $1.00 \mathrm{FT}(0.30 \mathrm{M})$ ABOVE LAND-SURFACE DATUM.

REMARKS. --TEST HOLE NO. 07.

PERIOD OF RECORD.-- 1980 TO CURRENT YEAR.

EXTREMES FOR PERIOD OF RECORD.--HIGHEST WATER LEVEL, $7.51 \mathrm{fT}(2.289 \mathrm{M})$ BELOW LAND-SURFACE DATUM.

MAR. 6, 1983; LOWEST WATER LEVEL, 13.28 FT (4.047M) BELOW LAND-SURFACE DATUM, OCT. 24, 1980.

WATER LEVEL, IN FEET BELOW LAND SURFACE DATUM, CLIMATIC YEAR APRIL 1982 TO MARCH 1983 MEAN VALUES

\begin{tabular}{|c|c|c|c|c|c|c|c|c|c|c|c|c|}
\hline DAY & APR & MAY & JUN & JUL & AUG & SEP & $O C T$ & NOV & DEC & JAN & FEB & MAR \\
\hline $\begin{array}{l}1 \\
2 \\
3 \\
4 \\
5\end{array}$ & $\begin{array}{l}8.96 \\
8.71 \\
9.05 \\
9.06 \\
9.03\end{array}$ & -. & $\begin{array}{l}9.37 \\
9.18 \\
9.10 \\
9.02 \\
8.83\end{array}$ & $\begin{array}{l}9.16 \\
9.20 \\
9.21 \\
9.25 \\
9.32\end{array}$ & $\begin{array}{r}9.98 \\
9.98 \\
10.03 \\
10.10 \\
10.18\end{array}$ & $\begin{array}{l}11.16 \\
11.17 \\
11.23 \\
11.22 \\
11.24\end{array}$ & $\begin{array}{l}11.69 \\
11.69 \\
11.73 \\
11.74 \\
11.77\end{array}$ & $\begin{array}{l}12.15 \\
12.18 \\
12.26 \\
12.31 \\
12.32\end{array}$ & $\begin{array}{l}12.23 \\
12.16 \\
12.12 \\
12.04 \\
11.88\end{array}$ & $\begin{array}{l}9.38 \\
9.30 \\
9.31 \\
9.22 \\
9.16\end{array}$ & $\begin{array}{l}8.40 \\
8.36 \\
8.59 \\
8.73 \\
8.57\end{array}$ & $\begin{array}{l}7.67 \\
7.73 \\
7.76 \\
7.68 \\
7.54\end{array}$ \\
\hline $\begin{array}{r}6 \\
7 \\
8 \\
9 \\
10\end{array}$ & $\begin{array}{l}9.42 \\
9.26 \\
9.20 \\
9.48 \\
9.39\end{array}$ & $\begin{array}{l}10.19 \\
10.27 \\
10.31 \\
10.33 \\
10.31\end{array}$ & $\begin{array}{l}8.63 \\
8.62 \\
8.68 \\
8.72 \\
8.96\end{array}$ & $\begin{array}{l}9.40 \\
9.47 \\
9.38 \\
9.22 \\
9.15\end{array}$ & $\begin{array}{l}10.20 \\
10.21 \\
10.26 \\
10.29 \\
10.33\end{array}$ & $\begin{array}{l}11.29 \\
11.34 \\
11.35 \\
11.35 \\
11.32\end{array}$ & $\begin{array}{l}11.80 \\
11.81 \\
11.76 \\
11.81 \\
11.91\end{array}$ & $\begin{array}{l}12.29 \\
12.29 \\
12.32 \\
12.35 \\
12.35\end{array}$ & $\begin{array}{l}11.83 \\
11.71 \\
11.63 \\
11.54 \\
11.32\end{array}$ & $\begin{array}{l}9.11 \\
9.08 \\
9.11 \\
9.05 \\
9.08\end{array}$ & $\begin{array}{l}8.40 \\
8.36 \\
8.22 \\
8.10 \\
8.03\end{array}$ & $\begin{array}{l}7.51 \\
7.69 \\
7.81 \\
7.94 \\
8.03\end{array}$ \\
\hline $\begin{array}{l}11 \\
12 \\
13 \\
14 \\
15\end{array}$ & $\begin{array}{l}9.43 \\
9.46 \\
9.66 \\
9.98 \\
9.93\end{array}$ & $\begin{array}{r}10.33 \\
10.32 \\
10.24 \\
9.97 \\
9.04\end{array}$ & $\begin{array}{l}8.96 \\
8.84 \\
8.77 \\
8.68 \\
8.58\end{array}$ & $\begin{array}{l}9.23 \\
9.32 \\
9.37 \\
9.38 \\
9.42\end{array}$ & $\begin{array}{l}10.35 \\
10.38 \\
10.34 \\
10.32 \\
10.40\end{array}$ & $\begin{array}{l}11.31 \\
11.34 \\
11.41 \\
11.45 \\
11.51\end{array}$ & $\begin{array}{l}11.94 \\
11.95 \\
11.95 \\
11.99 \\
11.95\end{array}$ & $\begin{array}{l}12.31 \\
12.37 \\
12.48 \\
12.43 \\
12.51\end{array}$ & $\begin{array}{l}11.15 \\
11.05 \\
10.82 \\
10.53 \\
10.53\end{array}$ & $\begin{array}{l}9.24 \\
9.47 \\
9.36 \\
9.38 \\
9.57\end{array}$ & $\begin{array}{l}8.07 \\
8.22 \\
7.86 \\
8.00 \\
7.95\end{array}$ & $\begin{array}{l}8.17 \\
8.20 \\
8.09 \\
8.11 \\
8.14\end{array}$ \\
\hline $\begin{array}{l}16 \\
17 \\
18 \\
19 \\
20\end{array}$ & $\begin{array}{c}9.82 \\
\ldots- \\
\ldots- \\
\cdots\end{array}$ & $\begin{array}{l}9.47 \\
9.01 \\
9.30 \\
9.26 \\
9.24\end{array}$ & $\begin{array}{l}8.62 \\
8.55 \\
8.47 \\
8.57 \\
8.66\end{array}$ & $\begin{array}{l}9.46 \\
9.52 \\
9.59 \\
9.64 \\
9.72\end{array}$ & $\begin{array}{l}10.46 \\
10.53 \\
10.57 \\
10.63 \\
10.70\end{array}$ & $\begin{array}{l}11.49 \\
11.47 \\
11.50 \\
11.51 \\
11.54\end{array}$ & $\begin{array}{l}12.04 \\
12.02 \\
11.99 \\
12.01 \\
12.12\end{array}$ & $\begin{array}{l}12.45 \\
12.44 \\
12.45 \\
12.44 \\
12.44\end{array}$ & $\begin{array}{l}10.53 \\
10.41 \\
10.20 \\
10.30 \\
10.28\end{array}$ & $\begin{array}{l}9.51 \\
9.49 \\
9.65 \\
9.69 \\
9.58\end{array}$ & $\begin{array}{l}7.99 \\
8.10 \\
8.19 \\
8.11 \\
8.18\end{array}$ & $\begin{array}{l}8.22 \\
8.38 \\
8.50 \\
8.57 \\
8.63\end{array}$ \\
\hline $\begin{array}{l}21 \\
22 \\
23 \\
24 \\
25\end{array}$ & $\begin{array}{l}-- \\
\cdots- \\
\cdots- \\
--\end{array}$ & $\begin{array}{l}9.29 \\
9.41 \\
9.46 \\
9.45 \\
9.40\end{array}$ & $\begin{array}{l}8.69 \\
8.76 \\
8.59 \\
8.99 \\
9.02\end{array}$ & $\begin{array}{l}9.77 \\
9.88 \\
9.86 \\
9.88 \\
9.86\end{array}$ & $\begin{array}{l}10.73 \\
10.70 \\
10.70 \\
10.75 \\
10.84\end{array}$ & $\begin{array}{l}11.61 \\
11.61 \\
11.57 \\
11.56 \\
11.59\end{array}$ & $\begin{array}{l}12.12 \\
12.13 \\
12.13 \\
12.14 \\
12.15\end{array}$ & $\begin{array}{l}12.52 \\
12.51 \\
12.53 \\
12.65 \\
12.62\end{array}$ & $\begin{array}{r}10.20 \\
10.11 \\
10.06 \\
9.99 \\
10.17\end{array}$ & $\begin{array}{l}9.43 \\
9.38 \\
9.44 \\
9.45 \\
9.46\end{array}$ & $\begin{array}{l}8.26 \\
8.09 \\
7.93 \\
7.78 \\
7.80\end{array}$ & $\begin{array}{l}8.81 \\
8.90 \\
8.86 \\
8.83 \\
8.79\end{array}$ \\
\hline $\begin{array}{l}26 \\
27 \\
28 \\
29 \\
30 \\
31\end{array}$ & $\begin{array}{c}\cdots- \\
\cdots \\
\cdots \\
\cdots \\
\cdots\end{array}$ & $\begin{array}{l}9.40 \\
9.48 \\
9.47 \\
9.34 \\
9.27 \\
9.31\end{array}$ & $\begin{array}{l}8.97 \\
8.90 \\
8.89 \\
8.94 \\
9.06 \\
-.-\end{array}$ & $\begin{array}{l}9.86 \\
9.84 \\
9.83 \\
9.84 \\
9.86 \\
9.64\end{array}$ & $\begin{array}{l}10.86 \\
10.91 \\
11.09 \\
11.15 \\
11.05 \\
11.16\end{array}$ & $\begin{array}{r}11.59 \\
11.58 \\
11.63 \\
11.67 \\
11.69 \\
\ldots--\end{array}$ & $\begin{array}{l}12.15 \\
12.10 \\
12.07 \\
12.14 \\
12.15 \\
12.16\end{array}$ & $\begin{array}{r}12.57 \\
12.50 \\
12.41 \\
12.36 \\
12.31 \\
. \ldots\end{array}$ & $\begin{array}{r}10.18 \\
9.83 \\
9.75 \\
9.76 \\
9.61 \\
9.47\end{array}$ & $\begin{array}{l}9.37 \\
9.38 \\
9.15 \\
8.88 \\
8.85 \\
8.70\end{array}$ & $\begin{array}{c}7.80 \\
7.74 \\
7.68 \\
\ldots- \\
\ldots- \\
-\end{array}$ & $\begin{array}{l}8.58 \\
8.76 \\
9.02 \\
8.97 \\
8.88 \\
8.70\end{array}$ \\
\hline
\end{tabular}




\section{GROUND-WATER LEVELS}

KAY COUNTY

364210097025401 . LOCAL NUMBER, 26N-02E-26 BDD 1.

LOCATION.--LAT 36 42'10", LONG $09702^{\prime} 54^{\prime \prime}$, HYDROLOGIC UNIT 11060001, OWNER: CITY OF PONCA CITY. AQUIFER.--ALLUVIAM.

WELL CHARACTERISTICS.--DUG PUBLIC SUPPLY WELL, NUMBER 5, DIAMETER 30 IN (0.76M), DEPTH 38 FT $(11.6 \mathrm{M})$.

DATUM.--ALTITUDE OF LAND-SURFACE DATUM IS $925 \mathrm{FT}$ (282M). MEASURING POINT: BOTTOM OF NUMBER AT PUMP BASE OPENING 6.70 FT (2.04M) ABOVE LAND-SURFACE DATUM. PERIOD OF RECORD.-- 1948 TO CURRENT YEAR.

EXTREMES FOR PERIOD OF RECORD.--HIGHEST WATER LEVEL, +3.30 FT $(+1.006 M)$ ABOVE LAND-SURFACE DATUM, AUG. 11, 1976; LOWEST, 29.13 FT (8.879M) BELOW LAND-SURFACE DATUM, FEB. 24, 1955.

WATER LEVELS IN FEET BELOW LAND SURFACE DATUM, CLIMATIC YEAR APRIL 1982 TO MARCH 1983

\begin{tabular}{cc}
\multicolumn{1}{c}{ DATE } & $\begin{array}{c}\text { WATER } \\
\text { LEVEL }\end{array}$ \\
APR 07,1982 & 20.13 \\
14 & 20.14 \\
21 & 20.13 \\
28 & 19.88 \\
MAY 05 & 19.71 \\
12 & 19.63 \\
19 & 19.13 \\
28 & 19.04 \\
JUN 02 & 19.38 \\
09 & 19.13
\end{tabular}

\begin{tabular}{|c|c|c|}
\hline & DATE & $\begin{array}{l}\text { WATER } \\
\text { LEVEL }\end{array}$ \\
\hline JUN & $\begin{array}{l}16,1982 \\
23\end{array}$ & $\begin{array}{l}18.96 \\
18.30\end{array}$ \\
\hline & 30 & 17.38 \\
\hline JUL & 07 & 17.46 \\
\hline & 14 & 17.13 \\
\hline & 21 & 17.00 \\
\hline & 28 & 16.88 \\
\hline UG & 04 & 17.13 \\
\hline & 11 & 17.30 \\
\hline & 18 & 17.13 \\
\hline
\end{tabular}

$\begin{array}{cc}\text { DATE } & \begin{array}{c}\text { WATER } \\ \text { LEVEL }\end{array} \\ \text { AUG 25, 1982 } & 18.30 \\ \text { SEP } 01 & 17.30 \\ 08 & 17.88 \\ 15 & 19.08 \\ 22 & 19.21 \\ 29 & 19.46 \\ \text { OCT } 06 & 20.04 \\ 13 & 20.13 \\ 20 & 20.38 \\ 27 & 20.54\end{array}$

DATE

WATER LEVEL

17.46

17.13

16.88

17.30

17.13

20.54

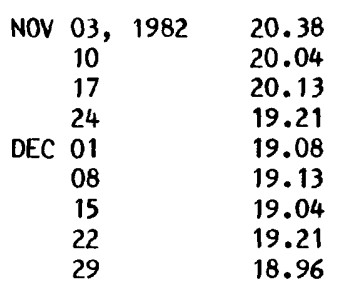


GROUND WATER LEVELS

\author{
LATIMER COUNTY
}

345908905013001. LOCAL NUMBER, 06N-22E-18 DCC 1.

LOCATION.--LAT $3459^{\prime} 08^{\prime \prime}$ LONG $09501^{\prime} 30^{\prime}$ ', HYDROLOGIC UNIT 11110105, OWNER; BUREAU OF LAND MANAGEMENT.

AQUIFER.--HARTSHORNE SANDSTONE.

WELL CHARACTERISTICS.--DRILLED TEST HOLE, DIAMETER 6 IN. (0.15M), DEPTH 138 FT (42.1M).

DATUM.--MEASURING POINT: TOP OF CASING $1.00 \mathrm{FT}(0.30 \mathrm{M})$ ABOVE LAND-SURFACE DATUM.

REMARKS. - TEST HOLE NO. 10.

PERIOD OF RECORD.--1980 TO CURRENT YEAR.

EXTREMES FOR PERIOD OF RECORD.--HIGHEST WATER LEVEL, 1.03 FT (0.314M) BELOW LAND-SURFACE DATUM, JUNE 01, 1982; LOWEST WATER LEVEL 8.78 FT (2.676M) BELOW LAND-SURFACE, NOV. 19, 1980.

WATER LEVEL, IN FEET BELOW LAND SURFACE DATUM, CLIMATIC YEAR APRIL 1982 TO MARCH 1983

MEAN VALUES

\begin{tabular}{|c|c|c|c|c|c|c|c|c|c|c|c|c|}
\hline DAY & APR & MAY & JUN & JUL & AUG & SEP & OCT & NOV & DEC & JAN & FEB & MAR \\
\hline $\begin{array}{l}1 \\
2 \\
3 \\
4 \\
5\end{array}$ & $\begin{array}{l}3.04 \\
2.89 \\
3.16 \\
3.09 \\
3.17\end{array}$ & $\begin{array}{l}3.46 \\
3.47 \\
3.46 \\
3.46 \\
3.47\end{array}$ & $\begin{array}{l}1.69 \\
1.73 \\
2.83 \\
2.82 \\
1.82\end{array}$ & $\begin{array}{l}2.54 \\
2.58 \\
2.62 \\
2.68 \\
2.73\end{array}$ & $\begin{array}{l}4.44 \\
4.48 \\
4.56 \\
4.63 \\
4.72\end{array}$ & $\begin{array}{l}5.19 \\
5.24 \\
5.37 \\
5.42 \\
5.45\end{array}$ & $\begin{array}{l}6.56 \\
6.53 \\
6.59 \\
6.61 \\
6.67\end{array}$ & $\begin{array}{l}7.21 \\
7.26 \\
7.42 \\
7.47 \\
7.46\end{array}$ & $\begin{array}{l}4.81 \\
4.47 \\
3.06 \\
2.77 \\
2.72\end{array}$ & $\begin{array}{l}2.66 \\
2.68 \\
2.79 \\
2.83 \\
2.90\end{array}$ & $\begin{array}{l}1.59 \\
1.70 \\
1.93 \\
2.02 \\
1.87\end{array}$ & $\begin{array}{l}2.00 \\
2.12 \\
2.18 \\
1.79 \\
1.40\end{array}$ \\
\hline $\begin{array}{r}6 \\
7 \\
8 \\
9 \\
10\end{array}$ & $\begin{array}{l}3.36 \\
3.24 \\
3.29 \\
3.42 \\
3.47\end{array}$ & $\begin{array}{l}3.52 \\
3.59 \\
3.64 \\
3.67 \\
3.68\end{array}$ & $\begin{array}{l}--- \\
1.33 \\
1.45 \\
1.66 \\
1.31\end{array}$ & $\begin{array}{l}2.80 \\
2.63 \\
2.04 \\
2.29 \\
2.54\end{array}$ & $\begin{array}{l}4.74 \\
4.80 \\
4.87 \\
4.94 \\
5.01\end{array}$ & $\begin{array}{l}5.52 \\
5.58 \\
5.62 \\
5.64 \\
5.62\end{array}$ & $\begin{array}{l}6.71 \\
6.73 \\
6.64 \\
6.70 \\
6.85\end{array}$ & $\begin{array}{l}7.39 \\
7.38 \\
7.43 \\
7.45 \\
7.45\end{array}$ & $\begin{array}{l}2.91 \\
3.03 \\
3.26 \\
3.35 \\
3.14\end{array}$ & $\begin{array}{l}2.90 \\
2.93 \\
2.97 \\
2.92 \\
3.04\end{array}$ & $\begin{array}{l}1.80 \\
1.82 \\
1.79 \\
1.78 \\
1.78\end{array}$ & $\begin{array}{l}1.54 \\
1.72 \\
1.87 \\
2.04 \\
2.20\end{array}$ \\
\hline $\begin{array}{l}11 \\
12 \\
13 \\
14 \\
15\end{array}$ & $\begin{array}{l}3.43 \\
3.31 \\
3.43 \\
3.48 \\
3.44\end{array}$ & $\begin{array}{l}3.72 \\
3.29 \\
1.66 \\
1.51 \\
1.53\end{array}$ & $\begin{array}{l}1.16 \\
1.04 \\
1.15 \\
1.28 \\
1.40\end{array}$ & $\begin{array}{l}2.79 \\
2.95 \\
3.04 \\
3.11 \\
3.19\end{array}$ & $\begin{array}{l}5.07 \\
4.95 \\
2.78 \\
2.79 \\
3.13\end{array}$ & $\begin{array}{l}5.65 \\
5.71 \\
5.84 \\
5.92 \\
6.02\end{array}$ & $\begin{array}{l}6.92 \\
6.90 \\
6.92 \\
6.99 \\
6.92\end{array}$ & $\begin{array}{l}7.30 \\
7.50 \\
7.56 \\
7.54 \\
7.60\end{array}$ & $\begin{array}{l}2.74 \\
2.72 \\
2.63 \\
2.61 \\
2.81\end{array}$ & $\begin{array}{l}3.19 \\
3.31 \\
3.22 \\
3.33 \\
3.44\end{array}$ & $\begin{array}{l}1.91 \\
2.05 \\
2.04 \\
1.97 \\
2.07\end{array}$ & $\begin{array}{l}2.36 \\
2.38 \\
2.38 \\
2.43 \\
2.46\end{array}$ \\
\hline $\begin{array}{l}16 \\
17 \\
18 \\
19 \\
20\end{array}$ & $\begin{array}{l}3.40 \\
2.59 \\
2.51 \\
2.48 \\
2.80\end{array}$ & $\begin{array}{l}1.68 \\
1.66 \\
1.71 \\
1.82 \\
1.97\end{array}$ & $\begin{array}{l}1.03 \\
1.08 \\
1.25 \\
1.51 \\
1.73\end{array}$ & $\begin{array}{l}3.27 \\
3.35 \\
3.44 \\
3.52 \\
3.62\end{array}$ & $\begin{array}{l}3.45 \\
3.70 \\
3.88 \\
4.03 \\
4.18\end{array}$ & $\begin{array}{l}6.04 \\
6.03 \\
6.08 \\
6.11 \\
6.18\end{array}$ & $\begin{array}{l}7.04 \\
7.02 \\
6.98 \\
7.00 \\
7.19\end{array}$ & $\begin{array}{l}7.48 \\
7.45 \\
7.46 \\
7.41 \\
7.45\end{array}$ & $\begin{array}{l}2.95 \\
2.97 \\
2.94 \\
3.21 \\
3.26\end{array}$ & $\begin{array}{l}3.33 \\
3.37 \\
3.49 \\
3.45 \\
3.29\end{array}$ & $\begin{array}{l}2.16 \\
2.31 \\
2.38 \\
2.36 \\
2.48\end{array}$ & $\begin{array}{l}2.56 \\
2.67 \\
2.75 \\
2.77 \\
2.72\end{array}$ \\
\hline $\begin{array}{l}21 \\
22 \\
23 \\
24 \\
25\end{array}$ & $\begin{array}{l}3.06 \\
3.19 \\
3.21 \\
3.16 \\
3.10\end{array}$ & $\begin{array}{l}2.15 \\
1.96 \\
2.04 \\
1.91 \\
1.91\end{array}$ & $\begin{array}{l}1.87 \\
2.03 \\
2.16 \\
2.23 \\
2.26\end{array}$ & $\begin{array}{l}3.73 \\
3.81 \\
3.90 \\
3.99 \\
4.06\end{array}$ & $\begin{array}{l}4.28 \\
4.33 \\
4.41 \\
4.51 \\
4.66\end{array}$ & $\begin{array}{l}6.30 \\
6.31 \\
6.26 \\
6.26 \\
6.33\end{array}$ & $\begin{array}{l}7.20 \\
7.23 \\
7.24 \\
7.25 \\
7.25\end{array}$ & $\begin{array}{l}7.55 \\
7.51 \\
7.62 \\
7.80 \\
7.73\end{array}$ & $\begin{array}{l}3.27 \\
3.25 \\
3.24 \\
3.20 \\
3.46\end{array}$ & $\begin{array}{l}3.15 \\
3.15 \\
3.21 \\
3.27 \\
3.29\end{array}$ & $\begin{array}{l}1.84 \\
1.53 \\
1.60 \\
1.65 \\
1.79\end{array}$ & $\begin{array}{l}2.83 \\
2.91 \\
2.91 \\
2.93 \\
2.91\end{array}$ \\
\hline $\begin{array}{l}26 \\
27 \\
28 \\
29 \\
30 \\
31\end{array}$ & $\begin{array}{r}3.25 \\
3.34 \\
3.33 \\
3.33 \\
3.42 \\
-.-\end{array}$ & $\begin{array}{l}2.14 \\
2.31 \\
1.54 \\
1.47 \\
1.64 \\
1.67\end{array}$ & $\begin{array}{r}2.29 \\
2.33 \\
2.37 \\
2.39 \\
2.47 \\
-.-\end{array}$ & $\begin{array}{l}4.13 \\
4.19 \\
4.23 \\
4.28 \\
4.36 \\
4.42\end{array}$ & $\begin{array}{l}4.71 \\
4.80 \\
4.97 \\
5.05 \\
5.07 \\
5.16\end{array}$ & $\begin{array}{r}6.31 \\
6.29 \\
6.36 \\
6.46 \\
6.54 \\
. \ldots\end{array}$ & $\begin{array}{l}7.25 \\
7.17 \\
7.14 \\
7.23 \\
7.21 \\
7.23\end{array}$ & $\begin{array}{r}7.68 \\
6.44 \\
4.53 \\
4.46 \\
4.62 \\
-.-\end{array}$ & $\begin{array}{l}3.53 \\
2.99 \\
2.57 \\
2.58 \\
2.56 \\
2.61\end{array}$ & $\begin{array}{l}3.06 \\
2.54 \\
2.32 \\
2.09 \\
2.07 \\
1.93\end{array}$ & $\begin{array}{c}1.86 \\
1.88 \\
1.91 \\
- \\
- \\
-\end{array}$ & $\begin{array}{l}2.60 \\
2.61 \\
2.76 \\
2.79 \\
2.77 \\
2.65\end{array}$ \\
\hline
\end{tabular}




\section{GROUND WATER LEVELS}

LE FLORE COUNTY

351002094314401. LOCAL NUMBER, 08N-26E-14 ACC 1.

LOCATION.--LAT 35 10'02", LONG 094 29'15", HYDROLOGIC UNIT 11110105, OWNER: BUREAU OF LAND

MANAGEMENT .

AOUIFER .--ATOKA FORMATION.

WELL CHARACTERISTICS.--DRILLED TLST HOLE, DIAMETER 6 IN (0.15M), DEPTH 277 FT (84.4M).

DATUM.--MEASURING POINT: TOP OF CASING $1.60 \mathrm{FT}(0.48 \mathrm{M})$ ABOVE LAND-SURFACE DATUM.

REMARKS.--TEST HOLE NO. 17.

PLRIOD OF RECORD.--1980 TO CURRENT YEAR.

EXTREMES FOR PERIOD OF RECORD.--HIGHEST WATER LEVEL, 1.54 FT (0.469M) ABOVE LAND-SURFACE DATUM.

JUNE 8,1981 ; LOWEST WATER LEVEL, $-3.43 \mathrm{FT}(1.045 \mathrm{M})$ BELOW LAND-SURFACE DATUM, NOV. 16, 1980.

WATER LEVEL, IN FEET ABOVE OR BELOW (-) LAND-SURFACE DATUM, CLIMATIC YEAR APRIL 1982 TO MARCH 1983 MEAN VALUES

\begin{tabular}{|c|c|c|c|c|c|c|c|c|c|c|c|c|}
\hline DAY & APR & MAY & JUN & JUL & AUG & SEP & OCT & NOV & $\mathrm{DEC}$ & JAN & FEB & MAR \\
\hline $\begin{array}{l}1 \\
2 \\
3 \\
4 \\
5\end{array}$ & $\begin{array}{l}1.07 \\
1.22 \\
1.09 \\
0.99 \\
1.06\end{array}$ & $\begin{array}{l}0.26 \\
0.24 \\
0.24 \\
0.23 \\
0.21\end{array}$ & $\begin{array}{l}1.54 \\
1.54 \\
1.53 \\
1.53 \\
1.53\end{array}$ & $\begin{array}{l}1.29 \\
1.19 \\
1.13 \\
1.04 \\
0.95\end{array}$ & $\begin{array}{l}-0.49 \\
-0.50 \\
-0.52 \\
-0.53 \\
-0.56\end{array}$ & $\begin{array}{l}-0.63 \\
-0.63 \\
-0.66 \\
-0.70 \\
-0.71\end{array}$ & $\begin{array}{l}-1.12 \\
-1.11 \\
-1.11 \\
-1.10 \\
-1.10\end{array}$ & $\begin{array}{l}1.16 \\
1.13 \\
1.14 \\
1.17 \\
1.17\end{array}$ & $\begin{array}{r}0.45 \\
0.41 \\
0.18 \\
-0.05 \\
-0.16\end{array}$ & $\begin{array}{l}-1.06 \\
-1.08 \\
-1.12 \\
-1.12 \\
-1.12\end{array}$ & $\begin{array}{l}-1.69 \\
-1.78 \\
-1.61 \\
-1.39 \\
-1.37\end{array}$ & $\begin{array}{l}-1.70 \\
-1.70 \\
-1.70 \\
-1.82 \\
-2.09\end{array}$ \\
\hline $\begin{array}{r}6 \\
7 \\
8 \\
9 \\
10\end{array}$ & $\begin{array}{l}0.88 \\
0.75 \\
0.80 \\
0.90 \\
0.76\end{array}$ & $\begin{array}{l}0.21 \\
0.17 \\
0.12 \\
0.07 \\
0.05\end{array}$ & $\begin{array}{l}1.53 \\
1.54 \\
1.54 \\
1.54 \\
1.54\end{array}$ & $\begin{array}{l}0.86 \\
0.80 \\
0.86 \\
0.89 \\
0.77\end{array}$ & $\begin{array}{l}-0.58 \\
-0.58 \\
-0.58 \\
-0.55 \\
-0.56\end{array}$ & $\begin{array}{l}-0.71 \\
-0.73 \\
-0.73 \\
-0.74 \\
-0.74\end{array}$ & $\begin{array}{r}-1.10 \\
-1.10 \\
-1.08 \\
0.94 \\
0.94\end{array}$ & $\begin{array}{l}1.17 \\
1.17 \\
1.17 \\
1.18 \\
1.18\end{array}$ & $\begin{array}{l}-0.10 \\
-0.06 \\
-0.05 \\
-0.06 \\
-0.25\end{array}$ & $\begin{array}{l}-1.12 \\
-1.13 \\
-1.13 \\
-1.15 \\
-1.18\end{array}$ & $\begin{array}{l}-1.44 \\
-1.44 \\
-1.43 \\
-1.42 \\
-1.42\end{array}$ & $\begin{array}{l}-2.13 \\
-2.01 \\
-1.92 \\
-1.88 \\
-1.87\end{array}$ \\
\hline $\begin{array}{l}11 \\
12 \\
13 \\
14 \\
15\end{array}$ & $\begin{array}{l}0.68 \\
0.64 \\
0.73 \\
0.73 \\
0.62\end{array}$ & $\begin{array}{l}0.02 \\
0.04 \\
0.53 \\
0.46 \\
1.07\end{array}$ & $\begin{array}{l}1.54 \\
1.54 \\
1.54 \\
1.54 \\
1.54\end{array}$ & $\begin{array}{l}0.67 \\
0.58 \\
0.53 \\
0.48 \\
0.41\end{array}$ & $\begin{array}{l}-0.55 \\
-0.50 \\
-0.50 \\
-0.51 \\
-0.51\end{array}$ & $\begin{array}{l}-0.74 \\
-0.74 \\
-0.76 \\
-0.79 \\
-0.82\end{array}$ & $\begin{array}{l}0.96 \\
0.97 \\
0.97 \\
0.97 \\
0.98\end{array}$ & $\begin{array}{l}1.13 \\
1.09 \\
1.29 \\
1.20 \\
1.28\end{array}$ & $\begin{array}{l}-0.46 \\
-0.50 \\
-0.55 \\
-0.68 \\
-0.65\end{array}$ & $\begin{array}{l}-1.16 \\
-1.04 \\
-1.06 \\
-1.09 \\
-1.02\end{array}$ & $\begin{array}{l}-1.39 \\
-1.29 \\
-1.27 \\
-1.37 \\
-1.38\end{array}$ & $\begin{array}{l}-1.85 \\
-1.85 \\
-1.91 \\
-1.93 \\
-1.94\end{array}$ \\
\hline $\begin{array}{l}16 \\
17 \\
18 \\
19 \\
20\end{array}$ & $\begin{array}{l}0.62 \\
0.62 \\
0.50 \\
0.48 \\
0.64\end{array}$ & $\begin{array}{l}1.08 \\
1.07 \\
1.05 \\
1.05 \\
1.04\end{array}$ & $\begin{array}{l}1.54 \\
1.54 \\
1.54 \\
1.54 \\
1.54\end{array}$ & $\begin{array}{l}0.35 \\
0.27 \\
0.21 \\
0.14 \\
0.05\end{array}$ & $\begin{array}{l}-0.53 \\
-0.55 \\
-0.59 \\
-0.60 \\
-0.61\end{array}$ & $\begin{array}{l}-0.84 \\
-0.85 \\
-0.84 \\
-0.86 \\
-0.87\end{array}$ & $\begin{array}{l}1.00 \\
1.03 \\
1.03 \\
1.03 \\
1.12\end{array}$ & $\begin{array}{l}1.19 \\
1.17 \\
1.17 \\
1.17 \\
1.17\end{array}$ & $\begin{array}{l}-0.59 \\
-0.59 \\
-0.75 \\
-0.72 \\
-0.66\end{array}$ & $\begin{array}{l}-1.02 \\
-1.08 \\
-1.05 \\
-1.02 \\
-1.07\end{array}$ & $\begin{array}{l}-1.37 \\
-1.37 \\
-1.37 \\
-1.42 \\
-1.40\end{array}$ & $\begin{array}{l}-1.94 \\
-1.94 \\
-1.93 \\
-1.93 \\
-1.93\end{array}$ \\
\hline $\begin{array}{l}21 \\
22 \\
23 \\
24 \\
25\end{array}$ & $\begin{array}{l}0.63 \\
0.47 \\
0.39 \\
0.38 \\
0.46\end{array}$ & $\begin{array}{l}1.10 \\
1.22 \\
1.26 \\
1.31 \\
1.40\end{array}$ & $\begin{array}{l}1.54 \\
1.54 \\
1.54 \\
1.54 \\
1.54\end{array}$ & $\begin{array}{r}0.03 \\
-0.03 \\
-0.10 \\
-0.17 \\
-0.24\end{array}$ & $\begin{array}{l}-0.61 \\
-0.61 \\
-0.61 \\
-0.61 \\
-0.63\end{array}$ & $\begin{array}{l}-0.92 \\
-0.96 \\
-0.94 \\
-0.92 \\
-0.93\end{array}$ & $\begin{array}{l}1.18 \\
1.19 \\
1.19 \\
1.19 \\
1.19\end{array}$ & $\begin{array}{l}1.21 \\
1.22 \\
1.10 \\
1.24 \\
1.26\end{array}$ & $\begin{array}{l}-0.66 \\
-0.70 \\
-0.70 \\
-0.82 \\
-0.93\end{array}$ & $\begin{array}{l}-1.14 \\
-1.16 \\
-1.16 \\
-1.16 \\
-1.14\end{array}$ & $\begin{array}{l}-1.39 \\
-1.62 \\
-1.71 \\
-1.71 \\
-1.69\end{array}$ & $\begin{array}{l}-1.90 \\
-1.85 \\
-1.85 \\
-1.85 \\
-1.85\end{array}$ \\
\hline $\begin{array}{l}26 \\
27 \\
28 \\
29 \\
30 \\
31\end{array}$ & $\begin{array}{r}0.56 \\
0.53 \\
0.36 \\
0.36 \\
0.34 \\
.--\end{array}$ & $\begin{array}{l}1.43 \\
1.41 \\
1.49 \\
1.53 \\
1.54 \\
1.54\end{array}$ & $\begin{array}{l}1.54 \\
1.54 \\
1.54 \\
1.51 \\
1.41 \\
.--\end{array}$ & $\begin{array}{l}-0.30 \\
-0.35 \\
-0.38 \\
-0.38 \\
-0.40 \\
-0.46\end{array}$ & $\begin{array}{l}-0.65 \\
-0.65 \\
-0.65 \\
-0.65 \\
-0.63 \\
-0.63\end{array}$ & $\begin{array}{r}-0.90 \\
-0.93 \\
-0.95 \\
-1.02 \\
-1.09 \\
--\end{array}$ & $\begin{array}{l}1.19 \\
1.19 \\
1.17 \\
1.16 \\
1.16 \\
1.16\end{array}$ & $\begin{array}{r}1.22 \\
0.94 \\
0.53 \\
0.45 \\
0.45 \\
-.-\end{array}$ & $\begin{array}{l}-0.87 \\
-1.01 \\
-1.17 \\
-1.06 \\
-1.06 \\
-1.06\end{array}$ & $\begin{array}{l}-1.18 \\
-1.25 \\
-1.33 \\
-1.46 \\
-1.48 \\
-1.49\end{array}$ & $\begin{array}{r}-1.68 \\
-1.67 \\
-1.69 \\
--- \\
--- \\
---\end{array}$ & $\begin{array}{l}-1.94 \\
-1.99 \\
-1.82 \\
-1.80 \\
-1.80 \\
-1.86\end{array}$ \\
\hline
\end{tabular}


LE FLORE COUNTY -- CONTINUED

351122094403901. LOCAL NUMBER, 08N-25E-04 CDC 1.

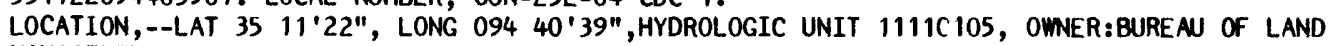
MANAGEMENT.

AQUIFER.--HARTSHORNE SANDSTONE.

WELL CHARACTERISTICS.--DRILLED TEST HOLE, DIAMETER 6 IN (0.15M), DEPTH 110 FT (33.5M).

DATUM.--MEASURING POINT: TOP OF CASING $1.40 \mathrm{FT}(0.36 \mathrm{M})$ ABOVE LAND-SURFACE DATUM.

REMARKS.--TEST HOLE NO. 11.

PERIOD OF RECORD. -- 1980 TO CURRENT YEAR.

EXTREMES FOR PERIOD OF RECORD.--HIGHEST WATER LEVEL, 5.26 FT (1.603M) BELOW LAND-SURFACE DATUM.

JUNE 20, 1982; LOWEST, 13.96 FT (4.255M) BELOW LAND-SURFACE DATUM, AUG. 9, 1980.

WATER LEVEL, IN FEET BELOW LAND SURFACE DATUM, CLIMATIC YEAR APRIL 1982 TO MARCH 1983 MEAN VALUES

\begin{tabular}{|c|c|c|c|c|c|c|c|c|c|c|c|c|}
\hline DAY & APR & MAY & JUN & JUL & AUG & SEP & OCT & NOV & DEC & JAN & FEB & MAR \\
\hline $\begin{array}{l}1 \\
2 \\
3 \\
4 \\
5\end{array}$ & $\begin{array}{l}6.22 \\
5.81 \\
6.19 \\
6.11 \\
5.75\end{array}$ & $\begin{array}{l}6.63 \\
6.74 \\
6.42 \\
6.49 \\
6.28\end{array}$ & $\begin{array}{l}5.95 \\
5.46 \\
5.61 \\
5.48 \\
5.93\end{array}$ & $\begin{array}{l}5.97 \\
6.28 \\
6.44 \\
6.60 \\
6.38\end{array}$ & $\begin{array}{l}7.30 \\
7.25 \\
7.29 \\
7.25 \\
6.88\end{array}$ & $\begin{array}{l}8.61 \\
8.98 \\
8.77 \\
9.12 \\
8.94\end{array}$ & $\begin{array}{r}9.66 \\
9.72 \\
10.00 \\
9.79 \\
9.66\end{array}$ & $\begin{array}{l}9.43 \\
9.21 \\
9.51 \\
9.35 \\
9.46\end{array}$ & $\begin{array}{l}7.85 \\
7.42 \\
7.42 \\
7.88 \\
7.87\end{array}$ & $\begin{array}{l}6.84 \\
6.81 \\
6.87 \\
6.49 \\
6.57\end{array}$ & $\begin{array}{l}6.40 \\
6.21 \\
6.82 \\
7.00 \\
7.16\end{array}$ & $\begin{array}{l}6.38 \\
6.38 \\
6.38 \\
6.11 \\
6.36\end{array}$ \\
\hline $\begin{array}{r}6 \\
7 \\
8 \\
9 \\
10\end{array}$ & $\begin{array}{l}6.28 \\
6.16 \\
5.83 \\
6.32 \\
6.61\end{array}$ & $\begin{array}{l}6.33 \\
6.58 \\
6.92 \\
6.51 \\
6.38\end{array}$ & $\begin{array}{l}5.75 \\
5.46 \\
5.54 \\
5.44 \\
5.69\end{array}$ & $\begin{array}{l}6.21 \\
6.47 \\
6.50 \\
6.30 \\
5.99\end{array}$ & $\begin{array}{l}7.10 \\
7.63 \\
7.42 \\
7.59 \\
7.88\end{array}$ & $\begin{array}{l}8.99 \\
8.90 \\
8.76 \\
9.06 \\
8.90\end{array}$ & $\begin{array}{r}9.78 \\
9.64 \\
9.49 \\
9.59 \\
10.14\end{array}$ & $\begin{array}{l}9.55 \\
9.68 \\
9.55 \\
9.26 \\
9.10\end{array}$ & $\begin{array}{l}7.57 \\
7.53 \\
7.54 \\
7.73 \\
7.67\end{array}$ & $\begin{array}{l}6.54 \\
6.65 \\
6.51 \\
6.61 \\
6.33\end{array}$ & $\begin{array}{l}6.85 \\
6.83 \\
6.94 \\
6.58 \\
6.59\end{array}$ & $\begin{array}{l}6.42 \\
6.11 \\
6.19 \\
6.36 \\
6.45\end{array}$ \\
\hline $\begin{array}{l}11 \\
12 \\
13 \\
14 \\
15\end{array}$ & $\begin{array}{l}6.58 \\
6.31 \\
6.10 \\
6.13 \\
6.12\end{array}$ & $\begin{array}{l}6.41 \\
6.32 \\
6.22 \\
6.14 \\
6.25\end{array}$ & $\begin{array}{l}5.78 \\
5.55 \\
5.72 \\
5.86 \\
5.83\end{array}$ & $\begin{array}{l}5.97 \\
6.08 \\
6.37 \\
6.39 \\
6.15\end{array}$ & $\begin{array}{l}7.87 \\
7.93 \\
7.96 \\
8.13 \\
8.40\end{array}$ & $\begin{array}{l}9.13 \\
9.03 \\
8.80 \\
9.32 \\
9.38\end{array}$ & $\begin{array}{r}9.85 \\
9.71 \\
9.79 \\
9.74 \\
10.13\end{array}$ & $\begin{array}{l}9.41 \\
9.12 \\
9.48 \\
9.68 \\
9.59\end{array}$ & $\begin{array}{l}7.49 \\
7.59 \\
7.23 \\
6.85 \\
6.75\end{array}$ & $\begin{array}{l}6.65 \\
7.06 \\
6.80 \\
6.59 \\
7.01\end{array}$ & $\begin{array}{l}6.66 \\
7.41 \\
7.02 \\
6.91 \\
6.72\end{array}$ & $\begin{array}{l}6.75 \\
6.67 \\
6.34 \\
6.41 \\
6.48\end{array}$ \\
\hline $\begin{array}{l}16 \\
17 \\
18 \\
19 \\
20\end{array}$ & $\begin{array}{l}6.61 \\
6.61 \\
6.77 \\
6.36 \\
6.35\end{array}$ & $\begin{array}{l}6.42 \\
6.01 \\
5.95 \\
5.74 \\
5.98\end{array}$ & $\begin{array}{l}6.06 \\
5.81 \\
5.54 \\
5.32 \\
5.26\end{array}$ & $\begin{array}{l}6.19 \\
6.46 \\
6.38 \\
6.29 \\
6.93\end{array}$ & $\begin{array}{l}8.38 \\
8.29 \\
8.31 \\
8.12 \\
8.46\end{array}$ & $\begin{array}{l}9.02 \\
8.78 \\
9.07 \\
9.33 \\
9.21\end{array}$ & $\begin{array}{r}9.88 \\
10.04 \\
9.71 \\
9.59 \\
9.60\end{array}$ & $\begin{array}{l}8.99 \\
8.97 \\
8.99 \\
8.97 \\
9.06\end{array}$ & $\begin{array}{l}7.24 \\
7.09 \\
6.83 \\
6.86 \\
6.74\end{array}$ & $\begin{array}{l}7.11 \\
6.72 \\
6.77 \\
7.00 \\
6.91\end{array}$ & $\begin{array}{l}6.61 \\
6.87 \\
6.93 \\
7.35 \\
7.27\end{array}$ & $\begin{array}{l}6.31 \\
6.53 \\
6.71 \\
6.84 \\
6.86\end{array}$ \\
\hline $\begin{array}{l}21 \\
22 \\
23 \\
24 \\
25\end{array}$ & $\begin{array}{l}6.64 \\
7.08 \\
6.75 \\
6.57 \\
6.69\end{array}$ & $\begin{array}{l}6.00 \\
6.03 \\
5.97 \\
5.69 \\
5.51\end{array}$ & $\begin{array}{l}5.64 \\
5.98 \\
6.10 \\
6.41 \\
6.38\end{array}$ & $\begin{array}{l}6.75 \\
6.58 \\
6.63 \\
6.70 \\
6.97\end{array}$ & $\begin{array}{l}8.84 \\
8.84 \\
8.58 \\
8.54 \\
8.58\end{array}$ & $\begin{array}{r}9.03 \\
9.10 \\
9.26 \\
9.42 \\
10.57\end{array}$ & $\begin{array}{r}9.87 \\
9.69 \\
10.13 \\
10.38 \\
9.95\end{array}$ & $\begin{array}{l}9.31 \\
8.94 \\
8.79 \\
9.30 \\
9.55\end{array}$ & $\begin{array}{l}6.81 \\
6.78 \\
6.84 \\
6.66 \\
6.98\end{array}$ & $\begin{array}{l}6.81 \\
6.80 \\
7.17 \\
6.92 \\
6.94\end{array}$ & $\begin{array}{l}6.93 \\
7.00 \\
6.66 \\
6.75 \\
6.81\end{array}$ & $\begin{array}{l}6.71 \\
6.67 \\
6.76 \\
6.66 \\
7.02\end{array}$ \\
\hline $\begin{array}{l}26 \\
27 \\
28 \\
29 \\
30 \\
31\end{array}$ & $\begin{array}{r}6.50 \\
6.47 \\
6.40 \\
6.32 \\
6.31 \\
. .-\end{array}$ & $\begin{array}{l}5.55 \\
5.52 \\
5.48 \\
5.47 \\
6.11 \\
5.79\end{array}$ & $\begin{array}{l}6.06 \\
5.83 \\
5.73 \\
5.77 \\
5.82 \\
. .-\end{array}$ & $\begin{array}{l}6.94 \\
7.06 \\
6.92 \\
6.77 \\
6.66 \\
7.07\end{array}$ & $\begin{array}{l}8.48 \\
8.98 \\
9.15 \\
9.08 \\
8.74 \\
8.91\end{array}$ & $\begin{array}{r}10.55 \\
9.43 \\
9.34 \\
9.34 \\
9.99 \\
. .-\end{array}$ & $\begin{array}{r}9.75 \\
9.68 \\
9.39 \\
9.39 \\
9.67 \\
10.04\end{array}$ & $\begin{array}{r}9.30 \\
9.34 \\
8.63 \\
8.06 \\
7.99 \\
. .-\end{array}$ & $\begin{array}{l}7.43 \\
6.92 \\
6.90 \\
7.28 \\
7.04 \\
6.66\end{array}$ & $\begin{array}{l}6.80 \\
7.14 \\
7.04 \\
6.80 \\
7.14 \\
6.86\end{array}$ & $\begin{array}{r}7.07 \\
6.88 \\
6.55 \\
. .- \\
\ldots- \\
.--\end{array}$ & $\begin{array}{l}6.65 \\
6.69 \\
6.95 \\
6.87 \\
6.79 \\
6.53\end{array}$ \\
\hline
\end{tabular}


GROUND WATER LEVELS

\section{LINCOLN COUNTY}

354442096400801. LOCAL NUMBER, 15N-06E-29 AAA 1

LOCATION.--LAT 35 44'42", LONG 096 40'08", HYDROLOGIC UNIT 11100303, OWNER: CITY OF STROUD. AQUIFER.--VAMOOSA FORMATION.

WELL CHARACTERISTICS.--DRILLED UNUSED PUBLIC SUPPLY WELL, DIAMETER 6 IN (0.15M), DEPTH 339 FT (103.3M).

DATUM.--ALTITUDE OF LAND-SURFACE DATUM IS $890 \mathrm{FT}$ (271M). MEASURING POINT: TOP OF CASING $1.00 \mathrm{FT}(0.30 \mathrm{M})$ ABOVE LAND-SURFACE DATUM.

PERIOD OF RECORD.--1977 TO CURRENT YEAR.

EXTREMES FOR PERIOD OF RECORD.--HIGHEST WATER LEVEL, 163.03 FT (49.692M) BELOW LAND-SURFACE DATUM, NOV. 30, 1982; LOWEST, 184.01 FT (56.086M) BELOW LAND-SURFACE DATUM, NOV. 10, 1977.

WATER LEVEL, IN FEET BELOW LAND SURFACE DATUM, CLIMATIC YEAR APRIL 1982 TO MARCH 1983 MEAN VALUES

\begin{tabular}{|c|c|c|c|c|c|c|c|c|c|c|c|c|}
\hline DAY & APR & MAY & JUN & JUL & AUG & SEP & OCT & NOV & DEC & JAN & FEB & MAR \\
\hline $\begin{array}{l}1 \\
2 \\
3 \\
4 \\
5\end{array}$ & $\begin{array}{l}165.43 \\
164.90 \\
165.09 \\
164.90 \\
164.91\end{array}$ & $\begin{array}{l}165.30 \\
165.30 \\
165.30 \\
165.30 \\
165.30\end{array}$ & $\begin{array}{l}165 \cdot 30 \\
165 \cdot 30 \\
165 \cdot 30 \\
165 \cdot 30 \\
165.30\end{array}$ & $\begin{array}{l}164.09 \\
164.04 \\
164.09 \\
164.04 \\
163.98\end{array}$ & $\begin{array}{l}164.20 \\
163.99 \\
163.81 \\
163.80 \\
163.69\end{array}$ & $\begin{array}{l}164.06 \\
164.06 \\
164.15 \\
164.19 \\
165.22\end{array}$ & $\begin{array}{l}163.54 \\
163.46 \\
163.47 \\
163.41 \\
163.40\end{array}$ & $\begin{array}{l}163.20 \\
163.32 \\
163.49 \\
163.54 \\
163.54\end{array}$ & $\begin{array}{l}163.10 \\
163.09 \\
163.13 \\
163.12 \\
163.12\end{array}$ & $\begin{array}{l}165.15 \\
165.24 \\
165.45 \\
165.34 \\
165.32\end{array}$ & $\begin{array}{l}-- \\
--- \\
--- \\
---\end{array}$ & $\begin{array}{l}--- \\
--- \\
--- \\
--\end{array}$ \\
\hline $\begin{array}{r}6 \\
7 \\
8 \\
9 \\
10\end{array}$ & $\begin{array}{l}165.11 \\
164.89 \\
164.85 \\
164.92 \\
164.95\end{array}$ & $\begin{array}{l}165.30 \\
165.30 \\
165.30 \\
165.30 \\
165.30\end{array}$ & $\begin{array}{l}165.30 \\
165.32 \\
164.26 \\
163.87 \\
164.01\end{array}$ & $\begin{array}{l}163.94 \\
164.00 \\
163.91 \\
163.76 \\
163.69\end{array}$ & $\begin{array}{l}163.76 \\
163.71 \\
163.70 \\
163.77 \\
164.08\end{array}$ & $\begin{array}{l}164.41 \\
164.34 \\
164.19 \\
164.19 \\
163.98\end{array}$ & $\begin{array}{l}163.39 \\
163.42 \\
163.25 \\
163.33 \\
163.46\end{array}$ & $\begin{array}{l}163.46 \\
163.37 \\
163.44 \\
163.45 \\
163.41\end{array}$ & $\begin{array}{l}163.31 \\
163.27 \\
163.62 \\
163.72 \\
163.36\end{array}$ & $\begin{array}{l}164.42 \\
163.93 \\
163.89 \\
163.58 \\
163.53\end{array}$ & $\begin{array}{l}--- \\
--- \\
--- \\
--- \\
---\end{array}$ & $\begin{array}{l}--- \\
--- \\
---\end{array}$ \\
\hline $\begin{array}{l}11 \\
12 \\
13 \\
14 \\
15\end{array}$ & $\begin{array}{l}164.89 \\
164.57 \\
164.86 \\
165.30 \\
165.30\end{array}$ & $\begin{array}{l}165.30 \\
165.30 \\
165.30 \\
165.30 \\
165.30\end{array}$ & $\begin{array}{l}164.00 \\
163.94 \\
163.92 \\
163.83 \\
163.69\end{array}$ & $\begin{array}{l}163.79 \\
163.69 \\
163.73 \\
163.69 \\
163.75\end{array}$ & $\begin{array}{l}164.28 \\
164.16 \\
163.88 \\
163.92 \\
164.11\end{array}$ & $\begin{array}{l}163.80 \\
163.80 \\
163.88 \\
163.96 \\
164.01\end{array}$ & $\begin{array}{l}163.57 \\
163.48 \\
163.50 \\
163.59 \\
163.48\end{array}$ & $\begin{array}{l}163.19 \\
163.45 \\
163.59 \\
163.62 \\
163.69\end{array}$ & $\begin{array}{l}163.30 \\
163.64 \\
163.80 \\
163.5 \\
164.22\end{array}$ & $\begin{array}{l}163.78 \\
163.96 \\
163.71 \\
163.71 \\
163.73\end{array}$ & $\begin{array}{l}--- \\
--- \\
--- \\
--- \\
---\end{array}$ & $\begin{array}{l}--- \\
--- \\
--- \\
---\end{array}$ \\
\hline $\begin{array}{l}16 \\
17 \\
18 \\
19 \\
20\end{array}$ & $\begin{array}{l}165.30 \\
165.30 \\
165.30 \\
165.30 \\
165.30\end{array}$ & $\begin{array}{l}165 \cdot 30 \\
165.30 \\
165.30 \\
165.30 \\
165.30\end{array}$ & $\begin{array}{l}163.85 \\
163.77 \\
163.79 \\
163.92 \\
164.01\end{array}$ & $\begin{array}{l}163.57 \\
163.61 \\
163.67 \\
163.55 \\
163.57\end{array}$ & $\begin{array}{l}164.28 \\
164.29 \\
164.34 \\
164.22 \\
164.20\end{array}$ & $\begin{array}{l}164.05 \\
163.92 \\
163.91 \\
163.79 \\
163.94\end{array}$ & $\begin{array}{l}163.68 \\
163.56 \\
163.47 \\
163.41 \\
163.75\end{array}$ & $\begin{array}{l}163.49 \\
163.28 \\
163.27 \\
163.12 \\
163.11\end{array}$ & $\begin{array}{l}164.40 \\
164.37 \\
164.12 \\
164.41 \\
164.40\end{array}$ & $\begin{array}{r}163.59 \\
163.62 \\
163.68 \\
. .- \\
.-\end{array}$ & $\begin{array}{l}--- \\
--- \\
--- \\
---\end{array}$ & \\
\hline $\begin{array}{l}21 \\
22 \\
23 \\
24 \\
25\end{array}$ & $\begin{array}{l}165.30 \\
165.31 \\
165.30 \\
165.30 \\
165.30\end{array}$ & $\begin{array}{l}165 \cdot 30 \\
165 \cdot 30 \\
165 \cdot 30 \\
165 \cdot 30 \\
165 \cdot 30\end{array}$ & $\begin{array}{l}163.99 \\
163.91 \\
164.06 \\
163.92 \\
163.84\end{array}$ & $\begin{array}{l}163.65 \\
163.79 \\
163.78 \\
163.76 \\
163.98\end{array}$ & $\begin{array}{l}164.09 \\
163.92 \\
163.96 \\
163.88 \\
164.01\end{array}$ & $\begin{array}{l}163.95 \\
163.91 \\
163.74 \\
163.59 \\
163.60\end{array}$ & $\begin{array}{l}163.80 \\
163.77 \\
163.75 \\
163.81 \\
163.75\end{array}$ & $\begin{array}{l}163.28 \\
163.33 \\
163.46 \\
163.76 \\
163.68\end{array}$ & $\begin{array}{l}164.38 \\
164.45 \\
164.38 \\
164.28 \\
164.69\end{array}$ & $\begin{array}{l}--- \\
--- \\
--- \\
---\end{array}$ & $\begin{array}{l}--- \\
--- \\
--- \\
---\end{array}$ & $\begin{array}{l}--- \\
--- \\
--- \\
--\end{array}$ \\
\hline $\begin{array}{l}26 \\
27 \\
28 \\
29 \\
30 \\
31\end{array}$ & $\begin{array}{r}165.30 \\
165.30 \\
165.30 \\
165.30 \\
165.30 \\
. .\end{array}$ & $\begin{array}{l}165.30 \\
165.30 \\
165.30 \\
165.30 \\
165.30 \\
165.30\end{array}$ & $\begin{array}{r}163.82 \\
163.84 \\
163.79 \\
163.86 \\
164.07 \\
\\
.\end{array}$ & $\begin{array}{l}164.76 \\
164.16 \\
163.99 \\
163.82 \\
163.83 \\
164.56\end{array}$ & $\begin{array}{l}163.90 \\
163.86 \\
164.08 \\
164.05 \\
163.95 \\
163.98\end{array}$ & $\begin{array}{r}163.75 \\
163.50 \\
163.44 \\
163.38 \\
163.56 \\
.\end{array}$ & $\begin{array}{l}163.55 \\
163.29 \\
163.26 \\
163.32 \\
163.24 \\
163.26\end{array}$ & $\begin{array}{r}163.58 \\
163.35 \\
163.18 \\
163.10 \\
163.03 \\
\\
.\end{array}$ & $\begin{array}{l}164.85 \\
164.51 \\
164.79 \\
165.06 \\
164.95 \\
165.04\end{array}$ & $\begin{array}{l}--- \\
-\cdots \\
--- \\
-\cdots \\
---\end{array}$ & $\begin{array}{l}--- \\
--- \\
--- \\
--- \\
---\end{array}$ & $\begin{array}{l}--- \\
--- \\
--- \\
--\end{array}$ \\
\hline
\end{tabular}


GROUND WATER LEVELS

\section{MUSKOGEE COUNTY}

351833095155401 . LOCAL NUMBER, 11N-19E-35 BBB 1.

LOCATION.--LAT 35 18'33" LONG 095 15'40", HYDROLOGIC UNIT 11110102 , OWNER: ARCHIE SIPE.

AQUIFER.--MCALESTER FORMATION.

WELL CHARACTERISTICS.--DRILLED WELL , DIAMETER 6 IN $(0.15 \mathrm{M})$, DEPTH 24 FT $(7.31)$.

DATUM.--ALTITUDE OF LAND-SURFACE DATUM IS $571 \mathrm{FT}(174 \mathrm{M})$. MEASURING POINT: TOP OF

CASING $1.00 \mathrm{FT}(0.30 \mathrm{M})$ ABOVE LAND-SURFACE DATUM.

PERIOD OF RECORD--1981 TO CURRENT YEAR.

EXTREMES FOR PERIOD OF RECORD.--HIGHEST WATER LEVEL, 4.89 FT (1.49M) BELOW

LAND-SURFACE DATUM, MAR. 5, 1983; LOWEST, $13.41 \mathrm{FT}(4.09)$ BELOW LAND-SURFACE

DATUM, OCT. 14,1981.

WATER LEVEL, IN FEET BELOW LAND SURFACE DATUM, CLIMATIC YEAR APRIL 1982 TO MARCH 1983

MEAN VALUES

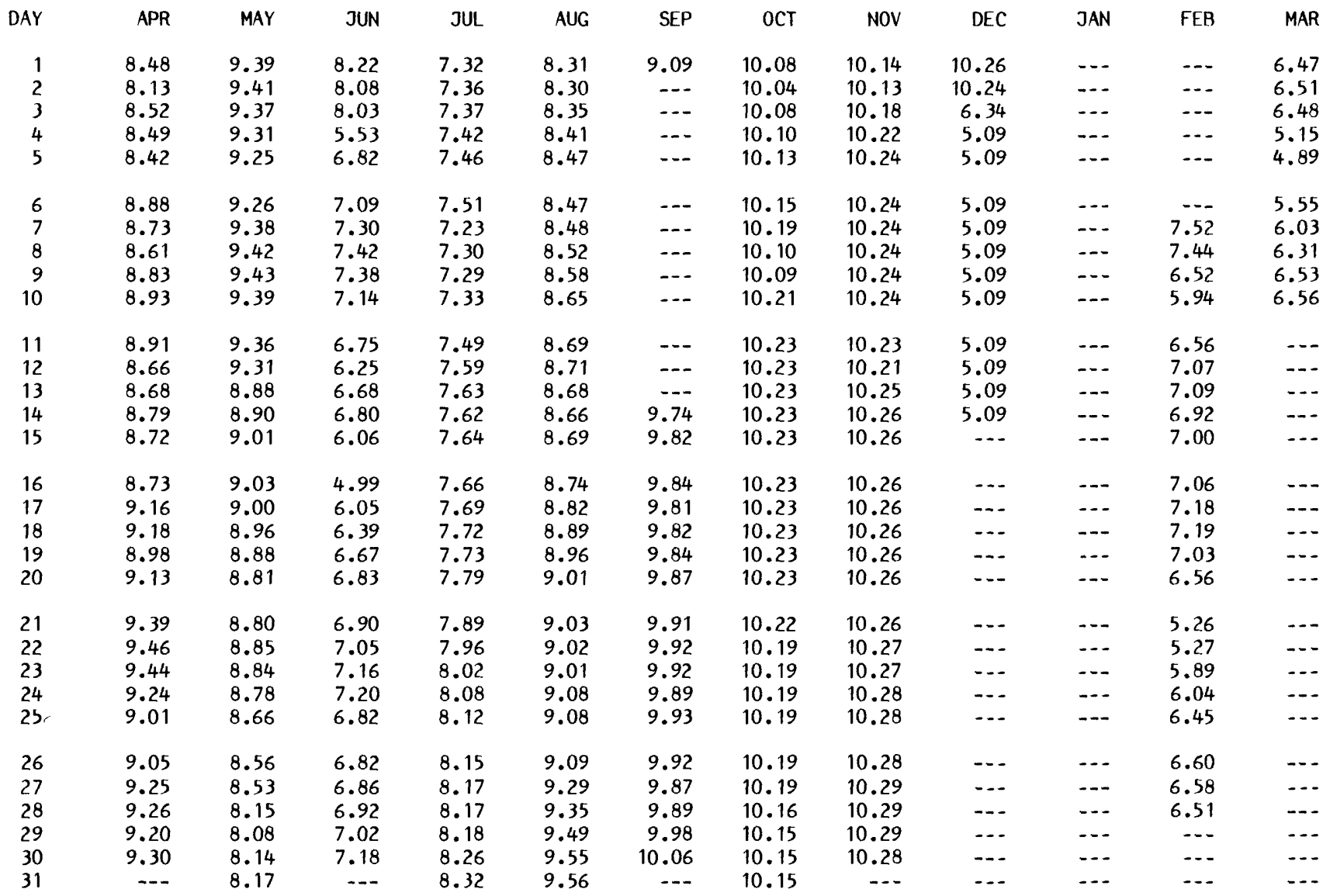


MUSKOGEE COUNTY -- CONTINUED

354613095161001. LOCAL NUMBER, 15N-19E-15 DDD 1.

LOCATION.--LAT 35 46'13", LONG 095 16'10", HYDROLOGIC UNIT 11110102, OWNER: U.S. GEOLOGICAL SURVEY. AQUIFER.--ALLUVIUM.

WELL CHARACTERISTICS.--DRILLED UNUSED WELL, DIAMETER $11 / 4$ IN $(0.03 \mathrm{M})$, DEPTH 29 FT $(8.84 \mathrm{M})$. DATUM.--ALTITUDE OF LAND-SURFACE DATUM IS $509 \mathrm{FT}$ (155M). MEASURING POINT: TOP OF PIPE $2.55 \mathrm{FT}$ (0.78M) ABOVE LAND-SURFACE DATUM.

PERIOD OF RECORD.--1958, 1974 TO CURRENT YEAR.

EXTREMES FOR PERIOD OF RECORD.--HIGHEST WATER LEVEL, 6.24 FT (1.902M) BELOW LAND-SURFACE DATUM, MAY 26, 1975; LOWEST, 15.84 FT (4.828M) BELOW LAND-SURFACE DATUM, AUG. 19, 1980.

WATER LEVELS IN FEET BELOW LAND SURFACE DATUM, CLIMATIC YEAR APRIL 1982 TO MARCH 1983

\begin{tabular}{|c|c|c|c|c|c|c|}
\hline DATE & $\begin{array}{l}\text { WATER } \\
\text { LEVEL }\end{array}$ & DATE & $\begin{array}{l}\text { WATER } \\
\text { LEVEL }\end{array}$ & DATE & $\begin{array}{l}\text { WATER } \\
\text { LEVEL }\end{array}$ & DATE \\
\hline 25,1982 & 11.19 & SEP 14,1982 & 10.87 & ОСТ 13,1982 & 11.04 & FEB 22,1983 \\
\hline
\end{tabular}

GROUND-WATER LEVELS

OKLAHOMA COUNTY

352750097223001. LOCAL NUMBER, 11N-02W-02 ABA 1.

LOCATION.--LAT 35 27'50", LONG 097 22'30", HYDROLOGIC UNIT 11100302, OWNER: MIDWEST CITY, WELL NO.

50.

AQUIFER.--GARBER SANDSTONE.

WELL CHARACTERISTICS.--DRILLED WELL, DIAMETER 11 IN (3.35M), DEPTH 751 FT (229M).

DATUM.--ALTITUDE OF LAND-SURFACE DATUM IS $1202 \mathrm{FT}$ (366M). MEASURING POINT: TOP OF CONCRETE

SLAB $1.5 \mathrm{FT}(0.46 \mathrm{M})$ ABOVE LAND-SURFACE DATUM.

REMARKS.--MEASURE WITH AIRLINE GAGE, AIRLINE IS SET AT $580 \mathrm{FT}$ (177M) BELOW LAND-SURFACE DATUM.

PERIOD OF RECORD.--1976 TO CURRENT YEAR.

EXTREMES FOR PERIOD OF RECORD.-- HIGHEST WATER LEVEL, 208.00 FT (63.398M) BELOW LAND-SURFACE

DATUM, JUNE 4, 1979; LOWEST, 446.02 FT (135.947M) BELOW LAND-SURFACE DATUM, DEC. 08, 1982.

WATER LEVELS IN FEET BELOW LAND SURFACE DATUM, CLIMATIC YEAR APRIL 1982 TO MARCH 1983

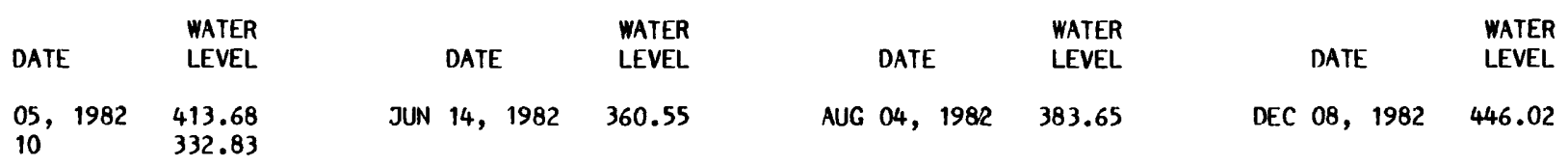


352725097224701. LOCAL NUMBER, 11N-02W-02 BDD 1.

LOCATION.--LAT 35 27'25", LONG 097 22'47", HYDROLOGIC UNIT 11100302, OWNER: MIDWEST CITY, WELL NO. 49.

AQUIFER.--GARBER SANDSTONE.

WELL CHARACTERISTICS.--DRILLED WELL, DIAMETER 11 IN (3.35M), DEPTH 274 FT (83.5M).

DATUM.--ALTITUDE OF LAND-SURFACE DATUM IS 1190 FT (363M). MEASURING POINT: TOP OF CONCRETE

SLAB $1.5 \mathrm{FT}$ (0.46M) ABOVE LAND-SURFACE DATUM.

REMARKS.--MEASURE WITH AIRLINE GAGE; AIRLINE IS SET AT 562 FT (171M) BELOW LAND-SURFACE DATUM. PERIDD OF RECORD. - 1976 TO CURRENT YEAR.

EXTREMES FOR PERIOD OF RECORD.--HIGHEST WATER LEVEL, $104.00 \mathrm{FT}$ (31.699M) BELOW LAND-SURFACE DATUM, FEB. 1, 1979; LOWEST, 273.00 FT (83.2 10M) BELOW LAND-SURFACE DATUM, OCT. 4, 1979.

WATER LEVELS IN FEET BELOW LAND SURFACE DATUM, CLIMATIC YEAR APRIL 1982 TO MARCH 1983

$\begin{array}{lrcccr}\text { DATE } & \begin{array}{l}\text { WATER } \\ \text { LEVEL }\end{array} & \text { DATE } & \begin{array}{c}\text { WATER } \\ \text { LEVEL }\end{array} & \text { DATE } & \begin{array}{r}\text { WATER } \\ \text { LEVEL }\end{array} \\ 14,1982 & 261.70 & \text { DEC 09, 1982 } & 250.15 & \text { JUN 06, } 1983 & 253.84\end{array}$

GROUND-WATER LEVELS

352705097281201. LOCAL NUMBER, 11N-03W-01 CDD 1.

LOCATION.--LAT 35 27'05", LONG 097 28'12", HYDROLOGIC UNIT 11100302, OWNER: OKLAHOMA CITY. AQUIFER.--GARBER SANDSTONE.

WELL CHARACTERISTICS.--DRILLED UNUSED WELL, DIAMETER 8 IN (0.20M), DEPTH 354 FT (108M). DATUM.--ALTITUDE OF LAND-SURFACE DATUM IS $1290 \mathrm{FT}$ (393M). MEASURING POINT: TOP OF CASING $1.3 \mathrm{FT}(0.40 \mathrm{M})$ ABOVE LAND-SURFACE DATUM. PERIOD OF RECORD.--1976 TO CURRENT YEAR.

EXTREMES FOR PERIOD OF RECORD.-- HIGHEST WATER LEVEL, 208.82 FT (63.648M) BELOW LAND-SURFACE DATUM, JUNE 15, 1976; LOWEST, 226.58 FT (69.060M) BELOW LAND-SURFACE DATUM, FEB. $25,1983$.

WATER LEVELS IN FEET BELOW LAND SURFACE DATUM, CLIMATIC YEAR APRIL 1982 TO MARCH 1983

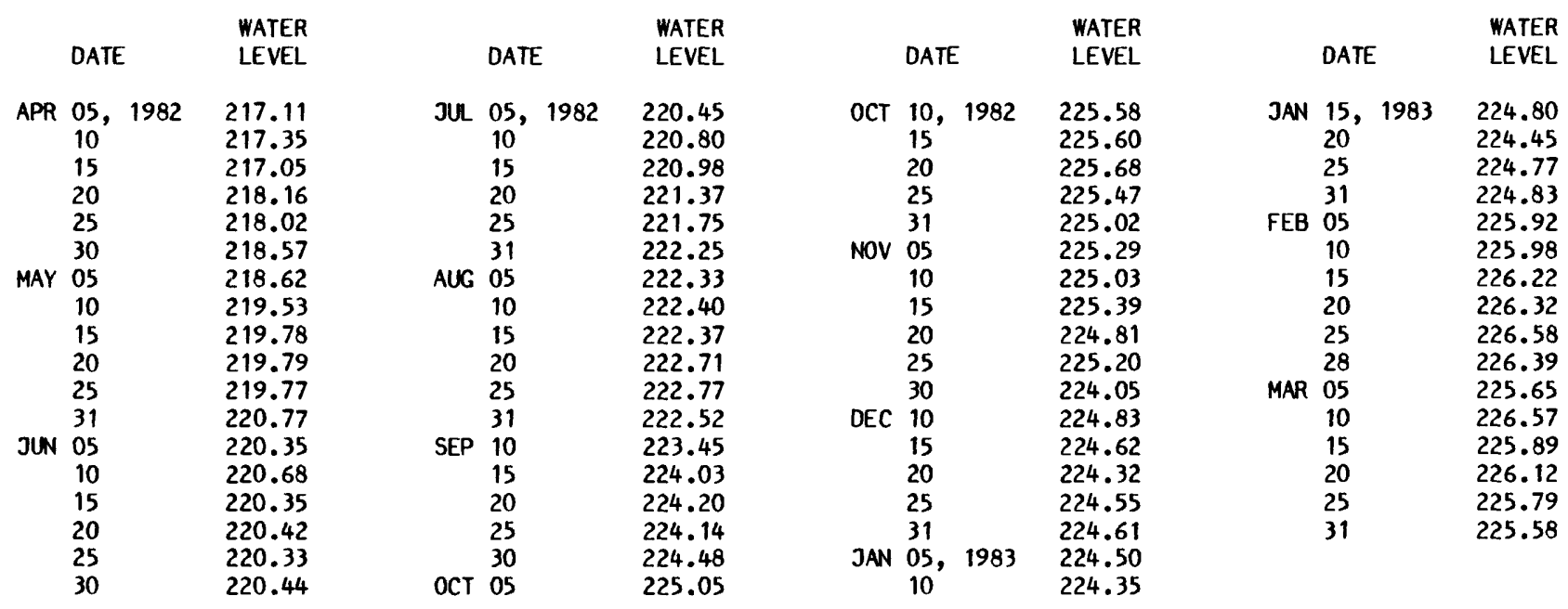


352449097293201. LOCAL NUMBER, 11N-03W-23 BCD 1. LOCATION.--LAT 35 24'49", LONG 097 29'32", HYDROLOGIC LNIT 11100302, OWNER: CITY OF OKLAHOMA CITY. AQUIFER.--GARBER SANDSTONE.

WELL CHARACTERISTICS.--DRILLED UNUSED WELL, DIAMETER 8 IN $(0.20 \mathrm{M})$, DEPTH 26 FT (7.92M). DATUM.--ALTITUDE OF LAND-SURFACE DATUM IS $1250 \mathrm{FT}$ (381M). MEASURING POINT: TOP OF CASING 0.5 FT (0.15M) ABOVE LAND-SURFACE DATUM. PERIOD OF RECORD..-1976 TO CURRENT YEAR.

EXTREMES FOR PERIOD OF RECORD.-- HIGHEST WATER LEVEL, 107.38 FT (32.729M) BELOW LAND-SURFACE DATUM, MARCH 15, 1983; LOWEST, 114.59 FT (34.927M) BELOW LAND-SURFACE DATUM, MAR. 10, 1980.

WATER LEVELS IN FEET BELOW LAND SURFACE DATUM, CLIMATIC YEAR APRIL 1982 TO MARCH 1983

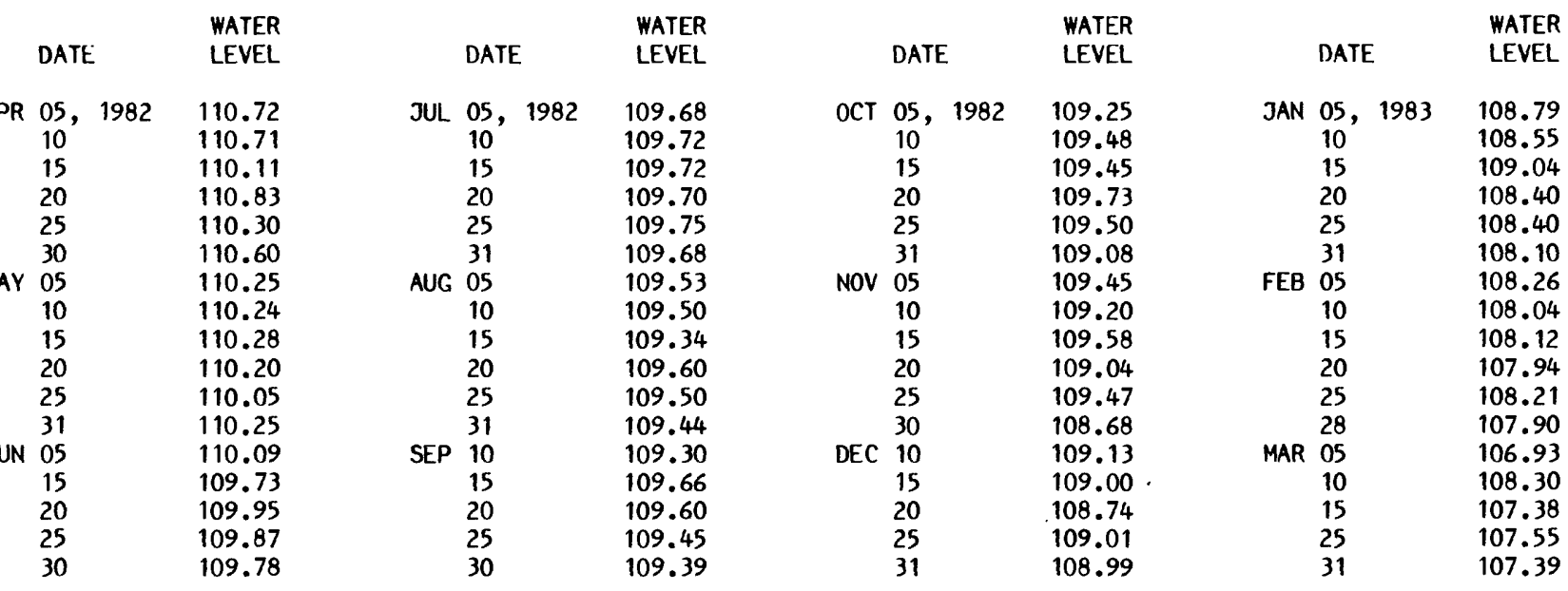

GROUND-WATER LEVELS

352910097232001. LOCAL NUMBER, 12N-02W-26 CBB 1.

LOCATION.--LAT 35 29'10", LONG 097 23'20", HYDROLOGIC UNIT 11100302, OWNER: MIDWEST CITY, WELL NO.

51.

AQUIFER.--GARBER SANDSTONE.

WELL CHARACTERISTICS.--DRILLED WELL, DIAMETER 11 IN (3.35M), DEPTH 748 FT (228M).

DATUM.--ALTITUDE OF LAND-SURFACE DATUM IS $1190 \mathrm{FT}$ (363M). MEASURING POINT: TOP OF CONCRETE

SLAB $1.5 \mathrm{FT}(0.46 \mathrm{M})$ ABOVE LAND-SURFACE DATUM.

REMARKS.--MEASURE WITH AIRLINE GAGE, AIRLINE IS SET AT 578 FT (176M) BELOW LAND-SURFACE DATUM.

PERIOD OF RECORD.-- 1976 TO CURRENT YEAR.

EXTREMES FOR PERIOD OF RECORD. --HIGHEST WATER LEVEL, $189.00 \mathrm{FT}$ (57.607M) BELOW LAND-SURFACE

DATUM, JULY 5, 1979; LOWEST, 360.00 FT (109.728M) BELOW LAND-SURFACE DATUM, JULY 7. 1980.

WATER LEVELS IN FEET BELOW LAND SURFACE DATUM, CLIMATIC YEAR APRIL 1982 TO MARCH 1983

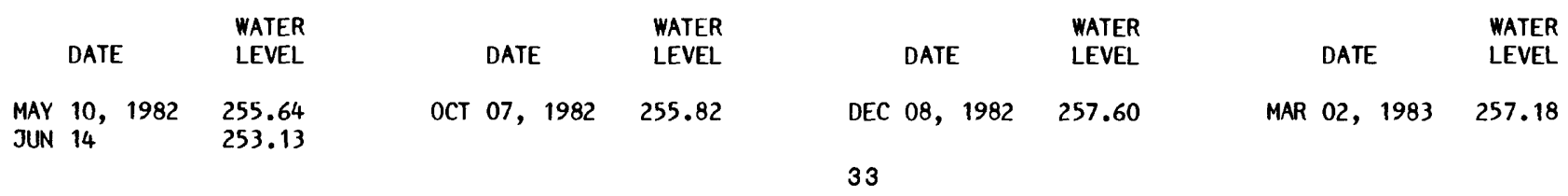


GROUND WATER LEVELS

OSAGE COUNTY

362935096291501. LOCAL NUMBER (REVISED), 23N-09E-10 AAD 1.

LOCATION.--LAT 36 29'35", LONG 096 29'15", HYDROLOGIC UNIT 11070107, OWNER: LESLIE DRUMMOND.

AQUIFER.--VAMOOSA FORMATION.

WELL CHARACTERISTICS.--DRILLED UNUSED WELL, DIAMETER 13 IN $(0.33 \mathrm{M})$, DEPTH 55 FT $(16,8 \mathrm{M})$.

DATUM.--ALTITUDE OF LAND-SURFACE DATUM IS $835 \mathrm{FT}$ (255M). MEASURING POINT: TOP OF CASING

$2.40 \mathrm{FT}$ (0.73M) ABOVE LAND-SURFACE DATUM.

PERIOD OF RECORD.--1971 TO CURRENT YEAR.

EXTREMES FOR PERIOD OF RECORD.--HIGHEST WATER LEVEL, 5.37 FT (1.637M) BELOW LAND-SURFACE

DATUM, JUNE 10, 1975; LOWEST, 9.26 FT (2.822M) BELOW LAND-SURFACE DATUM, AUG. 20, 1972.

WATER LEVEL, IN FEET BELOW LAND SURFACE DATUM, CLIMATIC YEAR APRIL 1982 TO MARCH 1983

MEAN VALUES

\begin{tabular}{|c|c|c|c|c|c|c|c|c|c|c|c|c|}
\hline DAY & APR & MAY & JUN & JUL & AUG & SEP & OCT & NOV & DEC & JAN & FEB & MAR \\
\hline $\begin{array}{l}1 \\
2 \\
3 \\
4 \\
5\end{array}$ & $\begin{array}{l}7.92 \\
7.80 \\
8.19 \\
7.89 \\
8.20\end{array}$ & $\begin{array}{l}8.28 \\
8.24 \\
8.18 \\
8.15 \\
8.14\end{array}$ & $\begin{array}{l}7.37 \\
7.27 \\
7.26 \\
7.28 \\
7.20\end{array}$ & $\begin{array}{l}7.30 \\
7.26 \\
7.26 \\
7.28 \\
7.28\end{array}$ & $\begin{array}{l}7.71 \\
7.70 \\
7.76 \\
7.82 \\
7.87\end{array}$ & $\begin{array}{l}8.28 \\
8.37 \\
8.44 \\
8.40 \\
8.40\end{array}$ & $\begin{array}{l}8.70 \\
8.65 \\
8.70 \\
8.64 \\
8.68\end{array}$ & $\begin{array}{l}8.66 \\
8.80 \\
8.92 \\
8.87 \\
8.83\end{array}$ & $\begin{array}{l}8.39 \\
8.41 \\
8.46 \\
8.36 \\
8.30\end{array}$ & $\begin{array}{l}8.06 \\
8.10 \\
8.09 \\
8.00 \\
8.00\end{array}$ & $\begin{array}{l}7.36 \\
7.49 \\
7.74 \\
7.82 \\
7.59\end{array}$ & $\begin{array}{l}7.34 \\
7.34 \\
7.27 \\
7.10 \\
7.07\end{array}$ \\
\hline $\begin{array}{r}6 \\
7 \\
8 \\
9 \\
10\end{array}$ & $\begin{array}{l}8.21 \\
7.93 \\
8.10 \\
8.18 \\
8.17\end{array}$ & $\begin{array}{l}8.19 \\
8.15 \\
8.12 \\
8.11 \\
8.08\end{array}$ & $\begin{array}{l}7.16 \\
7.24 \\
7.20 \\
7.30 \\
7.46\end{array}$ & $\begin{array}{l}7.32 \\
7.42 \\
7.38 \\
7.30 \\
7.37\end{array}$ & $\begin{array}{l}7.85 \\
7.86 \\
7.85 \\
7.84 \\
7.84\end{array}$ & $\begin{array}{l}8.46 \\
8.48 \\
8.47 \\
8.45 \\
8.40\end{array}$ & $\begin{array}{l}8.72 \\
8.69 \\
8.53 \\
8.73 \\
8.84\end{array}$ & $\begin{array}{l}8.71 \\
8.72 \\
8.78 \\
8.80 \\
8.76\end{array}$ & $\begin{array}{l}8.53 \\
8.49 \\
8.68 \\
8.45 \\
8.20\end{array}$ & $\begin{array}{l}7.93 \\
7.98 \\
7.95 \\
7.80 \\
7.98\end{array}$ & $\begin{array}{l}7.62 \\
7.57 \\
7.45 \\
7.42 \\
7.38\end{array}$ & $\begin{array}{l}7.28 \\
7.47 \\
7.52 \\
7.56 \\
7.54\end{array}$ \\
\hline $\begin{array}{l}11 \\
12 \\
13 \\
14 \\
15\end{array}$ & $\begin{array}{l}8.03 \\
7.89 \\
8.14 \\
8.04 \\
7.94\end{array}$ & $\begin{array}{l}8.08 \\
7.92 \\
7.79 \\
7.82 \\
7.90\end{array}$ & $\begin{array}{l}7.25 \\
7.23 \\
7.26 \\
7.11 \\
7.10\end{array}$ & $\begin{array}{l}7.46 \\
7.48 \\
7.46 \\
7.43 \\
7.45\end{array}$ & $\begin{array}{l}7.87 \\
7.82 \\
7.79 \\
7.83 \\
7.89\end{array}$ & $\begin{array}{l}8.43 \\
8.47 \\
8.53 \\
8.58 \\
8.67\end{array}$ & $\begin{array}{l}8.83 \\
8.78 \\
8.81 \\
8.81 \\
8.76\end{array}$ & $\begin{array}{l}8.52 \\
8.94 \\
8.81 \\
8.87 \\
8.82\end{array}$ & $\begin{array}{l}8.37 \\
8.34 \\
8.09 \\
8.17 \\
8.43\end{array}$ & $\begin{array}{l}8.15 \\
8.08 \\
7.91 \\
8.13 \\
8.11\end{array}$ & $\begin{array}{l}7.59 \\
7.77 \\
7.53 \\
7.39 \\
7.55\end{array}$ & $\begin{array}{c}7.58 \\
-.- \\
-.- \\
-.- \\
-.-\end{array}$ \\
\hline $\begin{array}{l}16 \\
17 \\
18 \\
19 \\
20\end{array}$ & $\begin{array}{l}8.11 \\
8.37 \\
8.12 \\
8.07 \\
8.34\end{array}$ & $\begin{array}{l}7.90 \\
7.72 \\
7.61 \\
7.58 \\
7.52\end{array}$ & $\begin{array}{l}7.28 \\
7.23 \\
7.24 \\
7.35 \\
7.27\end{array}$ & $\begin{array}{l}7.46 \\
7.49 \\
7.52 \\
7.53 \\
7.56\end{array}$ & $\begin{array}{l}7.99 \\
8.02 \\
8.01 \\
8.04 \\
8.08\end{array}$ & $\begin{array}{l}8.55 \\
8.50 \\
8.63 \\
8.55 \\
8.63\end{array}$ & $\begin{array}{l}8.88 \\
8.75 \\
8.72 \\
8.97 \\
8.98\end{array}$ & $\begin{array}{l}8.68 \\
8.71 \\
8.69 \\
8.60 \\
8.72\end{array}$ & $\begin{array}{l}8.40 \\
8.18 \\
8.16 \\
8.35 \\
8.27\end{array}$ & $\begin{array}{l}7.91 \\
8.02 \\
8.07 \\
7.98 \\
7.94\end{array}$ & $\begin{array}{l}7.57 \\
7.62 \\
7.51 \\
7.37 \\
7.62\end{array}$ & $\begin{array}{l}-- \\
-- \\
-- \\
-- \\
--\end{array}$ \\
\hline $\begin{array}{l}21 \\
22 \\
23 \\
24 \\
25\end{array}$ & $\begin{array}{l}8.38 \\
8.33 \\
8.21 \\
8.07 \\
8.04\end{array}$ & $\begin{array}{l}7.55 \\
7.58 \\
7.55 \\
7.45 \\
7.29\end{array}$ & $\begin{array}{l}7.26 \\
7.34 \\
7.35 \\
7.28 \\
7.22\end{array}$ & $\begin{array}{l}7.58 \\
7.60 \\
7.61 \\
7.61 \\
7.64\end{array}$ & $\begin{array}{l}8.08 \\
8.00 \\
8.04 \\
8.09 \\
8.20\end{array}$ & $\begin{array}{l}8.68 \\
8.59 \\
8.51 \\
8.61 \\
8.66\end{array}$ & $\begin{array}{l}8.92 \\
8.91 \\
8.87 \\
8.87 \\
8.85\end{array}$ & $\begin{array}{l}8.82 \\
8.65 \\
8.86 \\
8.94 \\
8.74\end{array}$ & $\begin{array}{l}8.17 \\
8.10 \\
8.07 \\
8.09 \\
8.51\end{array}$ & $\begin{array}{l}7.90 \\
7.92 \\
7.96 \\
8.04 \\
7.96\end{array}$ & $\begin{array}{l}7.58 \\
7.46 \\
7.38 \\
7.40 \\
7.51\end{array}$ & $\begin{array}{l}--- \\
--- \\
-- \\
--- \\
--\end{array}$ \\
\hline $\begin{array}{l}26 \\
27 \\
28 \\
29 \\
30 \\
31\end{array}$ & $\begin{array}{r}8.24 \\
8.28 \\
8.16 \\
8.21 \\
8.31 \\
. .-\end{array}$ & $\begin{array}{l}7.22 \\
7.21 \\
7.05 \\
7.17 \\
7.22 \\
7.41\end{array}$ & $\begin{array}{r}7.20 \\
7.18 \\
7.18 \\
7.23 \\
7.28 \\
.\end{array}$ & $\begin{array}{l}7.66 \\
7.66 \\
7.67 \\
7.67 \\
7.73 \\
7.73\end{array}$ & $\begin{array}{l}8.05 \\
8.15 \\
8.29 \\
8.23 \\
8.22 \\
8.28\end{array}$ & $\begin{array}{r}8.56 \\
8.52 \\
8.61 \\
8.71 \\
8.75 \\
. .-\end{array}$ & $\begin{array}{l}8.82 \\
8.70 \\
8.72 \\
8.79 \\
8.70 \\
8.68\end{array}$ & $\begin{array}{r}8.73 \\
8.50 \\
8.39 \\
8.45 \\
8.46 \\
-.-\end{array}$ & $\begin{array}{l}8.35 \\
7.90 \\
8.25 \\
8.22 \\
8.09 \\
8.09\end{array}$ & $\begin{array}{l}8.01 \\
7.98 \\
7.71 \\
7.79 \\
7.89 \\
7.74\end{array}$ & $\begin{array}{r}7.46 \\
7.38 \\
7.33 \\
--- \\
--- \\
---\end{array}$ & $\begin{array}{l}--- \\
--- \\
--- \\
-- \\
--- \\
---\end{array}$ \\
\hline
\end{tabular}


GROUND WATER LEVELS

OTTAWA COUNTY

365229094520201. LOCAL NUMBER, 28N-23E-30 DCC 1.

LOCATION.--LAT $3652^{\prime} 29^{\prime \prime}$, LONG $09452^{\prime} 02^{\prime}$ ', HYDROLOGIC UNIT 11070206, OWNER: CITY OF MIAMI, OKLA. AQUIFER. --ROUBIDOUX FORMATION.

WELL CHARACTERISTICS.--DRILLED UNUSED WELL, DIAMETER 6 IN. (0.15M),

REDUCED TO 3.5 IN (0.09M), DEPTH 1495 FT (456M).

DATUM.--MEASURING POINT: TOP OF $3.5 \mathrm{IN} .(0.09 \mathrm{M})$ CASING $1.00 \mathrm{FT}(0.30 \mathrm{M})$ BELOW LAND-SURFACE DATUM.

PERIOD OF RECORD.--1980 TO CURRENT YEAR.

EXTREMES FOR PERIOD OF RECORD.--HIGHEST WATER LEVEL, 427.74 FT (130.375M) BELOW

LAND-SURFACE DATUM, MAR. 18, 1981; LOWEST, 457.50 FT (139.446 M) BELOW LAND-SURFACE

DATUM, AUG. 02, 1980.

WATER LEVEL, IN FEET BELOW LAND SURFACE DATUM, CLIMATIC YEAR APRIL 1982 TO MARCH 1983

MEAN VALUES

\begin{tabular}{|c|c|c|c|c|c|c|c|c|c|c|c|c|}
\hline DAY & APR & MAY & JUN & JUL & AUG & SEP & OCT & NOV & DEC & JAN & FEB & MAR \\
\hline $\begin{array}{l}1 \\
2 \\
3 \\
4 \\
5\end{array}$ & $\begin{array}{l}442.22 \\
441.79 \\
441.59 \\
439.95 \\
439.94\end{array}$ & $\begin{array}{l}442.66 \\
441.98 \\
441.33 \\
441.57 \\
441.77\end{array}$ & $\begin{array}{l}442.85 \\
443.26 \\
442.13 \\
441.86 \\
442.23\end{array}$ & $\begin{array}{l}444.64 \\
444.96 \\
445.21 \\
444.60 \\
443.98\end{array}$ & $\begin{array}{l}-- \\
-- \\
-- \\
--\end{array}$ & $\begin{array}{l}442.30 \\
442.71 \\
443.23 \\
443.37 \\
442.74\end{array}$ & $\begin{array}{l}441.86 \\
441.62 \\
441.33 \\
441.05 \\
441.18\end{array}$ & $\begin{array}{l}443.54 \\
443.85 \\
444.17 \\
444.13 \\
443.92\end{array}$ & $\begin{array}{l}443.07 \\
443.10 \\
443.44 \\
444.03 \\
443.39\end{array}$ & $\begin{array}{l}432.86 \\
431.43 \\
432.46 \\
432.32 \\
432.95\end{array}$ & $\begin{array}{l}441.68 \\
441.58 \\
444.31 \\
442.31 \\
441.63\end{array}$ & $\begin{array}{l}439.34 \\
439.87 \\
439.67 \\
439.87 \\
438.47\end{array}$ \\
\hline $\begin{array}{r}6 \\
7 \\
8 \\
9 \\
10\end{array}$ & $\begin{array}{l}441.01 \\
441.08 \\
441.73 \\
442.19 \\
442.08\end{array}$ & $\begin{array}{l}441.73 \\
441.60 \\
441.72 \\
441.13 \\
440.53\end{array}$ & $\begin{array}{l}442.71 \\
442.47 \\
443.26 \\
444.10 \\
444.88\end{array}$ & $\begin{array}{l}444.76 \\
445.46 \\
445.95 \\
446.99 \\
447.55\end{array}$ & $\begin{array}{l}-- \\
-- \\
\cdots \\
-- \\
--\end{array}$ & $\begin{array}{l}442.02 \\
442.09 \\
442.48 \\
442.96 \\
443.35\end{array}$ & $\begin{array}{l}441.38 \\
441.60 \\
441.78 \\
441.60 \\
440.48\end{array}$ & $\begin{array}{l}444.03 \\
443.50 \\
443.29 \\
444.80 \\
445.55\end{array}$ & $\begin{array}{l}443.21 \\
443.33 \\
443.59 \\
443.26 \\
440.92\end{array}$ & $\begin{array}{l}432.95 \\
433.97 \\
434.07 \\
433.69 \\
433.21\end{array}$ & $\begin{array}{l}440.80 \\
439.89 \\
440.21 \\
440.07 \\
441.21\end{array}$ & $\begin{array}{r}437.20 \\
436.50 \\
-.- \\
-.- \\
-.-\end{array}$ \\
\hline $\begin{array}{l}11 \\
12 \\
13 \\
14 \\
13\end{array}$ & $\begin{array}{l}441.45 \\
440.83 \\
441.36 \\
442.23 \\
442.51\end{array}$ & $\begin{array}{l}440.78 \\
441.04 \\
440.97 \\
442.40 \\
443.29\end{array}$ & $\begin{array}{l}444.88 \\
442.92 \\
442.87 \\
442.92 \\
443.33\end{array}$ & $\begin{array}{l}447.65 \\
447.46 \\
447.32 \\
447.24 \\
447.60\end{array}$ & $\begin{array}{l}-+- \\
-- \\
-- \\
--\end{array}$ & $\begin{array}{l}442.90 \\
442.04 \\
442.30 \\
442.49 \\
442.86\end{array}$ & $\begin{array}{l}439.81 \\
439.88 \\
440.14 \\
440.19 \\
440.20\end{array}$ & $\begin{array}{l}444.89 \\
445.36 \\
445.44 \\
445.12 \\
444.69\end{array}$ & $\begin{array}{l}435.73 \\
435.30 \\
434.55 \\
435.70 \\
436.07\end{array}$ & $\begin{array}{l}433.36 \\
434.61 \\
434.83 \\
435.41 \\
435.38\end{array}$ & $\begin{array}{l}442.00 \\
441.31 \\
440.60 \\
439.93 \\
440.49\end{array}$ & $\begin{array}{l}-- \\
-- \\
-- \\
-- \\
--\end{array}$ \\
\hline $\begin{array}{l}16 \\
17 \\
18 \\
19 \\
20\end{array}$ & $\begin{array}{l}442.88 \\
443.28 \\
442.27 \\
441.62 \\
442.45\end{array}$ & $\begin{array}{l}442.24 \\
441.33 \\
442.58 \\
443.26 \\
443.54\end{array}$ & $\begin{array}{l}443.62 \\
443.78 \\
444.11 \\
444.22 \\
443.40\end{array}$ & $\begin{array}{l}447.85 \\
448.15 \\
447.93 \\
447.71 \\
448.48\end{array}$ & $\begin{array}{l}-- \\
-- \\
-- \\
-- \\
--\end{array}$ & $\begin{array}{l}442.70 \\
442.49 \\
442.35 \\
441.52 \\
440.88\end{array}$ & $\begin{array}{l}440.13 \\
438.66 \\
437.88 \\
439.00 \\
439.60\end{array}$ & $\begin{array}{l}444.41 \\
444.58 \\
444.55 \\
444.30 \\
443.97\end{array}$ & $\begin{array}{l}435.81 \\
435.11 \\
434.16 \\
434.58 \\
435.07\end{array}$ & $\begin{array}{l}432.95 \\
433.18 \\
434.70 \\
436.30 \\
438.78\end{array}$ & $\begin{array}{l}442.67 \\
444.03 \\
444.58 \\
442.49 \\
440.26\end{array}$ & $\begin{array}{l}--- \\
-- \\
-- \\
--\end{array}$ \\
\hline $\begin{array}{l}21 \\
22 \\
23 \\
24 \\
25\end{array}$ & $\begin{array}{l}443.02 \\
443.58 \\
443.88 \\
443.99 \\
443.18\end{array}$ & $\begin{array}{l}443.73 \\
444.33 \\
443.87 \\
443.87 \\
442.00\end{array}$ & $\begin{array}{l}442.54 \\
443.40 \\
444.03 \\
444.46 \\
444.72\end{array}$ & $\begin{array}{l}--- \\
--- \\
--- \\
---\end{array}$ & $\begin{array}{l}--- \\
--- \\
--- \\
--- \\
---\end{array}$ & $\begin{array}{l}441.27 \\
441.45 \\
441.66 \\
441.55 \\
441.50\end{array}$ & $\begin{array}{l}439.81 \\
440.20 \\
439.99 \\
439.34 \\
438.87\end{array}$ & $\begin{array}{l}444.10 \\
443.82 \\
444.53 \\
445.12 \\
444.84\end{array}$ & $\begin{array}{l}433.72 \\
431.12 \\
431.12 \\
431.58 \\
431.19\end{array}$ & $\begin{array}{l}442.03 \\
440.97 \\
442.03 \\
442.23 \\
442.79\end{array}$ & $\begin{array}{l}440.79 \\
442.86 \\
442.59 \\
442.57 \\
442.43\end{array}$ & $\begin{array}{l}--- \\
--- \\
--- \\
--\end{array}$ \\
\hline $\begin{array}{l}26 \\
27 \\
28 \\
29 \\
30 \\
31\end{array}$ & $\begin{array}{r}443.08 \\
443.15 \\
443.46 \\
443.16 \\
443.06 \\
\ldots-\end{array}$ & $\begin{array}{l}440.87 \\
441.43 \\
441.63 \\
443.20 \\
443.08 \\
442.63\end{array}$ & $\begin{array}{r}444.79 \\
444.19 \\
443.56 \\
443.87 \\
444.32 \\
\ldots--\end{array}$ & $\begin{array}{l}--- \\
--- \\
--- \\
-- \\
-- \\
--\end{array}$ & $\begin{array}{l}--- \\
-- \\
--- \\
--- \\
--- \\
---\end{array}$ & $\begin{array}{r}441.12 \\
440.66 \\
440.72 \\
441.39 \\
441.75 \\
\ldots\end{array}$ & $\begin{array}{l}439.20 \\
439.22 \\
439.36 \\
439.93 \\
443.42 \\
443.65\end{array}$ & $\begin{array}{r}444.10 \\
443.28 \\
442.34 \\
442.16 \\
442.53 \\
. .-\end{array}$ & $\begin{array}{l}431.00 \\
430.93 \\
433.13 \\
433.80 \\
435.62 \\
435.02\end{array}$ & $\begin{array}{l}443.06 \\
443.76 \\
445.45 \\
444.41 \\
442.75 \\
442.36\end{array}$ & $\begin{array}{r}440.72 \\
438.97 \\
438.81 \\
\ldots- \\
.--\end{array}$ & $\begin{array}{l}--- \\
--- \\
--- \\
--- \\
---\end{array}$ \\
\hline
\end{tabular}


PAYNE COUNTY

360615097100501 . LOCAL NUMBER, 19N-01E-23 CDC 1.

LOCATION.--LAT 36 06'15", LONG 097 10'05", HYDROLOGIC UNIT 11050003, OWNER: E.T. POOL. AQUIFER.--ROCKS OF PERMIAN AGE.

WELL CHARACTERISTICS.--DRILLED WELL, DIAMETER 7 IN (0.18M), DEPTH 47 FT (14.3M).

DATUM.--ALTITUDE OF LAND-SURFACE DATUM IS $1030 \mathrm{FT}(314 \mathrm{M})$. MEASURING POINT: TOP OF CASING $1.20 \mathrm{FT}(0.37 \mathrm{M})$ ABOVE LAND-SURFACE DATUM.

PERIOD OF RECORD.--1934 TO CURRENT YEAR.

EXTREMES FOR PERIOD OF RECORD.--HIGHEST WATER LEVEL, 18.10 FT (5.517M) BELOW LAND-SURFACE

DATUM, DEC. 24, 1962; LOWEST, 28.70 FT (8.748M) BELOW LAND-SURFACE DATUM, MAR. 25, 1974.

WATER LEVELS IN FEET BELOW LAND SURFACE DATUM, CLIMATIC YEAR APRIL 1982 TO MARCH 1983

\begin{tabular}{|c|c|c|c|c|}
\hline DATE & $\begin{array}{l}\text { MATER } \\
\text { WAFEFI }\end{array}$ & DATE & $\begin{array}{l}\text { MATER } \\
\text { FYFI }\end{array}$ & DATE \\
\hline & 26.50 & SEP 28,1982 & 26.10 & DEC 30,1982 \\
\hline
\end{tabular}

GROUND-WATER LEVELS

360725096521501 . LOCAL NUMBER, 19N-04E-15 CBB 1.

LOCATION.--LAT 36 07'25", LONG 096 52'15", HYDROLOGIC UNIT 11050003, OWNER: V.G. PHELPS. AQUIFER.--ROCKS OF EARLY PERMIAN AGE.

WELL CHARACTERISTICS.--DRILLED WELL, DIAMETER 6 IN (0.15M), DEPTH 49 FT (14.9M).

DATUM.--ALTITUDE OF LAND-SURFACE DATUM IS $880 \mathrm{FT}$ (268M). MEASURING POINT: TOP OF CASING $2.20 \mathrm{FT}(0.67 \mathrm{M})$ ABOVE LAND-SURFACE DATUM.

PERIOD OF RECORD.--1934 TO CURRENT YEAR.

EXTREMES FOR PERIOD OF RECORD.--HIGHEST WATER LEVEL, $1.00 \mathrm{FT}(0.305 \mathrm{M})$ BELOW LAND-SURFACE DATUM, APR. 1, 1975; LOWEST, 7.92 FT (2.414M) BELOW LAND-SURFACE DATUM, OCT. 26, 1956.

WATER LEVELS IN FEET EELOW LAND SURFACE DATUM, CLIMATIC YEAR APRIL 1982 TO MARCH 1983

$\begin{array}{lrcrlcr}\text { DATE } & \begin{array}{r}\text { MATER } \\ \text { LEVEL }\end{array} & \text { DATE } & \text { MATER } & \text { MEVEL } & \text { DATE } & \text { MATER } \\ \text { LEVEL }\end{array}$


PAYNE COUNTY -- CONTINUED

360930096573001 . LOCAL NUMBER, 19N-03E-02 BBA 1.

LOCATION.--LAT 36 09'30", LONG 096 57'30", HYDROLOGIC UNIT 11050003, OWNER: W.0. SNYDER. AQUIFER.--ROCKS OF PERMIAN AGE.

WELL CHARACTERISTICS.--DRILLED WELL, DIAMETER 6 IN (0.15M), DEPTH 34 FT (10.4M).

DATUM.--ALTITUDE OF LAND-SURFACE DATUM IS $920 \mathrm{FT}$ (280M). MEASURING POINT: TOP OF CASING $0.90 \mathrm{FT}$ (0.27M) ABOVE LAND-SURFACE DATUM.

PERIOD OF RECORD.--1934 TO CURRENT YEAR.

EXTREMES FOR PERIOD OF RECORD.--HIGHEST WATER LEVEL 6.73 FT (2.051M) BELOW LAND-SURFACE

DATUM, APR. 27, 1942; LOWEST, 25.08 FT (7.644M) BELOW LAND-SURFACE DATUM, OCT. 26, 1956.

WATER LEVELS IN FEET BELOW LAND SURFACE DATUM, CLIMATIC YEAR APRIL 1982 TO MARCH 1983

\begin{tabular}{|c|c|c|c|c|}
\hline DATE & $\begin{array}{l}\text { WATER } \\
\text { LEVEL }\end{array}$ & DATE & $\begin{array}{l}\text { WATER } \\
\text { LEVEL }\end{array}$ & DATE \\
\hline
\end{tabular}

$\begin{array}{llllll}\text { JUL } 02,1982 & 16.90 & \text { SEP } 28,1982 & 19.80 & \text { DEC } 30,1982 & 20.60\end{array}$

\section{GROUND-WATER LEVELS}

361120097055001 . LOCAL NUMBER, 20N-02E-21 CCD 1.

LOCATION.--LAT $3611^{\prime} 20^{\prime \prime}$, LONG 097 05'50", HYDROLOGIC UNIT 11050003, OWNER: A.L. SIMON. AQUIFER.--ROCKS OF PERMIAN AGE.

WELL CHARACTERISTICS.--DRILLED WELL, DIAMETER 6 IN (0.15M) DEPTH 41 FT (12.5M).

DATUM.--ALTITUDE OF LAND-SURFACE DATUM IS $1010 \mathrm{FT}$ (308M). MEASURING POINT: TOP OF CASING $1.30 \mathrm{FT}(0.40 \mathrm{M})$ ABOVE LAND-SURFACE DATUM.

PERIOD OF RECORD.--1934 TO CURRENT YEAR.

EXTREMES FOR PERIOD OF RECORD.--HIGHEST WATER LEVEL, 10.95 FT (3.338M) BELOW LAND-SURFACE DATUM, APR. 29, 1942; LOWEST, 36.29 FT (11.061M) BELOW LAND-SURFACE DATUM, APR. 5, 1937.

WATER LEVELS IN FEET BELOW LAND SURFACE DATUM, CLIMATIC YEAR APRIL 1982 TO MARCH 1983

$\begin{array}{lccccc}\text { DATE } & \begin{array}{l}\text { WATER } \\ \text { LEVEL }\end{array} & \text { DATE } & \begin{array}{l}\text { WATER } \\ \text { LEVEL }\end{array} & \text { DATE } & \text { WATER } \\ \text { LEVEL }\end{array}$




\section{GROUND-WATER LEVELS}

PAYNE COUNTY -- CONTINUED

360245096562001. LOCAL NUMBER, 18N-03E-12 CDC 1.

LOCATION.--LAT 36 02'45", LONG 096 56'20", HYDROLOGIC UNIT 11050003, OWNER: J. WOLF. AQUIFER.--ROCKS OF EARLY PERMIAN AGE.

WELL CHARACTERISTICS.--DRILLED WELL, DIAMETER 6 IN (0.15M), DEPTH 39 FT (11.9M). DATUM.--ALTITUDE OF LAND-SURFACE DATUM IS $830 \mathrm{FT}$ (253M). MEASURING POINT: TOP OF NORTH EDGE OF CASING $0.40 \mathrm{FT}(0.12 \mathrm{M})$ ABOVE LAND-SURFACE DATUM.

PERIOD OF RECORD.--1951 TO CURRENT YEAR.

EXTREMES FOR PERIOD OF RECORD.--HIGHEST WATER LEVEL, 7.40 FT (2.256M) BELOW LAND-SURFACE DATUM, APR. 1, 1975; LOWEST, 30.70 FT (9.357M) BELOW LAND-SURFACE DATUM, JULY 2, 1977.

WATER LEVELS IN FEET BELOW LAND SURFACE DATUM, CLIMATIC YEAR APRIL 1982 TO MARCH 1983

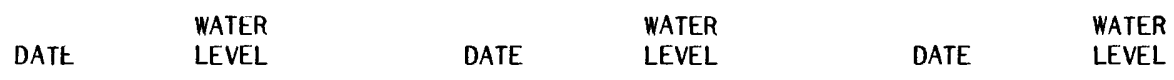

$\begin{array}{llllll}\text { JUL 03, } 1982 & 15.10 & \text { SEP 28, } 1982 & 18.50 & \text { DEC 31, } 1982 & 18.60\end{array}$

GROUND-WATER LEVELS

360515096564501. LOCAL NUMBER, 19N-03E-35 AAB 1.

LOCATION.--LAT 36 05'15", LONG 096 56'45", HYDROLOGIC UNIT 11050003, OWNER: LOVELL BROS. AQUIFER.--ROCKS OF EARLY PERMIAN AGE.

WELL CHARACTERISTICS.--DRILLED WELL, DIAMETER 6 IN $(0.15 \mathrm{M})$, DEPTH 49 FT (14.9M).

DATUM.--ALTITUDE OF LAND-SURFACE DATUM IS $900 \mathrm{FT}(274 \mathrm{M})$. MEASURING POINT: TOP OF CASING

$2.47 \mathrm{FT}(0.75 \mathrm{M})$ ABOVE LAND-SURFACE DATUM.

PERIOD OF RECORD.--1934 TO CURRENT YEAR.

EXTREMES FOR PERIOD OF RECORD..- HIGHEST WATER LEVEL, 11.33 FT (3.453M) BELOW LAND-SURFACE

DATUM, APR. 1, 1975; LOWEST, 39.73 FT (12.110M) BELOW LAND-SURFACE DATUM, MAY 24, 1939.

WATER LEVELS IN FEET BELOW LAND SURFACE DATUM, CLIMATIC YEAR APRIL 1982 TO MARCH 1983

$\begin{array}{lccccr}\text { WATER } & \text { WATER } & \text { WATER } & & \text { WATER } \\ \text { LEVEL } & \text { DATE } & \text { LEVEL } & \text { DATE } & \text { LEVEL } \\ \text { 03, 1982 } & 13.03 & \text { SEP 28, 1982 } & 20.33 & \text { DEC 31, } 1982 & 20.43\end{array}$




\section{GROUND-WATER LEVELS}

PAYNE COUNTY -- CONTINUED

361205096572501. LOCAL NUMBER, 20N-03E-23 BAB 1.

LOCATION.--LAT 36 12'05", LONG 096 57'25", HYDROLOGIC UNIT 11050003, OWNER: V.D. HESSER. AQUIFER.--ROCKS OF LARLY PERMIAN AGE.

WELL CHARACTERISTICS.--DRILLED WELL, DIAMETER 6 IN (0.15M) DEPTH 27 FT (8.23M).

DATUM.--ALTITUDE OF LAND-SURFACE DATUM IS 1045 FT (319M). MEASURING POINT: TOP OF CASING 0.77

FT (0.23M) ABOVE LAND-SURFACE DATUM.

PERIOD OF RECORD. 1934 TO CURRENT YEAR.

EXTREMES FOR PERIOD OF RECORD.--HICHEST WATER LEVEL, $1.20 \mathrm{FT}$ (0.366M) BELOW LAND-SURFACE

DATUM, MAY 27, 1943; LOWEST, 14.41 FT (4.392M) BELOW LAND-SURFACE DATUM, MARCH 1, 1957.

WATER LEVELS IN FEET BELOW LAND SURFACE DATUM, CLIMATIC YEAR APRIL 1982 TO MARCH 1983

$\begin{array}{lccccc}\text { DATE } & \begin{array}{c}\text { WATER } \\ \text { LEVEL }\end{array} & \text { DATE } & \begin{array}{l}\text { WATER } \\ \text { LEVEL }\end{array} & \text { DATE } & \begin{array}{l}\text { WATER } \\ \text { LEVEL }\end{array} \\ 02,1982 & 4.23 & \text { SEP 28, 1982 } & 9.23 & \text { DEC 30, 1982 } & 10.33\end{array}$


GROUND WATER LEVELS

PITTSBURG COUNTY

350241095341101. LOCAL NUMBER; 07N-16E-25 CDC 1.

LOCATION.--LAT $3505^{\prime} 41^{\prime \prime}$, LONG 095 34'11", HYDROLOGIC UNIT 11090204, OWNER: BUREAU OF LAND

MANAGEMENT.

AQUIFER.--BOGGY SHALE.

WELL CHARACTERISTICS.--DRILLED TEST HOLE, DIAMETER 6 IN $(0.15 \mathrm{M})$. DEPTH $140 \mathrm{FT}(42.7 \mathrm{M})$.

DATUM.--MEASURING POINT: TOP OF CASING $1.00 \mathrm{FT}(0.30 \mathrm{M})$ ABOVE LAND-SURFACE DATUM.

REMARKS.--TEST HOLE NO. 02.

PERIOD OF RECORD.--1980 TO CURRENT YEAR.

EXTREMES FOR PERIOD OF RECORD.--HIGHEST WATER LEVEL, $5.37 \mathrm{FT}$ (1.637M) BELOW LAND-SURFACE DATUM.

JUNE 9, 1981; LOWEST WATER LEVEL, 10.70 FT (3.261M) BELOW LAND-SURFACE DATUM, SEPT. $26,1980$.

WATER LEVEL, IN FEET BELOW LAND SURFACE DATUM, CLIMATIC YEAR APRIL 1982 TO MARCH 1983

MEAN VALUES

\begin{tabular}{|c|c|c|c|c|c|c|c|c|c|c|c|c|}
\hline DAY & APR & MAY & JUN & JUL & AUG & SEP & OCT & NOV & DEC & JAN & FEB & MAR \\
\hline $\begin{array}{l}1 \\
2 \\
3 \\
4 \\
5\end{array}$ & $\begin{array}{l}7.30 \\
7.11 \\
7.20 \\
7.23 \\
7.12\end{array}$ & $\begin{array}{l}7.38 \\
7.43 \\
7.37 \\
7.33 \\
7.24\end{array}$ & $\begin{array}{l}6.37 \\
6.30 \\
6.20 \\
6.09 \\
6.11\end{array}$ & $\begin{array}{l}6.45 \\
6.46 \\
6.46 \\
6.48 \\
6.45\end{array}$ & $\begin{array}{l}7.38 \\
7.39 \\
7.42 \\
7.45 \\
7.50\end{array}$ & $\begin{array}{l}8.14 \\
8.14 \\
8.21 \\
8.25 \\
8.27\end{array}$ & $\begin{array}{l}8.65 \\
8.63 \\
8.66 \\
8.68 \\
8.67\end{array}$ & $\begin{array}{l}8.83 \\
8.83 \\
8.96 \\
9.01 \\
8.98\end{array}$ & $\begin{array}{l}6.67 \\
6.62 \\
6.47 \\
6.17 \\
6.01\end{array}$ & $\begin{array}{l}6.44 \\
6.39 \\
6.45 \\
6.43 \\
6.40\end{array}$ & $\begin{array}{l}5.38 \\
5.37 \\
5.72 \\
6.00 \\
5.90\end{array}$ & $\begin{array}{l}-- \\
-- \\
-- \\
--\end{array}$ \\
\hline $\begin{array}{r}6 \\
7 \\
8 \\
9 \\
10\end{array}$ & $\begin{array}{l}7.34 \\
7.28 \\
7.09 \\
7.13 \\
7.18\end{array}$ & $\begin{array}{l}7.09 \\
7.24 \\
7.30 \\
7.28 \\
7.25\end{array}$ & $\begin{array}{l}6.10 \\
6.14 \\
6.18 \\
6.16 \\
6.18\end{array}$ & $\begin{array}{l}6.45 \\
6.38 \\
6.34 \\
6.36 \\
6.32\end{array}$ & $\begin{array}{l}7.52 \\
7.52 \\
7.56 \\
7.58 \\
7.59\end{array}$ & $\begin{array}{l}8.29 \\
8.31 \\
8.33 \\
8.32 \\
8.31\end{array}$ & $\begin{array}{l}8.63 \\
8.69 \\
8.68 \\
8.70 \\
8.80\end{array}$ & $\begin{array}{l}8.91 \\
8.83 \\
8.88 \\
8.93 \\
8.93\end{array}$ & $\begin{array}{l}6.23 \\
6.35 \\
6.52 \\
6.59 \\
6.34\end{array}$ & $\begin{array}{l}6.36 \\
6.29 \\
6.22 \\
6.10 \\
6.09\end{array}$ & $\begin{array}{l}5.82 \\
5.85 \\
5.71 \\
5.57 \\
5.41\end{array}$ & $\begin{array}{l}-- \\
-- \\
-- \\
--\end{array}$ \\
\hline $\begin{array}{l}11 \\
12 \\
13 \\
14 \\
15\end{array}$ & $\begin{array}{l}7.26 \\
7.08 \\
6.92 \\
6.99 \\
6.87\end{array}$ & $\begin{array}{l}7.21 \\
7.12 \\
6.86 \\
6.79 \\
6.82\end{array}$ & $\begin{array}{l}6.20 \\
6.28 \\
6.40 \\
6.44 \\
6.33\end{array}$ & $\begin{array}{l}6.35 \\
6.38 \\
6.40 \\
6.40 \\
6.40\end{array}$ & $\begin{array}{l}7.62 \\
7.63 \\
7.58 \\
7.57 \\
7.61\end{array}$ & $\begin{array}{l}8.33 \\
8.34 \\
8.41 \\
8.48 \\
8.54\end{array}$ & $\begin{array}{l}8.85 \\
8.86 \\
8.87 \\
8.87 \\
8.80\end{array}$ & $\begin{array}{l}8.85 \\
8.93 \\
9.04 \\
8.84 \\
8.94\end{array}$ & $\begin{array}{l}6.16 \\
6.23 \\
6.07 \\
5.83 \\
6.09\end{array}$ & $\begin{array}{l}6.22 \\
6.46 \\
6.34 \\
6.29 \\
6.50\end{array}$ & $\begin{array}{c}5.38 \\
5.56 \\
5.60 \\
5.43 \\
\ldots-\end{array}$ & $\begin{array}{l}-- \\
-- \\
-- \\
--\end{array}$ \\
\hline $\begin{array}{l}16 \\
17 \\
18 \\
19 \\
20\end{array}$ & $\begin{array}{l}6.83 \\
7.00 \\
7.09 \\
6.98 \\
7.01\end{array}$ & $\begin{array}{l}6.84 \\
6.83 \\
6.84 \\
6.77 \\
6.71\end{array}$ & $\begin{array}{l}6.16 \\
6.17 \\
6.17 \\
6.19 \\
6.23\end{array}$ & $\begin{array}{l}6.42 \\
6.43 \\
6.45 \\
6.47 \\
6.48\end{array}$ & $\begin{array}{l}7.66 \\
7.75 \\
7.79 \\
7.83 \\
7.89\end{array}$ & $\begin{array}{l}8.54 \\
8.51 \\
8.50 \\
8.44 \\
8.45\end{array}$ & $\begin{array}{l}8.84 \\
8.83 \\
8.76 \\
8.73 \\
8.88\end{array}$ & $\begin{array}{l}8.62 \\
8.73 \\
8.73 \\
8.55 \\
8.49\end{array}$ & $\begin{array}{l}6.50 \\
6.52 \\
6.30 \\
6.46 \\
6.52\end{array}$ & $\begin{array}{l}6.39 \\
6.24 \\
6.28 \\
6.30 \\
6.13\end{array}$ & $\begin{array}{l}\cdots \\
-- \\
-- \\
-- \\
--\end{array}$ & $\begin{array}{l}-- \\
-- \\
-- \\
--\end{array}$ \\
\hline $\begin{array}{l}21 \\
22 \\
23 \\
24 \\
25\end{array}$ & $\begin{array}{l}7.12 \\
7.14 \\
7.15 \\
7.03 \\
6.85\end{array}$ & $\begin{array}{l}6.72 \\
6.77 \\
6.78 \\
6.65 \\
6.56\end{array}$ & $\begin{array}{l}6.20 \\
6.23 \\
6.28 \\
6.28 \\
6.30\end{array}$ & $\begin{array}{l}6.56 \\
6.60 \\
6.63 \\
6.65 \\
6.74\end{array}$ & $\begin{array}{l}7.91 \\
7.90 \\
7.88 \\
7.90 \\
7.97\end{array}$ & $\begin{array}{l}8.48 \\
8.48 \\
8.44 \\
8.45 \\
8.54\end{array}$ & $\begin{array}{l}8.92 \\
8.93 \\
8.94 \\
8.96 \\
8.96\end{array}$ & $\begin{array}{l}8.51 \\
8.17 \\
8.18 \\
8.44 \\
8.07\end{array}$ & $\begin{array}{l}6.44 \\
6.30 \\
6.19 \\
6.05 \\
6.36\end{array}$ & $\begin{array}{l}5.97 \\
5.97 \\
5.96 \\
6.00 \\
6.11\end{array}$ & $\begin{array}{l}--- \\
-\ldots \\
--- \\
--- \\
--\end{array}$ & $\begin{array}{l}-- \\
\ldots \\
\cdots \\
--\end{array}$ \\
\hline $\begin{array}{l}26 \\
27 \\
28 \\
29 \\
30 \\
31\end{array}$ & $\begin{array}{r}6.82 \\
7.31 \\
7.39 \\
7.30 \\
7.40 \\
-.-\end{array}$ & $\begin{array}{l}6.54 \\
6.52 \\
6.38 \\
6.32 \\
6.35 \\
6.34\end{array}$ & $\begin{array}{r}6.29 \\
6.30 \\
6.32 \\
6.33 \\
6.39 \\
.\end{array}$ & $\begin{array}{l}6.82 \\
7.18 \\
7.30 \\
7.31 \\
7.36 \\
7.40\end{array}$ & $\begin{array}{l}7.99 \\
7.99 \\
8.07 \\
8.12 \\
8.10 \\
8.14\end{array}$ & $\begin{array}{r}8.53 \\
8.48 \\
8.49 \\
8.59 \\
8.64 \\
-.--\end{array}$ & $\begin{array}{l}8.99 \\
8.93 \\
8.86 \\
8.90 \\
8.89 \\
8.87\end{array}$ & $\begin{array}{r}7.75 \\
7.37 \\
6.90 \\
6.69 \\
6.67 \\
. .-\end{array}$ & $\begin{array}{l}6.63 \\
6.28 \\
6.22 \\
6.45 \\
6.48 \\
6.45\end{array}$ & $\begin{array}{l}6.07 \\
6.15 \\
6.07 \\
5.86 \\
5.96 \\
5.86\end{array}$ & $\begin{array}{l}--- \\
--- \\
\cdots- \\
-- \\
---\end{array}$ & $\begin{array}{l}-- \\
--- \\
--- \\
-- \\
--\end{array}$ \\
\hline
\end{tabular}




\section{GROUND WATER LEVELS}

PONTOTOC COUNTY

343457096404501. LOCAL NUMBER, 01N-06E-04 CAD 1.

LOCATION.--LAT 34 34'57", LONG 096 40'45", HYDROLOGIC UNIT 11140102, OWNER: J.H. BRENTZ.

AQUIFER.--ARBUCKLE GROUP.

WELL CHARACTERISTICS.--DRILLED OIL TEST WELL, DIAMETER 18 IN $(0.46 \mathrm{M})$, DEPTH 396 FT (121 M). DATUM.--ALTITUDE OF LAND-SURFACE DATUM IS 1157 FT (353M). MEASURING POINT: BASE OF RECORDER SHELTER AT LAND-SURFACE DATUM.

REMARKS.-- WELL ORIGINALLY 1,707 FT (520 M) DEEP.

PERIOD OF RECORD. - 1959 TO CURRENT YEAR.

EXTREMES FOR PERIOD OF RECORD.--HIGHEST WATER LEVEL, 83.49 FT (25.448M) BELOW LAND-SURFACE

DATUM, APR. 30, 1973; LOWEST, 126.52 FT (38.563M) BELOW LAND-SURFACE DATUM, AUG. 31, 1980.

WATER LEVEL, IN FEET BELOW LAND SURFACE DATUM, CLIMATIC YEAR APRIL 1982 TO MARCH 1983 MEAN VALUES

\begin{tabular}{|c|c|c|c|c|c|c|c|c|c|c|c|c|}
\hline DAY & APR & MAY & JUN & JUL & AUG & SEP & $\mathrm{OCT}$ & NOV & DEC & JAN & FEB & MAR \\
\hline $\begin{array}{l}1 \\
2 \\
3 \\
4 \\
5\end{array}$ & $\begin{array}{l}103.21 \\
103.19 \\
103.41 \\
103.41 \\
103.53\end{array}$ & $\begin{array}{l}106.11 \\
106.19 \\
106.25 \\
106.32 \\
106.38\end{array}$ & $\begin{array}{l}98.69 \\
98.05 \\
97.55 \\
97.19 \\
96.74\end{array}$ & $\begin{array}{l}95.14 \\
95.15 \\
95.18 \\
95.22 \\
95.27\end{array}$ & $\begin{array}{r}99.48 \\
99.64 \\
99.83 \\
100.02 \\
100.20\end{array}$ & $\begin{array}{l}104.43 \\
104.57 \\
104.74 \\
104.87 \\
105.00\end{array}$ & $\begin{array}{l}108.03 \\
108.12 \\
108.23 \\
108.32 \\
108.41\end{array}$ & $\begin{array}{l}110.70 \\
110.80 \\
110.94 \\
111.02 \\
111.08\end{array}$ & $\begin{array}{l}112.65 \\
112.72 \\
112.79 \\
112.86 \\
112.92\end{array}$ & $\begin{array}{l}114.29 \\
114.32 \\
114.32 \\
114.26 \\
114.21\end{array}$ & $\begin{array}{l}114.21 \\
113.72 \\
113.33 \\
112.96 \\
112.56\end{array}$ & $\begin{array}{l}109.75 \\
109.66 \\
109.56 \\
109.42 \\
109.32\end{array}$ \\
\hline $\begin{array}{r}6 \\
7 \\
8 \\
9 \\
10\end{array}$ & $\begin{array}{l}103.71 \\
103.70 \\
103.80 \\
103.95 \\
104.08\end{array}$ & $\begin{array}{c}106.47 \\
106.53 \\
106.59 \\
106.65 \\
106.69\end{array}$ & $\begin{array}{l}96.27 \\
95.90 \\
95.57 \\
95.31 \\
95.18\end{array}$ & $\begin{array}{l}95.36 \\
95.49 \\
95.59 \\
95.65 \\
95.78\end{array}$ & $\begin{array}{l}100.36 \\
100.52 \\
100.70 \\
100.87 \\
101.04\end{array}$ & $\begin{array}{c}105.14 \\
105.27 \\
105.40 \\
105.51 \\
105.60\end{array}$ & $\begin{array}{l}108.60 \\
108.68 \\
108.68 \\
108.81 \\
108.94\end{array}$ & $\begin{array}{l}111.12 \\
111.18 \\
111.27 \\
111.34 \\
111.41\end{array}$ & $\begin{array}{l}113.05 \\
113.10 \\
113.22 \\
113.27 \\
113.24\end{array}$ & $\begin{array}{l}114.15 \\
114.11 \\
114.09 \\
114.04 \\
114.08\end{array}$ & $\begin{array}{l}112.29 \\
112.03 \\
111.76 \\
111.54 \\
111.36\end{array}$ & $\begin{array}{l}109.33 \\
109.36 \\
109.36 \\
109.37 \\
109.35\end{array}$ \\
\hline $\begin{array}{l}11 \\
12 \\
13 \\
14 \\
15\end{array}$ & $\begin{array}{l}104.13 \\
104.11 \\
104.25 \\
104.34 \\
104.38\end{array}$ & $\begin{array}{l}106.75 \\
106.78 \\
106.55 \\
106.19 \\
105.89\end{array}$ & $\begin{array}{l}95.00 \\
94.89 \\
94.84 \\
94.74 \\
94.75\end{array}$ & $\begin{array}{l}95.94 \\
96.09 \\
96.23 \\
96.36 \\
96.51\end{array}$ & $\begin{array}{l}101.21 \\
101.37 \\
101.52 \\
101.66 \\
101.83\end{array}$ & $\begin{array}{l}105.71 \\
105.84 \\
105.99 \\
106.14 \\
106.30\end{array}$ & $\begin{array}{l}109.03 \\
109.11 \\
109.18 \\
109.29 \\
109.35\end{array}$ & $\begin{array}{l}111.40 \\
111.59 \\
111.66 \\
111.74 \\
111.81\end{array}$ & $\begin{array}{l}113.32 \\
113.39 \\
113.38 \\
113.40 \\
113.54\end{array}$ & $\begin{array}{l}114.13 \\
114.17 \\
114.13 \\
114.18 \\
114.23\end{array}$ & $\begin{array}{l}111.26 \\
111.17 \\
111.01 \\
110.83 \\
110.75\end{array}$ & $\begin{array}{l}109.35 \\
109.28 \\
109.20 \\
109.17 \\
109.13\end{array}$ \\
\hline $\begin{array}{l}16 \\
17 \\
18 \\
19 \\
20\end{array}$ & $\begin{array}{l}104.52 \\
104.75 \\
104.79 \\
104.82 \\
105.09\end{array}$ & $\begin{array}{l}105.61 \\
105.36 \\
105.05 \\
104.75 \\
104.46\end{array}$ & $\begin{array}{l}94.83 \\
94.83 \\
94.85 \\
94.96 \\
95.02\end{array}$ & $\begin{array}{l}96.66 \\
96.82 \\
96.98 \\
97.14 \\
97.32\end{array}$ & $\begin{array}{c}102.00 \\
102.24 \\
102.42 \\
102.58 \\
102.73\end{array}$ & $\begin{array}{l}106.40 \\
106.50 \\
106.63 \\
106.74 \\
106.89\end{array}$ & $\begin{array}{l}109.47 \\
109.52 \\
109.58 \\
109.68 \\
109.82\end{array}$ & $\begin{array}{l}111.82 \\
111.88 \\
111.99 \\
112.04 \\
112.11\end{array}$ & $\begin{array}{l}113.58 \\
113.59 \\
113.60 \\
113.74 \\
113.76\end{array}$ & $\begin{array}{l}114.19 \\
114.20 \\
114.26 \\
114.28 \\
114.28\end{array}$ & $\begin{array}{l}110.67 \\
110.62 \\
110.54 \\
110.41 \\
110.41\end{array}$ & $\begin{array}{l}109.15 \\
109.18 \\
109.20 \\
109.21 \\
109.26\end{array}$ \\
\hline $\begin{array}{l}21 \\
22 \\
23 \\
24 \\
25\end{array}$ & $\begin{array}{l}105.29 \\
105.38 \\
105.42 \\
105.40 \\
105.43\end{array}$ & $\begin{array}{l}104.23 \\
104.04 \\
103.83 \\
103.64 \\
103.47\end{array}$ & $\begin{array}{l}95.08 \\
95.19 \\
95.26 \\
95.33 \\
95.34\end{array}$ & $\begin{array}{l}97.57 \\
97.77 \\
97.93 \\
98.11 \\
98.27\end{array}$ & $\begin{array}{l}102.87 \\
102.98 \\
103.11 \\
103.25 \\
103.42\end{array}$ & $\begin{array}{l}107.11 \\
107.22 \\
107.26 \\
107.36 \\
107.46\end{array}$ & $\begin{array}{l}109.91 \\
109.99 \\
110.07 \\
110.14 \\
110.21\end{array}$ & $\begin{array}{l}112.20 \\
112.22 \\
112.37 \\
112.51 \\
112.53\end{array}$ & $\begin{array}{l}113.78 \\
113.86 \\
113.92 \\
113.94 \\
114.10\end{array}$ & $\begin{array}{l}114.29 \\
114.33 \\
114.38 \\
114.42 \\
114.45\end{array}$ & $\begin{array}{l}110.37 \\
110.30 \\
110.23 \\
110.13 \\
110.09\end{array}$ & $\begin{array}{l}109.34 \\
109.40 \\
109.41 \\
109.42 \\
109.39\end{array}$ \\
\hline $\begin{array}{l}26 \\
27 \\
28 \\
29 \\
30 \\
31\end{array}$ & $\begin{array}{r}105.60 \\
105.72 \\
105.77 \\
105.87 \\
106.00 \\
.\end{array}$ & $\begin{array}{r}103.31 \\
103.13 \\
102.29 \\
101.15 \\
100.15 \\
99.39\end{array}$ & $\begin{array}{r}95.28 \\
95.20 \\
95.13 \\
95.10 \\
95.11 \\
.\end{array}$ & $\begin{array}{l}98.45 \\
98.63 \\
98.79 \\
98.95 \\
99.15 \\
99.33\end{array}$ & $\begin{array}{l}103.54 \\
103.68 \\
103.88 \\
104.02 \\
104.15 \\
104.30\end{array}$ & $\begin{array}{r}107.52 \\
107.59 \\
107.71 \\
107.83 \\
107.95 \\
\ldots . .\end{array}$ & $\begin{array}{l}110.27 \\
110.36 \\
110.46 \\
110.54 \\
110.58 \\
110.65\end{array}$ & $\begin{array}{r}112.57 \\
112.56 \\
112.54 \\
112.58 \\
112.63 \\
.\end{array}$ & $\begin{array}{l}114.14 \\
114.10 \\
114.20 \\
114.25 \\
114.25 \\
114.27\end{array}$ & $\begin{array}{l}114.53 \\
114.61 \\
114.57 \\
114.59 \\
114.65 \\
114.59\end{array}$ & $\begin{array}{r}110.01 \\
109.90 \\
109.82 \\
\ldots \\
-.- \\
\ldots\end{array}$ & $\begin{array}{l}109.33 \\
109.47 \\
109.54 \\
109.53 \\
109.56 \\
109.49\end{array}$ \\
\hline
\end{tabular}


GROUND WATER LEVELS

ROGER MILLS COUNTY

354527099470501. LOCAL NUMBER, 15N-24W-19 DDA 1.

LOCATION.--LAT 35 45'27", LONG 099 47'05", HYDROLOGIC UNIT 11130301, OWNER: CHESTER WRIGHT. AQUIFER.--OGALLALA FORMATION.

WELL CHARACTERISTICS.--DRILLED UNUSED IRRIGATION WELL, DIAMETER 12 IN (0.30M), DEPTH 122 FT (37.2M).

DATUM.--ALTITUDE OF LAND-SURFACE DATUM IS 2315 FT (706M). MEASURING POINT: TOP OF WOOD RECORDER

BASE AT LAND-SURFACE DATUM.

PERIOD OF RECORD. - 1970 TO CURRENT YEAR.

EXTREMES FOR PERIOD OF RECORD.-- HIGHEST WATER LEVEL, 44.77 FT (13.646M) BELOW LAND-SURFACE

DATUM, MARCH 31, 1983; LOWEST, 57.27 FT (17.435M) BELOW LAND-SURFACE DATUM, JUNE 5, 1973.

WATER LEVEL, IN FEET BELOW LAND SURFACE DATUM, CLIMATIC YEAR APRIL 1982 TO MARCH 1983

MEAN VALUES

\begin{tabular}{|c|c|c|c|c|c|c|c|c|c|c|c|c|}
\hline DAY & APR & MAY & JUN & JUL & AUG & SEP & OCT & NOV & $D E C$ & JAN & FEB & MAR \\
\hline $\begin{array}{l}1 \\
2 \\
3 \\
4 \\
5\end{array}$ & $\begin{array}{l}55.40 \\
55.54 \\
55.58 \\
55.40 \\
55.75\end{array}$ & $\begin{array}{l}55.66 \\
55.62 \\
55.56 \\
55.54 \\
55.56\end{array}$ & $\begin{array}{l}55.62 \\
55.61 \\
55.68 \\
55.70 \\
55.60\end{array}$ & $\begin{array}{l}55.51 \\
55.50 \\
55.49 \\
55.44 \\
55.47\end{array}$ & $\begin{array}{l}55.27 \\
55.30 \\
55.33 \\
55.30 \\
55.28\end{array}$ & $\begin{array}{l}55.17 \\
55.09 \\
55.07 \\
55.13 \\
55.16\end{array}$ & $\begin{array}{l}54.98 \\
55.00 \\
54.90 \\
54.92 \\
55.03\end{array}$ & $\begin{array}{l}54.97 \\
55.00 \\
54.89 \\
54.81 \\
54.77\end{array}$ & $\begin{array}{l}--- \\
--- \\
--- \\
--- \\
---\end{array}$ & $\begin{array}{l}-- \\
--- \\
--- \\
--- \\
--\end{array}$ & $\begin{array}{l}54.54 \\
54.69 \\
54.77 \\
54.67 \\
54.57\end{array}$ & $\begin{array}{l}54.65 \\
54.52 \\
54.44 \\
54.38 \\
54.46\end{array}$ \\
\hline $\begin{array}{r}6 \\
7 \\
8 \\
9 \\
10\end{array}$ & $\begin{array}{l}55.56 \\
55.42 \\
55.65 \\
55.62 \\
55.66\end{array}$ & $\begin{array}{l}55.68 \\
55.59 \\
55.57 \\
55.59 \\
55.56\end{array}$ & $\begin{array}{l}55.63 \\
55.67 \\
55.59 \\
55.75 \\
55.71\end{array}$ & $\begin{array}{l}55.55 \\
55.48 \\
55.43 \\
55.52 \\
55.49\end{array}$ & $\begin{array}{l}55.29 \\
55.26 \\
55.25 \\
55.26 \\
55.22\end{array}$ & $\begin{array}{l}55.09 \\
55.07 \\
55.03 \\
54.98 \\
55.01\end{array}$ & $\begin{array}{l}54.89 \\
54.83 \\
55.05 \\
55.07 \\
55.00\end{array}$ & $\begin{array}{l}54.79 \\
54.86 \\
54.84 \\
54.82 \\
54.67\end{array}$ & $\begin{array}{l}-\cdots \\
--- \\
--- \\
---\end{array}$ & $\begin{array}{l}-.- \\
-- \\
--- \\
---\end{array}$ & $\begin{array}{l}54.70 \\
54.82 \\
54.56 \\
54.59 \\
54.61\end{array}$ & $\begin{array}{l}54.71 \\
54.70 \\
54.73 \\
54.74 \\
54.70\end{array}$ \\
\hline $\begin{array}{l}11 \\
12 \\
13 \\
14 \\
15\end{array}$ & $\begin{array}{l}55.46 \\
55.47 \\
55.62 \\
55.48 \\
55.44\end{array}$ & $\begin{array}{l}55.56 \\
55.52 \\
55.53 \\
55.64 \\
55.64\end{array}$ & $\begin{array}{l}55.65 \\
55.68 \\
55.66 \\
55.51 \\
55.65\end{array}$ & $\begin{array}{l}55.45 \\
55.40 \\
55.38 \\
55.38 \\
55.38\end{array}$ & $\begin{array}{l}55.19 \\
55.19 \\
55.23 \\
55.28 \\
55.27\end{array}$ & $\begin{array}{l}55.03 \\
55.08 \\
55.13 \\
55.17 \\
55.02\end{array}$ & $\begin{array}{l}54.96 \\
54.98 \\
54.94 \\
54.93 \\
54.96\end{array}$ & $\begin{array}{l}55.13 \\
54.77 \\
55.03 \\
54.76 \\
54.74\end{array}$ & $\begin{array}{l}--- \\
--- \\
--- \\
--- \\
--\end{array}$ & $\begin{array}{l}54.88 \\
54.72 \\
54.61 \\
54.85 \\
54.69\end{array}$ & $\begin{array}{l}54.75 \\
54.70 \\
54.51 \\
54.53 \\
54.68\end{array}$ & $\begin{array}{l}51.40 \\
54.50 \\
54.95 \\
54.45 \\
54.60\end{array}$ \\
\hline $\begin{array}{l}16 \\
17 \\
18 \\
19 \\
20\end{array}$ & $\begin{array}{l}55.69 \\
55.75 \\
55.50 \\
55.58 \\
55.76\end{array}$ & $\begin{array}{l}55.62 \\
55.66 \\
55.61 \\
55.58 \\
55.61\end{array}$ & $\begin{array}{l}55.66 \\
55.58 \\
55.67 \\
55.66 \\
55.58\end{array}$ & $\begin{array}{l}55.39 \\
55.37 \\
55.38 \\
55.41 \\
55.42\end{array}$ & $\begin{array}{l}55.21 \\
55.22 \\
55.23 \\
55.21 \\
55.12\end{array}$ & $\begin{array}{l}55.04 \\
55.08 \\
54.99 \\
55.11 \\
55.09\end{array}$ & $\begin{array}{l}54.81 \\
54.82 \\
55.03 \\
55.04 \\
54.97\end{array}$ & $\begin{array}{l}54.74 \\
54.73 \\
54.76 \\
54.92 \\
54.78\end{array}$ & $\begin{array}{l}-- \\
--- \\
--- \\
-\cdots\end{array}$ & $\begin{array}{l}54.57 \\
54.64 \\
54.66 \\
54.60 \\
54.60\end{array}$ & $\begin{array}{l}54.19 \\
54.63 \\
54.53 \\
54.43 \\
54.73\end{array}$ & $\begin{array}{l}54.67 \\
54.60 \\
54.53 \\
54.56 \\
54.66\end{array}$ \\
\hline $\begin{array}{l}21 \\
22 \\
23 \\
24 \\
25\end{array}$ & $\begin{array}{l}55.75 \\
55.71 \\
55.58 \\
55.42 \\
55.51\end{array}$ & $\begin{array}{l}55.69 \\
55.67 \\
55.62 \\
55.61 \\
55.60\end{array}$ & $\begin{array}{l}55.60 \\
55.62 \\
55.57 \\
55.58 \\
55.55\end{array}$ & $\begin{array}{l}55.39 \\
55.36 \\
55.35 \\
55.35 \\
55.35\end{array}$ & $\begin{array}{l}55.12 \\
55.17 \\
55.22 \\
55.06 \\
55.15\end{array}$ & $\begin{array}{l}54.97 \\
54.93 \\
55.06 \\
54.99 \\
54.87\end{array}$ & $\begin{array}{l}54.94 \\
54.90 \\
54.90 \\
54.85 \\
54.80\end{array}$ & $\begin{array}{l}54.85 \\
55.04 \\
54.85 \\
54.78 \\
54.80\end{array}$ & $\begin{array}{l}--- \\
-\cdots \\
-\cdots \\
-\cdots \\
---\end{array}$ & $\begin{array}{l}54.63 \\
54.62 \\
54.61 \\
54.72 \\
54.57\end{array}$ & $\begin{array}{l}54.68 \\
54.65 \\
54.72 \\
54.66 \\
52.04\end{array}$ & $\begin{array}{l}54.63 \\
47.41 \\
54.57 \\
54.51 \\
54.39\end{array}$ \\
\hline $\begin{array}{l}26 \\
27 \\
28 \\
29 \\
30 \\
31\end{array}$ & $\begin{array}{r}55.66 \\
55.63 \\
55.57 \\
55.59 \\
55.70 \\
.\end{array}$ & $\begin{array}{l}55.57 \\
55.58 \\
55.54 \\
55.64 \\
55.59 \\
55.79\end{array}$ & $\begin{array}{r}55.55 \\
55.54 \\
55.50 \\
55.54 \\
55.54 \\
.\end{array}$ & $\begin{array}{l}55.35 \\
55.33 \\
55.37 \\
55.33 \\
55.27 \\
55.25\end{array}$ & $\begin{array}{l}55.22 \\
55.13 \\
55.11 \\
55.12 \\
55.10 \\
55.17\end{array}$ & $\begin{array}{r}54.87 \\
54.97 \\
55.04 \\
55.04 \\
54.96 \\
. \ldots\end{array}$ & $\begin{array}{l}54.74 \\
54.93 \\
54.85 \\
54.81 \\
54.81 \\
54.85\end{array}$ & $\begin{array}{r}54.60 \\
54.65 \\
\ldots- \\
-.- \\
\ldots- \\
\ldots-\end{array}$ & $\begin{array}{l}-\ldots \\
--- \\
--- \\
--- \\
--- \\
--\end{array}$ & $\begin{array}{l}54.75 \\
54.59 \\
54.47 \\
54.73 \\
54.61 \\
54.57\end{array}$ & $\begin{array}{r}54.60 \\
54.55 \\
54.52 \\
\ldots- \\
\ldots-\end{array}$ & $\begin{array}{l}54.51 \\
54.74 \\
50.94 \\
54.60 \\
49.64 \\
44.77\end{array}$ \\
\hline
\end{tabular}


GROUND WATER LEVELS

SEQUOYAH COUNTY

352419094270401 . LOCAL NUMBER, 11N-27E-21 CDD 1.

LOCATION.--LAT 35 24'19", LONG 094 27'04", HYDROLOGIC UNIT 11110104, OWNER: U.S. GEOLOGICAL SURVEY. AQUIFER. --ALLUVIUM.

WELL CHARACTERISTICS.--DRILLED WELL, DIAMETER 8 IN $(0.20 \mathrm{M})$, DEPTH 48 FT $(14.6 \mathrm{M})$.

DATUM.--ALTITUDE OF LAND-SURFACE DATUM IS 412 FT (126M). MEASURING POINT: TOP OF RECORDER

PLATFORM 2.60 FT (0.79M) ABOVE LAND-SURFACE DATUM.

PERIOD OF RECORD.-- 1960 TO CURRENT YEAR.

EXTREMES FOR PERIOD OF RECORD.--HIGHEST WATER LEVEL 3.18 FT (0.969M) BELOW LAND-SURFACE

DATUM, JUNE 20, 1973; LOWEST, 18.72 FT (5.706M) BELOW LAND-SURFACE DATUM, OCT. 10, 1967.

WATER LEVEL, IN FEET BELOW LAND SURFACE DATUM, Cl_IMATIC YEAR APRIL 1982 TO MARCH 1983

MEAN VALUES

\begin{tabular}{|c|c|c|c|c|c|c|c|c|c|c|c|c|}
\hline DAY & APR & MAY & JUN & JUL & AUG & SEP & OCT & NOV & DEC & JAN & FEB & MAR \\
\hline 1 & 13.03 & 13.25 & 12.48 & 11.60 & 11.20 & 11.22 & 12.18 & 12.14 &.- & $\ldots$ & -.. & -- \\
\hline 2 & 12.96 & 13.24 & 12.46 & 11.56 & 11.14 & 11.25 & 11.75 & 11.95 & -- &.- & $\ldots$ & -.. \\
\hline 3 & 13.15 & 13.22 & 12.42 & 11.54 & 10.70 & 10.94 & 12.22 & 12.00 & -.. & -- & -- & -.. \\
\hline 4 & 13.04 & 13.22 & 12.33 & 11.54 & 11.12 & 10.95 & 12.23 & 11.19 & -.. & ... & -. & -.. \\
\hline 3 & 13.11 & 13.22 & 12.30 & 11.53 & 10.72 & 11.37 & 12.26 & 11.18 & $\ldots$ & $\ldots$ & -- & -.. \\
\hline 6 & 13.17 & 13.24 & 12.29 & 11.54 & 11.14 & 11.42 & 11.87 & 11.95 & - & - & -- & -- \\
\hline 7 & 13.05 & 13.27 & 12.30 & 11.45 & 10.71 & 11.45 & 11.43 & 11.96 & -.. & ... & $-\ldots$ & \\
\hline 8 & 13.07 & 13.29 & 12.28 & 11.29 & 11.13 & 11.47 & 10.93 & 12.00 & $\ldots$ & $\ldots$ & $\ldots$ & -.. \\
\hline 9 & 13.13 & 12.87 & 12.32 & 11.24 & 11.02 & 11.46 & 11.67 & 12.03 & $\ldots$ & $\ldots$ & $\ldots$ & ... \\
\hline 10 & 13.13 & 13.28 & 12.30 & 11.24 & 10.91 & 11.44 & 12.12 & 12.03 & $\cdots$ & -- & $\cdots$ & - \\
\hline 11 & 13.08 & 13.28 & 12.12 & 11.27 & 10.48 & 11.47 & 12.13 & 11.91 & $\ldots$ & $\ldots$ & $\ldots$ & $\ldots$ \\
\hline 12 & 13.00 & 13.20 & 12.05 & 11.27 & 10.06 & 11.51 & 11.26 & 12.16 & $\cdots$ & $\cdots$ & $\cdots$ & $\cdots$ \\
\hline 13 & 13.08 & 12.56 & 12.05 & 11.25 & 10.88 & 11.18 & 11.70 & 11.77 & -.. & $\ldots$ & $\ldots$ & - \\
\hline 14 & 13.08 & 12.57 & 12.00 & 11.23 & 10.83 & 11.64 & 12.15 & 12.19 & -.. & -.- & -.. & - \\
\hline 15 & 13.03 & 12.59 & 11.98 & 11.23 & 10.86 & 11.69 & 11.27 & 11.82 & $\cdots$ & -- & -- & - \\
\hline 16 & 13.09 & 12.63 & 11.87 & 11.22 & 10.88 & 11.69 & 12.21 & 11.72 & $\ldots$ & -.. & $\ldots$ & \\
\hline 17 & 13.21 & 12.66 & 11.84 & 11.22 & 10.08 & 11.67 & 12.18 & 11.72 & $\ldots$ & -. & $\ldots$ & - \\
\hline 18 & 13.13 & 12.66 & 11.43 & 11.21 & 10.97 & 11.72 & 11.74 & 11.16 & $\cdots$ & -- & -- & - \\
\hline 19 & 13.09 & 12.66 & 11.88 & 11.20 & 10.58 & 11.73 & 12.19 & -- & -- & --- & -- & - \\
\hline 20 & 13.21 & 12.65 & 11.85 & 11.22 & 11.03 & 11.79 & 12.33 & $-\cdots$ & --- & -- & --- & \\
\hline 21 & 13.25 & 12.67 & 11.84 & 11.24 & 11.06 & 11.86 & 12.32 & -- & -- & --- & -- & - \\
\hline 22 & 13.24 & 12.67 & 11.85 & 11.25 & 10.64 & 11.85 & 11.91 & --- & -- & $\cdots$ & -- & - \\
\hline 23 & 13.19 & 12.65 & 11.41 & 11.26 & 11.01 & 11.84 & 12.33 & -- & -- & $-\cdot$ & -- & - \\
\hline 24 & 13.12 & 12.20 & 11.81 & 11.27 & 11.02 & 11.89 & 12.33 & $\ldots$ & -.. & -.. & $\ldots$ & \\
\hline 25 & 13.08 & 12.61 & 11.78 & 11.27 & $-\cdots$ & 11.96 & 12.33 & -- & $\cdots$ &.- & --- & \\
\hline 26 & 13.19 & 12.60 & 11.74 & 11.28 & $\ldots$ & 11.96 & 12.33 & $\ldots$ & -.. & -.. & -- & \\
\hline 27 & 13.22 & 12.62 & 11.70 & 11.29 & - & 11.57 & 12.27 & -. & -. & $\cdots$ & $\ldots$ & \\
\hline 28 & 13.18 & 12.31 & 11.62 & 11.28 & -- & 12.06 & 12.26 & -- & -.- & -- & $\cdots$ & \\
\hline 29 & 13.20 & 12.49 & 11.19 & 11.28 & $\cdots$ & 12.13 & 12.24 & $\cdots$ & $\cdots$ & -- & -- & \\
\hline 30 & 13.25 & 12.47 & 11.61 & 11.28 & -- & 12.18 & 12.17 & $\cdots$ & -. & $\cdots$ & $\cdots$ & \\
\hline 31 & -.- & 12.52 & -- & 11.30 & $\ldots$ & $\ldots$ & 11.75 & $\ldots$ & $\cdots$ & $\ldots$ & $\ldots$ & \\
\hline
\end{tabular}


GROUND WATER LEVELS

TEXAS COUNTY

363033101440701 . LOCAL NUMBER, 01N-12E-35 BDD 1.

LOCATION.--LAT 36 30'33", LONG 101 44'07", HYDROLOGIC UNIT 11101033, OWNER: OTTO A. HARLAND. AQUIFER.-- OGALLALA FORMATION.

WELL CHARACTERISTICS.--DRILLED WELL, DIAMETER 7 IN (0.18M), DEPTH 386 FT (118M).

DATUM.--ALTITUDE OF LAND-SURFACE DATUM IS $3430 \mathrm{FT}$ (1045M). MEASURING POINT: TOP OF FLOAT LINE HOLE ON NORTH SIDE $1.70 \mathrm{FT}$ (0.52M) ABOVE LAND-SURFACE DATUM.

REMARKS.--DIGITAL RECORDER INSTALLED MAR. 17, 1980. MEAN-DAILY WATER LEVELS

PUBLISHED THEREAFTER.

PERIOD OF RECORD.-- 1956 TO CURRENT YEAR.

EXTREMES FOR PERIOD OF RECORD.-- HIGHEST WATER LEVEL, 191.87 FT (58.482M) BELOW LAND-SURFACE

DATUM, JAN. 10, 1971; LOWEST, 205.12 FT (62.521M) BELOW LAND-SURFACE DATUM, SEPT. 15,1982.

WATER LEVEL, IN FEET BELOW LAND SURFACE DATUM, CLIMATIC YEAR APRIL 1982 TO MARCH 1983 MEAN VALUES

\begin{tabular}{|c|c|c|c|c|c|c|c|c|c|c|c|c|}
\hline DAY & APR & MAY & JUN & JUL & AUG & SEP & OCT & NOV & DEC & JAN & FEB & MAR \\
\hline $\begin{array}{l}1 \\
2 \\
3 \\
4 \\
5\end{array}$ & $\begin{array}{l}203.94 \\
203.78 \\
204.20 \\
203.98 \\
204.22\end{array}$ & $\begin{array}{l}204.64 \\
204.46 \\
204.31 \\
204.19 \\
204.21\end{array}$ & $\begin{array}{l}204.31 \\
204.25 \\
204.38 \\
204.42 \\
204.21\end{array}$ & $\begin{array}{l}204.09 \\
204.06 \\
204.07 \\
204.05 \\
204.00\end{array}$ & $\begin{array}{l}204.28 \\
204.19 \\
204.18 \\
204.27 \\
204.40\end{array}$ & $\begin{array}{l}204.85 \\
204.96 \\
204.99 \\
204.86 \\
204.82\end{array}$ & $\begin{array}{l}204.99 \\
204.94 \\
204.97 \\
204.78 \\
204.67\end{array}$ & $\begin{array}{l}204.50 \\
204.66 \\
204.79 \\
204.68 \\
204.55\end{array}$ & $\begin{array}{l}204.21 \\
204.36 \\
204.62 \\
204.57 \\
204.54\end{array}$ & $\begin{array}{l}204.61 \\
204.65 \\
204.52 \\
204.43 \\
204.48\end{array}$ & $\begin{array}{l}204.34 \\
204.41 \\
204.57 \\
204.56 \\
204.39\end{array}$ & $\begin{array}{l}204.45 \\
204.44 \\
204.29 \\
204.11 \\
204.10\end{array}$ \\
\hline $\begin{array}{r}6 \\
7 \\
8 \\
9 \\
10\end{array}$ & $\begin{array}{l}204.27 \\
203.87 \\
204.08 \\
204.15 \\
204.30\end{array}$ & $\begin{array}{l}204.32 \\
204.24 \\
204.17 \\
204.26 \\
204.32\end{array}$ & $\begin{array}{l}204.13 \\
204.23 \\
204.07 \\
204.24 \\
204.31\end{array}$ & $\begin{array}{l}204.10 \\
204.25 \\
204.19 \\
204.07 \\
204.18\end{array}$ & $\begin{array}{l}204.52 \\
204.57 \\
204.60 \\
204.60 \\
204.61\end{array}$ & $\begin{array}{l}204.97 \\
205.00 \\
204.93 \\
204.85 \\
204.72\end{array}$ & $\begin{array}{l}206.49 \\
204.69 \\
204.48 \\
204.70 \\
204.86\end{array}$ & $\begin{array}{l}204.44 \\
204.41 \\
204.51 \\
204.47 \\
204.52\end{array}$ & $\begin{array}{l}204.62 \\
204.70 \\
204.97 \\
204.77 \\
204.44\end{array}$ & $\begin{array}{l}204.45 \\
204.43 \\
204.45 \\
204.30 \\
204.46\end{array}$ & $\begin{array}{l}204.53 \\
204.43 \\
204.40 \\
204.41 \\
204.45\end{array}$ & $\begin{array}{l}204.44 \\
204.60 \\
204.67 \\
204.78 \\
204.68\end{array}$ \\
\hline $\begin{array}{l}11 \\
12 \\
13 \\
14 \\
15\end{array}$ & $\begin{array}{l}204.11 \\
203.97 \\
204.24 \\
204.16 \\
204.07\end{array}$ & $\begin{array}{l}204.40 \\
204.35 \\
204.30 \\
204.36 \\
204.37\end{array}$ & $\begin{array}{l}204.21 \\
204.22 \\
204.18 \\
203.99 \\
204.02\end{array}$ & $\begin{array}{l}204.26 \\
204.24 \\
204.16 \\
203.99 \\
203.99\end{array}$ & $\begin{array}{l}204.69 \\
204.60 \\
204.58 \\
204.58 \\
204.65\end{array}$ & $\begin{array}{l}204.67 \\
204.77 \\
204.89 \\
204.98 \\
205.12\end{array}$ & $\begin{array}{l}204.82 \\
204.77 \\
204.78 \\
204.75 \\
204.72\end{array}$ & $\begin{array}{l}204.26 \\
204.69 \\
204.54 \\
204.70 \\
204.56\end{array}$ & $\begin{array}{l}204.60 \\
204.49 \\
204.16 \\
204.31 \\
204.59\end{array}$ & $\begin{array}{l}204.63 \\
204.65 \\
204.48 \\
204.66 \\
204.61\end{array}$ & $\begin{array}{l}204.62 \\
204.68 \\
204.45 \\
204.30 \\
204.55\end{array}$ & $\begin{array}{l}204.61 \\
204.43 \\
204.42 \\
204.31 \\
204.31\end{array}$ \\
\hline $\begin{array}{l}16 \\
17 \\
18 \\
19 \\
20\end{array}$ & $\begin{array}{l}204.33 \\
204.64 \\
204.39 \\
204.42 \\
204.69\end{array}$ & $\begin{array}{l}204.31 \\
204.29 \\
204.20 \\
204.04 \\
204.08\end{array}$ & $\begin{array}{l}204.19 \\
204.07 \\
204.16 \\
204.26 \\
204.16\end{array}$ & $\begin{array}{l}203.98 \\
204.01 \\
204.06 \\
204.07 \\
204.14\end{array}$ & $\begin{array}{l}204.77 \\
204.83 \\
204.80 \\
204.74 \\
204.78\end{array}$ & $\begin{array}{l}204.99 \\
204.95 \\
205.05 \\
204.94 \\
205.06\end{array}$ & $\begin{array}{l}204.76 \\
204.58 \\
204.53 \\
204.69 \\
204.90\end{array}$ & $\begin{array}{l}204.44 \\
204.43 \\
204.40 \\
204.40 \\
204.72\end{array}$ & $\begin{array}{l}204.58 \\
204.42 \\
204.42 \\
204.58 \\
204.48\end{array}$ & $\begin{array}{l}204.38 \\
204.37 \\
204.39 \\
204.33 \\
204.33\end{array}$ & $\begin{array}{l}204.50 \\
204.49 \\
204.47 \\
204.16 \\
204.50\end{array}$ & $\begin{array}{l}204.54 \\
204.58 \\
204.46 \\
204.44 \\
204.60\end{array}$ \\
\hline $\begin{array}{l}21 \\
22 \\
23 \\
24 \\
25\end{array}$ & $\begin{array}{l}204.76 \\
204.71 \\
204.52 \\
204.26 \\
204.33\end{array}$ & $\begin{array}{l}204.26 \\
204.29 \\
204.21 \\
204.14 \\
204.10\end{array}$ & $\begin{array}{l}204.12 \\
204.13 \\
204.13 \\
204.12 \\
204.16\end{array}$ & $\begin{array}{l}204.21 \\
204.24 \\
204.21 \\
204.16 \\
204.15\end{array}$ & $\begin{array}{l}204.79 \\
204.67 \\
204.64 \\
204.72 \\
204.81\end{array}$ & $\begin{array}{l}205.09 \\
204.98 \\
204.84 \\
204.93 \\
204.93\end{array}$ & $\begin{array}{l}204.78 \\
204.81 \\
204.73 \\
204.71 \\
204.63\end{array}$ & $\begin{array}{l}204.92 \\
204.85 \\
205.04 \\
205.11 \\
204.75\end{array}$ & $\begin{array}{l}204.34 \\
204.29 \\
204.22 \\
204.10 \\
204.72\end{array}$ & $\begin{array}{l}204.39 \\
204.39 \\
204.38 \\
204.52 \\
204.38\end{array}$ & $\begin{array}{l}204.65 \\
204.58 \\
204.47 \\
204.49 \\
204.49\end{array}$ & $\begin{array}{l}204.64 \\
204.53 \\
204.54 \\
204.48 \\
204.28\end{array}$ \\
\hline $\begin{array}{l}26 \\
27 \\
28 \\
29 \\
30 \\
31\end{array}$ & $\begin{array}{r}204.56 \\
204.60 \\
204.57 \\
204.55 \\
204.68 \\
-.--\end{array}$ & $\begin{array}{l}203.96 \\
203.96 \\
203.88 \\
204.04 \\
204.08 \\
204.37\end{array}$ & $\begin{array}{r}204.11 \\
204.13 \\
204.07 \\
204.01 \\
204.07 \\
. .-\end{array}$ & $\begin{array}{l}204.14 \\
204.22 \\
204.34 \\
204.36 \\
204.39 \\
204.36\end{array}$ & $\begin{array}{l}204.58 \\
204.66 \\
204.84 \\
204.82 \\
204.80 \\
204.83\end{array}$ & $\begin{array}{r}204.76 \\
204.66 \\
204.79 \\
204.99 \\
205.07 \\
.\end{array}$ & $\begin{array}{l}204.52 \\
204.39 \\
204.54 \\
204.51 \\
204.44 \\
204.45\end{array}$ & $\begin{array}{r}204.79 \\
204.63 \\
204.32 \\
204.33 \\
204.32 \\
.-.\end{array}$ & $\begin{array}{l}204.74 \\
204.39 \\
204.63 \\
204.68 \\
204.51 \\
204.61\end{array}$ & $\begin{array}{l}204.55 \\
204.44 \\
204.18 \\
204.44 \\
204.40 \\
204.43\end{array}$ & $\begin{array}{r}204.44 \\
204.41 \\
204.40 \\
. . . \\
. . .\end{array}$ & $\begin{array}{l}204.35 \\
204.69 \\
204.64 \\
204.60 \\
204.62 \\
204.38\end{array}$ \\
\hline
\end{tabular}


GROUND-WATER LEVELS

WASHITA COUNTY

352142099122501 . LOCAL NUMBER, 10N-19W-10 BBB 1.

LOCATION.--LAT 35 35'05", LONG 099 12'25", HYDROLOGIC UNIT 11120302, OWNER: MIDWEST OKLA

DEVELOPMENT AUTHORITY.

AQUIFER.--ELK CITY SANDSTONE.

WELL CHARACTERISTICS.--DRILLED WELL, DIAMETER 8 IN (0.20M), DEPTH 107 FT (32.6M).

DATUM.--ALTITUDE OF LAND-SURFACE DATUM IS $1920 \mathrm{FT}$ (585M). MEASURING POINT: TOP OF CASING 1.35

FT (0.41M) ABOVE LAND-SURFACE DATUM.

PERIOD OF RECORD. --APRIL TO SEPTEMBER 1979

EXTREMES FOR CURRENT YEAR.-- HIGHEST WATER LEVEL 33.33 FT (10.159M) BELOW LAND-SURFACE DATUM

APR. 30, 1979; LOWEST, 34.82 FT (10.613M) BELOW LAND-SURFACE DATUM, APR. 4, 1982.

WATER LEVEL, IN FEET BELOW LAND SURFACE DATUM, CLIMATIC YEAR APRIL 1982 TO MARCH 1983

MEAN VALUUES

\begin{tabular}{|c|c|c|c|c|c|c|c|c|c|c|c|c|}
\hline DAY & APR & MAY & JUN & JUL & AUG & SEP & OCT & NOV & DEC & JAN & FEB & MAR \\
\hline $\begin{array}{l}1 \\
2 \\
3 \\
4 \\
5\end{array}$ & $\begin{array}{l}34.55 \\
34.68 \\
34.77 \\
34.82 \\
34.72\end{array}$ & $\begin{array}{r}34.57 \\
34.57 \\
34.56 \\
34.55 \\
34.55\end{array}$ & $\begin{array}{l}34.07 \\
34.07 \\
34.07 \\
34.07 \\
34.07\end{array}$ & $\begin{array}{l}33.69 \\
33.68 \\
33.67 \\
33.66 \\
33.65\end{array}$ & $\begin{array}{l}33.52 \\
33.51 \\
33.51 \\
33.54 \\
33.57\end{array}$ & $\begin{array}{l}33.54 \\
33.53 \\
33.54 \\
33.52 \\
33.51\end{array}$ & $\begin{array}{l}33.48 \\
33.47 \\
33.48 \\
33.47 \\
33.47\end{array}$ & $\begin{array}{l}33.51 \\
33.53 \\
33.55 \\
33.53 \\
33.51\end{array}$ & $\begin{array}{l}33.44 \\
33.45 \\
33.46 \\
33.45 \\
33.46\end{array}$ & $\begin{array}{l}33.45 \\
33.45 \\
33.45 \\
33.44 \\
33.44\end{array}$ & $\begin{array}{l}33.52 \\
33.55 \\
33.56 \\
33.55 \\
33.54\end{array}$ & $\begin{array}{l}33.54 \\
33.54 \\
33.52 \\
33.51 \\
33.52\end{array}$ \\
\hline $\begin{array}{r}6 \\
7 \\
8 \\
9 \\
10\end{array}$ & $\begin{array}{l}34.68 \\
34.64 \\
34.66 \\
34.67 \\
34.68\end{array}$ & $\begin{array}{l}34.56 \\
34.56 \\
34.56 \\
34.56 \\
34.55\end{array}$ & $\begin{array}{l}34.07 \\
34.07 \\
34.05 \\
34.03 \\
34.00\end{array}$ & $\begin{array}{l}33.65 \\
33.65 \\
33.64 \\
33.63 \\
33.63\end{array}$ & $\begin{array}{l}33.57 \\
33.55 \\
33.55 \\
33.54 \\
33.55\end{array}$ & $\begin{array}{l}33.52 \\
33.52 \\
33.52 \\
33.51 \\
33.50\end{array}$ & $\begin{array}{l}33.48 \\
33.47 \\
33.46 \\
33.49 \\
33.50\end{array}$ & $\begin{array}{l}33.50 \\
33.50 \\
33.50 \\
33.50 \\
33.51\end{array}$ & $\begin{array}{l}33.47 \\
33.48 \\
33.51 \\
33.48 \\
33.45\end{array}$ & $\begin{array}{l}33.43 \\
33.43 \\
33.43 \\
33.42 \\
33.43\end{array}$ & $\begin{array}{l}33.55 \\
33.54 \\
33.54 \\
33.54 \\
33.54\end{array}$ & $\begin{array}{l}33.55 \\
33.55 \\
33.56 \\
33.57 \\
33.57\end{array}$ \\
\hline $\begin{array}{l}11 \\
12 \\
13 \\
14 \\
15\end{array}$ & $\begin{array}{l}34.77 \\
34.65 \\
34.67 \\
34.66 \\
34.66\end{array}$ & $\begin{array}{l}34.55 \\
34.51 \\
34.54 \\
34.56 \\
34.56\end{array}$ & $\begin{array}{l}33.97 \\
33.95 \\
33.93 \\
33.87 \\
33.83\end{array}$ & $\begin{array}{l}33.63 \\
33.62 \\
33.61 \\
33.60 \\
33.59\end{array}$ & $\begin{array}{l}33.53 \\
33.51 \\
33.50 \\
33.50 \\
33.50\end{array}$ & $\begin{array}{l}33.50 \\
33.50 \\
33.50 \\
33.52 \\
33.53\end{array}$ & $\begin{array}{l}33.50 \\
33.49 \\
33.50 \\
33.50 \\
33.51\end{array}$ & $\begin{array}{l}33.52 \\
33.59 \\
33.53 \\
33.55 \\
33.51\end{array}$ & $\begin{array}{l}33.47 \\
33.46 \\
33.43 \\
33.45 \\
33.48\end{array}$ & $\begin{array}{l}33.45 \\
33.44 \\
33.47 \\
33.57 \\
33.56\end{array}$ & $\begin{array}{l}33.56 \\
33.56 \\
33.54 \\
33.53 \\
33.55\end{array}$ & $\begin{array}{l}33.56 \\
33.55 \\
33.55 \\
33.54 \\
33.54\end{array}$ \\
\hline $\begin{array}{l}16 \\
17 \\
18 \\
19 \\
20\end{array}$ & $\begin{array}{l}34.68 \\
34.70 \\
34.68 \\
34.67 \\
34.70\end{array}$ & $\begin{array}{l}34.52 \\
34.49 \\
34.45 \\
34.42 \\
34.36\end{array}$ & $\begin{array}{l}33.83 \\
33.81 \\
33.80 \\
33.79 \\
33.78\end{array}$ & $\begin{array}{l}33.59 \\
33.58 \\
33.58 \\
33.57 \\
33.57\end{array}$ & $\begin{array}{l}33.51 \\
33.50 \\
33.50 \\
33.50 \\
33.51\end{array}$ & $\begin{array}{l}33.51 \\
33.50 \\
33.51 \\
33.50 \\
33.52\end{array}$ & $\begin{array}{l}33.51 \\
33.49 \\
33.48 \\
33.55 \\
33.60\end{array}$ & $\begin{array}{l}33.49 \\
33.49 \\
33.52 \\
33.50 \\
33.51\end{array}$ & $\begin{array}{l}33.47 \\
33.45 \\
33.45 \\
33.46 \\
33.44\end{array}$ & $\begin{array}{l}33.54 \\
33.55 \\
33.55 \\
33.54 \\
33.54\end{array}$ & $\begin{array}{l}33.55 \\
33.55 \\
33.54 \\
33.52 \\
33.55\end{array}$ & $\begin{array}{l}33.56 \\
33.55 \\
33.55 \\
33.55 \\
33.57\end{array}$ \\
\hline $\begin{array}{l}21 \\
22 \\
23 \\
24 \\
25\end{array}$ & $\begin{array}{l}34.70 \\
34.70 \\
34.68 \\
34.66 \\
34.66\end{array}$ & $\begin{array}{l}34.34 \\
34.32 \\
34.30 \\
34.28 \\
34.24\end{array}$ & $\begin{array}{l}33.77 \\
33.77 \\
33.75 \\
33.75 \\
33.74\end{array}$ & $\begin{array}{l}33.57 \\
33.56 \\
33.56 \\
33.56 \\
33.57\end{array}$ & $\begin{array}{l}33.50 \\
33.49 \\
33.50 \\
33.51 \\
33.51\end{array}$ & $\begin{array}{l}33.52 \\
33.50 \\
33.49 \\
33.50 \\
33.50\end{array}$ & $\begin{array}{l}33.61 \\
33.59 \\
33.56 \\
33.55 \\
33.54\end{array}$ & $\begin{array}{l}33.50 \\
33.48 \\
33.52 \\
33.52 \\
33.50\end{array}$ & $\begin{array}{l}33.43 \\
33.43 \\
33.42 \\
33.43 \\
33.48\end{array}$ & $\begin{array}{l}33.54 \\
33.53 \\
33.54 \\
33.55 \\
33.53\end{array}$ & $\begin{array}{l}33.55 \\
33.55 \\
33.54 \\
33.55 \\
33.55\end{array}$ & $\begin{array}{l}33.57 \\
33.56 \\
33.56 \\
33.56 \\
33.54\end{array}$ \\
\hline $\begin{array}{l}26 \\
27 \\
28 \\
29 \\
30 \\
31\end{array}$ & $\begin{array}{r}34.69 \\
34.65 \\
34.56 \\
34.57 \\
34.57 \\
.--\end{array}$ & $\begin{array}{l}34.19 \\
34.17 \\
34.12 \\
34.07 \\
34.07 \\
34.07\end{array}$ & $\begin{array}{r}33.73 \\
33.72 \\
33.71 \\
33.70 \\
33.70 \\
=--\end{array}$ & $\begin{array}{l}33.55 \\
33.54 \\
33.54 \\
33.53 \\
33.53 \\
33.52\end{array}$ & $\begin{array}{l}33.49 \\
33.53 \\
33.57 \\
33.56 \\
33.55 \\
33.55\end{array}$ & $\begin{array}{r}33.47 \\
33.47 \\
33.48 \\
33.49 \\
33.49 \\
=-.\end{array}$ & $\begin{array}{l}33.53 \\
33.51 \\
33.52 \\
33.52 \\
33.50 \\
33.51\end{array}$ & $\begin{array}{r}33.50 \\
33.47 \\
33.45 \\
33.45 \\
33.45 \\
\ldots\end{array}$ & $\begin{array}{l}33.45 \\
33.43 \\
33.47 \\
33.46 \\
33.45 \\
33.45\end{array}$ & $\begin{array}{l}33.55 \\
33.54 \\
33.52 \\
33.55 \\
33.53 \\
33.52\end{array}$ & $\begin{array}{r}33.55 \\
33.55 \\
33.54 \\
=-- \\
=-- \\
=--\end{array}$ & $\begin{array}{l}33.54 \\
33.57 \\
33.56 \\
33.56 \\
33.56 \\
33.53\end{array}$ \\
\hline
\end{tabular}


GROUND WATER LEVELS

WOODS COUNTY

365143098404201. LOCAL NUMBER, 28N-14W-35 BCC 1.

LOCATION.--LAT 36 51'43". LONG 098 40'42", HYDROLOGIC UNIT 11060002, QWNER: WILCOX.

AQUIFER.--CEDAR HILLS SANDSTONE.

WELL CHARACTERISTICS.--DRILLED UNUSED MUNICIPAL WELL, DIAMETER 13 IN (0.33M), DEPTH 54FT $(16.5 \mathrm{M})$.

DATUM.--ALTITUDE OF LAND-SURFACE DATUM IS 1360 FT (415M). MEASURE POINT: EDGE OF

LARGE HOLE IN STEEL PLATE 2.60 FT (0.79M) ABOVE LAND-SURFACE DATUM.

REMARKS.--DIGITAL RECORDER INSTALLED JULY 30, 1980. MEAN-DAILY WATER LEVELS

PUBLISHED THEREAFTER.

PERIOD OF RECORD.--1972 TO CURRENT YEAR.

EXTREMES FOR PERIOD OF RECORD.-- HIGHEST WATER LEVEL, $18.01 \mathrm{FT}$ (5.489M) BELOW

LAND-SURFACE DATUM, JUNE 18, 1982; LOWEST, $24.25 \mathrm{FT}(7.391 \mathrm{M})$ BELOW LAND-SURFACE

DATUM, MAR. 15, 1979.

WATER LEVEL, IN FEET BELOW LAND SURFACE DATUM, CLIMATIC YEAR APRIL 1982 T0 MARCH 1983

MEAN VALUES

\begin{tabular}{|c|c|c|c|c|c|c|c|c|c|c|c|c|}
\hline DAY & APR & MAY & JUN & JUL & AUG & SEP & OCT & NOV & DEC & JAN & FEB & MAR \\
\hline $\begin{array}{l}1 \\
2 \\
3 \\
4 \\
5\end{array}$ & $\begin{array}{l}21.54 \\
21.50 \\
21.62 \\
21.52 \\
21.62\end{array}$ & $\begin{array}{l}21.61 \\
21.59 \\
21.56 \\
21.54 \\
21.55\end{array}$ & $\begin{array}{l}19.63 \\
19.52 \\
19.33 \\
18.87 \\
18.37\end{array}$ & $\begin{array}{l}18.09 \\
18.08 \\
18.10 \\
18.10 \\
18.09\end{array}$ & $\begin{array}{l}18.26 \\
18.27 \\
18.29 \\
18.33 \\
18.36\end{array}$ & $\begin{array}{l}18.79 \\
18.84 \\
18.86 \\
18.84 \\
18.86\end{array}$ & $\begin{array}{l}19.26 \\
19.27 \\
19.30 \\
19.27 \\
19.30\end{array}$ & $\begin{array}{l}19.70 \\
19.74 \\
19.76 \\
19.76 \\
19.75\end{array}$ & $\begin{array}{l}20.00 \\
20.06 \\
20.09 \\
20.06 \\
20.08\end{array}$ & $\begin{array}{l}20.38 \\
20.40 \\
20.38 \\
20.38 \\
20.39\end{array}$ & $\begin{array}{l}20.58 \\
20.63 \\
20.67 \\
20.66 \\
20.62\end{array}$ & $\begin{array}{l}20.83 \\
20.82 \\
20.80 \\
20.76 \\
20.77\end{array}$ \\
\hline $\begin{array}{r}6 \\
7 \\
8 \\
9 \\
10\end{array}$ & $\begin{array}{l}21.62 \\
21.51 \\
21.59 \\
21.61 \\
21.61\end{array}$ & $\begin{array}{l}21.60 \\
21.58 \\
21.57 \\
21.57 \\
21.55\end{array}$ & $\begin{array}{l}18.22 \\
18.24 \\
18.22 \\
18.31 \\
18.34\end{array}$ & $\begin{array}{l}18.12 \\
18.16 \\
18.14 \\
18.12 \\
18.15\end{array}$ & $\begin{array}{l}18.38 \\
18.39 \\
18.40 \\
18.41 \\
18.42\end{array}$ & $\begin{array}{l}18.90 \\
18.91 \\
18.92 \\
18.92 \\
18.92\end{array}$ & $\begin{array}{l}19.35 \\
19.33 \\
19.29 \\
19.39 \\
19.42\end{array}$ & $\begin{array}{l}19.73 \\
19.75 \\
19.79 \\
19.80 \\
19.81\end{array}$ & $\begin{array}{l}20.12 \\
20.14 \\
20.19 \\
20.14 \\
20.08\end{array}$ & $\begin{array}{l}20.39 \\
20.41 \\
20.41 \\
20.38 \\
20.43\end{array}$ & $\begin{array}{l}20.68 \\
20.66 \\
20.66 \\
20.68 \\
20.69\end{array}$ & $\begin{array}{l}20.85 \\
20.87 \\
20.88 \\
20.89 \\
20.88\end{array}$ \\
\hline $\begin{array}{l}11 \\
12 \\
13 \\
14 \\
15\end{array}$ & $\begin{array}{l}21.55 \\
21.50 \\
21.58 \\
21.54 \\
21.50\end{array}$ & $\begin{array}{l}21.56 \\
21.56 \\
21.54 \\
21.57 \\
21.59\end{array}$ & $\begin{array}{l}18.32 \\
18.34 \\
18.34 \\
18.30 \\
18.32\end{array}$ & $\begin{array}{l}18.17 \\
18.16 \\
18.15 \\
18.14 \\
18.14\end{array}$ & $\begin{array}{l}18.44 \\
18.43 \\
18.43 \\
18.46 \\
18.50\end{array}$ & $\begin{array}{l}18.94 \\
18.96 \\
19.00 \\
19.04 \\
19.06\end{array}$ & $\begin{array}{l}19.43 \\
19.43 \\
19.46 \\
19.46 \\
19.48\end{array}$ & $\begin{array}{l}19.74 \\
19.90 \\
19.82 \\
19.91 \\
19.85\end{array}$ & $\begin{array}{l}20.17 \\
20.16 \\
20.08 \\
20.14 \\
20.22\end{array}$ & $\begin{array}{l}20.48 \\
20.45 \\
20.43 \\
20.51 \\
20.49\end{array}$ & $\begin{array}{l}20.73 \\
20.74 \\
20.68 \\
20.67 \\
20.74\end{array}$ & $\begin{array}{l}20.86 \\
20.79 \\
20.76 \\
20.74 \\
20.73\end{array}$ \\
\hline $\begin{array}{l}16 \\
17 \\
18 \\
19 \\
20\end{array}$ & $\begin{array}{l}21.59 \\
21.66 \\
21.56 \\
21.57 \\
21.65\end{array}$ & $\begin{array}{l}21.58 \\
21.10 \\
20.50 \\
20.35 \\
20.24\end{array}$ & $\begin{array}{l}18.31 \\
18.06 \\
18.01 \\
18.03 \\
18.02\end{array}$ & $\begin{array}{l}18.14 \\
18.15 \\
18.16 \\
18.17 \\
18.19\end{array}$ & $\begin{array}{l}18.54 \\
18.56 \\
18.56 \\
18.58 \\
18.61\end{array}$ & $\begin{array}{l}19.03 \\
19.05 \\
19.09 \\
19.08 \\
19.14\end{array}$ & $\begin{array}{l}19.50 \\
19.46 \\
19.48 \\
19.55 \\
19.58\end{array}$ & $\begin{array}{l}19.83 \\
19.86 \\
19.87 \\
19.85 \\
19.94\end{array}$ & $\begin{array}{l}20.22 \\
20.17 \\
20.19 \\
20.24 \\
20.23\end{array}$ & $\begin{array}{l}20.45 \\
20.49 \\
20.52 \\
20.50 \\
20.53\end{array}$ & $\begin{array}{l}20.74 \\
20.77 \\
20.74 \\
20.68 \\
20.79\end{array}$ & $\begin{array}{l}20.80 \\
20.80 \\
20.77 \\
20.76 \\
20.78\end{array}$ \\
\hline $\begin{array}{l}21 \\
22 \\
23 \\
24 \\
25\end{array}$ & $\begin{array}{l}21.66 \\
21.65 \\
21.59 \\
21.53 \\
21.53\end{array}$ & $\begin{array}{l}20.17 \\
20.11 \\
20.07 \\
20.04 \\
20.02\end{array}$ & $\begin{array}{l}18.04 \\
18.05 \\
18.06 \\
18.07 \\
18.07\end{array}$ & $\begin{array}{l}18.21 \\
18.22 \\
18.22 \\
18.22 \\
18.23\end{array}$ & $\begin{array}{l}18.62 \\
18.61 \\
18.63 \\
18.66 \\
18.69\end{array}$ & $\begin{array}{l}19.14 \\
19.11 \\
19.12 \\
19.18 \\
19.18\end{array}$ & $\begin{array}{l}19.59 \\
19.60 \\
19.60 \\
19.61 \\
19.62\end{array}$ & $\begin{array}{l}19.96 \\
19.94 \\
20.01 \\
20.02 \\
19.97\end{array}$ & $\begin{array}{l}20.21 \\
20.20 \\
20.21 \\
20.23 \\
20.34\end{array}$ & $\begin{array}{l}20.53 \\
20.54 \\
20.54 \\
20.58 \\
20.55\end{array}$ & $\begin{array}{l}20.80 \\
20.80 \\
20.78 \\
20.81 \\
20.82\end{array}$ & $\begin{array}{l}20.80 \\
20.78 \\
20.77 \\
20.74 \\
20.68\end{array}$ \\
\hline $\begin{array}{l}26 \\
27 \\
28 \\
29 \\
30 \\
31\end{array}$ & $\begin{array}{r}21.60 \\
21.60 \\
21.57 \\
21.59 \\
21.62 \\
-\end{array}$ & $\begin{array}{l}20.00 \\
19.99 \\
19.95 \\
19.97 \\
19.94 \\
19.93\end{array}$ & $\begin{array}{r}18.06 \\
18.07 \\
18.07 \\
18.08 \\
18.09 \\
\end{array}$ & $\begin{array}{l}18.24 \\
18.25 \\
18.26 \\
18.26 \\
18.28 \\
18.27\end{array}$ & $\begin{array}{l}18.64 \\
18.72 \\
18.75 \\
18.74 \\
18.76 \\
18.79\end{array}$ & $\begin{array}{r}19.15 \\
19.14 \\
19.19 \\
19.24 \\
19.26 \\
-\end{array}$ & $\begin{array}{l}19.61 \\
19.59 \\
19.65 \\
19.66 \\
19.65 \\
19.67\end{array}$ & $\begin{array}{r}20.02 \\
19.97 \\
19.93 \\
19.97 \\
19.99 \\
-\end{array}$ & $\begin{array}{l}20.31 \\
20.23 \\
20.36 \\
20.36 \\
20.35 \\
20.38\end{array}$ & $\begin{array}{l}20.60 \\
20.57 \\
20.52 \\
20.59 \\
20.61 \\
20.61\end{array}$ & $\begin{array}{r}20.82 \\
20.81 \\
20.82 \\
-.- \\
-.-\end{array}$ & $\begin{array}{l}20.67 \\
20.77 \\
20.78 \\
20.75 \\
20.73 \\
20.65\end{array}$ \\
\hline
\end{tabular}


GROUND WATER LEVELS

WOODWARD COUNTY

361256099102101. LOCAL NUMBER, 20N-19W-13 ABB 1.

LOCATION.--LAT 36 12'56", LONG 099 10'21", HYDROLOGIC UNIT 11100301, OWNER: M. JAZEN. AQUIFER.--RUSH SPRINGS FORMATION.

WELL CHARACTERISTICS.--DRILLED STOCK WELL, DIAMETER 6 IN $(0.15 \mathrm{M})$, DEPTH $40 \mathrm{FT}(12.2 \mathrm{M})$.

DATUM. --ALTITUDE OF LAND-SURFACE DATUM IS $1895 \mathrm{FT}$ (578M). MEASURING POINT: EDGE OF PLYWOOD SHELTER BASE $1.10 \mathrm{FT}(0.34 \mathrm{M})$ ABOVE LAND-SURFACE DATUM.

PERIOD OF RECORD.--1972 TO CURRENT YEAR.

EXTREMES FOR PERIOD OF RECORD.-- HIGHEST WATER LEVEL, 9.43 FT (2.874M) BELOW LAND-SURFACE

DATUM, MAR. 31, 1983; LOWEST, 17.44 FT (5.316M) BELOW LAND-SURFACE DATUM, JULY 5, 1972.

WATER LEVEL, IN FEET BELOW LAND SURFACE DATUM, CLIMATIC YEAR APRIL 1982 TO MARCH 1983

MEAN VALUES

\begin{tabular}{|c|c|c|c|c|c|c|c|c|c|c|c|c|}
\hline DAY & APR & MAY & JUN & JUL & AUG & SEP & OCT & Nov & DEC & JAN & FEB & MAR \\
\hline 1 & ... & - & $\ldots$ & $\ldots$ & -.. & $\ldots$ & 10.19 & 10.37 & 10.44 & 10.95 & 10.92 & 10.98 \\
\hline 2 & $\ldots$ & $\ldots$ & $\ldots$ & $\ldots$ & -.. & -.. & 10.19 & 10.49 & 10.57 & 11.01 & 11.05 & 10.94 \\
\hline 3 & -.. & --- & --- & -.- & -.. &.- & 10.26 & 10.58 & 10.71 & 10.94 & 11.21 & 10.83 \\
\hline 4 &.- & -- & -.. & -.. & --. & -.- & 10.17 & 10.51 & 10.63 & 10.89 & 11.18 & 10.44 \\
\hline 5 & 10.58 & 11.60 & 10.74 & 10.25 &.- & 10.20 & 10.17 & 10.45 & 10.66 & 10.92 & 11.01 & 10.29 \\
\hline 6 & $\ldots$ & -- & -- &.- & $\ldots$ & -- & 10.28 & 10.37 & 10.79 & 10.88 & 11.16 & 10.48 \\
\hline 7 & $\ldots$ & -.. & -.. & $\ldots$ & -.. & $\ldots$ & 10.20 & 10.38 & 10.82 & 10.91 & 11.07 & 10.49 \\
\hline 8 & $\ldots$ & ... & -- & -.. & -.. & $\ldots$ & 10.09 & 10.46 & 11.01 & 10.91 & 11.03 & 10.46 \\
\hline 9 & $\ldots$ & -.. & -.. & $\ldots$ & $\ldots$ & $\ldots$ & 10.28 & 10.46 & 10.81 & 10.80 & 11.06 & 10.45 \\
\hline 10 & 10.66 & 11.67 & 10.78 & 10.29 & $\ldots$ & 10.12 & 10.35 & 10.48 & 10.63 & 10.96 & 11.07 & 10.34 \\
\hline 11 & $\ldots$ & -.. &.- & -.. & -.. & $\ldots$ & 10.32 & 10.29 & 10.82 & 11.12 & 11.23 & 10.27 \\
\hline 12 & $\ldots$ &.- & -.. & -.- & -.. & -.. & 10.29 & 10.73 & 10.73 & 11.04 & 11.25 & 10.07 \\
\hline 13 & $\ldots$ & -.. & -.. & -.- & -.. & -.. & 10.32 & 10.50 & 10.51 & 10.93 & 11.06 & 10.03 \\
\hline 14 & $\ldots$ & -.. & -.. & -.- & -.. & -.- & 10.32 & 10.67 & 10.67 & 11.16 & 10.96 & 9.95 \\
\hline 15 & 10.64 & 11.82 & 10.56 & 10.18 &.- & 10.27 & 10.30 & 10.51 & 10.87 & 11.09 & 11.16 & 9.93 \\
\hline 16 & $\ldots$ & $\ldots$ & -.. & $\ldots$ &.- & -.. & 10.36 & 10.43 & 10.83 & 10.90 & 11.11 & 10.07 \\
\hline 17 & $\ldots$ & $\ldots$ & -.. & -.. &.- & $\ldots$ & 10.23 & 10.44 & 10.67 & 10.98 & 11.15 & 10.01 \\
\hline 18 & $\ldots$ & -.. & -.. & -.. & $\ldots$ & $\ldots$ & 10.22 & 10.43 & 10.74 & 11.03 & 11.08 & 9.91 \\
\hline 19 & $\ldots$ & -.. & -.. & -.. & -.. & $=-$ & 10.37 & 10.37 & 10.86 & 10.95 & 10.86 & 9.88 \\
\hline 20 & 11.30 & $\ldots$ & 10.48 & 10.20 & 10.16 & 10.28 & 10.48 & 10.56 & 10.79 & 10.96 & 11.14 & 9.95 \\
\hline 21 & $\ldots$ & 11.52 &.- & $\ldots$ & -.. & -.. & 10.41 & 10.61 & 10.69 & 10.97 & 11.18 & 9.93 \\
\hline 22 & $\ldots$ & ... & - & $\ldots$ & $\cdots$ & -.. & 10.41 & 10.52 & 10.65 & 10.96 & 11.13 & .87 \\
\hline 23 & $\ldots$ & $\ldots$ & $\ldots$ & -.. & -.. & -.. & 10.38 & 10.75 & 10.61 & 10.97 & 11.05 & 9.82 \\
\hline 24 &.- & ..- &.- & $\ldots$ & -.- & -.. & 10.39 & 10.79 & 10.62 & 11.09 & 11.12 & 9.77 \\
\hline 25 & 11.34 & 11.40 & 10.42 & $\ldots$ & 10.19 & $\ldots$ & 10.37 & 10.60 & 11.08 & 10.98 & 11.14 & 9.63 \\
\hline 26 & $\ldots$ & $\ldots$ & ... & $\ldots$ & -.. & $-\cdots$ & 10.32 & 10.68 & 10.95 & 11.14 & 11.08 & 9.64 \\
\hline 27 & -.. & -.. & -.. & -.. & $\ldots$ &.- & 10.23 & 10.55 & 10.69 & 11.05 & 11.01 & 9.91 \\
\hline 28 & $\cdots$ & -.. & -- & $\ldots$ & -- & -.- & 10.37 & 10.42 & 11.02 & 10.85 & 10.96 & 9.80 \\
\hline 29 & -.. & -.. &.- & $\cdots$ & -.. &.- & 10.37 & 10.46 & 10.99 & 11.04 & $-\cdots$ & 9.69 \\
\hline 30 & 11.61 & -.. & 10.35 &.- & -.- & 10.23 & 10.32 & 10.47 & 10.90 & 11.04 & -- & 9.64 \\
\hline 31 & $\ldots$ & 11.18 & $\ldots$ & $\ldots$ & 10.12 &.- & 10.34 & -.. & 10.97 & 11.00 & - & 9.43 \\
\hline
\end{tabular}


361714099315101. LOCAL NUMBER, 21N-22W-23 BBB 1.

LOCATION.--LAT 36 17'14", LONG 099 31'51", HYDROLOGIC UNIT 11100203, OWNER: U.S. GEOLOGICAL SURVEY. AQUIFER.--OGALLALA FORMATION.

WELL CHARACTERISTICS.--DRILLED TEST HOLE, DIAMETER 6 IN (0.15M), DEPTH 322 FT (98.1M).

DATUM.--ALTITUDE OF LAND-SURFACE DATUM IS $2340 \mathrm{FT}$ (713M). MEASURING POINT: TOP OF PLYWOOD WHELF

2.00FT (0.61M) ABOVE LAND-SURFACE DATUM.

PERIOD OF RECORD.-- 1957 TO 1963, 1965 TO CURRENT YEAR.

EXTREMES FOR PERIOD OF RECORD.--HIGHEST WATER LEVEL, 26.00 FT (7.925M) BELOW LAND-SURFACE

DATUM, JAN. 27, 1983; LOWEST, 32.64 FT (9.949M) BELOW LAND-SURFACE DATUM, MAY 19, 1971.

WATER LEVEL, IN FEET BELOW LAND SURFACE DATUM, CLIMATIC YEAR APRIL 1982 TO MARCH 1983 MEAN VALUES

\begin{tabular}{|c|c|c|c|c|c|c|c|c|c|c|c|c|}
\hline DAY & APR & MAY & JUN & JUL & AUG & SEP & OCT & NOV & DEC & JAN & FEB & MAR \\
\hline 1 & -.. & $\ldots$ &.- & -.. & -- &.-- & 26.23 & 26.13 & 26.05 & 26.16 & 26.06 & 26.17 \\
\hline 2 & ... & -.. & -.- & 26.64 &.-- & -.. & 26.21 & 26.18 & 26.08 & 26.16 & 26.07 & 26.17 \\
\hline 3 & -.- & -- & -- & -.. & --- &.-- & 26.23 & 26.22 & 26.13 & 26.15 & 26.14 & 26.13 \\
\hline 4 & $\ldots$ & $\ldots$ & $\ldots$ & -- & -- & --- & 26.19 & 26.19 & 26.11 & 26.11 & 26.16 & 26.09 \\
\hline 5 & 27.15 & 27.05 & $-\cdots$ & 26.61 & - & 26.35 & 26.18 & 26.16 & 26.10 & 26.12 & 26.10 & 26.08 \\
\hline 6 & -- & -- & -- & -- & -- & -.- & 26.22 & 26.12 & 26.15 & 26.11 & 26.01 & 26.17 \\
\hline 7 & -.- & -- & -- & -.. & -.. &.- & 26.19 & 26.11 & 26.15 & 26.11 & 26.13 & 26.21 \\
\hline 8 & --- & $-\cdots$ & -- & -- &.- & -.- & 26.14 & 26.14 & 26.25 & 26.11 & 26.10 & 26.24 \\
\hline 9 & -.. & -. &.- & -- &.-- &.-- & 26.22 & 26.14 & 26.19 & 26.05 & 26.10 & 26.15 \\
\hline 10 & 27.13 & 27.04 &.-- &.-- & -- & 26.31 & 26.26 & 26.14 & 26.09 & 26.11 & 26.10 & 26.14 \\
\hline 11 & --- &.- & -.- & -.. &.- & -.. & 26.25 & 26.07 & 26.15 & 26.15 & 26.15 & 26.01 \\
\hline 12 & --. & -. & $\ldots$ & -.. & -.. & -.. & 26.23 & 26.24 & 26.13 & 26.16 & 26.20 & 26.20 \\
\hline 13 & --- & -- & -- & --- & -- & $\ldots$ & 26.23 & 26.17 & 26.04 & 26.11 & 26.15 & 26.20 \\
\hline 14 & --- & -- & -- & -- & $\ldots$ & --- & 26.23 & 26.22 & 26.07 & 26.15 & 26.09 & 26.18 \\
\hline 15 & 26.99 & 27.09 &.- & 26.57 &.- & 26.35 & 26.21 & 26.17 & 26.16 & 26.06 & 26.16 & 26.17 \\
\hline 16 & --- & -- & --- & --- & -- & --- & 26.24 & 26.11 & 26.15 & 26.10 & 26.17 & 26.25 \\
\hline 17 & -- & -- & $\cdots$ & -- & -- & -- & 26.17 & 26.12 & 26.09 & 26.11 & 26.16 & 26.26 \\
\hline 18 & --- & --- & -- & -- & -- & -- & 26.14 & 26.10 & 26.08 & 26.12 & 26.16 & 26.24 \\
\hline 19 & --- & -- &.- & -.. &.-- & -- & 26.20 & 26.07 & 26.14 & 26.10 & 26.08 & 26.23 \\
\hline 20 & 27.16 & --- & --- & 26.54 & 26.40 & 26.33 & 26.26 & 26.13 & 26.10 & 26.09 & 26.15 & 26.12 \\
\hline 21 &.- & 27.05 &.-- &.- & $\ldots$ &.-- & 26.22 & 26.15 & 26.06 & 26.10 & 26.21 & 26.27 \\
\hline 22 & -- & -- & -- & -- & -- & -- & 26.22 & 26.11 & 26.04 & 26.09 & 26.20 & 26.27 \\
\hline 23 & --- & -- & -- & -- & --- & -- & 26.20 & 26.19 & 26.02 & 26.09 & 26.20 & 26.26 \\
\hline 24 & --- & -.- & -- & --- & --- & -- & 26.19 & 26.23 & 26.01 & 26.12 & 26.21 & 26.25 \\
\hline 25 & 27.04 & 27.02 & 26.69 & --- & 26.40 & 26.28 & 26.17 & 26.14 & 26.04 & 26.10 & 26.23 & 26.18 \\
\hline 26 &.-- & -.- & -.- & -.. & -.. &.- & 26.15 & 26.16 & 26.18 & 26.12 & 26.20 & 26.17 \\
\hline 27 & --- & -- & --- & -- & -- & -- & 26.11 & 26.12 & 26.07 & 26.00 & 26.18 & 26.33 \\
\hline 28 & -- & -- & -- & -- & -- & $\cdots$ & 26.16 & 26.06 & 26.14 & 26.04 & 26.17 & 26.34 \\
\hline 29 & -- & -- & -- & $\ldots$ & $\ldots$ & -- & 26.16 & 26.07 & 26.18 & 26.08 & --- & 26.32 \\
\hline 30 & 27.12 & -- & 26.67 & -- & --- & 26.23 & 26.12 & 26.07 & 26.14 & 26.11 & -- & 26.32 \\
\hline 31 & -- & -- & --- & -- & 26.36 & -- & 26.13 & - & 26.15 & 26.09 & -- & 26.21 \\
\hline
\end{tabular}


Table 2. List of wells and water-levels measured annually.

GROUND-WATER LEVELS

ADAIR COUNTY

355914094340001. LOCAL NUMBER;

$17 N-26 E-05$ BAA 1.

LOCATION,--LAT 35 59'14", LONG 094 34'00", HYDROLOGIC UNIT 11110103.

AQUIFER.--BOONE FORMATION.

PERIOD OF RECORD .--1979 TO CURRENT YEAR.

EXTREMES FOR PERIOD OF RECORD.--HIGHEST WATER LEVEL, 10.23 FT(3.12 M) BELOW LAND-SURFACE DATUM,MAR 9, 1983; LOWEST WATER LEVEL,24.23 FT(7.39 M) BELOW LAND-SURFACE DATUM, FEB 26, 1980.

WATER LEVELS IN FEET BELOW LAND SURFACE DATUM, CLIMATIC YEARS APRIL 1981 TO MARCH 1983

$\begin{array}{lll} & \text { WATER } & \\ \text { DATE } & \text { WEVEL } & \text { WATER } \\ \text { LEVEL }\end{array}$

MAR 29, $1982 \quad 12.48 \quad$ MAR 09, $1983 \quad 10.23$

GROUND-WATER LEVELS

355742094331201. LOCAL NUMBER; 17N-26E-09 CCA 1.

LOCATION,--LAT 35 57'42", LONG 094 33'12", HYDROLOGIC UNIT 11110103.

AQUIFER.--BOONE FORMATION.

PERIOD OF RECORD.--1979 TO CURRENT YEAR.

EXTREMES FOR PERIOD OF RECORD.--HIGHEST WATER LEVEL, $52.59 \mathrm{FT}(16.03 \mathrm{M})$ BELOW LAND-SURFACE DATUM,MAR 9, 1983; LOWEST WATER LEVEL,58.87 FT(17.94 M) BELOW LAND-SURFACE DATUM, MAR 10, 1981.

WATER LEVELS IN FEET BELOW LAND SURFACE DATUM, CLIMATIC YEARS APRIL 1981 TO MARCH 1983

$\begin{array}{lll} & \text { WATER } & \\ \text { DATE } & \text { LEVEL } & \text { WATER } \\ & \text { DATE } & \text { LEVEL }\end{array}$

MAR 29, $1982 \quad 57.31 \quad$ MAR 09, $1983 \quad 52.59$ 


\section{GROUND-WATER LEVELS}

\section{ADAIR COUNTY -- CONTINUED}

\section{LOCAL NUMBER;}

LOCATION,--LAT $3600^{\prime} 00^{\prime \prime}$, LONG 094

AQUIFER.--BOONE FORMATION.

PERIOD OF RECORD.--1977 TO CURRENT YEAR.

EXTREMES FOR PERIOD OF RECORD.--HIGHEST WATER LEVEL, $18.70 \mathrm{FT}(5.70 \mathrm{M})$ BELOW LAND-SURFACE DATUM,MAR 22, 1978; LOWEST WATER LEVEL,37.17 FT(11.33 M) BELOW LAND-SURFACE DATUM, FEB 26, 1980.

WATER LEVELS IN FEET BELOW LAND SURFACE DATUM, CLIMATIC YEARS APRIL 1981 TO MARCH 1983

\begin{tabular}{|c|c|}
\hline & WATER \\
\hline DATE & LEVEL \\
\hline
\end{tabular}

MAR 29, $1982 \quad Z \quad$ MAR 09, $1983 \quad 24.25$

GROUND-WATER LEVELS

360006094340001. LOCAL NUMBER; 18N-26E-32 BAA 1.

LOCATION,--LAT $3600^{\prime} 06^{\prime \prime}$, LONG 094 34'00", HYDROLOGIC UNIT 11110103.

AQUIFER .--BOONE FORMATION.

PERIOD OF RECORD.--1982 TO CURRENT YEAR.

EXTREMES FOR PERIOD OF RECORD.--HIGHEST WATER LEVEL, 32.82 FT(10.00 M) BELOW LAND-SURFACE DATUM,MAR 9, 1983; LOWEST WATER LEVEL, 32.99 FT(10.06 M) BELOW LAND-SURFACE DATUM, MAR 29, 1982.

WATER LEVELS IN FEET BELOW LAND SURFACE DATUM, CLIMATIC YEARS APRIL 1981 TO MARCH 1983

\begin{tabular}{|c|c|}
\hline & WATER \\
\hline DATE & LEVEL \\
\hline
\end{tabular}

MAR 29, $1982 \quad 32.99 \quad$ MAR 09, $1983 \quad 32.82$


362953098245401. LOCAL NUMBER;

$23 \mathrm{~N}-11 \mathrm{~W}-06$ DBB 1.

LOCATION,--LAT 36 29'53", LONG 098 24'54", HYDROLOGIC UNIT 11050001. AQUIFER.--ALLUVIUM.

PERIOD OF RECORD.--1983 TO CURRENT YEAR.

EXTREMES FOR PERIOD OF RECORD.--HIGHEST WATER LEVEL,7.40 FT(2.26 M) BELOW LAND-SURFACE DATUM,MAR 25, 1983; LOWEST WATER LEVEL,7.40 FT(2.26 M) BELOW LAND-SURFACE DATUM, MAR 25, 1983.

WATER LEVELS IN FEET BELOW LAND SURFACE DATUM, CLIMATIC YEARS APRIL 1981 TO MARCH 1983

$\begin{array}{ll} & \text { WATER } \\ \text { DATE } & \text { LEVEL }\end{array}$

MAR $25,1983 \quad 7.40$

GROUND-WATER LEVELS

363025098291101. LOCAL NUMBER; 24N-12W-33 DCC 1.

LOCATION,--LAT 36 30'25", LONG 098 29'11", HYDROLOGIC UNIT 11050001.

AQUIFER .--ALLUVIUM.

PERIOD OF RECORD.--1983 TO CURRENT YEAR.

EXTREMES FOR PERIOD OF RECORD.--HIGHEST WATER LEVEL, 15.05 FT(4.59 M) BELOW LAND-SURFACE DATUM, MAR 25, 1983; LOWEST WATER LEVEL, 15.05 FT(4.59 M) BELOW LAND-SURFACE DATUM, MAR 25, 1983.

WATtR LEVELS IN FELT BELOW LAND SURFACE DATUM, CLIMATIC YEARS APRIL 1981 TO MARCH 1983

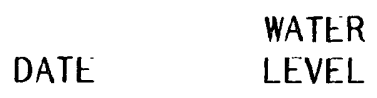

MAR 25, $1983 \quad 15.05$ 
363823098233701. LOCAL NUMBER; LOCATION,--LAT 36 38'23", LONG 098 AQUIFER.--TERRACE DEPOSITS. PERIOD OF RECORD.--1974 TO CURRENT YEAR. EXTREMES FOR PERIOD OF RECORD.--HIGHEST WATER LEVEL, 3.64 FT(1.11 M) BELOW LAND-SURFACE DATUM,MAR 2, 1983; LOWEST WATER LEVEL,8.25 FT(2.51 M) BELOW LAND-SURFACE DATUM, JAN 4, 1978.

WATER LEVELS IN FEET BELOW LAND SURFACE DATUM, CLIMATIC YEARS APRIL 1981 TO MARCH 1983

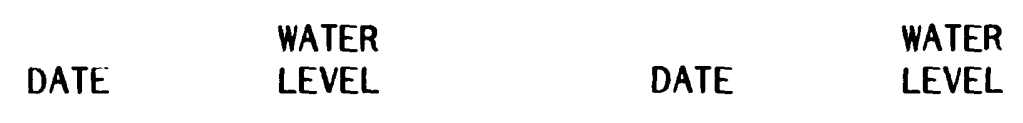

MAR 05, $1982 \quad 3.87 \quad$ MAR 02, $1983 \quad 3.64$
365028098104001 . LOCAL NUMBER; LOCATION,--LAT 36 50'28", LONG 098 AQUIFER.--TERRACE DEPOSITS. PERIOD OF RECORD.--1966 TO CURRENT YEAR. EXTREMES FOR PERIOD OF RECORD.--HIGHEST WATER LEVEL, $3.01 \mathrm{FT}(.92 \mathrm{M})$ BELOW LAND-SURFACE DATUM, MAR 2, 1983; LOWEST WATER LEVEL, 10.40 FT(3.17 M) BELOW LAND-SURFACE DATUM, MAR $04,1982$.

WATER LEVELS IN FEET BELOW LAND SURFACE DATUM, CLIMATIC YEARS APRIL 1981 TO MARCH 1983
DATE
WATER
LEVEL
DATE
WATER
LEVEL

MAR 04, $1982 \quad 10.40$ 
ALFALFA COUNTY -- CONTINUED

364909098210301. LOCAL NUMBER; 27N-11W-14 DBB 1. LOCATION,--LAT 36 49'09", LONG 098 21'03", HYDROLOGIC UNIT 11060002. AQUIFER.--TERRACE DEPOSITS. PERIOD OF RECORD.--1974 TO CURRENT YEAR. EXTREMES FOR PERIOD OF RECORD.--HIGHEST WATER LEVEL, $5.00 \mathrm{FT}(1.52 \mathrm{M})$ BELOW LAND-SURFACE DATUM,JAN 24, 1975; LOWEST WATER LEVEL, 10.73 FT(3.27 M) BELOW LAND-SURFACE DATUM, DEC 16, 1976.

WATER LEVELS IN FEET BELOW LAND SURFACE DATUM, CLIMATIC YEARS APRIL 1981 TO MARCH 1983

\begin{tabular}{|c|c|c|}
\hline & WATER & \\
\hline DATE & LEVEL & DATE \\
\hline
\end{tabular}

MAR OS, $1982 \quad 6.92 \quad$ MAR 02, $1983 \quad 5.99$

GROUND-WATER LEVELS

364837098205501. LOCAL NUMBER; 27N-11W-23 ABD 1. LOCATION,--LAT 36 48'37", LONG 098 20'55", HYDROLOGIC UNIT 11060004.

AQUIFER.--TERRACE DEPOSITS.

PERIOD OF RECORD.--1974 TO CURRENT YEAR.

EXTREMES FOR PERIOD OF RECORD.--HIGHEST WATER LEVEL,7.48 FT(2.28 M) BELOW LAND-SURFACE DATUM, MAR 2, 1983; LOWEST WATER LLVEL, 12.45 FT(3.79 M) BELOW LAND-SURFACE DATUM, DEC 16, 1976.

WATER LEVELS IN FEET BELOW LAND SURFACE DATUM, CLIMATIC YEARS APRIL 1981 TO MARCH 1983

$\begin{array}{lll} & \text { WATER } & \text { WATER } \\ \text { DATE } & & \\ \text { LEVEL } & \text { DATE } & \text { LEVEL }\end{array}$

MAR 05, $1982 \quad 8.20 \quad$ MAR $02,1983 \quad 7.48$ 


\section{ALFALFA COUNTY -- CONTINUED}

365916098125001. LOCAL NUMBER;

LOCATION,--LAT 36 59'16", LONG 098

AQUIFER.--TERRACE DEPOSITS.

PERIOD OF RECORD.--1974 TO CURRENT

EXTREMES FOR PERIOD OF RECORD .--HIGHEST WATER LEVEL,6.49 FT(1.98 M) BELOW LAND-SURFACE DATUM,JAN 24, 1975; LOWEST WATER LEVEL,8.75 FT(2.67 M) BELOW LAND-SURFACE DATUM, JAN 22, 1981. WATER LEVELS IN FEET BELOW LAND SURFACE DATUM, CLIMATIC YEARS APRIL 1981 TO MARCH 1983
$29 \mathrm{~N}-09 \mathrm{~W}-18 \mathrm{CDD} 1$. 12 '50", HYDROLOGIC UNIT 11060004.

YEAR.

$\begin{array}{lrrrr} & \text { WATER } & & \text { WATER } \\ \text { DATE } & \text { LEVEL } & \text { DATE } & \text { LEVEL } \\ & & & & \\ \text { MAR 05, 1982 } & 7.05 & \text { MAR 02, 1983 } & 7.58\end{array}$


ATOKA COUNTY

341938096090001. LOCAL NUMBER;

03S-11E-04 ACA 1. LOCATION,--LAT 34 19'38", LONG 096 09'00", HYDROLOGIC UNIT 11140104. AQUIFER.--ANTLERS SAND.

PERIOD OF RECORD.--1976 TO CURRENT YEAR.

EXTREMES FOR PERIOD OF RECORD.--HIGHEST WATER LEVEL, 30.00 FT(9.14 M) BELOW LAND-SURFACE DATUM, APR 21, 1976; LOWEST WATER LEVEL,40.76 FT(12.42 M) BELOW LAND-SURFACE DATUM, JAN $4,1977$.

WATER LEVELS IN FEET BELOW LAND SURFACE DATUM, CLIMATIC YEARS APRIL 1981 TO MARCH 1983

$\begin{array}{lll} & \text { WATER } & \text { WATER } \\ \text { DATE } & \text { LEVEL } & \text { DATE }\end{array}$

MAR $10,1982 \quad 34.96 \quad$ MAR 08, $1983 \quad 54.92 \mathrm{P}$ 
BEAVER COUNTY

363035100490201. LOCAL NUMBER; 01N-21E-32 ABC 1. LOCATION,--LAT 36 30'35", LONG 100 49'02", HYDROLOGIC UNIT 11100104. AQUIFER .--OGALLALA. PERIOD OF RECORD.--1968 TO CURRENT YEAR. EXTREMES FOR PERIOD OF RECORD.--HIGHEST WATER LEVEL,239.57 FT(73.02 M) BELOW LAND-SURFACE DATUM,FEB 5, 1968; LOWEST WATER LEVEL,243.50 FT(74.22 M) BELOW LAND-SURFACE DATUM, JAN 12, 1977.

WATER LEVELS IN FEET BELOW LAND SURFACE DATUM, CLIMATIC YEARS APRIL 1981 TO MARCH 1983

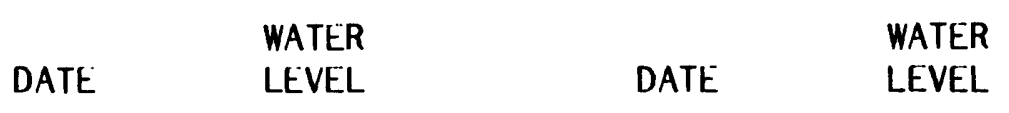

JAN $18,1982 \quad 243.00 \quad$ JAN $10,1983 \quad P$

GROUND-WATER LEVELS

363411100384701. LOCAL NUMBER; 01N-22E-12 BCB 1. LOCATION,--LAT 36 34'11", LONG 100 38'47", HYDROLOGIC UNIT 11100201. AQUIFER . - OGALLALA.

PERIOD OF RECORD.-- 1967 TO CURRENT YEAR.

EXTREMES FOR PERIOD OF RECORD.--HIGHEST WATER LEVEL, 182.33 FT(55.57 M) BELOW LAND-SURFACE DATUM,JAN 19, 1968; LOWEST WATER LEVEL,204.07 FT(62.20 M) BELOW LAND-SURFACE DATUM, JAN 18, 1982.

WATER LEVELS IN FEET BELOW LAND SURFACE DATUM, CLIMATIC YEARS APRIL 1981 TO MARCH 1983

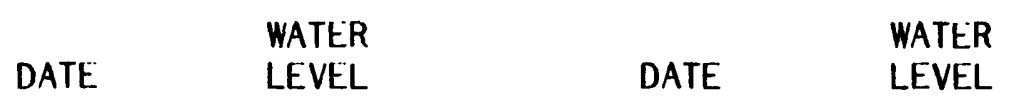

JAN 18, $1982 \quad 204.07 \quad$ JAN 13, $1983 \quad 199.85$ 
BEAVER COUNTY -- CONTINUED

363136100430001. LOCAL NUMBER; $\quad 01 \mathrm{~N}-22 \mathrm{E}-29$ BBD 1. LOCATION,--LAT 36 31'36", LONG 100 43'00", HYDROLOGIC UNIT 11100201. AQUIFER.--OGALLALA.

PERIOD OF RECORD.--1980 TO CURRENT YEAR.

EXTREMES FOR PERIOD OF RECORD.--HIGHEST WATER LEVEL,211.16 FT(64.36 M) BELOW LAND-SURFACE DATUM, JAN 27, 1981; LOWEST WATER LEVEL,222.35 FT(67.77 M) BELOW LAND-SURFACE DATUM, MAY 22, 1980.

WATER LEVELS IN FEET BELOW LAND SURFACE DATUM, CLIMATIC YEARS APRIL 1981 TO MARCH 1983

$\begin{array}{cccc}\text { DATE } & \text { WATER } & & \text { WATER } \\ \text { LEVEL } & \text { DATE } & \text { LEVEL } \\ \text { JAN 18, 1982 } & 211.47 & \text { JAN 10, 1983 } & 211.56\end{array}$

GROUND-WATER LEVELS

363011100410601. LOCAL NUMBER; O1N-22E-33 DDD 1.

LOCATION,--LAT 36 30'11", LONG 100 41'06", HYDROLOGIC UNIT 11100201.

AQUIFER.--OGALLALA.

PERIOD OF RECORD.--1970 TO CURRENT YEAR.

EXTREMES FOR PERIOD OF RECORD.--HIGHEST WATER LEVEL,236.06 FT(71.95 M) BELOW LAND-SURFACE DATUM, JAN 12, 1971; LOWEST WATER LEVEL,243.16 FT(74.12 M) BELOW LAND-SURFACE DATUM, JAN 18, 1978.

WATER LEVELS IN FEET BELOW LAND SURFACE DATUM, CLIMATIC YEARS APRIL 1981 TO MARCH 1983

$\begin{array}{cccc}\text { DATE } & \begin{array}{l}\text { WATER } \\ \text { LEVEL }\end{array} & \text { DATE } & \text { WATER } \\ \text { LEVEL }\end{array}$


BEAVER COUNTY -- CONTINUED

363006100404201. LOCAL NUMBER; LOCATION,--LAT 36 30'06", LONG 100 AQUIFER.--OGALLALA. PERIOD OF RECORD.--1967 TO CURRENT YEAR. EXTREMES FOR PERIOD OF RECORD.--HIGHEST WATER LEVEL,235.50 FT(71.78 M) BELOW LAND-SURFACE DATUM,FEB 8, 1967; LOHEST WATER JAN 12, 1977.

WATER LEVELS IN FEET BELOW LAND SURFACE DATUM, CLIMATIC YEARS APRIL 1981 TO MARCH 1983
$01 \mathrm{~N}-22 \mathrm{E}-34 \mathrm{CDC} 1$ 40'42", HYDROLOGIC UNIT 11100201. LEVT WATER LEVEL, $235.50 \mathrm{FT}(71.78 \mathrm{M})$ BELOW LAND-SURFACE
LEVEL,248.90 FT(75.86 M) BELOW LAND-SURFACE DATUM,

LEVEL, 248.90 FT(75.86 M) BELON LAND-SURFACE DATUM, WATER LEVELS IN FEET BELOW LAND SURFACE DATUM, CLIMATIC YEARS APRIL 1981 TO MARCH 1983

$\begin{array}{lll} & \text { WATER } & \\ \text { DATE } & \text { LEVEL } & \text { WATER } \\ & & \text { LEVEL }\end{array}$

JAN $18,1982 \quad 244.71 \quad$ JAN $10,1983 \quad 244.67$

GROUND-WATER LEVELS

363342100360801. LOCAL NUMBER; 01N-23E-08 DCB 1. LOCATION,--LAT 36 33'42", LONG 100 36'08", HYDROLOGIC UNIT 11100201. AQUIFER .--OGALLALA.

PERIOD OF RECORD.--1970 TO CURRENT YEAR.

EXTREMES FOR PERIOD OF RECORD.--HIGHEST WATER LEVEL, 202.81 FT(61.82 M) BELOW LAND-SURFACE DATUM, JAN 13, 1970; LOWEST WATER LEVEL,215.65 FT(65.73 M) BELOW LAND-SURFACE DATUM, JAN 14, 1977.

WATER LEVELS IN FEET BELOW LAND SURFACE DATUM, CLIMATIC YEARS APRIL 1981 TO MARCH 1983

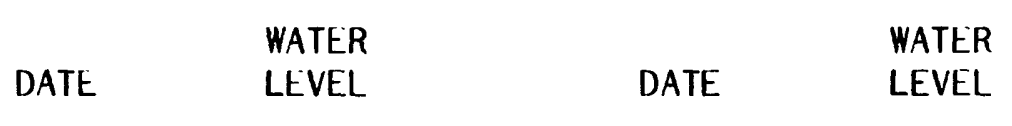

JAN $17,1982 \quad 209.95 \quad$ JAN 09, $1983 \quad 209.29$ 


\section{GROUND-WATER LEVELS}

BEAVER COUNTY -- CONTINUED

363207100365101. LOCAL NUMBER;

$01 \mathrm{~N}-23 \mathrm{E}-19$ DAB 1. LOCATION,--LAT 36 32'07', LONG 100 AQUIFER.--OGALLALA.

PERIOD OF RECORD.--1967 TO CURRENT YEAR.

EXTREMES FOR PERIOD OF RECORD.--HIGHEST WATER LEVEL,220.28 FT(67.14 M) BELOW LAND-SURFACE DATUM, JAN 18, 1968; LOWEST WATER LEVEL,241.61 FT(73.64 M) BELOW LAND-SURFACE DATUM, JAN 19, 1979.

WATER LEVELS IN FEET BELOW LAND SURFACE DATUM, CLIMATIC YEARS APRIL 1981 TO MARCH 1983

$\begin{array}{lll} & \text { WATER } & \\ \text { DATE } & \text { WEVEL } & \text { WATER } \\ & \text { LEVEL }\end{array}$

JAN 18, $1982 \quad 236.46 \quad$ JAN 11, $1983 \quad 236.33$

\section{GROUND-WATER LEVELS}

363233100324001. LOCAL NUMBER;

$01 \mathrm{~N}-23 \mathrm{E}-23$ AAB 1.

LOCATION,--LAT 36 32'33", LONG 100 32'40", HYDROLOGIC UNIT 11100201.

AQUIFER .--OGALLALA.

PERIOD OF RECORD.--1968 TO CURRENT YEAR.

EXTREMES FOR PERIOD OF RECORD.--HIGHEST WATER LEVEL, 163.58 FT(49.86 M) BELOW LAND-SURFACE DATUM,FEB 6, 1968; LOWEST WATER LEVEL, 171.02 FT(52.13 M) BELOW LAND-SURFACE DATUM, JAN 20, 1972.

WATER LEVELS IN FEET BELOW LAND SURFACE DATUM, CLIMATIC YEARS APRIL 1981 TO MARCH 1983

$\begin{array}{cccc}\text { DATE } & \text { WATER } & & \text { WATER } \\ \text { LEVEL } & \text { DATE } & \text { LEVEL } \\ \text { JAN 18, 1982 } & 166.70 & \text { JAN 11, 1983 } & 167.94\end{array}$


BEAVER COUNTY -- CONTINUED

363138100332701. LOCAL NUMBER; 01N-23E-26 BBB 1. LOCATION,--LAT 36 31'38", LONG 100 33'27", HYDROLOGIC UNIT 11100201.

AQUIFER.--OGALLALA.

PERIOD OF RECORD.--1967 TO CURRENT YEAR.

EXTREMES FOR PERIOD OF RECORD.--HIGHEST WATER LEVEL,212.42 FT(64.75 M) BELOW LAND-SURFACE DATUM,FEB 8, 1967; LOWEST WATER LEVEL,228.74 FT(69.72 M) BELOW LAND-SURFACE DATUM, JAN $27,1969$.

WATER LEVELS IN FEET BELOW LAND SURFACE DATUM, CLIMATIC YEARS APRIL 1981 TO MARCH 1983

$\begin{array}{lll} & \text { WATER } & \text { WATER } \\ \text { DATE } & \text { LEVEL } & \text { DATE }\end{array}$

JAN $18,1982 \quad 223.57 \quad$ JAN $11,1983 \quad 222.87$

GROUND-WATER LEVELS

363136100363701. LOCAL NUMBER; 01N-23E-29 BBC 1.

LOCATION,--LAT 36 31'36", LONG 100 36'37", HYDROLOGIC UNIT 11100201.

AQUIFER .--OGALLALA.

PERIOD OF RECORD.--1967 TO CURRENT YEAR.

EXTREMES FOR PERIOD OF RECORD.--HIGHEST WATER LEVEL,219.07 FT(66.77 M) BELOW LAND-SURFACE DATUM,FEB 8, 1967; LOWEST WATER LEVEL,247.80 FT(75.53 M) BELOW LAND-SURFACE DATUM, JAN 9, 1981.

WATER LEVELS IN FEET BELOW LAND SURFACE DATUM, CLIMATIC YEARS APRIL 1981 TO MARCH 1983

$\begin{array}{lll} & \text { WATER } & \text { WATER } \\ \text { DATE } & \text { LEVEL } & \text { DATE }\end{array}$

JAN 18, $1982 \quad 243.92 \quad$ JAN $11,1983 \quad 243.37$ 
BEAVER COUNTY -- CONTINUED

363514100251801. LOCAL NUMBER; 01N-24E-01 ABB 1.

LOCATION,--LAT 36 35'14", LONG 100 25'18", HYOROLOGIC UNIT 11100201.

AQUIFER, --OGALLALA.

PERIOD OF RECORD.-- 1967 TO CURRENT YEAR.

EXTREMES FOR PERIOD OF RECORD.--HIGHEST WATER LEVEL,215.57 FT(65.71 M) BELOW LAND-SURFACE DATUM, JAN 17, 1978; LOWEST WATER LEVEL,255.55 FT(77.89 M) BELOW LAND-SURFACE DATUM, JAN 18,1982 .

WATER LEVELS IN FEET BELOW LAND SURFACE DATUM, CLIMATIC YEARS APRIL 1981 TO MARCH 1983

\begin{tabular}{|c|c|}
\hline & $\begin{array}{l}\text { WATER } \\
\text { LEVEL }\end{array}$ \\
\hline
\end{tabular}

JAN 18, $1982 \quad 255.55 \mathrm{R} \quad$ JAN 11, $1983 \quad 220.03$

GROUND-WATER LEVELS

363017100250501. LOCAL NUMBER; 01N-24E-36 DAC 1.

LOCATION,--LAT 36 30'17", LONG 100 . 25'05", HYDROLOGIC UNIT 11100201.

AQUIFER .--OGALLALA.

PERIOD OF RECORD.--1980 TO CURRENT YEAR.

EXTREMES FOR PERIOD OF RECORD.--HIGHEST WATER LEVEL, 78.28 FT $(23.86 \mathrm{M})$ BELOW LAND-SURFACE DATUM, JUNE 3, 1980; LOWEST WATER LEVEL, 80.30 FT(24.48 M) BELOW LAND-SURFACE DATUM, JAN 18, 1982 .

WATER LEVELS IN FEET BELOW LAND SURFACE DATUM, CLIMATIC YEARS APRIL 1981 T0 MARCH 1983

\begin{tabular}{cccc}
\multicolumn{2}{c}{ WATER } & & \multicolumn{1}{c}{ WATER } \\
DATE & LEVEL & DATE & LEVEL \\
JAN 18, 1982 & 80.30 & JAN 11, 1983 & 79.45
\end{tabular}




\section{GROUND-WATER LEVELS}

BEAVER COUNTY -- CONTINUED

363424100192501. LOCAL NUMBER; 01N-25E-01 CCC 1. LOCATION,--LAT 36 34'24", LONG 100 19'25", HYDROLOGIC UNIT 11100201. AQUIFER.--OGALLALA.

PERIOD OF RECORD.--1980 TO CURRENT YEAR.

EXTREMES FOR PERIOD OF RECORD.--HIGHEST WATER LEVEL, 149.10 FT(45.45 M) BELOW LAND-SURFACE DATUM, JUNE 4, 1980; LOWEST WATER LEVEL, 159.38 FT(48.58 M) BELOW LAND-SURFACE DATUM, JAN 11, 1983.

WATER LEVELS IN FEET BELOW LAND SURFACE DATUM, CLIMATIC YEARS APRIL 1981 TO MARCH 1983

$\begin{array}{lll} & \text { WATER } & \text { WATER } \\ \text { DATE } & \text { LEVEL } & \text { DATE }\end{array}$

JAN 18, $1982159.06 \quad$ JAN $11,1983 \quad 159.38$

GROUND-WATER LEVELS

363356100241801. LOCAL NUMBER; 01N-25E-07 CAA 1.

LOCATION,--LAT 36 33'56", LONG 100 24'18", HYDROLOGIC UNIT 11100201.

AQUIFER.--OGALLALA.

PERIOD OF RECORD.--1968 TO CURRENT YEAR.

EXTREMES FOR PERIOD OF RECORD.--HIGHEST WATER LEVEL,59.38 FT(18.10 M) BELOW LAND-SURFACE DATUM,JAN 12, 1980; LOWEST WATER LEVEL,62.67 FT(19.10 M) BELOW LAND-SURFACE DATUM, FEB 3, 1978.

WATER LEVELS IN FEET BELOW LAND SURFACE DATUM, CLIMATIC YEARS APRIL 1981 TO MARCH 1983

$\begin{array}{lll} & \text { WATER } & \text { WATER } \\ \text { DATE } & \text { LEVEL } & \text { DATE }\end{array}$
JAN 18, 1982
Z JAN 11,1983
$P$ 
BEAVER COUNTY -- CONTINUED

363319100142401. LOCAL NUMBER; LOCATION,--LAT 36 33'19", LONG 100 AQUIFER.--OGALLALA.

PERIOD OF RECORD.--1980 TO CURRENT YEAR.

EXTREMES FOR PERIOD OF RECORD.--HIGHEST WATER LEVEL, 35.02 FT(10.67 M) BELOW LAND-SURFACE DATUM,JAN 11, 1983; LOWEST WATER LEVEL, 44.48 FT(13.56 M) BELOW LAND-SURFACE DATUM, JUNE $6,1980$.

WATER LEVELS IN FEET BELOW LAND SURFACE DATUM, CLIMATIC YEARS APRIL 1981 TO MARCH 1983

\begin{tabular}{|c|c|c|}
\hline & WATER & \\
\hline DATE & LEVEL & DATE \\
\hline
\end{tabular}

JAN 18, $1982 \quad 37.10 \quad$ JAN 11, $1983 \quad 35.02$

GROUND-WATER LEVELS

363028100124101 . LOCAL NUMBER; $\quad 01 \mathrm{~N}-26 \mathrm{E}-36$ BDC 1.

LOCATION,--LAT 36 30'28", LONG $10012^{\prime} 41^{\prime \prime}$, HYDROLOGIC UNIT 11100201.

AQUIFER .--OGALLALA.

PERIOD OF RECORD.--1967 TO CURRENT YEAR.

EXTREMES FOR PERIOD OF RECORD.-- HIGHEST WATER LEVEL, 167.79 FT(51.14 M) BELOW LAND-SURFACE DATUM,JAN 14, 1970; LOWEST WATER LEVEL, 171.52 FT(52.28 M) BELOW LAND-SURFACE DATUM, JAN 17, 1978.

WATER LEVELS IN FEET BELOW LAND SURFACE DATUM, CLIMATIC YEARS APRIL 1981 TO MARCH 1983

\begin{tabular}{|c|c|c|}
\hline & WATER & \\
\hline DATE & LEVEL & DATE \\
\hline
\end{tabular}

JAN 18, $1982 \quad 170.48 \quad$ JAN 11, $1983 \quad$ Z 
BEAVER COUNTY -- CONTINUED

363451100111801. LOCAL NUMBER; 01N-27E-06 ACB 1.

LOCATION,--LAT 36 34'51", LONG 100 11'18", HYDROLOGIC UNIT 11100201.

AQUIFER .--OGALLALA.

PERIOD OF RECORD.--1970 TO CURRENT YEAR.

EXTREMES FOR PERIOD OF RECORD.--HIGHEST WATER LEVEL, $19.30 \mathrm{FT}(5.88 \mathrm{M})$ BELOW LAND-SURFACE DATUM, JAN 12, 1976; LOWEST WATER LEVEL,21.57 FT(6.57 M) BELOW LAND-SURFACE DATUM, JAN 19, 1972.

WATER LEVELS IN FEET BELOW LAND SURFACE DATUM, CLIMATIC YEARS APRIL 1981 TO MARCH 1983

\begin{tabular}{cccc}
\multicolumn{1}{c}{ WATER } & & WATER \\
DATE & LEVEL & DATE & LEVEL \\
JAN 17, 1982 & 20.37 & JAN 09, 1983 & 20.03
\end{tabular}

GROUND-WATER LEVELS

363235100093301. LOCAL NUMBER; $01 \mathrm{~N}-27 \mathrm{E}-21 \mathrm{BBA} 1$. LOCATION,--LAT 36 32'35", LONG 100 09'33", HYDROLOGIC UNIT 11100201. AQUIFER .--OGALLALA.

PERIOD OF RECORD.--1967 TO CURRENT YÉAR.

EXTREMES FOR PERIOD OF RECORD.--HIGHEST WATER LEVEL, 37.15 FT(11.32 M) BELOW LAND-SURFACE DATUM,FEB 10, 1967; LOWEST WATER LEVEL,41.22 FT(12.56 M) BELOW LAND-SURFACE DATUM, JAN 18,1982 .

WATER LEVELS IN FEET BELOW LAND SURFACE DATUM, CLIMATIC YEARS APRIL 1981 TO MARCH 1983

\begin{tabular}{|c|c|c|}
\hline & WATER & \\
\hline DATE & LEVEL & DATE \\
\hline
\end{tabular}

JAN 18, $1982 \quad 41.22 \quad$ JAN $11,1983 \quad 41.08$ 
BEAVER COUNTY -- CONTINUED

363600100144701 . LOCAL NUMBER;

$01 \mathrm{~N}-27 \mathrm{E}-35$ DAC 1 LOCATION,--LAT $3636^{\prime} 00^{\prime \prime}$, LONG 100 AQUIFER.--OGALLALA.

PERIOD OF RECORD.--1967 TO CURRENT YEAR.

EXTREMES FOR PERIOD OF RECORD.--HIGHEST WATER LEVEL, 124.05 FT(37.81 M) BELOW LAND-SURFACE DATUM,JAN 18, 1968; LOWEST WATER LEVEL, 147.88 FT(45.07 M) BELOW LAND-SURFACE DATUM, JAN 14, 1980.

WATER LEVELS IN FEET BELOW LAND SURFACE DATUM, CLIMATIC YEARS APRIL 1981 TO MARCH 1983

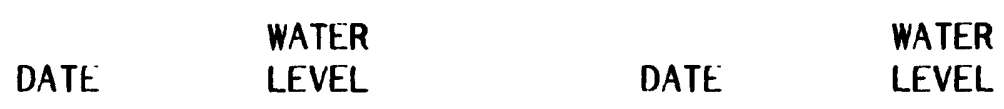

JAN $18,1982 \quad 137.21 \quad$ JAN $11,1983 \quad 137.02$

\section{GROUND-WATER LEVELS}

363503100010001. LOCAL NUMBER; 01N-28E-02 BCA 1. LOCATION,--LAT 36 35'03", LONG 100 01'00", HYDROLOGIC UNIT 11100201. AQUIFER. - OGALLALA.

PERIOD OF RECORD .--1968 TO CURRENT YEAR. EXTREMES FOR PERIOD OF RECORD.--HIGHEST WATER LEVEL, 151.75 FT(46.25 M) BELOW LAND-SURFACE DATUM, JAN 19, 1982; LOWEST WATER LEVEL,156.05 FT(47.56 M) BELOW LAND-SURFACE DATUM, JAN 14, 1970 .

WATER LEVELS IN FEET BELOW LAND SURFACE DATUM, CLIMATIC YEARS APRIL 1981 TO MARCH 1983

\begin{tabular}{|c|c|}
\hline$A$ & $\begin{array}{l}\text { WATER } \\
\text { LEVEL }\end{array}$ \\
\hline
\end{tabular}

JAN 19, $1982 \quad 151.75$ JAN 11, $1983 \quad 152.80$ 
GROUND-WATER LEVELS

BEAVER COUNTY -- CONTINUED

364015100505401. LOCAL NUMBER; 02N-20E-01 AAA 1.

LOCATION,--LAT 36 40'15", LONG 100 50'54", HYDROLOGIC UNIT 11100102.

AQUIFER.--OGALLALA.

PERIOD OF RECORD.--1968 TO CURRENT YEAR.

EXTREMES FOR PERIOD OF RECORD.--HIGHEST WATER LEVEL, 49.83 FT(15.19 M) BELOW LAND-SURFACE DATUM,JAN 10, 1983; LOWEST WATER LEVEL,55.77 FT(17.00 M) BELOW LAND-SURFACE DATUM, FEB 5, 1968.

WATER LEVELS IN FEET BELOW LAND SURFACE DATUM, CLIMATIC YEARS APRIL 1981 TO MARCH 1983

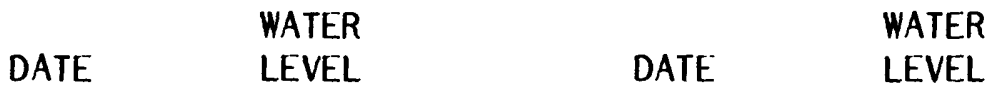

JAN $20,1982 \quad 50.10 \quad$ JAN $10,1983 \quad 49.83$

GROUND-WATER LEVELS

363847100460601. LOCAL NUMBER; 02N-21E-11 CDD 1. LOCATION,--LAT 36 38'47", LONG 100 46'06", HYDROLOGIC UNIT 11100102. AQUIFER.--OGALLALA.

PERIOD OF RECORD.--1967 TO CURRENT YEAR.

EXTREMES FOR PERIOD OF RECORD.--HIGHEST WATER LEVEL, 162.05 FT(49.39 M) BELOW LAND-SURFACE DATUM,FEB 9, 1967; LOWEST WATER LEVEL, 179.97 FT(54.85 M) BELOW LAND-SURFACE DATUM, JAN 20, 1982.

WATER LEVELS IN FEET BELOW LAND SURFACE DATUM, CLIMATIC YEARS APRIL 1981 T0 MARCH 1983

$\begin{array}{lll} & \text { WATER } & \\ \text { DATE } & \text { LEVEL } & \text { WATER } \\ & \text { DATE } & \text { LEVEL }\end{array}$

JAN 20, $1982179.97 \quad$ JAN $10,1983 \quad 178.75$ 
BEAVER COUNTY -- CONTINUED

363759100391901. LOCAL NUMBER; LOCATION,--LAT 36 37'59", LONG 100 AQUIFER. - - OGALLALA. PERIOD OF RECORD.--1967 TO CURRENT YEAR.

EXTREMES FOR. PERIOD OF RECORD.--HIGHEST WATER LEVEL,95.14 FT(29.00 M) BELOW LAND-SURFACE DATUM,JAN 13, 1976; LOWEST WATER LEVEL, $101.73 \mathrm{FT}(31.01 \mathrm{M})$ BELOW LAND-SURFACE DATUM, JAN 10, 1983.

WATER LEVELS IN FEET BELOW LAND SURFACE DATUM, CLIMATIC YEARS APRIL 1981 TO MARCH 1983

JAN $20,1982101.72 \quad$ JAN $10,1983 \quad 101.73$

\section{GROUND-WATER LEVELS}

363816100321901. LOCAL NUMBER;

$02 \mathrm{~N}-23 \mathrm{E}-13$ BCC 1 .

LOCATION,--LAT 36 38'16", LONG 100 32'19", HYDROLOGIC UNIT 11100201.

AQUIFER.--OGALLALA.

PERIOD OF RECORD.--1977 TO CURRENT YEAR.

EXTREMES FOR PERIOD OF RECORD.--HIGHEST WATER LEVEL, $12.58 \mathrm{FT}(3.83 \mathrm{M})$ BELOW LAND-SURFACE DATUM, JAN 11, 1977; LOWEST WATER LEVEL,13.15 FT(4.01 M) BELOW LAND-SURFACE DATUM, JAN 20, 1982.

WATER LEVELS IN FEET BELOW LAND SURFACE DATUM, CLIMATIC YEARS APRIL 1981 TO MARCH 1983

$\begin{array}{lll} & \text { WATER } & \text { WATER } \\ \text { DATE } & \text { LEVEL } & \text { DATE }\end{array}$

JAN 20, $1982 \quad 13.15 \quad$ JAN 13, $1983 \quad 13.15$ 
BEAVER COUNTY -- CONTINUED

364018100283001 . LOCAL NUMBER;

02N-24E-04 ABD 1.

LOCATION,--LAT 36 40'18", LONG 100

28'30", HYDROLOGIC UNIT 11100201. AQUIFER . - OGALLALA.

PERIOD OF RECORD.-- 1980 TO CURRENT YEAR.

EXTREMES FOR PERIOD OF RECORD.--HIGHEST WATER LEVEL, $11.60 \mathrm{FT}(3.54 \mathrm{M})$ BELOW LAND-SURFACE DATUM, MAY 13, 1980; LOWEST WATER LEVEL,11.87 FT(3.62 M) BELOW LAND-SURFACE DATUM, JAN 27, 1981.

WATER LEVELS IN FEET BELOW LAND SURFACE DATUM, CLIMATIC YEARS APRIL 1981 TO MARCH 1983

$\begin{array}{cccc}\text { DATE } & \begin{array}{c}\text { WATER } \\ \text { LEVEL }\end{array} & \text { DATE } & \text { WATER } \\ \text { LEVEL }\end{array}$

GROUND-WATER LEVELS

364036100240301. LOCAL NUMBER; 02N-25E-06 AAB 1. LOCATION,--LAT 36 40'36", LONG 100 24'03", HYDROLOGIC UNIT 11100201. AQUIFER .--OGALLALA.

PERIOD OF RECORD.--1980 TO CURRENT YEAR.

EXTREMES FOR PERIOD OF RECORD.--HIGHEST WATER LEVEL, 181.60 FT(55.35 M) BELOW LAND-SURFACE DATUM, JAN 29, 1981; LOWEST WATER LEVEL, 183.87 FT(56.04 M) BELOW LAND-SURFACE DATUM, JAN 13, 1983.

WATER LEVELS IN FEET BELOW LAND SURFACE DATUM, CLIMATIC YEARS APRIL 1981 TO MARCH 1983

$\begin{array}{lll} & \text { WATER } & \text { WATER } \\ \text { DATE } & \text { LEVEL } & \text { DATE }\end{array}$

JAN 19, $1982 \quad 181.79 \quad$ JAN $13,1983 \quad 183.87$ 
BEAVER COUNTY -- CONTINUED

363925100202501. LOCAL NUMBER; 02N-25E-11 BBD 1. LOCATION,--LAT 36 39'25", LONG 100 20'25", HYDROLOGIC UNIT 11100201. AQUIFER .--OGALLALA. PERIOD OF RECORD.--1980 TO CURRENT YEAR. EXTREMES FOR PERIOD OF RECORD.--HIGHEST WATER LEVEL, $56.91 \mathrm{FT}(17.35 \mathrm{M})$ BELOW LAND-SURFACE DATUM, JAN 13, 1983; LOWEST WATER LEVEL,71.25 FT(21.72 M) BELOW LAND-SURFACE DATUM, MAY 13, 1980.

WATER LEVELS IN FEET BELOW LAND SURFACE DATUM, CLIMATIC YEARS APRIL 1981 TO MARCH 1983

\begin{tabular}{|c|c|}
\hline & WATER \\
\hline DATE & LEVEL \\
\hline
\end{tabular}

JAN 19, $1982 \quad 57.51 \quad$ JAN $13,1983 \quad 56.91$

GROUND-WATER LEVELS

363740100235001. LOCAL NUMBER; O2N-25E-19 ADD 2. LOCATION,--LAT 36 37'40", LONG 100 23'50", HYDROLOGIC UNIT 11100201. AQUIFER . --OGALLALA. PERIOD OF RECORD.--1967 TO CURRENT YEAR. EXTREMES FOR PERIOD OF RECORD.--HIGHEST WATER LEVEL, 84.20 FT(25.66 M) BELOW LAND-SURFACE DATUM,JAN 16, 1974; LOWEST WATER LEVEL,88.27 FT(26.90 M) BELOW LAND-SURFACE DATUM, JAN 19, 1982.

WATER LEVELS IN FEET BELOW LAND SURFACE DATUM, CLIMATIC YEARS APRIL 1981 TO MARCH 1983

$\begin{array}{lll} & \text { WATER } & \text { WATER } \\ \text { DATE } & \text { LEVEL } & \text { DATE }\end{array}$

$\begin{array}{lllll}\text { JAN } 19,1982 & 88.27 & \text { JAN } 13,1983 & \text { Z }\end{array}$


BEAVER COUNTY -- CONTINUED

363720100185301 . LOCAL NUMBER; LOCATION,--LAT 36 37'20", LONG 100 AQUIFER.--OGALLALA.

PERIOD OF RECORD.--1980 TO CURRENT YEAR.

EXTREMES FOR PERIOD OF RECORD.--HIGHEST WATER LEVEL, 147.95 FT(45.10 M) BELOW LAND-SURFACE DATUM, JAN 13, 1983; LOWEST WATER LEVEL, $157.45 \mathrm{FT}(47.99 \mathrm{M})$ BELOW LAND-SURFACE DATUM, JAN 29, 1981 .

WATER LEVELS IN FEET BELOW LAND SURFACE DATUM, CLIMATIC YEARS APRIL 1981 TO MARCH 1983
02N-25E-24 DBB 1.

18'53", HYDROLOGIC UNIT 11100201.

$\begin{array}{cccc}\text { DATE } & \begin{array}{l}\text { WATER } \\ \text { LEVEL }\end{array} & \text { DATE } & \begin{array}{l}\text { WATER } \\ \text { LEVEL }\end{array} \\ \text { JAN 19, 1982 } & 148.12 & \text { JAN 13,1983 } & 147.95\end{array}$

GROUND-WATER LEVELS

363558100221001 . LOCAL NUMBER; 02N-25E-33 ACA 1. LOCATION,--LAT 36 35'58", LONG 100 22'10", HYDROLOGIC UNIT 11100201. AQUIFER . - OGALLALA. PERIOD OF RECORD.--1971 TO CURRENT YEAR. EXTREMES FOR PERIOD OF RECORD.--HIGHEST WATER LEVEL, 208.14 FT(63.44 M) BELOW LAND-SURFACE DATUM, JAN 27, 1983; LOWEST WATER LEVEL,212.94 FT(64.90 M) BELOW LAND-SURFACE DATUM, FEB 3, 1978.

WATER LEVELS IN FEET BELOW LAND SURFACE DATUM, CLIMATIC YEARS APRIL 1981 TO MARCH 1983

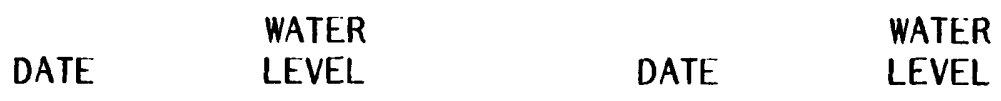

JAN 18, $1982 \quad 208.83 \quad$ JAN 27, $1983 \quad 208.14$ 
BEAVER COUNTY -- CONTINUED

364026100134901. LOCAL NUMBER; 02N-26E-02 BBA 1.

LOCATION,--LAT 36 40'26", LONG 100 13'49", HYDROLOGIC UNIT 11100201.

AQUIFER.--OGALLALA.

PERIOD OF RECORD.--1967 TO CURRENT YEAR.

EXTREMES FOR PERIOD OF RECORD.--HIGHEST WATER LEVEL, 54.49 FT(16.61 M) BELOW LAND-SURFACE DATUM, JAN 13, 1971; LOWEST WATER LEVEL,59.13 FT(18.02 M) BELOW LAND-SURFACE DATUM, JAN 15, 1980.

WATER LEVELS IN FEET BELOW LAND SURFACE DATUM, CLIMATIC YEARS APRIL 1981 TO MARCH 1983

\begin{tabular}{cccc}
\multicolumn{1}{c}{ WATER } & & WATER \\
DATE & LEVEL & DATE & LEVEL \\
JAN 19, 1982 & 58.87 & JAN 13, 1983 & 58.67
\end{tabular}

\section{GROUND-WATER LEVELS}

364008100063901. LOCAL NUMBER; 02N-27E-02 AAD 2. LOCATION,--LAT 36 40'08", LONG 100 06'39", HYDROLOGIC UNIT 11100201. AQUIFER .--OGALLALA.

PERIOD OF RECORD.--1967 TO CURRENT YEAR.

EXTREMES FOR PERIOD OF RECORD.--HIGHEST WATER LEVEL, $17.69 \mathrm{FT}(5.39 \mathrm{M})$ BELOW LAND-SURFACE DATUM,JAN 13, 1971; LOWEST WATER LEVEL,21.15 FT(6.45 M) BELOW LAND-SURFACE DATUM, JAN $8,1981$.

WATER LEVELS IN FEET BELOW LAND SURFACE DATUM, CLIMATIC YEARS APRIL 1981 TO MARCH 1983

\begin{tabular}{|c|c|c|}
\hline & WATER & \\
\hline DATE & LEVEL & DATE \\
\hline
\end{tabular}

JAN 19, $1982 \quad 20.45$ JAN 11, $1983 \quad 20.20$ 
BEAVER COUNTY -- CONTINUED

363831100082301. LOCAL NUMBER;

LOCATION,--LAT 36 38'31", LONG 100 AQUIFER.--OGALLALA.

PERIOD OF RECORD.--1967 TO CURRENT YEAR.

EXTREMES FOR PERIOD OF RECORD.--HIGHEST WATER LEVEL,9.20 FT(2.80 M) BELOW LAND-SURFACE DATUM, JAN 14, 1980; LOWEST WATER LEVEL, 10.45 FT(3.19 M) BELOW LAND-SURFACE DATUM, JAN $10,1977$.

WATER LEVELS IN FEET BELOW LAND SURFACE DATUM, CLIMATIC YEARS APRIL 1981 TO MARCH 1983

JAN 21, $1982 \quad 9.62 \quad$ JAN 11, $1983 \quad Z$

\section{GROUND-WATER LEVELS}

363705100093101. LOCAL NUMBER; 02N-27E-21 CDB 2. LOCATION,--LAT 36 37'05", LONG 100 09'31", HYDROLOGIC UNIT 11100201. AQUIFER.--OGALLALA.

PERIOD OF RECORD.--1968 TO CURRENT YEAR.

EXTREMES FOR PERIOD OF RECORD.--HIGHEST WATER LEVEL, $7.90 \mathrm{FT}(2.41 \mathrm{M})$ BELOW LAND-SURFACE DATUM, JAN 15, 1970; LOWEST WATER LEVEL,8.77 FT(2.67 M) BELOW LAND-SURFACE DATUM, FEB 24, 1982.

WATER LEVELS IN FEET BELOW LAND SURFACE DATUM, CLIMATIC YEARS APRIL 1981 TO MARCH 1983

\begin{tabular}{|c|c|}
\hline & WATER \\
\hline DATE & LEVEL \\
\hline
\end{tabular}

FEB $24,1982 \quad 8.77 \quad$ JAN $11,1983 \quad 8.38$




\section{GROUND-WATER LEVELS}

\section{BEAVER COUNTY -- CONTINUED}

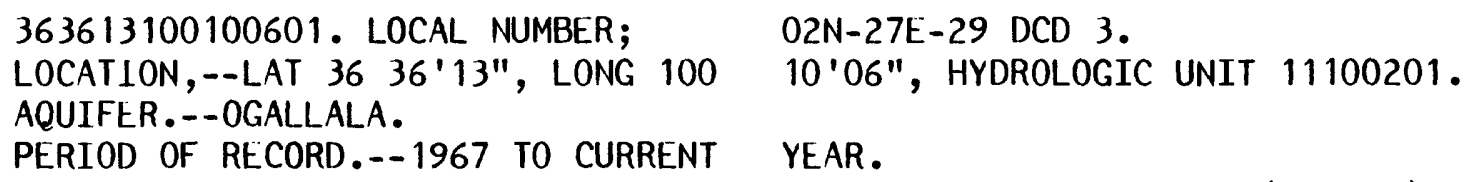

WATER LEVELS IN FEET BELOW LAND SURFACE DATUM, CLIMATIC YEARS APRIL 1981 TO MARCH 1983

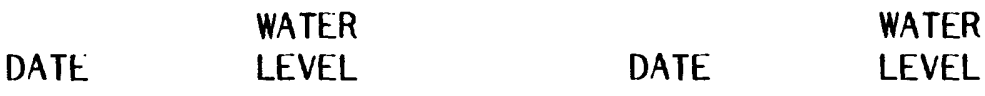

JAN $19,1982 \quad 11.32 \quad$ JAN $11,1983 \quad 10.72$

\section{GROUND-WATER LEVELS}

363527100105501. LOCAL NUMBER; 02N-27E-31 DDA 1. LOCATION,--LAT 36 35'27", LONG 100 10'55", HYDROLOGIC UNIT 11100201. AQUIFER .--OGALLALA.

PERIOD OF RECORD.--1967 TO CURRENT YEAR.

EXTREMES FOR PERIOD OF RECORD. --HIGHEST WATER LEVEL, $8.80 \mathrm{FT}(2.68 \mathrm{M})$ BELOW LAND-SURFACE DATUM,FEB 11, 1967; LOWEST WATER LEVEL, 10.70 FT(3.26 M) BELOW LAND-SURFACE DATUM, JAN 14, 1971.

WATER LEVELS IN FEET BELOW LAND SURFACE DATUM, CLIMATIC YEARS APRIL 1981 TO MARCH 1983

$\begin{array}{lll} & \text { WATER } & \\ \text { DATE } & \text { LEVEL } & \text { WATER } \\ & \text { LATE } & \text { LEVEL }\end{array}$

JAN 19, $198210.27 \quad$ JAN 11, $1983 \quad 9.57$


BEAVER COUNTY -- CONTINUED

364228100341701. LOCAL NUMBER; LOCATION,--LAT 36 42'28', LONG 100 AQUIFER .--OGALLALA.

PERIOD OF RECORD.--1980 TO CURRENT YEAR.

EXTREMES FOR PERIOD OF RECORD.--HIGHEST WATER LEVEL,87.20 FT(26.58 M) BELOW LAND-SURFACE DATUM, MAY 13, 1980; LOWEST WATER LEVEL,88.43 FT(26.95 M) BELOW LAND-SURFACE DATUM, JAN 11, 1983.

WATER LEVELS IN FEET BELOW LAND SURFACE DATUM, CLIMATIC YEARS APRIL 1981 TO MARCH 1983
$03 \mathrm{~N}-23 \mathrm{E}-22$ CAC 1.

34 '17', HYDROLOGIC UNIT 11100102.

\begin{tabular}{|c|c|c|}
\hline & WATER & \\
\hline DATE & LEVEL & DATE \\
\hline
\end{tabular}

JAN 20, $1982 \quad 87.80 \quad$ JAN $11,1983 \quad 88.43$

GROUND-WATER LEVELS

364110100374501. LOCAL NUMBER; $\quad 03 \mathrm{~N}-23 \mathrm{E}-31 \mathrm{BBC} 1$.

LOCATION,--LAT 36 41'10", LONG 100 37'45", HYDROLOGIC UNIT 11100201.

AQUIFER.--OGALLALA.

PERIOD OF RECORD.--1980 TO CURRENT YEAR.

EXTREMES FOR PERIOD OF RECORD.--HIGHEST WATER LEVEL,79.79 FT(24.32 M) BELOW LAND-SURFACE DATUM, MAY 13, 1980; LOWEST WATER LEVEL,80.94 FT(24.67 M) BELOW LAND-SURFACE DATUM, JAN 20, 1982 .

WATER LEVELS IN FEET BELOW LAND SURFACE DATUM, CLIMATIC YEARS APRIL 1981 TO MARCH 1983

\begin{tabular}{|c|c|}
\hline & WATER \\
\hline DATE & LEVEL \\
\hline
\end{tabular}

JAN 20, $1982 \quad 80.94 \quad$ JAN $13,1983 \quad 80.80$ 
BEAVER COUNTY -- CONTINUED

364348100305101 . LOCAL NUMBER;

03N-24E-18 BAA 1.

LOCATION,--LAT 36 43'48", LONG 100, 30'51", HYDROLOGIC UNIT 11100201.

AQUIFER .--OGALLALA.

PERIOD OF RECORD.--1968 TO CURRENT YEAR.

EXTREMES FOR PERIOD OF RECORD.--HIGHEST WATER LEVEL, 87.08 FT(26.54 M) BELOW LAND-SURFACE DATUM, JAN 13, 1983; LOWEST WATER LEVEL, 119.10 FT(36.30 M) BELOW LAND-SURFACE DATUM, JAN 13, 1976.

WATER LEVELS IN FEET BELOW LAND SURFACE DATUM, CLIMATIC YEARS APRIL 1981 TO MARCH 1983

$\begin{array}{lll} & \text { WATER } & \text { WATER } \\ \text { DATE } & \text { LEVEL } & \text { DATE }\end{array}$

JAN $10,1982 \quad 107.17 \quad$ JAN $13,1983 \quad 87.08$

GROUND-WATER LEVELS

364036100202601. LOCAL NUMBER; 03N-25E-35 CCA 1.

LOCATION,--LAT 36 40'36", LONG 100 20'26", HYDROLOGIC UNIT 11100201.

AQUIFER . - OGALLALA.

PERIOD OF RECORD.--1980 TO CURRENT YEAR.

EXTREMES FOR PERIOD OF RECORD.--HIGHEST WATER LEVEL, $81.01 \mathrm{FT}(24.69 \mathrm{M})$ BELOW LAND-SURFACE DATUM, MAY 14, 1980; LOWEST WATER LEVEL,81.73 FT(24.91 M) BELOW LAND-SURFACE DATUM, JAN 29, 1981.

WATER LEVELS IN FEET BELOW LAND SURFACE DATUM, CLIMATIC YEARS APRIL 1981 TO MARCH 1983

$\begin{array}{lccc}\text { DATE } & \begin{array}{c}\text { WATER } \\ \text { LEVEL }\end{array} & \text { DATE } & \text { WATER } \\ \text { LEVEL }\end{array}$


BEAVER COUNTY -- CONTINUED

364500100181201 . LOCAL NUMBER; LOCATION,--LAT 36 45'00", LONG 100 AQUIFER.--OGALLALA. PERIOD OF RECORD.--1968 TO CURRENT YEAR.

EXTREMES FOR PERIOD OF RECORD.--HIGHEST WATER LEVEL,82.43 FT(25.12 M) BELOW LAND-SURFACE DATUM, JAN 20, 1975; LOWEST WATER LEVEL,87.50 FT(26.67 M) BELOW LAND-SURFACE DATUM, JAN 16, 1969.

WATER LEVELS IN FEET BELOW LAND SURFACE DATUM, CLIMATIC YEARS APRIL 1981 TO MARCH 1983
03N-26E-06 CCC 1. 18'12", HYDROLOGIC UNIT 11100201. 
GROUND-WATER LEVELS

BEAVER COUNTY -- CONTINUED

364316100073301. LOCAL NUMBER; 03N-27E-14 CCB 1.

LOCATION,--LAT 36 43'16", LONG 100 07'33", HYDROLOGIC UNIT 11100201.

AQUIFER.--OGALLALA.

PERIOD OF RECORD.--1967 TO CURRENT YEAR.

EXTREMES FOR PERIOD OF RECORD.--HIGHEST WATER LEVEL, 85.24 FT(25.98 M) BELOW LAND-SURFACE

DATUM, JAN 15, 1970; LOWEST WATER LEVEL,94.70 FT(28.86 M) BELOW LAND-SURFACE DATUM,

JAN 15, 1980.

WATER LEVELS IN FEET BELOW LAND SURFACE DATUM, CLIMATIC YEARS APRIL 1981 TO MARCH 1983

$\begin{array}{lll} & \text { WATER } & \text { WATER } \\ \text { DATE } & \text { LEVEL } & \text { DATE }\end{array}$

JAN 19, $1982 \quad 88.99 \quad$ JAN $13,1983 \quad$ Z

GROUND-WATER LEVELS

364226100085901. LOCAL NUMBER; 03N-27E-21 DBD 1.

LOCATION,--LAT 36 42'26", LONG 100 08'59", HYDROLOGIC UNIT 11100201.

AQUIFER .--OGALLALA.

PERIOD OF RECORD.--1980 TO CURRENT YEAR.

EXTREMES FOR PERIOD OF RECORD.--HIGHEST WATER LEVEL,88.70 FT(27.04 M) BELOW LAND-SURFACE DATUM, MAY 20, 1980; LOWEST WATER LEVEL,89.13 FT(27.17 M) BELOW LAND-SURFACE DATUM, JAN 19, 1982.

WATER LEVELS IN FEET BELOW LAND SURFACE DATUM, CLIMATIC YEARS APRIL 1981 TO MARCH 1983

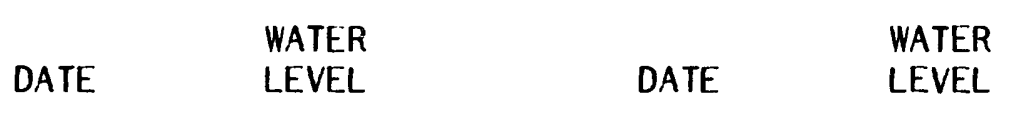

JAN $19,1982 \quad 89.13 \quad$ JAN 13, $1983 \quad 89.04$ 
BEAVER COUNTY -- CONTINUED

364206100030901. LOCAL NUMBER; 03N-28E-28 BAB 1.

LOCATION,--LAT 36 42'06", LONG 100 03'09", HYDROLOGIC UNIT 11100201.

AQUIFER . --OGALLALA.

PLRIOD OF RECORD.--1978 TO CURRENT YEAR.

EXTREMES FOR PERIOD OF RECORD.--HIGHEST WATER LEVEL, 29.29 FT(8.93 M) BELOW LAND-SURFACF

DATUM, APR 14, 1978; LOWEST WATER LEVEL,29.97 FT(9.13 M) BELOW LAND-SURFACE DATUM, JAN 19, 1982.

WATER LEVELS IN FEET BELOW LAND SURFACE DATUM, CLIMATIC YEARS APRIL 1981 TO MARCH 1983

\begin{tabular}{|c|c|}
\hline & WATER \\
\hline DATE & LEVEL \\
\hline
\end{tabular}

JAN 19, $1982 \quad 29.97 \quad$ JAN 13, $1983 \quad 29.52$

GROUND-WATER LEVELS

364734100571301. LOCAL NUMBER; 04N-20E-19 CBB 1.

LOCATION,--LAT 36 47'34", LONG 100 57'13", HYDROLOGIC UNIT 11100102.

AQUIFER .--OGALLALA.

PERIOD OF RECORD.--1968 TO CURRENT YEAR.

EXTREMES FOR PERIOD OF RECORD.--HIGHEST WATER LEVEL, 94.08 FT(28.68 M) BELOW LAND-SURFACE DATUM,FEB 8, 1968; LOWEST WATER LEVEL,111.92 FT(34.11 M) BELOW LAND-SURFACE DATUM, JAN $12,1983$.

WATER LEVELS IN FEET BELOW LAND SURFACE DATUM, CLIMATIC YEARS APRIL 1981 TO MARCH 1983

$\begin{array}{lll} & \text { WATER } & \\ \text { DATE } & \text { LEVEL } & \text { WATER } \\ \text { LEVEL }\end{array}$

JAN 20, $1982 \quad 110.84 \quad$ JAN 12, $1983 \quad 111.92$ 


\section{GROUND-WATER LEVELS}

BEAVER COUNTY -- CONTINUED

364728100533101. LOCAL NUMBER; LOCATION, --LAT 36 47'28", LONG 100 AQUIFER . - - OGALLALA.

PERIOD OF RECORD.--1967 TO CURRENT YEAR.

EXTREMES FOR PERIOD OF RECORD.--HIGHEST WATER LEVEL, $114.49 \mathrm{FT}(34.90 \mathrm{M})$ BELOW LAND-SURFACE DATUM, JAN 18, 1968; LOWEST WATER LEVEL, 121.78 FT(37.12 M) BELOW LAND-SURFACE DATUM, JAN $12,1983$.

WATER LEVELS IN FEET BELOW LAND SURFACE DATUM, CLIMATIC YEARS APRIL 1981 TO MARCH 1983

\begin{tabular}{|c|c|}
\hline & WATER \\
\hline DATE & LEVEL \\
\hline
\end{tabular}

JAN 20, $1982 \quad 121.16 \quad$ JAN $12,1983 \quad 121.78$

GROUND-WATER LEVELS

364901100381901. LOCAL NUMBER; 04N-22E-13 ABB 1.

LOCATION,--LAT 36 49'01", LONG 100 38'19", HYDROLOGIC UNIT 11100102.

AQUIFER .--OGALLALA.

PERIOD OF RECORD.--1967 TO CURRENT YEAR.

EXTREMES FOR PERIOD OF RECORD.--HIGHEST WATER LEVEL, $10.59 \mathrm{FT}(3.23 \mathrm{M})$ BELOW LAND-SURFACE DATUM, JAN 21, 1975; LOWEST WATER LEVEL, 14.53 FT(4.43 M) BELOW LAND-SURFACE DATUM, JAN 8, 1981.

WATER LEVELS IN FEET BELOW LAND SURFACE DATUM, CLIMATIC YEARS APRIL 1981 TO MARCH 1983

$\begin{array}{lll} & \text { WATER } & \\ \text { DATE } & \text { LEVEL } & \text { WATER } \\ & \text { DATE } & \text { LEVEL }\end{array}$

FEB 24, $1982 \quad 12.68$ JAN 13, $1983 \quad 13.20$ 
BEAVER COUNTY -- CONTINUED

364807100400601 . LOCAL NUMBER;

$04 \mathrm{~N}-22 \mathrm{E}-22$ AAD 1.

LOCATION,--LAT $3648^{\prime} 07^{\prime \prime}$, LONG 100

AQUIFER .--OGALLALA.

PERIOD OF RECORD.--1980 TO CURRENT YEAR.

EXTREMES FOR PERIOD OF RECORD.--HIGHEST WATER LEVEL, 27.18 FT(8.28 M) BELOW LAND-SURFACE DATUM, JAN 10, 1983; LOWEST WATER LEVEL,29.88 FT(9.11 M) BELOW LAND-SURFACE DATUM, JAN 20, 1982.

WATER LEVELS IN FEET BELOW LAND SURFACE DATUM, CLIMATIC YEARS APRIL 1981 TO MARCH 1983

$\begin{array}{lll} & \text { WATER } & \text { WATER } \\ \text { DATE } & \text { LEVEL } & \text { DATE }\end{array}$

JAN $20,1982 \quad 29.88 \quad$ JAN $10,1983 \quad 27.18$

GROUND-WATER LEVELS

364734100254501. LOCAL NUMBER; 04N-24E-24 CCC 1.

LOCATION,--LAT 36 47'34", LONG 100 25'45", HYDROLOGIC UNIT 11100201.

AQUIFER .--OGALLALA.

PERIOD OF RECORD .--1967 TO CURRENT YEAR.

EXTREMES FOR PERIOD OF RECORD.--HIGHEST WATER LEVEL, 20.88 FT(6.36 M) BELOW LAND-SURFACI DATUM,JAN 15, 1969; LOWEST WATER LEVEL,23.44 FT(7.14 M) BELOW LAND-SURFACE DATUM, JAN 13, 1976.

WATER LEVELS IN FEET BELOW LAND SURFACE DATUM, CLIMATIC YEARS APRIL 1981 TO MARCH 1983

$\begin{array}{cccc} & \text { WATER } & & \text { WATER } \\ \text { DATE } & \text { LEVEL } & \text { DATE } & \text { LEVEL } \\ \text { B 24, 1982 } & 22.66 & \text { JAN 13, 1983 } & 22.95\end{array}$


BEAVER COUNTY -- CONTINUED

364719100223501. LOCAL NUMBER;

LOCATION,--LAT 36 47'19", LONG 100 AQUIFER.--OGALLALA.

PERIOD OF RECORD.--1968 TO CURRENT YEAR.

EXTREMES FOR PERIOD OF RECORD.--HIGHEST WATER LEVEL, $22.83 \mathrm{FT}(6.96 \mathrm{M})$ BELOW LAND-SURFACE

DATUM, JAN 15, 1969; LOWEST WATER LEVEL, 28.93 FT(8.82 M) BELOW LAND-SURFACE DATUM, JAN 13, 1983.

WATER LEVELS IN FEET BELOW LAND SURFACE DATUM, CLIMATIC YEARS APRIL 1981 TO MARCH 1983
$04 \mathrm{~N}-25 \mathrm{E}-28$ BBB 1.

22 '35", HYDROLOGIC UNIT 11100201.
(1)

DATE $\quad$ WATER

FEB $24,1982 \quad 27.87$ $\begin{array}{ll} & \text { WATER } \\ \text { DATE } & \text { LEVEL }\end{array}$

JAN 13, $1983 \quad 28.93$

GROUND-WATER LEVELS

364626100242601. LOCAL NUMBER; 04N-25E-31 ABC 1.

LOCATION,--LAT 36 46'26", LONG 100 24'26", HYDROLOGIC UNIT 11100201.

AQUIFER.--OGALLALA.

PERIOD OF RECORD.--1977 TO CURRENT YEAR.

EXTREMES FOR PERIOD OF RECORD.--HIGHEST WATER LEVEL, 64.96 FT(19.80 M) BELOW LAND-SURFACE DATUM, JAN 17, 1978; LOWEST WATER LEVEL,72.42 FT(22.07 M) BELOW LAND-SURFACE DATUM, JAN 13, 1983.

WATER LEVELS IN FEET BELOW LAND SURFACE DATUM, CLIMATIC YEARS APRIL 1981 TO MARCH 1983

$\begin{array}{ccccc}\text { DATE } & \begin{array}{c}\text { WATER } \\ \text { LEVEL }\end{array} & \text { DATE } & \begin{array}{c}\text { WATER } \\ \text { LEVEL }\end{array} \\ \text { B 24, 1982 } & & \mathrm{P} & \text { JAN 13, 1983 } & 72.42\end{array}$


BEAVER COUNTY -- CONTINUED

365014100070901. LOCAL NUMBER; LOCATION,--LAT 36 50'14", LONG 100 AQUIFER .--OGALLALA.

PERIOD OF RECORD.--1980 TO CURRENT YEAR. EXTREMES FOR PERIOD OF RECORD.--HIGHEST WATER LEVEL,26.07 FT(7.95 M) BELOW LAND-SURFACE DATUM, MAY 22, 1980; LOWEST WATER LEVEL,27.23 FT(8.30 M) BELOW LAND-SURFACE DATUM, JAN 28, 1981.

WATER LEVELS IN FEET BELOW LAND SURFACE DATUM, CLIMATIC YEARS APRIL 1981 TO MARCH 1983

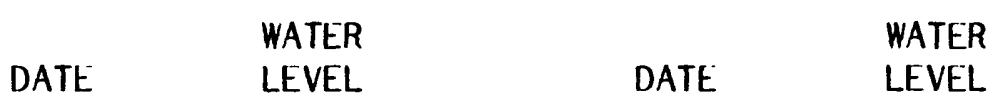

JAN 19, $1982 \quad 26.67 \quad$ JAN $10,1983 \quad 27.05$ $\begin{array}{ll}364551100064401 . \text { LOCAL NUMBER; } & 04 \mathrm{~N}-27 \mathrm{E}-35 \text { DDB } 1 . \\ \text { LOCATION,--LAT } 36 \text { 45'51", LONG } 100 \quad 06^{\prime} 44^{\prime \prime}, \text { HYDROLOGIC UNIT } 11100201 .\end{array}$

AQUIFER .--OGALLALA.

PERIOD OF RECORD.--1980 TO CURRENT YEAR.

EXTREMES FOR PERIOD OF RECORD.--HIGHEST WATER LEVEL, $12.33 \mathrm{FT}(3.76 \mathrm{M})$ BELOW LAND-SURFACE DATUM, MAY 22, 1980; LOWEST WATER LEVEL, $13.98 \mathrm{FT}(4.26 \mathrm{M})$ BELOW LAND-SURFACE DATUM, JAN 29, 1981.

WATER LEVELS IN FEET BELOW LAND SURFACE DATUM, CLIMATIC YEARS APRIL 1981 TO MARCH 1983 
365045100002501. LOCAL NUMBER; LOCATION,--LAT $3650^{\prime} 45^{\prime \prime}$, LONG 100 AQUIFER .--OGALLALA.

PERIOD OF RECORD.--1980 TO CURRENT YEAR. EXTREMES FOR PERIOD OF RECORD.--HIGHEST WATER LEVEL, $11.19 \mathrm{FT}(3.41 \mathrm{M})$ BELOW LAND-SURFACE DATUM, JAN 18, 1982; LOWEST WATER LEVEL, 12.70 FT(3.87 M) BELOW LAND-SURFACE DATUM, JAN 10, 1983.

WATER LEVELS IN FEET BELOW LAND SURFACE DATUM, CLIMATIC YEARS APRIL 1981 TO MARCH 1983
$04 \mathrm{~N}-28 \mathrm{E}-02$ AAC 1.

00 '25", HYDROLOGIC UNIT 11100201. 
BEAVER COUNTY -- CONTINUED

365508100514801 . LOCAL NUMBER;

05N-20E-02 CDD 1.

LOCATION,--LAT 36 55'08", LONG 100

AQUIFER .--OGALLALA.

PERIOD OF RECORD.--1967 TO CURRENT YEAR.

EXTREMES FOR PERIOD OF RECORD.--HIGHEST WATER LEVEL, $140.80 \mathrm{FT}(42.92 \mathrm{M})$ BELOW LAND-SURFACE DATUM, JAN 25, 1967; LOWEST WATER LEVEL, 155.08 FT(47.27 M) BELOW LAND-SURFACE DATUM, JAN 20, 1982.

WATER LEVELS IN FEET BELOW LAND SURFACE DATUM, CLIMATIC YEARS APRIL 1981 TO MARCH 1983

$\begin{array}{lll} & \text { WATER } & \text { WATER } \\ \text { DATE } & \text { LEVEL } & \text { DATE }\end{array}$

JAN 20, $1982 \quad 155.00 \quad$ JAN 11, $1983 \quad 0$

GROUND-WATER LEVELS

365507100515301. LOCAL NUMBER; O5N-20E-02 DCC 1.

LOCATION,--LAT 36 55'07", LONG 100 51'53", HYDROLOGIC UNIT 11100102.

AQUIFER .--OGALLALA.

PERIOD OF RECORD.--1968 TO CURRENT YEAR.

EXTREMES FOR PERIOD OF RECORD.--HIGHEST WATER LEVEL, $142.57 \mathrm{FT}(43.46 \mathrm{M})$ BELOW LAND-SURFACE DATUM, JAN 15, 1974; LOWEST WATER LEVEL, 162.37 FT(49.49 M) BELOW LAND-SURFACE DATUM, JAN 17, 1978.

WATER LEVELS IN FEET BELOW LAND SURFACE DATUM, CLIMATIC YEARS APRIL 1981 TO MARCH 1983

$\begin{array}{lll} & \text { WATER } & \text { WATER } \\ \text { DATE } & \text { LEVEL } & \text { DATE }\end{array}$

JAN 20, $1982 \quad 153.08 \quad$ JAN $11,1983 \quad 156.48$ 
BEAVER COUNTY -- CONTINUED

365141100542101. LOCAL NUMBER; $05 \mathrm{~N}-20 \mathrm{E}-28$ CCC 1. LOCATION,--LAT $3651^{\prime 4} 1^{\prime \prime}$, LONG 100 54'21", HYDROLOGIC UNIT 11100102. AQUIFER .--OGALLALA.

PERIOD OF RECORD.--1968 TO CURRENT YEAR. EXTREMES FOR PERIOD OF RECORD.--HIGHEST WATER LEVEL, $119.75 \mathrm{FT}(36.50 \mathrm{M})$ BELOW LAND-SURFACE DATUM,FEB 8, 1968; LOWEST WATER LEVEL, 145.90 FT(44.47 M) BELOW LAND-SURFACE DATUM, JAN 17, 1979.

WATER LEVELS IN FEET BELOW LAND SURFACE DATUM, CLIMATIC YEARS APRIL 1981 TO MARCH 1983

\begin{tabular}{|c|c|}
\hline & WATER \\
\hline DATE & LEVEL \\
\hline
\end{tabular}

JAN 20, $1982138.34 \quad$ JAN 12, $1983 \quad 141.65$

GROUND-WATER LEVELS

365450100475501. LOCAL NUMBER; OSN-21E-09 BBC 1. LOCATION,--LAT 36 54'50", LONG 100 47'55", HYDROLOGIC UNIT 11100102. AQUIFER.--OGALLALA.

PERIOD OF RECORD.--1970 TO CURRENT YEAR.

EXTREMES FOR PERIOD OF RECORD.--HIGHEST WATER LEVEL, 165.16 FT(50.34 M) BELOW LAND-SURFACE DATUM, JAN 11, 1983; LOWEST WATER LEVEL, 171.90 FT(52.40 M) BELOW LAND-SURFACE DATUM, JAN 18, 1978.

WATER LEVELS IN FEET BELOW LAND SURFACE DATUM, CLIMATIC YEARS APRIL 1981 TO MARCH 1983

\begin{tabular}{|c|c|c|}
\hline DATF & $\begin{array}{l}\text { WATER } \\
\text { I FVFI }\end{array}$ & A] \\
\hline & & $A_{1}$ \\
\hline
\end{tabular}

JAN 20, $1982 \quad 169.71 \quad$ JAN $11,1983 \quad 165.16$ 
BEAVER COUNTY -- CONTINUED

365419100472001. LOCAL NUMBER; LOCATION, --LAT 36 54'19", LONG 100 AQUIFER.--OGALLALA. PERIOD OF RECORD.--1967 TO CURRENT YEAR. EXTREMES FOR PERIOD OF RECORD.--HIGHEST WATER LEVEL, $160.01 \mathrm{FT}(48.77 \mathrm{M})$ BELOW LAND-SURFACE DATUM, JAN 18, 1968; LOWEST WATER LEVEL, $164.80 \mathrm{FT}(50.23 \mathrm{M})$ BELOW LAND-SURFACE DATUM, JAN 11, 1983.

WATER LEVELS IN FEET BELOW LAND SURFACE DATUM, CLIMATIC YEARS APRIL 1981 TO MARCH 1983
05N-21E-09 DCD 1. 47'20", HYDROLOGIC UNIT 11100102.

\section{WATER LEVELS IN}

$\begin{array}{ll}\text { WATER } \\ \text { DATE } & \text { LEVEL }\end{array}$

JAN 20, $1982 \quad 164.09$

JAN $11,1983 \quad 164.80$

GROUND-WATER LEVELS

365418100465001. LOCAL NUMBER; LOCATION,--LAT 36 54'18", LONG 100 46'50", HYDROLOGIC UNIT 11100102. AQUIFER.--OGALLALA. PERIOD OF RECORD.--1967 TO CURRENT YEAR. EXTREMES FOR PERIOD OF RECORD.--HIGHEST WATER LEVEL, 173.30 FT(52.82 M) BELOW LAND-SURFACE DATUM, JAN 14, 1970; LOWEST WATER LEVEL, 178.69 FT(54.46 M) BELOW LAND-SURFACE DATUM, JAN 11, 1983.

WATER LEVELS IN FEET BELOW LAND SURFACE DATUM, CLIMATIC YEARS APRIL 1981 TO MARCH 1983

$\begin{array}{cccc} & \text { WATER } & & \text { WATER } \\ \text { DATE } & \text { LEVEL } & \text { DATE } & \text { LEVEL } \\ \text { LN 20, 1982 } & 177.92 & \text { JAN 11, 1983 } & 178.69\end{array}$


BEAVER COUNTY -- CONTINUED

365343100483001. LOCAL NUMBER;

LOCATION,--LAT $3653^{\prime} 43^{\prime \prime}$, LONG 100 AQUIFER.--OGALLALA.

PERIOD OF RECORD.--1967 TO CURRENT YEAR.

EXTREMES FOR PERIOD OF RECORD.--HIGHEST WATER LEVEL, 149.80 FT(45.66 M) BELOW LAND-SURFACE DATUM,JAN 14, 1970; LOWEST WATER LEVEL, $155.50 \mathrm{FT}(47.40 \mathrm{M})$ BELOW LAND-SURFACE DATUM, JAN 11, 1983.

WATER LEVELS IN FEET BELOW LAND SURFACE DATUM, CLIMATIC YEARS APRIL 1981 TO MARCH 1983
$05 \mathrm{~N}-21 \mathrm{E}-17$ DBB 1.

48'30", HYDROLOGIC UNIT 11100102.
WATER

DATE

LEVEL

JAN $20,1982 \quad 155.04$

$\begin{array}{ll} & \text { WATER } \\ \text { DATE } & \text { LEVEL }\end{array}$

JAN 11, $1983 \quad 155.50$

GROUND-WATER LEVELS

365206100422901. LOCAL NUMBER; OSN-22E-29 CBA 1.

LOCATION,--LAT $3652^{\prime} 06^{\prime \prime}$, LONG $100 \quad 42^{\prime 2}$ ', HYDROLOGIC UNIT 11100102.

AQUIFER .--OGALLALA.

PERIOD OF RECORD.--1967 TO CURRENT YEAR.

EXTREMES FOR PERIOD OF RECORD.--HIGHEST WATER LEVEL, 133.41 FT(40.66 M) BELOW LAND-SURFACE DATUM, JAN 15, 1974; LOWEST WATER LEVEL, 140.68 FT(42.88 M) BELOW LAND-SURFACE DATUM, JAN 14, 1971.

WATER LEVELS IN FEET BELOW LAND SURFACE DATUM, CLIMATIC YEARS APRIL 1981 TO MARCH 1983

$\begin{array}{lll} & \text { WATER } & \text { WATER } \\ \text { DATE } & \text { LEVEL } & \text { DATE }\end{array}$

JAN 20, $1982134.76 \quad$ JAN 11, $1983 \quad 136.50$ 


\section{GROUND-WATER LEVELS}

BEAVER COUNTY -- CONTINUED

365316100335201. LOCAL NUMBER; LOCATION,--LAT $3653^{\prime} 16^{\prime \prime}$, LONG 100 AQUIFER .--OGALLALA.

PERIOD OF RECORD.--1967 TO CURRENT YEAR.

EXTREMES FOR PERIOD OF RECORD.--HIGHEST WATER LEVEL, 102.14 FT(31.13 M) BELOW LAND-SURFACE DATUM, JAN 15, 1974; LOWEST WATER LEVEL, 108.67 FT(33.12 M) BELOW LAND-SURFACE DATUM, JAN 20, 1982 .

WATER LEVELS IN FEET BELOW LAND SURFACE DATUM, CLIMATIC YEARS APRIL 1981 TO MARCH 1983

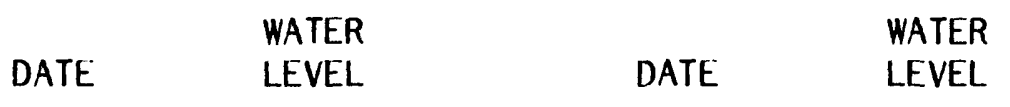

JAN $20,1982108.67 \quad$ JAN $11,1983 \quad 104.52$

365323100303101. LOCAL NUMBER; OSN-24E-19 BBD 1. LOCATION,--LAT 36 53'23", LONG 100 30'31", HYDROLOGIC UNIT 11100201. AQUIFER . --OGALLALA.

PERIOD OF RECORD.--1980 TO CURRENT YEAR. EXTREMES FOR PERIOD OF RECORD.--HIGHEST WATER LEVEL, 18.02 FT(5.49 M) BELOW LAND-SURFACE DATUM, JUNE 11, 1980; LOWEST WATER LEVEL,20.87 FT(6.36 M) BELOW LAND-SURFACE DATUM, JAN 10, 1983.

WATER LEVELS IN FEET BELOW LAND SURFACE DATUM, CLIMATIC YEARS APRIL 1981 TO MARCH 1983

\begin{tabular}{lccc}
\multicolumn{1}{c}{} & WATER & & WATER \\
DATE & LEVEL & DATE & LEVEL \\
& & & \\
LAN 10, 1983 & 20.87
\end{tabular}


BEAVER COUNTY -- CONTINUED

365553100214301. LOCAL NUMBER; 05N-25E-04 BAC 1.

LOCATION,--LAT 36 55'53", LONG 100 21'43", HYDROLOGIC UNIT 11040006.

AQUIFER.--OGALLALA.

PERIOD OF RECORD.--1967 TO CURRENT YEAR.

EXTREMES FOR PERIOD OF RECORD.--HIGHEST WATER LEVEL, 55.62 FT(16.95 M) BELOW LAND-SURFACE DATUM, JAN 24, 1967; LOWEST WATER LEVEL,60.84 FT(18.54 M) BELOW LAND-SURFACE DATUM, JAN $10,1983$.

WATER LEVELS IN FEET BELOW LAND SURFACE DATUM, CLIMATIC YEARS APRIL 1981 TO MARCH 1983

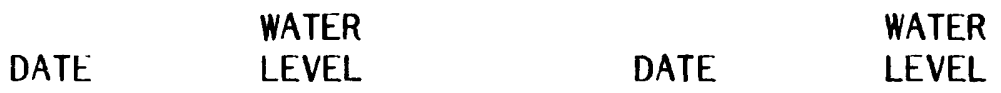

JAN $19,1982 \quad 60.47 \quad$ JAN $10,1983 \quad 60.84$

GROUND-WATER LEVELS

365201100232001. LOCAL NUMBER; 05N-25E-30 DBC 1. LOCATION,--LAT $3652^{\prime} 01^{\prime \prime}$, LONG 100 23'20", HYDROLOGIC UNIT 11100201. AQUIFER.--OGALLALA.

PERIOD OF RECORD.--1967 TO CURRENT YEAR.

EXTREMES FOR PERIOD OF RECORD.--HIGHEST WATER LEVEL, $14.17 \mathrm{FT}(4.32 \mathrm{M})$ BELOW LAND-SURFACE DATUM, JAN 27, 1969; LOWEST WATER LEVEL,23.32 FT(7.11 M) BELOW LAND-SURFACE DATUM, JAN 11, 1983.

WATER LEVELS IN FEET BELOW LAND SURFACE DATUM, CLIMATIC YEARS APRIL 1981 TO MARCH 1983

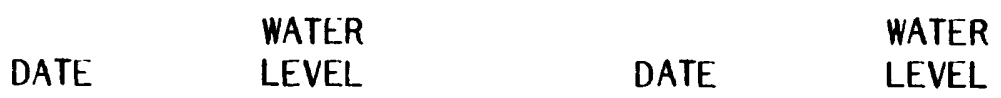

JAN $19,1982 \quad 23.04 \quad$ JAN 11, $1983 \quad 23.32$ 
BEAVER COUNTY -- CONTINUED

365138100203201. LOCAL NUMBER;

05N-25E-34 BAD 1.

LOCATION,--LAT 3651 '38", LONG 100 20'32", HYDROLOGIC UNIT 11100201. AQUIFER.--OGALLALA.

PERIOD OF RECORD.--1980 TO CURRENT YEAR.

EXTREMES FOR PERIOD OF RECORD.--HIGHEST WATER LEVEL, 66.48 FT(20.26 M) BELOW LAND-SURFACE DATUM, JUNE 11, 1980; LOWEST WATER LEVEL,67.35 FT(20.53 M) BELOW LAND-SURFACE DATUM, JAN 11, 1983.

WATER LEVELS IN FEET BELOW LAND SURFACE DATUM, CLIMATIC YEARS APRIL 1981 TO MARCH 1983

$\begin{array}{cccc} & \text { WATER } & & \text { WATER } \\ \text { DATE } & \text { LEVEL } & \text { DATE } & \text { LEVEL } \\ \text { JAN 19, 1982 } & 67.09 & \text { JAN 11, 1983 } & 67.35\end{array}$

GROUND-WATER LEVELS

365331100135801. LOCAL NUMBER; OSN-26E-15 CDD 1.

LOCATION,--LAT 3653 '31", LONG 100 13'58", HYDROLOGIC UNIT 11040006.

AQUIFER.--OGALLALA.

PERIOD OF RECORD.--1970 TO CURRENT YEAR.

EXTREMES FOR PERIOD OF RECORD.--HIGHEST WATER LEVEL, 188.09 FT(57.33 M) BELOW LAND-SURFACE DATUM, NOV 19, 1970; LOWEST WATER LEVEL, 193.12 FT(58.86 M) BELOW LAND-SURFACE DATUM, JAN 10, 1983.

WATER LEVELS IN FEET BELOW LAND SURFACE DATUM, CLIMATIC YEARS APRIL 1981 TO MARCH 1983

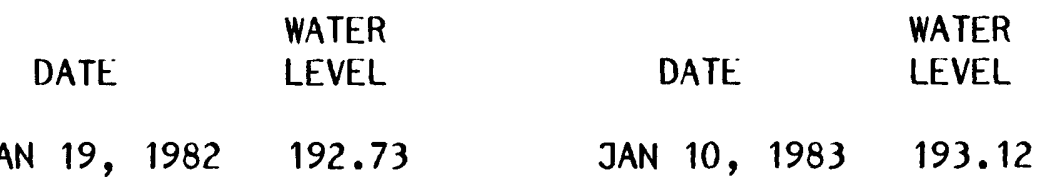


BEAVER COUNTY -- CONTINUED

365524100063401. LOCAL NUMBER;

05N-27E-02 CDB 1. LOCATION,--LAT 36 5''24", LONG 100 AQUIFER. - OGALLALA.

PERIOD OF RECORD .--1980 TO CURRENT YEAR.

EXTREMES FOR PERIOD OF RECORD.--HIGHEST WATER LEVEL,51.53 FT(15.71 M) BELOW LAND-SURFACE DATUM, JAN 10, 1983; LOWEST WATER LEVEL,58.77 FT(17.91 M) BELOW LAND-SURFACE DATUM, JUNE $11,1980$.

WATER LEVELS IN FEET BELOW LAND SURFACE DATUM, CLIMATIC YEARS APRIL 1981 TO MARCH 1983

$\begin{array}{cccc}\text { DATE } & \begin{array}{l}\text { WATER } \\ \text { LEVEL }\end{array} & \text { DATE } & \begin{array}{c}\text { WATER } \\ \text { LEVEL }\end{array} \\ \text { N 19, 1982 } & 52.18 & \text { JAN 10, 1983 } & 51.53\end{array}$

GROUND-WATER LEVELS

365321100013201. LOCAL NUMBER; 05N-28E-22 ABA 1. LOCATION,--LAT 36 53'21", LONG 100 01'32", HYDROLOGIC UNIT 11040008. AQUIFER . - OGALLALA.

PERIOD OF RECORD.--1968 TO CURRENT YEAR.

EXTREMES FOR PERIOD OF RECORD.--HIGHEST WATER LEVEL,20.19 FT(6.15 M) BELOW LAND-SURFACE DATUM, JAN 21, 1975; LOWEST WATER LEVEL,25.08 FT(7.64 M) BELOW LAND-SURFACE DATUM, JAN 13, 1979.

WATER LEVELS IN FEET BELOW LAND SURFACE DATUM, CLIMATIC YEARS APRIL 1981 TO MARCH 1983

$\begin{array}{lll} & \text { WATER } & \text { WATER } \\ \text { DATE } & \text { LEVEL } & \text { DATE }\end{array}$

JAN 19, $1982 \quad 20.90 \quad$ JAN 10, $1983 \quad 21.58$ 
GROUND-WATER LEVELS

BEAVER COUNTY -- CONTINUED

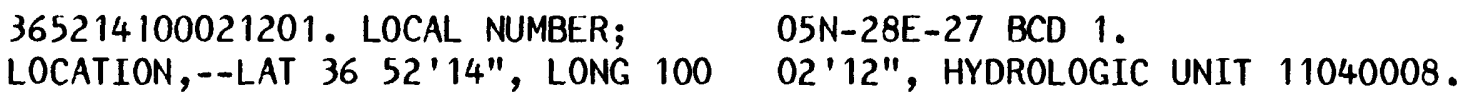

AQUIFER.--OGALLALA.

PERIOD OF RECORD.--1967 TO CURRENT YEAR.

EXTREMES FOR PERIOD OF RECORD.--HIGHEST WATER LEVEL, $32.50 \mathrm{FT}(9.91 \mathrm{M})$ BELOW LAND-SURFACE DATUM, JAN 13, 1970; LOWEST WATER LEVEL,64.30 FT(19.60 M) BELOW LAND-SURFACE DATUM, JAN 10, 1983.

WATER LEVELS IN FEET BELOW LAND SURFACE DATUM, CLIMATIC YEARS APRIL 1981 TO MARCH 1983

\begin{tabular}{|c|c|}
\hline & WATER \\
\hline DATF & LEVEL \\
\hline
\end{tabular}

JAN 19, $1982 \quad 58.77 \quad$ JAN $10,1983 \quad 64.30$

GROUND-WATER LEVELS

365600100515101. LOCAL NUMBER; 06N-20E-35 DCC 1.

LOCATION,--LAT 36 56'00", LONG 100 51'51", HYDROLOGIC UNIT 11100102.

AQUIFER.--OGALLALA.

PERIOD OF RECORD.--1967 TO CURRENT YEAR.

EXTREMES FOR PERIOD OF RECORD.--HIGHEST WATER LEVEL, $144.60 \mathrm{FT}(44.07 \mathrm{M})$ BELOW LAND-SURFACE DATUM, JAN 14, 1970; LOWEST WATER LEVEL, 161.22 FT(49.14 M) BELOW LAND-SURFACE DATUM, JAN 11, 1983.

WATER LEVELS IN FEET BELOW LAND SURFACE DATUM, CLIMATIC YEARS APRIL 1981 TO MARCH 1983

\begin{tabular}{|c|c|c|}
\hline DATE & $\begin{array}{l}\text { WATER } \\
\text { LEVEL }\end{array}$ & DATE \\
\hline
\end{tabular}

JAN 20, 1982157.33 JAN 11, $1983 \quad 161.22$ 
BEAVER COUNTY -- CONTINUED

365841100500801. LOCAL NUMBER;

$06 \mathrm{~N}-21 \mathrm{E}-18$ CCC 1.

LOCATION,--LAT 36 58'41", LONG 100 50'08", HYDROLOGIC UNIT 11040006.

AQUIFER.--OGALLALA.

PERIOD OF RECORD.--1967 TO CURRENT YEAR.

EXTREMES FOR PERIOD OF RECORD.--HIGHEST WATER LEVEL, 152.92 FT (46.61 M) BELOW LAND-SURFACE DATUM,JAN 14, 1969; LOWEST WATER LEVEL, 164.48 FT(50.13 M) BELOW LAND-SURFACE DATUM, JAN 10, 1980.

WATER LEVELS IN FEET BELOW LAND SURFACE DATUM, CLIMATIC YEARS APRIL 1981 TO MARCH 1983

\begin{tabular}{|c|c|c|}
\hline & WATER & \\
\hline DATE & LEVEL & DATE \\
\hline
\end{tabular}

JAN 20, $1982 \quad \mathrm{~J} \quad$ JAN 11, $1983 \quad \mathrm{~N}$

GROUND-WATER LEVELS

365748100371901. LOCAL NUMBER; 06N-22E-24 DDD 1. LOCATION,--LAT 36 57'48", LONG 100 37'19", HYDROLOGIC UNIT 11040006. AQUIFER .--OGALLALA.

PERIOD OF RECORD.--1967 TO CURRENT YEAR.

EXTREMES FOR PERIOD OF RECORD.--HIGHEST WATER LEVEL, 101.20 FT(30.85 M) BELOW LAND-SURFACE DATUM, JAN 13, 1976; LOWEST WATER LEVEL,106.87 FT(32.57 M) BELOW LAND-SURFACE DATUM, JAN 20, 1982 .

WATER LEVELS IN FEET BELOW LAND SURFACE DATUM, CLIMATIC YEARS APRIL 1981 TO MARCH 1983

$\begin{array}{lll} & \text { WATER } & \\ \text { DATE } & \text { LEVEL } & \text { WATER } \\ & \text { DATE } & \text { LEVEL }\end{array}$

JAN 20, 1982106.87 JAN 11, $1983 \quad 102.82$ 
BEAVER COUNTY -- CONTINUED

365632100423701. LOCAL NUMBER; LOCATION,--LAT 36 56'32", LONG 100 AQUIFER . - OGALLALA.

PERIOD OF RECORD.--1980 TO CURRENT YEAR.

EXTREMES FOR PERIOD OF RECORD.--HIGHEST WATER LEVEL, 190.16 FT(57.96 M) BELOW LAND-SURFACE DATUM, JAN 20, 1982; LOWEST WATER LEVEL, 191.28 FT(58.30 M) BELOW LAND-SURFACE DATUM, JAN 11, 1983.

WATER LEVELS IN FEET BELOW LAND SURFACE DATUM, CLIMATIC YEARS APRIL 1981 TO MARCH 1983
$06 \mathrm{~N}-22 \mathrm{E}-32$ CBB 1.

42'37", HYDROLOGIC UNIT 11040006.

\section{GROUND-WATER LEVELS}

365701100272001. LOCAL NUMBER; LOCATION,--LAT $3657^{\prime} 01^{\prime \prime}$, LONG 100 AQUIFER .--OGALLALA.

PERIOD OF RECORD.--1967 TO CURRENT YEAR.

EXTREMES FOR PERIOD OF RECORD.--HIGHEST WATER LEVEL, 116.07 FT(35.38 M) BELOW LAND-SURFACE DATUM, JAN 13, 1976; LOWEST WATER LEVEL,118.65 FT(36.16 M) BELOW LAND-SURFACE DATUM, JAN 10, 1983.

WATER LEVELS IN FEET BELOW LAND SURFACE DATUM, CLIMATIC YEARS APRIL 1981 TO MARCH 1983
$06 \mathrm{~N}-24 \mathrm{E}-27$ CCC 1 $27^{\prime} 20^{\prime \prime}$, HYDROLOGIC UNIT 11040006.

JAN 20, $1982190.16 \quad$ JAN $11,1983 \quad 191.28$

$\begin{array}{lll} & \text { WATER } & \text { WATER } \\ \text { DATE } & \text { LEVEL } & \text { DATE }\end{array}$

$\begin{array}{llll}\text { JAN 20, } 1982 & 116.72 & \text { JAN } 10,1983 & 118.65\end{array}$ 
BEAVER COUNTY -- CONTINUED

365947100241101. LOCAL NUMBER; 06N-25E-07 CCB 1. LOCATION,--LAT 36 59'47", LONG 100 24'11", HYDROLOGIC UNIT 11040006. AQUIFER.--OGALLALA. PERIOD OF RECORD.--1980 TO CURRENT YEAR. EXTREMES FOR PERIOD OF RECORD.--HIGHEST WATER LEVEL,6.18 FT (1.88 M) BELOW LAND-SURFACE DATUM, MAY 20, 1980; LOWEST WATER LEVEL, 7.08 FT(2.16 M) BELOW LAND-SURFACE DATUM, JAN 28, 1981.

WATER LEVELS IN FEET BELOW LAND SURFACE DATUM, CLIMATIC YEARS APRIL 1981 TO MARCH 1983

$\begin{array}{lll} & \text { WATER } & \text { WATER } \\ \text { DATE } & \text { LEVEL } & \text { DATE }\end{array}$

JAN 20, $1982 \quad 6.98 \quad$ JAN $10,1983 \quad 7.02$

\section{GROUND-WATER LEVELS}

365757100221001. LOCAL NUMBER; O6N-25E-20 DDD 1. LOCATION,--LAT 36 57'57", LONG 100 22'10", HYDROLOGIC UNIT 11040006. AQUIFER .--OGALLALA.

PERIOD OF RECORD .--1980 TO CURRENT YEAR.

EXTREMES FOR PERIOD OF RECORD.--HIGHEST WATER LEVEL,43.07 FT(13.13 M) BELOW LAND-SURFACE DATUM, MAY 20, 1980; LOWEST WATER LEVEL,44.02 FT(13.42 M) BELOW LAND-SURFACE DATUM, JAN $10,1983$.

WATER LEVELS IN FEET BELOW LAND SURFACE DATUM, CLIMATIC YEARS APRIL 1981 TO MARCH 1983

$\begin{array}{lll} & \text { WATER } & \\ \text { DATE } & \text { LEVEL } & \text { WATER } \\ & \text { DATE } & \text { LEVEL }\end{array}$

JAN 20, $1982 \quad 43.77$ JAN $10,1983 \quad 44.02$ 


\section{GROUND-WATER LEVELS}

\section{BECKHAM COUNTY}

350727099274901. LOCAL NUMBER;

$08 N-21 W-31$ CBB 1.

LOCATION,--LAT 35 07'27", LONG 099 27'49", HYDROLOGIC UNIT 11120302.

AQUIFER.--ALLUVIUM.

PERIOD OF RECORD.--1980 TO CURRENT YEAR.

EXTREMES FOR PERIOD OF RECORD.--HIGHEST WATER LEVEL,23.42 FT(7.14 M) BELOW LAND-SURFACE DATUM,MAR 5, 1980; LOWEST WATER LEVEL, $25.65 \mathrm{FT}(7.82 \mathrm{M})$ BELOW LAND-SURFACE DATUM, MAR 19, 1981.

WATER LEVELS IN FEET BELOW LAND SURFACE DATUM, CLIMATIC YEARS APRIL 1981 TO MARCH 1983

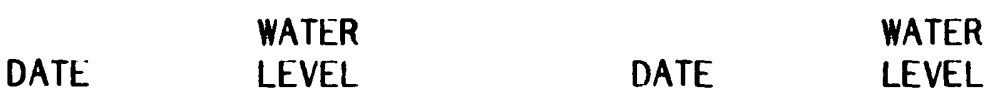

FEB 16, $1982 \quad 25.15 \quad$ JAN 27, $1983 \quad 23.53$

GROUND-WATER LEVELS

351215099292401. LOCAL NUMBER;

08N-22W-02 ABB 1.

LOCATION,--LAT 35 12'15", LONG 099 29'24", HYDROLOGIC UNIT 11120302.

AQUIFER.--RUSH SPRINGS FORMATION.

PERIOD OF RECORD.--1980 TO CURRENT YEAR.

EXTREMES FOR PERIOD OF RECORD.--HIGHEST WATER LEVEL, $52.03 \mathrm{FT}(15.86 \mathrm{M})$ BELOW LAND-SURFACE DATUM,JAN 27, 1983; LOWEST WATER LEVEL,64.68 FT(19.71 M) BELOW LAND-SURFACE DATUM, MAR 19, 1981.

WATER LEVELS IN FEET BELOW LAND SURFACE DATUM, CLIMATIC YEARS APRIL 1981 TO MARCH 1983

\begin{tabular}{|c|c|}
\hline \multicolumn{2}{|l|}{. } \\
\hline DATE & LEVEL \\
\hline
\end{tabular}

FEB $16,1982 \quad 60.82$ JAN 27, $1983 \quad 52.03$ 
BECKHAM COUNTY -- CONTINUED

350839099303001. LOCAL NUMBER; 08N-22W-27 AAD 1. LOCATION,--LAT 35 08'39", LONG 099 30'30", HYDROLOGIC UNIT 11120302. AQUIFER.--ALLUVIUM.

PERIOD OF RECORD . - 1980 TO CURRENT YEAR.

EXTREMES FOR PERIOD OF RECORD.--HIGHEST WATER LEVEL, 19.72 FT(6.01 M) BELOW LAND-SURFACE DATUM, JAN 27, 1983; LOWEST WATER LEVEL,22.85 FT(6.96 M) BELOW LAND-SURFACE DATUM, MAR 19, 1981.

WATER LEVELS IN FEET BELOW LAND SURFACE DATUM, CLIMATIC YEARS APRIL 1981 TO MARCH 1983

\begin{tabular}{|c|c|}
\hline & WATER \\
\hline DATE & LEVEL \\
\hline
\end{tabular}

FEB $16,1982 \quad 21.94 \quad$ JAN 27, $1983 \quad 19.72$

GROUND-WATER LEVELS

351030099385401. LOCAL NUMBER; 08N-23W-17 AAB 1.

LOCATION,--LAT 35 10'30", LONG 099 38'54", HYDROLOGIC UNIT 11120302.

AQUIFER .--ALLUVIUM.

PERIOD OF RECORD.--1980 TO CURRENT YEAR.

EXTREMES FOR PERIOD OF RECORD.--HIGHEST WATER LEVEL, $17.41 \mathrm{FT}(5.31 \mathrm{M})$ BELOW LAND-SURFACE DATUM, JAN 27, 1983; LOWEST WATER LEVEL, 19.26 FT(5.87 M) BELOW LAND-SURFACE DATUM, MAR 17, 1981.

WATER LEVELS IN FEET BELOW LAND SURFACE DATUM, CLIMATIC YEARS APRIL 1981 TO MARCH 1983

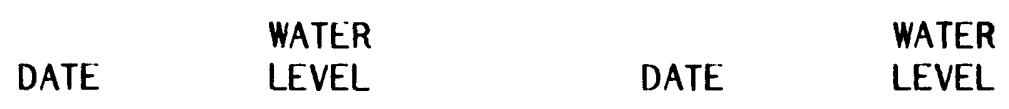

FEB $16,1982 \quad 18.66 \quad$ JAN $27,1983 \quad 17.41$ 
BECKHAM COUNTY -- CONTINUED

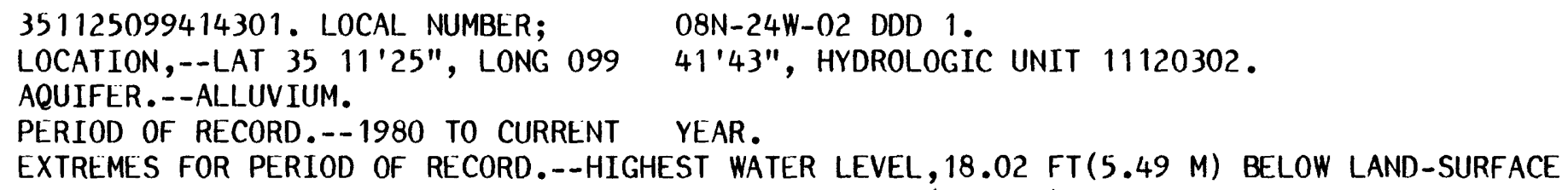

WATER LEVELS IN FEET BELOW LAND SURFACE DATUM, CLIMATIC YEARS APRIL 1981 TO MARCH 1983

$\begin{array}{lll} & \text { WATER } & \text { WATER } \\ \text { DATE } & \text { LEVEL } & \text { DATE }\end{array}$

FEB 16, $1982 \quad 21.53 \quad$ MAR 02, $1983 \quad 20.68$

GROUND-WATER LEVELS

351122099435501. LOCAL NUMBER; 08N-24W-09 AAB 1.

LOCATION,--LAT $3511^{\prime 2}$ ", LONG 099 43'55", HYDROLOGIC UNIT 11120302.

AQUIFER .--ALLUVIUM.

PERIOD OF RECORD.--1980 TO CURRENT YEAR.

EXTREMES FOR PERIOD OF RECORD.--HIGHEST WATER LEVEL, 44.25 FT(13.49 M) BELOW LAND-SURFACE DATUM, MAR 6, 1980; LOWEST WATER LEVEL, 49.26 FT(15.01 M) BELOW LAND-SURFACE DATUM, FEB 16, 1982 .

WATER LEVELS IN FEET BELOW LAND SURFACE DATUM, CLIMATIC YEARS APRIL 1981 TO MARCH 1983

\begin{tabular}{|c|c|c|}
\hline & WATER & \\
\hline DATE & LEVEL & DATE \\
\hline
\end{tabular}

FEB 16, $1982 \quad 49.26 \quad$ MAR 02, $1983 \quad 46.85$ 
BECKHAM COUNTY -- CONTINUED

351119099494101. LOCAL NUMBER; LOCATION,--LAT 3511 '19", LONG 099 AQUIFER.--DOG CREEK SHALE.

PERIOD OF RECORD. - 1980 TO CURRENT YEAR.

EXTREMES FOR PERIOD OF RECORD.--HIGHEST WATER LEVEL, 42.22 FT(12.87 M) BELOW LAND-SURFACE DATUM, MAR 6, 1980; LOWEST WATER LEVEL,48.15 FT(14.68 M) BELOW LAND-SURFACE DATUM, FEB $16,1982$.

WATER LEVELS IN FEET BELOW LAND SURFACE DATUM, CLIMATIC YEARS APRIL 1981 TO MARCH 1983

\begin{tabular}{|c|c|c|}
\hline & WATER & \\
\hline DATE & LEVEL & DATE \\
\hline
\end{tabular}

FEB $16,1982 \quad 48.15 \quad$ MAR $01,1983 \quad 43.77$

351347099324101. LOCAL NUMBER; O9N-22W-29 AAD 1. LOCATION,--LAT 35 13'47", LONG $09932^{\prime} 41^{\prime \prime}$, HYDROLOGIC UNIT 11120302. AQUIFER.--RUSH SPRINGS FORMATION. PERIOD OF RECORD.--1980 TO CURRENT YEAR.

EXTREMES FOR PERIOD OF RECORD.--HIGHEST WATER LEVEL, 32.88 FT(10.02 M) BELOW LAND-SURFACE DATUM, MAR 5, 1980; LOWEST WATER LEVEL, $39.38 \mathrm{FT}(12.00 \mathrm{M})$ BELOW LAND-SURFACE DATUM, FEB $16,1982$.

WATER LEVELS IN FEET BELOW LAND SURFACE DATUM, CLIMATIC YEARS APRIL 1981 TO MARCH 1983

$\begin{array}{lll}\text { WATER } & \text { WATER } \\ \text { DATE } & \text { LEVEL } & \text { DATE } \\ \text { LEVEL }\end{array}$

FEB $16,1982 \quad 39.38 \quad$ JAN 27, $1983 \quad 37.43$


BECKHAM COUNTY -- CONTINUED

351334099344001. LOCAL NUMBER; 09N-22W-30 BCC 1. LOCATION,--LAT 35 13'34", LONG 099 34'40", HYDROLOGIC UNIT 11120302. AQUIFER.--ALLUVIUM.

PERIOD OF RECORD.--1980 TO CURRENT YEAR. EXTREMES FOR PERIOD OF RECORD.--HIGHEST WATER LEVEL,25.78 FT(7.86 M) BELOW LAND-SURFACE DATUM,MAR 5, 1980; LOWEST WATER LEVEL,28.20 FT $(8.60 \mathrm{M})$ BELOW LAND-SURFACE DATUM, FEB 18, 1982.

WATER LEVELS IN FEET BELOW LAND SURFACE DATUM, CLIMATIC YEARS APRIL 1981 TO MARCH 1983

\begin{tabular}{|c|c|c|}
\hline & WATER & \\
\hline DATE & LEVEL & DATE \\
\hline
\end{tabular}

FEB $18,1982 \quad 28.20 \quad$ JAN $27,1983 \quad 27.14$

GROUND-WATER LEVELS

351301099323301. LOCAL NUMBER; 09N-22W-33 BBB 1.

LOCATION,--LAT 35 13'01", LONG 099 32'33", HYDROLOGIC UNIT 11120302.

AQUIFER.--RUSH SPRINGS FORMATION.

PERIOD OF RECORD.--1980 TO CURRENT YEAR.

EXTREMES FOR PERIOD OF RECORD.--HIGHEST WATER LEVEL, $38.65 \mathrm{FT}(11.78 \mathrm{M})$ BELOW LAND-SURFACE DATUM, MAR 5, 1980; LOWEST WATER LEVEL,44.83 FT(13.66 M) BELOW LAND-SURFACE DATUM, FEB $16, .1982$.

WATER LEVELS IN FEET BELOW LAND SURFACE DATUM, CLIMATIC YEARS APRIL 1981 TO MARCH 1983

\begin{tabular}{|c|c|c|}
\hline DATE & $\begin{array}{l}\text { WATER } \\
\text { LEVEL }\end{array}$ & DATE \\
\hline
\end{tabular}

FEB $16,1982 \quad 44.83 \quad$ JAN $27,1983 \quad 43.74$ 


\section{GROUND-WATER LEVELS}

BECKHAM COUNTY -- CONTINUED

351518099375901. LOCAL NUMBER; 09N-23W-16 ADD 1. LOCATION,--LAT 35 15'18", LONG 099 37'59", HYDROLOGIC UNIT 11120302. AQUIFER.--ALLUVIUM.

PERIOD OF RECORD.--1980 TO CURRENT YEAR. EXTREMES FOR PERIOD OF RECORD.--HIGHEST WATER LEVEL,54.00 FT(16.46 M) BELOW LAND-SURFACE DATUM,FEB 18, 1982; LOWEST WATER LEVEL,58.53 FT(17.84 M) BELOW LAND-SURFACE DATUM, MAR 02, 1983.

WATER LEVELS IN FEET BELOW LAND SURFACE DATUM, CLIMATIC YEARS APRIL 1981 TO MARCH 1983

$\begin{array}{lll} & \text { WATER } & \text { WATER } \\ \text { DATE } & \text { LEVEL } & \text { DATE }\end{array}$

FEB 18, $1982 \quad 54.00 \quad$ MAR 02, $1983 \quad 58.53$

\section{GROUND-WATER LEVELS}

351426099372701. LOCAL NUMBER; 09N-23W-22 BDD 1. LOCATION,--LAT 35 14'26", LONG 099 37'27", HYDROLOGIC UNIT 11120302.

AQUIFER.--ALLUVIUM.

PERIOD OF RECORD.--1980 TO CURRENT YEAR.

EXTREMES FOR PERIOD OF RECORD.--HIGHEST WATER LEVEL, $77.75 \mathrm{FT}(23.70 \mathrm{M})$ BELOW LAND-SURFACE DATUM,FEB 18, 1982; LOWEST WATER LEVEL,80.27 FT(24.47 M) BELOW LAND-SURFACE DATUM, MAR 5, 1980.

WATER LEVELS IN FEET BELOW LAND SURFACE DATUM, CLIMATIC YEARS APRIL 1981 TO MARCH 1983

\begin{tabular}{|c|c|}
\hline & $\begin{array}{l}\text { WATER } \\
\text { LFVFI }\end{array}$ \\
\hline
\end{tabular}

FEB 18, $1982 \quad 77.75 \quad$ MAR 02, $1983 \quad P$


BECKHAM COUNTY -- CONTINUED

351446099362401. LOCAL NUMBER; LOCATION,--LAT $3514^{\prime} 46^{\prime \prime}$, LONG 099 AQUIFER.--ALLUVIUM.

PERIOD OF RECORD.--1980 TO CURRENT YEAR.

EXTREMES FOR PERIOD OF RECORD.--HIGHEST WATER LEVEL, 67.32 FT(20.52 M) BELOW LAND-SURFACE DATUM, MAR 2, 1983; LOWEST WATER MAR 5, 1980.

WATER LEVELS IN FEET BELOW LAND SURFACE DATUM, CLIMATIC YEARS APRIL 1981 TO MARCH 1983
09N-23W-23 BAA 1.

$36^{\prime} 24^{\prime \prime}$, HYDROLOGIC UNIT 11120302. LEVEL, 75.62 FT(23.05 M) BELOW LAND-SURFACE DATUM,

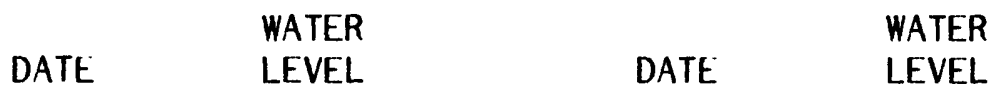

FEB $18,1982 \quad 71.74 \quad$ MAR $02,1983 \quad 67.32$

\section{GROUND-WATER LEVELS}

351308099353601. LOCAL NUMBER; 09N-23W-25 CCD 1. LOCATION,--LAT 35 13'08", LONG 099 35'36", HYDROLOGIC UNIT 11120302. AQUIFER.--ALLUVIUM.

PERIOD OF RECORD.--1980 TO CURRENT YEAR.

EXTREMES FOR PERIOD OF RECORD.--HIGHEST WATER LEVEL, $64.50 \mathrm{FT}(19.66 \mathrm{M})$ BELOW LAND-SURFACE DATUM, MAR 17, 1981; LOWEST WATER LEVEL,74.10 FT(22.59 M) BELOW LAND-SURFACE DATUM, FEB 18, 1982 .

WATER LEVELS IN FEET BELOW LAND SURFACE DATUM, CLIMATIC YEARS APRIL 1981 TO MARCH 1983

\begin{tabular}{|c|c|}
\hline & $\begin{array}{l}\text { WATER } \\
\text { LEVEL }\end{array}$ \\
\hline
\end{tabular}

FEB $18,1982 \quad 74.10 \quad$ JAN 27, $1983 \quad 65.00$


BECKHAM COUNTY -- CONTINUED

351353099383101. LOCAL NUMBER; 09N-23W-28 BAA 1. LOCATION,--LAT 35 13'53", LONG 099 38'31", HYDROLOGIC UNIT 11120302. AQUIFER.--ALLUVIUM. PERIOD OF RECORD.--1980 TO CURRENT YEAR. EXTREMES FOR PERIOD OF RECORD.--HIGHEST WATER LEVEL,50.50 FT(15.39 M) BELOW LAND-SURFACE DATUM, MAR 5, 1980; LOWEST WATER LEVEL,52.16 FT(15.85 M) BELOW LAND-SURFACE DATUM, JAN 27, 1983.

WATER LEVELS IN FEET BELOW LAND SURFACE DATUM, CLIMATIC YEARS APRIL 1981 TO MARCH 1983

\begin{tabular}{|c|c|}
\hline & WATER \\
\hline DATE & LEVEL \\
\hline
\end{tabular}

FEB 18, $1982 \quad 51.80 \quad$ JAN 27, $1983 \quad 52.16$

GROUND-WATER LEVELS

351222099395801. LOCAL NUMBER; 09N-23W-32 CCB 1. LOCATION,--LAT 35 12'22", LONG 099 39'58", HYDROLOGIC UNIT 11120302. AQUIFER.--ALLUVIUM.

PERIOD OF RECORD.--1980 TO CURRENT YEAR.

EXTREMES FOR PERIOD OF RECORD.--HIGHEST WATER LEVEL, $24.05 \mathrm{FT}(7.33 \mathrm{M})$ BELOW LAND-SURFACE DATUM, MAR 5, 1980; LOWEST WATER LEVEL,26.62 FT(8.11 M) BELOW LAND-SURFACE DATUM, JAN 27, 1983.

WATER LEVELS IN FEET BELOW LAND SURFACE DATUM, CLIMATIC YEARS APRIL 1981 TO MARCH 1983

$\begin{array}{lll} & \text { WATER } & \text { WATER } \\ \text { DATE } & \text { LEVEL } & \text { DATE }\end{array}$

FEB $16,1982 \quad Z \quad$ JAN 27, $1983 \quad 26.62$ 
BECKHAM COUNTY -- CONTINUED

351512099411001. LOCAL NUMBER; 09N-24W-13 DAA 1.

LOCATION,--LAT 35 15'12", LONG 099 41'10", HYDROLOGIC UNIT 11120302.

AQUIFER.--ALLUVIUM.

PERIOD OF RECORD.--1980 TO CURRENT YEAR.

EXTREMES FOR PERIOD OF RECORD.--HIGHEST WATER LEVEL, 12.45 FT(3.79 M) BELOW LAND-SURFACE DATUM, MAR 19, 1981; LOWEST WATER LEVEL, 16.06 FT(4.90 M) BELOW LAND-SURFACE DATUM, MAR 2, 1983.

WATER LEVELS IN FEET BELOW LAND SURFACE DATUM, CLIMATIC YEARS APRIL 1981 TO MARCH 1983

\begin{tabular}{|c|c|}
\hline & WATER \\
\hline DATE & LEVEL \\
\hline
\end{tabular}

FEB 16, $1982 \quad 14.72 \quad$ MAR 02, $1983 \quad 16.06$

GROUND-WATER LEVELS

351512099472401. LOCAL NUMBER; 09N-24W-18 CBB 1. LOCATION,--LAT 35 15'12", LONG 099 47'24", HYDROLOGIC UNIT 11120302. AQUIFER .--ALLUVIUM.

PERIOD OF RECORD.--1980 TO CURRENT YEAR.

EXTREMES FOR PERIOD OF RECORD.--HIGHEST WATER LEVEL, 22.58 FT (6.88 M) BELOW LAND-SURFACE DATUM, MAR 6, 1980; LOWEST WATER LEVEL,25.28 FT(7.71 M) BELOW LAND-SURFACE DATUM, FEB $16,1982$.

WATER LEVELS IN FEET BELOW LAND SURFACE DATUM, CLIMATIC YEARS APRIL 1981 TO MARCH 1983

\begin{tabular}{|c|c|c|}
\hline & WATER & \\
\hline DATE & LEVEL & DATE \\
\hline
\end{tabular}

FEB $16,1982 \quad 25.28 \quad$ MAR $01,1983 \quad 24.46$ 
BECKHAM COUNTY -- CONTINUED

351413099451601 . LOCAL NUMBER;

LOCATION,--LAT 35 14'13", LONG 099 AQUIFER .--ALLUVIUM.

PERIOD OF RECORD.--1980 TO CURRENT YEAR.

EXTREMES FOR PERIOD OF RECORD.--HIGHEST WATER LEVEL, $46.22 \mathrm{FT}(14.09 \mathrm{M})$ BELOW LAND-SURFACE DATUM, FEB 16, 1982; LOWEST WATER LEVEL,46.52 FT(14.18 M) BELOW LAND-SURFACE DATUM, MAR 7, 1980 .

WATER LEVELS IN FEET BELOW LAND SURFACE DATUM, CLIMATIC YEARS APRIL 1981 TO MARCH 1983
$09 N-24 W-21$ CBC 1

45'16", HYDROLOGIC UNIT 11120302.

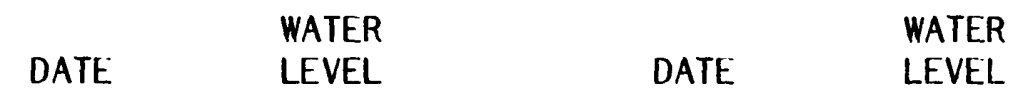

FEB 16, $1982 \quad 46.22$ MAR 01, $1983 \quad 46.30$

\section{GROUND-WATER LEVELS}

351242099420601. LOCAL NUMBER; 09N-24W-36 BCC 1. LOCATION,--LAT 35 12'42", LONG 099 42'06", HYDROLOGIC UNIT 11120302. AQUIFER.--ALLUVIUM.

PERIOD OF RECORD.--1980 TO CURRENT YEAR.

EXTREMES FOR PERIOD OF RECORD.--HIGHEST WATER LEVEL, $35.80 \mathrm{FT}(10.91 \mathrm{M})$ BELOW LAND-SURFACE DATUM, MAR 19, 1981; LOWEST WATER LEVEL,36.85 FT(11.23 M) BELOW LAND-SURFACE DATUM, FEB 16, 1982 .

WATER LEVELS IN FEET BELOW LAND SURFACE DATUM, CLIMATIC YEARS APRIL 1981 TO MARCH 1983

\begin{tabular}{|c|c|c|}
\hline DATE & $\begin{array}{l}\text { WATER } \\
\text { LEVEL }\end{array}$ & DAT \\
\hline UATE & & UAI \\
\hline
\end{tabular}

FEB $16,1982 \quad 36.85 \quad$ MAR 02, $1983 \quad 0$ 
BECKHAM COUNTY -- CONTINUED

351637099521001. LOCAL NUMBER; 09N-25W-05 DCC 1.

LOCATION,--LAT 35 16'37", LONG 099 52'10", HYDROLOGIC UNIT 11120302.

AQUIFER.--ALLUVIUM.

PLRIOD OF RECORD.--1980 TO CURRENT YEAR.

EXTREMES FOR PERIOD OF RECORD.--HIGHEST WATER LEVEL,44.05 FT(13.43 M) BELOW LAND-SURFACE DATUM,MAR 6, 1980; LOWEST WATER LEVEL,49.52 FT(15.09 M) BELOW LAND-SURFACE DATUM, MAR 1, 1983.

WATER LEVELS IN FEET BELOW LAND SURFACE DATUM, CLIMATIC YEARS APRIL 1981 TO MARCH 1983

$\begin{array}{lll} & \text { WATER } & \text { WATER } \\ \text { DATE } & \text { LEVEL } & \text { DATE }\end{array}$

FEB 16, $1982 \quad P \quad$ MAR 01, $1983 \quad 49.52$

GROUND-WATER LEVELS

351557099522601. LOCAL NUMBER; 09N-25W-08 CAC 1.

LOCATION,--LAT 35 15'57", LONG 099 52'26", HYDROLOGIC UNIT 11120302.

AQUIFER.--TERRACE DEPOSITS.

PERIOD OF RECORD.--1980 TO CURRENT YEAR.

EXTREMES FOR PERIOD OF RECORD.--HIGHEST WATER LEVEL, 57.62 FT(17.56 M) BELOW LAND-SURFACE DATUM, JAN 27, 1983; LOWEST WATER LEVEL,57.62 FT(17.56 M) BELOW LAND-SURFACE DATUM, JAN 27, 1983.

WATER LEVELS IN FEET BELOW LAND SURFACE DATUM, CLIMATIC YEARS APRIL 1981 TO MARCH 1983

$$
\begin{array}{ll}
\text { WATER } \\
\text { DATE } & \text { LEVEL }
\end{array}
$$

JAN 27, $1983 \quad 57.72$ 
BECKHAM COUNTY -- CONTINUED

351617099482701. LOCAL NUMBER; LOCATION,--LAT $3516^{\prime} 17^{\prime \prime}$, LONG 099 AQUIFER.--ALLUVIUM.

PERIOD OF RECORD.--1980 TO CURRENT

EXTREMES FOR PERIOD OF RECORD.- HIGHEST WA

\section{DATUM, MAR 6, 1980; LOWEST WATER} MAR 1, 1983.

WATER LEVELS IN FEET BELOW LAND SURFACE DATUM, CLIMATIC YEARS APRIL 1981 TO MARCH 1983
$09 \mathrm{~N}-25 \mathrm{~W}-12$ BCB 1 .

48'27', HYDROLOGIC UNIT 11120302.

YEAR.

ST WATER LEVEL, 38.20 FT(11.64 M) BELOW LAND-SURFACE LEVEL, 40.32 FT(12.29 M) BELOW LAND-SURFACE DATUM,

\section{WATER}

DATE

LEVEL

FEB 16, $1982 \quad 39.51 \quad$ MAR 01, $1983 \quad 40.32$

\section{GROUND-WATER LEVELS}

351452099494701. LOCAL NUMBER; 09N-25W-15 DDC 1. LOCATION,--LAT 35 14'52", LONG 099 49'47", HYDROLOGIC UNIT 11120302. AQUIFER.--ALLUVIUM.

PERIOD OF RECORD.--1980 TO CURRENT YEAR.

EXTREMES FOR PERIOD OF RECORD.--HIGHEST WATER LEVEL, $46.93 \mathrm{FT}(14.30 \mathrm{M})$ BELOW LAND-SURFACE DATUM,MAR 6, 1980; LOWEST WATER LEVEL,47.51 FT(14.48 M) BELOW LAND-SURFACE DATUM, MAR $1,1983$.

WATER LEVELS IN FEET BELOW LAND SURFACE DATUM, CLIMATIC YEARS APRIL 1981 TO MARCH 1983

$\begin{array}{lll} & \text { WATER } & \text { WATER } \\ \text { DATE } & \text { LEVEL } & \text { DATE }\end{array}$
FEB 16, 1982
47.35
MAR 01, 1983
47.51 


\section{GROUND-WATER LEVELS}

BECKHAM COUNTY -- CONTINUED

351402099533501. LOCAL NUMBER; 09N-25W-19 CCC 1. LOCATION,--LAT 35 14'02", LONG 099 53'35", HYDROLOGIC UNIT 11120302. AQUIFER.--ALLUVIUM.

PERIOD OF RECORD.--1980 TO CURRENT YEAR.

EXTREMES FOR PERIOD OF RECORD.--HIGHEST WATER LEVEL, $17.63 \mathrm{FT}(5.37 \mathrm{M})$ BELOW LAND-SURFACE DATUM, MAR 6, 1980; LOWEST WATER LEVEL, 19.49 FT(5.94 M) BELOW LAND-SURFACE DATUM, JAN 27, 1983.

WATER LEVELS IN FEET BELOW LAND SURFACE DATUM, CLIMATIC YEARS APRIL 1981 TO MARCH 1983

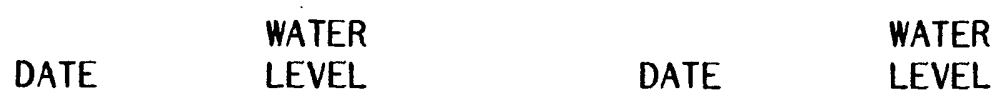

FEB $16,1982 \quad 18.88 \quad$ JAN 27, $1983 \quad 19.49$

GROUND-WATER LEVELS

351353099482701. LOCAL NUMBER; 09N-25W-25 BBB 1. LOCATION,--LAT 35 13'53", LONG 099 48'27", HYDROLOGIC UNIT 11120302. AQUIFER.--ALLUVIUM.

PERIOD OF RECORD.--1980 TO CURRENT YEAR.

EXTREMES FOR PERIOD OF RECORD.--HIGHEST WATER LEVEL,53.30 FT(16.25 M) BELOW LAND-SURFACE DATUM,FEB 16, 1982; LOWEST WATER LEVEL,54.15 FT(16.50 M) BELOW LAND-SURFACE DATUM, MAR 6,1980 .

WATER LEVELS IN FEET BELOW LAND SURFACE DATUM, CLIMATIC YEARS APRIL 1981 TO MARCH 1983

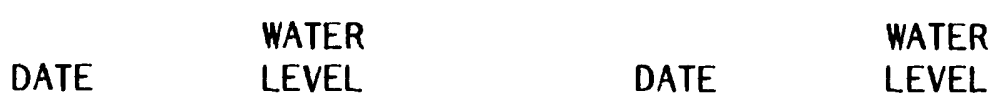

FEB 16, $1982 \quad 53.30 \quad$ MAR 01, $1983 \quad 53.57$ 
BE.CKHAM COUNTY -- CONTINUED

351400099533501. LOCAL NUMBER; 09N-25W-31 BCB 1. LOCATION,--LAT 35 14'00", LONG 099 53'35", HYDROLOGIC UNIT 11120302. AQUIFER.--WHITEHORSE GROUP. PERIOD OF RECORD.--1980 TO CURRENT YEAR. EXTREMES FOR PERIOD OF RECORD.--HIGHEST WATER LEVEL, 11.62 FT(3.54 M) BELOW LAND-SURFACE DATUM,MAR 6, 1980; LOWEST WATER LEVEL, $14.90 \mathrm{FT}(4.54 \mathrm{M})$ BELOW LAND-SURFACE DATUM, FEB 16, 1982.

WATER LEVELS IN FEET BELOW LAND SURFACE DATUM, CLIMATIC YEARS APRIL 1981 TO MARCH 1983

\begin{tabular}{|c|c|c|}
\hline & WATER & \\
\hline DATE & LEVEL & DATE \\
\hline
\end{tabular}

FEB $16,1982 \quad 14.90 \quad$ JAN $27,1983 \quad 12.97$

\section{GROUND-WATER LEVELS}

351216099483501 . LOCAL NUMBER;

09N-25W-35 DDD 1. LOCATION,--LAT 35 12'16", LONG 099 48'35", HYDROLOGIC UNIT 11120302. AQUIFER.--ALLUVIUM.

PERIOD OF RECORD.--1980 TO CURRENT YEAR.

EXTREMES FOR PERIOD OF RECORD.--HIGHEST WATER LEVEL, $42.62 \mathrm{FT}(12.99 \mathrm{M})$ BELOW LAND-SURFACE DATUM, MAR 7, 1980; LOWEST WATER LEVEL,47.15 FT(14.37 M) BELOW LAND-SURFACE DATUM, FEB 16, 1982.

WATER LEVELS IN FEET BELOW LAND SURFACE DATUM, CLIMATIC YEARS APRIL 1981 TO MARCH 1983

$\begin{array}{lll} & \text { WATER } & \text { WATER } \\ \text { DATE } & \text { LEVEL } & \text { DATE }\end{array}$

FEB 16, $1982 \quad 47.15 \quad$ MAR 01, $1983 \quad 44.30$ 
BECKHAM COUNTY -- CONTINUED

351617099590301. LOCAL NUMBER; LOCATION,--LAT $3516^{\prime} 17^{\prime \prime}$, LONG 099 AQUIFER.--ALLUVIUM.

PERIOD OF RECORD. - 1980 TO CURRENT YEAR.

EXTREMES FOR PERIOD OF RECORD.--HIGHEST WATER LEVEL,56.80 FT(17.31 M) BELOW LAND-SURFACE DATUM, MAR 18, 1981; LOWEST WATER LEVEL,58.00 FT(17.68 M) BELOW LAND-SURFACE DATUM, MAR 6, 1980.

WATER LEVELS IN FEET BELOW LAND SURFACE DATUM, CLIMATIC YEARS APRIL 1981 TO MARCH 1983
GROUND-WATER LEVELS

351525099560801. LOCAL NUMBER; LOCATION,--LAT 35 15'25", LONG 099 56'08", HYDROLOGIC UNIT 11120302. AQUIFER .--ALLUVIUM.

PERIOD OF RECORD.--1980 TO CURRENT YEAR.

EXTREMES FOR PERIOD OF RECORD.--HIGHEST WATER LEVEL, $16.56 \mathrm{FT}(5.05 \mathrm{M})$ BELOW LAND-SURFACE DATUM,MAR 6, 1980; LOWEST WATER LEVEL, 18.34 FT(5.59 M) BELOW LAND-SURFACE DATUM, JAN 27, 1983.

WATER LEVELS IN FEET BELOW LAND SURFACE DATUM, CLIMATIC YEARS APRIL 1981 TO MARCH 1983
FEB 16, 1982
18.30
JAN 27, 1983
18.34 


\section{GROUND-WATER LEVELS}

BECKHAM COUNTY -- CONTINUED

351433099544101. LOCAL NUMBER; LOCATION,--LAT 35 14'33", LONG 099 AQUIFER.--ALLUVIUM. PERIOD OF RECORD.--1980 TO CURRENT YEAR. EXTREMES FOR PERIOD OF RECORD.--HIGHEST WATER LEVEL,53.42 FT(16.28 M) BELOW LAND-SURFACE DATUM,MAR 6, 1980; LOWEST WATER LEVEL,55.49 FT(16.91 M) BELOW LAND-SURFACE DATUM, JAN $27,1983$.

WATER LEVELS IN FEET BELOW LAND SURFACE DATUM, CLIMATIC YEARS APRIL 1981 TO MARCH 1983

$09 \mathrm{~N}-26 \mathrm{~W}-24 \mathrm{BCA} 1$. $54^{\prime} 41^{\prime \prime}$, HYDROLOGIC UNIT 11120302.

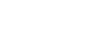
WATER LEVELS IN FEET BELOW LAND SURFACE DATU

\begin{tabular}{|c|c|c|}
\hline & WATER & \\
\hline DATE & LEVEL & DATE \\
\hline
\end{tabular}

FEB 16, $1982 \quad 55.05$

JAN 27, $1983 \quad 55.49$

GROUND-WATER LEVELS

351327099595901. LOCAL NUMBER; 09N-26W-30 CBA 1. LOCATION,--LAT 35 13'27", LONG 099 59'59", HYDROLOGIC UNIT 11120302. AQUIFER.--WHITEHORSE GROUP.

PERIOD OF RECORD.--1980 TO CURRENT YEAR. EXTREMES FOR PERIOD OF RECORD.--HIGHEST WATER LEVEL, 40.30 FT(12.28 M) BELOW LAND-SURFACE DATUM, JAN 27, 1983; LOWEST WATER LEVEL,46.46 FT(14.16 M) BELOW LAND-SURFACE DATUM, FEB 16, 1982.

WATER LEVELS IN FEET BELOW LAND SURFACE DATUM, CLIMATIC YEARS APRIL 1981 TO MARCH 1983

\begin{tabular}{|c|c|c|}
\hline & WATER & \\
\hline DATE & LEVEL & DATE \\
\hline
\end{tabular}

FEB 16, $1982 \quad 46.46 \quad$ JAN 27, $1983 \quad 40.30$ 
BECKHAM COUNTY -- CONTINUED

351306099515501. LOCAL NUMBER; 09N-26W-33 BBB 1. LOCATION,--LAT 35 13'06", LONG 099 51'55", HYDROLOGIC UNIT 11120302.

AQUIFER.--WHITEHORSE GROUP.

PERIOD OF RECORD.--1980 TO CURRENT YEAR.

EXTREMES FOR PERIOD OF RECORD.--HIGHEST WATER LEVEL, 10.48 FT(3.19 M) BELOW LAND-SURFACE DATUM, JAN 27, 1983; LOWEST WATER LEVEL, 13.46 FT(4. 10 $\mathrm{M}$ ) BELOW LAND-SURFACE DATUM, FEB $16,1982$.

WATER LEVELS IN FEET BELOW LAND SURFACE DATUM, CLIMATIC YEARS APRIL 1981 TO MARCH 1983

$\begin{array}{lll} & \text { WATER } & \text { WATER } \\ \text { DATE } & \text { LEVEL } & \text { DATE }\end{array}$

FEB $16,1982 \quad 13.46 \quad$ JAN 27, $1983 \quad 10.48$

GROUND-WATER LEVELS

352426099572801. LOCAL NUMBER; 11N-26W-21 DCC 1. LOCATION,--LAT 35 24'26", LONG 099 57'28", HYDROLOGIC UNIT 11120302.

AQUIFER.--OGALLALA.

PERIOD OF RECORD.--1980 TO CURRENT YEAR.

EXTREMES FOR PERIOD OF RECORD.--HIGHEST WATER LEVEL, $8.53 \mathrm{FT}(2.60 \mathrm{M})$ BELOW LAND-SURFACE DATUM,MAR 6, 1980; LOWEST WATER LEVEL, 11.40 FT(3.47 M) BELOW LAND-SURFACE DATUM, MAR 18, 1981.

WATER LEVELS IN FEET BELOW LAND SURFACE DATUM, CLIMATIC YEARS APRIL 1981 TO MARCH 1983

$\begin{array}{lll} & \text { WATER } & \text { WATER } \\ \text { DATE } & \text { LEVEL } & \text { DATE }\end{array}$

$\begin{array}{lllll}\text { FEB } 17,1982 & 10.47 & \text { MAR } 01,1983 & 8.77\end{array}$ 
BECKHAM COUNTY -- CONTIANED

352328099560001. LOCAL NUMBER; LOCATION,--LAT 35 23'28", LONG 099 AQUIFER.--ALLUVIUM.

PERIOD OF RECORD.--1980 TO CURRENT YEAR.

EXTREMES FOR PERIOD OF RECORD.--HIGHEST WATER LEVEL, $19.15 \mathrm{FT}(5.84 \mathrm{M})$ BELOW LAND-SURFACE DATUM,MAR 6, 1980; LOWEST WATER LEVEL,21.96 FT(6.69 M) BELOW LAND-SURFACE DATUM, FEB 17, 1982.

WATER LEVELS IN FEET BELOW LAND SURFACE DATUM, CLIMATIC YEARS APRIL 1981 TO MARCH 1983

FEB $17,1982 \quad 21.96 \quad$ MAR $01,1983 \quad 19.18$

GROUND-WATER LEVELS

351755099563201. LOCAL NUMBER; 10N-26W-34 BDD 1.

LOCATION,--LAT 35 17'55", LONG 099 56'32", HYDROLOGIC UNIT 11120302. AQUIFER.--ALLUVIUM.

PERIOD OF RECORD.--1980 TO CURAENT VEAR.

EXTREMES FOR PERIOD OF RECORD.--HIGWEST WATER LEVEL, 32.56 FT(9.92 M) BELOW LAND-SURFACE DATUM, MAR 6, 1980; LOWEST WATER LEVEL, 34.12 FT(10.40 M) BELOW LAND-SURFACE DATUM, FEB 17, 1982 .

WATER LEVELS IN FEET BELOW LAND SURFACE DATUM, CLIMATIC YEARS APRIL 1981 TO MARCH 1983

\begin{tabular}{|c|c|}
\hline & WATER \\
\hline ATE & LEVEL \\
\hline
\end{tabular}

FEB $17,1982 \quad 34.12 \quad$ MAR $01,1983 \quad 32.68$ 


\section{GROUND-WATER LEVELS}

BLAINE COUNTY

354506098225501. LOCAL NUMBER;

$15 \mathrm{~N}-11 \mathrm{~W}-28 \mathrm{BBC} 1$.

LOCATION,--LAT 35 45'06", LONG 098 22'55", HYDROLOGIC UNIT 11100301.

AQUIFER.--ALLUVIUM.

PERIOD OF RECORD.--1977 TO CURRENT YEAR.

EXTREMES FOR PERIOD OF RECORD.--HIGHEST WATER LEVEL, 12.11 FT(3.69 M) BELOW LAND-SURFACE DATUM,MAR 11, 1982; LOWEST WATER LEVEL, 14.22 FT(4.33 M) BELOW LAND-SURFACE DATUM, MAR 13, 1979.

WATER LEVELS IN FEET BELOW LAND SURFACE DATUM, CLIMATIC YEARS APRIL 1981 TO MARCH 1983

\begin{tabular}{|c|c|}
\hline & WATER \\
\hline DATE & LEVEL \\
\hline
\end{tabular}
MAR $31,1982 \quad 12.11$
FEB 28, 1983
Z

GROUND-WATER LEVELS

355223098220701. LOCAL NUMBER; 16N-11W-09 DDB 1.

LOCATION,--LAT 35 52'23", LONG 098 22'07", HYDROLOGIC UNIT 11100301.

AQUIFER.--TERRACE DEPOSITS.

PERIOD OF RECORD.--1977 TO CURRENT YEAR.

EXTREMES FOR PERIOD OF RECORD.--HIGHEST WATER LEVEL, 29.70 FT(9.05 M) BELOW LAND-SURFACE DATUM,FEB 28, 1983; LOWEST WATER LEVEL, $35.50 \mathrm{FT}(10.82 \mathrm{M})$ BELOW LAND-SURFACE DATUM, MAR 31, 1982.

WATER LEVELS IN FEET BELOW LAND SURFACE DATUM, CLIMATIC YEARS APRIL 1981 TO MARCH 1983

$\begin{array}{lll} & \text { WATER } & \text { WATER } \\ \text { DATE } & \text { LEVEL } & \text { DATE }\end{array}$

$\begin{array}{llll}\text { MAR } 31,1982 \quad 35.50 & \text { FEB 28, } 1983 \quad 29.70\end{array}$ 
BLAINE COUNTY -- CONTINUED

355151098215101. LOCAL NUMBER; LOCATION,--LAT 3551 '51", LONG 098 AQUIFER.--TERRACE DEPOSITS. PERIOD OF RECORD.--1981 TO CURRENT YEAR. EXTREMES FOR PERIOD OF RECORD.--HIGHEST WATER LEVEL, 34.44 FT(10.50 M) BELOW LAND-SURFACE DATUM, MAY 28, 1981; LOWEST WATER LEVEL, $34.90 \mathrm{FT}(10.64 \mathrm{M})$ BELOW LAND-SURFACE DATUM, MAR 31, 1982.

WATER LEVELS IN FEET BELOW LAND SURFACE DATUM, CLIMATIC YEARS APRIL 1981 TO MARCH 1983

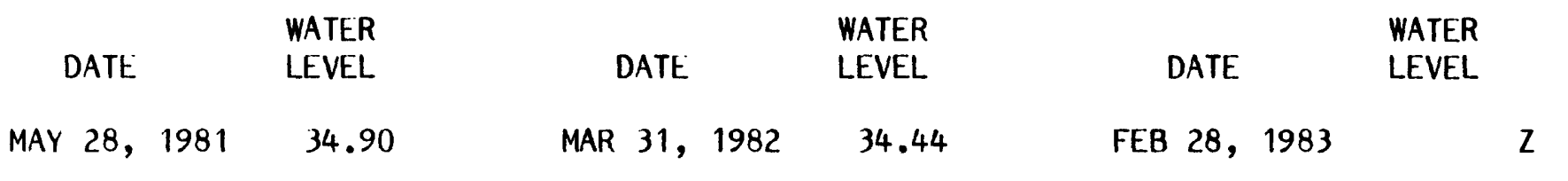

GROUND-WATER LEVELS

355045098243101. LOCAL NUMBER; 16N-11W-19 DBC 1. LOCATION,--LAT 35 50'45", LONG 098 24'31", HYDROLOGIC UNIT 11100301. AQUIFER.--TERRACE DEPOSITS. PERIOD OF RECORD.--1976 TO CURRENT YEAR. EXTREMES FOR PERIOD OF RECORD.--HIGHEST WATER LEVEL, $15.85 \mathrm{FT}(4.83 \mathrm{M})$ BELOW LAND-SURFACE DATUM,JAN 28, 1981; LOWEST WATER LEVEL, 32.07 FT(9.77 M) BELOW LAND-SURFACE DATUM, APR 12, 1977.

WATER LEVELS IN FEET BELOW LAND SURFACE DATUM, CLIMATIC YEARS APRIL 1981 TO MARCH 1983

\begin{tabular}{|c|c|c|}
\hline & WATER & \\
\hline DATE & LEVEL & DATE \\
\hline
\end{tabular}

JAN 28, $1982 \quad Z \quad$ FEB 28, $1983 \quad 16.90$


BLAINE COUNTY -- CONTINUED

355724098283501. LOCAL NUMBER; LOCATION,--LAT 35 57'24", LONG 098 AQUIFER.--TERRACE DEPOSITS. PLRIOD OF RECORD. --1977 TO CURRENT YEAR. EXTREMES FOR PERIOD OF RECORD.--HIGHEST WATER LEVEL,49.80 FT(15.18 M) BELOW LAND-SURFACE DATUM,FEB 28, 1983; LOWEST WATER LEVEL,53.93 FT(16.44 M) BELOW LAND-SURFACE DATUM, MAR $14,1979$.

WATER LEVELS IN FEET BELOW LAND SURFACE DATUM, CLIMATIC YEARS APRIL 1981 TO MARCH 1983

\begin{tabular}{|c|c|}
\hline & WATER \\
\hline DATE & LEVEL \\
\hline
\end{tabular}

MAR 31, $1982 \quad 51.87 \quad$ FEB 28, $1983 \quad 49.80$

\section{GROUND-WATER LEVELS}

360145098303501. LOCAL NUMBER;

$18 \mathrm{~N}-12 \mathrm{~W}-17 \mathrm{CCC} 1$

LOCATION,--LAT 36 01'45", LONG 098 30'35", HYDROLOGIC UNIT 11100301.

AQUIFER.--TERRACE DEPOSITS.

PERIOD OF RECORD.-- 1977 TO CURRENT YEAR.

EXTREMES FOR PERIOD OF RECORD.--HIGHEST WATER LEVEL, $32.86 \mathrm{FT}(10.02 \mathrm{M})$ BELOW LAND-SURFACE DATUM,APR 6, 1977; LOWEST WATER LEVEL, 34.05 FT(10.38 M) BELOW LAND-SURFACE DATUM, FEB 19, 1980.

WATER LEVELS IN FEET BELOW LAND SURFACE DATUM, CLIMATIC YEARS APRIL 1981 TO MARCH 1983

$\begin{array}{lll} & \text { WATER } & \text { WATER } \\ \text { DATE } & \text { LEVEL } & \text { DATE }\end{array}$

MAR $31,1982 \quad 33.90$

FEB 28, 1983

Z 


\section{GROUND-WATER LEVELS}

BLAINE COUNTY -- CONTINUED

360927098354701. LOCAL NUMBER; LOCATION,--LAT $3609^{\prime} 27$ ", LONG 098 AQUIFER.--TERRACE DEPOSITS.

PERIOD OF RECORD.--1976 TO CURRENT YEAR.

EXTREMES FOR PERIOD OF RECORD.--HIGHEST WATER LEVEL, 18.00 FT(5.49 M) BELOW LAND-SURFACE DATUM,MAR 11, 1976; LOWEST WATER LEVEL,21.65 FT(6.60 M) BELOW LAND-SURFACE DATUM, FEB 19, 1980.

WATER LEVELS IN FEET BELOW LAND SURFACE DATUM, CLIMATIC YEARS APRIL 1981 TO MARCH 1983
$19 \mathrm{~N}-13 \mathrm{~W}-04$ BBA 1.

35'47", HYDROLOGIC UNIT 11100301.

\section{$\begin{array}{lll} & & \\ & & \\ \text { DATE } & \text { WATER } & \text { WATER } \\ \text { LEVEL } & \text { DATE } & \text { LEVEL }\end{array}$}

MAR 31, $1982 \quad 20.24 \quad$ FEB 28, $1983 \quad P$

\section{GROUND-WATER LEVELS}

\section{LOCAL NUMBER; 19N-13W-23 ACC 1.}

LOCATION,--LAT 36 06'31", LONG 098 33'15", HYDROLOGIC UNIT 11100301.

AQUIFER.--TERRACE DEPOSITS.

PERIOD OF RECORD.--1976 TO CURRENT YEAR.

EXTREMES FOR PERIOD OF RECORD.--HIGHEST WATER LEVEL, 28.04 FT (8.55 M) BELOW LAND-SURFACE DATUM,MAR 11, 1976; LOWEST WATER LEVEL,29.26 FT(8.92 M) BELOW LAND-SURFACE DATUM, APR 12, 1977.

WATER LEVELS IN FEET BELOW LAND SURFACE DATUM, CLIMATIC YEARS APRIL 1981 TO MARCH 1983

\begin{tabular}{|c|c|c|}
\hline & WATER & \\
\hline DATE & LEVEL & DATE \\
\hline
\end{tabular}

MAR 31, $1982 \quad Z \quad$ FEB 28, $1983 \quad 28.55$ 
GROUND-WATER LEVELS

BLAINE COUNTY -- CONTINUED

360600098324701. LOCAL NUMBER; 19N-13W-25 BBB 1.

LOCATION,--LAT 36 06'00", LONG 098 32'47", HYDROLOGIC UNIT 11100301.

AQUIFER.--TERRACE DEPOSITS.

PERIOD OF RECORD.--1978 TO CURRENT YEAR.

EXTREMES FOR PERIOD OF RECORD.--HIGHEST WATER LEVEL,29.99 FT(9.14 M) BELOW LAND-SURFACE DATUM,FEB 28, 1983; LOWEST WATER LEVEL, 30.63 FT(9.34 M) BELOW LAND-SURFACE DATUM, APR 20, 1978.

WATER LEVELS IN FEET BELOW LAND SURFACE DATUM, CLIMATIC YEARS APRIL 1981 TO MARCH 1983

$\begin{array}{lll} & \text { WATER } & \text { WATER } \\ \text { DATE } & \text { LEVEL } & \text { DATE }\end{array}$

$\begin{array}{lll}\text { MAR } 31,1982 \quad 30.58 & \text { FEB 28, } 1983 \quad 29.99\end{array}$ 


\section{GROUND-WATER LEVELS}

BRYAN COUNTY

340135096013601. LOCAL NUMBER; 06S-12E-23 BBB 1. LOCATION,--LAT 3401'35", LONG 096 01'36", HYDROLOGIC UNIT 11140101. AQUIFER.--ANTLERS SAND.

PERIOD OF RECORD.--1977 TO CURRENT YEAR.

EXTREMES FOR PERIOD OF RECORD.--HIGHEST WATER LEVEL, 155.26 FT(47.32 M) BELOW LAND-SURCACE DATUM,MAR 8, 1983; LOWEST WATER LEVEL, 161.87 FT(49.34 M) BELOW LAND-SURFACE DATUM, MAR 29, 1978.

WATER LEVELS IN FEET BELOW LAND SURFACE DATUM, CLIMATIC YEARS APRIL 1981 TO MARCH 1983

\begin{tabular}{|c|c|c|}
\hline & WATER & \\
\hline DATE & LEVEL & DATE \\
\hline
\end{tabular}

MAR 09, $1982 \quad 157.80 \quad$ MAR 08, $1983 \quad 155.26$ 


\section{GROUND-WATER LEVELS}

\section{CADDO COUNTY}

350902098235501. LOCAL NUMBER;

08N-11W-19 CAD 1.

LOCATION,--LAT $3509^{\prime}$ '02", LONG 098

23'55", HYDROLOGIC UNIT 11130302.

AQUIFER.--RUSH SPRINGS FORMATION.

PERIOD OF RECORD.--1974 TO CURRENT YEAR.

EXTREMES FOR PERIOD OF RECORD.--HIGHEST WATER LEVEL, $77.50 \mathrm{FT}(23.62 \mathrm{M})$ BELOW LAND-SURFACE DATUM,DEC 2, 1975; LOWEST WATER LEVEL, 117.70 FT(35.87 M) BELOW LAND-SURFACE DATUM, MAR 22, 1982.

WATER LEVELS IN FEET BELOW LAND SURFACE DATUM, CLIMATIC YEARS APRIL 1981 TO MARCH 1983

$\begin{array}{lll} & \text { WATER } & \text { WATER } \\ \text { DATE } & \text { LEVEL } & \text { DATE }\end{array}$
MAR 22, $1982 \quad 117.70$
FEB $25,1983 \quad 85.64$

GROUND-WATER LEVELS

350930098352501. LOCAL NUMBER; 08N-13W-20 ABA 1.

LOCATION,--LAT 35 09'30", LONG 098 35'25", HYDROLOGIC UNIT 11130302.

AQUIFER.--RUSH SPRINGS FORMATION.

PERIOD OF RECORD.--1974 TO CURRENT YEAR.

EXTREMES FOR PERIOD OF RECORD.--HIGHEST WATER LEVEL, $69.03 \mathrm{FT}(21.04 \mathrm{M})$ BELOW LAND-SURFACE DATUM,MAR 8, 1978; LOWEST WATER LEVEL,71.25 FT(21.72 M) BELOW LAND-SURFACE DATUM, JAN 30, 1981.

WATER LEVELS IN FEET BELOW LAND SURFACE DATUM, CLIMATIC YEARS APRIL 1981 TO MARCH 1983

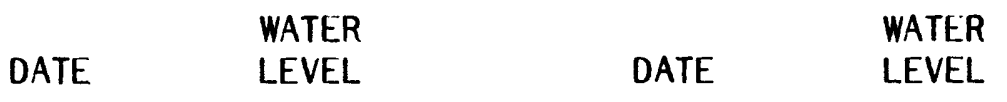

MAR 22, $1982 \quad 71.06 \quad$ FEB 24, $1983 \quad 70.42$ 
CADDO COUNTY -- CONTINUED

351342098240001. LOCAL NUMBER; LOCATION,--LAT 3513 '42", LONG 098 AQUIFER.--RUSH SPRINGS FORMATION. PERIOD OF RECORD.--1974 TO CURRENT EXTREMES FOR PERIOD OF RECORD.--HIGHEST WATER LEVEL,74.00 FT(22.56 M) BELOW LAND-SURFACE DATUM,DEC 3, 1975; LOWEST WATER LEVEL,86.65 FT(26.41 M) BELOW LAND-SURFACE DATUM, FEB 23, 1983.

WATER LEVELS IN FEET BELOW LAND SURFACE DATUM, CLIMATIC YEARS APRIL 1981 TO MARCH 1983
$09 \mathrm{~N}-11 \mathrm{~W}-30$ ACA 1 $24^{\prime} 00^{\prime \prime}$, HYDROLOGIC UNIT 11130302.

$\begin{array}{lll} & & \\ \text { DATE } & \text { WATER } & \\ \text { LEVEL } & \text { DATER } & \text { LEVEL }\end{array}$

MAR 22, $1982 \quad 86.14 \quad$ FEB 23, $1983 \quad 86.65$

\section{GROUND-WATER LEVELS}

351459098260201. LOCAL NUMBER;

09N-12W-14 DDB 1.

LOCATION,--LAT $3514^{\prime} 59^{\prime \prime}$, LONG 098

AQUIFER .--RUSH SPRINGS FORMATION.

26'02", HYDROLOGIC UNIT 11130302.

PERIOD OF RECORD.--1974 TO CURRENT ' YEAR.

EXTREMES FOR PERIOD OF RECORD.--HIGHEST WATER LEVEL,74.00 FT(22.56 M) BELOW LAND-SURFACE DATUM,DEC 3, 1975; LOWEST WATER LEVEL,84.50 FT(25.76 M) BELOW LAND-SURFACE DATUM, MAR 22, 1982.

WATER LEVELS IN FEET BELOW LAND SURFACE DATUM, CLIMATIC YEARS APRIL 1981 TO MARCH 1983

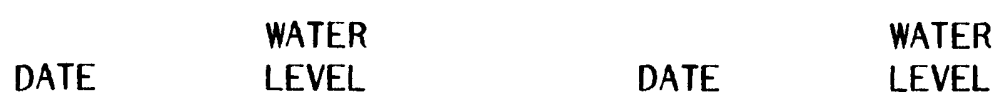

MAR 22, $1982 \quad 84.50 \quad$ FEB 23, $1983 \quad 84.01$ 
CADDO COUNTY -- CONTINUED

351439098263401. LOCAL NUMBER; O9N-12W-23 BAC 1. LOCATION,--LAT 35 14'39", LONG 098 26'34", HYDROLOGIC UNIT 11130302. AQUIFER.--RUSH SPRINGS FORMATION. PERIOD OF RECORD.--1974 TO CURRENT YEAR. EXTREMES FOR PERIOD OF RECORD.--HIGHEST WATER LEVEL, $72.90 \mathrm{FT}(22.22 \mathrm{M})$ BELOW LAND-SURFACE DATUM,MAR 8, 1978; LOWEST WATER LEVEL,80.50 FT(24.54 M) BELOW LAND-SURFACE DATUM, JAN 29, 1981.

WATER LEVELS IN FEET BELOW LAND SURFACE DATUM, CLIMATIC YEARS APRIL 1981 TO MARCH 1983

$\begin{array}{lll} & \text { WATER } & \text { WATER } \\ \text { DATE } & \text { LEVEL } & \text { DATE }\end{array}$

MAR 22, $1982 \quad 80.30 \quad$ FEB 23, $1983 \quad 80.39$

GROUND-WATER LEVELS

351623098312001. LOCAL NUMBER; 09N-13W-12 AAC 1. LOCATION,--LAT 35 16'23", LONG 098 31'20", HYDROLOGIC UNIT 11130302. AQUIFER.--RUSH SPRINGS FORMATION. PERIOD OF RECORD.--1974 TO CURRENT YEAR.

EXTREMES FOR PERIOD OF RECORD.--HIGHEST WATER LEVEL, 36.95 FT(11.26 M) BELOW LAND-SURFACE DATUM,DEC 3, 1975; LOWEST WATER LEVEL,47.60 FT(14.51 M) BELOW LAND-SURFACE DATUM, MAR 22, 1982.

WATER LEVELS IN FEET BELOW LAND SURFACE DATUM, CLIMATIC YEARS APRIL 1981 TO MARCH 1983

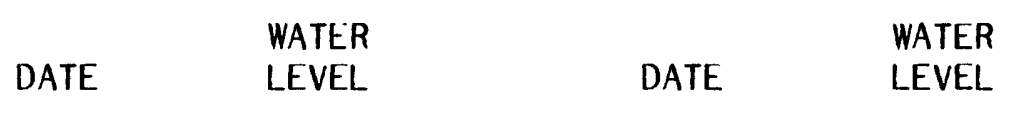

MAR 22, $1982 \quad 47.60 \quad$ FEB 25, $1983 \quad 46.72$ 


\section{GROUND-WATER LEVELS}

CADDO COUNTY -- CONTINUED

351302098351501. LOCAL NUMBER;

09N-13W-33 BBB 1.

LOCATION,--LAT 35 13'02", LONG 098

35'15", HYDROLOGIC UNIT 11130302.

AQUIFER.--RUSH SPRINGS FORMATION.

PERIOD OF RECORD.--1974 TO CURRENT YEAR.

EXTREMES FOR PERIOD OF RECORD.--HIGHEST WATER LEVEL, $31.00 \mathrm{FT}(9.45 \mathrm{M})$ BELOW LAND-SURFACE DATUM,DEC 3, 1975; LOWEST WATER LEVEL, $37.25 \mathrm{FT}(11.35 \mathrm{M})$ BELOW LAND-SURFACE DATUM, JAN 30, 1981.

WATER LEVELS IN FEET BELOW LAND SURFACE DATUM, CLIMATIC YEARS APRIL 1981 TO MARCH 1983

\begin{tabular}{|c|c|c|}
\hline & WATER & \\
\hline DATE & LEVEL & DATE \\
\hline
\end{tabular}

MAR 22, $1982 \quad 37.07 \quad$ FEB 24, $1983 \quad 36.23$

\section{GROUND-WATER LEVELS}

352225098274001. LOCAL NUMBER;

10N-12W-03 BBD 1 .

LOCATION,--LAT 35 22'25", LONG 098 27'40", HYDROLOGIC UNIT 11130302.

AQUIFER.--RUSH SPRINGS FORMATION.

PERIOD OF RECORD.--1974 TO CURRENT YEAR.

EXTREMES FOR PERIOD OF RECORD.--HIGHEST WATER LEVEL, 124.95 FT(38.08 M) BELOW LAND-SURFACE DATUM,MAR 7, 1979; LOWEST WATER LEVEL,136.99 FT(41.75 M) BELOW LAND-SURFACE DATUM, MAR 8, 1983.

WATER LEVELS IN FEET BELOW LAND SURFACE DATUM, CLIMATIC YEARS APRIL 1981 TO MARCH 1983

\begin{tabular}{|c|c|c|}
\hline & WATER & \\
\hline DATE & LEVEL & DATE \\
\hline
\end{tabular}

MAR 25, $1982 \quad 135.85 \quad$ MAR 08, $1983 \quad 136.99$ 
CADDO COUNTY -- CONTINUED

352229098300901. LOCAL NUMBER; LOCATION,--LAT 35 22'29", LONG 098 AQUIFER.--RUSH SPRINGS FORMATION. PERIOD OF RECORD.-- 1974 TO CURRENT EXTREMES FOR PERIOD OF RECORD.--HIGHEST WATER LEVEL, 79.54 FT (24.24 M) BELOW LAND-SURFACE DATUM,FEB 9, 1976; LOWEST WATER LEVEL,83.22 FT(25.37 M) BELOW LAND-SURFACE DATUM, MAR 25, 1982.

WATER LEVELS IN FEET BELOW LAND SURFACE DATUM, CLIMATIC YEARS APRIL 1981 TO MARCH 1983
MAR 25, 1982
83.22
MAR 08, 1983
83.18

\section{GROUND-WATER LEVELS}

352107098273801. LOCAL NUMBER;

$10 \mathrm{~N}-12 \mathrm{~W}-10 \mathrm{CCA} 1$. LOCATION,--LAT 35 21'07", LONG 098 27'38"., HYDROLOGIC UNIT 11130302.

AQUIFER.--RUSH SPRINGS FORMATION.

PERIOD OF RECORD.--1974 TO CURRENT YEAR.

EXTREMES FOR PERIOD OF RECORD.--HIGHEST WATER LEVEL, 79.54 FT(24.24 M) BELOW LAND-SURFACE DATUM,FEB 9, 1976; LOWEST WATER LEVEL,112.92 FT(34.42 M) BELOW LAND-SURFACE DATUM, MAR 8, 1983.

WATER LEVELS IN FEET BELOW LAND SURFACE DATUM, CLIMATIC YEARS APRIL 1981 TO MARCH 1983

$\begin{array}{lll} & \text { WATER } & \text { WATER } \\ \text { DATE } & \text { LEVEL } & \text { DATE }\end{array}$

MAR 23, $1982 \quad 111.00 \quad$ MAR 08, $1983 \quad 112.92$ 


\section{GROUND-WATER LEVELS}

CADDO COUNTY -- CONTINUED

352015098253001. LOCAL NUMBER;

LOCATION,--LAT $3520^{\prime} 15^{\prime \prime}$, LONG 098 AQUIFER.--RUSH SPRINGS FORMATION.

PERIOD OF RECORD. - 1974 TO CURRENT

EXTREMES FOR PERIOD OF RECORD.--HIGHEST WATER LEVEL,62.77 FT(19.13 M) BELOW LAND-SURFACE DATUM,DEC 1, 1975; LOWEST WATER

FEB 25, 1983.

WATER LEVELS IN FEET BELOW LAND SURFACE DATUM, CLIMATIC YEARS APRIL 1981 TO MARCH 1983
$10 \mathrm{~N}-12 \mathrm{~W}-13 \mathrm{CCA} 1$

$25^{\prime} 30^{\prime \prime}$, HYDROLOGIC UNIT 11130302. LEVEL, 69.70 FT (21.24 M) BELOW LAND-SURFACE DATUM,

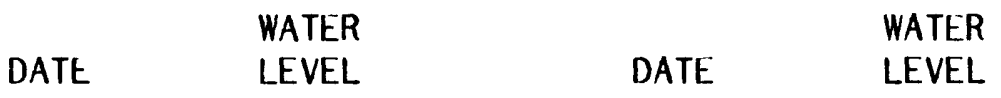

$\begin{array}{llll}\text { MAR } 23,1982 & 68.29 & \text { FEB } 25,1983 \quad 69.70\end{array}$

GROUND-WATER LEVELS

352017098263301. LOCAL NUMBER; 10N-12W-14 CBD 1.

LOCATION,--LAT 35 20'17", LONG 098 26'33", HYDROLOGIC UNIT 11130302.

AQUIFER.--RUSH SPRINGS FORMATION.

PERIOD OF RECORD.--1974 TO CURRENT YEAR.

EXTREMES FOR PERIOD OF RECORD.--HIGHEST WATER LEVEL,88.39 FT(26.94 M) BELOW LAND-SURFACE DATUM,DEC 18, 1974; LOWEST WATER LEVEL,103.91 FT(31.67 M) BELOW LAND-SURFACE DATUM, FEB 25, 1983.

WATER LEVELS IN FEET BELOW LAND SURFACE DATUM, CLIMATIC YEARS APRIL 1981 TO MARCH 1983

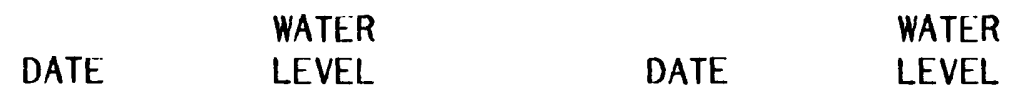

MAR 23, $1982 \quad 97.65 \quad$ FEB $25,1983 \quad 103.91$ 
CADDO COUNTY -- CONTINUED

352046098285901. LOCAL NUMBER; LOCATION,--LAT 35 20'46", LONG 098 AQUIFER.--RUSH SPRINGS FORMATION. PERIOD OF RECORD .--1974 TO CURRENT EXTREMES FOR PERIOD OF RECORD.--HIGHEST WATER LEVEL, 82.25 FT(25.07 M) BELOW LAND-SURFACE DATUM,FEB 9, 1977; LOWEST WATER LEVEL,91.87 FT(28.00 M) BELOW LAND-SURFACE DATUM, FEB 23, 1983.

WATER LEVELS IN FEET BELOW LAND SURFACE DATUM, CLIMATIC YEARS APRIL 1981 TO MARCH 1983
10N-12W-17 AAD 1.

28'59", HYDROLOGIC UNIT 11130302.
YEAR.

\begin{tabular}{|c|c|c|}
\hline & WATER & \\
\hline DATE & LEVEL & DATE \\
\hline
\end{tabular}

MAR 23, $1982 \quad 91.10 \quad$ FEB 23, $1983 \quad 91.87$

GROUND-WATER LEVELS

351923098291301. LOCAL NUMBER; LOCATION,--LAT $3519^{\prime} 23^{\prime \prime}$, LONG 098 AQUIFER.--RUSH SPRINGS FORMATION.

PERIOD OF RECORD.--1974 TO CURRENT YEAR. EXTREMES FOR PERIOD OF RECORD.--HIGHEST WATER LEVEL, $79.58 \mathrm{FT}(24.26 \mathrm{M})$ BELOW LAND-SURFACE DATUM, JAN 1, 1975; LOWEST WATER LEVEL,99.10 FT(30.21 M) BELOW LAND-SURFACE DATUM, MAR 22, 1982. WATER LEVELS IN FEET BELOW LAND SURFACE DATUM, CLIMATIC YEARS APRIL 1981 TO MARCH 1983
10N-12W-20 DAC 1 .

29'13", HYDROLOGIC UNIT 11130302.

$\begin{array}{lll} & \text { WATER } & \\ \text { DATE } & \text { LEVEL } & \text { WATER } \\ & \text { LEVEL }\end{array}$

$\begin{array}{llll}\text { MAR 22, } 1982 \quad 99.10 & \text { FEB 25, } 1983 \quad 89.32\end{array}$ 
GROUND-WATER LEVELS

CADDO COUNTY -- CONTINUED

351950098263601. LOCAL NUMBER; LOCATION,--LAT $3519^{\prime} 50^{\prime \prime}$, LONG 098 AQUIFER.--RUSH SPRINGS FORMATION.

PERIOD OF RECORD.--1978 TO CURRENT

EXTREMES FOR PERIOD OF RECORD.--HIGHEST WATER LEVEL, 84.35 FT(25.71 M) BELOW LAND-SURFACE JAN 29, 1981.

WATER LEVELS IN FEET BELOW LAND SURFACE DATUM, CLIMATIC YEARS APRIL 1981 TO MARCH 1983

$10 \mathrm{~N}-12 \mathrm{~W}-23$ BBD 1.

26'36", HYDROLOGIC UNIT 11130302.
GROUND-WATER LEVELS

351730098300301. LOCAL NUMBER;

$10 \mathrm{~N}-12 \mathrm{~W}-31$ DDB 2 .

LOCATION,--LAT $3517^{\prime} 30^{\prime \prime}$, LONG 098 30'03", HYDROLOGIC UNIT 11130302.

AQUIFER.--RUSH SPRINGS FORMATION.

PERIOD OF RECORD.--1974 TO CURRENT YEAR.

EXTREMES FOR PERIOD OF RECORD.--HIGHEST WATER LEVEL, 71.85 FT(21.90 M) BELOW LAND-SURFACE DATUM,FEB 9, 1977; LOWEST WATER LEVEL,89.45 FT(27.26 M) BELOW LAND-SURFACE DATUM, MAR 24, 1982 .

WATER LEVELS IN FEET BELOW LAND SURFACE DATUM, CLIMATIC YEARS APRIL 1981 TO MARCH 1983
MAR 24, 1982
89.45
FEB 23, 1983
83.56

WATER

LEVEL 
CADDO COUNTY -- CONTINUED

351805098281201. LOCAL NUMBER; LOCATION, --LAT 35 18'05", LONG 098 AQUIFER.--RUSH SPRINGS FORMATION. PERIOD OF RECORD.--1974 TO CURRENT EXTREMES FOR PERIOD OF RECORD.--HIGHEST WATER LEVEL,91.74 FT(27.96 M) BELOW LAND-SURFACE FEBB 24, 1983.

WATER LEVELS IN FEET BELOW LAND SURFACE DATUM, CLIMATIC YEARS APRIL 1981 TO MARCH 1983
10N-12W-33 ABD 1. 28 '12", HYDROLOGIC UNIT 11130302.

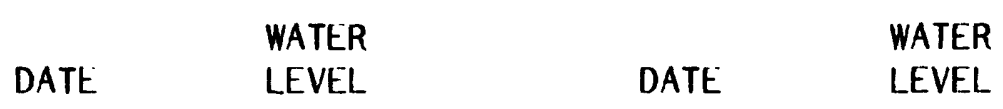

MAR 22, $1982 \quad 99.40 \quad$ FEB 24, $1983 \quad 99.92$

\section{GROUND-WATER LEVELS}

351755098265801. LOCAL NUMBER; 10N-12W-34 ADD 1.

LOCATION,--LAT 35 17'55", LONG 098 26'58", HYDROLOGIC UNIT 11130302.

AQUIFER.--RUSH SPRINGS FORMATION.

PERIOD OF RECORD.--1974 TO CURRENT YEAR.

EXTREMES FOR PERIOD OF RECORD.--HIGHEST WATER LEVEL, 58.27 FT(17.76 M) BELOW LAND-SURFACE DATUM,DEC 1, 1975; LOWEST WATER LEVEL,70.66 FT(21.54 M) BELOW LAND-SURFACE DATUM, MAR 22, 1982.

WATER LEVELS IN FEET BELOW LAND SURFACE DATUM, CLIMATIC YEARS APRIL 1981 TO MARCH 1983

\begin{tabular}{|c|c|c|}
\hline & WATER & \\
\hline DATE & LEVEL & DATE \\
\hline
\end{tabular}

MAR 22, $1982 \quad 70.66 \quad$ FEB 23, $1983 \quad 66.20$ 
GROUND-WATER LEVELS

CADDO COUNTY -- CONTINUED

351748098264201. LOCAL NUMBER; LOCATION,--LAT 3517 '48", LONG 098 AQUIFER.--RUSH SPRINGS FORMATION. PERIOD OF RECORD. --1974 TO CURREN EXTREMES FOR PERIOD OF RECORD.--HIGHEST WATER LEVEL, $34.18 \mathrm{FT}(10.42 \mathrm{M})$ BELOW LAND-SURFACE MAR 22, 1982.

WATER LEVELS IN FEET BELOW LAND SURFACE DATUM, CLIMATIC YEARS APRIL 1981 TO MARCH 1983

10N-12W-35 CBA 1. 26 '42", HYDROLOGIC UNIT 11130302.

\section{DATE}

WATER

LEVEL

DATE

WATER

LEVEL

MAR 22, $1982 \quad 42.83$
41.64

\section{GROUND-WATER LEVELS}

352203098322401. LOCAL NUMBER; LOCATION,--LAT 35 22'03", LONG 098 AQUIFER.--RUSH SPRINGS FORMATION. PERIOD OF RECORD.--1975 TO CURRENT EXTREMES FOR PERIOD OF RECORD.--HIGHEST WATER LEVEL, $72.40 \mathrm{FT}(22.07 \mathrm{M})$ BELOW LAND-SURFACE DATUM,MAR 9, 1978; LOWEST WATER LEVEL,81.18 FT(24.74 M) BELOW LAND-SURFACE DATUM, MAR 8, 1983.

WATER LEVELS IN FEET BELOW LAND SURFACE DATUM, CLIMATIC YEARS APRIL 1981 TO MARCH 1983
10N-13W-02 DAC 1 . 32 '24", HYDROLOGIC UNIT 11130302.

WATER LEVELS I

WATER

DATE LEVEL

MAR 23, $1982 \quad 80.36$ $\begin{array}{ll} & \text { WATER } \\ \text { DATE } & \text { LEVEL }\end{array}$

MAR $08,1983 \quad 81.18$ 
GROUND-WATER LEVELS

CADDO COUNTY -- CONTINUED

351952098332801. LOCAL NUMBER; LOCATION,--LAT 35 19'52", LONG 098 AQUIFER.--RUSH SPRINGS FORMATION. PERIOD OF RECORD.--1975 TO CURRENT EXTREMES FOR PERIOD OF RECORD.--HIGHEST WATER LEVEL,60.61 FT(18.47 M) BELOW LAND-SURFACE DATUM,DEC 1, 1975; LOWEST WATER LEVEL,71.27 FT(21.72 M) BELOW LAND-SURFACE DATUM, FEB 24, 1983.

WATER LEVELS IN FEET BELOW LAND SURFACE DATUM, CLIMATIC YEARS APRIL 1981 TO MARCH 1983
10N-13W-22 AAC 1. 33'28", HYDROLOGIC UNIT 11130302.

GROUND-WATER LEVELS

352715098234901. LOCAL NUMBER;

$11 \mathrm{~N}-11 \mathrm{~W}-06 \mathrm{DAC} 1$

LOCATION,--LAT 35 27'15", LONG 098 23'49", HYDROLOGIC UNIT 11130302. AQUIFER ---RUSH SPRINGS FORMATION.

PERIOD OF RECORD.--1982 TO CURRENT YEAR.

EXTREMES FOR PERIOD OF RECORD.--HIGHEST WATER LEVEL, 109.12 FT(33.26 M) BELOW LAND-SURFACE DATUM, MAR 8, 1983; LOWEST WATER LEVEL, $109.12 \mathrm{FT}(33.26 \mathrm{M})$ BELOW LAND-SURFACE DATUM, MAR 8, 1983.

WATER LEVELS IN FEET BELOW LAND SURFACE DATUM, CLIMATIC YEARS APRIL 1981 TO MARCH 1983

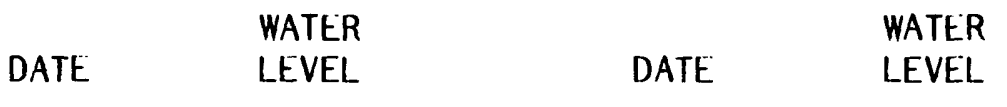

$\begin{array}{llll}\text { MAR 22, } 1982 & \mathrm{Z} & \text { MAR } 08,1983 & 109.12\end{array}$ 
352308098241101. LOCAL NUMBER; LOCATION,--LAT 35 23'08", LONG 098 AQUIFER.--RUSH SPRINGS FORMATION.

PERIOD OF RECORD.--1981 TO CURRENT

EXTREMES FOR PERIOD OF RECORD.--HIGHEST WATER LEVEL,89.05 FT(27.14 M) BELOW LAND-SURFACE

DATUM, MAR 8, 1983; LOWEST WATER LEVEL,92.93 FT(28.33 M) BELOW LAND-SURFACE DATUM, JAN 27, 1981.

WATER LEVELS IN FEET BELOW LAND SURFACE DATUM, CLIMATIC YEARS APRIL 1981 TO MARCH 1983
$11 \mathrm{~N}-11 \mathrm{~W}-31$ ACC 1 24'11", HYDROLOGIC UNIT 11130302.

\section{YEAR.}

$\begin{array}{lll} & \text { WATER } & \\ \text { DATE } & \text { LEVEL } & \text { WATER } \\ & \text { DATE } & \text { LEVEL }\end{array}$

MAR 25, $198290.18 \quad$ MAR 08, $1983 \quad 89.05$

GROUND-WATER LEVELS

352316098284101. LOCAL NUMBER; LOCATION,--LAT 35 23'16", LONG 098 AQUIFER.--RUSH SPRINGS FORMATION. PERIOD OF RECORD.--1974 TO CURRENT YEAR. EXTREMES FOR PERIOD OF RECORD.--HIGHEST WATER LEVEL, $130.58 \mathrm{FT}(39.80 \mathrm{M})$ BELOW LAND-SURFACE DATUM,FEB 9, 1977; LOWEST WATER LEVEL,135.66 FT(41.35 M) BELOW LAND-SURFACE DATUM, JAN 27, 1981.

WATER LEVELS IN FEET BELOW LAND SURFACE DATUM, CLIMATIC YEARS APRIL 1981 TO MARCH 1983
MAR 25, 1982
134.80
MAR 08, $1983 \quad 134.97$ 
CADDO COUNTY -- CONTINUED

352515098263001. LOCAL NUMBER; 11N-12W-14 CDC 1. LOCATION,--LAT 35 25'15", LONG 098 26'30", HYDROLOGIC UNIT 11130302. AQUIFER.--RUSH SPRINGS FORMATION. PERIOD OF RECORD.--1974 TO CURRENT YEAR. EXTREMES FOR PERIOD OF RECORD.--HIGHEST WATER LEVEL,78.69 FT(23.98 M) BELOW LAND-SURFACE DATUM,FEB 9, 1977; LOWEST WATER LEVEL,94.44 FT(28.79 M) BELOW LAND-SURFACE DATUM, MAR 7, 1979.

WATER LEVELS IN FEET BELOW LAND SURFACE DATUM, CLIMATIC YEARS APRIL 1981 TO MARCH 1983

\begin{tabular}{|c|c|c|}
\hline & WATER & \\
\hline DATE & LEVEL & DATE \\
\hline
\end{tabular}

MAR 25, $1982 \quad 85.32 \quad$ MAR 08, $1983 \quad 85.87$

GROUND-WATER LEVELS

352321098281001. LOCAL NUMBER; 11N-12W-33 AAC 1. LOCATION,--LAT 35 23'21", LONG 098 28'10", HYDROLOGIC UNIT 11130302. AQUIFER.--RUSH SPRINGS FORMATION. PERIOD OF RECORD.--1974 TO CURRENT YEAR.

EXTREMES FOR PERIOD OF RECORD.--HIGHEST WATER LEVEL, 127.88 FT(38.98 M) BELOW LAND-SURFAC DATUM,FEB 9, 1977; LOWEST WATER LEVEL, 137.31 FT(41.85 M) BELOW LAND-SURFACE DATUM, MAR 8, 1983.

WATER LEVELS IN FEET BELOW LAND SURFACE DATUM, CLIMATIC YEARS APRIL 1981 T0 MARCH 1983

$\begin{array}{lll} & \text { WATER } & \\ \text { DATE } & \text { LEVEL } & \text { WATER } \\ & \text { LEVEL }\end{array}$

MAR 25, $1982 \quad 135.73 \quad$ MAR 08, $1983 \quad 137.31$ 
GROUND-WATER LEVELS

CADDO COUNTY -- CONTINUED

352551098343901. LOCAL NUMBER; 11N-13W-16 ACA 1.

LOCATION,--LAT 35 25'51", LONG 098 34'39", HYDROLOGIC UNIT 11130302.

AQUIFER.--RUSH SPRINGS FORMATION.

PERIOD OF RECORD.--1974 TO CURRENT YEAR.

EXTREMES FOR PERIOD OF RECORD.--HIGHEST WATER LEVEL,88.13 FT(26.86 M) BELOW LAND-SURFACE DATUM,DEC 2, 1975; LOWEST WATER LEVEL,91.30 FT(27.83 M) BELOW LAND-SURFACE DATUM, JAN $27,1981$.

WATER LEVELS IN FEET BELOW LAND SURFACE DATUM, CLIMATIC YEARS APRIL 1981 TO MARCH 1983

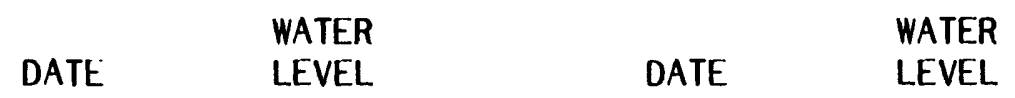

MAR 25, $1982 \quad 90.67 \quad$ MAR 04, $1983 \quad 89.80$

GROUND-WATER LEVELS

353012098225901. LOCAL NUMBER; 12N-11W-20 ACA 1.

LOCATION,--LAT 35 30'12", LONG 098 22'59", HYDROLOGIC UNIT 11090202.

AQUIFER.--RUSH SPRINGS FORMATION.

PERIOD OF RECORD.--1981 TO CURRENT YEAR.

EXTREMES FOR PERIOD OF RECORD.--HIGHEST WATER LEVEL, 111.28 FT(33.92 M) BELOW LAND-SURFACE DATUM,MAR 8, 1983; LOWEST WATER LEVEL,112.69 FT(34.35 M) BELOW LAND-SURFACE DATUM, JAN 27, 1981.

WATER LEVELS IN FEET BELOW LAND SURFACE DATUM, CLIMATIC YEARS APRIL 1981 TO MARCH 1983

\begin{tabular}{|c|c|}
\hline & WATER \\
\hline DATE & LEVEL \\
\hline
\end{tabular}

MAR 25, $1982 \quad 111.95 \quad$ MAR 08, $1983 \quad 111.28$ 
CADDO COUNTY - CONTINUED

352922098225801. LOCAL NUMBER; LOCATION,--LAT 35 29'22", LONG 098 AQUIFER.--RUSH SPRINGS FORMATION. PERIOD OF RECORD.--1981 TO CURRENT EXTREMES FOR PERIOD OF RECORD.- - HIGHEST WATER LEVEL, 109.31 FT(33.32 M) BELOW LAND-SURFACE

DATUM,MAR 8, 1983; LOWEST WATER LEVEL, $110.70 \mathrm{FT}(33.74 \mathrm{M})$ BELOW LAND-SURFACE DATUM, MAR 25, 1982.

WATER LEVELS IN FEET BELOW LAND SURFACE DATUM, CLIMATIC YEARS APRIL 1981 TO MARCH 1983
12N-11W-29 AAA 1. 22 '58", HYDROLOGIC UNIT 11090202.

\section{YEAR.}

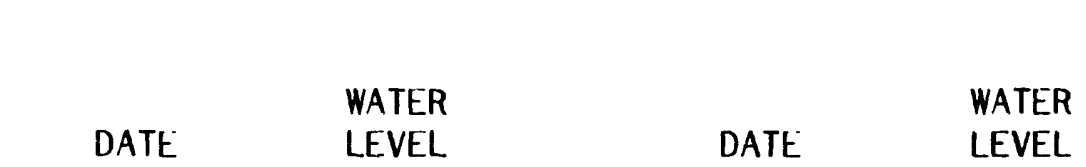

MAR $25,1982 \quad 110.70 \quad$ MAR 08, $1983 \quad 109.31$

GROUND-WATER LEVELS

353137098265801. LOCAL NUMBER; 12N-12W-10 DAD 1

LOCATION,--LAT 35 31'37", LONG 098 26'58", HYDROLOGIC UNIT 11090201.

AQUIFER.--RUSH SPRINGS FORMATION.

PERIOD OF RECORD.--1974 TO CURRENT YEAR.

EXTREMES FOR PERIOD OF RECORD.--HIGHEST WATER LEVEL, $62.00 \mathrm{FT}(18.90 \mathrm{M})$ BELOW LAND-SURFACE DATUM,DEC 2, 1975; LOWEST WATER LEVEL,66.67 FT(20.32 M) BELOW LAND-SURFACE DATUM, DEC 17, 1974.

WATER LEVELS IN FEET BELOW LAND SURFACE DATUM, CLIMATIC YEARS APRIL 1981 TO MARCH 1983

$\begin{array}{lll} & \text { WATER } & \text { WATER } \\ \text { DATE } & \text { LEVEL } & \text { DATE }\end{array}$

MAR 25, $1982 \quad 0 \quad$ MAR 04, $1983 \quad 63.02$ 


\section{GROUND-WATER LEVELS}

CADDO COUNTY -- CONTINUED

352924098335601. LOCAL NUMBER; LOCATION,--LAT 35 29'24", LONG 098 AQUIFER.--RUSH SPRINGS FORMATION. PERIOD OF RECORD.--1974 TO CURRENT EXTREMES FOR PERIOD OF RECORD.--HIGHEST WATER LEVEL, $64.80 \mathrm{FT}(19.75 \mathrm{M})$ BELOW LAND-SURFACE DATUM,DEC 17, 1974; LOWEST WATER LEVEL,67.77 FT(20.66 M) BELOW LAND-SURFACE DATUM, MAR 25, 1982. WATER LEVELS IN FEET BELOW LAND SURFACE DATUM, CLIMATIC YEARS APRIL 1981 TO MARCH 1983
$12 \mathrm{~N}-13 \mathrm{~W}-27 \mathrm{BAC} 1$ $33^{\prime} 56^{\prime \prime}$, HYDROLOGIC UNIT 11090201.

YEAR
WATER

DATE

MAR 25, 1982
LEVEL

67.77

DATE LEVEL

MAR 04, $1983 \quad 67.31$ 
GROUND-WATER LEVELS

\section{CANADIAN COUNTY}

353130097415501. LOCAL NUMBER;

$12 \mathrm{~N}-05 \mathrm{~W}-11$ CDA 1

LOCATION,--LAT 3531 '30", LONG 097

41'55", HYDROLOGIC UNIT 11100301.

AQUIFER.--ALLUVIUM.

PERIOD OF RECORD. - -1977 TO CURRENT YEAR.

EXTREMES FOR PERIOD OF RECORD.--HIGHEST WATER LEVEL,6.08 FT(1.85 M) BELOW LAND-SURFACE DATUM, MAR 14, 1983; LOWEST WATER LEVEL,11.34 FT(3.46 M) BELOW LAND-SURFACE DATUM, MAR 15, 1977.

WATER LEVELS IN FEET BELOW LAND SURFACE DATUM, CLIMATIC YEARS APRIL 1981 TO MARCH 1983

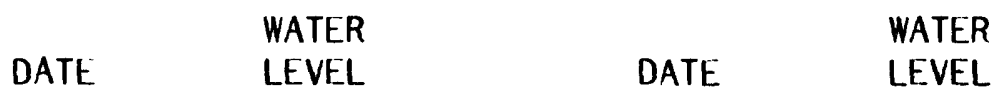

MAR $31,1982 \quad 7.85 \quad$ MAR $14,1983 \quad 6.08$

GROUND-WATER LEVELS

353105097454001. LOCAL NUMBER; 12N-05W-18 ADA 1.

LOCATION,--LAT $3531^{\prime}$ 'S", LONG 097 45'40", HYDROLOGIC UNIT 11100301.

AQUIFER.--ALLUVIUM.

PERIOD OF RECORD.--1977 TO CURRENT YEAR.

EXTREMES FOR PERIOD OF RECORD.--HIGHEST WATER LEVEL, $10.83 \mathrm{FT}(3.30 \mathrm{M})$ BELOW LAND-SURFACE DATUM,MAR 19, 1983; LOWEST WATER LEVEL,18.48 FT(5.63 M) BELOW LAND-SURFACE DATUM, MAR 13, 1978.

WATER LEVELS IN FEET BELOW LAND SURFACE DATUM, CLIMATIC YEARS APRIL 1981 TO MARCH 1983

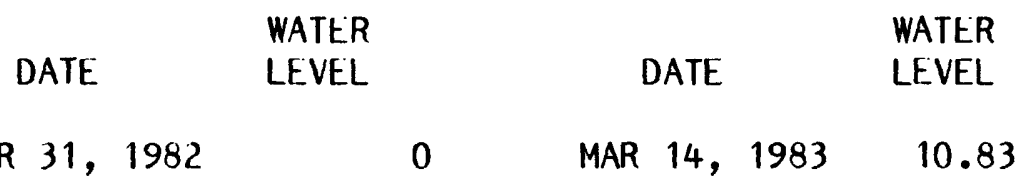


CANADIAN COUNTY -- CONTINUED

353210097485601. LOCAL NUMBER; 12N-06W-10 AAB 1. LOCATION,--LAT 35 32'10", LONG 097 48'56", HYDROLOGIC UNIT 11100301. AQUIFER.--ALLUVIUM.

PERIOD OF RECORD.--1978 TO CURRENT YEAR.

EXTREMES FOR PERIOD OF RECORD.--HIGHEST WATER LEVEL, $15.31 \mathrm{FT}(4.67 \mathrm{M})$ BELOW LAND-SURFACE DATUM, MAR 14, 1983; LOWEST WATER LEVEL, 16.80 FT(5.12 M) BELOW LAND-SURFACE DATUM, FEB 20, 1979 .

WATER LEVELS IN FEET BELOW LAND SURFACE DATUM, CLIMATIC YEARS APRIL 1981 TO MARCH 1983

$\begin{array}{lll} & \text { WATER } & \text { WATER } \\ \text { DATE } & \text { LEVEL } & \text { DATE }\end{array}$
MAR 31, 1982
16.42
MAR 14, $1983 \quad 15.31$

GROUND-WATER LEVELS

353236097551801. LOCAL NUMBER; 12N-07W-03 DAB 1. LOCATION,--LAT 35 32'36", LONG 097 55'18", HYDROLOGIC UNIT 11100301. AQUIFER .--ALLUVIUM.

PERIOD OF RECORD.--1978 TO CURRENT YEAR. EXTREMES FOR PERIOD OF RECORD.--HIGHEST WATER LEVEL, $18.62 \mathrm{FT}(5.68 \mathrm{M})$ BELOW LAND-SURFACE DATUM, MAR 14, 1983; LOWEST WATER LEVEL,20.83 FT(6.35 M) BELOW LAND-SURFACE DATUM, JAN 26, 1981.

WATER LEVELS IN FEET BELOW LAND SURFACE DATUM, CLIMATIC YEARS APRIL 1981 TO MARCH 1983

\begin{tabular}{|c|c|c|}
\hline & $\begin{array}{l}\text { WATER } \\
\text { IFVFI }\end{array}$ & \\
\hline DAIE & & DATE \\
\hline
\end{tabular}

MAR $31,1982 \quad 19.18 \quad$ MAR $14,1983 \quad 18.62$ 


\section{GROUND-WATER LEVELS}

CANADIAN COUNTY -- CONTINUED

353315097521001. LOCAL NUMBER; LOCATION,--LAT $3533^{\prime} 15^{\prime \prime}$, LONG 097 AQUIFER.--ALLUVIUM.

PERIOD OF RECORD.--1977 TO CURRENT YEAR.

EXTREMES FOR PERIOD OF RECORD.--HIGHEST WATER LEVEL, $10.69 \mathrm{FT}(3.26 \mathrm{M})$ BELOW LAND-SURFACE DATUM,MAR 14, 1983; LOWEST WATER LEVEL, 14.65 FT(4.47 M) BELOW LAND-SURFACE DATUM, FEB 20, 1979.

WATER LEVELS IN FEET BELOW LAND SURFACE DATUM, CLIMATIC YEARS APRIL 1981 TO MARCH 1983

$\begin{array}{lcccc}\text { DATE } & \begin{array}{c}\text { WATER } \\ \text { LEVEL }\end{array} & \text { DATE } & \text { WATER } \\ \text { LEVEL }\end{array}$

\section{GROUND-WATER LEVELS}

353446098033701. LOCAL NUMBER; 13N-08W-28 BBA 1. LOCATION,--LAT 35 34'46", LONG 098 03'37", HYDROLOGIC UNIT 11100301. AQUIFER.--ALLUVIUM.

PERIOD OF RECORD.--1977 TO CURRENT YEAR.

EXTREMES FOR PERIOD OF RECORD.--HIGHEST WATER LEVEL, $12.16 \mathrm{FT}(3.71 \mathrm{M})$ BELOW LAND-SURFACE DATUM,FEB 22, 1980; LOWEST WATER LEVEL, 16.49 FT(5.03 M) BELOW LAND-SURFACE DATUM, MAR 31, 1982.

WATER LEVELS IN FEET BELOW LAND SURFACE DATUM, CLIMATIC YEARS APRIL 1981 TO MARCH 1983

\begin{tabular}{|c|c|}
\hline & WATER \\
\hline DATE & LEVEL \\
\hline
\end{tabular}

MAR $31,1982 \quad 16.49 \quad$ MAR 14, $1983 \quad 14.35$ 
GROUND-WATER LEVELS

CANADIAN COUNTY -- CONTINUED

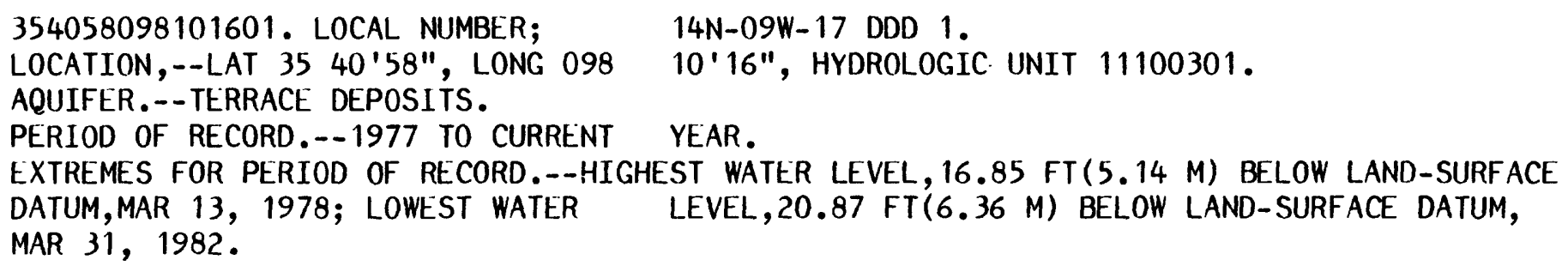

WATER LEVELS IN FEET BELOW LAND SURFACE DATUM, CLIMATIC YEARS APRIL 1981 TO MARCH 1983

\begin{tabular}{cccc}
\multicolumn{1}{c}{ WATER } & & \multicolumn{1}{c}{ WATER } \\
DATE & LEVEL & DATE & LEVEL \\
MAR 31, 1982 & 20.87 & MAR 14, 1983 & 18.55
\end{tabular}

139 


\section{GROUND-WATER LEVELS}

CARTER COUNTY

341526097165101. LOCAL NUMBER;

03S-01W-35 BBC 1.

LOCATION,--LAT 34 15'26", LONG 097

16 '51", HYDROLOGIC UNIT 11130303.

AQUIFER.--WICHITA FORMATION.

PERIOD OF RECORD. - 1976 TO CURRENT YEAR.

EXTREMES FOR PERIOD OF RECORD.--HIGHEST WATER LEVEL,21.14 FT(6.44 M) BELOW LAND-SURFACE DATUM, JAN 24, 1980; LOWEST WATER LEVEL,43.16 FT(13.16 M) BELOW LAND-SURFACE DATUM, JAN 4, 1977.

WATER LEVELS IN FEET BELOW LAND SURFACE DATUM, CLIMATIC YEARS APRIL 1981 TO MARCH 1983

\begin{tabular}{cccc}
\multicolumn{1}{c}{ WATER } & & WATER \\
DATE & LEVEL & DATE & LEVEL \\
MAR 08, 1982 & 27.72 & MAR 07, 1983 & 27.58
\end{tabular}


GROUND-WATER LEVELS

CHEROKEE COUNTY

355920095111001. LOCAL NUMBER; 18N-20E-33 CDD 1. LOCATION,--LAT 35 59'20", LONG 095 11'10", HYDROLOGIC UNIT 11070209.

AQUIFER.--SAVANNA FORMATION.

PERIOD OF RECORD.--1979 TO CURRENT YEAR.

EXTREMES FOR PERIOD OF RECORD.--HIGHEST WATER LEVEL,1.99 FT(.61 M) BELOW LAND-SURFACE DATUM,FEB 26, 1980; LOWEST WATER LEVEL, 17.15 FT(5.23 M) BELOW LAND-SURFACE DATUM, MAR 9, 1981.

WATER LEVELS IN FEET BELOW LAND SURFACE DATUM, CLIMATIC YEARS APRIL 1981 TO MARCH 1983

\begin{tabular}{|c|c|c|}
\hline & WATER & \\
\hline DATE & LEVEL & DATE \\
\hline
\end{tabular}

MAR 29, $1982 \quad 10.83 \quad$ MAR 08, $1983 \quad 9.05$

GROUND-WATER LEVELS

360151094594501. LOCAL NUMBER; 18N-22E-17 CCA 1.

LOCATION,--LAT 3601 '51", LONG 094 59'45", HYDROLOGIC UNIT 11070209.

AQUIFER.--BOONE FORMATION.

PERIOD OF RECORD.--1981 TO CURRENT YEAR.

EXTREMES FOR PERIOD OF RECORD.--HIGHEST WATER LEVEL, 40.28 FT(12.28 M) BELOW LAND-SURFACE DATUM,MAR 8, 1983; LOWEST WATER LEVEL,53.13 FT(16.19 M) BELOW LAND-SURFACE DATUM, MAR 11, 1981.

WATER LEVELS IN FEET BELOW LAND SURFACE DATUM, CLIMATIC YEARS APRIL 1981 TO MARCH 1983

\begin{tabular}{|c|c|c|}
\hline & WATER & \\
\hline DATE & LEVEL & DATE \\
\hline
\end{tabular}

MAR $30,1982 \quad 45.46 \quad$ MAR 08, $1983 \quad 40.28$ 


\section{GROUND-WATER LEVELS}

CHOCTAW COUNTY

340201095531501. LOCAL NUMBER;

06S-14E-18 CBB 1.

LOCATION,--LAT 3402 '01", LONG 095

53'15", HYDROLOGIC UNIT 11140104.

AQUIFER.--ANTLERS SAND.

PERIOD OF RECORD. - -1976 TO CURRENT YEAR.

EXTREMES FOR PERIOD OF RECORD.--HIGHEST WATER LEVEL, $24.95 \mathrm{FT}(7.60 \mathrm{M})$ BELOW LAND-SURFACE DATUM,MAR 8, 1983; LOWEST WATER LEVEL,28.05 FT(8.55 M) BELOW LAND-SURFACE DATUM, MAR 15, 1978.

WATER LEVELS IN FEET BELOW LAND SURFACE DATUM, CLIMATIC YEARS APRIL 1981 TO MARCH 1983

$\begin{array}{lll} & \text { WATER } & \\ \text { DATE } & \text { WEVEL } & \text { WATER } \\ & \text { LEVEL }\end{array}$

MAR 09, $1982 \quad 27.48 \quad$ MAR 08, $1983 \quad 24.95$

GROUND-WATER LEVELS

340326095164001. LOCAL NUMBER; 06S-19E-01 CCC 1.

LOCATION,--LAT 34 03'26", LONG 095 16'40", HYDROLOGIC UNIT 11140105.

AQUIFER.--ANTLERS SAND.

PERIOD OF RECORD.--1976 TO CURRENT YEAR.

EXTREMES FOR PERIOD OF RECORD.--HIGHEST WATER LEVEL, 62.06 FT(18.92 M) BELOW LAND-SURFACE DATUM, MAR 10, 1982; LOWEST WATER LEVEL,85.00 FT(25.91 M) BELOW LAND-SURFACE DATUM, FEB 26, 1980.

WATER LEVELS IN FEET BELOW LAND SURFACE DATUM, CLIMATIC YEARS APRIL 1981 TO MARCH 1983

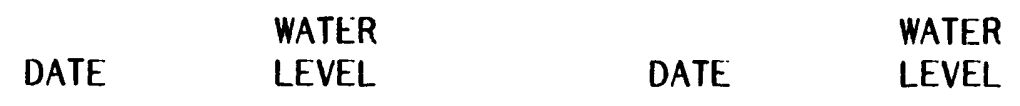

MAR $10,1982 \quad 62.06 \quad$ MAR 09, $1983 \quad 66.47$ 
GROUND-WATER LEVELS

CHOCTAW COUNTY -- CONTINUED

340326095171901 . LOCAL NUMBER;

06S-19E-02 CDD 1.

LOCATION,--LAT 34 03'26", LONG 095

AQUIFER.--ANTLERS SAND.

PERIOD OF RECORD.--1976 TO CURRENT YEAR.

EXTREMES FOR PERIOD OF RECORD.--HIGHEST WATER LEVEL, $51.09 \mathrm{FT}(15.57 \mathrm{M})$ BELOW LAND-SURFACE DATUM, JUNE 15, 1976; LOWEST WATER LEVEL,81.36 FT(24.80 M) BELOW LAND-SURFACE DATUM, MAR 10, 1982.

WATER LEVELS IN FEET BELOW LAND SURFACE DATUM, CLIMATIC YEARS APRIL 1981 TO MARCH 1983

$\begin{array}{lll} & \text { WATER } & \text { WATER } \\ \text { DATE } & \text { LEVEL } & \text { DATE }\end{array}$

$\begin{array}{llll}\text { MAR } 10,1982 \quad 81.36 & \text { MAR 09, } 1983 \quad 79.78\end{array}$

GROUND-WATER LEVELS

340412095175101. LOCAL NUMBER; 06S-19E-03 AAA 1.

LOCATION,--LAT 34 04'12", LONG 095 17'51", HYDROLOGIC UNIT 11140105.

AQUIFER.--ANTLERS SAND.

PERIOD OF RECORD.--1976 TO CURRENT YEAR.

EXTREMES FOR PERIOD OF RECORD.--HIGHEST WATER LEVEL, 34.49 FT(10.51 M) BELOW LAND-SURFACE DATUM, JUNE 15, 1976; LOWEST WATER LEVEL,45.13 FT(13.76 M) BELOW LAND-SURFACE DATUM, MAR 10, 1982.

WATER LEVELS IN FEET BELOW LAND SURFACE DATUM, CLIMATIC YEARS APRIL 1981 TO MARCH 1983

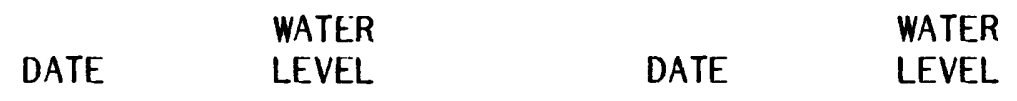

MAR 10, $1982 \quad 45.13 \quad$ MAR 09, $1983 \quad 41.95$ 
CHOCTAW COUNTY -- CONTINUED

340326095182201. LOCAL NUMBER; 06S-19E-03 CDD 1. LOCATION,--LAT 34 03'26", LONG 095 18'22", HYDROLOGIC UNIT 11140105. AQUIFER.--ANTLERS SAND.

PERIOD OF RECORD.--1976 TO CURRENT YEAR.

EXTREMES FOR PERIOD OF RECORD.--HIGHEST WATER LEVEL, $18.45 \mathrm{FT}(5.62 \mathrm{M})$ BELOW LAND-SURFACE DATUM,MAR 26, 1979; LOWEST WATER LEVEL,28.09 FT(8.56 M) BELOW LAND-SURFACE DATUM, MAR $5,1981$.

WATER LEVELS IN FEET BELOW LAND SURFACE DATUM, CLIMATIC YEARS APRIL 1981 TO MARCH 1983

\begin{tabular}{|c|c|}
\hline & WATER \\
\hline DATE & LEVEL \\
\hline
\end{tabular}

MAR $10,1982 \quad 27.40 \quad$ MAR 09, $1983 \quad 24.57$

GROUND-WATER LEVELS

340346095175101. LOCAL NUMBER; 06S-19E-03 DAA 1.

LOCATION,--LAT 34 03'46", LONG 095 17'51", HYDROLOGIC UNIT 11140105.

AQUIFER.--ANTLERS SAND.

PERIOD OF RECORD.--1976 TO CURRENT YEAR.

EXTREMES FOR PERIOD OF RECORD.--HIGHEST WATER LEVEL,48.63 FT(14.82 M) BELOW LAND-SURFACE DATUM, JUNE 15, 1976; LOWEST WATER LEVEL,57.09 FT(17.40 M) BELOW LAND-SURFACE DATUM, MAR 5, 1981.

WATER LEVELS IN FEET BELOW LAND SURFACE DATUM, CLIMATIC YEARS APRIL 1981 TO MARCH 1983

\begin{tabular}{|c|c|}
\hline & WATER \\
\hline DATE & LEVEL \\
\hline
\end{tabular}

MAR 09, $1983 \quad$ Z MAR 10, $1983 \quad Z$


CHOCTAW COUNTY -- CONTINUED

340326095175101. LOCAL NUMBER; 06S-19E-03 DDD 2.

LOCATION,--LAT 34 03'26", LONG 095 17'51", HYDROLOGIC UNIT 11140105.

AQUIFER.--ANTLERS SAND.

PERIOD OF RECORD.--1976 TO CURRENT YEAR.

EXTREMES FOR PERIOD OF RECORD.--HIGHEST WATER LEVEL,53.21 FT(16.22 M) BELOW LAND-SURFACE DATUM, MAR 9, 1983; LOWEST WATER LEVEL,59.83 FT(18.24 M) BELOW LAND-SURFACE DATUM, FEB 26, 1980.

WATER LEVELS IN FEET BELOW LAND SURFACE DATUM, CLIMATIC YEARS APRIL 1981 TO MARCH 1983

$\begin{array}{lll} & \text { WATER } & \text { WATER } \\ \text { DATE } & \text { LEVEL } & \text { DATE }\end{array}$

MAR $10,1982 \quad 58.55 \quad$ MAR 09, $1983 \quad 53.21$

GROUND-WATER LEVELS

340320095174301. LOCAL NUMBER; 06S-19E-11 BBB 1.

LOCATION,--LAT 34 03'20", LONG 095 17'43", HYDROLOGIC UNIT 11140105.

AQUIFER.--ANTLERS SAND.

PERIOD OF RECORD.--1976 TO CURRENT YEAR.

EXTREMES FOR PERIOD OF RECORD.--HIGHEST WATER LEVEL, $74.31 \mathrm{FT}(22.65 \mathrm{M})$ BELOW LAND-SURFACE DATUM, JUNE 15, 1976; LOWEST WATER LEVEL,81.81 FT(24.94 M) BELOW LAND-SURFACE DATUM, MAR 10, 1982.

WATER LEVELS IN FEET BELOW LAND SURFACE DATUM, CLIMATIC YEARS APRIL 1981 TO MARCH 1983

$\begin{array}{lll} & \text { WATER } & \text { WATER } \\ \text { DATE } & \text { LEVEL } & \text { DATE }\end{array}$

MAR 10, $1982 \quad 81.81 \quad$ MAR 09, $1983 \quad$ W 
GROUND-WATER LEVELS.

CHOCTAW COUNTY -- CONTINUED

335805095374201. LOCAL NUMBER; 07S-16E-09 AAA 1. LOCATION,--LAT 33 58'05", LONG 095 37'42", HYDROLOGIC UNIT 11140105. AQUIFER.--ANTLERS SAND.

PERIOD OF RECORD.--1981 TO CURRENT YEAR.

EXTREMES FOR PERIOD OF RECORD.--HIGHEST WATER LEVEL, $32.36 \mathrm{FT}(9.86 \mathrm{M})$ BELOW LAND-SURFACE DATUM, MAR 10, 1982; LOWEST WATER LEVEL, 35.57 FT(10.84 M) BELOW LAND-SURFACE DATUM, MAR 17, 1981.

WATER LEVELS IN FEET BELOW LAND SURFACE DATUM, CLIMATIC YEARS APRIL 1981 TO MARCH 1983

\begin{tabular}{|c|c|c|}
\hline & WATER & \\
\hline DATE & LEVEL & DATE \\
\hline
\end{tabular}

MAR 10, $1982 \quad 32.36 \quad$ MAR 09, $1983 \quad 32.80$

GROUND-WATER LEVELS

335752095380601. LOCAL NUMBER; 07S-16E-09 ACB 1.

LOCATION,--LAT 33 57'52", LONG 095 38'06", HYDROLOGIC UNIT 11140105.

AQUIFER.--ANTLERS SAND.

PERIOD OF RECORD.--1981 TO CURRENT YEAR.

EXTREMES FOR PERIOD OF RECORD.--HIGHEST WATER LEVEL, $18.30 \mathrm{FT}(5.58 \mathrm{M})$ BELOW LAND-SURFACE DATUM, MAR 17, 1981; LOWEST WATER LEVEL, 19.20 FT(5.85 M) BELOW LAND-SURFACE DATUM, MAR 10, 1982 .

WATER LEVELS IN FEET BELOW LAND SURFACE DATUM, CLIMATIC YEARS APRIL 1981 TO MARCH 1983

\begin{tabular}{|c|c|c|}
\hline & WATER & \\
\hline DATE & LEVEL & DATE \\
\hline
\end{tabular}

MAR 10, $1982 \quad 19.20 \quad$ MAR 09, $1983 \quad 18.90$ 
GROUND-WATER LEVELS

CIMARRON COUNTY

363410102560001 . LOCAL NUMBER;

01N-01E-10 AAD 1.

LOCATION,--LAT 36 34'10", LONG 102

AQUIFER.--MORRISON FORMATION.

PERIOD OF RECORD.--1970 TO CURRENT YEAR.

EXTREMES FOR PERIOD OF RECORD.--HIGHEST WATER LEVEL, $162.35 \mathrm{FT}(49.48 \mathrm{M})$ BELOW LAND-SURFACE DATUM, NOV 24, 1970; LOWEST WATER LEVEL, $173.24 \mathrm{FT}(52.80 \mathrm{M})$ BELOW LAND-SURFACE DATUM, JAN 7, 1980.

WATER LEVELS IN FEET BELOW LAND SURFACE DATUM, CLIMATIC YEARS APRIL 1981 TO MARCH 1983

\begin{tabular}{|c|c|c|}
\hline DATE & $\begin{array}{l}\text { WATER } \\
\text { LEVEL }\end{array}$ & \\
\hline & & DAIE \\
\hline
\end{tabular}

FEB 23, $1982 \quad 172.58 \quad$ JAN 11, $1983 \quad$ Z

GROUND-WATER LEVELS

363240102560001. LOCAL NUMBER; O1N-01E-15 BBB 1.

LOCATION,--LAT 36 32'40", LONG 102 56'00", HYDROLOGIC UNIT 11100101.

AQUIFER.--DAKOTA FORMATION.

PERIOD OF RECORD.--1967 TO CURRENT YEAR.

EXTREMES FOR PERIOD OF RECORD.--HIGHEST WATER LEVEL, 120.00 FT(36.58 M) BELOW LAND-SURFACE DATUM, JAN 7, 1980; LOWEST WATER LEVEL, 197.65 FT(60.24 M) BELOW LAND-SURFACE DATUM, JAN 18, 1982.

WATER LEVELS IN FEET BELOW LAND SURFACE DATUM, CLIMATIC YEARS APRIL 1981 TO MARCH 1983

$\begin{array}{cccc}\text { DATE } & \begin{array}{l}\text { WATER } \\ \text { LEVEL }\end{array} & \text { DATE } & \begin{array}{l}\text { WATER } \\ \text { LEVEL }\end{array} \\ \text { JAN 18, 1982 } & 197.65 & \text { JAN 11, 1983 } & 195.15\end{array}$


CIMARRON COUNTY -- CONTINUED

363120102545001. LOCAL NUMBER; 01N-01E-25 CBB 1.

LOCATION,--LAT 36 31'20", LONG 102 54'50", HYDROLOGIC UNIT 11100103.

AQUIFER .--CHEYENNE SANDSTONE.

PERIOD OF RECORD.--1967 TO CURRENT YEAR.

EXTREMES FOR PERIOD OF RECORD.--HIGHEST WATER LEVEL, 158.52 FT(48.32 M) BELOW LAND-SURFACE DATUM, JAN 14, 1970; LOWEST WATER LEVEL, 184.15 FT(56.13 M) BELOW LAND-SURFACE DATUM, JAN 5, 1981.

WATER LEVELS IN FEET BELOW LAND SURFACE DATUM, CLIMATIC YEARS APRIL 1981 TO MARCH 1983

$\begin{array}{cccc} & \text { WATER } & & \text { WATER } \\ \text { DATE } & \text { LEVEL } & \text { DATE } & \text { LEVEL } \\ \text { JAN 18, } 1982 & 173.79 & \text { JAN 11, 1983 } & 178.34\end{array}$

GROUND-WATER LEVELS

363030102571001. LOCAL NUMBER; 01N-01E-33 ACD 1. LOCATION,--LAT 36 30'30", LONG 102 57'10", HYDROLOGIC UNIT 11100103. AQUIFER .--DAKOTA FORMATION. PERIOD OF RECORD.--1966 TO CURRENT YEAR.

EXTREMES FOR PERIOD OF RECORD.--HIGHEST WATER LEVEL, 175.92 FT(53.62 M) BELOW LAND-SURFACE DATUM, AUG 15, 1966; LOWEST WATER LEVEL, $195.32 \mathrm{FT}(59.53 \mathrm{M})$ BELOW LAND-SURFACE DATUM, JAN 11, 1983.

WATER LEVELS IN FEET BELOW LAND SURFACE DATUM, CLIMATIC YEARS APRIL 1981 TO MARCH 1983

\begin{tabular}{|c|c|c|}
\hline & WATER & \\
\hline DATE & LEVEL & DATE \\
\hline
\end{tabular}

JAN 18, $1982195.30 \quad$ JAN 11, $1983 \quad 195.32$ 
GROUND-WATER LEVELS

CIMARRON COUNTY -- CONTINUED

363050102551001. LOCAL NUMBER; 01N-01E-35 ABC 1. LOCATION,--LAT 36 30'50", LONG 102 55'10", HYDROLOGIC UNIT 11100103.

AQUIFER.--CHEYENNE SANDSTONE.

PERIOD OF RECORD.--1967 TO CURRENT YEAR.

EXTREMES FOR PERIOD OF RECORD.--HIGHEST WATER LEVEL, 159.15 FT(48.51 M) BELOW LAND-SURFACE DATUM,JAN 24, 1967; LOWEST WATER LEVEL, $177.22 \mathrm{FT}(54.02 \mathrm{M})$ BELOW LAND-SURFACE DATUM, JAN 17, 1978.

WATER LEVELS IN FEET BELOW LAND SURFACE DATUM, CLIMATIC YEARS APRIL 1981 T0 MARCH 1983

$\begin{array}{lll} & \text { WATER } & \text { WATER } \\ \text { DATE } & \text { LEVEL } & \text { DATE }\end{array}$

JAN $18,1982 \quad 174.82 \quad$ JAN $11,1983 \quad 174.82$

GROUND-WATER LEVELS

363400102525001. LOCAL NUMBER; 01N-02E-07 DAA 1.

LOCATION,--LAT 36 34'00", LONG 102 52'50", HYDROLOGIC UNIT 11100101.

AQUIFER.--CHEYENNE SANDSTONE.

PERIOD OF RECORD.-- 1967 TO CURRENT YEAR.

EXTREMES FOR PERIOD OF RECORD.--HIGHEST WATER LEVEL, 142.95 FT(43.57 M) BELOW LAND-SURFACE DATUM, JAN 17, 1979; LOWEST WATER LEVEL, 159.65 FT(48.66 M) BELOW LAND-SURFACE DATUM, JAN 12, 1971.

WATER LEVELS IN FEET BELOW LAND SURFACE DATUM, CLIMATIC YEARS APRIL 1981 T0 MARCH 1983

$\begin{array}{lll} & \text { WATER } & \text { WATER } \\ \text { DATE } & \text { LEVEL } & \text { DATE }\end{array}$

JAN 18, $1982 \quad 143.80 \quad$ JAN 11, $1983 \quad 145.45$


VATER LEVELS IN FEET BELOW LAND SURFACE DATUM, CLIMATIC YEARS APRIL 1981 TO MARCH 1983

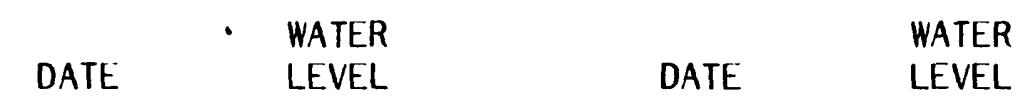

JAN 18, $1982131.09 \quad$ JAN $11,1983 \quad 130.17$

GROUND-WATER LEVELS

363400102500001. LOCAL NUMBER; 01N-02E-10 BDD 1.

OCATION,--LAT 36 34'00", LONG 102 50'00", HYDROLOGIC UNIT 11100101.

QQUIFER.--CHEYENNE SANDSTONE.

'ERIOD OF RECORD.--1966 TO CURRENT YEAR.

XTREMES FOR PERIOD OF RECORD.--HIGHEST WATER LEVEL, 103.76 FT(31.63 M) BELOW LAND-SURFACE IATUM, JAN 22, 1973; LOWEST WATER LEVEL, 141.70 FT(43.19 M) BELOW LAND-SURFACE DATUM, IUG $18,1966$.

IATER LEVELS IN FEET BELOW LAND SURFACE DATUM, CLIMATIC YEARS APRIL 1981 TO MARCH 1983

$\begin{array}{lll} & \text { WATER } & \\ \text { DATE } & \text { WEVEL } & \text { WATER } \\ & \text { DATE } & \text { LEVEL }\end{array}$

IAN 18, $1982 \quad 117.82 \quad$ JAN $11,1983 \quad$ Z


CIMARRON COUNTY -- CONTINUED

363430102474001. LOCAL NUMBER; 01N-02E-12 ABB 1. LOCATION,--LAT 36 34'30", LONG 102 47'40", HYDROLOGIC UNIT 11100103.

AQUIFER.--CHEYENNE SANDSTONE.

PERIOD OF RECORD.--1967 TO CURRENT YEAR.

EXTREMES FOR PERIOD OF RECORD.--HIGHEST WATER LEVEL, $95.81 \mathrm{FT}(29.20 \mathrm{M})$ BELOW LAND-SURFACE DATUM,JAN 17, 1979; LOWEST WATER LEVEL, 114.50 FT(34.90 M) BELOW LAND-SURFACE DATUM, JAN 5, 1981.

WATER LEVELS IN FEET BELOW LAND SURFACE DATUM, CLIMATIC YEARS APRIL 1981 TO MARCH 1983

\begin{tabular}{|c|c|c|}
\hline & WATER & \\
\hline DATE & LEVEL & DATE \\
\hline
\end{tabular}

JAN $18,1982 \quad 98.60 \quad$ JAN $11,1983 \quad 103.68$

GROUND-WATER LEVELS

363330102482001. LOCAL NUMBER; 01N-02E-13 BBC 1.

LOCATION,--LAT 36 33'30", LONG 102 48'20", HYDROLOGIC UNIT 11100103.

AQUIFER ---CHEYENNE SANDSTONE.

PERIOD OF RECORD.--1967 TO CURRENT YEAR.

EXTREMES FOR PERIOD OF RECORD.--HIGHEST WATER LEVEL, 86.25 FT(26.29 M) BELOW LAND-SURFACE DATUM, JAN 15, 1974; LOWEST WATER LEVEL, 105.88 FT(32.27 M) BELOW LAND-SURFACE DATUM, JAN 11, 1977.

WATER LEVELS IN FEET BELOW LAND SURFACE DATUM, CLIMATIC YEARS APRIL 1981 TO MARCH 1983

\begin{tabular}{cccc}
\multicolumn{1}{c}{} & WATER & & WATER \\
DATK & LEVEL & DATE & LEVEL \\
JAN 18, 1982 & 103.63 & JAN 11, 1983 & 96.35
\end{tabular}


GROUND-WATER LEVELS

CIMARRON COUNTY -- CONTINUED

363310102511001. LOCAL NUMBER; 01N-02E-16 BDC 1.

LOCATION,--LAT 36 33'10", LONG 102 51'10", HYDROLOGIC UNIT 11100103.

AQUIFER.--CHEYENNE SANDSTONE.

PERIOD OF RECORD.-- 1967 TO CURRENT YEAR.

EXTREMES FOR PERIOD OF RECORD.--HIGHEST WATER LEVEL, 83.35 FT(25.41 M) BELOW LAND-SURFACE DATUM, JAN 16, 1974; LOWEST WATER LEVEL, 142.76 FT(43.51 M) BELOW LAND-SURFACE DATUM, JAN 11, 1977.

WATER LEVELS IN FEET BELOW LAND SURFACE DATUM, CLIMATIC YEARS APRIL 1981 TO MARCH 1983

$\begin{array}{lll} & \text { WATER } & \text { WATER } \\ \text { DATE } & \text { LEVEL } & \text { DATE }\end{array}$

JAN 18, $1982 \quad 85.95 \quad$ JAN 11, $1983 \quad 85.55$

GROUND-WATER LEVELS

363220102482001. LOCAL NUMBER; 01N-02E-24 CBB 1. LOCATION,--LAT 36 32'20", LONG 102 48'20", HYDROLOGIC UNIT 11100103. AQUIFER.--CHEYENNE SANDSTONE.

PERIOD OF RECORD.--1967 TO CURRENT YEAR.

EXTREMES FOR PERIOD OF RECORD. --HIGHEST WATER LEVEL, 109.45 FT(33.36 M) BELOW LAND-SURFACE DATUM, JAN 15, 1970; LOWEST WATER LEVEL, $125.80 \mathrm{FT}(38.34 \mathrm{M})$ BELOW LAND-SURFACE DATUM, JAN 11, 1977.

WATER LEVELS IN FEET BELOW LAND SURFACE DATUM, CLIMATIC YEARS APRIL 1981 TO MARCH 1983

\begin{tabular}{|c|c|c|}
\hline & WATER & \\
\hline DATE & LEVEL & DATE \\
\hline
\end{tabular}

JAN 18, $1982 \quad 115.56 \quad$ JAN 11, $1983 \quad 114.10$ 
CIMARRON COUNTY -- CONTINUED

363120102502001. LOCAL NUMBER; 01N-02E-27 BCC 1. LOCATION,--LAT 36 31'20", LONG 102 50'20", HYDROLOGIC UNIT 11100103. AQUIFER .--DAKOTA FORMATION. PERIOD OF RECORD.--1966 TO CURRENT YEAR. EXTREMES FOR PERIOD OF RECORD.--HIGHEST WATER LEVEL, 88.52 FT(26.98 M) BELOW LAND-SURFACE DATUM, JAN 24, 1967; LOWEST WATER LEVEL,98.54 FT(30.03 M) BELOW LAND-SURFACE DATUM, JAN 18, 1982.

WATER LEVELS IN FEET BELOW LAND SURFACE DATUM, CLIMATIC YEARS APRIL 1981 TO MARCH 1983

$\begin{array}{lccc}\text { DATE } & \begin{array}{l}\text { WATER } \\ \text { LEVEL }\end{array} & \text { DATE } & \begin{array}{c}\text { WATER } \\ \text { LEVEL }\end{array} \\ \text { DATE } & \text { DAN 11, 1983 } & 98.18\end{array}$

GROUND-WATER LEVELS

363150102522001. LOCAL NUMBER; 01N-02E-29 BBA 1. LOCATION,--LAT 36 31'50", LONG 102 52'20", HYDROLOGIC UNIT 11100103. AQUIFER.--CHEYENNE SANDSTONE. PERIOD OF RECORD.--1970 TO CURRENT YEAR. EXTREMES FOR PERIOD OF RECORD.--HIGHEST WATER LEVEL, 108.98 FT(33.22 M) BELOW LAND-SURFACE DATUM, JAN 23, 1973; LOWEST WATER LEVEL, 116.26 FT(35.44 M) BELOW LAND-SURFACE DATUM, JAN 11, 1983.

WATER LEVELS IN FEET BELOW LAND SURFACE DATUM, CLIMATIC YEARS APRIL 1981 T0 MARCH 1983

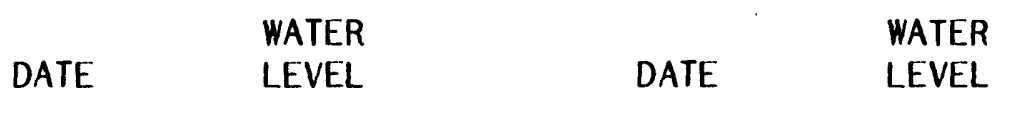

JAN 18, $1982 \quad 116.02 \quad$ JAN $11,1983 \quad 116.26$ 
GROUND-WATER LEVELS

CIMARRON COUNTY -- CONTINUED

363130102534001. LOCAL NUMBER; 01N-02E-30 BCC 1.

LOCATION,--LAT 36 31'30", LONG 102 53'40", HYDROLOGIC UNIT 11100103.

AQUIFER.--OGALLALA.

PERIOD OF RECORD.--1967 TO CURRENT YEAR.

EXTREMES FOR PERIOD OF RECORD.--HIGHEST WATER LEVEL, $133.56 \mathrm{FT}(40.71 \mathrm{M})$ BELOW LAND-SURFACE DATUM, JAN 16, 1968; LOWEST WATER LEVEL, 154.46 FT(47.08 M) BELOW LAND-SURFACE DATUM, JAN $5,1981$.

WATER LEVELS IN FEET BELOW LAND SURFACE DATUM, CLIMATIC YEARS APRIL 1981 TO MARCH 1983

$\begin{array}{ccccc}\text { DATE } & \begin{array}{l}\text { WATER } \\ \text { LEVEL }\end{array} & \text { DATE } & \begin{array}{c}\text { WATER } \\ \text { LEVEL }\end{array} \\ \text { JAN 18, 1982 } & 144.72 & \text { JAN 11, 1983 } & 144.05\end{array}$

GROUND-WATER LEVELS

363410102452001. LOCAL NUMBER; 01N-03E-08 ADB 1. LOCATION,--LAT 36 34'10", LONG 102 45'20", HYDROLOGIC UNIT 11100103. AQUIFER .--DAKOTA CHEYENNE. PERIOD OF RECORD.--1967 TO CURRENT YEAR. EXTREMES FOR PERIOD OF RECORD.--HIGHEST WATER LEVEL, 77.41 FT(23.59 M) BELOW LAND-SURFACE DATUM,JAN 15, 1970; LOWEST WATER LEVEL,94.50 FT(28.80 M) BELOW LAND-SURFACE DATUM, JAN 23, 1973.

WATER LEVELS IN FEET BELOW LAND SURFACE DATUM, CLIMATIC YEARS APRIL 1981 TO MARCH 1983

$\begin{array}{ccccc}\text { DATE } & \begin{array}{c}\text { WATER } \\ \text { LEVEL }\end{array} & \text { DATE } & \text { WATER } \\ \text { LEVEL }\end{array}$


GROUND-WATER LEVELS

CIMARRON COUNTY -- CONTINUED

363254102460701. LOCAL NUMBER;

01N-03E-17 CBC 1.

LOCATION,--LAT 36 32'54", LONG 102

AQUIFER.--DAKOTA CHEYENNE.

$46^{\prime}$ O7', HYDROLOGIC UNIT 11100103.

PERIOD OF RECORD . - 1968 TO CURRENT YEAR.

EXTREMES FOR PERIOD OF RECORD.--HIGHEST WATER LEVEL,96.32 FT(29.36 M) BELOW LAND-SURFACE DATUM, JAN 15, 1970; LOWEST WATER LEVEL,110.27 FT(33.61 M) BELOW LAND-SURFACE DATUM, JAN 17, 1979.

WATER LEVELS IN FEET BELOW LAND SURFACE DATUM, CLIMATIC YEARS APRIL 1981 TO MARCH 1983

\begin{tabular}{|c|c|}
\hline & WATER \\
\hline DATE & LEVEL \\
\hline
\end{tabular}

JAN $19,1982 \quad 105.88 \quad$ JAN $10,1983 \quad 104.15$

GROUND-WATER LEVELS

363150102414001. LOCAL NUMBER; $\quad 01 \mathrm{~N}-03 E-25$ BBB 1.

LOCATION,--LAT 36 31'50", LONG 102 41'40", HYDROLOGIC UNIT 11100103.

AQUIFER .--DAKOTA CHEYENNE.

PERIOD OF RECORD.-- 1966 TO CURRENT YEAR.

EXTREMES FOR PERIOD OF RECORD.--HIGHEST WATER LEVEL, $79.93 \mathrm{FT}(24.36 \mathrm{M})$ BELOW LAND-SURFACE DATUM, JAN 15, 1970; LOWEST WATER LEVEL, 107.10 FT(32.64 M) BELOW LAND-SURFACE DATUM, JAN 6, 1981.

WATER LEVELS IN FEET BELOW LAND SURFACE DATUM, CLIMATIC YEARS APRIL 1981 TO MARCH 1983

\begin{tabular}{|c|c|c|}
\hline & WATER & \\
\hline DATE & LEVEL & DATE \\
\hline
\end{tabular}

JAN 19, $1982 \quad 89.65 \quad$ JAN 10, $1983 \quad 88.09$ 
363110102461001. LOCAL NUMBER; 01N-03E-29 CCB 1.

LOCATION,--LAT 36 31'10", LONG 102 46'10", HYDROLOGIC UNIT 11100103.

AQUIFER.--DAKOTA CHEYENNE.

PERIOD OF RECORD.--1967 TO CURRENT YEAR.

EXTREMES FOR PERIOD OF RECORD.--HIGHEST WATER LEVEL, $133.79 \mathrm{FT}(40.78 \mathrm{M})$ BELOW LAND-SURFACE DATUM, JAN 14, 1970; LOWEST WATER LEVEL, $181.52 \mathrm{FT}(55.33 \mathrm{M})$ BELOW LAND-SURFACE DATUM, JAN 17, 1979.

WATER LEVELS IN FEET BELOW LAND SURFACE DATUM, CLIMATIC YEARS APRIL 1981 TO MARCH 1983

$\begin{array}{lll} & \text { WATER } & \text { WATER } \\ \text { DATE } & \text { LEVEL } & \text { DATE }\end{array}$

JAN 19, $1982152.35 \quad$ JAN $10,1983 \quad 138.72$

GROUND-WATER LEVELS

363014102443001. LOCAL NUMBER; 01N-03E-33 DCC 1

LOCATION,--LAT 36 30'14", LONG 102 44'30", HYDROLOGIC UNIT 11100103.

AQUIFER .--OGALLALA.

PERIOD OF RECORD.--1938 TO CURRENT YEAR.

EXTREMES FOR PERIOD OF RECORD.--HIGHEST WATER LEVEL, 37.03 FT(11.29 M) BELOW LAND-SURFACE DATUM, JAN 20, 1975; LOWEST WATER LEVEL,47.93 FT(14.61 M) BELOW LAND-SURFACE DATUM, JAN 11, 1983.

WATER LEVELS IN FEET BELOW LAND SURFACE DATUM, CLIMATIC YEARS APRIL 1981 TO MARCH 1983

$\begin{array}{cccc}\text { WATE } & \text { WATER } & & \text { WATER } \\ \text { LEVEL } & \text { DATE } & \text { LEVEL } \\ \text { JAN 19, 1982 } & 46.10 & \text { JAN 11, 1983 } & 47.93 \mathrm{R}\end{array}$




\section{GROUND-WATER LEVELS}

CIMARRON COUNTY -- CONTINUED

363030102423001. LOCAL NUMBER; 01N-03E-35 CAA 1.

LOCATION,--LAT 36 30'30", LONG 102 42'30", HYDROLOGIC UNIT 11100103.

AQUIFER. - OGALLALA.

PERIOD OF RECORD.--1967 TO CURRENT YEAR.

EXTREMES FOR PERIOD OF RECORD.--HIGHEST WATER LEVEL,75.05 FT(22.88 M) BELOW LAND-SURFACE DATUM, JAN 14, 1970; LOWEST WATER LEVEL,87.27 FT(26.60 M) BELOW LAND-SURFACE DATUM, JAN 17, 1979.

WATER LEVELS IN FEET BELOW LAND SURFACE DATUM, CLIMATIC YEARS APRIL 1981 TO MARCH 1983

\begin{tabular}{|c|c|}
\hline & WATER \\
\hline DATE & LEVEL \\
\hline
\end{tabular}

JAN 19, $1982 \quad 81.48 \quad$ JAN $10,1983 \quad 80.87$

GROUND-WATER LEVELS

363120102354001. LOCAL NUMBER; 01N-04E-26 ADC 1. LOCATION,--LAT 36 31'20", LONG 102 35'40", HYDROLOGIC UNIT 11100103. AQUIFER .--OGALLALA.

PERIOD OF RECORD.--1967 TO CURRENT YEAR.

EXTREMES FOR PERIOD OF RECORD.--HIGHEST WATER LEVEL, 52.36 FT(15.96 M) BELOW LAND-SURFACE DATUM, JAN 16, 1974; LOWEST WATER LEVEL,58.58 FT(17.86 M) BELOW LAND-SURFACE DATUM, JAN 7, 1981.

WATER LEVELS IN FEET BELOW LAND SURFACE DATUM, CLIMATIC YEARS APRIL 1981 TO MARCH 1983

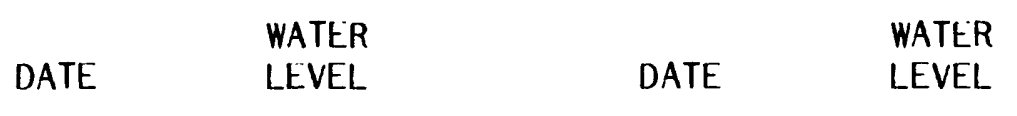

JAN 19, $1982 \quad 56.10 \quad$ JAN $10,1983 \quad 55.63$ 


\section{GROUND-WATER LEVELS}

CIMARRON COUNTY -- CONTINUED

363120102284001. LOCAL NUMBER; 01N-05E-25 CBB 1. LOCATION,--LAT 36 31'20", LONG 102 28'40", HYDROLOGIC UNIT 11100101. AQUIFER .--OGALLALA.

PERIOD OF RECORD.--1967 TO CURRENT YEAR.

EXTREMES FOR PERIOD OF RECORD.--HIGHEST WATER LEVEL,53.46 FT(16.29 M) BELOW LAND-SURFACE DATUM,JAN 12, 1971; LOWEST WATER LEVEL,70.06 FT(21.35 M) BELOW LAND-SURFACE DATUM, JAN 23, 1973.

WATER LEVELS IN FEET BELOW LAND SURFACE DATUM, CLIMATIC YEARS APRIL 1981 TO MARCH 1983

\begin{tabular}{|c|c|c|}
\hline & WATER & \\
\hline DATE & LEVEL & DATE \\
\hline
\end{tabular}

JAN 19, $1982 \quad Z \quad$ JAN 10, $1983 \quad 65.37$

GROUND-WATER LEVELS

363120102301001. LOCAL NUMBER; 01N-05E-27 DBD 1.

LOCATION,--LAT 36 31'20", LONG 102 30'10", HYDROLOGIC UNIT 11100101. AQUIFER.--OGALLALA.

PERIOD OF RECORD.--1967 TO CURRENT YEAR.

EXTREMES FOR PERIOD OF RECORD.--HIGHEST WATER LEVEL,61.66 FT(18.79 M) BELOW LAND-SURFACE DATUM, JAN 12, 1971; LOWEST WATER LEVEL,71.93 FT(21.92 M) BELOW LAND-SURFACE DATUM, JAN 18, 1979.

NATER LEVELS IN FEET BELOW LAND SURFACE DATUM, CLIMATIC YEARS APRIL 1981 TO MARCH 1983
DATE
WATER
DATE
WATER
LEVEL

JAN 19, $1982 \quad 71.25$

JAN $10,1983 \quad 70.95$ 
363100102330001. LOCAL NUMBER; 01N-05E-32 BBA 1. LOCATION,--LAT 36 31'00", LONG 102 33'00", HYDROLOGIC UNIT 11100103. AQUIFER.--OGALLALA.

PERIOD OF RECORD.--1966 TO CURRENT YEAR.

EXTREMES FOR PERIOD OF RECORD.--HIGHEST WATER LEVEL,62.96 FT(19.19 M) BELOW LAND-SURFACE DATUM,SEP 21, 1966; LOWEST WATER LEVEL,70.18 FT(21.39 M) BELOW LAND-SURFACE DATUM, JAN $9,1980$.

WATER LEVELS IN FEET BELOW LAND SURFACE DATUM, CLIMATIC YEARS APRIL 1981 TO MARCH 1983

$\begin{array}{lll} & \text { WATER } & \text { WATER } \\ \text { DATE } & \text { LEVEL } & \text { DATE } \\ \end{array}$

JAN $19,1982 \quad 69.80 \quad$ JAN $10,1983 \quad 69.90$

GROUND-WATER LEVELS

$363010102303001 . \quad$ LOCAL NUMBER; 01N-05E-34 CDD 1.

LOCATION,--LAT 36 30'10", LONG 102 30'30", HYDROLOGIC UNIT 11100101.

AQUIFER.--OGALLALA.

PERIOD OF RECORD .--1967 TO CURRENT YEAR.

EXTREMES FOR PERIOD OF RECORD.--HIGHEST WATER LEVEL, 77.29 FT(23.56 M) BELOW LAND-SURFACE DATUM, JAN 16, 1974; LOWEST WATER LEVEL,85.61 FT(26.09 M) BELOW LAND-SURFACE DATUM, JAN 23, 1973.

WATER LEVELS IN FEET BELOW LAND SURFACE DATUM, CLIMATIC YEARS APRIL 1981 TO MARCH 1983

$\begin{array}{lll} & \text { WATER } & \text { WATER } \\ \text { DATE } & \text { LEVEL } & \text { DATE }\end{array}$

JAN 19, $1982 \quad 81.81 \quad$ JAN $10,1983 \quad 79.83$ 


\section{CIMARRON COUNTY -- CONTINUED}

363030102292001. LOCAL NUMBER; 01N-05E-35 DBC 1. LOCATION,--LAT 36 30'30", LONG 102 29'20", HYDROLOGIC UNIT 11100101. AQUIFER . - OGALLALA.

PERIOD OF RECORD.--1967 TO CURRENT YEAR.

EXTREMES FOR PERIOD OF RECORD.--HIGHEST WATER LEVEL,68.53 FT(20.89 M) BELOW LAND-SURFACE DATUM,JAN 20, 1975; LOWEST WATER LEVEL,71.48 FT(21.79 M) BELOW LAND-SURFACE DATUM, JAN 18, 1979.

WATER LEVELS IN FEET BELOW LAND SURFACE DATUM, CLIMATIC YEARS APRIL 1981 TO MARCH 1983

$\begin{array}{lll} & \text { WATER } & \text { WATER } \\ \text { DATE } & \text { LEVEL } & \text { DATE }\end{array}$

JAN 19, $1982 \quad \mathrm{Z} \quad$ JAN 10, $1983 \quad 70.48$

GROUND-WATER LEVELS

363517102262701. LOCAL NUMBER; 01N-06E-05 BAA 1.

LOCATION,--LAT 36 35'17", LONG 102 26'27", HYDROLOGIC UNIT 11100101.

AQUIFER .--OGALLALA.

PERIOD OF RECORD.--1938 TO CURRENT YEAR.

EXTREMES FOR PERIOD OF RECORD.--HIGHEST WATER LEVEL,99.80 FT(30.42 M) BELOW LAND-SURFACE DATUM, JAN 12, 1971; LOWEST WATER LEVEL, $107.73 \mathrm{FT}(32.84 \mathrm{M})$ BELOW LAND-SURFACE DATUM, OCT 27, 1938.

WATER LEVELS IN FEET BELOW LAND SURFACE DATUM, CLIMATIC YEARS APRIL 1981 TO MARCH 1983

$\begin{array}{lll} & \text { WATER } & \\ \text { DATE } & \text { LEVEL } & \text { WATER } \\ & & \text { LEVEL }\end{array}$

JAN 19, $1982 \quad Z \quad$ JAN 10, $1983 \quad 101.08$ 
CIMARRON COUNTY -- CONTINUED

363110102230001. LOCAL NUMBER; 01N-06E-26 CDD 1.

LOCATION,--LAT 36 31'10", LONG 102 23'00", HYDROLOGIC UNIT 11100101.

AQUIFER .--OGALLALA.

PERIOD OF RECORD.--1967 TO CURRENT YEAR.

EXTREMES FOR PERIOD OF RECORD.--HIGHEST WATER LEVEL, 102.15 FT(31.14 M) BELOW LAND-SURFACE DATUM, JAN 10, 1983; LOWEST WATER LEVEL, 109.59 FT(33.40 M) BELOW LAND-SURFACE DATUM, JAN 23, 1973.

WATER LEVELS IN FEET BELOW LAND SURFACE DATUM, CLIMATIC YEARS APRIL 1981 TO MARCH 1983

$\begin{array}{cccc}\text { DATE } & \begin{array}{l}\text { WATER } \\ \text { LEVEL }\end{array} & \text { DATE } & \text { WATER } \\ \text { LEVEL }\end{array}$

GROUND-WATER LEVELS

363130102184001. LOCAL NUMBER; 01N-07E-28 BAD 1. LOCATION,--LAT 36 31'30", LONG 102 18'40", HYDROLOGIC UNIT 11100101. AQUIFER.--DOCKUM FORMATION. PERIOD OF RECORD.--1968 TO CURRENT YEAR. EXTREMES FOR PERIOD OF RECORD.--HIGHEST WATER LEVEL, 191.45 FT(58.35 M) BELOW LAND-SURFACE DATUM, JAN 15, 1969; LOWEST WATER LEVEL, 194.10 FT(59.16 M) BELOW LAND-SURFACE DATUM, JAN 19, 1972 .

WATER LEVELS IN FEET BELOW LAND SURFACE DATUM, CLIMATIC YEARS APRIL 1981 TO MARCH 1983

\begin{tabular}{|c|c|c|}
\hline & WATER & \\
\hline DATE & LEVEL & DATE \\
\hline
\end{tabular}

JAN 19, $1982191.60 \quad$ JAN $10,1983 \quad 191.60$ 
363440102115001. LOCAL NUMBER; $01 \mathrm{~N}-08 \mathrm{E}-04$ DDB 1. LOCATION,--LAT 36 34'40", LONG 102 11'50", HYDROLOGIC UNIT 11100101. AQUIFER.--OGALLALA. PERIOD OF RECORD.--1967 TO CURRENT YEAR. EXTREMES FOR PERIOD OF RECORD.--HIGHEST WATER LEVEL, $304.90 \mathrm{FT}(92.93 \mathrm{M})$ BELOW LAND-SURFACE DATUM, JAN 15, 1968; LOWEST WATER LEVEL,317.45 FT(96.76 M) BELOW LAND-SURFACE DATUM, JAN 21, 1982 .

WATER LEVELS IN FEET BELOW LAND SURFACE DATUM, CLIMATIC YEARS APRIL 1981 TO MARCH 1983

$\begin{array}{lll} & \text { WATER } & \text { WATER } \\ \text { DATE } & \text { LEVEL } & \text { DATE }\end{array}$

JAN 21, $1982 \quad 317.45 \quad$ JAN 12, $1983 \quad 316.90$

GROUND-WATER LEVELS

363351102050701. LOCAL NUMBER; 01N-09E-10 CBC 1. LOCATION,--LAT 36 33'51", LONG 102 05'07", HYDROLOGIC UNIT 11100101. AQUIFER .--OGALLALA.

PERIOD OF RECORD.--1970 TO CURRENT YEAR. EXTREMES FOR PERIOD OF RECORD.--HIGHEST WATER LEVEL,256.67 FT(78.23 M) BELOW LAND-SURFACE DATUM, JAN 15, 1979; LOWEST WATER LEVEL,285.85 FT(87.13 M) BELOW LAND-SURFACE DATUM, JAN 12, 1983.

WATER LEVELS IN FEET BELOW LAND SURFACE DATUM, CLIMATIC YEARS APRIL 1981 TO MARCH 1983

$\begin{array}{lll} & \text { WATER } & \text { WATER } \\ \text { DATE } & \text { LEVEL } & \text { DATE }\end{array}$

JAN 21, $1982 \quad 284.32$ JAN 12, $1983 \quad 285.85$ 
363348102035201. LOCAL NUMBER; 01N-09E-11 CBD 1. LOCATION,--LAT 36 33'48", LONG 102 03'52", HYDROLOGIC UNIT 11100101.

AQUIFER.--OGALLALA.

PERIOD OF RECORD.--1967 TO CURRENT YEAR.

EXTREMES FOR PERIOD OF RECORD.--HIGHEST WATER LEVEL, $273.69 \mathrm{FT}(83.42 \mathrm{M})$ BELOW LAND-SURFACE DATUM,JAN 14, 1969; LOWEST WATER LEVEL,310.62 FT(94.68 M) BELOW LAND-SURFACE DATUM, JAN $9,1980$.

WATER LEVELS IN FEET BELOW LAND SURFACE DATUM, CLIMATIC YEARS APRIL 1981 TO MARCH 1983

$\begin{array}{lll} & \text { WATER } & \\ \text { DATE } & \text { LEVEL } & \text { WATER } \\ & \text { DEVEL }\end{array}$

JAN 21, $1982 \quad 0 \quad$ JAN 12, $1983 \quad 302.70$

GROUND-WATER LEVELS

363110102040001. LOCAL NUMBER; 01N-09E-27 DDA 1.

LOCATION,--LAT 36 31'10", LONG 102 04'00", HYDROLOGIC UNIT 11100101.

AQUIFER.--OGALLALA.

PERIOD OF RECORD.--1967 TO CURRENT YEAR.

EXTREMES FOR PERIOD OF RECORD.--HIGHEST WATER LEVEL, $225.93 \mathrm{FT}(68.86 \mathrm{M})$ BELOW LAND-SURFACE DATUM, JAN 24, 1967; LOWEST WATER LEVEL,257.80 FT(78.58 M) BELOW LAND-SURFACE DATUM, JAN $18,1978$.

WATER LEVELS IN FEET BELOW LAND SURFACE DATUM, CLIMATIC YEARS APRIL 1981 T0 MARCH 1983

$\begin{array}{lll} & \text { WATER } & \\ \text { DATE } & \text { WEVEL } & \text { WATER } \\ \text { LEVEL }\end{array}$

JAN 21, $1982 \quad 249.35$ JAN 12, $1983 \quad 253.14$ 
CIMARRON COUNTY -- CONTINUED

363110102064001. LOCAL NUMBER; LOCATION,--LAT 3631 '10", LONG 102 AQUIFER.--OGALLALA.

PERIOD OF RECORD.--1967 TO CURRENT YEAR.

EXTREMES FOR PERIOD OF RECORD.--HIGHEST WATER LEVEL,271.19 FT(82.66 M) BELOW LAND-SURFACE DATUM,JAN 24, 1967; LOWEST WATER LEVEL, 300.70 FT(91.65 M) BELOW LAND-SURFACE DATUM, JAN 7, 1981.

WATER LEVELS IN FEET BELOW LAND SURFACE DATUM, CLIMATIC YEARS APRIL 1981 TO MARCH 1983

WATER

DATE LEVEL

JAN 20, $1982 \quad 290.38$
$01 \mathrm{~N}-09 \mathrm{E}-29 \mathrm{DCB} 1$. $06^{\prime} 40$ ", HYDROLOGIC UNIT 11100101. 


\section{CIMARRON COUNTY -- CONTINUED}

364020102473001. LOCAL NUMBER; 02N-02E-01 ADB 1. LOCATION,--LAT 36 40'20", LONG 102 47'30", HYDROLOGIC UNIT 11100101. AQUIFER.--OGALLALA. PERIOD OF RECORD. - - 1966 TO CURRENT YEAR. EXTREMES FOR PERIOD OF RECORD.--HIGHEST WATER LEVEL, 145.74 FT(44.42 M) BELOW LAND-SURFACE DATUM, JAN 10, 1983; LOWEST WATER LEVEL, 149.69 FT(45.63 M) BELOW LAND-SURFACE DATUM, JAN 15, 1974.

WATER LEVELS IN FEET BELOW LAND SURFACE DATUM, CLIMATIC YEARS APRIL 1981 TO MARCH 1983

\begin{tabular}{|c|c|}
\hline & WATER \\
\hline ATE & LEVEL \\
\hline
\end{tabular}

JAN 20, $1982 \quad 145.98 \quad$ JAN $10,1983 \quad 145.74$

GROUND-WATER LEVELS

363820102510001. LOCAL NUMBER; 02N-02E-16 CAA 1.

LOCATION,--LAT 36 38'20", LONG 102 51'00", HYDROLOGIC UNIT 11100101.

AQUIFER. - - OGALLALA.

PERIOD OF RECORD.--1967 TO CURRENT YEAR.

EXTREMES FOR PERIOD OF RECORD.--HIGHEST WATER LEVEL, 129.44 FT(39.45 M) BELOW LAND-SURFACE DATUM, JAN 10, 1983; LOWEST WATER LEVEL, 155.22 FT(47.31 M) BELOW LAND-SURFACE DATUM, JAN 17, 1978.

WATER LEVELS IN FEET BELOW LAND SURFACE DATUM, CLIMATIC YEARS APRIL 1981 TO MARCH 1983

$\begin{array}{lll} & \text { WATER } & \text { WATER } \\ \text { DATE } & \text { LEVEL } & \text { DATE }\end{array}$

JAN 19, $1982138.72 \quad$ JAN 10, $1983 \quad 129.44$ 
GROUND-WATER LEVELS

CIMARRON COUNTY -- CONTINUED

363700102480001. LOCAL NUMBER;

LOCATION,--LAT 36 37'00", LONG 102

AQUIFER.--CHEYENNE SANDSTONE.

PERIOD OF RECORD.--1967 TO CURRENT YEAR.

EXTREMES FOR PERIOD OF RECORD.--HIGHEST WATER LEVEL, $99.90 \mathrm{FT}(30.45 \mathrm{M})$ BELOW LAND-SURFACE

EXTREMES FOR PERIOD OF RECORD.--HIGHEST WATER LEVEL,99.90 FT(30.45 M) BELOW LAND-SURFACE
DATUM,JAN 24, 1967; LOWEST WATER LEVEL, 125.45 FT $(38.24 \mathrm{M})$ BELOW LAND-SURFACE DATUM, JAN 22, 1975.

$02 \mathrm{~N}-02 \mathrm{E}-25$ BAD 1.

$48^{\prime} 00^{\prime \prime}$, HYDROLOGIC UNIT 11100101. WATER LEVELS IN FEET BELOW LAND SURFACE DATUM, CLIMATIC YEARS APRIL 1981 TO MARCH 1983

\section{ER}

DATE

WATER

DATE

LEVEL

JAN 19,1982

Z

JAN $10,1983 \quad 104.06$

364030102253001. LOCAL NUMBER; 02N-06E-04 BBB 1. LOCATION,--LAT 36 40'30", LONG 102 25'30", HYDROLOGIC UNIT 11100101. AQUIFER.--OGALLALA. PERIOD OF RECORD.--1967 TO CURRENT YEAR. EXTREMES FOR PERIOD OF RECORD.--HIGHEST WATER LEVEL, $122.53 \mathrm{FT}(37.35 \mathrm{M})$ BELOW LAND-SURFACE DATUM, JAN 25, 1967; LOWEST WATER LEVEL, 159.45 FT(48.60 M) BELOW LAND-SURFACE DATUM, JAN 7, 1981.

WATER LEVELS IN FEET BELOW LAND SURFACE DATUM, CLIMATIC YEARS APRIL 1981 TO MARCH 1983

\begin{tabular}{cccc}
\multicolumn{1}{c}{} & WATER & & WATER \\
DATE & LEVEL & DATE & LEVEL \\
JAN 19, 1982 & 158.94 & JAN 11, 1983 & 134.25
\end{tabular}


363920102184001. LOCAL NUMBER; 02N-07E-09 ACC 1. LOCATION,--LAT 36 39'20", LONG 102 18'40", HYDROLOGIC UNIT 11100101. AQUIFER.--OGALLALA.

PERIOD OF RECORD.--1967 TO CURRENT YEAR.

EXTREMES FOR PERIOD OF RECORD.--HIGHEST WATER LEVEL, 163.49 FT(49.83 M) BELOW LAND-SURFACE DATUM, JAN 14, 1970; LOWEST WATER LEVEL, 169.76 FT(51.74 M) BELOW LAND-SURFACE DATUM, JAN 13, 1976.

WATER LEVELS IN FEET BELOW LAND SURFACE DATUM, CLIMATIC YEARS APRIL 1981 TO MARCH 1983

\begin{tabular}{|c|c|c|}
\hline & WATER & \\
\hline DATE & LEVEL & DATE \\
\hline
\end{tabular}

JAN 20, $1982 \quad 164.55 \quad$ JAN 12, $1983 \quad$ Z

\section{GROUND-WATER LEVELS}

363800102150001. LOCAL NUMBER; 02N-07E-13 DDD 1. LOCATION,--LAT $366^{\prime} 38^{\prime} 00^{\prime \prime}$, LONG 102 15'00", HYDROLOGIC UNIT 11100101. AQUIFER . - OGALLALA.

PERIOD OF RECORD.--1967 TO CURRENT YEAR.

EXTREMES FOR PERIOD OF RECORD.--HIGHEST WATER LEVEL,254.87 FT(77.68 M) BELOW LAND-SURFACE DATUM, JAN 16, 1968; LOWEST WATER LEVEL,271.18 FT(82.66 M) BELOW LAND-SURFACE DATUM, JAN 13, 1971 .

WATER LEVELS IN FEET BELOW LAND SURFACE DATUM, CLIMATIC YEARS APRIL 1981 TO MARCH 1983

$\begin{array}{lll} & \text { WATER } & \text { WATER } \\ \text { DATE } & \text { LEVEL } & \text { DATE }\end{array}$

JAN 20, $1982 \quad 270.85$ JAN 12, $1983 \quad 269.03$ 

JAN 13, 1976.

WATER LEVELS IN FEET BELOW LAND SURFACE DATUM, CLIMATIC YEARS APRIL 1981 TO MARCH 1983

\begin{tabular}{|c|c|c|c|}
\hline DATE & $\begin{array}{l}\text { WATER } \\
\text { LEVEL }\end{array}$ & DATE & $\begin{array}{l}\text { WATER } \\
\text { LEVEL }\end{array}$ \\
\hline 0, 198 & 228.93 & JAN 12,1983 & 228.38 \\
\hline
\end{tabular}

PERIOD OF RECORD.--1967 TO CURRENT YEAR.

EXTREMES FOR PERIOD OF RECORD.--HIGHEST WATER LEVEL,255.73 FT(77.95 M) BELOW LAND-SURFACE DATUM, JAN 25, 1967; LOWEST WATER LEVEL,278.70 FT(84.95 M) BELOW LAND-SURFACE DATUM, JAN 7, 1981.

WATER LEVELS IN FEET BELOW LAND SURFACE DATUM, CLIMATIC YEARS APRIL 1981 TO MARCH 1983

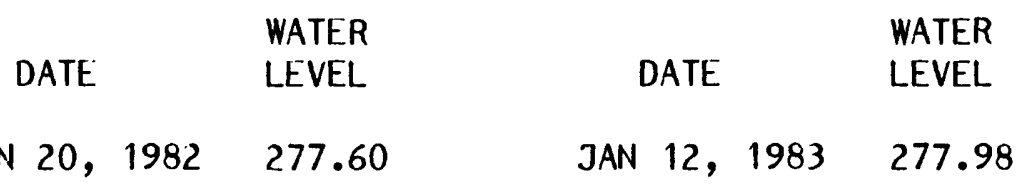


GROUND-WATER LEVELS

CIMARRON COUNTY -- CONTINUED

363730102142001. LOCAL NUMBER; 02N-08E-19 DBB 1.

LOCATION,--LAT 36 37'30", LONG 102 14'20", HYDROLOGIC UNIT 11100101.

AQUIFER.--OGALLALA.

PERIOD OF RECORD.--1967 TO CURRENT YEAR.

EXTREMES FOR PERIOD OF RECORD.--HIGHEST WATER LEVEL,226.90 FT(69.16 M) BELOW LAND-SURFACE DATUM,JAN 25, 1967; LOWEST WATER LEVEL,242.33 FT(73.86 M) BELOW LAND-SURFACE DATUM, JAN 16, 1979.

WATER LEVELS IN FEET BELOW LAND SURFACE DATUM, CLIMATIC YEARS APRIL 1981 TO MARCH 1983

$\begin{array}{lll} & \text { WATER } & \text { WATER } \\ \text { DATE } & \text { LEVEL } & \text { DATE }\end{array}$

JAN $20,1982 \quad 239.65 \quad$ JAN $12,1983 \quad 237.20$

GROUND-WATER LEVELS

363750102110001. LOCAL NUMBER; 02N-08E-22 ABC 1. LOCATION,--LAT 36 37'50", LONG 102 11'00", HYDROLOGIC UNIT' 11100101. AQUIFER . - OGALLALA.

PERIOD OF RECORD.--1967 TO CURRENT YEAR. EXTREMES FOR PERIOD OF RECORD.--HIGHEST WATER LEVEL, $260.15 \mathrm{FT}(79.29 \mathrm{M})$ BELOW LAND-SURFACE DATUM,JAN 24, 1967; LOWEST WATER LEVEL, 303.66 FT(92.56 M) BELOW LAND-SURFACE DATUM, JAN 20, 1982 .

WATER LEVELS IN FEET BELOW LAND SURFACE DATUM, CLIMATIC YEARS APRIL 1981 TO MARCH 1983

$\begin{array}{lll} & \text { WATER } & \text { WATER } \\ \text { DATE } & \text { LEVEL } & \text { DATE }\end{array}$

JAN 20, $1982 \quad 303.66 \quad$ JAN 12, $1983 \quad 292.52$ 
CIMARRON COUNTY -- CONTINUED

363700102100001. LOCAL NUMBER; 02N-08E-26 ABB 1.

LOCATION,--LAT 36 37'00", LONG 102 10'00", HYDROLOGIC UNIT 11100101.

AQUIFER.--OGALLALA.

PERIOD OF RECORD.--1967 TO CURRENT YEAR.

EXTREMES FOR PERIOD OF RECORD.--HIGHEST WATER LEVEL, 289.84 FT(88.34 M) BELOW LAND-SURFACE DATUM, JAN 24, 1967; LOWEST WATER LEVEL, 336.97 FT(102.71 M) BELOW LAND-SURFACE DATUM, JAN 15, 1979.

WATER LEVELS IN FEET BELOW LAND SURFACE DATUM, CLIMATIC YEARS APRIL 1981 TO MARCH 1983

$\begin{array}{lll} & \text { WATER } & \\ \text { DATE } & \text { LEVEL } & \text { WATER } \\ & \text { DATE } & \text { LEVEL }\end{array}$

JAN 20, $1982 \quad 334.80 \quad$ JAN 12, $1983 \quad 316.23$

GROUND-WATER LEVELS

364008102032601. LOCAL NUMBER; O2N-09E-02 ACC 1. LOCATION,--LAT 36 40'08", LONG 102 03'26", HYDROLOGIC UNIT 11100101. AQUIFER.--OGALLALA.

PERIOD OF RECORD.--1938 TO CURRENT YEAR.

EXTREMES FOR PERIOD OF RECORD.--HIGHEST WATER LEVEL, 108.32 FT(33.02 M) BELOW LAND-SURFACE DATUM, JAN 21, 1982; LOWEST WATER LEVEL, 120.49 FT(36.73 M) BELOW LAND-SURFACE DATUM, AUG 24, 1938.

WATER LEVELS IN FEET BELOW LAND SURFACE DATUM, CLIMATIC YEARS APRIL 1981 TO MARCH 1983

\begin{tabular}{|c|c|c|}
\hline & WATER & \\
\hline DATE & LEVEL & DATE \\
\hline
\end{tabular}

JAN 21, 1982 108.18 JAN 12, $1983 \quad 115.33$ 
363844102060101. LOCAL NUMBER;

$02 \mathrm{~N}-09 \mathrm{E}-16$ BBA 1. LOCATION,--LAT 36 38'44", LONG 102 06 '01", HYDROLOGIC UNIT 11100101. AQUIFER.--OGALLALA. PERIOD OF RECORD.--1967 TO CURRENT YEAR. EXTREMES FOR PERIOD OF RECORD.--HIGHEST WATER LEVEL, $159.65 \mathrm{FT}(48.66 \mathrm{M})$ BELOW LAND-SURFACE DATUM, JAN 12, 1983; LOWEST WATER LEVEL,160.40 FT(48.89 M) BELOW LAND-SURFACE DATUM, JAN 20, 1982.

WATER LEVELS IN FEET BELOW LAND SURFACE DATUM, CLIMATIC YEARS APRIL 1981 TO MARCH 1983

$\begin{array}{clcc} & \text { WATER } & & \text { WATER } \\ \text { DATE } & \text { LEVEL } & \text { DATE } & \text { LEVEL } \\ & & & \\ \text { LN 20, 1982 } & 160.40 & \text { JAN 12, 1983 } & 159.65\end{array}$

\section{GROUND-WATER LEVELS}

363610102054001. LOCAL NUMBER; 02N-09E-33 ABB 1. LOCATION,--LAT 36 36'10", LONG 102 05'40", HYDROLOGIC UNIT 11100101. AQUIFER .--OGALLALA. PERIOD OF RECORD.--1967 TO CURRENT YEAR. EXTREMES FOR PERIOD OF RECORD.--HIGHEST WATER LEVEL,261.00 FT(79.55 M) BELOW LAND-SURFACE DATUM, JAN 15, 1979; LOWEST WATER LEVEL,288.68 FT(87.99 M) BELOW LAND-SURFACE DATUM, JAN 20, 1982.

WATER LEVELS IN FEET BELOW LAND SURFACE DATUM, CLIMATIC YEARS APRIL 1981 TO MARCH 1983

$\begin{array}{lll} & \text { WATER } & \text { WATER } \\ \text { DATE } & \text { LEVEL } & \text { DATE }\end{array}$

JAN 20, $1982 \quad 288.68 \quad$ JAN $12,1983 \quad 284.55$ 
364130102512001. LOCAL NUMBER; LOCATION,--LAT 3641 '30", LONG 102 AQUIFER . - OGALLALA.

PERIOD OF RECORD.-- 1967 TO CURRENT YEAR.

EXTREMES FOR PERIOD OF RECORD.--HIGHEST WATER LEVEL,11.18 FT(3.41 M) BELOW LAND-SURFACE

EXTREMES FOR PERIOD OF RECORD.--HIGHEST WATER LEVEL, $11.18 \mathrm{FT}(3.41 \mathrm{M})$ BELOW LAND-SURFACE
DATUM,JAN 19, 1982; LOWEST WATER LEVEL, 38.15 FT(11.63 M) BELOW LAND-SURFACE DATUM, JAN 15, 1970.

WATER LEVELS IN FEET BELOW LAND SURFACE DATUM, CLIMATIC YEARS APRIL 1981 TO MARCH 1983
$03 \mathrm{~N}-02 \mathrm{E}-28 \mathrm{CCC} 1$.

$51^{\prime} 20^{\prime \prime}$, HYDROLOGIC UNIT 11040002.

$\begin{array}{cccc}\text { DATE } & \begin{array}{c}\text { WATER } \\ \text { LEVEL }\end{array} & \text { DATE } & \text { WATER } \\ \text { LEVEL }\end{array}$

GROUND-WATER LEVELS

364420102361001. LOCAL NUMBER; 03N-04E-11 CAC 1.

LOCATION,--LAT 36 44'20", LONG 102 36'10", HYDROLOGIC UNIT 11100101.

AQUIFER .--OGALLALA.

PERIOD OF RECORD.--1978 TO CURRENT YEAR.

EXTREMES FOR PERIOD OF RECORD.--HIGHEST WATER LEVEL, 159.84 FT(48.72 M) BELOW LAND-SURFACE DATUM, JAN 10, 1983; LOWEST WATER LEVEL, 160.86 FT(49.03 M) BELOW LAND-SURFACE. DATUM, JAN 5, 1981.

WATER LEVELS IN FEET BELOW LAND SURFACE DATUM, CLIMATIC YEARS APRIL 1981 TO MARCH 1983

\begin{tabular}{|c|c|c|}
\hline & WATER & \\
\hline DATE & LEVEL & DATE \\
\hline
\end{tabular}

JAN $19,1982 \quad 160.54 \quad$ JAN $10,1983 \quad 159.84$ 


\section{GROUND-WATER LEVELS}

CIMARRON COUNTY -- CONTINUED

364150102401001. LOCAL NUMBER;

$03 \mathrm{~N}-04 \mathrm{E}-30 \mathrm{BDD} 1$. LOCATION,--LAT 36 41'50", LONG 102 40'10", HYDROLOGIC UNIT 11100101. AQUIFER .--OGALLALA.

PERIOD OF RECORD.--1967 TO CURRENT YEAR.

EXTREMES FOR PERIOD OF RECORD.--HIGHEST WATER LEVEL, $131.21 \mathrm{FT}(39.99 \mathrm{M})$ BELOW LAND-SURFACE DATUM, JAN 24, 1967; LOWEST WATER LEVEL, 181.28 FT(55.25 M) BELOW LAND-SURFACE DATUM, JAN 15, 1979.

WATER LEVELS IN FEET BELOW LAND SURFACE DATUM, CLIMATIC YEARS APRIL 1981 TO MARCH 1983

\begin{tabular}{|c|c|}
\hline & WATER \\
\hline DATE & LEVEL \\
\hline
\end{tabular}

JAN 19, $1982 \quad 153.04 \quad$ JAN 10, $1983 \quad 151.68$

GROUND-WATER LEVELS

364100102383001. LOCAL NUMBER; 03N-04E-33 BCC 1. LOCATION,--LAT 36 41'00", LONG 102 38'30", HYDROLOGIC UNIT 11100101. AQUIFER .--OGALLALA.

PERIOD OF RECORD.--1967 TO CURRENT YEAR.

EXTREMES FOR PERIOD OF RECORD.--HIGHEST WATER LEVEL, 106.59 FT(32.49 M) BELOW LAND-SURFACE DATUM, JAN 24, 1967; LOWEST WATER LEVEL,135.86 FT(41.41 M) BELOW LAND-SURFACE DATUM, JAN 17, 1978.

WATER LEVELS IN FEET BELOW LAND SURFACE DATUM, CLIMATIC YEARS APRIL 1981 TO MARCH 1983

$\begin{array}{lll} & \text { WATER } & \text { WATER } \\ \text { DATE } & \text { LEVEL } & \text { DATE }\end{array}$

JAN 19, $1982 \quad 124.07 \quad$ JAN $10,1983 \quad 122.77$ 


\section{CIMARRON COUNTY -- CONTINUED}

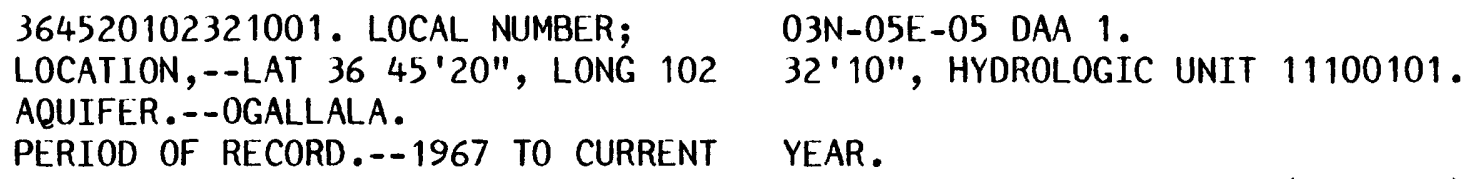

WATER LEVELS IN FEET BELOW LAND SURFACE DATUM, CLIMATIC YEARS APRIL 1981 TO MARCH 1983

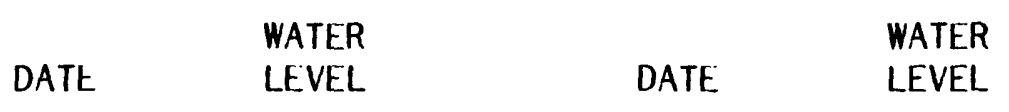

JAN $19,1982 \quad 221.39 \quad$ JAN $10,1983 \quad 219.82$

GROUND-WATER LEVELS

364420102295001. LOCAL NUMBER; 03N-05E-11 CCC 1.

LOCATION,--LAT 36 44'20", LONG 102 29'50", HYDROLOGIC UNIT 11100101.

AQUIFER .--OGALLALA.

PERIOD OF RECORD.--1967 TO CURRENT YEAR.

EXTREMES FOR PERIOD OF RECORD.--HIGHEST WATER LEVEL, $145.75 \mathrm{FT}(44.42 \mathrm{M})$ BELOW LAND-SURFACE DATUM,JAN 28, 1967; LOWEST WATER LEVEL,228.55 FT(69.66 M) BELOW LAND-SURFACE DATUM, JAN $6,1981$.

WATER LEVELS IN FEET BELOW LAND SURFACE DATUM, CLIMATIC YEARS APRIL 1981 T0 MARCH 1983

$\begin{array}{lll} & \text { WATER } & \text { WATER } \\ \text { DATE } & \text { LEVEL } & \text { DATE }\end{array}$

JAN 20, $1982 \quad 207.28 \quad$ JAN $10,1983 \quad 0$


364440102282001. LOCAL NUMBER; LOCATION,--LAT 36 44'40", LONG 102 AQUIFER.--OGALLALA. PERIOD OF RECORD.--1967 TO CURRENT YEAR. EXTREMES FOR PERIOD OF RECORD.--HIGHEST WATER LEVEL, 131.35 FT(40.04 M) BELOW LAND-SURFACE DATUM, JAN 28, 1967; LOWEST WATER LEVEL, 173.81 FT(52.98 M) BELOW LAND-SURFACE DATUM, JAN 10, 1983.

WATER LEVELS IN FEET BELOW LAND SURFACE DATUM, CLIMATIC YEARS APRIL 1981 TO MARCH 1983
$03 \mathrm{~N}-05 \mathrm{E}-12$ ACC 1. 28 '20", HYOROLOGIC UNIT 11100101.
ST WATER LEVEL, $131.35 \mathrm{FT}(40.04 \mathrm{M})$ BELOW LAND-SURFACE
LEVEL, $173.81 \mathrm{FT}(52.98 \mathrm{M})$ BELOW LAND-SURFACE DATUM,
WATER

DATE LEVEL

JAN $20,1982 \quad 171.44$

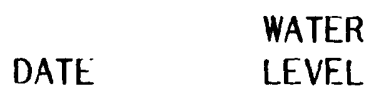

JAN $10,1983 \quad 173.81$

GROUND-WATER LEVELS

364340102231301. LOCAL NUMBER; 03N-05E-16 DBA 1.

LOCATION,--LAT 36 43'40", LONG 102 23'13", HYDROLOGIC UNIT 11100101.

AQUIFER .--OGALLALA.

PERIOD OF RECORD .-- 1967 TO CURRENT YEAR.

EXTREMES FOR PERIOD OF RECORD.--HIGHEST WATER LEVEL, 170.14 FT(51.86 M) BELOW LAND-SURFACE DATUM,JAN 24, 1967; LOWEST WATER LEVEL,228.88 FT(69.76 M) BELOW LAND-SURFACE DATUM, JAN 13, 1976.

WATER LEVELS IN FEET BELOW LAND SURFACE DATUM, CLIMATIC YEARS APRIL 1981 TO MARCH 1983

$\begin{array}{lll} & \text { WATER } & \text { WATER } \\ \text { DATE } & \text { LEVEL } & \text { DATE }\end{array}$

JAN 19, $1982 \quad 206.71 \quad$ JAN $10,1983 \quad 206.79$ 
GROUND-WATER LEVELS

CIMARRON COUNTY -- CONTINUED

364310102295001. LOCAL NUMBER;

03N-05E-23 BBB 1.

LOCATION,--LAT 36 43'10", LONG 102

29'50", HYDROLOGIC UNIT 11100101.

AQUIFER .--OGALLALA.

PERIOD OF RECORD.--1967 TO CURRENT YEAR.

EXTREMES FOR PERIOD OF RECORD.--HIGHEST WATER LEVEL, 148.25 FT(45.19 M) BELOW LAND-SURFACE DATUM, JAN 28, 1967; LOWEST WATER LEVEL, $199.16 \mathrm{FT}(60.70 \mathrm{M})$ BELOW LAND-SURFACE DATUM, JAN 10, 1983.

WATER LEVELS IN FEET BELOW LAND SURFACE DATUM, CLIMATIC YEARS APRIL 1981 TO MARCH 1983

$\begin{array}{lll} & \text { WATER } & \text { WATER } \\ \text { DATE } & \text { LEVEL } & \text { DATE }\end{array}$

JAN 19, $1982195.00 \quad$ JAN 10, $1983 \quad 199.16$

GROUND-WATER LEVELS

364539102214601. LOCAL NUMBER; 03N-06E-01 ABC 1.

LOCATION,--LAT 36 45'39", LONG 102 21'46", HYDROLOGIC UNIT 11100101.

AQUIFER.--OGALLALA.

PERIOD OF RECORD.--1967 TO CURRENT YEAR.

EXTREMES FOR PERIOD OF RECORD.--HIGHEST WATER LEVEL, $89.90 \mathrm{FT}(27.40 \mathrm{M})$ BELOW LAND-SURFACE DATUM, JAN 25, 1967; LOWEST WATER LEVEL, $112.01 \mathrm{FT}(34.14 \mathrm{M})$ BELOW LAND-SURFACE DATUM, JAN 11, 1983.

WATER LEVELS IN FEET BELOW LAND SURFACE DATUM, CLIMATIC YEARS APRIL 1981 TO MARCH 1983

\begin{tabular}{|c|c|}
\hline & $\begin{array}{l}\text { WATER } \\
\text { LEVEL }\end{array}$ \\
\hline
\end{tabular}

JAN 20, $1982 \quad 111.25$ JAN 11, $1983 \quad 112.01$ 
364440102253001. LOCAL NUMBER; 03N-06E-09 BCC 1. LOCATION,--LAT 36 44'40", LONG 102 25'30", HYDROLOGIC UNIT 11100101. AQUIFER.--OGALLALA. PERIOD OF RECORD.--1967 TO CURRENT YEAR. EXTREMES FOR PERIOD OF RECORD.--HIGHEST WATER LEVEL, $121.40 \mathrm{FT}(37.00 \mathrm{M})$ BELOW LAND-SURFACE DATUM, JAN 16, 1968; LOWEST WATER LEVEL, 163.10 FT(49.71 M) BELOW LAND-SURFACE DATUM, JAN 7, 1981.

WATER LEVELS IN FEET BELOW LAND SURFACE DATUM, CLIMATIC YEARS APRIL 1981 TO MARCH 1983

$\begin{array}{lll} & \text { WATER } & \text { WATER } \\ \text { DATE } & \text { LEVEL } & \text { DATE }\end{array}$

JAN 19, $1982162.38 \quad$ JAN 11, $1983 \quad 163.08$

GROUND-WATER LEVELS

364400102225001. LOCAL NUMBER; 03N-06E-14 ABB 1. LOCATION,--LAT 36 44'00", LONG 102 22'50", HYDROLOGIC UNIT 11100101. AQUIFER.--OGALLALA.

PERIOD OF RECORD.--1967 TO CURRENT YEAR.

EXTREMES FOR PERIOD OF RECORD.--HIGHEST WATER LEVEL, 98.73 FT(30.09 M) BELOW LAND-SURFACE DATUM, JAN 25, 1967; LOWEST WATER LEVEL, 125.10 FT(38.13 M) BELOW LAND-SURFACE DATUM, JAN $7,1981$.

WATER LEVELS IN FEET BELOW LAND SURFACE DATUM, CLIMATIC YEARS APRIL 1981 TO MARCH 1983

$\begin{array}{lll} & \text { WATER } & \text { WATER } \\ \text { DATE } & \text { LEVEL } & \text { DATE }\end{array}$

JAN 20, $1982 \quad 123.73 \quad$ JAN $11,1983 \quad 124.01$ 
GROUND-WATER LEVELS

CIMARRON COUNTY -- CONTINUED

364220102240401. LOCAL NUMBER;

03N-06E-15 DCA 1

LOCATION,--LAT 3642 '20", LONG 102

AQUIFER. --OGALLALA.

PERIOD OF RECORD.--1967 TO CURRENT YEAR.

EXTREMES FOR PERIOD OF RECORD.--HIGHEST WATER LEVEL, $115.45 \mathrm{FT}(35.19 \mathrm{M})$ BELOW LAND-SURFACE DATUM,JAN 16, 1968; LOWEST WATER LEVEL, 132.00 FT(40.23 M) BELOW LAND-SURFACE DATUM, JAN 7, 1981.

WATER LEVELS IN FEET BELOW LAND SURFACE DATUM, CLIMATIC YEARS APRIL 1981 TO MARCH 1983

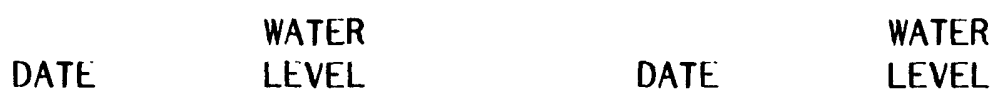

JAN 20, $1982 \quad 129.27 \quad$ JAN $11,1983 \quad 128.41$

GROUND-WATER LEVELS

364330102253001. LOCAL NUMBER; 03N-06E-16 CBB 1.

LOCATION,--LAT 36 43'30", LONG 102 25'30", HYDROLOGIC UNIT 11100101.

AQUIFER .--OGALLALA.

PERIOD OF RECORD.--1967 TO CURRENT YEAR.

EXTREMES FOR PERIOD OF RECORD .--HIGHEST WATER LEVEL, 107.14 FT(32.66 M) BELOW LAND-SURFACE DATUM, JAN 16, 1968; LOWEST WATER LEVEL, 129.26 FT(39.40 M) BELOW LAND-SURFACE DATUM, JAN 13, 1976.

WATER LEVELS IN FEET BELOW LAND SURFACE DATUM, CLIMATIC YEARS APRIL 1981 TO MARCH 1983

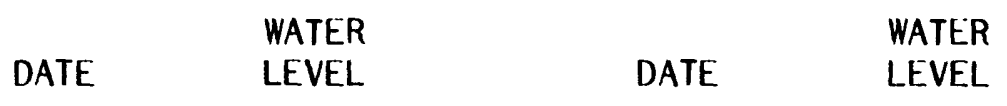

JAN 19, 1982121.30 JAN 11, $1983 \quad 124.15$ 
GROUND-WATER LEVELS

CIMARRON COUNTY -- CONTINUED

364130102195001. LOCAL NUMBER;

03N-07E-32 BAA 1.

LOCATION,--LAT 3641 '30", LONG 102

AQUIFER.--OGALLALA.

PERIOD OF RECORD. - 1967 TO CURRENT YEAR.

EXTREMES FOR PERIOD OF RECORD.--HIGHEST WATER LEVEL, $134.51 \mathrm{FT}(41.00 \mathrm{M})$ BELOW LAND-SURFACE DATUM,JAN 13, 1971; LOWEST WATER LEVEL, 143.57 FT(43.76 M) BELOW LAND-SURFACE DATUM, JAN 12, 1977.

WATER LEVELS IN FEET BELOW LAND SURFACE DATUM, CLIMATIC YEARS APRIL 1981 TO MARCH 1983

$\begin{array}{lll} & \text { WATER } & \text { WATER } \\ \text { DATE } & \text { LEVEL } & \text { DATE }\end{array}$

JAN 20, $1982 \quad 141.55 \quad$ JAN $11,1983 \quad 140.78$

GROUND-WATER LEVELS

364040102182001. LOCAL NUMBER; 03N-07E-33 DDC 1.

LOCATION,--LAT 36 40'40", LONG 102 18'20", HYDROLOGIC UNIT 11100101.

AQUIFER.--OGALLALA.

PERIOD OF RECORD.--1967 TO CURRENT YEAR.

EXTREMES FOR PERIOD OF RECORD.--HIGHEST WATER LEVEL, $133.67 \mathrm{FT}(40.74 \mathrm{M})$ BELOW LAND-SURFACE DATUM, JAN 13, 1971; LOWEST WATER LEVEL, 141.19 FT(43.03 M) BELOW LAND-SURFACE DATUM, JAN 11, 1983.

WATER LEVELS IN FEET BELOW LAND SURFACE DATUM, CLIMATIC YEARS APRIL 1981 TO MARCH 1983

$\begin{array}{lll} & \text { WATER } & \text { WATER } \\ \text { DATE } & \text { LEVEL } & \text { DATE }\end{array}$

JAN 20, $1982 \quad 140.52 \quad$ JAN 11, $1983 \quad 141.19$ 
CIMARRON COUNTY -- CONTINUED

364400102022001. LOCAL NUMBER; LOCATION,--LAT $3644^{\prime} 00$ ", LONG 102 AQUIFER .--OGALLALA. PERIOD OF RECORD.--1966 TO CURRENT YEAR. EXTREMES FOR PERIOD OF RECORD.--HIGHEST WATER LEVEL, 175.98 FT(53.64 M) BELOW LAND-SURFACE DATUM, JAN 9, 1980; LOWEST WATER LEVEL, 197.66 FT(60.25 M) BELOW LAND-SURFACE DATUM, JAN 14, 1969.

WATER LEVELS IN FEET BELOW LAND SURFACE DATUM, CLIMATIC YEARS APRIL 1981 TO MARCH 1983
02 '20", HYDROLOGIC UNIT 11100101.
$03 \mathrm{~N}-09 \mathrm{E}-13$ ABB 1.

\begin{tabular}{cccc}
\multicolumn{1}{c}{} & WATER & & WATER \\
DATE & LEVEL & DATE & LEVEL \\
JAN 20, 1982 & 186.00 & JAN 12, 1983 & 186.34
\end{tabular}

\section{GROUND-WATER LEVELS}

364918102415201. LOCAL NUMBER; 04N-03E-11 DDD 1. LOCATION,--LAT 36 49'18", LONG 102 41'52", HYDROLOGIC UNIT 11100101. AQUIFER.--OGALLALA.

PERIOD OF RECORD.--1980 TO CURRENT YEAR.

EXTREMES FOR PERIOD OF RECORD.--HIGHEST WATER LEVEL,96.89 FT(29.53 M) BELOW LAND-SURFACE DATUM, APR 3, 1980; LOWEST WATER LEVEL, 122.27 FT(37.27 M) BELOW LAND-SURFACE DATUM, JAN $27,1981$.

WATER LEVELS IN FEET BELOW LAND SURFACE DATUM, CLIMATIC YEARS APRIL 1981 TO MARCH 1983

\begin{tabular}{|c|c|c|}
\hline & WATER & \\
\hline DATE & LEVEL & DATE \\
\hline
\end{tabular}

JAN 21, $1982 \quad 115.20 \quad$ JAN 10, $1983 \quad 105.43$ 
GROUND-WATER LEVELS

CIMARRON COUNTY -- CONTINUED

364745102435501 . LOCAL NUMBER; LOCATION,--LAT 36 47'45", LONG 102 AQUIFER .--OGALLALA.

PERIOD OF RECORD.-- 1974 TO CURRENT EXTREMES FOR PERIOD OF RECORD.--HIGHEST WATER LEVEL, $12.29 \mathrm{FT}(3.75 \mathrm{M})$ BELOW LAND-SURFACE DATUM, JAN 15, 1974; LOWEST WATER LEVEL,29.20 FT(8.90 M) BELOW LAND-SURFACE DATUM, JAN 8, 1980.

WATER LEVELS IN FEET BELOW LAND SURFACE DATUM, CLIMATIC YEARS APRIL 1981 TO MARCH 1983

$04 \mathrm{~N}-03 \mathrm{E}-22 \mathrm{CBC} 1$

43'55", HYDROLOGIC UNIT 11100101.

YEAR 。

GROUND-WATER LEVELS

364720102362001. LOCAL NUMBER; 04N-04E-26 BCB 1. LOCATION,--LAT 36 47'20", LONG 102 36'20", HYDROLOGIC UNIT 11100101. AQUIFER .--OGALLALA. PERIOD OF RECORD.-- 1967 TO CURRENT YEAR. EXTREMES FOR PERIOD OF RECORD.--HIGHEST WATER LEVEL, 201.27 FT(61.35 M) BELOW LAND-SURFACE DATUM, JAN 24, 1967; LOWEST WATER LEVEL,218.60 FT(66.63 M) BELOW LAND-SURFACE DATUM, JAN $18,1972$.

WATER LEVELS IN FEET BELOW LAND SURFACE DATUM, CLIMATIC YEARS APRIL 1981 TO MARCH 1983

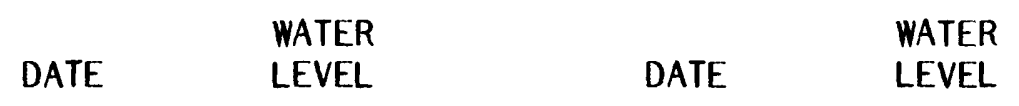

JAN 19, $1982 \quad 209.35 \quad$ JAN $10,1983 \quad 210.89$ 


\section{GROUND-WATER LEVELS}

CIMARRON COUNTY -- CONTINUED

364630102343001. LOCAL NUMBER; 04N-04E-36 AAC 1.

LOCATION,--LAT 36 46'30", LONG 102 34'30", HYDROLOGIC UNIT 11100101. AQUIFER.--OGALLALA.

PERIOD OF RECORD.--1967 TO CURRENT YEAR.

EXTREMES FOR PERIOD OF RECORD.--HIGHEST WATER LEVEL, $168.88 \mathrm{FT}(51.47 \mathrm{M})$ BELOW LAND-SURFACE DATUM,JAN 24, 1967; LOWEST WATER LEVEL, $196.98 \mathrm{FT}(60.04 \mathrm{M})$ BELOW LAND-SURFACE DATUM, JAN 7, 1980.

WATER LEVELS IN FEET BELOW LAND SURFACE DATUM, CLIMATIC YEARS APRIL 1981 TO MARCH 1983

\begin{tabular}{|c|c|c|}
\hline & WATER & \\
\hline DATE & LEVEL & DATE \\
\hline
\end{tabular}

JAN 19, $1982189.79 \quad$ JAN $10,1983 \quad 187.63$

\section{GROUND-WATER LEVELS}

364910102305501. LOCAL NUMBER; 04N-05E-15 BBB 1.

LOCATION,--LAT 36 49'10", LONG 102 30'55", HYDROLOGIC UNIT 11040002.

AQUIFER.--OGALLALA.

PERIOD OF RECORD.--1967 TO CURRENT YEAR.

EXTREMES FOR PERIOD OF RECORD.--HIGHEST WATER LEVEL, 122.14 FT(37.23 M) BELOW LAND-SURFACE DATUM, JAN 18, 1972; LOWEST WATER LEVEL, 127.58 FT(38.89 M) BELOW LAND-SURFACE DATUM, JAN 18, 1979.

WATER LEVELS IN FEET BELOW LAND SURFACE DATUM, CLIMATIC YEARS APRIL 1981 TO MARCH 1983

$\begin{array}{lll} & \text { WATER } & \text { WATER } \\ \text { DATE } & \text { LEVEL } & \text { DATE }\end{array}$

JAN 20, $1982 \quad P \quad$ JAN $10,1983 \quad 112.00$ 
CIMARRON COUNTY -- CONTINUED

364550102340001. LOCAL NUMBER; 04N-05E-31 CCD 1. LOCATION,--LAT 36 45'50", LONG 102 34'00", HYDROLOGIC UNIT 11100101. AQUIFER.--OGALLALA.

PERIOD OF RECORD.--1967 TO CURRENT YEAR.

EXTREMES FOR PERIOD OF RECORD.--HIGHEST WATER LEVEL, 174.05 FT(53.05 M) BELOW LAND-SURFACE DATUM, JAN 24, 1967; LOWEST WATER LEVEL,203.63 FT(62.07 M) BELOW LAND-SURFACE DATUM, JAN $5,1981$.

WATER LEVELS IN FEET BELOW LAND SURFACE DATUM, CLIMATIC YEARS APRIL 1981 TO MARCH 1983

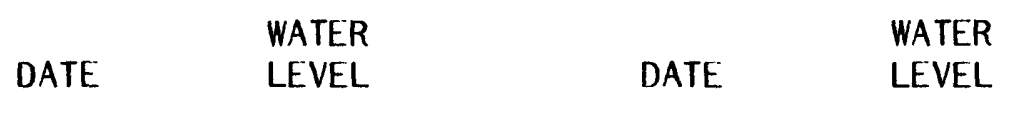

JAN 19, $1982197.94 \quad$ JAN 10, $1983 \quad 195.93$

GROUND-WATER LEVELS

364640102321001. LOCAL NUMBER; 04N-05E-32 AAA 1. LOCATION,--LAT 36 46'40", LONG 102 32'10", HYDROLOGIC UNIT 11100101. AQUIFER .--OGALLALA.

PERIOD OF RECORD.--1967 TO CURRENT YEAR.

EXTREMES FOR PERIOD OF RECORD.--HIGHEST WATER LEVEL, $176.33 \mathrm{FT}(53.75 \mathrm{M})$ BELOW LAND-SURFACE DATUM, JAN 24, 1967; LOWEST WATER LEVEL, 199.07 FT(60.68 M) BELOW LAND-SURFACE DATUM, JAN $10,1983$.

WATER LEVELS IN FEET BELOW LAND SURFACE DATUM, CLIMATIC YEARS APRIL 1981 TO MARCH 1983

\begin{tabular}{|c|c|c|}
\hline & WATER & \\
\hline DATE & LEVEL & DATE \\
\hline
\end{tabular}

JAN 19, $1982198.46 \quad$ JAN 10, $1983 \quad 199.07$ 
CIMARRON COUNTY -- CONTINUED

364620102282001. LOCAL NUMBER; 04N-05E-36 ACB 1. LOCATION,--LAT 36 46'20", LONG 102 28'20", HYDROLOGIC UNIT 11040002. AQUIFER . - OGALLALA.

PERIOD OF RECORD.--1967 TO CURRENT YEAR.

EXTREMES FOR PERIOD OF RECORD.--HIGHEST WATER LEVEL, $158.50 \mathrm{FT}(48.31 \mathrm{M})$ BELOW LAND-SURFACE DATUM, JAN 28, 1967; LOWEST WATER LEVEL,204.24 FT(62.25 M) BELOW LAND-SURFACE DATUM, JAN 10, 1983.

WATER LEVELS IN FEET BELOW LAND SURFACE DATUM, CLIMATIC YEARS APRIL 1981 TO MARCH 1983

\begin{tabular}{|c|c|c|}
\hline & WATER & DATE \\
\hline DATE & & DATE \\
\hline
\end{tabular}

JAN 20, $1982 \quad 204.14 \quad$ JAN 10, $1983 \quad 204.24$

GROUND-WATER LEVELS

364950102250001. LOCAL NUMBER; 04N-06E-09 ACB 1. LOCATION,--LAT 36 49'50", LONG 102 25'00", HYDROLOGIC UNIT 11040002.

AQUIFER .--DOCKUM FORMATION.

PERIOD OF RECORD.--1967 TO CURRENT YEAR.

EXTREMES FOR PERIOD OF RECORD.--HIGHEST WATER LEVEL, $191.60 \mathrm{FT}(58.40 \mathrm{M})$ BELOW LAND-SURFACE DATUM, JAN 25, 1967; LOWEST WATER LEVEL,273.38 FT(83.33 M) BELOW LAND-SURFACE DATUM, JAN 20, 1982.

WATER LEVELS IN FEET BELOW LAND SURFACE DATUM, CLIMATIC YEARS APRIL 1981 TO MARCH 1983

\begin{tabular}{|c|c|}
\hline & WATER \\
\hline DATE & LEVEL \\
\hline
\end{tabular}

JAN 20, $1982 \quad 273.38 \quad$ JAN $11,1983 \quad$ Z 


\section{GROUND-WATER LEVELS}

CIMARRON COUNTY -- CONTINUED

364830102262001. LOCAL NUMBER;

04N-06E-17 CDC 1.

LOCATION,--LAT 36 48'30", LONG 102 26'20", HYDROLOGIC UNIT 11040002.

AQUIFER.--DOCKUM FORMATION.

PERIOD OF RECORD.--1967 TO CURRENT YEAR.

EXTREMES FOR PERIOD OF RECORD.--HIGHEST WATER LEVEL, 194.90 FT(59.41 M) BELOW LAND-SURFACE DATUM, JAN 25, 1967; LOWEST WATER LEVEL,237.64 FT(72.43 M) BELOW LAND-SURFACE DATUM, JAN 10, 1983.

WATER LEVELS IN FEET BELOW LAND SURFACE DATUM, CLIMATIC YEARS APRIL 1981 TO MARCH 1983

\begin{tabular}{|c|c|c|}
\hline & WATER & \\
\hline DATE & LEVEL & DATE \\
\hline
\end{tabular}

JAN 20, $1982 \quad 231.84 \quad$ JAN $10,1983 \quad 237.64$

GROUND-WATER LEVELS

364800102243001. LOCAL NUMBER; 04N-06E-22 CBB 1.

LOCATION,--LAT 36 48'00", LONG 102 24'30", HYDROLOGIC UNIT 11100101.

AQUIFER .--OGALLALA.

PERIOD OF RECORD.--1967 TO CURRENT YEAR.

EXTREMES FOR PERIOD OF RECORD.--HIGHEST WATER LEVEL, 147.45 FT(44.94 M) BELOW LAND-SURFACE DATUM, JAN 27, 1967; LOWEST WATER LEVEL, 199.45 FT(60.79 M) BELOW LAND-SURFACE DATUM, JAN 14, 1976.

WATER LEVELS IN FEET BELOW LAND SURFACE DATUM, CLIMATIC YEARS APRIL 1981 TO MARCH 1983

\begin{tabular}{|c|c|}
\hline & WATER \\
\hline DATE & LEVEL \\
\hline
\end{tabular}

JAN 20, $1982 \quad 185.18 \quad$ JAN 10, $1983 \quad 193.40$ 
364820102231001. LOCAL NUMBER; 04N-06E-23 BAB 1. LOCATION,--LAT 36 48'20", LONG 102 23'10", HYDROLOGIC UNIT 11100101. AQUIFER. - OGALLALA.

PERIOD OF RECORD.--1967 TO CURRENT YEAR.

EXTREMES FOR PERIOD OF RECORD.--HIGHEST WATER LEVEL, $128.45 \mathrm{FT}(39.15 \mathrm{M})$ BELOW LAND-SURFACE DATUM,JAN 25, 1967; LOWEST WATER LEVEL,168.93 FT(51.49 M) BELOW LAND-SURFACE DATUM, JAN 11, 1983.

WATER LEVELS IN FEET BELOW LAND SURFACE DATUM, CLIMATIC YEARS APRIL 1981 TO MARCH 1983

\begin{tabular}{|c|c|c|c|}
\hline DATE & $\begin{array}{l}\text { WATER } \\
\text { LEVEL }\end{array}$ & DATE & $\begin{array}{l}\text { WATER } \\
\text { LEVEL }\end{array}$ \\
\hline 20,1982 & 163.13 & JAN 11,1983 & 168.93 \\
\hline
\end{tabular}

GROUND-WATER LEVELS

364720102222001. LOCAL NUMBER; 04N-06E-25 BBC 1. LOCATION,--LAT 36 47'20", LONG 102 22'20", HYDROLOGIC UNIT 11100101. AQUIFER .--OGALLALA. PERIOD OF RECORD.--1967 TO CURRENT YEAR. EXTREMES FOR PERIOD OF RECORD.--HIGHEST WATER LEVEL, $112.00 \mathrm{FT}(34.14 \mathrm{M})$ BELOW LAND-SURFACE DATUM, JAN 25, 1967; LOWEST WATER LEVEL, 149.79 FT(45.66 M) BELOW LAND-SURFACE DATUM, JAN 13, 1976.

WATER LEVELS IN FEET BELOW LAND SURFACE DATUM, CLIMATIC YEARS APRIL 1981 TO MARCH 1983

\begin{tabular}{llcc}
\multicolumn{1}{c}{} & WATER & & WATER \\
DATE & LEVEL & DATE & LEVEL \\
& & JAN 10, 1983 & 132.81
\end{tabular}


364650102232001. LOCAL NUMBER; 04N-06E-26 CBC 1. LOCATION,--LAT 36 46'50", LONG 102 23'20", HYDROLOGIC UNIT 11100101.

AQUIFER.--OGALLALA.

PERIOD OF RECORD.--1966 TO CURRENT YEAR.

EXTREMES FOR PERIOD OF RECORD.--HIGHEST WATER LEVEL, 111.20 FT(33.89 M) BELOW LAND-SURFACE DATUM, AUG 10, 1966; LOWEST WATER LEVEL, 148.78 FT(45.35 M) BELOW LAND-SURFACE DATUM, JAN $18,1979$.

WATER LEVELS IN FEET BELOW LAND SURFACE DATUM, CLIMATIC YEARS APRIL 1981 TO MARCH 1983

$\begin{array}{lll} & \text { WATER } & \text { WATER } \\ \text { DATE } & \text { LEVEL } & \text { DATE }\end{array}$

JAN 20, $1982146.35 \quad$ JAN $10,1983 \quad 145.39$

\section{GROUND-WATER LEVELS}

364640102271001. LOCAL NUMBER; 04N-06E-30 DCC 1. LOCATION,--LAT 36 46'40", LONG 102 27'10", HYDROLOGIC UNIT 11100101. AQUIFER .--OGALLALA. PERIOD OF RECORD.--1967 TO CURRENT YEAR. EXTREMES FOR PERIOD OF RECORD.--HIGHEST WATER LEVEL, $163.60 \mathrm{FT}$ (49.87 M) BELOW LAND-SURFACE DATUM, JAN 17, 1968; LOWEST WATER LEVEL, 199.55 FT(60.82 M) BELOW LAND-SURFACE DATUM, JAN 14, 1976.

WATER LEVELS IN FEET BELOW LAND SURFACE DATUM, CLIMATIC YEARS APRIL 1981 TO MARCH 1983

\begin{tabular}{|c|c|}
\hline & WATER \\
\hline ATE & LEVEL \\
\hline
\end{tabular}

JAN 20, $1982 \quad 195.82 \quad$ JAN 10, $1983 \quad 198.08$ 
365040102145001. LOCAL NUMBER; LOCATION,--LAT $3650^{\prime} 40^{\prime \prime}$, LONG 102 AQUIFER.--OGALLALA. PERIOD OF RECORD.--1967 TO CURRENT YEAR. EXTREMES FOR PERIOD OF RECORD.--HIGHEST WATER LEVEL,189.32 FT(57.70 M) BELOW LAND-SURFACE DATUM,JAN 25, 1967; LOWEST WATER LEVEL,207.27 FT(63.18 M) BELOW LAND-SURFACE DATUM, JAN 23, 1973.

WATER LEVELS IN FEET BELOW LAND SURFACE DATUM, CLIMATIC YEARS APRIL 1981 TO MARCH 1983

\begin{tabular}{|c|c|}
\hline & WATER \\
\hline ATE & LEVEL \\
\hline
\end{tabular}

JAN 21, $1982 \quad 197.68 \quad$ JAN 11, $1983 \quad 193.51$
365030102175001. LOCAL NUMBER; 04N-07E-03 CBA 1. LOCATION,--LAT 36 50'30", LONG 102 17'50", HYDROLOGIC UNIT 11100101. AQUIFER.--OGALLALA. PERIOD OF RECORD.--1967 TO CURRENT YEAR. EXTREMES FOR PERIOD OF RECORD.--HIGHEST WATER LEVEL, $179.25 \mathrm{FT}(54.64 \mathrm{M})$ BELOW LAND-SURFACE DATUM, JAN 25, 1967; LOWEST WATER LEVEL, 196.98 FT(60.04 M) BELOW LAND-SURFACE DATUM, JAN 19, 1972 .

WATER LEVELS IN FEET BELOW LAND SURFACE DATUM, CLIMATIC YEARS APRIL 1981 TO MARCH 1983
JAN 21, 1982
0
JAN 11, 1983
0 


\section{GROUND-WATER LEVELS}

CIMARRON COUNTY -- CONTINUED

365010102204001. LOCAL NUMBER; 04N-07E-07 ABB 1.

LOCATION,--LAT 36 50'10", LONG 102 20'40", HYDROLOGIC UNIT 11100101.

AQUIFER.--OGALLALA.

PERIOD OF RECORD.--1967 TO CURRENT YEAR.

EXTREMES FOR PERIOD OF RECORD.--HIGHEST WATER LEVEL, 197.10 FT(60.08 M) BELOW LAND-SURFACE DATUM,JAN 25, 1967; LOWEST WATER LEVEL,220.90 FT(67.33 M) BELOW LAND-SURFACE DATUM, JAN 17, 1979.

WATER LEVELS IN FEET BELOW LAND SURFACE DATUM, CLIMATIC YEARS APRIL 1981 TO MARCH 1983

$\begin{array}{lll} & \text { WATER } & \text { WATER } \\ \text { DATE } & \text { LEVEL } & \text { DATE }\end{array}$

JAN 21, $1982 \quad 215.13 \quad$ JAN 11, $1983 \quad 216.80$

GROUND-WATER LEVELS

364940102190001. LOCAL NUMBER; 04N-07E-09 CBB 1. LOCATION,--LAT 36 49'40", LONG 102 19'00", HYDROLOGIC UNIT 11100101. AQUIFER .--OGALLALA.

PERIOD OF RECORD.--1967 TO CURRENT YEAR.

EXTREMES FOR PERIOD OF RECORD.--HIGHEST WATER LEVEL, 176.95 FT(53.93 M) BELOW LAND-SURFACE DATUM,JAN 25, 1967; LOWEST WATER LEVEL,225.70 FT(68.79 M) BELOW LAND-SURFACE DATUM, JAN 21, 1982.

WATER LEVELS IN FEET BELOW LAND SURFACE DATUM, CLIMATIC YEARS APRIL 1981 TO MARCH 1983

\begin{tabular}{|c|c|}
\hline & WATER \\
\hline DATE & LEVEL \\
\hline
\end{tabular}

JAN 21, $1982 \quad 225.70 \quad$ JAN $11,1983 \quad 213.81$ 


\section{GROUND-WATER LEVELS}

CIMARRON COUNTY -- CONTINUED

364850102184001. LOCAL NUMBER; LOCATION,--LAT 36 48'50", LONG 102 AQUIFER. - OGALLALA.

PERIOD OF RECORD.--1967 TO CURRENT YEAR.

EXTREMES FOR PERIOD OF RECORD.--HIGHEST WATER LEVEL, 131.56 FT (40.10 M) BELOW LAND-SURFACE DATUM, JAN 25, 1967; LOWEST WATER LEVEL, 150.13 FT(45.76 M) BELOW LAND-SURFACE DATUM, JAN 22, 1975.

WATER LEVELS IN FEET BELOW LAND SURFACE DATUM, CLIMATIC YEARS APRIL 1981 TO MARCH 1983
04N-07E-16 ACC 1.

$18^{\prime} 40^{\prime \prime}$, HYDROLOGIC UNIT 11100101.
WATER

DATE LEVEL

JAN 21, $1982 \quad 145.90$

$\begin{array}{llcl} & \text { WATER } & & \\ \text { DATE } & \text { LEVEL } & \text { WATER } \\ & & \text { DATE } & \text { LEVEL } \\ \text { 21, 1982 } & 145.90 & \text { JAN 10, 1983 } & 144.04\end{array}$

\section{GROUND-WATER LEVELS}

364850102212001. LOCAL NUMBER; 04N-07E-18 CBB 1. LOCATION,--LAT 36 48'50", LONG 102 21'20", HYDROLOGIC UNIT 11100101. AQUIFER . - OGALLALA.

PERIOD OF RECORD.--1967 TO CURRENT YEAR.

EXTREMES FOR PERIOD OF RECORD.--HIGHEST WATER LEVEL, 154.23 FT(47.01 M) BELOW LAND-SURFACE DATUM,JAN 25, 1967; LOWEST WATER LEVEL, 178.98 FT(54.55 M) BELOW LAND-SURFACE DATUM, JAN $8,1980$.

WATER LEVELS IN FEET BELOW LAND SURFACE DATUM, CLIMATIC YEARS APRIL 1981 TO MARCH 1983

\begin{tabular}{|c|c|c|}
\hline & WATER & \\
\hline DATE & LEVEL & DATE \\
\hline
\end{tabular}

JAN 21, $1982 \quad 174.25$ JAN 10, $1983 \quad 163.02$ 
CIMARRON COUNTY -- CONTINUED

364610102194001. LOCAL NUMBER; LOCATION,--LAT 36 46'10", LONG 102 AQUIFER.--OGALLALA. PLRIOD OF RECORD.--1967 TO CURRENT YEAR. EXTREMES FOR PERIOD OF RECORD.--HIGHEST WATER LEVEL,80.83 FT (24.64 M) BELOW LAND-SURFACE

DATUM, JAN 8, 1980; LOWEST WATER LEVEL,96.50 FT(29.41 M) BELOW LAND-SURFACE DATUM, JAN 15, 1976.

WATER LEVELS IN FEET BELOW LAND SURFACE DATUM, CLIMATIC YEARS APRIL 1981 TO MARCH 1983
04N-07E-32 DBB 1.
$19^{\prime} 40^{\prime \prime}$, HYDROLOGIC UNIT 11100101.

04N-07E-32 DBB 1.
$19^{\prime} 40^{\prime \prime}$, HYDROLOGIC UNIT 11100101.
JAN 20, 1982
0
JAN 11, $1983 \quad 0$

WATER LEVEL

GROUND-WATER LEVELS

365100102125001. LOCAL NUMBER; LOCATION,--LAT 36 51'O0", LONG 102 AQUIFER.--OGALLALA. PERIOD OF RECORD.--1966 TO CURRENT YEAR. EXTREMES FOR PERIOD OF RECORD.--HIGHEST WATER LEVEL, 181.70 FT(55.38 M) BELOW LAND-SURFACE DATUM, NOV 4, 1966; LOWEST WATER LEVEL,205.40 FT(62.61 M) BELOW LAND-SURFACE DATUM, JAN 15, 1974.

WATER LEVELS IN FEET BELOW LAND SURFACE DATUM, CLIMATIC YEARS APRIL 1981 TO MARCH 1983

$04 \mathrm{~N}-08 \mathrm{E}-05$ AAB 1.

12 '50", HYDROLOGIC UNIT 11100101.
JAN 21, 1982

WATER

DATE

WATER

LEVEL

WATER

LEVEL 
GROUND-WATER LEVELS

CIMARRON COUNTY -- CONTINUED

364950102031001. LOCAL NUMBER; $04 \mathrm{~N}-09 \mathrm{E}-11$ ADD 1.

LOCATION,--LAT 36 49'50", LONG 102 03'10", HYDROLOGIC UNIT 11100101.

AQUIFER.--DOCKUM FORMATION.

PERIOD OF RECORD.--1967 TO CURRENT YEAR.

EXTREMES FOR PERIOD OF RECORD.--HIGHEST WATER LEVEL,217.49 FT(66.29 M) BELOW LAND-SURFACE DATUM,JAN 24, 1967; LOWEST WATER LEVEL,225.50 FT(68.73 M) BELOW LAND-SURFACE DATUM, JAN $13,1970$.

WATER LEVELS IN FEET BELOW LAND SURFACE DATUM, CLIMATIC YEARS APRIL 1981 TO MARCH 1983

\begin{tabular}{|c|c|c|c|}
\hline DATE & $\begin{array}{l}\text { WATER } \\
\text { LEVEL }\end{array}$ & DATE & $\begin{array}{l}\text { WATER } \\
\text { LEVEL }\end{array}$ \\
\hline 21,1982 & 220.97 & JAN 11,1983 & 217.88 \\
\hline
\end{tabular}

GROUND-WATER LEVELS

365110102024001. LOCAL NUMBER; 05N-09E-36 CBC 1.

LOCATION,--LAT 36 51'10", LONG 102 02'40", HYDROLOGIC UNIT 11100101.

AQUIFER .--OGALLALA.

PERIOD OF RECORD.--1967 TO CURRENT YEAR.

EXTREMES FOR PERIOD OF RECORD.--HIGHEST WATER LEVEL, 198.67 FT(60.55 M) BELOW LAND-SURFACE DATUM, JAN 18, 1979; LOWEST WATER LEVEL,216.55 FT(66.00 M) BELOW LAND-SURFACE DATUM, JAN $13,1970$.

WATER LEVELS IN FEET BELOW LAND SURFACE DATUM, CLIMATIC YEARS APRIL 1981 TO MARCH 1983

$\begin{array}{lll} & \text { WATER } & \text { WATER } \\ \text { DATE } & \text { LEVEL } & \text { DATE }\end{array}$

JAN 21, $1982198.90 \quad$ JAN $11,1983 \quad 198.77$ 


\section{CIMARRON COUNTY -- CONTINUED}

365730102132001. LOCAL NUMBER; 06N-08E-29 BDC 1. LOCATION,--LAT 36 57'30", LONG 102 13'20", HYDROLOGIC UNIT 11040002.

AQUIFER.--OGALLALA.

PERIOD OF RECORD.--1967 TO CURRENT YEAR.

EXTREMES FOR PERIOD OF RECORD.--HIGHEST WATER LEVEL, 158.46 FT(48.30 M) BELOW LAND-SURFACE DATUM, JAN 16, 1968; LOWEST WATER LEVEL, $193.58 \mathrm{FT}(59.00 \mathrm{M})$ BELOW LAND-SURFACE DATUM, JAN 11, 1983.

WATER LEVELS IN FEET BELOW LAND SURFACE DATUM, CLIMATIC YEARS APRIL 1981 TO MARCH 1983

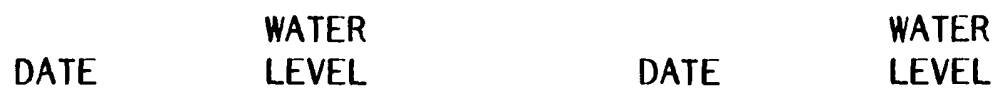

JAN 21, $1982 \quad 192.44 \quad$ JAN 11, $1983 \quad 193.58$

\section{GROUND-WATER LEVELS}

365740102054901. LOCAL NUMBER; 06N-09E-28 BCA 1.

LOCATION,--LAT 36 57'40", LONG 102 05'49", HYDROLOGIC UNIT 11040002.

AQUIFER .--OGALLALA.

PERIOD OF RECORD.-- 1980 TO CURRENT YEAR.

EXTREMES FOR PERIOD OF RECORD.--HIGHEST WATER LEVEL, $141.55 \mathrm{FT}(43.14 \mathrm{M})$ BELOW LAND-SURFACE DATUM, APR 10, 1980; LOWEST WATER LEVEL, 144.35 FT(44.00 M) BELOW LAND-SURFACE DATUM, JAN 13, 1983.

WATER LEVELS IN FEET BELOW LAND SURFACE DATUM, CLIMATIC YEARS APRIL 1981 TO MARCH 1983

\begin{tabular}{|c|c|c|}
\hline & WATER & \\
\hline DATE & LEVEL & DATE \\
\hline
\end{tabular}

JAN 21, $1982143.32 \quad$ JAN 13, $1983 \quad 144.35$ 
GROUND-WATER LEVELS

\section{CLEVELAND COUNTY}

350430097175501. LOCAL NUMBER; 07N-01W-15 CCC 1. LOCATION,--LAT 35 04'30", LONG 097 17'55", HYDROLOGIC UNIT 11090202. AQUIFER .--GARBER SANDSTONE. PERIOD OF RECORD .--1981 TO CURRENT YEAR. EXTREMES FOR PERIOD OF RECORD.--HIGHEST WATER LEVEL,43.71 FT(13.32 M) BELOW LAND-SURFACE DATUM, MAR 7, 1983; LOWEST WATER LEVEL,53.50 FT(16.31 M) BELOW LAND-SURFACE DATUM, SEP $18,1981$.

WATER LEVELS IN FEET BELOW LAND SURFACE DATUM, CLIMATIC YEARS APRIL 1981 TO MARCH 1983

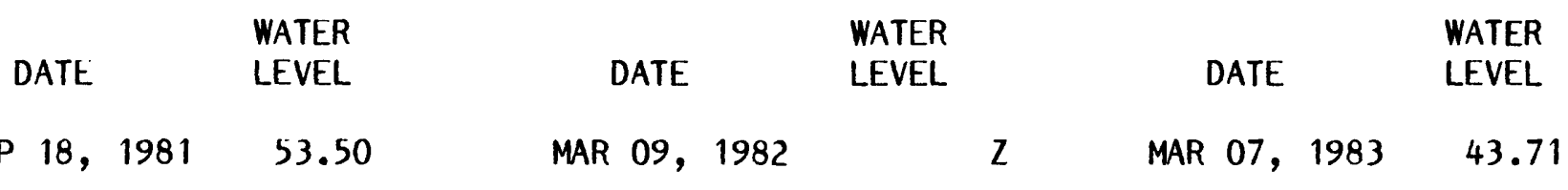

GROUND-WATER LEVELS

350351097165201. LOCAL NUMBER; 07N-01W-23 CBC 1. LOCATION,--LAT 35 03'51", LONG 097 16'52", HYDROLOGIC UNIT 11090202. AQUIFER.--GARBER SANDSTONE. PERIOD OF RECORD.--1981 TO CURRENT YEAR.

EXTREMES FOR PERIOD OF RECORD.--HIGHEST WATER LEVEL, 17.31 FT(5.28 M) BELOW LAND-SURFACE DATUM, MAR 7, 1983; LOWEST WATER LEVEL,63.00 FT(19.20 M) BELOW LAND-SURFACE DATUM, SEP 18, 1981.

WATER LEVELS IN FEET BELOW LAND SURFACE DATUM, CLIMATIC YEARS APRIL 1981 TO MARCH 1983

$\begin{array}{lll} & \text { WATER } & \\ \text { DATE } & \text { LEVEL } & \text { WATER } \\ \text { LEVEL }\end{array}$

$\begin{array}{llll}\text { MAR 09, } 1982 & \text { M } & \text { MAR 07, } 1983 & 17.31\end{array}$ 
GROUND-WATER LEVELS

CLEVELAND COUNTY -- CONTINUED

350812097180301. LOCAL NUMBER; 07N-01W-28 ADD 1.

LOCATION,--LAT 35 08'12", LONG 097 18'03", HYDROLOGIC UNIT 11090202.

AQUIFER .--GARBER SANDSTONE.

PERIOD OF RECORD .- 1981 TO CURRENT YEAR.

EXTREMES FOR PERIOD OF RECORD.--HIGHEST WATER LEVEL,43.29 FT(13.19 M) BELOW LAND-SURFACE DATUM,MAR 7, 1983; LOWEST WATER LEVEL, $49.98 \mathrm{FT}(15.23 \mathrm{M})$ BELOW LAND-SURFACE DATUM, SEP 18, 1981.

WATER LEVELS IN FEET BELOW LAND SURFACE DATUM, CLIMATIC YEARS APRIL 1981 TO MARCH 1983

\begin{tabular}{ccccccc}
\multicolumn{1}{c}{} & WATER & & & WATER & WATER \\
DATE & LEVEL & DATE & LEVEL & DATE & LEVEL \\
SEP 18, 1981 & 49.98 & MAR 09, 1982 & & Z & MAR 07, 1983 & 43.29
\end{tabular}

GROUND-WATER LEVELS

351149097210501. LOCAL NUMBER; 08N-01W-06 CBB 1.

LOCATION,--LAT 35 11'49", LONG 097 21'05", HYDROLOGIC UNIT 11090203.

AQUIFER.--GARBER SANDSTONE.

PERIOD OF RECORD.--1983 TO CURRENT YEAR.

EXTREMES FOR PERIOD OF RECORD.--HIGHEST WATER LEVEL, $51.00 \mathrm{FT}(15.54 \mathrm{M})$ BELOW LAND-SURFACE DATUM, APR 26, 1983; LOWEST WATER LEVEL,51.00 FT(15.54 M) BELOW LAND-SURFACE DATUM, APR 26, 1983.

WATER LEVELS IN FEET BELOW LAND SURFACE DATUM, CLIMATIC YEARS APRIL 1981 TO MARCH 1983

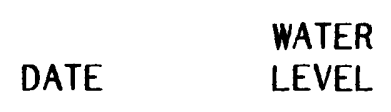

MAR 31, $1983 \quad 51.00$ 


\section{GROUND-WATER LEVELS}

CLEVELAND COUNTY -- CONTINUED

350845097214501. LOCAL NUMBER;

LOCATION,--LAT $3508^{\prime} 45^{\prime \prime}$, LONG 097

AQUIFER.--GARBER SANDSTONE.

PERIOD OF RECORD.--1979 TO CURRENT YEAR.

EXTREMES FOR PERIOD OF RECORD.--HIGHEST WATER LEVEL, 152.10 FT(46.36 M) BELOW LAND-SURFACE

DATUM,MAR 15, 1979; LOWEST WATER LEVEL, 156.18 FT(47.60 M) BELOW LAND-SURFACE DATUM, MAR 3, 1983.

WATER LEVELS IN FEET BELOW LAND SURFACE DATUM, CLIMATIC YEARS APRIL 1981 TO MARCH 1983
$08 \mathrm{~N}-02 \mathrm{~W}-25$ BAA 1.

$21^{\prime} 45^{\prime \prime}$, HYDROLOGIC UNIT 11090202.

\begin{tabular}{|c|c|c|}
\hline & WATER & \\
\hline DATE & LEVEL & DATE \\
\hline
\end{tabular}

MAR 22, $1982 \quad Z \quad$ MAR 03, $1983 \quad 156.18$

GROUND-WATER LEVELS

351301097102301. LOCAL NUMBER; 09N-01E-35 BBA 1.

LOCATION,--LAT 35 13'01", LONG 097 10'23", HYDROLOGIC UNIT 11090203.

AQUIFER.--GARBER SANDSTONE.

PERIOD OF RECORD.--1983 TO CURRENT YEAR.

EXTREMES FOR PERIOD OF RECORD.--HIGHEST WATER LEVEL, $94.10 \mathrm{FT}(28.68 \mathrm{M})$ BELOW LAND-SURFACE DATUM, APR 26, 1983; LOWEST WATER LEVEL,94.10 FT(28.68 M) BELOW LAND-SURFACE DATUM, APR 26, 1983.

WATER LEVELS IN FEET BELOW LAND SURFACE DATUM, CLIMATIC YEARS APRIL 1981 TO MARCH 1983

$\begin{array}{ll}\text { WATER } \\ \text { DATE } & \text { LEVEL }\end{array}$

MAR 31, $1983 \quad 94.10$ 
GROUND-WATER LEVELS

CLEVELAND COUNTY -- CONTINUED

351452097232201. LOCAL NUMBER; 09N-02W-15 DDD 1.

LOCATION,--LAT 35 14'52", LONG 097 23'22", HYDROLOGIC UNIT 11090203.

AQUIFER.--GARBER SANDSTONE.

PERIOD OF RECORD.--1975 TO CURRENT YEAR.

EXTREMES FOR PERIOD OF RECORD.--HIGHEST WATER LEVEL,235.00 FT(71.63 M) BELOW LAND-SURFACE DATUM,FEB 25, 1980; LOWEST WATER LEVEL, $428.00 \mathrm{FT}(130.45 \mathrm{M})$ BELOW LAND-SURFACE DATUM, JUNE 26, 1975.

WATER LEVELS IN FEET BELOW LAND SURFACE DATUM, CLIMATIC YEARS APRIL 1981 TO MARCH 1983

\begin{tabular}{|c|c|c|}
\hline DATE & $\begin{array}{l}\text { WATER } \\
\text { LEVEL }\end{array}$ & DATE \\
\hline
\end{tabular}

MAR 22, $1982 \quad 244.00 \quad$ MAR 09, $1983 \quad 261.00$

GROUND-WATER LEVELS

351426097232201. LOCAL NUMBER; 09N-02W-22 ADD 1. LOCATION,--LAT 35 14'26", LONG 097 23'22", HYDROLOGIC UNIT 11090203. AQUIFER .--GARBER SANDSTONE. PERIOD OF RECORD.--1976 TO CURRENT YEAR. EXTREMES FOR PERIOD OF RECORD.--HIGHEST WATER LEVEL, 170.00 FT(51.82 M) BELOW LAND-SURFACE DATUM, MAR 29, 1976; LOWEST WATER LEVEL,290.00 FT(88.39 M) BELOW LAND-SURFACE DATUM, JAN 2, 1981.

WATER LEVELS IN FEET BELOW LAND SURFACE DATUM, CLIMATIC YEARS APRIL 1981 TO MARCH 1983

$\begin{array}{lll}\text { WATER } & \text { WATER } \\ \text { DATE } & \text { LEVEL } & \text { DATE } \\ \text { LEVEL }\end{array}$

MAR 22, $1982 \quad 202.00 \quad$ MAR 09, $1983 \quad 215.00$ 
CLEVELAND COUNTY -- CONTINUED

351314097254501. LOCAL NUMBER; 09N-02W-29 DCA 1. LOCATION,--LAT 35 13'14", LONG 097 25'45", HYDROLOGIC UNIT 11090202.

AQUIFER.--GARBER SANDSTONE.

PERIOD OF RECORD.--1976 TO CURRENT YEAR.

EXTREMES FOR PERIOD OF RECORD.--HIGHEST WATER LEVEL, $105.20 \mathrm{FT}(32.06 \mathrm{M})$ BELOW LAND-SURFACE DATUM,MAR 29, 1976; LOWEST WATER LEVEL,465.00 FT(141.73 M) BELOW LAND-SURFACE DATUM, JAN 2, 1981.

WATER LEVELS IN FEET BELOW LAND SURFACE DATUM, CLIMATIC YEARS APRIL 1981 TO MARCH 1983

\begin{tabular}{|c|c|}
\hline & WATER \\
\hline DATE & LEVEL \\
\hline
\end{tabular}

MAR 22, $1982 \quad 460.00 \quad$ MAR 09, $1983 \quad 437.20$

GROUND-WATER LEVELS

351643097285601. LOCAL NUMBER; 09N-03W-02 DCA 1.

LOCATION,--LAT 35 16'43", LONG 097 28'56", HYDROLOGIC UNIT 11090203.

AQUIFER.--GARBER SANDSTONE.

PERIOD OF RECORD.--1975 TO CURRENT YEAR.

EXTREMES FOR PERIOD OF RECORD.--HIGHEST WATER LEVEL, $303.00 \mathrm{FT}(92.35 \mathrm{M})$ BELOW LAND-SURFACE DATUM, JUNE 26, 1975; LOWEST WATER LEVEL,467.00 FT(142.34 M) BELOW LAND-SURFACE DATUM, FEB 25, 1980.

WATER LEVELS IN FEET BELOW LAND SURFACE DATUM, CLIMATIC YEARS APRIL 1981 TO MARCH 1983

$\begin{array}{llcl} & \text { WATER } & & \\ \text { DATE } & \text { LEVEL } & \text { WATER } \\ \text { LATE } & \text { LEVEL } \\ \text { R 22, 1982 } & 403.00 & \text { MAR 09, 1983 } & 356.00\end{array}$


351557097292001. LOCAL NUMBER; LOCATION,--LAT 35 15'57", LONG 097 AQUIFER.--GARBER SANDSTONE. PERIOD OF RECORD.--1983 TO CURRENT EXTREMES FOR PERIOD OF RECORD.--HIGHEST WATER LEVEL, $407.50 \mathrm{FT}(124.21 \mathrm{M})$ BELOW LAND-SURFACE DATUM,MAR 3, 1983; LOWEST WATER LEVEL,407.50 FT(124.21 M) BELOW LAND-SURFACE DATUM, MAR 3, 1983.

WATER LEVELS IN FEET BELOW LAND SURFACE DATUM, CLIMATIC YEARS APRIL 1981 T0 MARCH 1983

\section{GROUND-WATER LEVELS}

351413097285601. LOCAL NUMBER; LOCATION, --LAT 35 14'13", LONG 097 AQUIFER.--GARBER SANDSTONE. PERIOD OF RECORD.--1975 TO CURRENT YEAR. EXTREMES FOR PERIOD OF RECORD.--HIGHEST WATER LEVEL, $336.50 \mathrm{FT}(102.57 \mathrm{M})$ BELOW LAND-SURFACE DATUM,MAR 3, 1983; LOWEST WATER LEVEL,444.00 FT(135.33 M) BELOW LAND-SURFACE DATUM, FEB 26, 1980.

WATER LEVELS IN FEET BELOW LAND SURFACE DATUM, CLIMATIC YEARS APRIL 1981 TO MARCH 1983
09N-03W-23 DBD 1. 28 '56", HYDROLOGIC UNIT 11090202.

$\begin{array}{ll} & \text { WATER } \\ \text { DATE } & \text { LEVEL }\end{array}$

MAR 03, $1983 \quad 336.50$ 


\section{GROUND-WATER LEVELS}

\section{CLEVELAND COUNTY -- CONTINUED}

352137097165201. LOCAL NUMBER; LOCATION,--LAT 35 21'37", LONG 097 AQUIFER.--GARBER SANDSTONE.

PERIOD OF RECORD.--1979 TO CURRENT YEAR. EXTREMES FOR PERIOD OF RECORD.--HIGHEST WATER LEVEL, 14.00 FT(4.27 M) BELOW LAND-SURFACE DATUM,MAR 30, 1979; LOWEST WATER LEVEL, 14.32 FT(4.36 M) BELOW LAND-SURFACE DATUM, APR 9, 1982.

WATER LEVELS IN FEET BELOW LAND SURFACE DATUM, CLIMATIC YEARS APRIL 1981 TO MARCH 1983

$10 \mathrm{~N}-01 \mathrm{~W}-11 \mathrm{BBC} 1$

16'52", HYDROLOGIC UNIT 11090203.

LEVEL, 14.32 FT(4.36 M) BELOW LAND-SURFACE DATUM,

\begin{abstract}
WATER LEVELS IN
\end{abstract}

$\begin{array}{ll} & \text { WATER } \\ \text { DATE } & \text { LEVEL }\end{array}$

MAR $31,1982 \quad 14.32$

$\begin{array}{ll} & \text { WATER } \\ \text { DATE } & \text { LEVEL }\end{array}$

MAR 07, 1983

Z

MAR 31,1983

Z

GROUND-WATER LEVELS

331913097262501. LOCAL NUMBER; 10N-02W-20 CCC 1. LOCATION,--LAT 35 19'13", LONG 097 26'25", HYDROLOGIC UNIT 11090203. AQUIFER.--GARBER SANDSTONE.

PERIOD OF RECORD.--1980 TO CURRENT YEAR.

EXTREMES FOR PERIOD OF RECORD.--HIGHEST WATER LEVEL, $125.55 \mathrm{FT}(38.27 \mathrm{M})$ BELOW LAND-SURFACE DATUM,FEB 26, 1980; LOWEST WATER LEVEL, 129.49 FT(39.47 M) BELOW LAND-SURFACE DATUM, APR 16, 1981.

WATER LLVELS IN FEET BELOW LAND SURFACE DATUM, CLIMATIC YEARS APRIL 1981 TO MARCH 1983
DATE
WATER
LEVEL
DATE
WATER
MAR 08, $1982 \quad 128.80$
MAR 07, 1983
Z 
GROUND-WATER LEVELS

CLEVELAND COUNTY -- CONTINUED

352137097302301. LOCAL NUMBER; 10N-03W-10 BAC 1. LOCATION,--LAT 35 21'37", LONG 097 30'23", HYDROLOGIC UNIT 11090203. AQUIFER.--GARBER SANDSTONE.

PERIOD OF RECORD.--1975 TO CURRENT YEAR. EXTREMES FOR PERIOD OF RECORD.--HIGHEST WATER LEVEL, 343.92 FT(104.83 M) BELOW LAND-SURFACE DATUM,DEC 29, 1977; LOWEST WATER LEVEL, $545.92 \mathrm{FT}(166.40 \mathrm{M})$ BELOW LAND-SURFACE DATUM, MAR 7, 1983.

WATER LEVELS IN FEET BELOW LAND SURFACE DATUM, CLIMATIC YEARS APRIL 1981 TO MARCH 1983

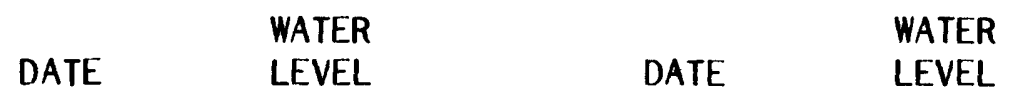

MAR 16, $1982 \quad 470.00 \mathrm{R} \quad$ MAR 07, $1983 \quad 545.92 \mathrm{R}$

GROUND-WATER LEVELS

352025097293601. LOCAL NUMBER; 10N-03W-14 CBB 1. LOCATION,--LAT 35 20'25", LONG 097 29'36", HYDROLOGIC UNIT 11090203. AQUIFER.--GARBER SANDSTONE. PERIOD OF RECORD.--1975 TO CURRENT YEAR. EXTREMES FOR PERIOD OF RECORD.--HIGHEST WATER LEVEL, 274.84 FT(83.77 M) BELOW LAND-SURFACE DATUM,DEC 28, 1976; LOWEST WATER LEVEL,471.00 FT(143.56 M) BELOW LAND-SURFACE DATUM, MAR 7, 1983.

WATER LEVELS IN FEET BELOW LAND SURFACE DATUM, CLIMATIC YEARS APRIL 1981 TO MARCH 1983

$\begin{array}{lll} & \text { WATER } & \\ \text { DATE } & \text { LEVEL } & \text { WATER } \\ & \text { LATE } & \text { LEVEL }\end{array}$

MAR $16,1982 \quad 410.00 \mathrm{R} \quad$ MAR 07, $1983 \quad 471.00$ 
351939097302301. LOCAL NUMBER; 10N-03W-22 BDC 1.

LOCATION,--LAT 35 19'39", LONG 097 30'23", HYDROLOGIC UNIT 11090202.

AQUIFER.--GARBER SANDSTONE.

PERIOD OF RECORD. --1975 TO CURRENT YEAR.

EXTREMES FOR PERIOD OF RECORD.--HIGHEST WATER LEVEL,295.50 FT(90.07 M) BELOW LAND-SURFACE DATUM,MAR 14, 1979; LOWEST WATER LEVEL,548.50 FT(167.18 M) BELOW LAND-SURFACE DATUM, MAR 23, 1983.

WATER LEVELS IN FEET BELOW LAND SURFACE DATUM, CLIMATIC YEARS APRIL 1981 TO MARCH 1983

$\begin{array}{lll} & \text { WATER } & \text { WATER } \\ \text { DATE } & \text { LEVEL } & \text { DATE }\end{array}$

FEB 16, $1982 \quad 380.00 \mathrm{R} \quad$ MAR 23, $1983 \quad 548.50$

GROUND-WATER LEVELS

351814097273601. LOCAL NUMBER; 10N-03W-36 AAA 1. LOCATION,--LAT 35 18'14", LONG 097 27'36", HYDROLOGIC UNIT 11090203. AQUIFER .--GARBER SANDSTONE. PERIOD OF RECORD.--1979 TO CURRENT YEAR. EXTREMES FOR PERIOD OF RECORD.--HIGHEST WATER LEVEL,214.50 FT(65.38 M) BELOW LAND-SURFACE DATUM,MAR 15, 1979; LOWEST WATER LEVEL,503.30 FT(153.41 M) BELOW LAND-SURFACE DATUM, MAR 23, 1983.

WATER LEVELS IN FEET BELOW LAND SURFACE DATUM, CLIMATIC YEARS APRIL 1981 TO MARCH 1983

$\begin{array}{lll} & \text { WATER } & \\ \text { DATE } & \text { LEVEL } & \text { WATER } \\ & \text { DATE } & \text { LEVEL }\end{array}$

MAR 16, $1982 \quad 410.00 \mathrm{R} \quad$ MAR 23, $1983 \quad 503.30 \mathrm{R}$ 
GROUND-WATER LEVELS

CLEVELAND COUNTY -- CONTINUED

351952097362101. LOCAL NUMBER;

10N-04W-22 ABD 1. LOCATION, --LAT 3519 '52", LONG 097 AQUIFER.--GARBER SANDSTONE. PERIOD OF RECORD.--1975 TO CURRENT YEAR.

EXTREMES FOR PERIOD OF RECORD.--HIGHEST WATER LEVEL,294.78 FT(89.85 M) BELOW LAND-SURFACE DATUM,FEB 4, 1976; LOWEST WATER LEVEL,402.96 FT(122.82 M) BELOW LAND-SURFACE DATUM, MAR 23, 1983.

WATER LEVELS IN FEET BELOW LAND SURFACE DATUM, CLIMATIC YEARS APRIL 1981 TO MARCH 1983

\begin{tabular}{ccccc}
\multicolumn{1}{c}{$\begin{array}{c}\text { WATER } \\
\text { LEVEL }\end{array}$} & DATE & WATER \\
DATE & LEVEL \\
LAR 31, 1982 & & $Z$ & MAR 23, 1983 & 402.96
\end{tabular}




\section{GROUND-WATER LEVELS}

COAL COUNTY

343634096204901 . LOCAL NUMBER

02N-09E-27 CDD 1.

LOCATION,--LAT 34 36'34", LONG 096 20'49", HYDROLOGIC UNIT 11140104.

AQUIFER.-.SAVANNA FORMATION.

PERIOD OF RECORD.--1981 TO CURRENT YEAR.

EXTREMES FOR PERIOD OF RECORD.--HIGHEST WATER LEVEL,1.52 FT(.46 M) BELOW LAND-SURFACE DATUM,MAR 11, 1983; LOWEST WATER LEVEL, 15.04 FT(4.58 M) BELOW LAND-SURFACE DATUM, MAR 6, 1981.

WATER LEVELS IN FEET BELOW LAND SURFACE DATUM, CLIMATIC YEARS APRIL 1981 TO MARCH 1983

\begin{tabular}{lrrrr} 
DATE & $\begin{array}{c}\text { WATER } \\
\text { LEVEL }\end{array}$ & DATE & \multicolumn{1}{l}{ WATER } \\
LEVEL
\end{tabular}




\section{GROUND-WATER LEVELS}

COMANCHE COUNTY

344630098095901. LOCAL NUMBER

04N-09W-32 DBB 1.

LOCATION,--LAT 34 46'30", LONG 098 09'59", HYDROLOGIC UNIT 11130208.

AQUIFER.--RUSH SPRINGS FORMATION.

PERIOD OF RECORD.--1983 TO CURRENT YEAR.

EXTREMES FOR PERIOD OF RECORD.--HIGHEST WATER LEVEL, 101.94 FT(31.07 M) BELOW LAND-SURFACE DATUM,JAN 24, 1983; LOWEST WATER LEVEL, $101.94 \mathrm{FT}(31.07 \mathrm{M})$ BELOW LAND-SURFACE DATUM, JAN 24, 1983.

WATER LEVELS IN FEET BELOW LAND SURFACE DATUM, CLIMATIC YEARS APRIL 1981 TO MARCH 1983

$\begin{array}{ll} & \text { WATER } \\ \text { DATE } & \text { LEVEL }\end{array}$

JAN 24, $1983 \quad 101.94$

\section{GROUND-WATER LEVELS}

344630098083201. LOCAL NUMBER; 04N-09W-33 DAA 1.

LOCATION,--LAT 34 46'30", LONG 098 08'32", HYDROLOGIC UNIT 11130208.

AQUIFER .--RUSH SPRINGS FORMATION.

PERIOD OF RECORD._-1983 TO CURRENT YEAR.

EXTREMES FOR PERIOD OF RECORD.--HIGHEST WATER LEVEL, $149.09 \mathrm{FT}(45.44 \mathrm{M})$ BELOW LAND-SURFACE DATUM,MAR 15, 1983; LOWEST WATER LEVEL, 149.09 FT(45.44 M) BELOW LAND-SURFACE DATUM, MAR 15, 1983.

WATER LEVELS IN FEET BELOW LAND SURFACE DATUM, CLIMATIC YEARS APRIL 1981 TO MARCH 1983

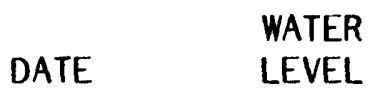

MAR $15,1983 \quad 149.09$ 
COMANCHE COUNTY -- CONTINUED

$\begin{array}{ll}344940098175201 . \text { LOCAL NUMBER; } & \text { 04N-10W-07 CCC 1. } \\ \text { LOCATION,--LAT } 34 \text { 49'40", LONG 098 } & 17^{\prime} 52^{\prime \prime}, \text { HYDROLOGIC UNIT } 11130202 .\end{array}$

AQUIFER.--ALLUVIUM.

PERIOD OF RECORD.--1979 TO CURRENT YEAR.

EXTREMES FOR PERIOD OF RECORD.--HIGHEST WATER LEVEL, 3.44 FT(1.05 M) BELOW LAND-SURFACE DATUM,FEB 2, 1983; LOWEST WATER LEVEL,8.30 FT(2.53 M) BELOW LAND-SURFACE DATUM, MAR 25, 1981.

WATER LEVELS IN FEET BELOW LAND SURFACE DATUM, CLIMATIC YEARS APRIL 1981 TO MARCH 1983

\begin{tabular}{crrrr}
\multicolumn{1}{c}{} & WATER & & \multicolumn{1}{c}{ WATER } \\
DATE & LEVEL & DATE & \multicolumn{1}{l}{ LEVEL } \\
MAR 25, 1982 & 5.50 & FEB 02, 1983 & 3.44
\end{tabular}

GROUND-WATER LEVELS

342911098374701. LOCAL NUMBER; 01S-14W-12 BCC 1. LOCATION,--LAT 34 29'11", LONG 098 37'47", HYDROLOGIC UNIT 11130203.

AQUIFER . - WANN FORMATION.

PERIOD OF RECORD.--1981 TO CURRENT YEAR.

EXTREMES FOR PERIOD OF RECORD.--HIGHEST WATER LEVEL,26.22 FT(7.99 M) BELOW LAND-SURFACE DATUM,FEB 8, 1983; LOWEST WATER LEVEL,27.17 FT(8.28 M) BELOW LAND-SURFACE DATUM, MAR 24, 1981.

WATER LEVELS IN FEET BELOW LAND SURFACE DATUM, CLIMATIC YEARS APRIL 1981 TO MARCH 1983

\begin{tabular}{|c|c|c|}
\hline & WATER & \\
\hline DATE & LEVEL & DATE \\
\hline
\end{tabular}

MAR 25, $1982 \quad 26.23 \quad$ FEB 08, $1983 \quad 26.22$ 
342232098281901. LOCAL NUMBER; 02S-12W-21 BBB 1. LOCATION,--LAT 34 22'32", LONG 098 28'19", HYDROLOGIC UNIT 11130203. AQUIFER.--ALLUVIUM. PERIOD OF RECORD.--1983 TO CURRENT YEAR. EXTREMES FOR PERIOD OF RECORD.--HIGHEST WATER LEVEL, 19.16 FT(5.84 M) BELOW LAND-SURFACE DATUM,FEB 8, 1983; LOWEST WATER LEVEL, 19.16 FT(5.84 M) BELOW LAND-SURFACE DATUM, FEB 8, 1983.

WATER LEVELS IN FEET BELOW LAND SURFACE DATUM, CLIMATIC YEARS APRIL 1981 TO MARCH 1983

$\begin{array}{ll} & \text { WATER } \\ \text { DATE } & \text { LEVEL }\end{array}$

FEB $08,1983 \quad 19.16$

GROUND-WATER LEVELS

341625098170201 . LOCAL NUMBER; 03S-10W-30 AAB 1. LOCATION,--LAT 34 16'25", LONG 098 17'02", HYDROLOGIC UNIT 11130202. AQUIFER .--ALLUVIUM.

PERIOD OF RECORD.--1979 TO CURRENT YEAR. EXTREMES FOR PERIOD OF RECORD.--HIGHEST WATER LEVEL, 10.57 FT(3.22 M) BELOW LAND-SURFACE DATUM,FEB 8, 1983; LOWEST WATER LEVEL, $14.90 \mathrm{FT}(4.54 \mathrm{M})$ BELOW LAND-SURFACE DATUM, MAR $25,1981$.

WATER LEVELS IN FEET BELOW LAND SURFACE DATUM, CLIMATIC YEARS APRIL 1981 TO MARCH 1983

\begin{tabular}{|c|c|c|}
\hline & WATER & \\
\hline DATE & LEVEL & DATE \\
\hline
\end{tabular}

MAR 25, $1982 \quad 13.35 \quad$ FEB $08,1983 \quad 10.57$ 


\section{GROUND-WATER LEVELS}

CRAIG COUNTY

363220095132701. LOCAL NUMBER;

$24 \mathrm{~N}-20 \mathrm{E}-30$ BBB 1.

LOCATION,--LAT 36 32'20", LONG 095

13'27", HYDROLOGIC UNIT 11070209.

AQUIFER.--BOONE FORMATION.

PERIOD OF RECORD.--1979 TO CURRENT YEAR.

EXTREMES FOR PERIOD OF RECORD.--HIGHEST WATER LEVEL, $1.05 \mathrm{FT}(.32 \mathrm{M})$ BELOW LAND-SURFACE DATUM,MAR 8, 1983; LOWEST WATER LEVEL,4.68 FT(1.43 M) BELOW LAND-SURFACE DATUM, MAR 11, 1981.

WATER LEVELS IN FEET BELOW LAND SURFACE DATUM, CLIMATIC YEARS APRIL 1981 TO MARCH 1983

$\begin{array}{lll} & \text { WATER } & \text { WATER } \\ \text { DATE } & \text { LEVEL } & \text { DATE }\end{array}$

MAR $30,1982 \quad Z \quad$ MAR 08, $1983 \quad 1.05$

GROUND-WATER LEVELS

363918093071601. LOCAL NUMBER; 25N-20E-13 BAA 1.

LOCATION,--LAT 36 39'18", LONG 095 07'16", HYDROLOGIC UNIT 11070209.

AQUIFER.--ALLUVIUM.

PERIOD OF RECORD.--1979 TO CURRENT YEAR.

EXTREMES FOR PERIOD OF RECORD.--HIGHEST WATER LEVEL, $1.69 \mathrm{FT}(.52 \mathrm{M})$ BELOW LAND-SURFACE DATUM,FEB 28, 1980; LOWEST WATER LEVEL,2.62 FT(.54 M) BELOW LAND-SURFACE DATUM MAR 30,1982 .

WATER LEVELS IN FEET BELOW LAND SURFACE DATUM, CLIMATIC YEARS APRIL 1981 TO MARCH 1983

\begin{tabular}{|c|c|}
\hline & WATER \\
\hline DATE & LEVEL \\
\hline
\end{tabular}

MAR 30, $1982 \quad 2.62 \quad$ MAR 08, $1983 \quad \mathrm{~N}$




\section{GROUND-WATER LEVELS}

CRAIG COUNTY -- CONTINUED

364438095043501. LOCAL NUMBER; LOCATION,--LAT 36 44'38", LONG 095 AQUIFER.--SAVANNA FORMATION. PERIOD OF RECORD.-- 1977 TO CURRENT YEAR. EXTREMES FOR PERIOD OF RECORD.--HIGHEST WATER LEVEL, $1.00 \mathrm{FT}(.30 \mathrm{M})$ BELOW LAND-SURFACE

DATUM,MAR 12, 1979; LOWEST WATER LEVEL,7.41 FT(2.26 M) BELOW LAND-SURFACE DATUM, MAR 11, 1981.

WATER LEVELS IN FEET BELOW LAND SURFACE DATUM, CLIMATIC YEARS APRIL 1981 TO MARCH 1983
26N-21E-08 DDD 1.

04'35", HYDROLOGIC UNIT 11070209.
WATER

DATE

MAR 30, 1982
LEVEL

2.70 
GROUND-WATER LEVELS

CREEK COUNTY

355749096352001. LOCAL NUMBER; 17N-07E-08 CBD 1.

LOCATION,--LAT 35 57'49", LONG 096 35'20", HYDROLOGIC UNIT 11050003.

AQUIFER.--VAMOOSA.

PERIOD OF RECORD.--1977 TO CURRENT YEAR.

EXTREMES FOR PERIOD OF RECORD.--HIGHEST WATER LEVEL, $37.50 \mathrm{FT}(11.43 \mathrm{M})$ BELOW LAND-SURFACE DATUM, MAR 30, 1981; LOWEST WATER LEVEL,212.50 FT(64.77 M) BELOW LAND-SURFACE DATUM, MAR 24, 1977.

WATER LEVELS IN FEET BELOW LAND SURFACE DATUM, CLIMATIC YEARS APRIL 1981 TO MARCH 1983

\begin{tabular}{|c|c|c|}
\hline DATF & $\begin{array}{l}\text { WATER } \\
\text { LFVEI }\end{array}$ & \\
\hline & & DAIE \\
\hline
\end{tabular}

MAR 05, $1982 \quad P \quad$ FEB 18, $1983 \quad 84.50$

GROUND-WATER LEVELS

360717096202801. LOCAL NUMBER; 19N-09E-15 CCA 1. LOCATION,--LAT 36 07'17", LONG 096 20'28", HYDROLOGIC UNIT 11050003. AQUIFER . --VAMOOSA.

PERIOD OF RECORD.--1980 TO CURRENT YEAR.

EXTREMES FOR PERIOD OF RECORD.--HIGHEST WATER LEVEL, $25.20 \mathrm{FT}(7.68 \mathrm{M})$ BELOW LAND-SURFACE DATUM,FEB 8, 1983; LOWEST WATER LEVEL,33.09 FT(10.09 M) BELOW LAND-SURFACE DATUM, MAR 25, 1981.

WATER LEVELS IN FEET BELOW LAND SURFACE DATUM, CLIMATIC YEARS APRIL 1981 TO MARCH 1983

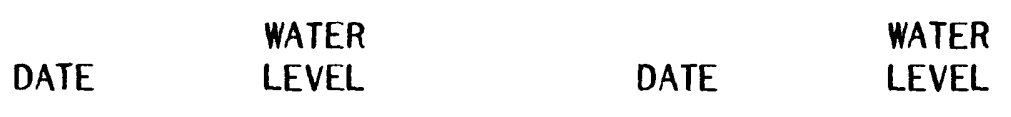

FEB 23, $1982 \quad 32.72 \quad$ FEB 08, $1983 \quad 25.20$ 


\section{GROUND-WATER LEVELS}

\section{CUSTER COUNTY}

353252098401901. LOCAL NUMBER;

$12 \mathrm{~N}-14 \mathrm{~W}-03$ BBD 1.

LOCATION,--LAT 35 32'52", LONG 098 40'19", HYDROLOGIC UNIT 11090201. AQUIFER.--RUSH SPRINGS FORMATION.

PERIOD OF RECORD.--1978 TO CURRENT YEAR.

EXTREMES FOR PERIOD OF RECORD.--HIGHEST WATER LEVEL, 70.22 FT (21.40 M) BELOW LAND-SURFACE DATUM, MAR 28, 1979; LOWEST WATER LEVEL,84.70 FT(25.82 M) BELOW LAND-SURFACE DATUM, MAR 22, 1983.

WATER LEVELS IN FEET BELOW LAND SURFACE DATUM, CLIMATIC YEARS APRIL 1981 TO MARCH 1983

\begin{tabular}{|c|c|}
\hline & WATER \\
\hline DATE & LEVEL \\
\hline
\end{tabular}

MAR 08, $1982 \quad 84.22 \mathrm{R} \quad$ MAR 22, $1983 \quad 84.70$

GROUND-WATER LEVELS

352828097475401. LOCAL NUMBER; 12N-15W-33 BCA 1.

LOCATION,--LAT 35 28'28", LONG 097 47'54", HYDROLOGIC UNIT 11130302.

AQUIFER.--RUSH SPRINGS FORMATION.

PERIOD OF RECORD.--1978 TO CURRENT YEAR.

EXTREMES FOR PERIOD OF RECORD.--HIGHEST WATER LEVEL, 67.39 FT(20.54 M) BELOW LAND-SURFACE DATUM,MAR 22, 1983; LOWEST WATER LEVEL,75.15 FT(22.91 M) BELOW LAND-SURFACE DATUM, MAR 28, 1979.

WATER LEVELS IN FEET BELOW LAND SURFACE DATUM, CLIMATIC YEARS APRIL 1981 TO MARCH 1983

$\begin{array}{lll} & \text { WATER } & \\ \text { DATE } & \text { LEVEL } & \text { WATER } \\ & \text { DATE } & \text { LEVEL }\end{array}$

$\begin{array}{llll}\text { MAR } 08,1982 & P & \text { MAR 22, } 1983 \quad 67.39\end{array}$ 
353650098430901 . LOCAL NUMBER; LOCATION,--LAT 35 36'50", LONG 098 AQUIFER.--RUSH SPRINGS FORMATION. PERIOD OF RECORD.--1979 TO CURRENT EXTREMES FOR PERIOD OF RECORD.--HIGHEST WATER LEVEL, 75.37 FT(22.97 M) BELOW LAND-SURFACE DATUM, MAR 22, 1983; LOWEST WATER LEVEL,76.70 FT(23.38 M) BELOW LAND-SURFACE DATUM, MAR 8, 1982. WATER LEVELS IN FEET BELOW LAND SURFACE DATUM, CLIMATIC YEARS APRIL 1981 TO MARCH 1983
$13 \mathrm{~N}-14 \mathrm{~W}-08 \mathrm{CBC} 1$.

43'09', HYDROLOGIC UNIT 11090201.

YEAR.

LEVEL,76.70 FT(23.38 M) BELOW LAND-SURFACE DATUM,
DATE
WATER
LEVEL
DATE
WATER
LEVEL
MAR 08, $1982 \quad 76.70$
MAR 22, $1983 \quad 75.37$

\section{GROUND-WATER LEVELS}

353716098384501. LOCAL NUMBER; 13N-14W-12 BBD 1. LOCATION,--LAT 35 37'16", LONG 098 38'45", HYDROLOGIC UNIT 11090201. AQUIFER.--ALLUVIUM.

PERIOD OF RECORD.--1979 TO CURRENT YEAR.

EXTREMES FOR PERIOD OF RECORD.--HIGHEST WATER LEVEL, 40.00 FT(12.19 M) BELOW LAND-SURFACE DATUM,MAR 29, 1979; LOWEST WATER LEVEL,41.51 FT(12.65 M) BELOW LAND-SURFACE DATUM, MAR $8,1982$.

WATER LEVELS IN FEET BELOW LAND SURFACE DATUM, CLIMATIC YEARS APRIL 1981 TO MARCH 1983

$\begin{array}{lll} & \text { WATER } & \text { WATER } \\ \text { DATE } & \text { LEVEL } & \text { DATE }\end{array}$

MAR 08, $1982 \quad 41.51 \quad$ MAR 22, $1983 \quad 40.05$


CUSTER COUNTY -- CONTINUED

353927098402101. LOCAL NUMBER; 14N-14W-27 DBD 1. LOCATION,--LAT 35 39'27", LONG 098 40'21", HYDROLOGIC UMIT 11090201. AQUIFER.--RUSH SPRINGS FORMATION. PERIOD OF RECORD.--1979 TO CURRENT YEAR. EXTREMES FOR PERIOD OF RECORD.--HIGHEST WATER LEVEL,28.60 FT(8.72 M) BELOW LAND-SURFACE DATUM, MAR 22, 1983; LOWEST WATER LEVEL, 30.62 FT(9.33 M) BELOW LAND-SURFACE DATUM, MAR 8, 1982.

WATER LEVELS IN FEET BELOW LAND SURFACE DATUM, CLIMATIC YEARS APRIL 1981 TO MARCH 1983

\begin{tabular}{|c|c|c|}
\hline ATE & $\begin{array}{l}\text { WATER } \\
\text { LEVEL }\end{array}$ & \\
\hline ATE & LEVEL & DATE \\
\hline
\end{tabular}

MAR 08, $1982 \quad 30.62 \quad$ MAR 22, $1983 \quad 28.60$

GROUND-WATER LEVELS

353920098415701. LOCAL NUMBER; 14N-14W-28 CCA 1. LOCATION,--LAT 35 39'20", LONG 098 41'57", HYDROLOGIC UNIT 11090201. AQUIFER.--ALLUVIUM.

PERIOD OF RECORD.--1979 TO CURRENT YEAR.

EXTREMES FOR PERIOD OF RECORD.--HIGHEST WATER LEVEL, 38.12 FT(11.62 M) BELOW LAND-SURFACE DATUM,MAR 28, 1979; LOWEST WATER LEVEL,39.25 FT(11.96 M) BELOW LAND-SURFACE DATUM, MAR 8, 1982.

WATER LEVELS IN FEET BELOW LAND SURFACE DATUM, CLIMATIC YEARS APRIL 1981 TO MARCH 1983

$\begin{array}{lll} & \text { WATER } & \\ \text { DATE } & \text { LEVEL } & \text { WATER } \\ & \text { DATE } & \text { LEVEL }\end{array}$

MAR 08, $1982 \quad 39.25 \quad$ MAR 22, $1983 \quad 38.30$ 


\section{GROUND-WATER LEVELS}

CUSTER COUNTY -- CONTINUED

354401098434901. LOCAL NUMBER;

LOCATION,--LAT 35 44'01", LONG 098

$15 N-14 W-31$ BDD 1 .

AQUIFER.--RUSH SPRINGS FORMATION.

PERIOD OF RECORD.--1979 TO CURRENT YEAR.

EXTREMES FOR PERIOD OF RECORD.--HIGHEST WATER LEVEL,46.86 FT(14.28 M) BELOW LAND-SURFACE DATUM, MAR 28, 1979; LOWEST WATER LEVEL,77.02 FT(23.48 M) BELOW LAND-SURFACE DATUM, MAR 8, 1982.

WATER LEVELS IN FEET BELOW LAND SURFACE DATUM, CLIMATIC YEARS APRIL 1981 TO MARCH 1983

\begin{tabular}{|c|c|}
\hline & WATER \\
\hline DATE & LEVEL \\
\hline
\end{tabular}

MAR 08, $1982 \quad 77.02 \mathrm{~S}$ MAR 22, $1983 \quad 63.30$ 
GROUND-WATER LEVELS

DELAWARE COUNTY

361415094452501 . LOCAL NUMBER;

20N-24E-04 DCA 1.

LOCATION,--LAT 36 14'15", LONG 094 45'25", HYDROLOGIC UNIT 11110103.

AQUIFER.--KEOKUK L.S..

PERIOD OF RECORD.--1977 TO CURRENT YEAR.

EXTREMES FOR PERIOD OF RECORD.--HIGHEST WATER LEVEL, 16.13 FT(4.92 M) BELOW LAND-SURFACE DATUM,MAR 22, 1978; LOWEST WATER LEVEL, $32.90 \mathrm{FT}(10.03 \mathrm{M})$ BELOW LAND-SURFACE DATUM, MAR 10, 1981.

WATER LEVELS IN FEET BELOW LAND SURFACE DATUM, CLIMATIC YEARS APRIL 1981 TO MARCH 1983

\begin{tabular}{|c|c|c|}
\hline & WATER & \\
\hline DATE & LEVEL & DATE \\
\hline
\end{tabular}

MAR 29, $1982 \quad 28.48 \quad$ MAR $11,1983 \quad 24.50$

GROUND-WATER LEVELS

362456094581801. LOCAL NUMBER; 22N-22E-04 CAB 1.

LOCATION,--LAT 36 24'56", LONG 094 58'18", HYDROLOGIC UNIT 11070209.

AQUIFER.--BOONE FORMATION.

PERIOD OF RECORD.--1979 TO CURRENT YEAR.

EXTREMES FOR PERIOD OF RECORD.--HIGHEST WATER LEVEL, $31.75 \mathrm{FT}(9.68 \mathrm{M})$ BELOW LAND-SURFACE DATUM, MAR 8, 1979; LOWEST WATER LEVEL, 34.08 FT(10.39 M) BELOW LAND-SURFACE DATUM, MAR 10, 1981.

WATER LEVELS IN FEET BELOW LAND SURFACE DATUM, CLIMATIC YEARS APRIL 1981 T0 MARCH 1983

$\begin{array}{lll} & \text { WATER } & \\ \text { DATE } & \text { LEVEL } & \text { WATER } \\ \text { LEVEL }\end{array}$
MAR 29, 1982
33.07
MAR 11, 1983
W 


\section{GROUND-WATER LEVELS}

DELAWARE COUNTY -- CONTINUED

362554094580201. LOCAL NUMBER;

$23 \mathrm{~N}-22 \mathrm{E}-33$ CCA 1 .

LOCATION,--LAT 36 25'54", LONG 094 58'02", HYDROLOGIC UNIT 11070206. AQUIFER.--BOONE FORMATION.

PERIOD OF RECORD.--1979 TO CURRENT YEAR.

EXTREMES FOR PERIOD OF RECORD.--HIGHEST WATER LEVEL, 25.36 FT(7.73 M) BELOW LAND-SURFACE DATUM, MAR 10, 1981; LOWEST WATER LEVEL, 35.23 FT(10.74 M) BELOW LAND-SURFACE DATUM, MAR 29, 1982.

WATER LEVELS IN FEET BELOW LAND SURFACE DATUM, CLIMATIC YEARS APRIL 1981 TO MARCH 1983

\begin{tabular}{|c|c|c|}
\hline DATE & $\begin{array}{l}\text { WATER } \\
\text { LEVEL }\end{array}$ & DATE \\
\hline & & \\
\hline
\end{tabular}

MAR 29, $1982 \quad 35.23 \quad$ MAR 11, $1983 \quad 33.89$ 
GROUND-WATER LEVELS

DEWEY COUNTY

355328098441301. LOCAL NUMBER;

$16 \mathrm{~N}-14 \mathrm{~W}-06$ CBB 1.

LOCATION,--LAT $3553^{\prime} 28^{\prime \prime}$, LONG 098

44'13", HYDROLOGIC UNIT 11090201.

AQUIFER .--RUSH SPRINGS FORMATION.

PERIOD OF RECORD.--1976 TO CURRENT YEAR.

EXTREMES FOR PERIOD OF RECORD.--HIGHEST WATER LEVEL,51.80 FT(15.79 M) BELOW LAND-SURFACE DATUM,FEB 6, 1981; LOWEST WATER LEVEL,54.54 FT(16.62 M) BELOW LAND-SURFACE DATUM, MAR 29, 1979. .

WATER LEVELS IN FEET BELOW LAND SURFACE DATUM, CLIMATIC YEARS APRIL 1981 TO MARCH 1983

\begin{tabular}{|c|c|c|}
\hline & WATER & \\
\hline DATE & LEVEL & DATE \\
\hline
\end{tabular}

MAR 22, $1982 \quad 51.83 \quad$ MAR 28, $1983 \quad$ Z

GROUND-WATER LEVELS

355816098432401. LOCAL NUMBER;

$17 \mathrm{~N}-14 \mathrm{~W}-05 \mathrm{CCC} 1$.

LOCATION,--LAT 35 58'16", LONG 098 43'24", HYDROLOGIC UNIT 11090201.

AQUIFER.--RUSH SPRINGS FORMATION.

PERIOD OF RECORD.--1976 TO CURRENT YEAR.

EXTREMES FOR PERI0D OF RECORD.--HIGHEST WATER LEVEL, $98.75 \mathrm{FT}(30.10 \mathrm{M})$ BELOW LAND-SURFACE DATUM,MAR 22, 1982; LOWEST WATER LEVEL, $105.18 \mathrm{FT}(32.06 \mathrm{M})$ BELOW LAND-SURFACE DATUM, MAR 10, 1976.

WATER LEVELS IN FEET BELOW LAND SURFACE DATUM, CLIMATIC YEARS APRIL 1981 TO MARCH 1983

\begin{tabular}{|c|c|c|}
\hline & WATER & \\
\hline DATE & LEVEL & DATE \\
\hline
\end{tabular}

MAR 22, $198298.75 \quad$ MAR 28, $1983 \quad 98.82$ 


\section{GROUND-WATER LEVELS}

DEWEY COUNTY -- CONTINUED

355546098390001. LOCAL NUMBER; LOCATION,--LAT 35 55'46", LONG 098 AQUIFER.--RUSH SPRINGS FORMATION. PERIOD OF RECORD.--1979 TO CURRENT EXTREMES FOR PERIOD OF RECORD.--HIGHEST WATER LEVEL,60.65 FT(18.49 M) BELOW LAND-SURFACE DATUM,FEB 6, 1981; LOWEST WATER LEVEL,62.10 FT(18.93 M) BELOW LAND-SURFACE DATUM, MAR 29, 1979.

WATER LEVELS IN FEET BELOW LAND SURFACE DATUM, CLIMATIC YEARS APRIL 1981 TO MARCH 1983

\section{DATE}

WATER LEVEL

Z
$17 \mathrm{~N}-14 \mathrm{~W}-24 \mathrm{CCA} 1$. 39'00", HYDROLOGIC UNIT 11090201.
MAR 22, 1982 MAR 28, $1983 \quad$ W WATER DATE LEVEL

\section{GROUND-WATER LEVELS}

355638098444401. LOCAL NUMBER;

$17 \mathrm{~N}-15 \mathrm{~W}-13$ DDB 1

LOCATION,--LAT 35 56'38", LONG 098 44'44", HYDROLOGIC UNIT 11090201. AQUIFER.--RUSH SPRINGS FORMATION. PERIOD OF RECORD.--1979 TO CURRENT YEAR. EXTREMES FOR PERIOD OF RECORD.--HIGHEST WATER LEVEL,94.13 FT(28.69 M) BELOW LAND-SURFACE DATUM, FEB 4, 1980; LOWEST WATER LEVEL, 94.26 FT(28.73 M) BELOW LAND-SURFACE DATUM, MAR 29, 1979.

WATER LEVELS IN FEET BELOW LAND SURFACE DATUM, CLIMATIC YEARS APRIL 1981 TO MARCH 1983

$\begin{array}{lll} & \text { WATER } & \text { WATER } \\ \text { DATE } & \text { LEVEL } & \text { DATE }\end{array}$

MAR 22, 1982

Z MAR 28,1983

W 


\section{GROUND-WATER LEVELS}

DEWEY COUNTY -- CONTINUED

360316098442001 . LOCAL NUMBER; LOCATION,--LAT 36 03'16", LONG 098 AQUIFER.--RUSH SPRINGS FORMATION. PERIOD OF RECORD.--1979 TO CURRENT EXTREMES FOR PERIOD OF RECORD.--HIGHEST WATER LEVEL, 55.29 FT(16.85 M) BELOW LAND-SURFACE DATUM,MAR 28, 1983; LOWEST WATER LEVEL,57.48 FT(17.52 M) BELOW LAND-SURFACE DATUM, MAR 17, 1980.

WATER LEVELS IN FEET BELOW LAND SURFACE DATUM, CLIMATIC YEARS APRIL 1981 TO MARCH 1983
$18 \mathrm{~N}-14 \mathrm{~W}-07$ BBD 1 44'20", HYDROLOGIC UNIT 11100301.
WATER
DATE LEVEL
DATE
WATER
LEVEL

MAR 22, $1982 \quad 57.26$

MAR 28, $1983 \quad 55.29$

\section{GROUND-WATER LEVELS}

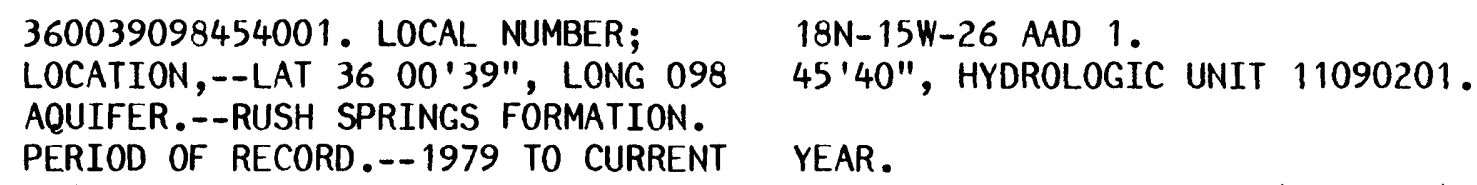




\section{GROUND-WATER LEVELS}

DEWEY COUNTY -- CONTINUED

360132098525301. LOCAL NUMBER; 18N-16W-23 BBD 1.

LOCATION,--LAT 3601 '32", LONG 098 52'53", HYDROLOGIC UNIT 11090201.

AQUIFER.--RUSH SPRINGS FORMATION.

PERIOD OF RECORD.-- 1979 TO CURRENT YEAR.

EXTREMES FOR PERIOD OF RECORD.--HIGHEST WATER LEVEL,20.50 FT(6.25 M) BELOW LAND-SURFACE DATUM,FEB 6, 1981; LOWEST WATER LEVEL,21.63 FT(6.59 M) BELOW LAND-SURFACE DATUM, MAR 29, 1979.

WATER LEVELS IN FEET BELOW LAND SURFACE DATUM, CLIMATIC YEARS APRIL 1981 TO MARCH 1983

\begin{tabular}{|c|c|}
\hline DATE & $\begin{array}{l}\text { WATER } \\
\text { LEVEL }\end{array}$ \\
\hline
\end{tabular}

MAR 22, $1982 \quad$ Z MAR 28, 1983 W

GROUND-WATER LEVELS

360753098423001. LOCAL NUMBER; 19N-14W-08 DDD 1.

LOCATION,--LAT 36 07'53", LONG 098 42'30", HYDROLOGIC UNIT 11100301.

AQUIFER.--TERRACE DEPOSITS.

PERIOD OF RECORD.--1978 TO CURRENT YEAR.

EXTREMES FOR PERIOD OF RECORD.--HIGHEST WATER LEVEL, 19.92 FT(6.07 M) BELOW LAND-SURFACE DATUM, JUNE 21, 1978; LOWEST WATER LEVEL,23.73 FT(7.23 M) BELOW LAND-SURFACE DATUM, MAR 12, 1979.

WATER LEVELS IN FEET BELOW LAND SURFACE DATUM, CLIMATIC YEARS APRIL 1981 TO MARCH 1983

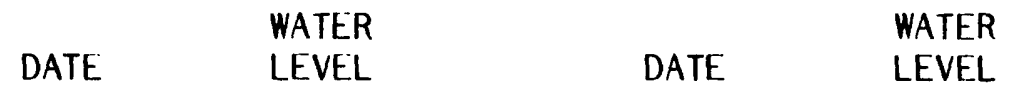

MAR 22, $1982 \quad 23.38 \quad$ MAR $28,1983 \quad 20.47$ 
DEWEY COUNTY -- CONTINUED

360539098402001. LOCAL NUMBER; LOCATION, --LAT 36 05'39", LONG 098 AQUIFER.--RUSH SPRINGS FORMATION. PERIOD OF RECORD.--1979 TO CURRENT EXTREMES FOR PERIOD OF RECORD.--HIGHEST WATER LEVEL,26.26 FT(8.00 M) BELOW LAND-SURFACE DATUM,FEB 4, 1981; LOWEST WATER LEVEL,27.38 FT(8.35 M) BELOW LAND-SURFACE DATUM, MAR 29, 1979.

WATER LEVELS IN FEET BELOW LAND SURFACE DATUM, CLIMATIC YEARS APRIL 1981 TO MARCH 1983

$\begin{array}{lll} & \text { WATER } & \text { WATER } \\ \text { DATE } & \text { LEVEL } & \text { DATE }\end{array}$

MAR 22, 1982
19N-14W-27 ADD 1. 40'20", HYDROLOGIC UNIT 11100301.
YEAR. LEVEL

MAR 28, 1983

\section{GROUND-WATER LEVELS}

360454098395601. LOCAL NUMBER; LOCATION,--LAT $3604^{\prime}$ '54", LONG 098 AQUIFER.--RUSH SPRINGS FORMATION. PERIOD OF RECORD.--1978 TO CURRENT EXTREMES FOR PERIOD OF RECORD.--HIGHEST WATER LEVEL, 69.72 FT(20.95 M) BELOW LAND-SURFACE DATUM,MAR 28, 1983; LOWEST WATER LEVEL,71.15 FT(21.69 M) BELOW LAND-SURFACE DATUM, MAR 15, 1979.

WATER LEVELS IN FEET BELOW LAND SURFACE DATUM, CLIMATIC YEARS APRIL 1981 TO MARCH 1983

\begin{tabular}{|c|c|c|}
\hline & WATER & \\
\hline DATE & LEVEL & DATE \\
\hline
\end{tabular}
MAR 22, 1982
69.72
MAR 28, 1983
68.74

$19 \mathrm{~N}-14 \mathrm{~W}-35$ BDB 1.

39'56", HYDROLOGIC UNIT 11100301. 
DEWEY COUNTY -- CONTINUED

360903099110201. LOCAL NUMBER;

LOCATION, --LAT 36 09'03", LONG 099

AQUIFER.--OGALLALA.

PERIOD OF RECORD.--1980 TO CURRENT YEAR.

EXTREMES FOR PERIOD OF RECORD.--HIGHEST WATER LEVEL, $18.17 \mathrm{FT}(5.54 \mathrm{M})$ BELOW LAND-SURFACE DATUM, MAR 18, 1980; LOWEST WATER LEVEL, 18.45 FT(5.62 M) BELOW LAND-SURFACE DATUM, FEB 4, 1981.

WATER LEVELS IN FEET BELOW LAND SURFACE DATUM, CLIMATIC YEARS APRIL 1981 TO MARCH 1983
19N-19W-02 DAA 1.

11 '02", HYDROLOGIC UNIT 11100301. LEVEL, 18.45 FT(5.62 M) BELON LAND-SURFACE DATUM,

$\begin{array}{lll} & \text { WATER } & \\ \text { DATE } & \text { LEVEL } & \text { WATER } \\ & \text { DATE } & \text { LEVEL }\end{array}$

MAR 22, 1982
MAR 22,1982
Z
MAR 28, 1983

W

\section{GROUND-WATER LEVELS}

360850099141001. LOCAL NUMBER; LOCATION, --LAT 3608 '50", LONG 099 AQUIFER .--OGALLALA.

PERIOD OF RECORD.--1981 TO CURRENT YEAR.

EXTREMES FOR PERIOD OF RECORD.--HIGHEST WATER LEVEL, $60.65 \mathrm{FT}(18.49 \mathrm{M})$ BELOW LAND-SURFACE

DATUM, FEB 4, 1981; LOWEST WATER LEVEL,67.90 FT(20.70 M) BELOW LAND-SURFACE DATUM, MAR 28, 1983.

WATER LEVELS IN FEET BELOW LAND SURFACE DATUM, CLIMATIC YEARS APRIL 1981 TO MARCH 1983

\section{DATE}

WATER

LEVEL

MAR 22, 1982
$19 \mathrm{~N}-19 \mathrm{~W}-04$ CCA 1.

14'10", HYDROLOGIC UNIT 11100301. 
DEWEY COUNTY -- CONTINUED

360640099130701. LOCAL NUMBER; LOCATION,--LAT $3606^{\prime} 40^{\prime \prime}$, LONG 099 AQUIFER.--OGALLALA. PERIOD OF RECORD.--1980 TO CURRENT YEAR. EXTREMES FOR PERIOD OF RECORD.--HIGHEST WATER LEVEL,93.45 FT(28.48 M) BELOW LAND-SURFACE DATUM,FEB 4, 1981; LOWEST WATER LEVEL,93.56 FT(28.52 M) BELOW LAND-SURFACE DATUM, MAR 18, 1980.

WATER LEVELS IN FEET BELOW LAND SURFACE DATUM, CLIMATIC YEARS APRIL 1981 T0 MARCH 1983

$\begin{array}{lll} & \text { WATER } & \\ \text { DATE } & \text { LEVEL } & \text { WATER } \\ & \text { DATE } & \text { LEVEL }\end{array}$

MAR 22, 1982
$19 \mathrm{~N}-19 \mathrm{~W}-22 \mathrm{BAC} 1$.

$13^{\prime} 07^{\prime \prime}$, HYDROLOGIC UNIT 11100301.
MAR 28, $1983 \quad$ W

\section{GROUND-WATER LEVELS}

360913099174101 . LOCAL NUMBER; LOCATION, --LAT 36 09'13", LONG 099 AQUIFER.--OGALLALA.

PERIOD OF RECORD.--1981 TO CURRENT YEAR. EXTREMES FOR PERIOD OF RECORD.--HIGHEST WATER LEVEL, 98.10 FT(29.90 M) BELOW LAND-SURFACE DATUM,FEB 4, 1981; LOWEST WATER LEVEL,98.10 FT(29.90 M) BELOW LAND-SURFACE DATUM, FEB 4, 1981 .

WATER LEVELS IN FEET BELOW LAND SURFACE DATUM, CLIMATIC YEARS APRIL 1981 TO MARCH 1983

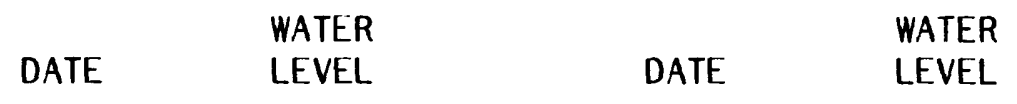

MAR 22, 1982

P $\quad$ MAR 28, 1983

Z 
DEWEY COUNTY - - CONTINUED

360759099195601. LOCAL NUMBER; LOCATION,--LAT 3607 '59", LONG 099 AQUIFER.--OGALLALA.

PERIOD OF RECORD.--1980 TO CURRENT

EXTREMES FOR PERIOD OF RECORD.--HIGHEST WATER LEVEL, 59.99 FT(18.28 M) BELOW LAND-SURFACE DATUM,MAR 28, 1983; LOWEST WATER LEVEL,61.70 FT(18.81 M) BELOW LAND-SURFACE DATUM, MAR 22, 1982.

WATER LEVELS IN FEET BELOW LAND SURFACE DATUM, CLIMATIC YEARS APRIL 1981 TO MARCH 1983

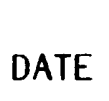

WATER LEVEL

DATE
19N-20W-09 DDB 1. 19'56", HYDROLOGIC UNIT 11090201.
MAR 28, $1983 \quad 59.99$
MAR 22, $1982 \quad 61.70$

WATER

LEVEL 


\section{GROUND-WATER LEVELS}

\section{ELLIS COUNTY}

360113099501601. LOCAL NUMBER;

$18 \mathrm{~N}-25 \mathrm{~W}-23$ CAA 1.

LOCATION,--LAT 3601 '13", LONG 099 50'16", HYDROLOGIC UNIT 11090201. AQUIFER.--ALLUVIUM.

PERIOD OF RECORD.--1980 TO CURRENT YEAR.

EXTREMES FOR PERIOD OF RECORD.--HIGHEST WATER LEVEL,9.16 FT(2.79 M) BELOW LAND-SURFACE DATUM,FEB 24, 1982; LOWEST WATER LEVEL, 10.33 FT(3.15 M) BELOW LAND-SURFACE DATUM, FEB 5, 1981.

WATER LEVELS IN FEET BELOW LAND SURFACE DATUM, CLIMATIC YEARS APRIL 1981 TO MARCH 1983

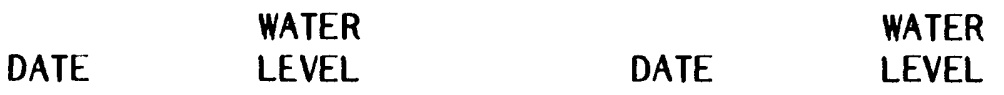

FEB 24, $1982 \quad 9.16 \quad$ JAN 27, $1983 \quad 10.23$

\section{GROUND-WATER LEVELS}

360137099490801. LOCAL NUMBER; LOCATION,--LAT 3601 '37", LONG 099 AQUIFER .--OGALLALA.

PERIOD OF RECORD.--1980 TO CURRENT YEAR.

EXTREMES FOR PERIOD OF RECORD.--HIGHEST WATER LEVEL,21.16 FT(6.45 M) BELOW LAND-SURFACE

DATUM, JAN 27, 1983; LOWEST WATER LEVEL,24.00 FT(7.32 M) BELOW LAND-SURFACE DATUM, FEB 6, 1980.

WATER LEVELS IN FEET BELOW LAND SURFACE DATUM, CLIMATIC YEARS APRIL 1981 TO MARCH 1983
$18 \mathrm{~N}-25 \mathrm{~W}-24$ BAA 1.

49'08", HYDROLOGIC UNIT 11090201.
WATER

DATE

FEB $24,1982 \quad 23.71$ $\begin{array}{ll} & \text { WATER } \\ \text { DATE } & \text { LEVEL }\end{array}$

JAN 27, $1983 \quad 21.16$ 


\section{ELLIS COUNTY -- CONTINUED}

360916099391001. LOCAL NUMBER; 19N-23W-04 ADB 1. LOCATION,--LAT 36 09'16", LONG 099 39'10", HYDROLOGIC UNIT 11090201. AQUIFER .--OGALLALA.

PERIOD OF RECORD.--1980 TO CURRENT YEAR.

EXTREMES FOR PERIOD OF RECORD.--HIGHEST WATER LEVEL, $175.80 \mathrm{FT}(53.58 \mathrm{M})$ BELOW LAND-SURFACE DATUM, JAN 29, 1980; LOWEST WATER LEVEL, $181.69 \mathrm{FT}(55.38 \mathrm{M})$ BELOW LAND-SURFACE DATUM, FEB 25, 1982 .

WATER LEVELS IN FEET BELOW LAND SURFACE DATUM, CLIMATIC YEARS APRIL 1981 TO MARCH 1983

$\begin{array}{lll} & \text { WATER } & \text { WATER } \\ \text { DATE } & \text { LEVEL } & \text { DATE }\end{array}$

FEB 25, $1982 \quad 181.69 \quad$ JAN 27, $1983 \quad 177.30$

GROUND-WATER LEVELS

360922099413401. LOCAL NUMBER; 19N-23W-06 ABC 1.

LOCATION,--LAT 36 09'22", LONG 099 41'34", HYDROLOGIC UNIT 11090201.

AQUIFER .--OGALLALA.

PERIOD OF RECORD.--1980 TO CURRENT YEAR.

EXTREMES FOR PERIOD OF RECORD.--HIGHEST WATER LEVEL, $170.45 \mathrm{FT}(51.95 \mathrm{M})$ BELOW LAND-SURFACE DATUM, JAN 27, 1983; LOWEST WATER LEVEL,173.85 FT(52.99 M) BELOW LAND-SURFACE DATUM, FEB 25, 1982 .

WATER LEVELS IN FEET BELOW LAND SURFACE DATUM, CLIMATIC YEARS APRIL 1981 TO MARCH 1983

\begin{tabular}{|c|c|c|}
\hline & WATER & \\
\hline DATE & LEVEL & DATE \\
\hline
\end{tabular}

FEB 25, $1982 \quad 173.85 \quad$ JAN 27, $1983 \quad 170.45$ 
GROUND-WATER LEVELS

ELLIS COUNTY -- CONTINUED

360756099401901. LOCAL NUMBER;

$19 \mathrm{~N}-23 \mathrm{~W}-08$ DDD 1. LOCATION,--LAT 36 07'56", LONG 099 40'19", HYDROLOGIC UNIT 11090201. AQUIFER.--OGALLALA.

PERIOD OF RECORD.--1980 TO CURRENT YEAR.

EXTREMES FOR PERIOD OF RECORD.--HIGHEST WATER LEVEL, $175.85 \mathrm{FT}(53.60 \mathrm{M})$ BELOW LAND-SURFACE DATUM, JAN 14, 1983; LOWEST WATER LEVEL, 177.64 FT(54.14 M) BELOW LAND-SURFACE DATUM, FEB 25, 1982.

WATER LEVELS IN FEET BELOW LAND SURFACE DATUM, CLIMATIC YEARS APRIL 1981 TO MARCH 1983

\begin{tabular}{|c|c|c|}
\hline & WATER & \\
\hline DATE & & DATE \\
\hline
\end{tabular}

FEB 25, $1982 \quad 177.64 \quad$ JAN $14,1983 \quad 175.85$

GROUND-WATER LEVELS

360758099382201. LOCAL NUMBER; 19N-23W-10 DCB 1. LOCATION,--LAT 36 07'58", LONG 099 38'22", HYDROLOGIC UNIT 11090201. AQUIFER.--OGALLALA.

PERIOD OF RECORD.--1980 TO CURRENT YEAR.

EXTREMES FOR PERIOD OF RECORD.--HIGHEST WATER LEVEL, $183.02 \mathrm{FT}(55.78 \mathrm{M})$ BELOW LAND-SIJRFACE DATUM,JAN 31, 1980; LOWEST WATER LEVEL, 194.90 FT(59.41 M) BELOW LAND-SURFACE DATUM, JAN 14, 1983.

WATER LEVELS IN FEET BELOW LAND SURFACE DATUM, CLIMATIC YEARS APRIL 1981 TO MARCH 1983

$\begin{array}{lll} & \text { WATER } & \\ \text { DATE } & \text { WEVEL } & \text { WATER } \\ \text { LEVEL }\end{array}$

FEB 2b, $1982 \quad 194.78 \quad$ JAN 14, $1983 \quad 194.90$ 
ELLIS COUNTY -- CONTINUED

360929099455901. LOCAL NUMBER; LOCATION,--LAT 36 09'29", LONG 099 AQUIFER.--OGALLALA.

PERIOD OF RECORD.--1980 TO CURRENT

EXTREMES FOR PERIOD OF RECORD.--HIGHEST WATER LEVEL, $141.04 \mathrm{FT}(42.99 \mathrm{M})$ BELOW LAND-SURFACE DATUM,MAR 5, 1981; LOWEST WATER LEVEL,160.50 FT(48.92 M) BELOW LAND-SURFACE DATUM, FEB 24, 1982.

WATER LEVELS IN FEET BELOW LAND SURFACE DATUM, CLIMATIC YEARS APRIL 1981 TO MARCH 1983
$19 \mathrm{~N}-24 \mathrm{~W}-04$ BAA 1.

45'59", HYDROLOGIC UNIT 11090201.

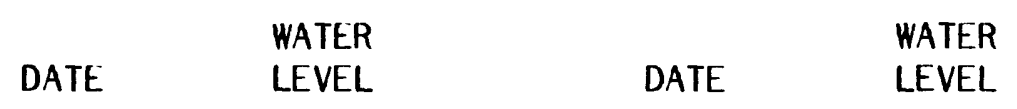

FEB 24, $1982 \quad 160.50$

GROUND-WATER LEVELS

360804099475001. LOCAL NUMBER; LOCATION,--LAT 3608 '04', LONG 099 AQUIFER.--OGALLALA.

PERIOD OF RECORD.--1980 TO CURRENT YEAR. EXTREMES FOR PERIOD OF RECORD.--HIGHEST WATER LEVEL, $74.03 \mathrm{FT}(22.56 \mathrm{M})$ BELOW LAND-SURFACE DATUM,FEB 25, 1980; LOWEST WATER LEVEL,91.91 FT(28.01 M) BELOW LAND-SURFACE DATUM, JAN 14, 1983.

WATER LEVELS IN FEET BELOW LAND SURFACE DATUM, CLIMATIC YEARS APRIL 1981 TO MARCH 1983
$19 \mathrm{~N}-24 \mathrm{~W}-07 \mathrm{DBC} 1$.

47'50", HYDROLOGIC UNIT 11090201. 


\section{ELLIS COUNTY -- CONTINUED}

360753099460901. LOCAL NUMBER; LOCATION,--LAT 3607 '53", LONG 099 AQUIFER.--OGALLALA.

PERIOD OF RECORD.--1980 TO CURRENT YEAR.

EXTREMES FOR PERIOD OF RECORD.--HIGHEST WATER LEVEL, $140.45 \mathrm{FT}(42.81 \mathrm{M})$ BELOW LAND-SURFACE DATUM, MAR 5, 1981; LOWEST WATER LEVEL, $148.96 \mathrm{FT}(45.40 \mathrm{M})$ BELOW LAND-SURFACE DATUM, JAN 31, 1980. WATER LEVELS IN FEET BELOW LAND SURFACE DATUM, CLIMATIC YEARS APRIL 1981 TO MARCH 1983
$19 N-24 W-09$ CBD 1. 46'09", HYDROLOGIC UNIT 11090201. $\begin{array}{lll} & \text { WATER } & \text { WATER } \\ \text { DATE } & \text { LEVEL } & \text { DATE }\end{array}$

FEB 24, $1982 \quad 147.88 \quad$ JAN 14, $1983 \quad 148.00$

GROUND-WATER LEVELS

360758099451001. LOCAL NUMBER; 19N-24W-10 CCA 1. LOCATION,--LAT 36 07'58", LONG 099 45'10", HYDROLOGIC UNIT 11090201. AQUIFER .--OGALLALA. PERIOD OF RECORD.--1980 TO CURRENT YEAR. EXTREMES FOR PERIOD OF RECORD.--HIGHEST WATER LEVEL, 146.97 FT(44.80 M) BELOW LAND-SURFACE DATUM, JAN 14, 1983; LOWEST WATER LEVEL, 147.71 FT(45.02 M) BELOW LAND-SURFACE DATUM, MAR 5, 1981.

WATER LEVELS IN FEET BELOW LAND SURFACE DATUM, CLIMATIC YEARS APRIL 1981 TO MARCH 1983

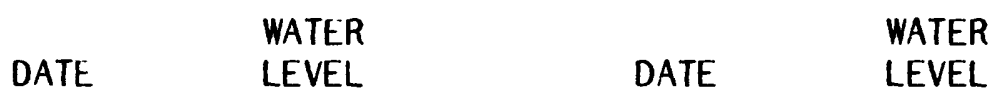

FEB 24, $1982 \quad 147.53 \quad$ JAN 14, $1983 \quad 146.97$ 
GROUND-WATER LEVELS

\section{ELLIS COUNTY -- CONTINUED}

360604099483101. LOCAL NUMBER; LOCATION,--LAT 3606 '04", LONG 099 AQUIFER .--OGALLALA.

PERIOD OF RECORD.--1980 TO CURRENT YEAR. EXTREMES FOR PERIOD OF RECORD.--HIGHEST WATER LEVEL, 82.55 FT(25.16 M) BELOW LAND-SURFACE DATUM,JAN 27, 1983; LOWEST WATER LEVEL,83.79 FT(25.54 M) BELOW LAND-SURFACE DATUM, JAN 31, 1980.

WATER LEVELS IN FEET BELOW LAND SURFACE DATUM, CLIMATIC YEARS APRIL 1981 TO MARCH 1983
$19 \mathrm{~N}-24 \mathrm{~W}-19$ CCC 1

48'31', HYDROLOGIC UNIT 11090201.

$\begin{array}{lll} & & \\ & & \\ \text { DATE } & \text { WATER } & \text { WATER } \\ \text { LEVEL } & \text { DATE } & \text { LEVEL }\end{array}$
FEB 24, 1982
83.37
JAN 27, 1983
82.55

GROUND-WATER LEVELS

360613099422801. LOCAL NUMBER; LOCATION,--LAT 36 06'13", LONG 099 AQUIFER .--OGALLALA.

PERIOD OF RECORD.--1980 TO CURRENT YEAR.

EXTREMES FOR PERIOD OF RECORD.--HIGHEST WATER LEVEL, 149.00 FT(45.42 M) BELOW LAND-SURFACE DATUM, JAN 27, 1983; LOWEST WATER LEVEL, 150.42 FT(45.85 M) BELOW LAND-SURFACE DATUM, MAR S, 1981.

WATER LEVELS IN FEET BELOW LAND SURFACE DATUM, CLIMATIC YEARS APRIL 1981 TO MARCH 1983
$19 \mathrm{~N}-24 \mathrm{~W}-23$ DCA 1 42'28", HYDROLOGIC UNIT 11090203.
$\begin{array}{ll} & \text { WATER } \\ \text { DATE } & \text { LEVEL }\end{array}$
LEVEL
DATE
WATER
LEVEL
FEB 24, $1982 \quad 150.04$
JAN 27, $1983 \quad 149.00$ 
ELLIS COUNTY -- CONTINUED

360802099530301. LOCAL NUMBER; 19N-25W-08 DAD 1.

LOCATION,--LAT 36 08'02", LONG 099 53'03", HYDROLOGIC UNIT 11090201.

AQUIFER. - OGALLALA.

PERIOD OF RECORD.--1980 TO CURRENT YEAR.

EXTREMES FOR PERIOD OF RECORD.--HIGHEST WATER LEVEL, 116.86 FT(35.62 M) BELOW LAND-SURFACE DATUM, MAR 5, 1981; LOWEST WATER LEVEL, 117.49 FT(35.81 M) BELOW LAND-SURFACE DATUM, FEB 5, 1980.

WATER LEVELS IN FEET BELOW LAND SURFACE DATUM, CLIMATIC YEARS APRIL 1981 TO MARCH 1983

$\begin{array}{lll} & \text { WATER } & \text { WATER } \\ \text { DATE } & \text { LEVEL } & \text { DATE }\end{array}$

FEB 24, $1982 \quad Z \quad$ JAN 22, $1983 \quad$ Z

GROUND-WATER LEVELS

360827099485001. LOCAL NUMBER; 19N-25W-12 AAD 1. LOCATION,--LAT 36 08'27", LONG 099 48'50", HYDROLOGIC UNIT 11090201. AQUIFER .--OGALLALA. PERIOD OF RECORD..-1980 TO CURRENT YEAR. EXTREMES FOR PERIOD OF RECORD.--HIGHEST WATER LEVEL, $112.00 \mathrm{FT}(34.14 \mathrm{M})$ BELOW LAND-SURFACE DATUM, JAN 13, 1983; LOWEST WATER LEVEL, 113.63 FT(34.63 M) BELOW LAND-SURFACE DATUM, FEB 5, 1980 .

WATER LEVELS IN FEET BELOW LAND SURFACE DATUM, CLIMATIC YEARS APRIL 1981 TO MARCH 1983

$\begin{array}{lll} & \text { WATER } & \text { WATER } \\ \text { DATE } & \text { LEVEL } & \text { DATE }\end{array}$

FEB 24, $1982112.56 \quad$ JAN 13, $1983 \quad 112.00$


360736099483901. LOCAL NUMBER; 19N-25W-13 DAA 1. LOCATION,--LAT 36 07'36", LONG 099 48'39", HYDROLOGIC UNIT 11090201. AQUIFER.--OGALLALA.

PERIOD OF RECORD.--1980 TO CURRENT YEAR. EXTREMES FOR PERIOD OF RECORD.--HIGHEST WATER LEVEL, $98.65 \mathrm{FT}(30.07 \mathrm{M})$ BELOW LAND-SURFACE DATUM,JAN 14, 1983; LOWEST WATER LEVEL,99.62 FT(30.36 M) BELOW LAND-SURFACE DATUM, FEB $5,1980$.

WATER LEVELS IN FEET BELOW LAND SURFACE DATUM, CLIMATIC YEARS APRIL 1981 TO MARCH 1983

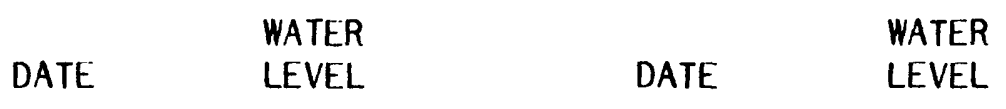

FEB 24, $198299.10 \quad$ JAN 14, $1983 \quad 98.65$

GROUND-WATER LEVELS

360606099514501. LOCAL NUMBER; 19N-25W-22 CCC 1. LOCATION,--LAT 36 06'06", LONG 099 51'45", HYDROLOGIC UNIT 11090201. AQUIFER .--OGALLALA.

PERIOD OF RECORD.--1980 TO CURRENT YEAR. EXTREMES FOR PERIOD OF RECORD.--HIGHEST WATER LEVEL, $24.58 \mathrm{FT}(7.49 \mathrm{M})$ BELOW LAND-SURFACE DATUM,FEB 24, 1982; LOWEST WATER LEVEL, 25.30 FT(7.71 M) BELOW LAND-SURFACE DATUM, JAN 14, 1983.

WATER LEVELS IN FEET BELOW LAND SURFACE DATUM, CLIMATIC YEARS APRIL 1981 TO MARCH 1983

$\begin{array}{lll} & \text { WATER } & \text { WATER } \\ \text { DATE } & \text { LEVEL } & \text { DATE }\end{array}$

FEB 24, $1982 \quad 24.58 \quad$ JAN $14,1983 \quad 25.30$ 
ELLIS COUNTY -- CONTINUED

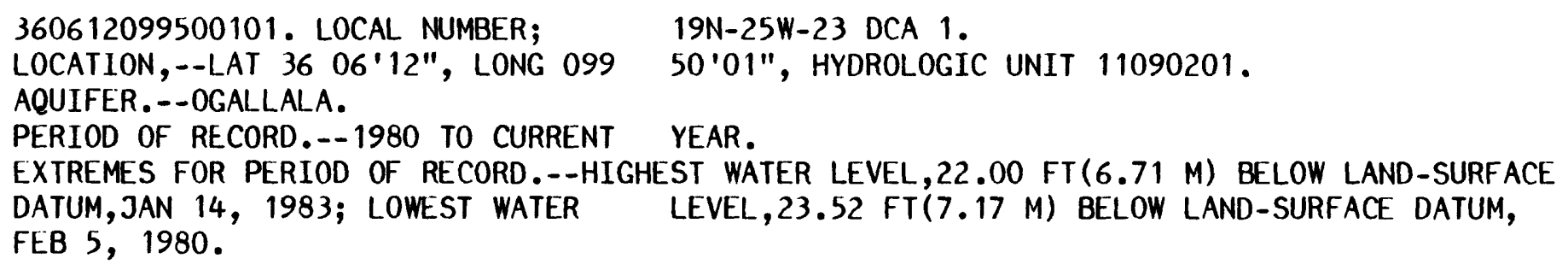

WATER LEVELS IN FEET BELOW LAND SURFACE DATUM, CLIMATIC YEARS APRIL 1981 TO MARCH 1983

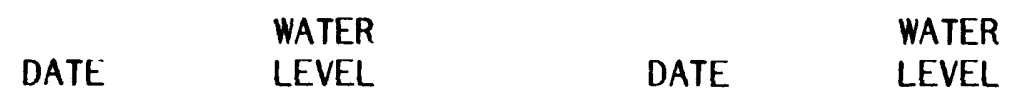

FEB 24, $1982 \quad 22.44 \quad$ JAN 14, $1983 \quad 22.00$

GROUND-WATER LEVELS

$360558099522601 . \quad L O C A L$ NUMBER; 19N-25W-28 ABB 1.

LOCATION,--LAT 36 05'58", LONG 099 52'26", HYDROLOGIC UNIT 11090201. AQUIFER .--OGALLALA.

PERIOD OF RECORD.--1980 TO CURRENT YEAR.

EXTREMES FOR PERIOD OF RECORD.--HIGHEST WATER LEVEL,66.36 FT(20.23 M) BELOW LAND-SURFACE DATUM, JAN 14, 1983; LOWEST WATER LEVEL,67.07 FT(20.44 M) BELOW LAND-SURFACE DATUM, FEB 5, 1980.

WATER LEVELS IN FEET BELOW LAND SURFACE DATUM, CLIMATIC YEARS APRIL 1981 TO MARCH 1983

$\begin{array}{lll} & \text { WATER } & \text { WATER } \\ \text { DATE } & \text { LEVEL } & \text { DATE }\end{array}$

FEB 24, 198266.52 JAN 14, $1983 \quad 66.36$ 
GROUND-WATER LEVELS

ELLIS COUNTY -- CONTINUED

360825099582701. LOCAL NUMBER; 19N-26W-04 DAC 1.

LOCATION,--LAT 36 08'25", LONG 099 58'27", HYDROLOGIC UNIT 11090201.

AQUIFER.--OGALLALA.

PERIOD OF RECORD.--1980 TO CURRENT YEAR.

EXTREMES FOR PERIOD OF RECORD.--HIGHEST WATER LEVEL, 142.80 FT(43.53 M) BELOW LAND-SURFACE DATUM,FEB 6, 1980; LOWEST WATER LEVEL, $169.00 \mathrm{FT}(51.51 \mathrm{M})$ BELOW LAND-SURFACE DATUM, JAN 14, 1983.

WATER LEVELS IN FEET BELOW LAND SURFACE DATUM, CLIMATIC YEARS APRIL 1981 TO MARCH 1983

\begin{tabular}{|c|c|}
\hline & WATER \\
\hline DATE & LEVEL \\
\hline
\end{tabular}

FEB 24, $1982168.66 \quad$ JAN 14, $1983 \quad 169.00$

GROUND-WATER LEVELS

360822099552201. LOCAL NUMBER; 19N-26W-12 ACA 1.

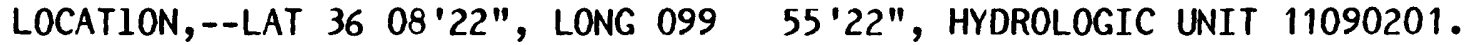

AQUIFER.--OGALLALA.

PERIOD OF RECORD.--1980 TO CURRENT YEAR.

EXTREMES FOR PERIOD OF RECORD.--HIGHEST WATER LEVEL, 165.88 FT(50.56 M) BELOW LAND-SURFACE DATUM,MAR 5, 1981; LOWEST WATER LEVEL,173.20 FT(52.79 M) BELOW LAND-SURFACE DATUM, JAN 13, 1983.

WATER LEVELS IN FEET BELOW LAND SURFACE DATUM, CLIMATIC YEARS APRIL 1981 TO MARCH 1983

\begin{tabular}{|c|c|c|}
\hline OAT & WATER & \\
\hline DAT & & DATE \\
\hline
\end{tabular}

FEB 24, $1982172.92 \quad$ JAN 13, $1983 \quad 173.20$ 


\section{ELLIS COUNTY -- CONTINUED}

360520099562601 . LOCAL NUMBER; LOCATION,--LAT 36 05'20", LONG 099 56'26", HYDROLOGIC UNIT 11090201. AQUIFER .--OGALLALA.

PERIOD OF RECORD.--1980 TO CURRENT YEAR.

EXTREMES FOR PERIOD OF RECORD.--HIGHEST WATER LEVEL,4.60 FT(1.40 M) BELOW LAND-SURFACE DATUM, JAN 14, 1983; LOWEST WATER LEVEL,8.00 FT(2.44 M) BELOW LAND-SURFACE DATUM, FEB 5, 1981.

WATER LEVELS IN FEET BELOW LAND SURFACE DATUM, CLIMATIC YEARS APRIL 1981 TO MARCH 1983

\begin{tabular}{crrrr}
\multicolumn{1}{c}{} & WATER & & & WATER \\
DATE & LEVEL & DATE & LEVEL \\
FEB 24, 1982 & 7.69 & JAN 14, 1983 & 4.60
\end{tabular}

GROUND-WATER LEVELS

360520099583401. LOCAL NUMBER;

$19 \mathrm{~N}-26 \mathrm{~W}-28$ DCA 1

LOCATION,--LAT 36 05'20", LONG 099 58'34", HYDROLOGIC UNIT 11090201.

AQUIFER .--OGALLALA.

PERIOD OF RECORD.--1980 TO CURRENT YEAR.

EXTREMES FOR PERIOD OF RECORD. --HIGHEST WATER LEVEL, $169.04 \mathrm{FT}(51.52 \mathrm{M})$ BELOW LAND-SURFACE DATUM, FEB 5, 1981; LOWEST WATER LEVEL, $170.81 \mathrm{FT}(52.06 \mathrm{M})$ BELOW LAND-SURFACE DATUM, JAN 14, 1983.

WATER LEVELS IN FEET BELOW LAND SURFACE DATUM, CLIMATIC YEARS APRIL 1981 TO MARCH 1983

$\begin{array}{cccc} & \text { WATER } & & \text { WATER } \\ \text { DATE } & \text { LEVEL } & \text { DATE } & \text { LEVEL } \\ \text { B 24, 1982 } & 170.71 & \text { JAN 14, 1983 } & 170.81\end{array}$


GROUND-WATER LEVELS

ELLIS COUNTY -- CONTINUED

360427099572801. LOCAL NUMBER;

LOCATION,--LAT $3604^{\prime} 27$ ', LONG 099

AQUIFER.--OGALLALA.

PERIOD OF RECORD. - 1980 TO CURRENT YEAR.

EXTREMES FOR PERIOD OF RECORD.--HIGHEST WATER LEVEL, 122.57 FT(37.36 M) BELOW LAND-SURFACE DATUM, JAN 14, 1983; LOWEST WATER LEVEL, 123.27 FT(37.57 M) BELOW LAND-SURFACE DATUM, FEB 5, 1980.

WATER LEVELS IN FEET BELOW LAND SURFACE DATUM, CLIMATIC YEARS APRIL 1981 T0 MARCH 1983
GROUND-WATER LEVELS

360420099560001. LOCAL NUMBER;

LOCATION,--LAT $3604^{\prime 20 ", ~ L O N G ~} 099$

AQUIFER.--OGALLALA.

PERIOD OF RECORD.--1980 TO CURRENT YEAR.

EXTREMES FOR PERIOD OF RECORD.--HIGHEST WATER LEVEL, 18.72 FT(5.71 M) BELOW LAND-SURFACE DATUM,FLB 5, 1981; LOWEST WATER FEB 5, 1980 .

WATER LEVELS IN FEET BELOW LAND SURFACE DATUM, CLIMATIC YEARS APRIL 1981 TO MARCH 1983
$19 \mathrm{~N}-26 \mathrm{~W}-36 \mathrm{CCC} 1$

$56^{\prime} 00^{\prime \prime}$, HYDROLOGIC UNIT 11090201.

LEVEL, 53.49 FT (16.30 M) BELOW LAND-SURFACE DATUM,
$19 \mathrm{~N}-26 \mathrm{~W}-34$ DCA 1

57'28", HYDROLOGIC UNIT 11090201.

WATER

122.57

$\begin{array}{cccc} & & & \\ \text { WATER } & \text { WATER } \\ \text { DATE } & \text { LEVEL } & \text { DATE } & \text { LEVEL } \\ \text { FEB 24, 1982 } & 52.99 & \text { JAN 14, 1983 }\end{array}$ 


\section{ELLIS COUNTY -- CONTINUED}

360936099332901. LOCAL NUMBER; LOCATION,--LAT 36 09'36", LONG 099 AQUIFER.--OGALLALA.

PERIOD OF RECORD.--1980 TO CURRENT EXTREMES FOR PERIOD OF RECORD.--HIGHEST WATER LEVEL, 226.10 FT(68.92 M) BELOW LAND-SURFACE DATUM, JAN 29, 1980; LOWEST WATER LEVEL,229.35 FT(69.91 M) BELOW LAND-SURFACE DATUM, MAR 17, 1981.

WATER LEVELS IN FEET BELOW LAND SURFACE DATUM, CLIMATIC YEARS APRIL 1981 TO MARCH 1983
$20 \mathrm{~N}-22 \mathrm{~W}-32$ DCA 1 . 33'29", HYDROLOGIC UNIT 11090201.

\section{WATER WATE WATE}

JAN 21, $1982 \quad 229.05 \quad$ JAN 27, $1983 \quad 227.76$

GROUND-WATER LEVELS

361420099411001. LOCAL NUMBER; LOCATION,--LAT 36 14'20", LONG 099 AQUIFER .--OGALLALA. PERIOD OF RECORD.--1980 TO CURRENT YEAR. EXTREMES FOR PERIOD OF RECORD.--HIGHEST WATER LEVEL, $17.98 \mathrm{FT}(5.48 \mathrm{M})$ BELOW LAND-SURFACE DATUM, JAN 28, 1980; LOWEST WATER LEVEL,20.38 FT(6.21 M) BELOW LAND-SURFACE DATUM, JAN 21, 1982.

WATER LEVELS IN FEET BELOW LAND SURFACE DATUM, CLIMATIC YEARS APRIL 1981 TO MARCH 1983 $20 \mathrm{~N}-23$ W-06 ADD 1.
$41^{\prime} 10^{\prime \prime}$, HYDROLOGI

41'10", HYOROLOGIC UNIT 11100203. 
ELLIS COUNTY -- CONTINUED

361154099415801. LOCAL NUMBER; LOCATION,--LAT 3611 '54", LONG 099 AQUIFER . - OGALLALA. PERIOD OF RECORD.--1980 TO CURRENT YEAR. EXTREMES FOR PERIOD OF RECORD.--HIGHEST WATER LEVEL,57.27 FT(17.46 M) BELOW LAND-SURFACE DATUM,JAN 27, 1983; LOWEST WATER LEVEL,58.77 FT(17.91 M) BELOW LAND-SURFACE DATUM, JAN 21, 1982 .

WATER LEVELS IN FEET BELOW LAND SURFACE DATUM, CLIMATIC YEARS APRIL 1981 TO MARCH 1983
$20 N-23 W-19$ BCA 1.

41'58", HYDROLOGIC UNIT 11100203.

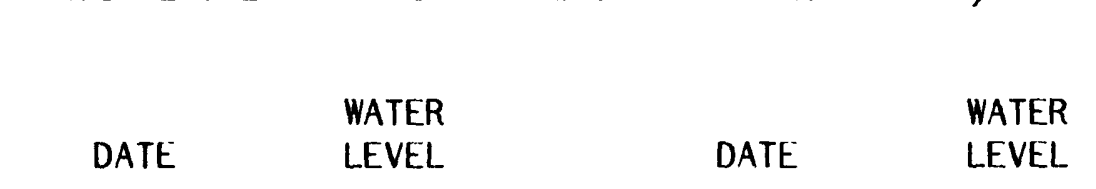

JAN $21,1982 \quad 58.77 \quad$ JAN $27,1983 \quad 57.27$

\title{
GROUND-WATER LEVELS
}

\begin{abstract}
361127099374301. LOCAL NUMBER; 2ON-23W-23 CCA 1. LOCATION,--LAT 36 11'27", LONG 099 37'43", HYDROLOGIC UNIT 11100203. AQUIFER .--OGALLALA. PERIOD OF RECORD.--1980 TO CURRENT YEAR. EXTREMES FOR PERIOD OF RECORD.--HIGHEST WATER LEVEL, 176.98 FT(53.94 M) BELOW LAND-SURFACE DATUM, JAN 27, 1983; LOWEST WATER LEVEL, 179.33 FT(54.66 M) BELOW LAND-SURFACE DATUM, MAR 17, 1981.

WATER LEVELS IN FEET BELOW LAND SURFACE DATUM, CLIMATIC YEARS APRIL 1981 TO MARCH 1983

\begin{tabular}{|c|c|c|}
\hline DATE & $\begin{array}{l}\text { WATER } \\
\text { LEVEL }\end{array}$ & DATE \\
\hline
\end{tabular}

JAN 21, $1982 \quad 178.98 \quad$ JAN $27,1983 \quad 176.98$
\end{abstract}


ELLIS COUNTY -- CONTINUED

361405099443301. LOCAL NUMBER; 20N-24W-03 DDB 1. LOCATION,--LAT 36 14'05", LONG 099 44'33", HYDROLOGIC UNIT 11100203. AQUIFER.--OGALLALA. PERIOD OF RECORD.--1980 TO CURRENT YEAR. EXTREMES FOR PERIOD OF RECORD.--HIGHEST WATER LEVEL,9.70 FT(2.96 M) BELOW LAND-SURFACE DATUM,JAN 31, 1980; LOWEST WATER LEVEL, $10.50 \mathrm{FT}(3.20 \mathrm{M})$ BELOW LAND-SURFACE DATUM, JAN 13, 1983.

WATER LEVELS IN FEET BELOW LAND SURFACE DATUM, CLIMATIC YEARS APRIL 1981 TO MARCH 1983

\begin{tabular}{|c|c|c|}
\hline & WATER & \\
\hline DATE & LEVEL & DATE \\
\hline
\end{tabular}

JAN 21, $198210.33 \quad$ JAN $13,1983 \quad 10.50$

GROUND-WATER LEVELS

361335099475301. LOCAL NUMBER; 20N-24W-07 ACA 1.

LOCATION,--LAT 36 13'35", LONG 099 47'53", HYDROLOGIC UNIT 11100203. AQUIFER .--OGALLALA.

PERIOD OF RECORD.--1980 TO CURRENT YEAR.

EXTREMES FOR PLRIOD OF RECORD.--HIGHEST WATER LEVEL, 42.48 FT(12.95 M) BELOW LAND-SURFACE DATUM,JAN 14, 1983; LOWEST WATER LEVEL,44.83 FT(13.66 M) BELOW LAND-SURFACE DATUM, JAN 21, 1982.

WATER LEVELS IN FEET BELOW LAND SURFACE DATUM, CLIMATIC YEARS APRIL 1981 TO MARCH 1983

$\begin{array}{lll} & \text { WATER } & \text { WATER } \\ \text { DATE } & \text { LEVEL } & \text { DATE }\end{array}$

JAN 21, $1982 \quad 44.83 \quad$ JAN $14,1983 \quad 42.48$ 
ELLIS COUNTY -- CONTINUED

361300099453801 . LOCAL NUMBER;

LOCATION,--LAT 36 13'00", LONG 099 AQUIFER .--OGALLALA.

PERIOD OF RECORD.--1980 TO CURRENT

EXTREMES FOR PERIOD OF RECORD.--HIGHEST WATER LEVEL, 88.46 FT(26.96 M) BELOW LAND-SURFACE DATUM, JAN 29, 1980; LOWEST WATER LEVEL,89.47 FT(27.27 M) BELOW LAND-SURFACE DATUM, JAN 21, 1982 .

WATER LEVELS IN FEET BELOW LAND SURFACE DATUM, CLIMATIC YEARS APRIL 1981 TO MARCH 1983

WATER

LEVEL

DATE

WATER

LEVEL

JAN 21, 1982

89.47

88.47

\section{GROUND-WATER LEVELS}

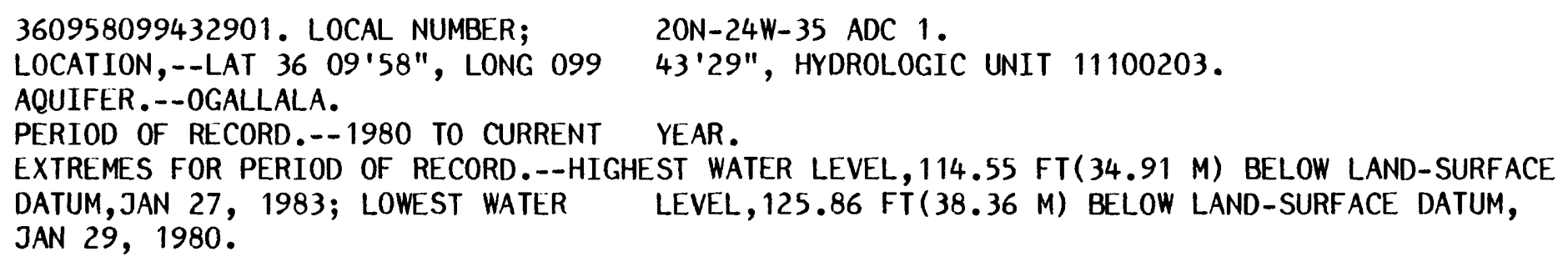

WATER LEVELS IN FEET BELOW LAND SURFACE DATUM, CLIMATIC YEARS APRIL 1981 TO MARCH 1983

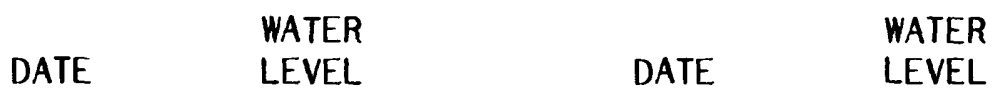

FEB 25, $1982 \quad 120.60 \quad$ JAN 27, $1983 \quad 114.55$


GROUND-WATER LEVELS

ELLIS COUNTY -- CONTINUED

361429099503501 . LOCAL NUMBER; LOCATION,--LAT 36 14'29", LONG 099 AQUIFER.--OGALLALA.

PERIOD OF RECORD.--1980 TO CURRENT YEAR. EXTREMES FOR PERIOD OF RECORD.--HIGHEST WATER LEVEL,67.22 FT(20.49 M) BELOW LAND-SURFACE DATUM, MAY 17, 1981; LOWEST WATER LEVEL,67.61 FT(20.61 M) BELOW LAND-SURFACE DATUM, JAN 13, 1983.

WATER LEVELS IN FEET BELOW LAND SURFACE DATUM, CLIMATIC YEARS APRIL 1981 TO MARCH 1983
$20 N-25 W-02$ BCA 1.

50'35", HYDROLOGIC UNIT 11100203.

\section{WATER WATER \\ DATE LEVEL DATE LEVEL \\ JAN $21,1982 \quad 67.83 \quad$ JAN $13,1983 \quad 67.61$ \\ GROUND-WATER LEVELS}

361431099523801. LOCAL NUMBER;

LOCATION,--LAT $3614^{\prime} 31^{\prime \prime}$, LONG 099

AQUIFER .--OGALLALA.

PERIOD OF RECORD.--1980 TO CURRENT YEAR.

EXTREMES FOR PERIOD OF RECORD.--HIGHEST WATER LEVEL, $57.50 \mathrm{FT}(17.53 \mathrm{M})$ BELOW LAND-SURFACE DATUM,FEB 7, 1980; LOWEST WATER JAN 21, 1982.

WATER LEVELS IN FEET BELOW LAND SURFACE DATUM, CLIMATIC YEARS APRIL 1981 TO MARCH 1983
$20 \mathrm{~N}-25 \mathrm{~W}-04$ BCA 2 .

$52^{\prime} 38^{\prime \prime}$, HYDROLOGIC UNIT 11100203. LEVEL, 59.44 FT(18.12 M) BELOW LAND-SURFACE DATUM,
DATE
WATER
LEVEL
DATE
WATER
LEVEL

JAN 21, $1982 \quad 59.44$

JAN 14, $1983 \quad 58.30$ 
ELLIS COUNTY -- CONTINUED

361403099533301. LOCAL NUMBER; LOCATION,--LAT 36 14'03", LONG 099 AQUIFER .--OGALLALA. PERIOD OF RECORD.-- 1980 TO CURRENT YEAR. EXTREMES FOR PERIOD OF RECORD.--HIGHEST WATER LEVEL,20.10 FT(6.13 M) BELOW LAND-SURFACE DATUM,FEB 7, 1980; LOWEST WATER LEVEL,23.53 FT(7.17 M) BELOW LAND-SURFACE DATUM, JAN 13, 1983.

WATER LEVELS IN FEET BELOW LAND SURFACE DATUM, CLIMATIC YEARS APRIL 1981 TO MARCH 1983
20N-25W-05 CAC 1.

53'33", HYDROLOGIC UNIT 11100203.
JAN 21, 1982
23.03
JAN $13,1983 \quad 23.53$

WATER

LEVEL

\section{DATE}

\section{GROUND-WATER LEVELS}

361429099544001. LOCAL NUMBER; 20N-25W-06 BDB 1.

LOCATION,--LAT 36 14'29", LONG 099 54'40", HYDROLOGIC UNIT 11100203. AQUIFER .--OGALLALA.

PERIOD OF RECORD.--1981 TO CURRENT YEAR.

EXTREMES FOR PERIOD OF RECORD.--HIGHEST WATER LEVEL, $100.08 \mathrm{FT}(30.50 \mathrm{M})$ BELOW LAND-SURFACE DATUM, MAR 17, 1981; LOWEST WATER LEVEL,101.69 FT(31.00 M) BELOW LAND-SURFACE DATUM, JAN 21, 1982.

WATER LEVELS IN FEET BELOW LAND SURFACE DATUM, CLIMATIC YEARS APRIL 1981 TO MARCH 1983

\begin{tabular}{|c|c|c|}
\hline & WATER & \\
\hline DATE & LEVEL & DATE \\
\hline
\end{tabular}
JAN 21, 1982
101.69
JAN 13, 1983
Z 
GROUND-WATER LEVELS

ELLIS COUNTY -- CONTINUED

361156099504001. LOCAL NUMBER; LOCATION,--LAT 36 11'56", LONG 099 AQUIFER .--OGALLALA. PERIOD OF RECORD.--1981 TO CURRENT YEAR.

EXTREMES FOR PERIOD OF RECORD.--HIGHEST WATER LEVEL, 113.10 FT(34.47 M) BELOW LAND-SURFACE DATUM, MAR 17, 1981; LOWEST WATER LEVEL, $113.68 \mathrm{FT}(34.65 \mathrm{M})$ BELOW LAND-SURFACE DATUM, JAN 21, 1982 .

WATER LEVELS IN FEET BELOW LAND SURFACE DATUM, CLIMATIC YEARS APRIL 1981 TO MARCH 1983
$20 \mathrm{~N}-25 W-23 \mathrm{BBC} 1$.

50 '40", HYDROLOGIC UNIT 11100203.
LEVEL, $113.68 \mathrm{FT}(34.65 \mathrm{M})$ BELOW LAND-SURFACE DATUM,

$\begin{array}{lll} & \text { WATER } & \\ \text { DATE } & \text { LEVEL } & \text { WATER } \\ \text { DATE } & \text { LEVEL }\end{array}$

JAN 21, 1982113.68 JAN 14, $1983 \quad 113.20$

GROUND-WATER LEVELS

361211099573801. LOCAL NUMBER; 20N-26W-15 DCC 1.

LOCATION,--LAT 36 12'11", LONG 099 57'38", HYDROLOGIC UNIT 11100203.

AQUIFER .--OGALLALA.

PERIOD OF RECORD.--1981 TO CURRENT YEAR.

EXTREMES FOR PERIOD OF RECORD.--HIGHEST WATER LEVEL, 175.76 FT(53.57 M) BELOW LAND-SURFACE DATUM,MAR 17, 1981; LOWEST WATER LEVEL,177.39 FT(54.07 M) BELOW LAND-SURFACE DATUM, JAN 13, 1983.

WATER LEVELS IN FEET BELOW LAND SURFACE DATUM, CLIMATIC YEARS APRIL 1981 TO MARCH 1983

$\begin{array}{lll} & \text { WATER } & \text { WATER } \\ \text { DATE LEVEL } & \text { DATE } & \text { LEVEL }\end{array}$

JAN 21, $1982 \quad 176.51 \quad$ JAN 13, $1983 \quad 177.39$ 
GROUND-WATER LEVELS

ELLIS COUNTY -- CONTINUED

361007099572201. LOCAL NUMBER; 20N-26W-34 ADB 1. LOCATION,--LAT 36 10'07", LONG 099 57'22", HYDROLOGIC UNIT 11100203.

AQUIFER. --OGALLALA.

PERIOD OF RECORD.--1981 TO CURRENT YEAR.

EXTREMES FOR PERIOD OF RECORD.--HIGHEST WATER LEVEL, $139.60 \mathrm{FT}(42.55 \mathrm{M})$ BELOW LAND-SURFACE DATUM, MAR 17, 1981; LOWEST WATER LEVEL, 140.41 FT(42.80 M) BELOW LAND-SURFACE DATUM, JAN 13, 1983.

WATER LEVELS IN FEET BELOW LAND SURFACE DATUM, CLIMATIC YEARS APRIL 1981 TO MARCH 1983

$\begin{array}{lll} & \text { WATER } & \text { WATER } \\ \text { DATE } & \text { LEVEL } & \text { DATE }\end{array}$

JAN 21, $1982 \quad 139.73 \quad$ JAN $13,1983 \quad 140.41$

GROUND-WATER LEVELS

360822099551301. LOCAL NUMBER; 20N-26W-36 DDB 1.

LOCATION,--LAT 36 08'22", LONG 099 55'13", HYDROLOGIC UNIT 11100203.

AQUIFER .--OGALLALA.

PERIOD OF RECORD.--1981 TO CURRENT YEAR.

EXTREMES FOR PERIOD OF RECORD.--HIGHEST WATER LEVEL, $185.55 \mathrm{FT}(56.56 \mathrm{M})$ BELOW LAND-SURFACE DATUM, JAN 21, 1981; LOWEST WATER LEVEL, 189.30 FT(57.70 M) BELOW LAND-SURFACE DATUM, MAR 17, 1981.

WATER LEVELS IN FEET BELOW LAND SURFACE DATUM, CLIMATIC YEARS APRIL 1981 T0 MARCH 1983

$\begin{array}{lll} & \text { WATER } & \text { WATER } \\ \text { DATE } & \text { LEVEL } & \text { DATE }\end{array}$

JAN 21, 1982185.55 JAN 13, $1983 \quad 186.70$ 


\section{GROUND-WATER LEVELS}

ELLIS COUNTY -- CONTINUED

361852099412801. LOCAL NUMBER; LOCATION,--LAT 36 18'52", LONG 099 AQUIFER.--OGALLALA.

PERIOD OF RECORD.--1980 TO CURRENT YEAR.

EXTREMES FOR PERIOD OF RECORD.--HIGHEST WATER LEVEL,53.54 FT(16.32 M) BELOW LAND-SURFACE DATUM,FEB 5, 1981 ; LOWEST WATER LEVEL,55.47 FT(16.91 M) BELOW LAND-SURFACE DATUM, JAN 31, 1980.

WATER LEVELS IN FEET BELOW LAND SURFACE DATUM, CLIMATIC YEARS APRIL 1981 TO MARCH 1983
$21 \mathrm{~N}-23 \mathrm{~W}-08 \mathrm{BCA} 1$. $41^{\prime} 28$ ", HYDROLOGIC UNIT 11100203.

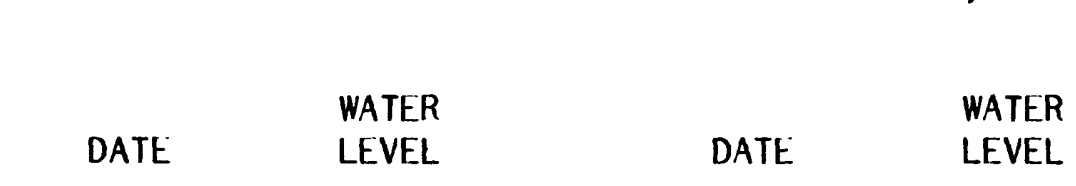
JAN 21, $1982 \quad 54.83$
JAN $12,1983 \quad 53.79$

GROUND-WATER LEVELS

361607099362601. LOCAL NUMBER;

$21 \mathrm{~N}-23 \mathrm{~W}-25$ DDA 1. LOCATION,--LAT 36 16'07", LONG 099 36'26", HYDROLOGIC UNIT 11100203. AQUIFER.--OGALLALA.

PERIOD OF RECORD.--1980 TO CURRENT YEAR.

EXTREMES FOR PERIOD OF RECORD.--HIGHEST WATER LEVEL, 48.29 FT(14.72 M) BELOW LAND-SURFACE DATUM, JAN 12, 1983; LOWEST WATER LEVEL,52.90 FT(16.12 M) BELOW LAND-SURFACE DATUM, JAN 21, 1982.

WATER LEVELS IN FEET BELOW LAND SURFACE DATUM, CLIMATIC YEARS APRIL 1981 TO MARCH 1983

$\begin{array}{lll} & \text { WATER } & \\ \text { DATE } & \text { LEVEL } & \text { WATER } \\ & \text { DATE } & \text { LEVEL }\end{array}$

JAN 21, $1982 \quad 52.90 \quad$ JAN 12, $1983 \quad 48.29$ 
GROUND-WATER LEVELS

ELLIS COUNTY -- CONTINUED

361629099424301. LOCAL NUMBER; 21N-23W-30 BBB 1. LOCATION,--LAT 36 16'29", LONG 099 42'43", HYDROLOGIC UNIT 11100203. AQUIFER.--OGALLALA. PERIOD OF RECORD.--1980 TO CURRENT YEAR. EXTREMES FOR PERIOD OF RECORD.--HIGHEST WATER LEVEL, 52.94 FT(16.14 M) BELOW LAND-SURFACE DATUM,JAN 12, 1983; LOWEST WATER LEVEL,54.12 FT(16.50 M) BELOW LAND-SURFACE DATUM, FEB 5, 1980.

WATER LEVELS IN FEET BELOW LAND SURFACE DATUM, CLIMATIC YEARS APRIL 1981 TO MARCH 1983

$\begin{array}{lll} & \text { WATER } & \text { WATER } \\ \text { DATE } & \text { LEVEL } & \text { DATE }\end{array}$

JAN 21, $1982 \quad 53.50 \quad$ JAN $12,1983 \quad 52.94$

361924099430701. LOCAL NUMBER; 21N-24W-01 DBD 2. LOCATION,--LAT 36 19'24", LONG 099 43'07", HYDROLOGIC UNIT 11100203. AQUIFER .--OGALLALA. PERIOD OF RECORD.--1980 TO CURRENT YEAR. EXTREMES FOR PERIOD OF RECORD.--HIGHEST WATER LEVEL, 31.94 FT(9.74 M) BELOW LAND-SURFACE DATUM,FEB 5, 1981; LOWEST WATER LEVEL,32.42 FT(9.88 M) BELOW LAND-SURFACE DATUM, JAN 21, 1982.

WATER LEVELS IN FEET BELOW LAND SURFACE DATUM, CLIMATIC YEARS APRIL 1981 TO MARCH 1983

$\begin{array}{lll} & \text { WATER } & \text { WATER } \\ \text { DATE } & \text { LEVEL } & \text { DATE }\end{array}$

JAN 21, $1982 \quad 32.42 \quad$ JAN 12, $1983 \quad 32.14$ 


\section{ELLIS COUNTY -- CONTINUED}

362017099455101. LOCAL NUMBER; 21N-24W-03 BBB 2. LOCATION,--LAT 36 20'17", LONG 099 45'51", HYDROLOGIC UNIT 11100203. AQUIFER.--ALLUVIUM.

PERIOD OF RECORD.--1980 TO CURRENT YEAR.

EXTREMES FOR PERIOD OF RECORD.--HIGHEST WATER LEVEL,5.46 FT(1.66 M) BELOW LAND-SURFACE DATUM,JAN 29, 1980; LOWEST WATER LEVEL,6.23 FT(1.90 M) BELOW LAND-SURFACE DATUM, JAN 21, 1982.

WATER LEVELS IN FEET BELOW LAND SURFACE DATUM, CLIMATIC YEARS APRIL 1981 TO MARCH 1983

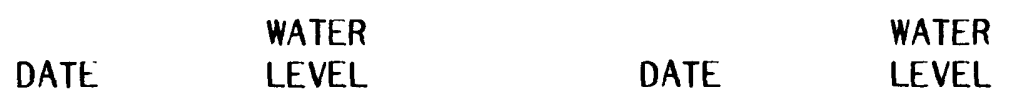

JAN 21, $1982 \quad 6.23 \quad$ FEB $17,1983 \quad 5.78$

\section{GROUND-WATER LEVELS}

361912099452701. LOCAL NUMBER; 21N-24W-03 CDD 1. LOCATION,--LAT 36 19'12", LONG 099 45'27", HYDROLOGIC UNIT 11100203. AQUIFER.--TERRACE DEPOSITS.

PERIOD OF RECORD.--1981 TO CURRENT YEAR.

EXTREMES FOR PERIOD OF RECORD.--HIGHEST WATER LEVEL, $16.95 \mathrm{FT}(5.17 \mathrm{M})$ BELOW LAND-SURFACE DATUM,FEB 5, 1981; LOWEST WATER LEVEL, $16.95 \mathrm{FT}(5.17 \mathrm{M})$ BELOW LAND-SURFACE DATUM, FEB 5, 1981.

WATER LEVELS IN FEET BELOW LAND SURFACE DATUM, CLIMATIC YEARS APRIL 1981 TO MARCH 1983

\begin{tabular}{|c|c|c|}
\hline & WATER & \\
\hline DATE & LEVEL & DATE \\
\hline
\end{tabular}

JAN 21, 1982 Z $\quad$ FEB 17, $1983 \quad 15.14 \mathrm{P}$


ELLIS COUNTY -- CONTINUED

361911099481901. LOCAL NUMBER; LOCATION,--LAT 36 19'11", LONG 099 AQUIFER .--OGALLALA.

PERIOD OF RECORD.--1980 TO CURRENT YEAR. EXTREMES FOR PERIOD OF RECORD.--HIGHEST WATER LEVEL,9.54 FT(2.91 M) BELOW LAND-SURFACE DATUM, MAR 25, 1980; LOWEST WATER LEVEL, 10.84 FT(3.30 M) BELOW LAND-SURFACE DATUM, JAN 21, 1982.

WATER LEVELS IN FEET BELOW LAND SURFACE DATUM, CLIMATIC YEARS APRIL 1981 TO MARCH 1983
$21 \mathrm{~N}-24 \mathrm{~W}-06$ DCD 2. 48'19", H:YDROLOGIC UNIT 11100203.

$\begin{array}{cccc} & & & \\ \text { WATER } & & \text { WATER } \\ \text { DATE } & \text { LEVEL } & \text { DATE } & \text { LEVEL } \\ \text { JAN 21, } 1982 & 10.84 & \text { JAN 12, 1983 } & 9.95\end{array}$

\section{GROUND-WATER LEVELS}

361817099461301. LOCAL NUMBER; LOCATION, --LAT $3618^{\prime} 17^{\prime \prime}$, LONG 099 AQUIFER .--OGALLALA.

PERIOD OF RECORD.--1980 TO CURRENT YEAR. EXTREMES FOR PERIOD OF RECORD.--HIGHEST WATER LEVEL, 39.10 FT(11.92 M) BELOW LAND-SURFACE JAN 31, 1980.
$21 \mathrm{~N}-24 \mathrm{~W}-09$ DDC 1

46 '13", HYDROLOGIC UNIT 11100203. 


\section{ELLIS COUNTY -- CONTINUED}

361905099455801. LOCAL NUMBER; LOCATION,--LAT 36 19'05", LONG 099 AQUIFER.--ALLUVIUM.

PERIOD OF RECORD.--1980 TO CURRENT YEAR. EXTREMES FOR PERIOD OF RECORD.--HIGHEST WATER LEVEL,9.56 FT(2.91 M) BELOW LAND-SURFACE DATUM, JAN 31, 1980; LOWEST WATER LEVEL, $10.82 \mathrm{FT}(3.30 \mathrm{M})$ BELOW LAND-SURFACE DATUM, JAN 21, 1982 .

WATER LEVELS IN FEET BELOW LAND SURFACE DATUM, CLIMATIC YEARS APRIL 1981 TO MARCH 1983
JAN $21,1982 \quad 10.82$
FEB $17,1983 \quad 10.74$

GROUND-WATER LEVELS

361815099444201. LOCAL NUMBER;

$21 \mathrm{~N}-24 \mathrm{~W}-11 \operatorname{CCC} 1$.

LOCATION,--LAT 36 18'15", LONG 099 44'42", HYDROLOGIC UNIT 11100203.

AQUIFER ---ALLUVIUM.

PERIOD OF RECORD.--1979 TO CURRENT YEAR.

EXTREMES FOR PERIOD OF RECORD.--HIGHEST WATER LEVEL, 38.04 FT(11.59 M) BELOW LAND-SURFACE

DATUM,APR 11, 1979; LOWEST WATER LEVEL,40.57 FT(12.37 M) BELOW LAND-SURFACE DATUM, JAN 18, 1982.

WATER LEVELS IN FEET BELOW LAND SURFACE DATUM, CLIMATIC YEARS APRIL 1981 TO MARCH 1983

\begin{tabular}{cccc}
\multicolumn{1}{c}{ WATER } & \multicolumn{3}{c}{ WATER } \\
DATE & LEVEL & DATE & LEVEL \\
JAN 18, 1982 & 40.57 & JAN 12, 1983 & $\mathbf{3 8 . 8 5}$
\end{tabular}


361648099442601. LOCAL NUMBER; 21N-24W-23 CAD 1. LOCATION,--LAT 36 16'48", LONG 099 44'26", HYDROLOGIC UNIT 11100203. AQUIFER.--OGALLALA.

PERIOD OF RECORD.--1980 TO CURRENT YEAR.

EXTREMES FOR PERIOD OF RECORD.--HIGHEST WATER LEVEL, 40.50 FT(12.34 M) BELOW LAND-SURFACE DATUM,FEB 5, 1981; LOWEST WATER LEVEL, 41.45 FT(12.63 M) BELOW LAND-SURFACE DATUM, JAN 21, 1982.

WATER LEVELS IN FEET BELOW LAND SURFACE DATUM, CLIMATIC YEARS APRIL 1981 TO MARCH 1983

$\begin{array}{lll} & \text { WATER } & \\ \text { DATE } & \text { WEVEL } & \text { DATER } \\ \text { LEVEL }\end{array}$

JAN 21, $1982 \quad 41.45$ FEB 17, $1983 \quad 40.57$

GROUND-WATER LEVELS

361646099434301. LOCAL NUMBER; 21N-24W-24 CBC 1. LOCATION, --LAT 36 16'46", LONG 099 43'43", HYDROLOGIC UNIT 11100203. AQUIFER.--OGALLALA.

PERIOD OF RECORD.--1980 TO CURRENT YEAR.

EXTREMES FOR PERIOD OF RECORD.--HIGHEST WATER LEVEL, $37.14 \mathrm{FT}(11.32 \mathrm{M})$ BELOW LAND-SURFACE DATUM,FEB 5, 1981; LOWEST WATER LEVEL, 37.96 FT(11.57 M) BELOW LAND-SURFACE DATUM, FEB 5, 1980.

WATER LEVELS IN FEET BELOW LAND SURFACE DATUM, CLIMATIC YEARS APRIL 1981 TO MARCH 1983

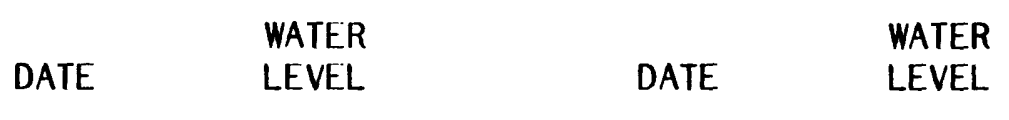

JAN 21, $1982 \quad 37.73 \quad$ JAN 12, $1983 \quad 37.15$ 


\section{ELLIS COUNTY -- CONTINUED}

361600099460301 . LOCAL NUMBER; LOCATION,--LAT 36 16'00", LONG 099 AQUIFER.--OGALLALA. PERIOD OF RECORD.--1980 TO CURRENT YEAR. EXTREMES FOR PERIOD OF RECORD.--HIGHEST WATER LEVEL, $35.46 \mathrm{FT}(10.81 \mathrm{M})$ BELOW LAND-SURFACE. DATUM,FEB 6, 1980; LOWEST WATER LEVEL,36.00 FT(10.97 M) BELOW LAND-SURFACE DATUM, FEB 5, 1981.

WATER LEVELS IN FEET BELOW LAND SURFACE DATUM, CLIMATIC YEARS APRIL 1981 TO MARCH 1983
JAN 18,1982
35.79
JAN 12,1983
0

GROUND-WATER LEVELS

361552099475201. LOCAL NUMBER; 21N-24W-29 CAC 1. LOCATION,--LAT 36 15'52", LONG 099 47'52", HYDROLOGIC UNIT 11100203. AQUIFER.--OGALLALA. PERIOD OF RECORD.--1980 TO CURRENT YEAR. EXTREMES FOR PERIOD OF RECORD.--HIGHEST WATER LEVEL, 69.90 FT(21.31 M) BELOW LAND-SURFACE DATUM,FEB 5, 1980; LOWEST WATER LEVEL,70.40 FT(21.46 M) BELOW LAND-SURFACE DATUM, JAN $18,1982$.

WATER LEVELS IN FEET BELOW LAND SURFACE DATUM, CLIMATIC YEARS APRIL 1981 TO MARCH 1983

\begin{tabular}{|c|c|c|}
\hline & WATER & \\
\hline DATE & LEVEL & DATE \\
\hline
\end{tabular}

JAN $18,1982 \quad 70.40 \quad$ JAN 12, $1983 \quad 70.34$




\section{ELLIS COUNTY -- CONTINUED}

361455099490201. LOCAL NUMBER; LOCATION,--LAT 36 14'55", LONG 099 AQUIFER.--OGALLALA.

PERIOD OF RECORD.--1980 TO CURRENT YEAR.

EXTREMES FOR PERIOD OF RECORD.--HIGHEST WATER LEVEL, $39.78 \mathrm{FT}(12.12 \mathrm{M})$ BELOW LAND-SURFACE DATUM,FEB 5, 1981; LOWEST WATER LEVEL,43.52 FT(13.26 M) BELOW LAND-SURFACE DATUM, FEB 5, 1980 .

WATER LEVELS IN FEET BELOW LAND SURFACE DATUM, CLIMATIC YEARS APRIL 1981 TO MARCH 1983

\section{GROUND-WATER LEVELS}

361735099513501. LOCAL NUMBER; LOCATION,--LAT 36 17'35", LONG 099 AQUIFER.--OGALLALA.

\section{PERIOD OF RECORD.--1980 TO CURRENT YEAR.}

EXTREMES FOR PERIOD OF RECORD.--HIGHEST WATER LEVEL,6.07 FT(1.85 M) BELOW LAND-SURFACE DATUM,FEB 5, 1980; LOWEST WATER LEVEL,7.10 FT(2.16 M) BELOW LAND-SURFACE DATUM, JAN 20, 1982.

WATER LEVELS IN FEET BELOW LAND SURFACE DATUM, CLIMATIC YEARS APRIL 1981 TO MARCH 1983

WATER

DATE

JAN 20, 1982
LEVEL

7.10
$21 \mathrm{~N}-25 \mathrm{~W}-15$ DDB 1.

51 '35", HYDROLOGIC UNIT 11100203.
WATER

LEVEL

JAN 19, 1982

Z JAN 12,1983

Z

JAN 13, $1983 \quad 0$ 


\section{ELLIS COUNTY -- CONTINUED}

361737099531301. LOCAL NUMBER; 21N-25W-16 CCA 1. LOCATION,--LAT 36 17'37", LONG 099 53'13", HYDROLOGIC UNIT 11100203. AQUIFER.--OGALLALA. PERIOD OF RECORD.--1980 TO CURRENT YEAR.

EXTREMES FOR PERIOD OF RECORD.--HIGHEST WATER LEVEL, 13.46 FT(4.10 M) BELOW LAND-SURFACE DATUM,FEB 6, 1980; LOWEST WATER LEVEL, 15.45 FT(4.71 M) BELOW LAND-SURFACE DATUM, JAN 19, 1982.

WATER LEVELS IN FEET BELOW LAND SURFACE DATUM, CLIMATIC YEARS APRIL 1981 TO MARCH 1983

\begin{tabular}{|c|c|c|}
\hline & WATER & \\
\hline DATE & LEVEL & DATE \\
\hline
\end{tabular}

JAN 19, $1982 \quad 15.45 \quad$ JAN 19, $1983 \quad 14.28$

\section{GROUND-WATER LEVELS}

361635099543101. LOCAL NUMBER;

$21 \mathrm{~N}-25 \mathrm{~W}-20 \operatorname{CCC} 1$.

LOCATION,--LAT 36 16'35", LONG 099 54'31", HYDROLOGIC UNIT 11100203.

AQUIFER .--OGALLALA.

PERIOD OF RECORD.--1980 TO CURRENT YEAR.

EXTREMES FOR PERIOD OF RECORD.--HIGHEST WATER LEVEL, 32.74 FT(9.98 M) BELOW LAND-SURFACE DATUM,FEB 6, 1980; LOWEST WATER LEVEL,36.95 FT(11.26 M) BELOW LAND-SURFACE DATUM, JAN 19, 1982.

WATER LEVELS IN FEET BELOW LAND SURFACE DATUM, CLIMATIC YEARS APRIL 1981 TO MARCH 1983

\begin{tabular}{|c|c|c|}
\hline & WATER & \\
\hline DATE & LEVEL & DATE \\
\hline
\end{tabular}

JAN 19, $1982 \quad 36.95 \quad$ JAN 18, $1983 \quad 34.07$ 
GROUND-WATER LEVELS

ELLIS COUNTY - - CONTINUED

361641099513801 . LOCAL NUMBER; LOCATION,--LAT 36 16'41', LONG 099 AQUIFER .--OGALLALA.

PERIOD OF RECORD.--1980 TO CURRENT YEAR.

EXTREMES FOR PERIOD OF RECORD.--HIGHEST WATER LEVEL, $29.21 \mathrm{FT}(8.90 \mathrm{M})$ BELOW LAND-SURFACE DATUM, JAN 18, 1983; LOWEST WATER LEVEL,46.12 FT(14.06 M) BELOW LAND-SURFACE DATUM, FEB $5,1980$.

WATER LEVELS IN FEET BELOW LAND SURFACE DATUM, CLIMATIC YEARS APRIL 1981 TO MARCH 1983

JAN 20, $1982 \quad 30.43 \quad$ JAN 18, $1983 \quad 29.21$

GROUND-WATER LEVELS

361634099501301. LOCAL NUMBER; 21N-25W-24 CCC 1.

LOCATION,--LAT 36 16'34", LONG 099 50'13", HYDROLOGIC UNIT 11100203.

AQUIFER.--OGALLALA.

PERIOD OF RECORD.--1980 TO CURRENT YEAR.

EXTREMES FOR PERIOD OF RECORD.--HIGHEST WATER LEVEL,40.62 FT(12.38 M) BELOW LAND-SURFACE

DATUM, MAR 18, 1981; LOWEST WATER LEVEL,42.45 FT(12.94 M) BELOW LAND-SURFACE DATUM, JAN 18,1982 .

WATER LEVELS IN FEET BELOW LAND SURFACE DATUM, CLIMATIC YEARS APRIL 1981 TO MARCH 1983

$\begin{array}{lll} & \text { WATER } & \\ \text { DATE } & \text { LEVEL } & \text { WATER } \\ \text { LEVEL }\end{array}$

JAN 18, $198242.45 \quad$ JAN 18, $1983 \quad 41.53$ 
ELLIS COUNTY -- CONTINUED

361550099530801. LOCAL NUMBER; LOCATION,--LAT 36 15'50", LONG 099 AQUIFER.--OGALLALA.

PERIOD OF RECORD.-- 1980 TO CURRENT YEAR.

EXTREMES FOR PERIOD OF RECORD.--HIGHEST WATER LEVEL,23.33 FT(7.11 M) BELOW LAND-SURFACE DATUM,FEB 6, 1980; LOWEST WATER LEVEL,28.83 FT(8.79 M) BELOW LAND-SURFACE DATUM, JAN 20, 1982.

WATER LEVELS IN FEET BELOW LAND SURFACE DATUM, CLIMATIC YEARS APRIL 1981 TO MARCH 1983

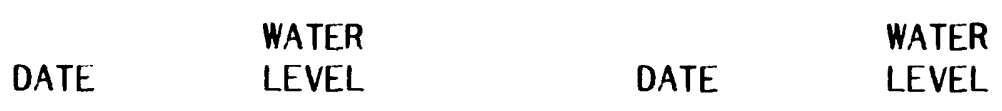

JAN $20,1982 \quad 28.83 \quad$ JAN $13,1983 \quad 25.04$

\section{GROUND-WATER LEVELS}

361558099524101. LOCAL NUMBER; 21N-25W-28 DBD 1. LOCATION,--LAT 36 15'58", LONG 099 52'41", HYOROLOGIC UNIT 11100203. AQUIFER.--OGALLALA. PERIOD OF RECORD.--1979 TO CURRENT YEAR. EXTREMES FOR PERIOD OF RECORD.--HIGHEST WATER LEVEL, 94.45 FT(28.79 M) BELOW LAND-SURFACE DATUM, JAN 18, 1983; LOWEST WATER LEVEL,95.99 FT(29.26 M) BELOW LAND-SURFACE DATUM, APR 12, 1979.

WATER LEVELS IN FEET BELOW LAND SURFACE DATUM, CLIMATIC YEARS APRIL 1981 TO MARCH 1983

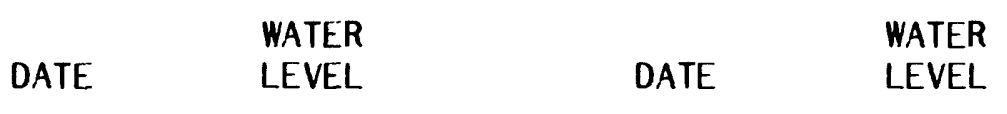

JAN 20, $198295.54 \quad$ JAN $18,1983 \quad 94.45$ 


\section{GROUND-WATER LEVELS}

\section{ELLIS COUNTY -- CONTINUED}

361622099552601 . LOCAL NUMBER; LOCATION,--LAT $3616^{\prime 22}$ ', LONG 099 AQUIFER.--OGALLALA.

PERIOD OF RECORD.--1980 TO CURRENT YEAR. EXTREMES FOR PERIOD OF RECORD.--HIGHEST WATER LEVEL,43.69 FT(13.32 M) BELOW LAND-SURFACE DATUM,FEB 5, 1980; LOWEST WATER LEVEL,46.05 FT(14.04 M) BELOW LAND-SURFACE DATUM, JAN 20, 1982.

WATER LEVELS IN FEET BELOW LAND SURFACE DATUM, CLIMATIC YEARS APRIL 1981 TO MARCH 1983
$21 \mathrm{~N}-25 \mathrm{~W}-30 \mathrm{BBD} 1$.

55'26", HYDROLOGIC UNIT 11100203.

\begin{tabular}{|c|c|}
\hline & WATER \\
\hline DATE & LEVEL \\
\hline
\end{tabular}
JAN 20, 1982
46.05
JAN 13, 1983
44.82

\section{GROUND-WATER LEVELS}

361456099542401. LOCAL NUMBER;

$21 \mathrm{~N}-25 W-32$ CCA 1.

LOCATION,--LAT 36 14'56", LONG 099 54'24", HYDROLOGIC UNIT 11100203.

AQUIFER .--OGALLALA.

PERIOD OF RECORD.--1980 TO CURRENT YEAR.

EXTREMES FOR PERIOD OF RECORD.--HIGHEST WATER LEVEL, $70.65 \mathrm{FT}(21.53 \mathrm{M})$ BELOW LAND-SURFACE DATUM, MAR 18, 1981; LOWEST WATER LEVEL,71.83 FT(21.89 M) BELOW LAND-SURFACE DATUM, JAN 20, 1982 .

WATER LEVELS IN FEET BELOW LAND SURFACE DATUM, CLIMATIC YEARS APRIL 1981 TO MARCH 1983

$\begin{array}{lll} & \text { WATER } & \\ \text { DATE } & \text { LEVEL } & \text { WATER } \\ \text { LATE } & \text { LEVEL }\end{array}$

JAN 20, $1982 \quad 71.83 \quad$ JAN 13, $1983 \quad 71.55$


361501099521201. LOCAL NUMBER; 21N-25W-34 CBD 1. LOCATION,--LAT 36 15'01", LONG 099 52'12", HYDROLOGIC UNIT 11100203. AQUIFER .- OGALLALA. PERIOD OF RECORD.--1980 TO CURRENT YEAR. EXTREMES FOR PERIOD OF RECORD.--HIGHEST WATER LEVEL, $62.55 \mathrm{FT}(19.07 \mathrm{M})$ BELOW LAND-SURFACE DATUM,JAN 20, 1982; LOWEST WATER LEVEL,69.43 FT(21.16 M) BELOW LAND-SURFACE DATUM, JAN 13, 1983.

WATER LEVELS IN FEET BELOW LAND SURFACE DATUM, CLIMATIC YEARS APRIL 1981 TO MARCH 1983

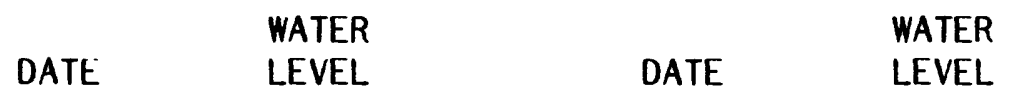

JAN 20, $198262.55 \quad$ JAN 13, $1983 \quad 69.43$

GROUND-WATER LEVELS

361534099501401. LOCAL NUMBER; 21N-25W-36 BBB 1. LOCATION,--LAT 36 15'34", LONG 099 50'14", HYOROLOGIC UNIT 11100203. AQUIFER .--OGALLALA. PERIOD OF RECORD.--1980 TO CURRENT YEAR. EXTREMES FOR PERIOD OF RECORD.--HIGHEST WATER LEVEL,77.20 FT(23.53 M) BELOW LAND-SURFACE DATUM, JAN 18, 1983; LOWEST WATER LEVEL,91.76 FT(27.97 M) BELOW LAND-SURFACE DATUM, FEB 5, 1980.

WATER LEVELS IN FEET BELOW LAND SURFACE DATUM, CLIMATIC YEARS APRIL 1981 TO MARCH 1983

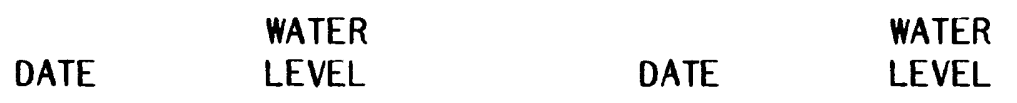

JAN 18, $1982 \quad 78.55 \quad$ JAN $18,1983 \quad 77.20$


GROUND-WATER LEVELS

ELLIS COUNTY -- CONTINUED

361831099590901. LOCAL NUMBER; 21N-26W-09 DAC 1.

LOCATION,--LAT 36 18'31", LONG 099 59'09", HYDROLOGIC UNIT 11100203.

AQUIFER.--OGALLALA.

PERIOD OF RECORD.--1980 TO CURRENT YEAR.

EXTREMES FOR PERIOD OF RECORD.--HIGHEST WATER LEVEL, $81.84 \mathrm{FT}(24.94 \mathrm{M})$ BELOW LAND-SURFACE

DATUM, MAR 17, 1981; LOWEST WATER LEVEL, 122.70 FT( $37.40 \mathrm{M})$ BELOW LAND-SURFACE DATUM, FEB $6,1980$.

WATER LEVELS IN FEET BELOW LAND SURFACE DATUM, CLIMATIC YEARS APRIL 1981 TO MARCH 1983

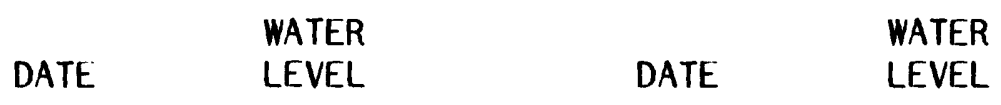

JAN $19,1982 \quad 83.37$ JAN $13,1983 \quad 82.62$

GROUND-WATER LEVELS

361720099590201. LOCAL NUMBER; 21N-26W-21 AAA 1.

LOCATION,--LAT 36 17'20", LONG 099 59'02", HYDROLOGIC UNIT 11100203.

AQUIFER .--OGALLALA.

PERIOD OF RECORD.--1980 TO CURRENT YEAR.

EXTREMES FOR PERIOD OF RECORD.--HIGHEST WATER LEVEL,2.53 FT(.77 M) BELOW LAND-SURFACE DATUM, JAN 13, 1983; LOWEST WATER LEVEL, 19.04 FT $(5.80 \mathrm{M})$ BELOW LAND-SURFACE DATUM, FEB $6,1980$.

WATER LEVELS IN FEET BELOW LAND SURFACE DATUM, CLIMATIC YEARS APRIL 1981 TO MARCH 1983

\begin{tabular}{|c|c|}
\hline & WATER \\
\hline DATE & LEVEL \\
\hline
\end{tabular}

JAN 19, $1982 \quad 3.10 \quad$ JAN $13,1983 \quad 2.53$


GROUND-WATER LEVELS

\section{ELLIS COUNTY -- CONTINUED}

361638099590301 . LOCAL NUMBER;

LOCATION,--LAT 36 16'38', LONG 099

AQUIFER. - OGALLALA.

PERIOD OF RECORD.--1980 TO CURRENT YEAR.

EXTREMES FOR PERIOD OF RECORD.--HIGHEST WATER LEVEL, 17.87 FT $(5.45 \mathrm{M})$ BELOW LAND-SURFACE DATUM,FEB 6, 1980; LOWEST WATER LEVEL,29.94 FT(9.13 M) BELOW LAND-SURFACE DATUM, JAN 13, 1983.

WATER LEVELS IN FEET BELOW LAND SURFACE DATUM, CLIMATIC YEARS APRIL 1981 TO MARCH 1983

$\begin{array}{ccccc} & \text { WATER } & & & \text { WATER } \\ \text { DATE } & \text { LEVEL } & & \text { DATE } & \text { LEVEL } \\ & & & & \\ & & \text { Z } & \text { JAN 13, 1983 } & 29.94\end{array}$

\section{GROUND-WATER LEVELS}

361647099554901. LOCAL NUMBER;

$21 \mathrm{~N}-26 \mathrm{~W}-24 \mathrm{DBD} 1$.

LOCATION,--LAT 36 16'47", LONG 099 55'49", HYDROLOGIC UNIT 11100203.

AQUIFER .--OGALLALA.

PERIOD OF RECORD.--1980 TO CURRENT YEAR.

EXTREMES FOR PERIOD OF RECORD.--HIGHEST WATER LEVEL, 16.64 FT(5.07 M) BELOW LAND-SURFACE DATUM,FEB 5, 1980; LOWEST WATER LEVEL, 18.50 FT(5.64 M) BELOW LAND-SURFACE DATUM, JAN 20, 1982 .

WATER LEVELS IN FEET BELOW LAND SURFACE DATUM, CLIMATIC YEARS APRIL 1981 TO MARCH 1983

$\begin{array}{lll} & \text { WATER } & \text { WATER } \\ \text { DATE } & \text { LEVEL } & \text { DATE }\end{array}$

JAN 20, $1982 \quad 18.50 \quad$ FEB 16, $1983 \quad 18.06$ 
362451099372701. LOCAL NUMBER;

LOCATION,--LAT 36 24'51', LONG 099

AQUIFER.--OGALLALA.

PERIOD OF RECORD - - 1980 TO CURRENT YEAR

EXTREMES FOR PERIOD OF RECORD.--HIGHEST WATER LEVEL, 3.78 FT(1.15 M) BELOW LAND-SURFACE DATUM, JAN 31, 1980; LOWEST WATER

FEB 4, 1981.

WATER LEVELS IN FEET BELOW LAND SURFACE DATUM, CLIMATIC YEARS APRIL 1981 TO MARCH 1983

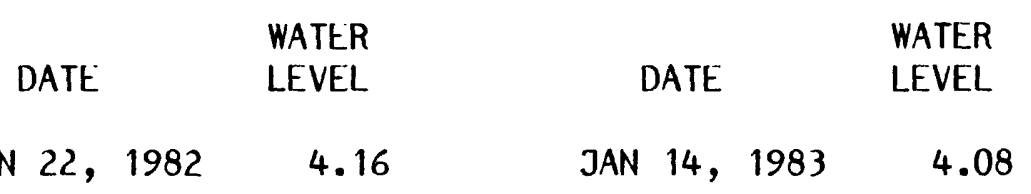

GROUND-WATER LEVELS

362450099383101. LOCAL NUMBER; 22N-23W-03 ADD 1.

LOCATION,--LAT 36 24'50", LONG 099 38'31", HYDROLOGIC UNIT 11100203. AQUIFER . - OGALLALA.

PERIOD OF RECORD.--1980 TO CURRENT YEAR.

EXTREMES FOR PERIOD OF RECORD.--HIGHEST WATER LEVEL, 13.87 FT(4.23 M) BELOW LAND-SURFACE DATUM, JAN 22, 1982; LOWEST WATER LEVEL, 14.57 FT(4.44 M) BELOW LAND-SURFACE DATUM, FEB $4,1981$.

WATER LEVELS IN FEET BELOW LAND SURFACE DATUM, CLIMATIC YEARS APRIL 1981 TO MARCH 1983

$\begin{array}{lll} & \text { WATER } & \text { WATER } \\ \text { DATE } & \text { LEVEL } & \text { DATE }\end{array}$

JAN 22, $1982 \quad 13.87 \quad$ FEB $15,1983 \quad 14.40$ 
ELLIS COUNTY -- CONTINUED

362415099393901. LOCAL NUMBER; 22N-23W-09 AAB 1.

LOCATION,--LAT 36 24'15", LONG 099 39'39", HYDROLOGIC UNIT 11100203.

AQUIFER. - OGALLALA.

PERIOD OF RECORD.--1980 TO CURRENT YEAR.

EXTREMES FOR PERIOD OF RECORD.--HIGHEST WATER LEVEL, 16.55 FT (5.04 M) BELOW LAND-SURFACE DATUM, FEB 4, 1981; LOWEST WATER LEVEL, $17.08 \mathrm{FT}(5.21 \mathrm{M})$ BELOW LAND-SURFACE DATUM, FEB 15, 1983.

WATER LEVELS IN FEET BELOW LAND SURFACE DATUM, CLIMATIC YEARS APRIL 1981 TO MARCH 1983

\begin{tabular}{ccccc}
\multicolumn{1}{c}{} & WATER & & & WATER \\
DATE & LEVEL & DATE & LEVEL \\
JAN 22, 1982 & 16.89 & FEB 15, 1983 & 17.08
\end{tabular}

GROUND-WATER LEVELS

362340099373501. LOCAL NUMBER; 22N-23W-11 DDB 1. LOCATION,--LAT 36 23'40", LONG 099 37'35", HYDROLOGIC UNIT 11100203. AQUIFER.--OGALLALA. PERIOD OF RECORD.--1980 TO CURRENT YEAR. EXTREMES FOR PERIOD OF RECORD.--HIGHEST WATER LEVEL, 6.47 FT(1.97 M) BELOW LAND-SURFACE DATUM, JAN 22, 1982; LOWEST WATER LEVEL,7.41 FT(2.26 M) BELOW LAND-SURFACE DATUM, FEB 15, 1983.

WATER LEVELS IN FEET BELOW LAND SURFACE DATUM, CLIMATIC YEARS APRIL 1981 TO MARCH 1983

\begin{tabular}{|c|c|c|}
\hline & WATER & \\
\hline DATE & LEVEL & DATE \\
\hline
\end{tabular}

JAN 22, $1982 \quad 6.47 \quad$ FEB 15, $1983 \quad 7.41$


GROUND-WATER LEVELS

ELLIS COUNTY -- CONTINUED

362254099364001. LOCAL NUMBER; 22N-23W-13 DBD 1.

LOCATION,--LAT 36 22'54", LONG 099 36'40", HYDROLOGIC UNIT 11100203. AQUIFER, - -OGALLALA.

PERIOD OF RECORD.--1980 TO CURRENT YEAR.

EXTREMES FOR PERIOD OF RECORD.--HIGHEST WATER LEVEL,9.74 FT(2.97 M) BELOW LAND-SURFACE DATUM, JAN 29, 1980; LOWEST WATER LEVEL,11.07 FT(3.37 M) BELOW LAND-SURFACE DATUM, FEB 4, 1981.

WATER LEVELS IN FEET BELOW LAND SURFACE DATUM, CLIMATIC YEARS APRIL 1981 TO MARCH 1983

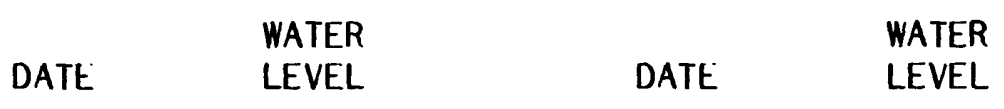

JAN 22, $1982 \quad 10.18 \quad$ JAN 14, $1983 \quad 9.89$

GROUND-WATER LEVELS

363753099405701. LOCAL NUMBER; 22N-23W-17 DBD 1.

LOCATION,--LAT 36 37'53", LONG 099 40'57', HYDROLOGIC UNIT 11100203.

AQUIFER .--OGALLALA.

PERIOD OF RECORD. --1980 TO CURRENT YEAR.

EXTREMES FOR PERIOD OF RECORD.--HIGHEST WATER LEVEL, $17.21 \mathrm{FT}(5.25 \mathrm{M})$ BELOW LAND-SURFACE DATUM,FEB 14, 1983; LOWEST WATER LEVEL, $17.85 \mathrm{FT}(5.44 \mathrm{M})$ BELOW LAND-SURFACE DATUM, JAN 21, 1982 .

WATER LEVELS IN FEET BELOW LAND SURFACE DATUM, CLIMATIC YEARS APRIL 1981 TO MARCH 1983

$\begin{array}{lll} & \text { WATER } & \text { WATER } \\ \text { DATE } & \text { LEVEL } & \text { DATE }\end{array}$

JAN 21, $1982 \quad 17.85 \quad$ FEB 14, $1983 \quad 17.21$ 
ELLIS COUNTY -- CONTINUED

362201099415501. LOCAL NUMBER; LOCATION,--LAT 3622 '01", LONG 099 AQUIFER.--OGALLALA. PERIOD OF RECORD.--1980 TO CURRENT EXTREMES FOR PERIOD OF RECORD.--HIGHEST WATER LEVEL,22.07 FT(6.73 M) BELOW LAND-SURFACE DATUM,FEB 4, 1981; LOWEST WATER LEVEL,23.03 FT(7.02 M) BELOW LAND-SURFACE DATUM, FEB 14, 1983.

WATER LEVELS IN FEET BELOW LAND SURFACE DATUM, CLIMATIC YEARS APRIL 1981 TO MARCH 1983

JAN 21, $1982 \quad 22.18 \quad$ FEB 14, $1983 \quad 23.03$

\section{GROUND-WATER LEVELS}

362221099383801. LOCAL NUMBER; 22N-23W-22 ADB 1.

LOCATION,--LAT 36 22'21", LONG 099 38'38", HYDROLOGIC UNIT 11100203.

AQUIFER .--OGALLALA.

PERIOD OF RECORD.--1980 TO CURRENT YEAR.

EXTREMES FOR PERIOD OF RECORD.--HIGHEST WATER LEVEL, 13.81 FT(4.21 M) BELOW LAND-SURFACE DATUM,FEB 15, 1983; LOWEST WATER LEVEL, 17.94 FT(5.47 M) BELOW LAND-SURFACE DATUM, JAN 31, 1980 .

WATER LEVELS IN FEET BELOW LAND SURFACE DATUM, CLIMATIC YEARS APRIL 1981 TO MARCH 1983

$\begin{array}{lll} & \text { WATER } & \text { WATER } \\ \text { DATE } & \text { LEVEL } & \text { DATE }\end{array}$

JAN 21, $1982 \quad 14.37 \quad$ FEB 15, $1983 \quad 13.81$ 
ELLIS COUNTY -- CONTINUED

363717099214701. LOCAL NUMBER; LOCATION,--LAT 36 37'17", LONG 099 AQUIFER.--OGALLALA.

PERIOD OF RECORD.--1980 TO CURRENT YEAR.

EXTREMES FOR PERIOD OF RECORD.--HIGHEST WATER LEVEL, $27.88 \mathrm{FT}(8.50 \mathrm{M})$ BELOW LAND-SURFACE DATUM,FEB 4, 1981; LOWEST WATER LEVEL, 32.10 FT(9.78 M) BELOW LAND-SURFACE DATUM, JAN 31, 1980.

WATER LEVELS IN FEET BELOW LAND SURFACE DATUM, CLIMATIC YEARS APRIL 1981 TO MARCH 1983

$\begin{array}{lll} & \text { WATER } & \\ \text { DATE } & \text { WEVEL } & \text { WATER } \\ & \text { DEVEL }\end{array}$

JAN 21, $1982 \quad 29.95 \quad$ FEB $15,1983 \quad 28.73$

GROUND-WATER LEVELS

362056099382001. LOCAL NUMBER; 22N-23W-26 DCC 1. LOCATION,--LAT 36 20'56", LONG 099 38'20", HYDROLOGIC UNIT 11100302. AQUIFER. - -OGALLALA. PERIOD OF RECORD.--1980 TO CURRENT YEAR. EXTREMES FOR PERIOD OF RECORD.--HIGHEST WATER LEVEL, $48.36 \mathrm{FT}(14.74 \mathrm{M})$ BELOW LAND-SURFACE DATUM,FEB 15, 1983; LOWEST WATER LEVEL,50.22 FT(15.31 M) BELOW LAND-SURFACE DATUM, JAN 21, 1982.

WATER LEVELS IN FEET BELOW LAND SURFACE DATUM, CLIMATIC YEARS APRIL 1981 TO MARCH 1983

$\begin{array}{lll} & \text { WATER } & \text { WATER } \\ \text { DATE } & \text { LEVEL } & \text { DATE }\end{array}$

JAN 21, $1982 \quad 50.22 \quad$ FEB $15,1983 \quad 48.36$ 


\section{GROUND-WATER LEVELS}

\section{ELLIS COUNTY -- CONTINUED}

362052099410901. LOCAL NUMBER; 22N-23W-32 ABB 1.

LOCATION,--LAT 36 20'52", LONG 099 41'09", HYDROLOGIC UNIT 11100203.

AQUIFER . - OGALLALA.

PERIOD OF RECORD.--1980 TO CURRENT YEAR.

EXTREMES FOR PERIOD OF RECORD.--HIGHEST WATER LEVEL, 10.61 FT(3.23 M) BELOW LAND-SURFACE DATUM,FEB 15, 1983; LOWEST WATER LEVEL, 11.89 FT(3.62 M) BELOW LAND-SURFACE DATUM, JAN 21, 1982.

WATER LEVELS IN FEET BELOW LAND SURFACE DATUM, CLIMATIC YEARS APRIL 1981 TO MARCH 1983

$\begin{array}{lll} & \text { WATER } & \text { WATER } \\ \text { DATE } & \text { LEVEL } & \text { DATE }\end{array}$

JAN 21, $1982 \quad 11.89 \quad$ FEB 15, $1983 \quad 10.61$

GROUND-WATER LEVELS

362003099365501. LOCAL NUMBER; 22N-23W-36 CDD 1. LOCATION,--LAT 36 20'03", LONG 099 36'55", HYDROLOGIC UNIT 11100203. AQUIFER .- OGALLALA.

PERIOD OF RECORD.--1980 TO CURRENT YEAR.

EXTREMES FOR PERIOD OF RECORD.--HIGHEST WATER LEVEL,33.38 FT(10.17 M) BELOW LAND-SURFACE DATUM,FEB 15, 1983; LOWEST WATER LEVEL, $34.92 \mathrm{FT}(10.64 \mathrm{M})$ BELOW LAND-SURFACE DATUM, JAN 31, 1980.

WATER LEVELS IN FEET BELOW LAND SURFACE DATUM, CLIMATIC YEARS APRIL 1981 TO MARCH 1983

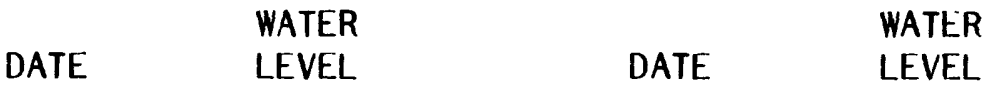

JAN 21, $1982 \quad 34.20 \quad$ FEB $15,1983 \quad 33.38$ 
GROUND-WATER LEVELS

ELLIS COUNTY -- CONTINUED

362349099443501. LOCAL NUMBER; 22N-24W-11 CBB 1. LOCATION,--LAT 36 23'49", LONG 099 44'35", HYDROLOGIC UNIT 11100203.

AQUIFER.--OGALLALA.

PERIOD OF RECORD.--1980 TO CURRENT YEAR.

EXTREMES FOR PERIOD OF RECORD.--HIGHEST WATER LEVEL, 100.10 FT(30.51 M) BELOW LAND-SURFACE DATUM,FEB 16, 1983; LOWEST WATER LEVEL,114.39 FT(34.87 M) BELOW LAND-SURFACE DATUM, JAN 29, 1980.

WATER LEVELS IN FEET BELOW LAND SURFACE DATUM, CLIMATIC YEARS APRIL 1981 TO MARCH 1983

$\begin{array}{lll} & \text { WATER } & \text { WATER } \\ \text { DATE } & \text { LEVEL } & \text { DATE }\end{array}$

JAN 20, $1982101.07 \quad$ FEB $16,1983 \quad 100.10$

GROUND-WATER LEVELS

362225099470601. LOCAL NUMBER; 22N-24W-20 ADA 1. LOCATION,--LAT 36 22'25", LONG 099 47'06", HYDROLOGIC UNIT 11100203. AQUIFER .--OGALLALA.

PERIOD OF RECORD.-- 1980 TO CURRENT YEAR. EXTREMES FOR PERIOD OF RECORD.--HIGHEST WATER LEVEL, 21.44 FT(6.53 M) BELOW LAND-SURFACE DATUM,FEB 16, 1983; LOWEST WATER LEVEL,22.59 FT(6.89 M) BELOW LAND-SURFACE DATUM, FEB 4, 1981.

WATER LEVELS IN FEET BELOW LAND SURFACE DATUM, CLIMATIC YEARS APRIL 1981 TO MARCH 1983

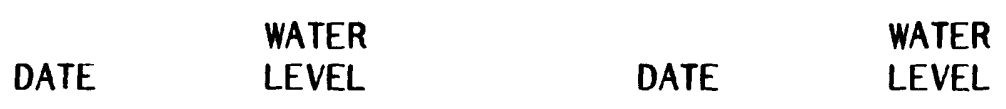

JAN 20, $1982 \quad 22.16 \quad$ FEB $16,1983 \quad 21.44$ 


\section{GROUND-WATER LEVELS}

\section{ELLIS COUNTY -- CONTINUED}

362129099424901. LOCAL NUMBER; LOCATION,--LAT 3621 '29", LONG 099 AQUIFER.--OGALLALA. PERIOD OF RECORD.-- 1980 TO CURRENT YEAR. EXTREMES FOR PERIOD OF RECORD.--HIGHEST WATER LEVEL, $19.52 \mathrm{FT}(5.95 \mathrm{M})$ BELOW LAND-SURFACE DATUM, FEB 14, 1983; LOWEST WATER LEVEL,23.63 FT(7.20 M) BELOW LAND-SURFACE DATUM, JAN 29, 1980.

WATER LEVELS IN FEET BELOW LAND SURFACE DATUM, CLIMATIC YEARS APRIL 1981 TO MARCH 1983
JAN 20, $1982 \quad 21.35$
FEB $14,1983 \quad 19.52$

\section{GROUND-WATER LEVELS}

362143099473501. LOCAL NUMBER;

$22 \mathrm{~N}-24 \mathrm{~W}-29$ ABB 1.

LOCATION,--LAT 36 21'43", LONG 099 47'35", HYDROLOGIC UNIT 11100203. AQUIFER . - OGALLALA. PERIOD OF RECORD .--1980 TO CURRENT YEAR. EXTREMES FOR PERIOD OF RECORD.--HIGHEST WATER LEVEL, 14.74 FT(4.49 M) BELOW LAND-SURFACE DATUM,MAR 25, 1980; LOWEST WATER LEVEL, $16.48 \mathrm{FT}(5.02 \mathrm{M})$ BELOW LAND-SURFACE DATUM, JAN 20, 1982.

WATER LEVELS IN FEET BELOW LAND SURFACE DATUM, CLIMATIC YEARS APRIL 1981 TO MARCH 1983

$\begin{array}{cccc} & \text { WATER } & & \text { WATER } \\ \text { DATE } & \text { LEVEL } & \text { DATE } & \text { LEVEL } \\ \text { L 20, 1982 } & 16.48 & \text { FEB 16, 1983 } & 15.41\end{array}$


GROUND-WATER LEVELS

\section{ELLIS COUNTY -- CONTINUED}

362050099475601. LOCAL NUMBER; LOCATION,--LAT 36 20'50", LONG 099 AQUIFER.--OGALLALA.

PERIOD OF RECORD.--1980 TO CURRENT

EXTREMES FOR PERIOD OF RECORD.--HIGHEST WATER LEVEL,73.04 FT(22.26 M) BELOW LAND-SURFACE

DATUM,MAR 25, 1980; LOWEST WATER LEVEL,73.79 FT(22.49 M) BELOW LAND-SURFACE DATUM, JAN 20, 1982.

WATER LEVELS IN FEET BELOW LAND SURFACE DATUM, CLIMATIC YEARS APRIL 1981 TO MARCH 1983
$22 \mathrm{~N}-24 \mathrm{~W}-32$ BBA 1. 47'56", HYDROLOGIC UNIT 11100203.
YEAR.

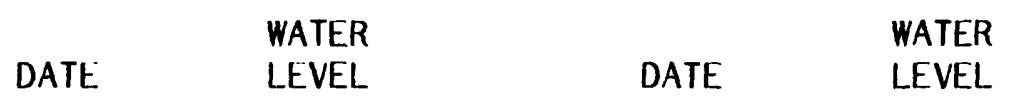

JAN 20, $1982 \quad 73.79 \quad$ FEB 16, $1983 \quad 73.19$

GROUND-WATER LEVELS

362017099450801. LOCAL NUMBER; 22N-24W-34 DAC 1.

LOCATION,--LAT 36 20'17", LONG 099 45'08", HYDROLOGIC UNIT 11100203.

AQUIFER .--OGALLALA.

PERIOD OF RECORD.--1980 TO CURRENT YEAR.

EXTREMES FOR PERIOD OF RECORD.--HIGHEST WATER LEVEL, 8.19 FT(2.50 M) BELOW LAND-SURFACE DATUM,FEB 16, 1983; LOWEST WATER LEVEL, 10.88 FT(3.32 M) BELOW LAND-SURFACE DATUM, JAN 29, 1980 .

WATER LEVELS IN FEET BELOW LAND SURFACE DATUM, CLIMATIC YEARS APRIL 1981 TO MARCH 1983

$\begin{array}{lll} & \text { WATER } & \text { WATER } \\ \text { DATE } & \text { LEVEL } & \text { DATE }\end{array}$
JAN 21, 1982
8.98
FEB 16, 1983
8.19 


\section{GROUND-WATER LEVELS}

ELLIS COUNTY -- CONTINUED

362226099544201. LOCAL NUMBER; 22N-25W-19 AAC 1.

LOCATION,--LAT 36 22'26", LONG 099 54'42", HYDROLOGIC UNIT 11100203.

AQUIFER.--OGALLALA.

PERIOD OF RECORD.--1980 TO CURRENT YEAR.

EXTREMES FOR PERIOD OF RECORD.--HIGHEST WATER LEVEL, $197.09 \mathrm{FT}(60.07 \mathrm{M})$ BELOW LAND-SURFACE DATUM,FEB 5, 1981; LOWEST WATER LEVEL, 198.49 FT(60.50 M) BELOW LAND-SURFACE DATUM, FEB 7, 1980 .

WATER LEVELS IN FEET BELOW LAND SURFACE DATUM, CLIMATIC YEARS APRIL 1981 TO MARCH 1983

$\begin{array}{cccc}\text { DATE } & \begin{array}{c}\text { WATER } \\ \text { LEVEL }\end{array} & \text { DATE } & \begin{array}{c}\text { WATER } \\ \text { LEVEL }\end{array} \\ \text { JAN 20, 1982 } & 198.21 & \text { JAN 19, 1983 } & 197.40\end{array}$

GROUND-WATER LEVELS

362236099494001. LOCAL NUMBER; 22N-25W-24 ABB 1. LOCATION,--LAT 36 22'36", LONG 099 49'40", HYDROLOGIC UNIT 11100203. AQUIFER.--OGALLALA.

PERIOD OF RECORD.--1980 TO CURRENT YEAR.

EXTREMES FOR PERIOD OF RECORD.--HIGHEST WATER LEVEL,70.74 FT(21.56 M) BELOW LAND-SURFACE DATUM,MAR 25, 1980; LOWEST WATER LEVEL,72.09 FT(21.97 M) BELOW LAND-SURFACE DATUM, JAN 19, 1982.

WATER LEVELS IN FEET BELOW LAND SURFACE DATUM, CLIMATIC YEARS APRIL 1981 TO MARCH 1983

$\begin{array}{lll} & \text { WATER } & \\ \text { DATE } & \text { WEVEL } & \text { DATE } \\ \text { LEVEL }\end{array}$

JAN 19, $1982 \quad 72.09 \quad$ FEB $16,1983 \quad 71.35$ 
GROUND-WATER LEVELS

ELLIS COUNTY -- CONTINUED

362049099524601. LOCAL NUMBER;

$22 \mathrm{~N}-25 \mathrm{~W}-33$ ABB 1.

LOCATION,--LAT 36 20'49", LONG 099 52'46", HYDROLOGIC UNIT 11100203.

AQUIFER. - OGALLALA.

PERIOD OF RECORD.--1980 TO CURRENT YEAR.

EXTREMES FOR PERIOD OF RECORD.--HIGHEST WATER LEVEL, 95.99 FT(29.26 M) BELOW LAND-SURFACE DATUM,FEB 7, 1980; LOWEST WATER LEVEL,217.12 FT(66.18 M) BELOW LAND-SURFACE DATUM, JAN 19, 1982.

WATER LEVELS IN FEET BELOW LAND SURFACE DATUM, CLIMATIC YEARS APRIL 1981 TO MARCH 1983

\begin{tabular}{clcl}
\multicolumn{1}{c}{ WATER } & & WATER \\
DATE & LEVEL & DATE & LEVEL \\
JAN 19, 1982 & 217.12 & FEB 16, 1983 & 216.30
\end{tabular}

GROUND-WATER LEVELS

362042099495901. LOCAL NUMBER; 22N-25W-36 BBD 1.

LOCATION,--LAT 36 20'42", LONG 099 49'59", HYDROLOGIC UNIT 11100203.

AQUIFER.--OGALLALA.

PERIOD OF RECORD.--1980 TO CURRENT YEAR.

EXTREMES FOR PERIOD OF RECORD.--HIGHEST WATER LEVEL, $154.53 \mathrm{FT}(47.10 \mathrm{M})$ BELOW LAND-SURFACE DATUM,MAR 25, 1980; LOWEST WATER LEVEL, 157.79 FT(48.09 M) BELOW LAND-SURFACE DATUM, JAN 19, 1982.

WATER LEVELS IN FEET BELOW LAND SURFACE DATUM, CLIMATIC YEARS APRIL 1981 TO MARCH 1983

\begin{tabular}{ccccc}
\multicolumn{1}{c}{ WATER } & & WATER \\
DATE & LEVEL & DATE & LEVEL \\
JAN 19, 1982 & 157.79 & FEB 16, 1983 & 154.72
\end{tabular}


GROUND-WATER LEVELS

ELLIS COUNTY -- CONTINUED

362500099562701. LOCAL NUMBER; 22N-26W-01 BBD 1.

LOCATION,--LAT $36^{2} 25^{\prime} 00^{\prime \prime}$, LONG 099 56'27", HYDROLOGIC UNIT 11100203.

AQUIFER.--OGALLALA.

PERIOD OF RECORD.--1980 TO CURRENT YEAR.

EXTREMES FOR PERIOD OF RECORD.--HIGHEST WATER LEVEL,217.04 FT(66.15 M) BELOW LAND-SURFACE DATUM, MAR 25, 1980; LOWEST WATER LEVEL,218.35 FT(66.55 M) BELOW LAND-SURFACE DATUM, JAN 19, 1982.

WATER LEVELS IN FEET BELOW LAND SURFACE DATUM, CLIMATIC YEARS APRIL 1981 TO MARCH 1983

$\begin{array}{lll} & \text { WATER } & \text { WATER } \\ \text { DATE } & \text { LEVEL } & \text { DATE }\end{array}$

JAN 19, $1982 \quad 218.35 \quad$ JAN 19, $1983 \quad$ W

GROUND-WATER LEVELS

362410099594601. LOCAL NUMBER; 22N-26W-09 BBD 1. LOCATION,--LAT 36 24'10", LONG 099 59'46", HYDROLOGIC UNIT 11100203.

AQUIFER.--OGALLALA.

PERIOD OF RECORD.--1980 TO CURRENT YEAR.

EXTREMES FOR PERIOD OF RECORD.--HIGHEST WATER LEVEL,53.38 FT(16.27 M) BELOW LAND-SURFACE DATUM,MAR 25, 1980; LOWEST WATER LEVEL,55.53 FT(16.93 M) BELOW LAND-SURFACE DATUM, JAN 19, 1982.

WATER LEVELS IN FEET BELOW LAND SURFACE DATUM, CLIMATIC YEARS APRIL 1981 TO MARCH 1983

$\begin{array}{lll} & \text { WATER } & \text { WATER } \\ \text { DATE } & \text { LEVEL } & \text { DATE }\end{array}$

JAN 19, $1982 \quad 55.53 \quad$ FEB 16, $1983 \quad 54.45$ 


\section{ELLIS COUNTY -- CONTINUED}

362148099594301. LOCAL NUMBER; 22N-26W-21 CCD 1. LOCATION,--LAT 36 21'48", LONG 099 59'43", HYDROLOGIC UNIT 11100203. AQUIFER.--OGALLALA. PERIOD OF RECORD.--1980 TO CURRENT YEAR. EXTREMES FOR PERIOD OF RECORD.--HIGHEST WATER LEVEL, 58.32 FT(17.78 M) BELOW LAND-SURFACE DATUM, MAR 17, 1981; LOWEST WATER LEVEL,65.00 FT(19.81 M) BELOW LAND-SURFACE DATUM, MAR 25, 1980 .

WATER LEVELS IN FEET BELOW LAND SURFACE DATUM, CLIMATIC YEARS APRIL 1981 TO MARCH 1983

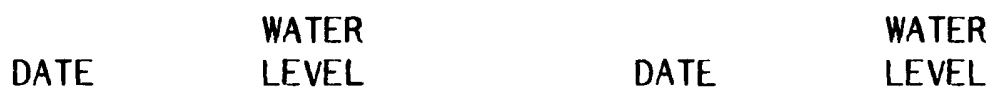

JAN 19, $1982 \quad 59.28 \quad$ FEB $14,1983 \quad 58.70$

GROUND-WATER LEVELS

362847099410401. LOCAL NUMBER; 23N-23W-08 DCC 1. LOCATION,--LAT 36 28'47", LONG 099 41'04", HYDROLOGIC UNIT 11100203. AQUIFER.--OGALLALA.

PERIOD OF RECORD.--1980 TO CURRENT YEAR.

EXTREMES FOR PERIOD OF RECORD.--HIGHEST WATER LEVEL, 56.71 FT(17.29 M) BELOW LAND-SURFACE DATUM,MAR 25, 1980; LOWEST WATER LEVEL,61.74 FT(18.82 M) BELOW LAND-SURFACE DATUM, JAN 21, 1982.

WATER LEVELS IN FEET BELOW LAND SURFACE DATUM, CLIMATIC YEARS APRIL 1981 TO MARCH 1983

$\begin{array}{lll} & \text { WATER } & \text { WATER } \\ \text { DATE } & \text { LEVEL } & \text { DATE }\end{array}$

JAN 21, $1982 \quad 61.74 \quad$ JAN 12, $1983 \quad 61.63$ 


\section{ELLIS COUNTY -- CONTINUED}

362750099392701. LOCAL NUMBER; LOCATION,--LAT 3627 '50", LONG 099 AQUIFER.--OGALLALA.

PERIOD OF RECORD. - 1980 TO CURRENT YEAR.

EXTREMES FOR PERIOD OF RECORD.--HIGHEST WATER LEVEL, 60.26 FT(18.37 M) BELOW LAND-SURFACE DATUM,JAN 12, 1983; LOWEST WATER LEVEL,60.55 FT(18.46 M) BELOW LAND-SURFACE DATUM, JAN 21, 1982.

WATER LEVELS IN FEET BELOW LAND SURFACE DATUM, CLIMATIC YEARS APRIL 1981 TO MARCH 1983
$23 \mathrm{~N}-23 \mathrm{~W}-22$ BBB 1. 39'27", HYDROLOGIC UNIT 11100203.

$\begin{array}{lll} & \\ & & \\ \text { DATE } & \text { WATER } & \text { WATER } \\ \text { LEVEL } & \text { DATE } & \text { LEVEL }\end{array}$

JAN 21, $198260.55 \quad$ JAN 12, $1983 \quad 60.26$

GROUND-WATER LEVELS

362518099421801. LOCAL NUMBER;

LOCATION, --LAT 36 25'18", LONG 099 AQUIFER.--OGALLALA.

PERIOD OF RECORD.--1980 TO CURRENT YEAR.

EXTREMES FOR PERIOD OF RECORD.--HIGHEST WATER LEVEL, 51.96 FT(15.84 M) BELOW LAND-SURFACE

DATUM, MAR 25, 1980; LOWEST WATER LEVEL,53.31 FT(16.25 M) BELOW LAND-SURFACE DATUM, JAN 21, 1982.

WATER LEVELS IN FEET BELOW LAND SURFACE DATUM, CLIMATIC YEARS APRIL 1981 TO MARCH 1983
$23 N-23 W-31$ CDD 1

42'18", HYDROLOGIC UNIT 11100203.

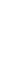


GROUND-WATER LEVELS

\section{ELLIS COUNTY -- CONTINUED}

362536099400901. LOCAL NUMBER; 23N-23W-33 CAA 1.

LOCATION,--LAT 36 25'36", LONG 099 40'09", HYDROLOGIC UNIT 11100203.

AQUIFER.--OGALLALA.

PERIOD OF RECORD.--1980 TO CURRENT YEAR.

EXTREMES FOR PERIOD OF RECORD.--HIGHEST WATER LEVEL, 96.04 FT(29.27 M) BELOW LAND-SURFACE DATUM, MAR 18, 1981; LOWEST WATER LEVEL, 108.16 FT(32.97 M) BELOW LAND-SURFACE DATUM, MAR 25, 1980 .

WATER LEVELS IN FEET BELOW LAND SURFACE DATUM, CLIMATIC YEARS APRIL 1981 TO MARCH 1983

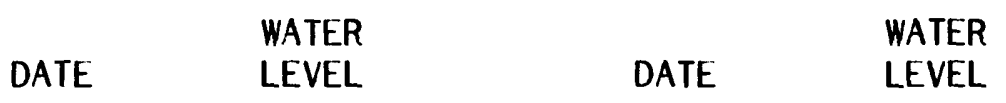

JAN 21, 198296.67 JAN 12, $1983 \quad 96.18$

GROUND-WATER LEVELS

363020099432901. LOCAL NUMBER; 23N-24W-01 BAC 1.

LOCATION,--LAT 36 30'20", LONG 099 43'29", HYDROLOGIC UNIT 11100201.

AQUIFER .- OGALLALA.

PERIOD OF RECORD.--1980 TO CURRENT YEAR.

EXTREMES FOR PERIOD OF RECORD.--HIGHEST WATER LEVEL,136.71 FT(41.67 M) BELOW LAND-SURFACE DATUM, MAR 26, 1980; LOWEST WATER LEVEL,138.92 FT(42.34 M) BELOW LAND-SURFACE DATUM, JAN 12, 1983.

WATER LEVELS IN FEET BELOW LAND SURFACE DATUM, CLIMATIC YEARS APRIL 1981 TO MARCH 1983

\begin{tabular}{|c|c|}
\hline & WATER \\
\hline DATE & LEVEL \\
\hline
\end{tabular}

FEB 24, $1982138.03 \quad$ JAN 12, $1983 \quad 138.92$ 


\section{ELLIS COUNTY -- CONTINUED}

362912099461801. LOCAL NUMBER; 23N-24W-09 ACD 1. LOCATION,--LAT 36 29'12", LONG 099 46'18", HYDROLOGIC UNIT 11100201. AQUIFER.--OGALLALA. PERIOD OF RECORD.--1980 TO CURRENT YEAR. EXTREMES FOR PERIOD OF RECORD.--HIGHEST WATER LEVEL, 122.75 FT(37.41 M) BELOW LAND-SURFACE DATUM,FEB 8, 1980; LOWEST WATER LEVEL, 198.32 FT(60.45 M) BELOW LAND-SURFACE DATUM, JAN 13, 1983.

WATER LEVELS IN FEET BELOW LAND SURFACE DATUM, CLIMATIC YEARS APRIL 1981 TO MARCH 1983

$\begin{array}{lll} & \text { WATER } & \\ \text { DATE } & \text { WEVEL } & \text { WATER } \\ & \text { DATE } & \text { LEVEL }\end{array}$

FEB 24, $1982198.09 \quad$ JAN 13, $1983 \quad 198.32$

\section{GROUND-WATER LEVELS}

362906099450601 . LOCAL NUMBER; LOCATION,--LAT 36 29'06", LONG 099 AQUIFER.--OGALLALA. PERIOD OF RECORD.--1980 TO CURRENT EXTREMES FOR PERIOD OF RECORD.--HIGHEST WATER LEVEL, 175.02 FT(53.35 M) BELOW LAND-SURFACE DATUM,FEB 8, 1980; LOWEST WATER LEVEL, 176.33 FT(53.75 M) BELOW LAND-SURFACE DATUM, JAN 12, 1983.

WATER LEVELS IN FEET BELOW LAND SURFACE DATUM, CLIMATIC YEARS APRIL 1981 TO MARCH 1983
$23 N-24 W-10$ DAB 1 45'06", HYDROLOGIC UNIT 11100203.

\begin{tabular}{|c|c|c|}
\hline & WATER & \\
\hline DATE & LEVEL & DATE \\
\hline
\end{tabular}

FEB 24, $1982 \quad 176.12$ JAN 12, $1983 \quad 176.33$ 


\section{GROUND-WATER LEVELS}

\section{ELLIS COUNTY -- CONTINUED}

362933099440101 . LOCAL NUMBER; LOCATION,--LAT 36 29'33", LONG 099 AQUIFER. --OGALLALA.

PERIOD OF RECORD.--1980 TO CURRENT EXTREMES FOR PERIOD OF RECORD.--HIGHEST WATER LEVEL, 142.30 FT(43.37 M) BELOW LAND-SURFACE

DATUM, JAN 12, 1983; LOWEST WATER LEVEL, $142.62 \mathrm{FT}(43.47 \mathrm{M})$ BELOW LAND-SURFACE DATUM, FEB 24, 1982 .

WATER LEVELS IN FEET BELOW LAND SURFACE DATUM, CLIMATIC YEARS APRIL 1981 TO MARCH 1983
$23 N-24 W-11$ AAB 1.
$44^{\prime} 01$ ", HYDROLOGIC

44'01", HYDROLOGIC UNIT 11100203.

YEAR

\begin{tabular}{ccccc}
\multicolumn{1}{c}{} & WATER & & & WATER \\
DATE & LEVEL & DATE & LEVEL \\
FEB 24, 1982 & 142.62 & JAN 12, 1983 & 142.30
\end{tabular}

GROUND-WATER LEVELS

362800099434001 . LOCAL NUMBER; 23N-24W-13 CCA 1.

LOCATION,--LAT 36 28'00", LONG 099 43'40", HYDROLOGIC UNIT 11100203.

AQUIFER.--OGALLALA.

PERIOD OF RECORD.--1980 TO CURRENT YEAR.

EXTREMES FOR PERIOD OF RECORD.--HIGHEST WATER LEVEL, 96.41 FT(29.39 M) BELOW LAND-SURFACE

DATUM,FEB 8, 1980; LOWEST WATER LEVEL,98.37 FT(29.98 M) BELOW LAND-SURFACE DATUM,

JAN 13, 1983.

WATER LEVELS IN FEET BELOW LAND SURFACE DATUM, CLIMATIC YEARS APRIL 1981 TO MARCH 1983

$\begin{array}{lll} & \text { WATER } & \text { WATER } \\ \text { DATE } & \text { LEVEL } & \text { DATE }\end{array}$

FEB 24, $198298.14 \quad$ JAN 13, $1983 \quad 98.37$ 


\section{ELLIS COUNTY -- CONTINUED}

362833099485901 . LOCAL NUMBER; LOCATION,--LAT 36 28'33", LONG 099 AQUIFER.--OGALLALA.

PERIOD OF RECORD.--1980 TO CURRENT YEAR.

EXTREMES FOR PERIOD OF RECORD.--HIGHEST WATER LEVEL, $151.39 \mathrm{FT}(46.14 \mathrm{M})$ BELOW LAND-SURFACE DATUM, JAN 13, 1983; LOWEST WATER LEVEL, 152.58 FT(46.51 M) BELOW LAND-SURFACE DATUM, FEB 24, 1982 .

WATER LEVELS IN FEET BELOW LAND SURFACE DATUM, CLIMATIC YEARS APRIL 1981 TO MARCH 1983
FEB 24, $1982 \quad 152.58$
JAN $13,1983 \quad 151.39$

GROUND-WATER LEVELS

362957099514501. LOCAL NUMBER;

23N-25W-03 DDA 1.

LOCATION,--LAT 36 29'57", LONG 099 51'45", HYDROLOGIC UNIT 11100203.

AQUIFER .--OGALLALA.

PERIOD OF RECORD.--1980 TO CURRENT YEAR.

EXTREMES FOR PERIOD OF RECORD.--HIGHEST WATER LEVEL, 134.27 FT(40.93 M) BELOW LAND-SURFACE DATUM,FEB 24, 1982; LOWEST WATER LEVEL, 134.99 FT(41.14 M) BELOW LAND-SURFACE DATUM, FEB 7, 1980 .

WATER LEVELS IN FEET BELOW LAND SURFACE DATUM, CLIMATIC YEARS APRIL 1981 TO MARCH 1983

\begin{tabular}{|c|c|c|}
\hline DATE & $\begin{array}{l}\text { WATER } \\
\text { LEVEL }\end{array}$ & DATE \\
\hline
\end{tabular}
FEB 24, $1982 \quad 134.27$
JAN $13,1983 \quad 134.56$ 
362939099525401. LOCAL NUMBER; LOCATION,--LAT 36 29'39", LONG 099 AQUIFER .--OGALLALA.

PERIOD OF RECORD.--1980 TO CURRENT YEAR.

EXTREMES FOR PERIOD OF RECORD.--HIGHEST WATER LEVEL, 189.14 FT(57.65 M) BELOW LAND-SURFACE DATUM,FEB 7, 1980; LOWEST WATER LEVEL,189.44 FT(57.74 M) BELOW LAND-SURFACE DATUM, JAN 12, 1983.

WATER LEVELS IN FEET BELOW LAND SURFACE DATUM, CLIMATIC YEARS APRIL 1981 TO MARCH 1983
23N-25W-04 DCC 1

52 '54", HYDROLOGIC UNIT 11100203.

LEVEL, 189.44 FT( 57.74 M) BELON LAND-SURFACE DATUM,
DATE WATER

LEVEL
189.36
FEB 24, 1982
JAN 12, $1983 \quad 189.44$

GROUND-WATER LEVELS

362800099524601. LOCAL NUMBER; 23N-25W-16 DCA 1.

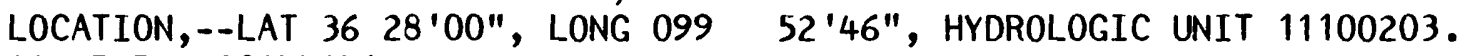

AQUIFER. $\rightarrow$ OGALLALA.

PERIOD OF RECORD.--1980 TO CURRENT YEAR.

EXTREMES FOR PERIOD OF RECORD.--HIGHEST WATER LEVEL, $159.08 \mathrm{FT}(48.49 \mathrm{M})$ BELOW LAND-SURFACE DATUM,FEB 7, 1980; LOWEST WATER LEVEL, 160.45 FT(48.91 M) BELOW LAND-SURFACE DATUM, JAN $13,1983$.

WATER LEVELS IN FEET BELOW LAND SURFACE DATUM, CLIMATIC YEARS APRIL 1981 TO MARCH 1983

\begin{tabular}{|c|c|c|}
\hline DATE & WATER & DATR \\
\hline DATE & & DATE \\
\hline
\end{tabular}

FEB 24, 1982160.21 JAN 13, $1983 \quad 160.45$ 


\section{ELLIS COUNTY -- CONTINUED}

362736099510801. LOCAL NUMBER; LOCATION,--LAT 36 27'36", LONG 099 AQUIFER.--OGALLALA.

PERIOD OF RECORD.--1980 TO CURRENT YEAR. EXTREMES FOR PERIOD OF RECORD.--HIGHEST WATER LEVEL, 120.84 FT(36.83 M) BELOW LAND-SURFACE DATUM, JAN 13, 1983; LOWEST WATER LEVEL, 120.98 FT(36.87 M) BELOW LAND-SURFACE DATUM, FEB 24, 1982 .

WATER LEVELS IN FEET BELOW LAND SURFACE DATUM, CLIMATIC YEARS APRIL 1981 TO MARCH 1983
FEB 24, $1982 \quad 120.98$
JAN 13, $1983 \quad 120.84$

GROUND-WATER LEVELS

362654099544601. LOCAL NUMBER; LOCATION,--LAT 36 26'54', LONG 099 AQUIFER .--OGALLALA.

PERIOD OF RECORD.--1980 TO CURRENT YEAR.

EXTREMES FOR PERIOD OF RECORD.--HIGHEST WATER LEVEL, 93.10 FT(28.38 M) BELOW LAND-SURFACE DATUM,FEB 7, 1980; LOWEST WATER LEVEL,94.19 FT(28.71 M) BELOW LAND-SURFACE DATUM, JAN 21, 1982.

WATER LEVELS IN FEET BELOW LAND SURFACE DATUM, CLIMATIC YEARS APRIL 1981 TO MARCH 1983

$\begin{array}{lccc} & \text { WATER } & & \\ \text { DATE } & \text { LEVEL } & \text { WATER } \\ \text { DATE } & \text { LEVEL } \\ \text { D 21, 1982 } & 94.19 & \text { JAN 13, 1983 } & 93.32\end{array}$




\section{GROUND-WATER LEVELS}

ELLIS COUNTY -- CONTINUED

362740099563001. LOCAL NUMBER; 23N-26W-24 BBD 1.

LOCATION,--LAT 36 27'40", LONG 099 56'30", HYDROLOGIC UNIT 11100203.

AQUIFER.--OGALLALA.

PERIOD OF RECORD.--1980 TO CURRENT YEAR.

EXTREMES FOR PERIOD OF RECORD.--HIGHEST WATER LEVEL, 144.07 FT(43.91 M) BELOW LAND-SURFACE DATUM,FEB 7, 1980; LOWEST WATER LEVEL, 145.43 FT(44.33 M) BELOW LAND-SURFACE DATUM, JAN 13, 1983.

WATER LEVELS IN FEET BELOW LAND SURFACE DATUM, CLIMATIC YEARS APRIL 1981 TO MARCH 1983

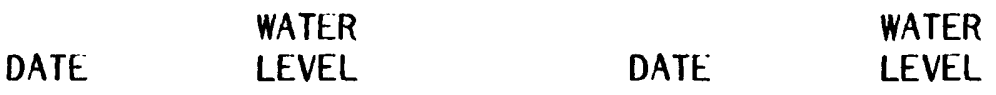

JAN 21, $1982 \quad 145.14 \quad$ JAN 13, $1983 \quad 145.43$

GROUND-WATER LEVELS

362647099554901. LOCAL NUMBER; 23N-26W-25 AAC 1. LOCATION,--LAT 36 26'47", LONG 099 55'49", HYDROLOGIC UNIT 11100203. AQUIFER. - -OGALLALA.

PERIOD OF RECORD.--1980 TO CURRENT YEAR.

EXTREMES FOR PERIOD OF RECORD.--HIGHEST WATER LEVEL, $174.50 \mathrm{FT}(53.19 \mathrm{M})$ BELOW LAND-SURFACE DATUM, JAN 13, 1983; LOWEST WATER LEVEL, 175.02 FT(53.35 M) BELOW LAND-SURFACE DATUM, FEB 7, 1980.

WATER LEVELS IN FEET BELOW LAND SURFACE DATUM, CLIMATIC YEARS APRIL 1981 TO MARCH 1983

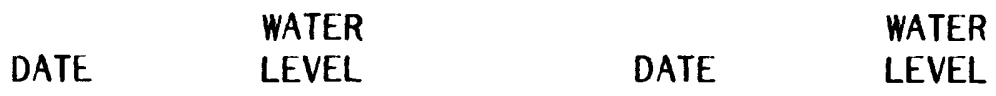

JAN 21, $1982 \quad 174.87 \quad$ JAN 13, $1983 \quad 174.50$ 
GROUND-WATER LEVELS

ELLIS COUNTY -- CONTINUED

362621099574601. LOCAL NUMBER; LOCATION,--LAT 36 26'21", LONG 099 AQUIFER.--OGALLALA.

PERIOD OF RECORD. -1980 TO CURRENT YEAR.

EXTREMES FOR PERIOD OF RECORD.--HIGHEST WATER LEVEL, 176.80 FT(53.89 M) BELOW LAND-SURFACE DATUM, JAN 21, 1982; LOWEST WATER LEVEL, 186.05 FT(56.71 M) BELOW LAND-SURFACE DATUM, FEB 7, 1980.

WATER LEVELS IN FEET BELOW LAND SURFACE DATUM, CLIMATIC YEARS APRIL 1981 TO MARCH 1983

$\begin{array}{lll} & \text { WATER } & \\ \text { DATE } & \text { WEVEL } & \text { WATER } \\ & & \text { LEVEL }\end{array}$

JAN 21, $1982 \quad 176.80 \quad$ JAN 13, $1983 \quad 185.20$

GROUND-WATER LEVELS

362522099573501. LOCAL NUMBER; 23N-26W-35 CCA 1.

LOCATION,--LAT 36 25'22", LONG 099 57'35", HYDROLOGIC UNIT 11100203.

AQUIFER.--OGALLALA.

PERIOD OF RECORD.--1980 TO CURRENT YEAR.

EXTREMES FOR PERIOD OF RECORD.--HIGHEST WATER LEVEL, 184.50 FT(56.24 M) BELOW LAND-SURFACE DATUM,FEB 7, 1980; LOWEST WATER LEVEL, 185.45 FT(56.53 M) BELOW LAND-SURFACE DATUM, JAN 21, 1982.

WATER LEVELS IN FEET BELOW LAND SURFACE DATUM, CLIMATIC YEARS APRIL 1981 TO MARCH 1983

\begin{tabular}{|c|c|c|}
\hline & WATER & \\
\hline DATE & LEVEL & DATE \\
\hline
\end{tabular}

JAN 21, $1982185.45 R \quad$ JAN 13, $1983 \quad \mathrm{~N}$ 


\section{ELLIS COUNTY -- CONTINUED}

363203099381401. LOCAL NUMBER; LOCATION,--LAT 3632 '03", LONG 099 AQUIFER.--TERRACE DEPOSITS.

PERIOD OF RECORD.--1980 TO CURRENT YEAR.

EXTREMES FOR PERIOD OF RECORD.--HIGHEST WATER LEVEL, 10.13 FT(3.09 M) BELOW LAND-SURFACE DATUM,FEB 15, 1983; LOWEST WATER LEVEL, 13.33 FT(4.06 M) BELOW LAND-SURFACE DATUM, JAN 21, 1982.

WATER LEVELS IN FEET BELOW LAND SURFACE DATUM, CLIMATIC YEARS APRIL 1981 TO MARCH 1983
DATE
WATER
LEVEL

JAN 21, $198213.33 R \quad$ FEB 15, $1983 \quad 10.13$

JAN 21, $198213.33 R \quad$ FEB 15, $1983 \quad 10.13$

\section{GROUND-WATER LEVELS}

363124099403201. LOCAL NUMBER; LOCATION,--LAT 36 31'24", LONG 099 AQUIFER.--TERRACE DEPOSITS.

$24 N-23 W-28$ CCC 1.

40'32", HYDROLOGIC UNIT 11100201.

PERIOD OF RECORD.--1980 TO CURRENT YEAR.

EXTREMES FOR PERIOD OF RECORD.--HIGHEST WATER LEVEL, $29.50 \mathrm{FT}(8.99 \mathrm{M})$ BELOW LAND-SURFACE DATUM, JAN 21, 1982; LOWEST WATER LEVEL, 30.44 FT(9.28 M) BELOW LAND-SURFACE DATUM, MAR 18, 1981.

WATER LEVELS IN FEET BELOW LAND SURFACE DATUM, CLIMATIC YEARS APRIL 1981 TO MARCH 1983
DATE
WATER
DATE
WATER
LEVEL

JAN 21, $1982 \quad 29.50$

FEB $15,1983 \quad 29.86$ 
GROUND-WATER LEVELS

ELLIS COUNTY -- CONTINUED

363325099481101. LOCAL NUMBER;

LOCATION,--LAT 36 33'25", LONG 099

AQUIFER.--TERRACE DEPOSITS.

PERIOD OF RECORD.--1980 TO CURRENT YEAR.

EXTREMES FOR PERIOD OF RECORD.--HIGHEST WATER LEVEL, 16.33 FT(4.98 M) BELOW LAND-SURFACE DATUM,MAR 26, 1980; LOWEST WATER LEVEL, 19.17 FT(5.84 M) BELOW LAND-SURFACE DATUM, JAN 21, 1982.

WATER LEVELS IN FEET BELOW LAND SURFACE DATUM, CLIMATIC YEARS APRIL 1981 T0 MARCH 1983

$\begin{array}{lll} & \text { WATER } & \text { WATER } \\ \text { DATE } & \text { LEVEL } & \text { DATE }\end{array}$

JAN 21, $1982 \quad 19.17 \quad$ FEB 15, $1983 \quad 20.52 \mathrm{P}$

GROUND-WATER LEVELS

363215099471301. LOCAL NUMBER; 24N-24W-20 DDC 1.

LOCATION,--LAT 36 32'15", LONG 099 47'13", HYDROLOGIC UNIT 11100201.

AQUIFER.--TERRACE DEPOSITS.

PERIOD OF RECORD.--1980 TO CURRENT YEAR.

EXTREMES FOR PERIOD OF RECORD.--HIGHEST WATER LEVEL, $35.59 \mathrm{FT}(10.85 \mathrm{M})$ BELOW LAND-SURFACE

- DATUM,MAR 25, 1980; LOWEST WATER LEVEL, 36.22 FT(11.04 M) BELOW LAND-SURFACE DATUM, FEB 15, 1983.

WATER LEVELS IN FEET BELOW LAND SURFACE DATUM, CLIMATIC YEARS APRIL 1981 TO MARCH 1983

\begin{tabular}{|c|c|c|}
\hline & WATER & \\
\hline DATE & LEVEL & DATE \\
\hline
\end{tabular}

JAN 21, $1982 \quad 35.94 \quad$ FEB 15, $1983 \quad 36.22$ 


\section{ELLIS COUNTY -- CONTINUED}

363159099471801. LOCAL NUMBER;

LOCATION,--LAT 3631 'S9", LONG 099

AQUIFER. - -OGALLALA.

PERIOD OF RECORD.--1981 TO CURRENT YEAR.

EXTREMES FOR PERIOD OF RECORD.--HIGHEST WATER LEVEL,45.72 FT(13.94 M) BELOW LAND-SURFACE

DATUM, JAN 21, 1982; LOWEST WATER LEVEL,45.86 FT(13.98 M) BELOW LAND-SURFACE DATUM, FEB 15, 1983.

WATER LEVELS IN FEET BELOW LAND SURFACE DATUM, CLIMATIC YEARS APRIL 1981 TO MARCH 1983
$24 \mathrm{~N}-24 \mathrm{~W}-29$ ACA 1.

47'18", HYDROLOGIC UNIT 11100201.
JAN 21,1982
45.72
FEB 15, 1983
45.86

GROUND-WATER LEVELS

363448099521801. LOCAL NUMBER; 24N-25W-03 CCC 1.

LOCATION,--LAT 36 34'48", LONG 099 52'18", HYDROLOGIC UNIT 11100201.

AQUIFER. --OGALLALA.

PERIOD OF RECORD.--1980 TO CURRENT YEAR.

EXTREMES FOR PERIOD OF RECORD.--HIGHEST WATER LEVEL, $11.27 \mathrm{FT}(3.44 \mathrm{M})$ BELOW LAND-SURFACE DATUM, FEB 15, 1983; LOWEST WATER LEVEL, 12.52 FT(3.82 M) BELOW LAND-SURFACE DATUM, MAR 25, 1980.

WATER LEVELS IN FEET BELOW LAND" SURFACE DATUM, CLIMATIC YEARS APRIL 1981 TO MARCH 1983

$\begin{array}{lll} & \text { WATER } & \\ \text { DATE } & \text { WEVEL } & \text { DATER } \\ \end{array}$

JAN 21, $1982 \quad 12.20 \quad$ FEB $15,1983 \quad 11.27$ 


\section{ELLIS COUNTY -- CONTINUED}

363511099532601. LOCAL NUMBER; LOCATION,--LAT 36 35'11", LONG 099 AQUIFER.--OGALLALA.

PERIOD OF RECORD.--1980 TO CURRENT YEAR.

EXTREMES FOR PERIOD OF RECORD.--HIGHEST WATER LEVEL, 3.56 FT(1.09 M) BELOW LAND-SURFACE DATUM, MAR 19, 1981; LOWEST WATER LEVEL,3.61 FT(1.10 M) BELOW LAND-SURFACE DATUM, JAN 21, 1982.

WATER LEVELS IN FEET BELOW LAND SURFACE DATUM, CLIMATIC YEARS APRIL 1981 TO MARCH 1983
$24 \mathrm{~N}-25 \mathrm{~W}-04$ CBB 1.

53'26", HYDROLOGIC UNIT 11100201.

$\begin{array}{lll} & & \\ & & \\ \text { DATE } & \text { WATER } & \text { WATER } \\ \text { LEVEL } & \text { DATE } & \text { LEVEL }\end{array}$
JAN 21, 1982
3.61
FEB 15, 1983
Z

GROUND-WATER LEVELS

363316099552501. LOCAL NUMBER;

LOCATION,--LAT 36 33'16", LONG 099

AQUIFER.--OGALLALA.

PERIOD OF RECORD.--1981 TO CURRENT YEAR.

EXTREMES FOR PERIOD OF RECORD.--HIGHEST WATER LEVEL, 19.60 FT(5.97 M) BELOW LAND-SURFACE DATUM, MAR 19, 1981; LOWEST WATER LEVEL, 19.82 FT(6.04 M) BELOW LAND-SURFACE DATUM, JAN 21, 1982.

WATER LEVELS IN FEET BELOW LAND SURFACE DATUM, CLIMATIC YEARS̊ APRIL 1981 TO MARCH 1983

$24 N-25 W-18$ CCA 1.

55'25", HYDROLOGIC UNIT 11100201.

LEVEL, 19.82 FT $(6.04$ M) BELOW LAND-SURFACE DATUM,

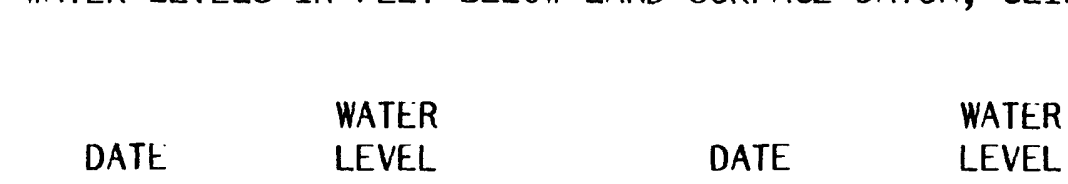

JAN 21, $1982 \quad 19.82 \quad$ FEB $15,1983 \quad 19.80$ 


\section{ELLIS COUNTY -- CONTINUED}

363254099513201. LOCAL NUMBER; 24N-25W-22 AAC 1. LOCATION,--LAT 36 32'54", LONG 099 51'32", HYDROLOGIC UNIT 11100201. AQUIFER .- OGALLALA. PERIOD OF RECORD.--1980 TO CURRENT YEAR. EXTREMES FOR PERIOD OF RECORD.--HIGHEST WATER LEVEL,67.19 FT(20.48 M) BELOW LAND-SURFACE DATUM,FEB 15, 1983; LOWEST WATER LEVEL,70.98 FT(21.63 M) BELOW LAND-SURFACE DATUM, JAN 21, 1982 .

WATER LEVELS IN FEET BELOW LAND SURFACE DATUM, CLIMATIC YEARS APRIL 1981 TO MARCH 1983

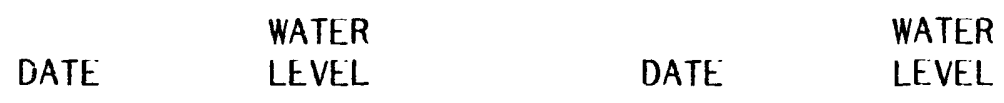

JAN 21, $198270.98 \quad$ FEB $15,1983 \quad 67.19$

\section{GROUND -WATER LEVELS}

363145099515201. LOCAL NUMBER; 24N-25W-27 DBB 1. LOCATION,--LAT 36 31'45", LONG 099 51'52", HYDROLOGIC UNIT 11100201. AQUIFER . --OGALLALA. PERIOD OF RECORD.--1981 TO CURRENT YEAR. EXTREMES FOR PERIOD OF RECORD.--HIGHEST WATER LEVEL, $169.46 \mathrm{FT}(51.65 \mathrm{M})$ BELOW LAND-SURFACE DATUM, JAN 21, 1982; LOWEST WATER LEVEL, 169.70 FT(51.72 M) BELOW LAND-SURFACE DATUM, FEB 15, 1983.

WATER LEVELS IN FEET BELOW LAND SURFACE DATUM, CLIMATIC YEARS APRIL 1981 TO MARCH 1983

$\begin{array}{lll} & \text { WATER } & \text { WATER } \\ \text { DATE } & \text { LEVEL } & \text { DATE }\end{array}$

JAN 21, $1982169.46 \quad$ FEB 15, $1983 \quad 169.70$ 


\section{GROUND-WATER LEVELS}

\section{ELLIS COUNTY -- CONTINUED}

363036099510801 . LOCAL NUMBER; LOCATION,--LAT 36 30'36", LONG 099 AQUIFER. - -OGALLALA.

PERIOD OF RECORD.--1980 TO CURRENT YEAR.

EXTREMES FOR PERIOD OF RECORD.--HIGHEST WATER LEVEL, 163.15 FT(49.73 M) BELOW LAND-SURFACE DATUM,MAR 25, 1980; LOWEST WATER LEVEL, $166.37 \mathrm{FT}(50.71 \mathrm{M})$ BELOW LAND-SURFACE DATUM, MAR 19, 1981.

WATER LEVELS IN FEET BELOW LAND SURFACE DATUM, CLIMATIC YEARS APRIL 1981 TO MARCH 1983
JAN 21, 1982
163.87
FEB 15, $1983 \quad 163.63$

GROUND-WATER LEVELS

363501099594201. LOCAL NUMBER; 24N-26W-04 CCA 1. LOCATION,--LAT 36 35'01", LONG 099 59'42", HYDROLOGIC UNIT 11100201. AQUIFER .--OGALLALA. PERIOD OF RLCORD.--1980 TO CURRENT YEAR. EXTREMES FOR PERIOD OF RECORD.--HIGHEST WATER LEVEL, 110.02 FT(33.53 M) BELOW LAND-SURFACE DATUM, JAN 21, 1982; LOWEST WATER LEVEL,110.72 FT(33.75 M) BELOW LAND-SURFACE DATUM, FEB 15, 1983 .

WATER LEVELS IN FEET BELOW LAND SURFACE DATUM, CLIMATIC YEARS APRIL 1981 TO MARCH 1983

\begin{tabular}{|c|c|c|}
\hline $\mathrm{D \triangle T}$ & WATER & DATE \\
\hline & & DAIE \\
\hline
\end{tabular}
FEB 15, $1983 \quad 110.72$ 


\section{GROUND-WATER LEVELS}

ELLIS COUNTY -- CONTINUED

363407099580101. LOCAL NUMBER; LOCATION,--LAT 36 34'07", LONG 099 AQUIFER.--OGALLALA.

PERIOD OF RECORD.--1980 TO CURRENT YEAR.

EXTREMES FOR PERIOD OF RECORD.--HIGHEST WATER LEVEL, 100.42 FT(30.61 M) BELOW LAND-SURFACE DATUM,MAR 26, 1980; LOWEST WATER LEVEL, $101.42 \mathrm{FT}(30.91 \mathrm{M})$ BELOW LAND-SURFACE DATUM, MAR 19, 1981.

WATER LEVELS IN FEET BELOW LAND SURFACE DATUM, CLIMATIC YEARS APRIL 1981 TO MARCH 1983
24N-26W-10 DDB 1.

58'01", HYDROLOGIC UNIT 11100201.

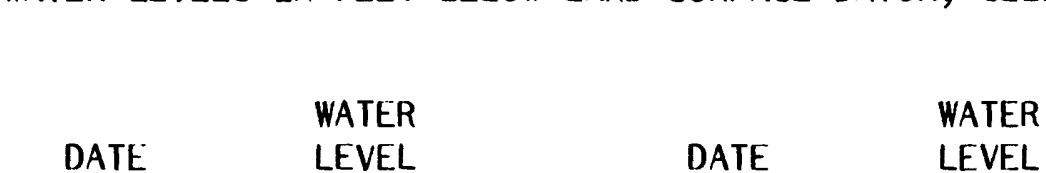

JAN 21, $1982101.30 \quad$ FEB 15, $1983 \quad 101.00$

GROUND-WATER LEVELS

363211099573401. LOCAL NUMBER; 24N-26W-26 BBA 1.

LOCATION,--LAT 36 32'11", LONG 099 57'34", HYDROLOGIC UNIT 11100201.

AQUIFER .--OGALLALA.

PERIOD OF RECORD.--1980 TO CURRENT YEAR.

EXTREMES FOR PERIOD OF RECORD.--HIGHEST WATER LEVEL, 31.86 FT(9.71 M) BELOW LAND-SURFACE DATUM, JAN 13, 1983; LOWEST WATER LEVEL,52.10 FT(15.88 M) BELOW LAND-SURFACE DATUM, JAN 21, 1982.

WATER LEVELS IN FEET BELOW LAND SURFACE DATUM, CLIMATIC YEARS APRIL 1981 TO MARCH 1983

\begin{tabular}{|c|c|c|}
\hline & WATER & \\
\hline DATE & LEVEL & DATE \\
\hline
\end{tabular}

JAN 21, $1982 \quad 52.10 \quad$ JAN 13, $1983 \quad 31.86$ 
GROUND-WATER LEVELS

ELLIS COUNTY -- CONTINUED

363211099580201. LOCAL NUMBER; 24N-26W-27 AAB 1. LOCATION,--LAT 36 32'11", LONG 099 58'02", HYDROLOGIC UNIT 11100201. AQUIFER.--OGALLALA. PERIOD OF RECORD.--1980 TO CURRENT YEAR. EXTREMES FOR PERIOD OF RECORD.--HIGHEST WATER LEVEL, $74.18 \mathrm{FT}(22.61 \mathrm{M})$ BELOW LAND-SURFACE DATUM, MAR 26, 1980; LOWEST WATER LEVEL,76.38 FT(23.28 M) BELOW LAND-SURFACE DATUM, JAN 21, 1982.

WATER LEVELS IN FEET BELOW LAND SURFACE DATUM, CLIMATIC YEARS APRIL 1981 TO MARCH 1983

$\begin{array}{lll} & \text { WATER } & \text { WATER } \\ \text { DATE } & \text { LEVEL } & \text { DATE }\end{array}$

JAN 21, $1982 \quad 76.38 \quad$ FEB $15,1983 \quad 75.82$

GROUND-WATER LEVELS

363118099595701. LOCAL NUMBER; 24N-26W-33 BBB 1. LOCATION,--LAT 36 31'18", LONG 099 59'57", HYDROLOGIC UNIT 11100201. AQUIFER.--OGALLALA.

PERIOD OF RECORD.--1980 TO CURRENT YEAR.

EXTREMES FOR PERIOD OF RECORD.--HIGHEST WATER LEVEL, $115.98 \mathrm{FT}(35.35 \mathrm{M})$ BELOW LAND-SURFACE DATUM,MAR 26, 1980; LOWEST WATER LEVEL,117.85 FT(35.92 M) BELOW LAND-SURFACE DATUM, MAR 19, 1981.

WATER LEVELS IN FEET BELOW LAND SURFACE DATUM, CLIMATIC YEARS APRIL 1981 TO MARCH 1983

\begin{tabular}{|c|c|c|}
\hline & WATER & \\
\hline DATE & LEVEL & DATE \\
\hline
\end{tabular}

JAN 21, $1982 \quad 116.82 \quad$ JAN 13, $1983 \quad 117.65$ 


\section{GROUND-WATER LEVELS}

GARFIELD COUNTY

361907097573601. LOCAL NUMBER; 21N-07W-04 CCC 1.

LOCATION,--LAT 36 19'07", LONG 097 57'36", HYDROLOGIC UNIT 11050002.

AQUIFER.--CEDAR HILL SANDSTONE.

PERIOD OF RECORD.--1977 TO CURRENT YEAR.

EXTREMES FOR PERIOD OF RECORD.--HIGHEST WATER LEVEL, $18.45 \mathrm{FT}(5.62 \mathrm{M})$ BELOW LAND-SURFACE DATUM,MAR 20, 1979; LOWEST WATER LEVEL,20.37 FT(6.21 M) BELOW LAND-SURFACE DATUM, MAR 10, 1982 .

WATER LEVELS IN FEET BELOW LAND SURFACE DATUM, CLIMATIC YEARS APRIL 1981 TO MARCH 1983

\begin{tabular}{|c|c|}
\hline DATE & $\begin{array}{l}\text { WATER } \\
\text { LEVEL }\end{array}$ \\
\hline
\end{tabular}

$\begin{array}{llll}\text { MAR } 10,1982 & 20.37 \quad \text { FEB 28, } 1983 \quad P\end{array}$

GROUND-WATER LEVELS

361907098053801. LOCAL NUMBER; 21N-08W-06 DCC 1.

LOCATION,--LAT 36 19'07", LONG 098 05'38", HYDROLOGIC UNIT 11050002.

AQUIFER.--ALLUVIUM.

PERIOD OF RECORD.--1975 TO CURRENT YEAR.

EXTREMES FOR PERIOD OF RECORD.--HIGHEST WATER LEVEL,2.10 FT(.64 M) BELOW LAND-SURFACE DATUM,MAR 10, 1982; LOWEST WATER LEVEL,5.20 FT(1.58 M) BELOW LAND-SURFACE DATUM, JAN 15, 1981.

WATER LEVELS IN FEET BELOW LAND SURFACE DATUM, CLIMATIC YEARS APRIL 1981 TO MARCH 1983

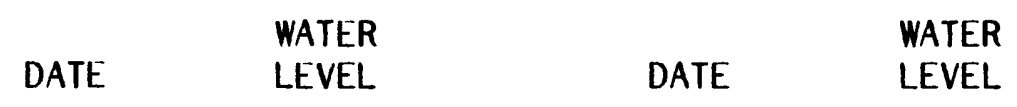

MAR 10, $1982 \quad 2.10 \quad$ FEB 28, $1983 \quad 3.37$ 
GARFIELD COUNTY -- CONTINUED

361725098052001. LOCAL NUMBER; 21N-08W-18 DCD 1.

LOCATION,--LAT 36 17'25", LONG 098 05'20", HYDROLOGIC UNIT 11050002.

AQUIFER.--TERRACE DEPOSITS.

PERIOD OF RECORD.--1975 TO CURRENT YEAR.

EXTREMES FOR PERIOD OF RECORD.--HIGHEST WATER LEVEL, $1.70 \mathrm{FT}(.52 \mathrm{M})$ BELOW LAND-SURFACE DATUM, JAN 22, 1975; LOWEST WATER LEVEL,11.17 FT(3.40 M) BELOW LAND-SURFACE DATUM, JAN 29, 1980.

WATER LEVELS IN FEET BELOW LAND SURFACE DATUM, CLIMATIC YEARS APRIL 1981 TO MARCH 1983

\begin{tabular}{cccc}
\multicolumn{1}{c}{ WATER } & & \multicolumn{2}{c}{ WATER } \\
DATE & LEVEL & DATE & LEVEL \\
MAR 10, 1982 & 10.42 & FEB 28, 1983 & 7.92
\end{tabular}

GROUND-WATER LEVELS

361651098061001. LOCAL NUMBER; 21N-08W-19 CBB 1.

LOCATION,--LAT 36 16'51", LONG 098 06'10", HYOROLOGIC UNIT 11050002.

AQUIFER.--TERRACE DEPOSITS.

PERIOD OF RECORD.--1975 TO CURRENT YEAR.

EXTREMES FOR PERIOD OF RECORD.--HIGHEST WATER LEVEL, 18.18 FT(5.54 M) BELOW LAND-SURFACE DATUM,DEC 12, 1976; LOWEST WATER LEVEL,27.22 FT(8.30 M) BELOW LAND-SURFACE DATUM, MAR 21, 1979 .

WATER LEVELS IN FELT BELOW LAND SURFACE DATUM, CLIMATIC YEARS APRIL 1981 TO MARCH 1983

\begin{tabular}{|c|c|c|}
\hline & WATER & \\
\hline DATE & LEVEL & DATE \\
\hline
\end{tabular}

MAR $10,1982 \quad 26.93 \quad$ FEB 28, $1983 \quad 21.92$ 
GARFIELD COUNTY -- CONTINUED

362736097563901. LOCAL NUMBER; 23N-07W-21 AAD 1.

LOCATION,--LAT 36 27'36", LONG 097 56'39", HYDROLOGIC UNIT 11050002.

AQUIFER.--TERRACE DEPOSITS.

PERIOD OF RECORD. --1975 TO CURRENT YEAR.

EXTREMES FOR PERIOD OF RECORD.--HIGHEST WATER LEVEL, 33.05 FT(10.07 M) BELOW LAND-SURFACE

DATUM,MAR 8, 1983; LOWEST WATER LEVEL, 36.32 FT(11.07 M) BELOW LAND-SURFACE DATUM, JAN 24, 1975.

WATER LEVELS IN FEET BELOW LAND SURFACE DATUM, CLIMATIC YEARS APRIL 1981 TO MARCH 1983

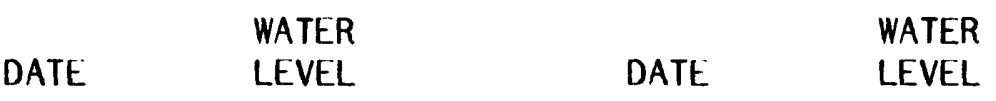

MAR $10,1982 \quad 33.27 \quad$ MAR 08, $1983 \quad 33.05$

GROUND-WATER LEVELS

362736097573601. LOCAL NUMBER; 23N-07W-21 BBC 1.

LOCATION,--LAT 36 27'36", LONG 097 57'36", HYDROLOGIC UNIT 11050002.

AQUIFER.--TERRACE DEPOSITS.

PERIOD OF RECORD.--1975 TO CURRENT YEAR.

EXTREMES FOR PERIOD OF RECORD.--HIGHEST WATER LEVEL,5.96 FT(1.82 M) BELOW LAND-SURFACE DATUM, JAN 24, 1975; LOWEST WATER LEVEL,9.95 FT(3.03 M) BELOW LAND-SURFACE DATUM, DEC 8, 1976.

WATER LEVELS IN FEET BELOW LAND SURFACE DATUM, CLIMATIC YEARS APRIL 1981 TO MARCH 1983

$\begin{array}{lll} & \text { WATER } & \text { WATER } \\ \text { DATE } & \text { LEVEL } & \text { DATE }\end{array}$

MAR 10, $1982 \quad 6.73 \quad$ MAR $08,1983 \quad 8.03$ 


\section{GROUND-WATER LEVELS}

GARF IELD COUNTY -- CONTINUED

362650097543901. LOCAL NUMBER; 23N-07W-26 AAB 1.

LOCATION,--LAT 36 26'50", LONG 097 54'39", HYDROLOGIC UNIT 11050002.

AQUIFER.--TERRACE DEPOSITS.

PERIOD OF RECORD.--1975 TO CURRENT YEAR.

EXTREMES FOR PERIOD OF RECORD.--HIGHEST WATER LEVEL, 27.74 FT(8.46 M) BELOW LAND-SURFACE DATUM,MAR 8, 1983; LOWEST WATER LEVEL, 30.50 FT(9.30 M) BELOW LAND-SURFACE DATUM, DEC 8, 1976.

WATER LEVELS IN FEET BELOW LAND SURFACE DATUM, CLIMATIC YEARS APRIL 1981 TO MARCH 1983

$\begin{array}{lll} & \text { WATER } & \\ \text { DATE } & \text { WEVEL } & \text { WATER } \\ \text { LATE } & \text { LEVEL }\end{array}$

MAR $10,1982 \quad 28.59 \quad$ MAR $08,1983 \quad 27.74$

GROUND-WATER LEVELS

362650097551901. LOCAL NUMBER; 23N-07W-26 BBA 1.

LOCATION,--LAT 36 26'50", LONG 097 55'19", HYDROLOGIC UNIT 11050002.

AQUIFER.--TERRACE DEPOSITS.

PERIOD OF RECORD. - - 1975 TO CURRENT YEAR.

EXTREMES FOR PERIOD OF RECORD.--HIGHEST WATER LEVEL, 38.42 FT(11.71 M) BELOW LAND-SURFACE DATUM,MAR 8, 1983; LOWEST WATER LEVEL, 39.49 FT(12.04 M) BELOW LAND-SURFACE DATUM, MAR 10, 1982.

WATER LEVELS IN FEET BELOW LAND SURFACE DATUM, CLIMATIC YEARS APRIL 1981 TO MARCH 1983

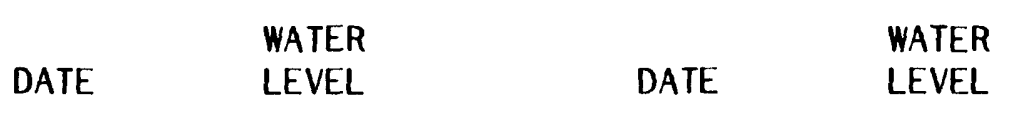

MAR $10,1982 \quad 39.49 \quad$ MAR 08, $1983 \quad 38.42$ 


\section{GARF IELD COUNTY -- CONTINUED}

362650097555101 . LOCAL NUMBER;

LOCATION,--LAT 36 26'50", LONG 097 AQUIFER.--TERRACE DEPOSITS.

PERIOD OF RECORD.--1950 TO CURRENT

EXTREMES FOR PERIOD OF RECORD.--HIGHEST WATER LEVEL, $48.70 \mathrm{FT}(14.84 \mathrm{M})$ BELOW LAND-SURFACE DATUM, JAN 16, 1976; LOWEST WATER LEVEL,55.79 FT(17.00 M) BELOW LAND-SURFACE DATUM, MAR 7, 1950.

WATER LEVELS IN FEET BELOW LAND SURFACE DATUM, CLIMATIC YEARS APRIL 1981 TO MARCH 1983

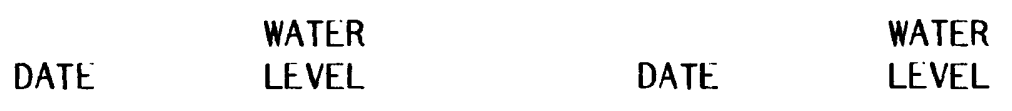

MAR $10,1982 \quad 50.98 \quad$ MAR $08,1983 \quad 50.25$

GROUND-WATER LEVELS

362650097562301. LOCAL NUMBER;

$23 N-07 W-27$ BBA 1.

LOCATION,--LAT 36 26'50", LONG 097

AQUIFER.--TERRACE DEPOSITS.

PERIOD OF RECORD. --1975 TO CURRENT YEAR.

EXTREMES FOR PERIOD OF RECORD.--HIGHEST WATER LEVEL,48.61 FT(14.82 M) BELOW LAND-SURFACE DATUM,DEC 8, 1976; LOWEST WATER LEVEL,50.50 FT(15.39 M) BELOW LAND-SURFACE DATUM, JAN 24, 1975.

WATER LEVELS IN FEET BELOW LAND SURFACE DATUM, CLIMATIC YEARS APRIL 1981 TO MARCH 1983

\begin{tabular}{|c|c|c|}
\hline & WATER & \\
\hline DATE & LEVEL & DATE \\
\hline
\end{tabular}

MAR $10,1982 \quad 49.27 \quad$ MAR 08, $1983 \quad 49.04$ 
GROUND-WATER LEVELS

GARF IELD COUNTY - - CONTINUED

362637097563101 . LOCAL NUMBER;

LOCATION,--LAT 36 26'37", LONG 097

AQUIFER. - -TERRACE DEPOSITS.

PERIOD OF RECORD.--1950 TO CURRENT YEAR.

EXTREMES FOR PERIOD OF RECORD.--HIGHEST WATER LEVEL, 44.85 FT (13.67 M) BELOW LAND-SURFACE DATUM,DEC 8, 1976; LOWEST WATER LEVEL,48.63 FT(14.82 M) BELOW LAND-SURFACE DATUM, MAR 7, 1950 .

23N-07W-27 BCB 1.

56'31", HYDROLOGIC UNIT 11050002.

WATER LEVELS IN FEET BELOW LAND SURFACE DATUM, CLIMATIC YEARS APRIL 1981 TO MARCH 1983

$\begin{array}{cccc}\text { DATE } & \text { WATER } & & \text { WATER } \\ \text { LEVEL } & \text { DATE } & \text { LEVEL } \\ \text { MAR 10, 1982 } & 45.35 & \text { MAR 08, 1983 } & 45.17\end{array}$

GROUND-WATER LEVELS

362650097565501. LOCAL NUMBER; 23N-07W-28 ABA 1.

LOCATION,--LAT 36 26'50", LONG 097 56'55", HYDROLOGIC UNIT 11050002.

AQUIFER.--TERRACE DEPOSITS.

PERIOD OF RECORD.--1975 TO CURRENT YEAR.

EXTREMES FOR PERIOD OF RECORD.--HIGHEST WATER LEVEL, $47.38 \mathrm{FT}(14.44 \mathrm{M})$ BELOW LAND-SURFACE DATUM, MAR 8, 1983; LOWEST WATER LEVEL,49.04 FT(14.95 M) BELOW LAND-SURFACE DATUM, JAN 24, 1975.

WATER LEVELS IN FEET BELOW LAND SURFACE DATUM, CLIMATIC YEARS APRIL 1981 TO MARCH 1983

\begin{tabular}{|c|c|c|c|}
\hline & WATER & & WATER \\
\hline DATE & LEVEL & DATE & LEVEL \\
\hline IAR 10,1982 & 47.50 & MAR 08, 1983 & 47.38 \\
\hline
\end{tabular}


GROUND-WATER LEVELS

GARFIELD COUNTY -- CONTINUED

362655097572101. LOCAL NUMBER; 23N-07W-28 BAB 1.

LOCATION, --LAT 36 26'55", LONG 097 57'21", HYDROLOGIC UNIT 11050002.

AQUIFER.--TERRACE DEPOSITS.

PERIOD OF RECORD.--1975 TO CURRENT YEAR.

EXTREMES FOR PERIOD OF RECORD.--HIGHEST WATER LEVEL, $55.13 \mathrm{FT}(16.80 \mathrm{M})$ BELOW LAND-SURFACE DATUM,DEC 8, 1976; LOWEST WATER LEVEL,57.04 FT(17.39 M) BELOW LAND-SURFACE DATUM, JAN 24, 1975.

WATER LEVELS IN FEET BELOW LAND SURFACE DATUM, CLIMATIC YEARS APRIL 1981 TO MARCH 1983

\begin{tabular}{lccr} 
& WATER & & \\
DATE & LEVEL & WATER \\
DATE & LEVEL \\
\hline 10,1982 & 55.88 & MAR 08, 1983 & 55.82
\end{tabular}




\section{GROUND-WATER LEVELS}

GARVIN COUNTY

345054096580501. LOCAL NUMBER; 04N-03E-03 ADD 1. LOCATION,--LAT 34 50'54", LONG 096 58'05", HYDROLOGIC UNIT 11090202.

AQUIFER.--TERRACE DEPOSITS.

PERIOD OF RECORD.--1975 TO CURRENT YEAR.

EXTREMES FOR PERIOD OF RECORD .--HIGHEST WATER LEVEL, $24.78 \mathrm{FT}(7.55 \mathrm{M})$ BELOW LAND-SURFACE DATUM,JAN 21, 1976; LOWEST WATER LEVEL,33.03 FT(10.07 M) BELOW LAND-SURFACE DATUM, MAR 18, 1982.

WATER LEVELS IN FEET BELOW LAND SURFACE DATUM, CLIMATIC YEARS APRIL 1981 TO MARCH 1983

\begin{tabular}{|c|c|}
\hline & WATER \\
\hline DATE & LEVEL \\
\hline
\end{tabular}

MAR 18, $1982 \quad 33.03 \quad$ MAR 21, $1983 \quad 31.84$

GROUND-WATER LEVELS

344844096575701. LOCAL NUMBER;

$04 \mathrm{~N}-03 \mathrm{E}-14$ CCC 2 .

LOCATION,--LAT 34 48'44", LONG 096 57'57", HYDROLOGIC UNIT 11090202.

AQUIFER .--TERRACE DEPOSITS.

PERIOD OF RECORD.--1975 TO CURRENT YEAR.

EXTREMES FOR PERIOD OF RECORD.--HIGHEST WATER LEVEL,65.07 FT(19.83 M) BELOW LAND-SURFACE DATUM, MAR 28, 1979; LOWEST WATER LEVEL,72.90 FT(22.22 M) BELOW LAND-SURFACE DATUM, JAN 23, 1981.

WATER LEVELS IN FEET BELOW LAND SURFACE DATUM, CLIMATIC YEARS APRIL 1981 TO MARCH 1983

$\begin{array}{lll} & \text { WATER } & \text { WATER } \\ \text { DATE } & \text { LEVEL } & \text { DATE }\end{array}$

MAR 18, $1982 \quad 72.74 \quad$ MAR 21, $1983 \quad 71.91$ 


\section{GROUND-WATER LEVELS}

GRADY COUNTY

344446097551501. LOCAL NUMBER; 03N-07W-10 DBB 1. LOCATION,--LAT 34 44'46", LONG 097 55'15", HYDROLOGIC UNIT 11130303. AQUIFER.--RUSH SPRINGS FORMATION. PERIOD OF RECORD.--1979 TO CURRENT YEAR. EXTREMES FOR PERIOD OF RECORD.--HIGHEST WATER LEVEL, $12.59 \mathrm{FT}(3.84 \mathrm{M})$ BELOW LAND-SURFACE DATUM, MAR 10, 1983; LOWEST WATER LEVEL, 15.12 FT(4.61 M) BELOW LAND-SURFACE DATUM, MAR 26, 1979.

WATER LEVELS IN FEET BELOW LAND SURFACE DATUM, CLIMATIC YEARS APRIL 1981 TO MARCH 1983

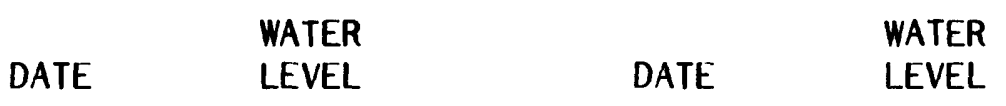

MAR 23, $1982 \quad Z \quad$ MAR $10,1983 \quad 12.59$

GROUND-WATER LEVELS

344353097572201. LOCAL NUMBER; 03N-07W-17 DBB 1.

LOCATION,--LAT 34 43'53", LONG 097 57'22", HYDROLOGIC UNIT 11130208.

AQUIFER.--RUSH SPRINGS FORMATION.

PERIOD OF RECORD.--1976 TO CURRENT YEAR.

EXTREMES FOR PERIOD OF RECORD.--HIGHEST WATER LEVEL, $32.83 \mathrm{FT}(10.01 \mathrm{M})$ BELOW LAND-SURFACE DATUM,DEC 10, 1976; LOWEST WATER LEVEL, $37.75 \mathrm{FT}(11.51 \mathrm{M})$ BELOW LAND-SURFACE DATUM, JAN 26, 1981.

WATER LEVELS IN FEET BELOW LAND SURFACE DATUM, CLIMATIC YEARS APRIL 1981 TO MARCH 1983

$\begin{array}{lll} & \text { WATER } & \\ \text { DATE } & \text { LEVEL } & \text { WATER } \\ \text { DATE } & \text { LEVEL }\end{array}$

MAR 23, $1982 \quad 37.08$ MAR 10, $1983 \quad 35.03$ 
GRADY COUNTY -- CONTINUED

344222098030101 . LOCAL NUMBER; LOCATION,--LAT 34 42'22", LONG 098 AQUIFER.--RUSH SPRINGS FORMATION. PERIOD OF RECORD.--1979 TO CURRENT YEAR.

EXTREMES FOR PERIOD OF RECORD.--HIGHEST WATER LEVEL, $117.93 \mathrm{FT}(35.95 \mathrm{M})$ BELOW LAND-SURFACE DATUM, MAR 10, 1983; LOWEST WATER LEVEL, 132.37 FT(40.35 M) BELOW LAND-SURFACE DATUM, JAN 26, 1981.

WATER LEVELS IN FEET BELOW LAND SURFACE DATUM, CLIMATIC YEARS APRIL 1981 TO MARCH 1983
03N-08W-28 BCA 1.

03'01", HYDROLOGIC UNIT 11130208.

$\begin{array}{lll} & & \\ & & \\ \text { DATE } & \text { WATER } & \text { WATER } \\ \text { LEVEL } & \text { DATE } & \text { LEVEL }\end{array}$
MAR 23, $1982 \quad 126.90$
MAR $10,1983 \quad 117.93$

\title{
GROUND-WATER LEVELS
}

\begin{abstract}
351907097510401. LOCAL NUMBER; 10N-06W-29 AAB 1.
LOCATION,--LAT 35 19'07", LONG 097 51'04", HYDROLOGIC UNIT 11090202. AQUIFER.--RUSH SPRINGS FORMATION. PERIOD OF RECORD.--1979 TO CURRENT YEAR.

EXTREMES FOR PERIOD OF RECORD.--HIGHEST WATER LEVEL, 7.50 FT(2.29 M) BELOW LAND-SURFACE DATUM, MAR 23, 1982; LOWEST WATER LEVEL, 10.22 FT(3.12 M) BELOW LAND-SURFACE DATUM, MAR $12,1979$.
\end{abstract}

WATER LEVELS IN FEET BELOW LAND SURFACE DATUM, CLIMATIC YEARS APRIL 1981 TO MARCH 1983

$\begin{array}{lll} & \text { WATER } & \text { WATER } \\ \text { DATE } & \text { LEVEL } & \text { DATE }\end{array}$

MAR 23, $1982 \quad 7.50 \quad$ MAR $10,1983 \quad 8.13$ 
GROUND-WATER LEVELS

GRANT COUNTY

364133097460901. LOCAL NUMBER;

$26 \mathrm{~N}-05 \mathrm{~W}-31$ ADA 1

LOCATION,--LAT 3641 '33", LONG 097 $46^{\prime}$ '09", HYDROL.OGIC UNIT 11060004.

AQUIFER.--TERRACE DEPOSITS.

PERIOD OF RECORD.--1975 TO CURRENT YEAR.

EXTREMES FOR PERIOD OF RECORD.--HIGHEST WATER LEVEL, 3.05 FT(.93 M) BELOW LAND-SURFACE DATUM,MAR 6, 1975; LOWEST WATER LEVEL, 18.08 FT(5.51 M) BELOW LAND-SURFACE DATUM, MAR 28, 1979.

WATER LEVELS IN FEET BELOW LAND SURFACE DATUM, CLIMATIC YEARS APRIL 1981 TO MARCH 1983

$\begin{array}{lll} & \text { WATER } & \\ \text { DATE } & \text { WEVEL } & \text { WATER } \\ & \text { DATE } & \text { LEVEL }\end{array}$

MAR 04, $1982 \quad 17.47 \quad$ MAR 03, $1983 \quad 16.09$

GROUND-WATER LEVELS

364244097471401. LOCAL NUMBER; 26N-06W-24 DDD 1.

LOCATION,--LAT 36 42'44", LONG 097 47'14", HYDROLOGIC UNIT 11060004.

AQUIFER.--ALLUVIUM.

PERIOD OF RECORD.--1975 TO CURRENT YEAR.

EXTREMES FOR PERIOD OF RECORD.--HIGHEST WATER LEVEL, $7.50 \mathrm{FT}(2.29 \mathrm{M})$ BELOW LAND-SURFACE DATUM,MAR 6, 1975; LOWEST WATER LEVEL, 16.33 FT(4.98 M) BELOW LAND-SURFACE DATUM, MAR 11, 1977.

WATER LEVELS IN FEET BELOW LAND SURFACE DATUM, CLIMATIC YEARS APRIL 1981 TO MARCH 1983

$\begin{array}{lll} & \text { WATER } & \text { WATER } \\ \text { DATE } & \text { LEVEL } & \text { DATE }\end{array}$

$\begin{array}{llll}\text { MAR 31, } 1982 & Z & \text { MAR 03, } 1983 & 11.91\end{array}$ 
GRANT COUNTY -- CONTINUED

364514097592101. LOCAL NUMBER; 26N-07W-07 ABA 1. LOCATION,--LAT 36 45'14", LONG 097 59'21", HYDROLOGIC UNIT 11060004. AQUIFER.--TERRACE DEPOSITS.

PERIOD OF RECORD.--1975 TO CURRENT YEAR.

EXTREMES FOR PERIOD OF RECORD.--HIGHEST WATER LEVEL, 3.48 FT(1.06 M) BELOW LAND-SURFACE DATUM,MAR 5, 1975; LOWEST WATER LEVEL, 12.10 FT(3.69 M) BELOW LAND-SURFACE DATUM, MAR 28, 1979.

WATER LEVELS IN FEET BELOW LAND SURFACE DATUM, CLIMATIC YEARS APRIL 1981 TO MARCH 1983

\begin{tabular}{|c|c|c|}
\hline & WATER & \\
\hline DATE & LEVEL & DATE \\
\hline
\end{tabular}

MAR 04, $1982 \quad 11.30 \quad$ MAR 03, $1983 \quad 9.31$

GROUND-WATER LEVELS

364238098025201. LOCAL NUMBER; 26N-08W-27 BAA 1.

LOCATION,--LAT 36 42'38", LONG 098 02'52", HYDROLOGIC UNIT 11060004.

AQUIFER.--TERRACE DEPOSITS.

PERIOD OF RECORD.--1975 TO CURRENT YEAR.

EXTREMES FOR PERIOD OF RECORD.--HIGHEST WATER LEVEL, 5.05 FT(1.54 M) BELOW LAND-SURFACE DATUM, MAR 5, 1975; LOWEST WATER LEVEL, 12.00 FT(3.66 M) BELOW LAND-SURFACE DATUM, JAN 21, 1981.

WATER LEVELS IN FEET BELOW LAND SURFACE DATUM, CLIMATIC YEARS APRIL 1981 TO MARCH 1983

\begin{tabular}{|c|c|c|}
\hline & WATER & \\
\hline DATE & LEVEL & DATE \\
\hline
\end{tabular}

MAR $04,1982 \quad 10.32 \quad$ MAR $03,1983 \quad 10.82$ 
GRANT COUNTY -- CONTINUED

364935097594601. LOCAL NUMBER; 27N-07W-18 BAB 1. LOCATION,--LAT 36 49'35", LONG 097 59'46", HYDROLOGIC UNIT 11060004. AQUIFER.--ALLUVIUM. PERIOD OF RECORD.--1975 TO CURRENT YEAR. EXTREMES FOR PERIOD OF RECORD.--HIGHEST WATER LEVEL, $5.39 \mathrm{FT}(1.64 \mathrm{M})$ BELOW LAND-SURFACE DATUM,MAR 5, 1975; LOWEST WATER LEVEL, $14.89 \mathrm{FT}(4.54 \mathrm{M})$ BELOW LAND-SURFACE DATUM, MAR 28, 1979.

WATER LEVELS IN FEET BELOW LAND SURFACE DATUM, CLIMATIC YEARS APRIL 1981 TO MARCH 1983

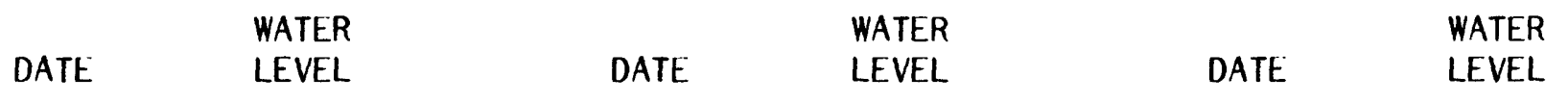

$\begin{array}{lllllll}\text { MAR 04, } 1982 & 13.92 & \text { MAR 03, } 1983 & 13.63 & \text { MAR 04, } 1983 & 13.92\end{array}$




\section{GROUND-WATER LEVELS}

GREER COUNTY

344630099370201. LOCAL NUMBER; 04N-23W-33 DAB 1.

LOCATION,--LAT 34 46'30", LONG 099 37'02", HYDROLOGIC UNIT 11120304.

AQUIFER.--BLAINE GYPSUM.

PERIOD OF RECORD.--1980 TO CURRENT YEAR.

EXTREMES FOR PERIOD OF RECORD.--HIGHEST WATER LEVEL, 33.48 FT(10.20 M) BELOW LAND-SURFACE DATUM,FEB 19, 1982; LOWEST WATER LEVEL, 38.70 FT(11.80 M) BELOW LAND-SURFACE DATUM, JAN 26, 1983.

WATER LEVELS IN FEET BELOW LAND SURFACE DATUM, CLIMATIC YEARS APRIL 1981 TO MARCH 1983

\begin{tabular}{|c|c|}
\hline & WATER \\
\hline DATE & LEVEL \\
\hline
\end{tabular}

FEB 19, $1982 \quad 33.48 \quad$ JAN 26, $1983 \quad 38.70$

GROUND-WATER LEVELS

345541099193001. LOCAL NUMBER; 05N-20W-08 AAA 1. LOCATION,--LAT 34 55'41", LONG 099 19'30", HYDROLOGIC UNIT 11120304. AQUIFER.--TERRACE DEPOSITS.

PERIOD OF RECORD.--1980 TO CURRENT YEAR.

EXTREMES FOR PERIOD OF RECORD.--HIGHEST WATER LEVEL,28.40 FT(8.66 M) BELOW LAND-SURFACE DATUM,FEB 27, 1980; LOWEST WATER LEVEL,34.79 FT(10.60 M) BELOW LAND-SURFACE DATUM, FEB 19, 1982.

WATER LEVELS IN FEET BELOW LAND SURFACE DATUM, CLIMATIC YEARS APRIL 1981 TO MARCH 1983

\begin{tabular}{|c|c|c|}
\hline & WATER & \\
\hline DATE & & DATE \\
\hline
\end{tabular}

FEB 19, $1982 \quad 34.79 \quad$ MAR $10,1983 \quad 32.80$


GREER COUNTY -- CONTINUED

345403099202501 . LOCAL NUMBER; LOCATION,--LAT 34 54'03", LONG 099 AQUIFER.--TERRACE DEPOSITS.

PERIOD OF RECORD.--1980 TO CURRENT YEAR.

EXTREMES FOR PERIOD OF RECORD.--HIGHEST WATER LEVEL, $39.97 \mathrm{FT}(12.18 \mathrm{M})$ BELOW LAND-SURFACE DATUM,MAR 10, 1983; LOWEST WATER LEVEL,49.84 FT(15.19 M) BELOW LAND-SURFACE DATUM, FEB 19, 1982.

WATER LEVELS IN FEET BELOW LAND SURFACE DATUM, CLIMATIC YEARS APRIL 1981 TO MARCH 1983
FEB 19, 1982
MAR $10,1983 \quad 39.97$

WATER

DATE

WATER

LEVEL

GROUND-WATER LEVELS

345356099212801. LOCAL NUMBER; 05N-20W-19 BBB 1. LOCATION,--LAT 34 53'56", LONG 099 21'28", HYDROLOGIC UNIT 11120304. AQUIFER.--TERRACE DEPOSITS. PERIOD OF RECORD.--1980 TO CURRENT YEAR. LXTREMES FOR PERIOD OF RECORD.--HIGHEST WATER LEVEL, 28.76 FT(8.77 M) BELOW LAND-SURFACE DATUM,FEB 28, 1980; LOWEST WATER LEVEL,30.20 FT(9.20 M) BELOW LAND-SURFACE DATUM, FEB $19,1982$.

WATER LEVELS IN FEET BELOW LAND SURFACE DATUM, CLIMATIC YEARS APRIL 1981 TO MARCH 1983

$\begin{array}{lll} & \text { WATER } & \text { WATER } \\ \text { DATE } & \text { LEVEL } & \text { DATE }\end{array}$

FEB 19, $1982 \quad 30.20 \quad$ MAR 10, $1983 \quad 29.29$ 
GREER COUNTY -- CONTINUED

345528099255801. LOCAL NUMBER;

LOCATION,--LAT 34 55'28", LONG 099

AQUIFER.--TERRACE DEPOSITS.

PERIOD OF RECORD.--1980 TO CURRENT YEAR.

EXTREMES FOR PERIOD OF RECORD.--HIGHEST WATER LEVEL, $27.98 \mathrm{FT}(8.53 \mathrm{M})$ BELOW LAND-SURFACE DATUM,FEB 27, 1980; LOWEST WATER LEVEL,28.18 FT(8.59 M) BELOW LAND-SURFACE DATUM, MAR 10, 1983.

WATER LEVELS IN FEET BELOW LAND SURFACE DATUM, CLIMATIC YEARS APRIL 1981 TO MARCH 1983

$\begin{array}{lll} & \text { WATER } & \text { WATER } \\ \text { DATE } & \text { LEVEL } & \text { DATE }\end{array}$

FEB 19, $1982 \quad 28.18 \quad$ MAR $10,1983 \quad 28.05$

GROUND-WATER LEVELS

345409099220801. LOCAL NUMBER; 05N-21W-13 CDA 1.

LOCATION,--LAT 34 54'09", LONG 099 22'08", HYDROLOGIC UNIT 11120304.

AQUIFER.--TERRACE DEPOSITS.

PERIOD OF RECORD.-- 1980 TO CURRENT YEAR.

EXTREMES FOR PERIOD OF RECORD.--HIGHEST WATER LEVEL, $32.30 \mathrm{FT}(9.85 \mathrm{M})$ BELOW LAND-SURFACE DATUM,MAR 10, 1983; LOWEST WATER LEVEL,35.34 FT(10.77 M) BELOW LAND-SURFACE DATUM, FEB 19, 1982 .

WATER LEVELS IN FEET BELOW LAND SURFACE DATUM, CLIMATIC YEARS APRIL 1981 TO MARCH 1983

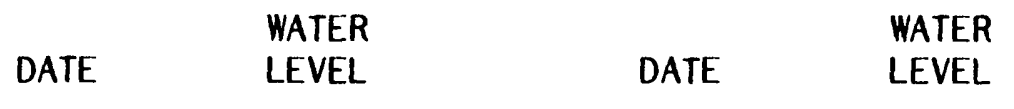

FEB 19, $1982 \quad 35.34 \quad$ MAR 10, $1983 \quad 32.30$


345356099224001. LOCAL NUMBER; LOCATION,--LAT $3453^{\prime} 56^{\prime \prime}$, LONG 099 AQUIFER.--TERRACE DEPOSITS. PERIOD OF RECORD.--1980 TO CURRENT YEAR. EXTREMES FOR PERIOD OF RECORD.--HIGHEST WATER LEVEL, 30.40 FT(9.27 M) BELOW LAND-SURFACE DATUM,MAR 10, 1983; LOWEST WATER LEVEL,31.26 FT(9.53 M) BELOW LAND-SURFACE DATUM, FEB 19, 1982 .

WATER LEVELS IN FEET BELOW LAND SURFACE DATUM, CLIMATIC YEARS APRIL 1981 TO MARCH 1983
05N-21W-23 AAA 1 $22^{\prime} 40$ ", HYDROLOGIC UNIT 11120304.

WATER LEVELS IN

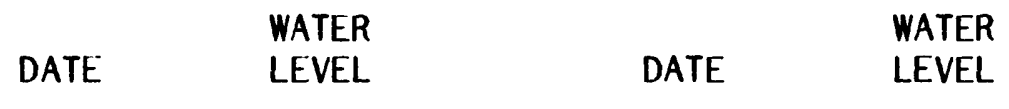

FEB 19, $1982 \quad 31.26 \quad$ MAR 10, $1983 \quad 30.40$

GROUND-WATER LEVELS

345547099302701. LOCAL NUMBER; 05N-22W-03 DCC 1.

LOCATION,--LAT 34 55'47", LONG 099 30'27", HYDROLOGIC UNIT 11120304.

AQUIFER.--ALLUVIUM.

PERIOD OF RECORD.--1980 TO CURRENT YEAR.

EXTREMES FOR PERIOD OF RECORD.--HIGHEST WATER LEVEL, 11.18 FT(3.41 M) BELOW LAND-SURFACE DATUM,FEB 27, 1980; LOWEST WATER LEVEL, 11.64 FT(3.55 M) BELOW LAND-SURFACE DATUM, FEB 16, 1982 .

WATER LEVELS IN FEET BELOW LAND SURFACE DATUM, CLIMATIC YEARS APRIL 1981 TO MARCH 1983

\begin{tabular}{|c|c|c|c|c|}
\hline DATE & $\begin{array}{l}\text { WATER } \\
\text { LEVEL }\end{array}$ & DATE & $\begin{array}{l}\text { WATER } \\
\text { LEVEL }\end{array}$ & DATE \\
\hline
\end{tabular}

$\begin{array}{llllllll}\text { FEB } 16,1982 & 11.64 & \text { MAR } & 02,1983 & 10.88 & \text { MAR } 10,1983 & 10.88\end{array}$ 
GREER COUNTY -- CONTINUED

345554099313001. LOCAL NUMBER;

LOCATION,--LAT $3455^{\prime} 54^{\prime \prime}$, LONG 099

AQUIFER .--ALLUVIUM.

PERIOD OF RECORD.--1980 TO CURRENT YEAR.

EXTREMES FOR PERIOD OF RECORD.--HIGHEST WATER LEVEL, 10.99 FT(3.35 M) BELOW LAND-SURFACE DATUM,FEB 27, 1980; LOWEST WATER LEVEL, 12.43 FT(3.79 M) BELOW LAND-SURFACE DATUM, FEB 16, 1982.

WATER LEVELS IN FEET BELOW LAND SURFACE DATUM, CLIMATIC YEARS APRIL 1981 TO MARCH 1983
$05 \mathrm{~N}-22 \mathrm{~W}-04$ DCB 1.

31 ' 30 ", HYDROLOGIC UNIT 11120304.

$\begin{array}{cccc}\text { DATE } & \text { WATER } & & \text { WATER } \\ \text { LEVEL } & \text { DATE } & \text { LEVEL } \\ \text { FEB 16, 1982 } & 12.43 & \text { MAR 10, 1983 } & 11.23\end{array}$

GROUND-WATER LEVELS

345251099363101. LOCAL NUMBER; 05N-23W-27 ADB 1. LOCATION,--LAT 34 52'51", LONG 099 36'31", HYDROLOGIC UNIT 11120304. AQUIFER.--BLAINE GYPSUM.

PERIOD OF RECORD.-- 1980 TO CURRENT YEAR.

EXTREMES FOR PERIOD OF RECORD.--HIGHEST WATER LEVEL,63.34 FT(19.31 M) BELOW LAND-SURFACE DATUM, JAN 26, 1983; LOWEST WATER LEVEL,64.26 FT(19.59 M) BELOW LAND-SURFACE DATUM, FEB 19, 1982 .

WATER LEVELS IN FEET BELOW LAND SURFACE DATUM, CLIMATIC YEARS APRIL 1981 TO MARCH 1983

$\begin{array}{lll} & \text { WATER } & \text { WATER } \\ \text { DATE } & \text { LEVEL } & \text { DATE }\end{array}$

FEB 19, $1982 \quad 64.26 \quad$ JAN 26, $1983 \quad 63.34$


GREER COUNTY -- CONTINUED

345554099281201. LOCAL NUMBER; LOCATION, --LAT 34 55'54', LONG 099 AQUIFER . - -ALLUVIUM.

PERIOD OF RECORD.--1980 TO CURRENT YEAR.

EXTREMES FOR PERIOD OF RECORD.--HIGHEST WATER LEVEL,27.10 FT(8.26 M) BELOW LAND-SURFACE DATUM,FEB 27, 1980; LOWEST WATER LEVEL,27.79 FT(8.47 M) BELOW LAND-SURFACE DATUM, FEB 19, 1982.

WATER LEVELS IN FEET BELOW LAND SURFACE DATUM, CLIMATIC YEARS APRIL 1981 TO MARCH 1983
FEB 19, 1982
27.79
MAR $10,1983 \quad 27.24$

\section{GROUND-WATER LEVELS}

350055099204901. LOCAL NUMBER; 06N-20W-07 ABA 1. LOCATION,--LAT $3505^{\prime} 55^{\prime \prime}$, LONG 099 20'49", HYDROLOGIC UNIT 11120302. AQUIFER.--TERRACE DEPOSITS. PERIOD OF RECORD.--1980 TO CURRENT YEAR. EXTREMES FOR PERIOD OF RECORD.--HIGHEST WATER LEVEL, $27.40 \mathrm{FT}(8.35 \mathrm{M})$ BELOW LAND-SURFACE DATUM,FEB 18, 1982; LOWEST WATER LEVEL,29.13 FT(8.88 M) BELOW LAND-SURFACE DATUM, MAR 10, 1983.

WATER LEVELS IN FEET BELOW LAND SURFACE DATUM, CLIMATIC YEARS APRIL 1981 TO MARCH 1983

$\begin{array}{lll} & \text { WATER } & \text { WATER } \\ \text { DATE } & \text { LEVEL } & \text { DATE }\end{array}$

FEB $18,1982 \quad 27.40 \quad$ MAR $10,1983 \quad 29.13$ 
350121099232701. LOCAL NUMBER; 06N-21W-02 CBA 1. LOCATION,--LAT 35 01'21", LONG 099 23'27", HYDROLOGIC UNIT 11120302. AQUIFER.--TERRACE DEPOSITS. PERIOD OF RECORD.--1980 TO CURRENT YEAR. EXTREMES FOR PERIOD OF RECORD.--HIGHEST WATER LEVEL, 32.70 FT(9.97 M) BELOW LAND-SURFACE DATUM,MAR 8, 1980; LOWEST WATER LEVEL,33.30 FT(10.15 M) BELOW LAND-SURFACE DATUM, MAR 10, 1983.

WATER LEVELS IN FEET BELOW LAND SURFACE DATUM, CLIMATIC YEARS APRIL 1981 TO MARCH 1983

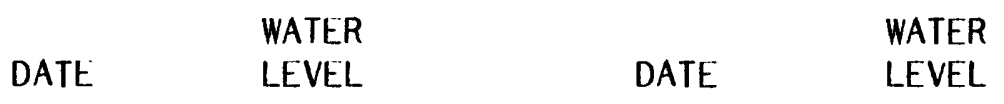

FEB 18, $1982 \quad 32.89 \quad$ MAR $10,1983 \quad 33.30$

GROUND-WATER LEVELS

345758099345601. LOCAL NUMBER; 06N-23W-25 BDC 1. LOCATION,--LAT 34 57'58", LONG 099 34'56", HYDROLOGIC UNIT 11120304. AQUIFER .--ALLUVIUM.

PERIOD OF RECORD.--1980 TO CURRENT YEAR.

EXTREMES FOR PERIOD OF RECORD.--HIGHEST WATER LEVEL,29.19 FT(8.90 M) BELOW LAND-SURFACE DATUM, JAN 26, 1983; LOWEST WATER LEVEL,30.83 FT(9.50 M) BELOW LAND-SURFACE DATUM, FEB 19,1982 .

WATER LEVELS IN FEET BELOW LAND SURFACE DATUM, CLIMATIC YEARS APRIL 1981 TO MARCH 1983

\begin{tabular}{|c|c|c|c|c|c|}
\hline & WATER & & WATER & & WATER \\
\hline DATE & LEVEL & DATE & LEVEL & DATE & LEVEL \\
\hline 19,1982 & 30.83 & JAN 23, 1983 & 29.19 & JAN 26, 1983 & 29.19 \\
\hline
\end{tabular}


GREER COUNTY -- CONTINUED

345818099413201. LOCAL NUMBER; $\quad 06 \mathrm{~N}-24 \mathrm{~W}-25$ BBB 1. LOCATION,--LAT 34 58'18", LONG 099 41'32", HYDROLOGIC UNIT 11120304. AQUIFER.--ALLUVIUM.

PERIOD OF RECORD.--1980 TO CURRENT YEAR.

EXTREMES FOR PERIOD OF RECORD.--HIGHEST WATER LEVEL, $13.85 \mathrm{FT}(4.22 \mathrm{M})$ BELOW LAND-SURFACE DATUM,JAN 26, 1983; LOWEST WATER LEVEL, 14.99 FT(4.57 M) BELOW LAND-SURFACE DATUM, FEB 19, 1982.

WATER LEVELS IN FEET BELOW LAND SURFACE DATUM, CLIMATIC YEARS APRIL 1981 TO MARCH 1983

$\begin{array}{lll} & \text { WATER } & \text { WATER } \\ \text { DATE } & \text { LEVEL } & \text { DATE }\end{array}$

FEB 19, $198214.99 \quad$ JAN 26, $1983 \quad 13.85$

GROUND-WATER LEVELS

350615099242301. LOCAL NUMBER; 07N-21W-03 CDC 1. LOCATION,--LAT 35 06'15", LONG 099 24'23", HYDROLOGIC UNIT 11120302. AQUIFER .--ALLUVIUM.

PERIOD OF RECORD.--1980 TO CURRENT YEAR.

EXTREMES FOR PERIOD OF RECORD.--HIGHEST WATER LEVEL, $27.88 \mathrm{FT}(8.50 \mathrm{M})$ BELOW LAND-SURFACE DATUM,MAR 5, 1980; LOWEST WATER LEVEL,28.43 FT(8.67 M) BELOW LAND-SURFACE DATUM, FEB 18, 1982.

WATER LEVELS IN FEET BELOW LAND SURFACE DATUM, CLIMATIC YEARS APRIL 1981 TO MARCH 1983

$\begin{array}{lll} & \text { WATER } & \text { WATER } \\ \text { DATE } & \text { LEVEL } & \text { DATE }\end{array}$

FEB $18,1982 \quad 28.43 \quad$ JAN $25,1983 \quad 28.22$ 
GREER COUNTY -- CONTINUED

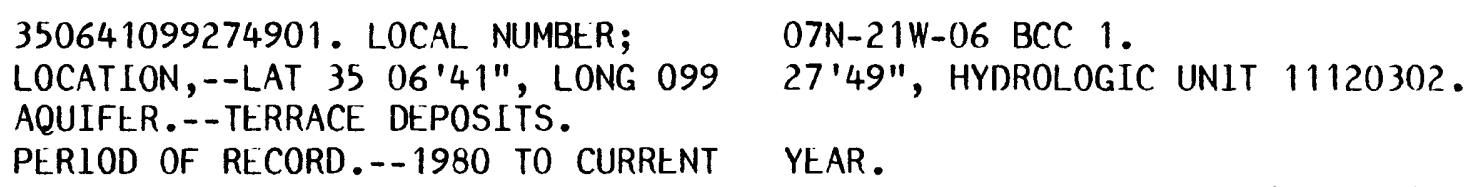

WATER LEVELS IN FEET BELOW LAND SURFACE DATUM, CLIMATIC YEARS APRIL 1981 TO MARCH 1983

\begin{tabular}{|c|c|c|}
\hline & WATER & \\
\hline DATE & LEVEL & DATE \\
\hline
\end{tabular}

FEB $18,1982 \quad 27.76 \quad$ JAN $25,1983 \quad 26.30$

GROUND-WATER LEVELS

350529099255801. LOCAL NUMBER; 07N-21W-08 DDB 1.

LOCATION,--LAT 35 05'29", LONG 099 25'58", HYDROLOGIC UNIT 11120302.

AQUIFER.--TERRACE DEPOSITS.

PERIOD OF RECORD.--1980 TO CURRENT YEAR.

EXTREMES FOR PERIOD OF RECORD.--HIGHEST WATER LEVEL, 23.76 FT $(7.24 \mathrm{M})$ BELOW LAND-SURFACE DATUM,MAR 5, 1980; LOWEST WATER LEVEL,25.76 FT(7.85 M) BELOW LAND-SIJRFACE DATUM, FEB 18, 1982 .

WATER LEVELS IN FEET BELOW LAND SURFACE DATUM, CLIMATIC YEARS APRIL 1981 TO MARCH 1983

$\begin{array}{lll} & \text { WATER } & \text { WATER } \\ \text { DATE } & \text { LEVEL } & \text { DATE } \\ & \text { LEVEL }\end{array}$

FtB $18,1982 \quad 25.76 \quad$ JAN 25, $1983 \quad 24.92$ 


\section{GROUND-WATER LEVELS}

GREER COUNTY -- CONTINUED

350516099243901. LOCAL NUMBER;

LOCATION,--LAT 35 05'16", LONG 099 AQUIFER.--TERRACE DEPOSITS.

PERIOD OF RECORD .--1980 TO CURRENT

EXTREMES FOR PERIOD OF RECORD.--HIGHEST WATER LEVEL, $24.03 \mathrm{FT}(7.32 \mathrm{M})$ BELOW LAND-SURFACE DATUM, MAR 5, 1980; LOWEST WATER LEVEL,29.68 FT(9.05 M) BELOW LAND-SURFACE DATUM, FEB 18, 1982.

WATER LEVELS IN FEET BELOW LAND SURFACE DATUM, CLIMATIC YEARS APRIL 1981 TO MARCH 1983

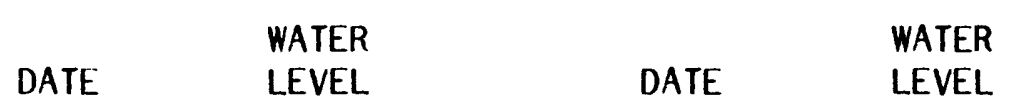

FEB 18, $1982 \quad 29.68 \mathrm{R} \quad$ JAN 25, $1983 \quad 28.54$
350457099254201. LOCAL NUMBER; 07N-21W-16 CCB 1.

LOCATION,--LAT $3505^{\prime}$ '57", LONG 099 25'42", HYDROLOGIC UNIT 11120302.

AQUIFER.--TERRACE DEPOSITS.

PERIOD OF RECORD.--1980 TO CURRENT YEAR.

EXTREMES FOR PERIOD OF RECORD.--HIGHEST WATER LEVEL, 19.27 FT(5.87 M) BELOW LAND-SURFACE DATUM,MAR 5, 1980; LOWEST WATER LEVEL,22.08 FT(6.73 M) BELOW LAND-SURFACE DATUM, FEB 18, 1982.

WATER LEVELS IN FEET BELOW LAND SURFACE DATUM, CLIMATIC YEARS APRIL 1981 TO MARCH 1983
FEB $18,1982 \quad 22.08$
JAN 25, 1983
21.26

WATER

LEVEL

DATE

WATER

DATE 
GREER COUNTY -- CONTINUED

350457099271701. LOCAL NUMBER;

$07 \mathrm{~N}-21 \mathrm{~W}-18$ ACC 1.

LOCATION,--LAT 35 04'57", LONG 099 27'17", HYDROLOGIC UNIT 11120304.

AQUIFER.--TERRACE DEPOSITS.

PERIOD OF RECORD.--1980 TO CURRENT YEAR.

EXTREMES FOR PERIOD OF RECORD.--HIGHEST WATER LEVEL, 39.78 FT(12.12 M) BELOW LAND-SURFACE DATUM, MAR 5, 1980; LOWEST WATER LEVEL,42.19 FT(12.86 M) BELOW LAND-SURFACE DATUM, FEB $18,1982$.

WATER LEVELS IN FEET BELOW LAND SURFACE DATUM, CLIMATIC YEARS APRIL 1981 TO MARCH 1983

\begin{tabular}{|c|c|c|}
\hline & WATER & \\
\hline DATE & LEVEL & DATE \\
\hline
\end{tabular}

FEB 18, $1982 \quad 42.19 \quad$ JAN 25, $1983 \quad 41.40$

GROUND-WATER LEVELS

350411099274101. LOCAL NUMBER;

$07 N-21 W-19$ BCA 1.

LOCATION,--LAT 35 04'11", LONG 099 27'41", HYDROLOGIC UNIT 11120302.

AQUIFER.--TERRACE DEPOSITS.

PERIOD OF RECORD.--1981 TO CURRENT YEAR.

EXTREMES FOR PERIOD OF RECORD.--HIGHEST WATER LEVEL, 36.89 FT(11.24 M) BELOW LAND-SURFACE DATUM,MAR 26, 1981; LOWEST WATER LEVEL, $37.82 \mathrm{FT}(11.53 \mathrm{M})$ BELOW LAND-SURFACE DATUM, FEB $18,1982$.

WATER LEVELS IN FEET BELOW LAND SURFACE DATUM, CLIMATIC YEARS APRIL 1981 TO MARCH 1983

\begin{tabular}{|c|c|}
\hline & WATER \\
\hline DATE & LEVEL \\
\hline
\end{tabular}

FEB $18,1982 \quad 37.82 \quad$ JAN $25,1983 \quad 0$ 
GREER COUNTY -- CONTINUED

350239099265301. LOCAL NUMBER; LOCATION,--LAT 35 02'39", LONG 099 AQUIFER.--TERRACE DEPOSITS. PERIOD OF RECORD.--1980 TO CURRENT YEAR.

EXTREMES FOR PERIOD OF RECORD.--HIGHEST WATER LEVEL,20.13 FT(6.14 M) BELOW LAND-SURFACE DATUM, MAR 3, 1980; LOWEST WATER LEVEL, 22.10 FT(6.74 M) BELOW LAND-SURFACE DATUM, FEB 18, 1982.

WATER LEVELS IN FEET BELOW LAND SURFACE DATUM, CLIMATIC YEARS APRIL 1981 TO MARCH 1983
07N-21W-31 AAA 1. 26 '53', HYDROLOGIC UNIT 11120302.

\begin{tabular}{|c|c|}
\hline & WATER \\
\hline DATE & LEVEL \\
\hline
\end{tabular}

FEB $18,1982 \quad 22.10 \quad$ JAN $25,1983 \quad 21.78$

GROUND-WATER LEVELS

350701099302701. LOCAL NUMBER; LOCATION,--LAT $3507^{\prime} 01^{\prime \prime}$, LONG 099 AQUIFER.--TERRACE DEPOSITS. PERIOD OF RECORD. - 1980 TO CURRENT EXTREMES FOR PERIOD OF RECORD.--HIGHEST WATER LEVEL, $26.35 \mathrm{FT}(8.03 \mathrm{M})$ BELOW LAND-SURFACE DATUM,MAR 5, 1980; LOWEST WATER LEVEL,27.48 FT(8.38 M) BELOW LAND-SURFACE DATUM, FEB 18, 1982.

WATER LEVELS IN FEET BELOW LAND SURFACE DATUM, CLIMATIC YEARS APRIL 1981 TO MARCH 1983
07N-22W-03 ABB 1. 30'27", HYDROLOGIC UNIT 11120304.

YEAR.

$\begin{array}{lll} & \text { WATER } & \text { WATER } \\ \text { DATE } & \text { LEVEL } & \text { DATE }\end{array}$

FEB $18,1982 \quad 27.48$

JAN 25, 1983 
GREER COUNTY -- CONTINUED

350523099303501. LOCAL NUMBER; 07N-22W-10 CDD 1. LOCATION,--LAT 35 05'23", LONG 099 30'35", HYDROLOGIC UNIT 11120304. AQUIFER.--TERRACE DEPOSITS.

PERIOD OF RECORD.--1980 TO CURRENT YEAR.

EXTREMES FOR PERIOD OF RECORD.--HIGHEST WATER LEVEL, 44.30 FT(13.50 M) BELOW LAND-SURFACE DATUM,MAR 5, 1980; LOWEST WATER LEVEL,48.14 FT (14.67 M) BELOW LAND-SURFACE DATUM, FEB 18, 1982.

WATER LEVELS IN FEET BELOW LAND SURFACE DATUM, CLIMATIC YEARS APRIL 1981 TO MARCH 1983

\begin{tabular}{|c|c|c|}
\hline & WATER & \\
\hline DATE & LEVEL & DATE \\
\hline
\end{tabular}

FEB $18,1982 \quad 48.14 \quad$ JAN 25, $1983 \quad 47.52$

GROUND-WATER LEVELS

350516099282801. LOCAL NUMBER; 07N-22W-13 BAA 1.

LOCATION,--LAT 35 05'16", LONG 099 28'28", HYDROLOGIC UNIT 11120304.

AQUIFER.--TERRACE DEPOSITS.

PERIOD OF RECORD.--1980 TO CURRENT YEAR.

EXTREMES FOR PERIOD OF RECORD.--HIGHEST WATER LEVEL, 46.60 FT(14.20 M) BELOW LAND-SURFACE DATUM,MAR 5, 1980; LOWEST WATER LEVEL,48.93 FT(14.91 M) BELOW LAND-SURFACE DATUM, JAN 25, 1983.

WATER LEVELS IN FEET BELOW LAND SURFACE DATUM, CLIMATIC YEARS APRIL 1981 T0 MARCH 1983

\begin{tabular}{|c|c|c|}
\hline & WATER & \\
\hline DATE & LEVEL & DATE \\
\hline
\end{tabular}

FEB 18, $1982 \quad 48.72 \quad$ JAN 25, $1983 \quad 48.93$ 


\section{GROUND-WATER LEVELS}

HARMON COUNTY

343020099422001. LOCAL NUMBER; LOCATION,--LAT 34 30'20", LONG 099 AQUIFER.--BLAINE GYPSUM.

PERIOD OF RECORD.--1976 TO CURRENT YEAR.

EXTREMES FOR PERIOD OF RECORD.--HIGHEST WATER LEVEL,12.83 FT(3.91 M) BELOW LAND-SURFACE DATUM,FEB 28, 1980; LOWEST WATER LEVEL, 14.10 FT(4.30 M) BELOW LAND-SURFACE DATUM, JAN 26, 1983.

WATER LEVELS IN FEET BELOW LAND SURFACE DATUM, CLIMATIC YEARS APRIL 1981 TO MARCH 1983
$01 \mathrm{~N}-24 \mathrm{~W}-34$ DCC 1 42'20', HYDROLOGIC UNIT 11130101.

$\begin{array}{cccc} & \text { WATER } & & \\ & & & \text { WATER } \\ \text { DATE } & \text { LEVEL } & \text { DATE } & \text { LEVEL } \\ \text { MAR 09, } 1982 & 13.86 & \text { JAN 26, 1983 } & 14.10\end{array}$

\section{GROUND-WATER LEVELS}

343419099462201. LOCAL NUMBER;

$01 \mathrm{~N}-25 \mathrm{~W}-12$ DAA 1 LOCATION,--LAT 34 34'19", LONG 099 46'22", HYDROLOGIC UNIT 11130101. AQUIFER.--BLAINE GYPSUM.

PERIOD OF RECORD.--1976 TO CURRENT YEAR.

EXTREMES FOR PERIOD OF RECORD.--HIGHEST WATER LEVEL, 33.92 FT(10.34 M) BELOW LAND-SURFACE DATUM, MAR 9, 1978; LOWEST WATER LEVEL,51.44 FT(15.68 M) BELOW LAND-SURFACE DATUM, JAN 26, 1983.

WATER LEVELS IN FEET BELOW LAND SURFACE DATUM, CLIMATIC YEARS APRIL 1981 TO MARCH 1983

\begin{tabular}{|c|c|c|}
\hline ATE & $\begin{array}{l}\text { WATER } \\
\text { LEVEL }\end{array}$ & DATE \\
\hline ATE & & DAIE \\
\hline
\end{tabular}

MAR 09, $1982 \quad 47.92 \quad$ JAN 26, $1983 \quad 51.44$


HARMON COUNTY -- CONTINUED

343042099474701. LOCAL NUMBER; LOCATION,--LAT 34 30'42", LONG 099 A UIFER.--BLAINE GYPSUM. PERIOD OF RECORD.--1976 TO CURRENT YEAR. EXTREMES FOR PERIOD OF RECORD.--HIGHEST WATER LEVEL, $16.60 \mathrm{FT}(5.06 \mathrm{M})$ BELOW LAND-SURFACE JAN 26, 1983.

WATER LEVELS IN FEET BELOW LAND SURFACE DATUM, CLIMATIC YEARS APRIL 1981 TO MARCH 1983
$01 \mathrm{~N}-25 \mathrm{~W}-35$ DDB 1.

47'47", HYDROLOGIC UNIT 11130101. 
343512099574701. LOCAL NUMBER;

LOCATION, --LAT $3435^{\prime} 12^{\prime \prime}$, LONG 099

AQUIFER.--BLAINE GYPSUM.

PERIOD OF RECORD.--1976 TO CURRENT YEAR.

EXTREMES FOR PERIOD OF RECORD.--HIGHEST WATER LEVEL, 44.84 FT(13.67 M) BELOW LAND-SURFACE DATUM, MAR 9, 1978; LOWEST WATER MAR $9,1982$.

WATER LEVELS IN FEET BELOW LAND SURFACE DATUM, CLIMATIC YEARS APRIL 1981 TO MARCH 1983
02N-26W-05 BCC 1.

57'47", HYDROLOGIC UNIT 11130101.
LET WATER LEVEL, $44.84 \mathrm{FT}(13.67 \mathrm{M})$ BELOW LAND-SURFACE
LEVEL, $61.98 \mathrm{FT}(18.89 \mathrm{M})$ BELOW LAND-SURFACE DATUM,

LEVEL, 61.98 FT $(18.89$ M) BELON LAND-SURFACE DATUM,

$\begin{array}{lccc}\text { DATE } & \begin{array}{l}\text { WATER } \\ \text { LEVEL }\end{array} & \text { DATE } & \text { WATER } \\ \text { LEVEL }\end{array}$

GROUND-WATER LEVELS

343855099544501. LOCAL NUMBER; LOCATION,--LAT 34 38'55", LONG 099 AQUIFER.--BLAINE GYPSUM.

PERIOD OF RECORD .--1976 TO CURRENT YEAR.

EXTREMES FOR PERIOD OF RECORD.--HIGHEST WATER LEVEL, 75.45 FT $(23.00 \mathrm{M})$ BELOW LAND-SURFACE DATUM,DEC 27, 1976; LOWEST WATER LEVEL, 89.78 FT(27.36 M) BELOW LAND-SURFACE DATUM, MAR 11, 1981.

WATER LEVELS IN FEET BELOW LAND SURFACE DATUM, CLIMATIC YEARS APRIL 1981 TO MARCH 1983
$02 \mathrm{~N}-26 \mathrm{~W}-15$ AAD 1

$54^{\prime} 45^{\prime \prime}$, HYDROLOGIC UNIT 11130101.

\begin{abstract}
WATER
\end{abstract}
DATE

LEVEL
88.46
MAR 09, 1982
JAN 26, $1983 \quad 89.10$

DATE

WATER

LEVEL 
HARMON COUNTY -- CONTINUED

343559099534901. LOCAL NUMBER; LOCATION,--LAT 34 35'59", LONG 099 AQUIFER.--BLAINE GYPSUM. PERIOD OF RECORD.--1976 TO CURRENT YEAR. EXTREMES FOR PERIOD OF RECORD.--HIGHEST WATER LEVEL, 33.17 FT(10.11 M) BELOW LAND-SURFACE DATUM,DEC 28, 1976; LOWEST WATER LEVEL,35.34 FT(10.77 M) BELOW LAND-SURFACE DATUM, MAR 11, 1981.

WATER LEVELS IN FEET BELOW LAND SURFACE DATUM, CLIMATIC YEARS APRIL 1981 TO MARCH 1983
DATE
WATER
LEVEL
DATE
WATER
LEVEL

MAR 09, $1982 \quad 34.55$

JAN 26, $1983 \quad 35.01$

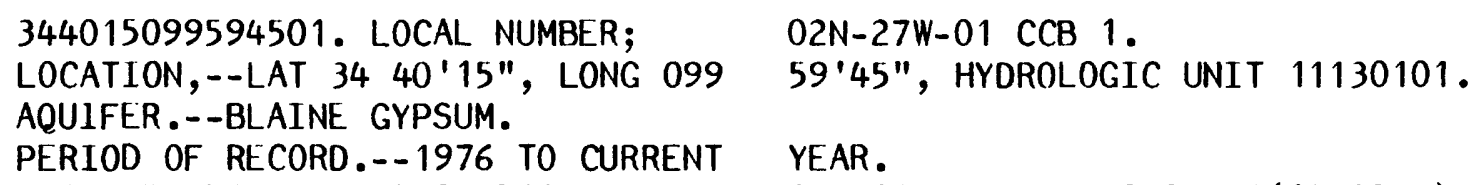


HARMON COUNTY -- CONTINUED

344220099441601. LOCAL NUMBER; 03N-24W-29 AAA 1. LOCATION,--LAT 34 42'20", LONG 099 44'16", HYDROLOGIC UNIT 11120202. AQUIFER.--BLAINE GYPSUM.

PERIOD OF RECORD.--1976 TO CURRENT YEAR. EXTREMES FOR PERIOD OF RECORD.--HIGHEST WATER LEVEL, $61.57 \mathrm{FT}(18.77 \mathrm{M})$ BELOW LAND-SURFACE DATUM,MAR 8, 1978; LOWEST WATER LEVEL,75.12 FT(22.90 M) BELOW LAND-SURFACE DATUM, MAR 11, 1981.

WATER LEVELS IN FEET BELOW LAND SURFACE DATUM, CLIMATIC YEARS APRIL 1981 TO MARCH 1983

$\begin{array}{lll} & \text { WATER } & \text { WATER } \\ \text { DATE } & \text { LEVEL } & \text { DATE }\end{array}$

MAR 09, $1982 \quad 73.67 \quad$ JAN 26, $1983 \quad 72.06$

GROUND-WATER LEVELS

344145099512501. LOCAL NUMBER; 03N-25W-32 BBB 1. LOCATION,--LAT 34 41'45", LONG 099 51'25", HYDROLOGIC UNIT 11130101. AQUIFER.--BLAINE GYPSUM. PERIOD OF RECORD.--1976 TO CURRENT YEAR. EXTREMES FOR PERIOD OF RECORD.--HIGHEST WATER LEVEL, 39.86 FT(12.15 M) BELOW LAND-SURFACE DATUM,MAR 9, 1978; LOWEST WATER LEVEL,65.52 FT(19.97 M) BELOW LAND-SURFACE DATUM, MAR 9, 1982.

WATER LEVELS IN FEET BELOW LAND SURFACE DATUM, CLIMATIC YEARS APRIL 1981 TO MARCH 1983

$\begin{array}{lll} & \text { WATER } & \\ \text { DATE } & \text { WEVEL } & \text { WATER } \\ \text { LATE } & \text { LEVEL }\end{array}$

MAR 09, $1982 \quad 65.52 \quad$ JAN 26, $1983 \quad 59.83$ 


\section{GROUND-WATER LEVELS}

HARMON COUNTY -- CONTINUED

344145099541501. LOCAL NUMBER;

$03 \mathrm{~N}-26 \mathrm{~W}-35$ BAB 1

LOCATION,--LAT 34 41'45", LONG 099

AQUIFER.--BLAINE GYPSUM.

PERIOD OF RECORD.--1976 TO CURRENT YEAR.

EXTREMES FOR PERIOD OF RECORD.--HIGHEST WATER LEVEL,51.14 FT(15.59 M) BELOW LAND-SURFACE

DATUM,MAR 8, 1978; LOWEST WATER LEVEL,59.40 FT(18.11 M) BELOW LAND-SURFACE DATUM, MAR 11, 1981.

WATER LEVELS IN FEET BELOW LAND SURFACE DATUM, CLIMATIC YEARS APRIL 1981 TO MARCH 1983

$\begin{array}{lll} & \text { WATER } & \text { WATER } \\ \text { DATE } & \text { LEVEL } & \text { DATE }\end{array}$

MAR 09, 1982 DRY JAN 26, $1983 \quad \mathrm{~N}$ 
363749099364301. LOCAL NUMBER;

LOCATION,--LAT 36 37'49", LONG 099 AQUIFER.--TERRACE DEPOSITS.

PERIOD OF RECORD.-- 1978 TO CURRENT YEAR.

EXTREMES FOR PERIOD OF RECORD.--HIGHEST WATER LEVEL,50.30 FT(15.33 M) BELOW LAND-SURFACE DATUM, JAN 20, 1982; LOWEST WATER LEVEL,51.93 FT(15.83 M) BELOW LAND-SURFACE DATUM, APR 17, 1979.

WATER LEVELS IN FEET BELOW LAND SURFACE DATUM, CLIMATIC YEARS APRIL 1981 TO MARCH 1983
$25 \mathrm{~N}-22 \mathrm{~W}-19$ BDD 1.

36'43", HYDROLOGIC UNIT 11100201.

\begin{tabular}{|c|c|c|}
\hline & WATER & \\
\hline DATE & LEVEL & DATE \\
\hline
\end{tabular}

JAN $20,198250.30 \quad$ JAN $11,1983 \quad 50.33$

GROUND-WATER LEVELS

363844099442301. LOCAL NUMBER; 25N-24W-13 BDC 1.

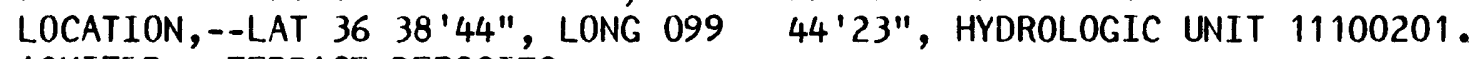

AQUIFER.--TERRACE DEPOSITS.

PERIOD OF RECORD.--1978 TO CURRENT YEAR.

EXTREMES FOR PERIOD OF RECORD.--HIGHEST WATER LEVEL, $35.75 \mathrm{FT}(10.90 \mathrm{M})$ BELOW LAND-SURFACE DATUM, JAN 11, 1983; LOWEST WATER LEVEL,42.62 FT(12.99 M) BELOW LAND-SURFACE DATUM, APR 17, 1979.

WATER LEVELS IN FEET BELOW LAND SURFACE DATUM, CLIMATIC YEARS APRIL 1981 TO MARCH 1983

$\begin{array}{lll} & \text { WATER } & \text { WATER } \\ \text { DATE } & \text { LEVEL } & \text { DATE }\end{array}$

JAN 20, $1982 \quad 36.49 \quad$ JAN 11, $1983 \quad 35.75$


HARPER COUNTY -- CONTINUED

363827099485001 . LOCAL NUMBER; 25N-24W-17 CCB 1. LOCATION,--LAT 36 38'27", LONG 099 48'50", HYDROLOGIC UNIT 11100203.

AQUIFER. - -ALLUVIUM.

PERIOD OF RECORD.--1977 TO CURRENT YEAR.

EXTREMES FOR PERIOD OF RECORD.--HIGHEST WATER LEVEL,5.80 FT(1.77 M) BELOW LAND-SURFACE DATUM,MAR 25, 1980; LOWEST WATER LEVEL,8.32 FT(2.54 M) BELOW LAND-SURFACE DATUM, JAN 12, 1983.

WATER LEVELS IN FEET BELOW LAND SURFACE DATUM, CLIMATIC YEARS APRIL 1981 TO MARCH 1983

\begin{tabular}{|c|c|}
\hline & WATER \\
\hline DATE & LEVEL \\
\hline
\end{tabular}

$\begin{array}{lllll}\text { JAN } 20,1982 & 7.61 & \text { JAN } 12, & 1983 & 8.32\end{array}$

\section{GROUND-WATER LEVELS}

363957099540201. LOCAL NUMBER; 25N-25W-09 BAA 1. LOCATION,--LAT 36 39'57", LONG 099 54'02", HYDROLOGIC UNIT 11100203. AQUIFER . - OGALLALA. PERIOD OF RECORD.--1977 TO CURRENT YEAR. EXTREMES FOR PERIOD OF RECORD.--HIGHEST WATER LEVEL, 15.39 FT(4.69 M) BELOW LAND-SURFACE DATUM,MAR 10, 1977; LOWEST WATER LEVEL,34.20 FT(10.42 M) BELOW LAND-SURFACE DATUM, FEB 3, 1981.

WATER LEVELS IN FEET BELOW LAND SURFACE DATUM, CLIMATIC YEARS APRIL 1981 T0 MARCH 1983
DATE
WATER
LEVEL
DATE
WATER

JAN 20, $1982 \quad 25.43$

JAN $12,1983 \quad 21.87$ 
HARPER COUNTY -- CONTINUED

363602099550601 . LOCAL NUMBER; 25N-25W-32 BDD 1.

LOCATION,--LAT 36 36'02", LONG 099 55'06", HYDROLOGIC UNIT 11100203.

AQUIFER.--OGALLALA.

PERIOD OF RECORD.--1981 TO CURRENT YEAR.

EXTREMES FOR PERIOD OF RECORD.--HIGHEST WATER LEVEL,74.32 FT(22.65 M) BELOW LAND-SURFACE DATUM, JAN 12, 1983; LOWEST WATER LEVEL,76.60 FT(23.35 M) BELOW LAND-SURFACE DATUM, MAR 19, 1981.

WATER LEVELS IN FEET BELOW LAND SURFACE DATUM, CLIMATIC YEARS APRIL 1981 TO MARCH 1983

$\begin{array}{cccc} & \text { WATER } & & \text { WATER } \\ \text { DATE } & \text { LEVEL } & \text { DATE } & \text { LEVEL } \\ & & & \\ \text { JAN 20, 1982 } & 74.70 & \text { JAN 12, 1983 } & 74.32\end{array}$

GROUND-WATER LEVELS

363714099564301. LOCAL NUMBER; 25N-26W-25 AAD 1.

LOCATION,--LAT 36 37'14", LONG 099 56'43", HYDROLOGIC UNIT 11100203.

AQUIFER .--OGALLALA.

PERIOD OF RECORD.--1977 TO CURRENT YEAR.

EXTREMES FOR PERIOD OF RECORD.--HIGHEST WATER LEVEL, $32.99 \mathrm{FT}(10.06 \mathrm{M})$ BELOW LAND-SURFACE

DATUM,MAR 10, 1977; LOWEST WATER LEVEL,35.79 FT(10.91 M) BELOW LAND-SURFACE DATUM, JAN 20, 1982.

WATER LEVELS IN FEET BELOW LAND SURFACE DATUM, CLIMATIC YEARS APRIL 1981 TO MARCH 1983

\begin{tabular}{|c|c|}
\hline & WATER \\
\hline DATE & LEVEL \\
\hline
\end{tabular}

JAN 20, $1982 \quad 35.79 \quad$ JAN $12,1983 \quad 34.30$ 
HARPER COUNTY -- CONTINUED

363538099575301. LOCAL NUMBER; LOCATION,--LAT 36 35'38', LONG 099 AQUIFER.--OGALLALA. PERIOD OF RECORD.--1980 TO CURRENT YEAR. EXTREMES FOR PERIOD OF RECORD.--HIGHEST WATER LEVEL, 54.26 FT(16.54 M) BELOW LAND-SURFACE DATUM,FEB 3, 1981; LOWEST WATER LEVEL,55.75 FT(16.99 M) BELOW LAND-SURFACE DATUM, JAN 20, 1982.

WATER LEVELS IN FEET BELOW LAND SURFACE DATUM, CLIMATIC YEARS APRIL 1981 TO MARCH 1983
$25 N-26 W-35$ DDD 1.

57 '53", HYDROLOGIC UNIT 11100203.

\section{WATER LEVELS IN}

$\begin{array}{lll} & \text { WATER } & \\ \text { DATE } & \text { LEVEL } & \text { WATER } \\ & & \text { LEVEL }\end{array}$

JAN 20, $1982 \quad 55.75 \quad$ JAN 12, $1983 \quad 55.40$

\section{GROUND-WATER LEVELS}

364118099444701. LOCAL NUMBER; 26N-24W-35 ADD 1. LOCATION,--LAT 36 41'18", LONG 099 44'47", HYDROLOGIC UNIT 11100201. AQUIFER.--TERRACE DEPOSITS. PERIOD OF RECORD.--1978 TO CURRENT YEAR. EXTREMES FOR PERIOD OF RECORD.--HIGHEST WATER LEVEL, 12.67 FT(3.86 M) BELOW LAND-SURFACE DATUM, APR 18, 1978; LOWEST WATER LEVEL,16.06 FT(4.90 M) BELOW LAND-SURFACE DATUM, JAN 11, 1983 .

WATER LEVELS IN FEET BELOW LAND SURFACE DATUM, CLIMATIC YEARS APRIL 1981 TO MARCH 1983

$\begin{array}{lll} & \text { WATER } & \\ \text { DATE } & \text { LEVEL } & \text { WATER } \\ & \text { DATE } & \text { LEVEL }\end{array}$

JAN 20, $1982 \quad 15.57 \quad$ JAN 11, $1983 \quad 16.06$




\section{GROUND-WATER LEVELS}

HARPER COUNTY -- CONTINUED

364336099542001. LOCAL NUMBER; 26N-25W-17 DDC 1.

LOCATION,--LAT 36 43'36", LONG 099 54'20", HYDROLOGIC UNIT 11100201.

AQUIFER.--ALLUVIUM.

PERIOD OF RECORD.--1977 TO CURRENT YEAR.

EXTREMES FOR PERIOD OF RECORD.--HIGHEST WATER LEVEL,7.38 FT (2.25 M) BELOW LAND-SURFACE DATUM, JAN 12, 1983; LOWEST WATER LEVEL,8.81 FT(2.69 M) BELOW LAND-SURFACE DATUM, JAN 20, 1982.

WATER LEVELS IN FEET BELOW LAND SURFACE DATUM, CLIMATIC YEARS APRIL 1981 TO MARCH 1983

\begin{tabular}{|c|c|c|}
\hline & $\begin{array}{l}\text { WATER } \\
\text { LEVEL }\end{array}$ & DATE \\
\hline
\end{tabular}

JAN 20, $1982 \quad 8.81 \quad$ JAN $12,1983 \quad 7.38$

GROUND-WATER LEVELS

364102099554601. LOCAL NUMBER; 26N-25W-31 DDA 1. LOCATION,--LAT 36 41'02", LONG 099 55'46", HYDROLOGIC UNIT 11100201. AQUIFER.--TERRACE DEPOSITS.

PERIOD OF RECORD.--1978 TO CURRENT YEAR.

EXTREMES FOR PERIOD OF RECORD.--HIGHEST WATER LEVEL, $13.73 \mathrm{FT}(4.18 \mathrm{M})$ BELOW LAND-SURFACE DATUM,JAN 12, 1983; LOWEST WATER LEVEL,17.38 FT(5.30 M) BELOW LAND-SURFACE DATUM, JAN 20, 1982.

WATER LEVELS IN FEET BELOW LAND SURFACE DATUM, CLIMATIC YEARS APRIL 1981 TO MARCH 1983

$\begin{array}{lll} & \text { WATER } & \\ \text { DATE } & \text { LEVEL } & \text { WATER } \\ & & \text { LEVEL }\end{array}$

$\begin{array}{llll}\text { JAN } 20,1982 & 17.38 & \text { JAN } 12,1983 & 13.73\end{array}$ 
GROUND-WATER LEVELS

HARPER COUNTY - - CONTINUED

364255099581301. LOCAL NUMBER;

$26 \mathrm{~N}-26 \mathrm{~W}-23 \mathrm{DBC} 1$.

LOCATION,--LAT 3642 '55", LONG 099

AQUIFER.--OGALLALA.

PERIOD OF RECORD.--1980 TO CURRENT YEAR.

EXTREMES FOR PERIOD OF RECORD.--HIGHEST WATER LEVEL, 55.16 FT(16.81 M) BELOW LAND-SURFACE DATUM,MAR 25, 1980; LOWEST WATER LEVEL,55.72 FT(16.98 M) BELOW LAND-SURFACE DATUM, JAN 12, 1983.

WATER LEVELS IN FEET BELOW LAND SURFACE DATUM, CLIMATIC YEARS APRIL 1981 TO MARCH 1983

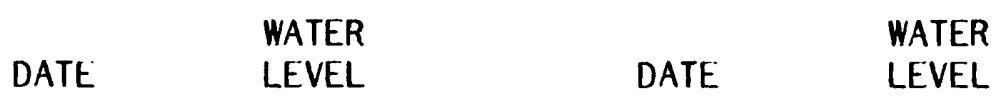

JAN 20, $1982 \quad 55.59 \quad$ JAN 12, $1983 \quad 55.72$

GROUND-WATER LEVELS

364825099494701. LOCAL NUMBER;

$27 \mathrm{~N}-24 \mathrm{~W}-19$ BDB 1.

LOCATION,--LAT 36 48'25", LONG 099 49'47", HYDROLOGIC UNIT 11050001.

AQUIFER .--ALLUVIUM.

PERIOD OF RECORD.--1978 TO CURRENT YEAR.

EXTREMES FOR PERIOD OF RECORD.--HIGHEST WATER LEVEL,22.30 FT(6.80 M) BELOW LAND-SURFACE DATUM, JAN 20, 1982; LOWEST WATER LEVEL,25.12 FT (7.66 M) BELOW LAND-SURFACE DATUM, MAR 26, 1980 .

WATER LEVELS IN FEET BELOW LAND SURFACE DATUM, CLIMATIC YEARS APRIL 1981 TO MARCH 1983

$\begin{array}{lll} & \text { WATER } & \text { WATER } \\ \text { DATE } & \text { LEVEL } & \text { DATE }\end{array}$

JAN 20, $1982 \quad 22.30 \quad$ JAN 12, $1983 \quad 22.79$ 


\section{GROUND-WATER LEVELS}

HARPER COUNTY -- CONTINUED

364829099553201. LOCAL NUMBER; 27N-25W-19 AAA 1. LOCATION,--LAT 36 48'29", LONG 099 55'32", HYDROLOGIC UNIT 11100201. AQUIFER. --ALLUVIUM.

PERIOD OF RECORD.--1977 TO CURRENT YEAR.

EXTREMES FOR PERIOD OF RECORD.--HIGHEST WATER LEVEL,6.40 FT(1.95 M) BELOW LAND-SURFACE DATUM, JAN 20, 1982; LOWEST WATER LEVEL,7.90 FT(2.41 M) BELOW LAND-SURFACE DATUM, MAR 8, 1978.

WATER LEVELS IN FEET BELOW LAND SURFACE DATUM, CLIMATIC YEARS APRIL 1981 TO MARCH 1983

$\begin{array}{lll} & \text { WATER } & \text { WATER } \\ \text { DATE } & \text { LEVEL } & \text { DATE }\end{array}$

JAN 20, $1982 \quad 6.40 \quad$ JAN 12, $1983 \quad 7.34$

GROUND-WATER LEVELS

364953099564001. LOCAL NUMBER; 27N-26W-12 DAD 1.

LOCATION,--LAT 36 49'53", LONG 099 56'40", HYDROLOGIC UNIT 11100201.

AQUIFER .--OGALLALA.

PERIOD OF RECORD.--1980 TO CURRENT YEAR.

EXTREMES FOR PERIOD OF RECORD.--HIGHEST WATER LEVEL, $28.05 \mathrm{FT}(8.55 \mathrm{M})$ BELOW LAND-SURFACE DATUM, JAN 12, 1983; LOWEST WATER LEVEL,29.78 FT(9.08 M) BELOW LAND-SURFACE DATUM, MAR 26, 1980.

WATER LEVELS IN FEET BELOW LAND SURFACE DATUM, CLIMATIC YEARS APRIL 1981 TO MARCH 1983

\begin{tabular}{|c|c|c|}
\hline & WATER & \\
\hline DATE & LEVEL & DATE \\
\hline
\end{tabular}

JAN 20, $1982 \quad 28.33 \quad$ JAN 12, $1983 \quad 28.05$ 


\section{GROUND-WATER LEVELS}

\section{HARPER COUNTY -- CONTINUED}

364923099570301 . LOCAL NUMBER; LOCATION,--LAT 36 49'23", LONG 099 AQUIFER.--ALLUVIUM.

PERIOD OF RECORD.--1978 TO CURRENT

EXTREMES FOR PERIOD OF RECORD.--HIGHEST WATER LEVEL, 24.32 FT (7.41 M) BELOW LAND-SURFACE

DATUM, JAN 12, 1983; LOWEST WATER LEVEL,26.12 FT(7.96 M) BELOW LAND-SURFACE DATUM, JAN 17, 1979. WATER LEVELS IN FEET BELOW LAND SURFACE DATUM, CLIMATIC YEARS APRIL 1981 TO MARCH 1983

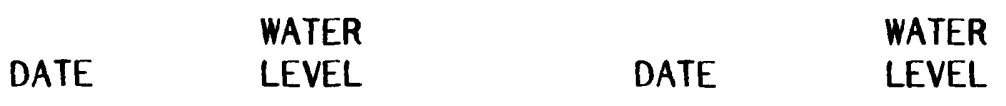

JAN 20, $1982 \quad 24.63 \quad$ JAN 12, $1983 \quad 24.32$
$27 \mathrm{~N}-26 \mathrm{~W}-13$ ABD 1.

57'03", HYDROLOGIC UNIT 11100201.

YEAR 


\section{GROUND-WATER LEVELS}

HASKELL COUNTY

350700095142801. LOCAL NUMBER;

$08 \mathrm{~N}-20 \mathrm{E}-31 \mathrm{CCD} 1$. LOCATION, --LAT $3507^{\prime} 00^{\prime \prime}$, LONG 095 14'28", HYDROLOGIC UNIT 11110104. AQUIFER.--MCALESTER FORMATION. PERIOD OF RECORD.--1976 TO CURRENT YEAR.

EXTREMES FOR PERIOD OF RECORD.--HIGHEST WATER LEVEL,.89 FT(.27 M) BELOW LAND-SURFACE DATUM,MAR 14, 1978; LOWEST WATER LEVEL,3.49 FT(1.06 M) BELOW LAND-SURFACE DATUM, JAN 5, 1977.

WATER LEVELS IN FEET BELOW LAND SURFACE DATUM, CLIMATIC YEARS APRIL 1981 TO MARCH 1983

$\begin{array}{lll} & \text { WATER } & \text { WATER } \\ \text { DATE } & \text { LEVEL } & \text { DATE }\end{array}$
MAR 22, 1982
1.52
MAR 22, 1983
W 


\section{GROUND-WATER LEVELS}

HUGHES COUNTY

344929096053501. LOCAL NUMBER; $04 \mathrm{~N}-11 \mathrm{E}-13$ AAA 1.

LOCATION,--LAT 34 49'29", LONG 096 05'35", HYDROLOGIC UNIT 11140103.

AQUIFER .--THURMAN FORMATION.

PERIOD OF RECORD.--1976 TO CURRENT YEAR.

EXTREMES FOR PERIOD OF RECORD.--HIGHEST WATER LEVEL, 3.89 FT(1.19 M) BELOW LAND-SURFACE. DATUM,MAR 21, 1983; LOWEST WATER LEVEL,55.50 FT(16.92 M) BELOW LAND-SURFACE DATUM, MAR 14, 1978.

WATER LEVELS IN FEET BELOW LAND SURFACE DATUM, CLIMATIC YEARS APRIL 1981 TO MARCH 1983

$\begin{array}{lll} & \text { WATER } & \text { WATER } \\ \text { DATE } & \text { LEVEL } & \text { DATE }\end{array}$

MAR 22, $1982 \quad 6.49 \quad$ MAR $21,1983 \quad 3.89$


GROUND-WATER LEVELS

JACKSON COUNTY

343913099313901. LOCAL NUMBER; 02N-22W-08 DDA 1.

LOCATION,--LAT 34 39'13", LONG 099 31'39", HYDROLOGIC UNIT 11120202.

AQUIFER.--BLAINE GYPSUM.

PERIOD OF RECORD.--1976 TO CURRENT YEAR.

EXTREMES FOR PERIOD OF RECORD.--HIGHEST WATER LEVEL, 30.20 FT(9.20 M) BELOW LAND-SURFACE DATUM,MAR 9, 1978; LOWEST WATER LEVEL,39.59 FT(12.07 M) BELOW LAND-SURFACE DATUM, JAN 26, 1983.

WATER LEVELS IN FEET BELOW LAND SURFACE DATUM, CLIMATIC YEARS APRIL 1981 TO MARCH 1983

\begin{tabular}{|c|c|c|c|}
\hline DATE & $\begin{array}{l}\text { WATER } \\
\text { LEVEL }\end{array}$ & DATE & $\begin{array}{l}\text { WATER } \\
\text { LEVEL }\end{array}$ \\
\hline 09,1982 & 36.85 & JAN 26, 1983 & 39.59 \\
\hline
\end{tabular}

GROUND-WATER LEVELS

343945099342501. LOCAL NUMBER; 02N-23W-12 BBD 1.

LOCATION,--LAT 34 39'45", LONG 099 34'25", HYDROLOGIC UNIT 11120202.

AQUIFER.--BLAINE GYPSUM.

PERIOD OF RECORD.--1976 TO CURRENT YEAR.

EXTREMES FOR PERIOD OF RECORD .--HIGHEST WATER LEVEL,27.53 FT(8.39 M) BELOW LAND-SURFACE DATUM,MAR 9, 1978; LOWEST WATER LEVEL,37.02 FT(11.28 M) BELOW LAND-SURFACE DATUM, MAR 11, 1981.

WATER LEVELS IN FEET BELOW LAND SURFACE DATUM, CLIMATIC YEARS APRIL 1981 TO MARCH 1983

$\begin{array}{lll} & \text { WATER } & \text { WATER } \\ \text { DATE } & \text { LEVEL } & \text { DATE }\end{array}$

MAR 09, $1982 \quad 29.70 \quad$ JAN 26, $1983 \quad 0$ 


\section{GROUND-WATER LEVELS}

\section{JACKSON COUNTY -- CONTINUED}

343810099365701. LOCAL NUMBER; LOCATION,--LAT 34 38'10", LONG 099 AQUIFER.--BLAINE GYPSUM. PERIOD OF RECORD.--1976 TO CURRENT YEAR. EXTREMES FOR PERIOD OF RECORD.--HIGHEST WATER LEVEL,22.68 FT(6.91 M) BELOW LAND-SURFACE DATUM,MAR 9, 1982; LOWEST WATER LEVEL,26.85 FT(8.18 M) BELOW LAND-SURFACE DATUM, JAN 26, 1983.

WATER LEVELS IN FEET BELOW LAND SURFACE DATUM, CLIMATIC YEARS APRIL 1981 TO MARCH 1983

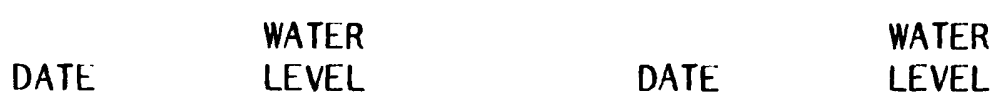

MAR 09, $1982 \quad 22.68 \quad$ JAN 26, $1983 \quad 26.85$

\section{GROUND-WATER LEVELS}

344114099323701. LOCAL NUMBER;

$03 \mathrm{~N}-22 \mathrm{~W}-32$ CBB 2.

LOCATION,--LAT 34 41'14", LONG 099 32'37", HYDROLOGIC UNIT 11120202.

AQUIFER.--BLAINE GYPSUM.

PERIOD OF RECORD..-1976 TO CURRENT YEAR.

EXTREMES FOR PERIOD OF RECORD.--HIGHEST WATER LEVEL, 37.00 FT(11.28 M) BELOW LAND-SURFACE DATUM, MAR 9, 1978; LOWEST WATER LEVEL,46.53 FT(14.18 M) BELOW LAND-SURFACE DATUM, JAN 26, 1983.

WATER LEVELS IN FEET BELOW LAND SURFACE DATUM, CLIMATIC YEARS APRIL 1981 TO MARCH 1983

$\begin{array}{lll} & \text { WATER } & \text { WATER } \\ \text { DATE } & \text { LEVEL } & \text { DATE }\end{array}$
MAR 09, 1982
43.82
JAN 26, $1983 \quad 46.53$ 
JACKSON COUNTY -- CONTINUED

344220099355501. LOCAL NUMBER; 03N-23W-27 AAD 1. LOCATION,--LAT 34 42'20", LONG 099 35'55", HYDROLOGIC UNIT 11120202.

AQUIFER.--BLAINE GYPSUM.

PERIOD OF RECORD.--1976 TO CURRENT YEAR.

EXTREMES FOR PERIOD OF RECORD.--HIGHEST WATER LEVEL, 34.94 FT(10.65 M) BELOW LAND-SURFACE DATUM,MAR 9, 1978; LOWEST WATER LEVEL,47.38 FT(14.44 M) BELOW LAND-SURFACE DATUM, MAR 11, 1981.

WATER LEVELS IN FEET BELOW LAND SURFACE DATUM, CLIMATIC YEARS APRIL 1981 TO MARCH 1983

$\begin{array}{lll} & \text { WATER } & \\ \text { DATE } & \text { WEVEL } & \text { DATER } \\ \text { LEVEL }\end{array}$

MAR 09, $1982 \quad 41.38 \quad$ JAN 26, $1983 \quad 41.06$

GROUND-WATER LEVELS

344032099390201. LOCAL NUMBER; 03N-23W-30 DAA 1.

LOCATION,--LAT 34 40'32", LONG 099 39'02", HYDROLOGIC UNIT 11120202.

AQUIFER.--BLAINE GYPSUM.

PERIOD OF RECORD .-1976 TO CURRENT YEAR.

EXTREMES FOR PERIOD OF RECORD.--HIGHEST WATER LEVEL, 19.75 FT(6.02 M) BELOW LAND-SURFACE DATUM,MAR 9, 1978; LOWEST WATER LEVEL,25.42 FT(7.75 M) BELOW LAND-SURFACE DATUM, MAR 9, 1982.

WATER LEVELS IN FEET BELOW LAND SURFACE DATUM, CLIMATIC YEARS APRIL 1981 TO MARCH 1983

\begin{tabular}{|c|c|c|}
\hline & WATER & \\
\hline DATE & LEVEL & DATE \\
\hline
\end{tabular}

MAR 09, $1982 \quad 25.42$ JAN 26, $1983 \quad 24.51$ 
344637099152801. LOCAL NUMBER; LOCATION,--LAT 34 46'37", LONG 099 AQUIFER.--TERRACE DEPOSITS.

PERIOD OF RECORD .-- 1979 TO CURRENT YEAR.

EXTREMES FOR PERIOD OF RECORD.--HIGHEST WATER LEVEL, 68.90 FT(21.00 M) BELOW LAND-SURFACE DATUM,MAR 4, 1980; LOWEST WATER LEVEL,72.82 FT(22.20 M) BELOW LAND-SURFACE DATUM, AUG 29, 1979.

WATER LEVELS IN FEET BELOW LAND SURFACE DATUM, CLIMATIC YEARS APRIL 1981 TO MARCH 1983
$04 \mathrm{~N}-20 \mathrm{~W}-36$ BDC 1.

15 '28", HYDROLOGIC UNIT 11120303.

\begin{tabular}{|c|c|c|}
\hline & WATER & \\
\hline DATE & LEVEL & DATE \\
\hline
\end{tabular}

MAR 11, 1982

P JAN 26, 1983

Z

GROUND-WATER LEVELS

343020099404501. LOCAL NUMBER; 015-24W-02 ABB 1. LOCATION,--LAT 34 30'20", LONG 099 40'45", HYDROLOGIC UNIT 11130101. AQUIFER.--BLAINE GYPSUM. PERIOD OF RECORD.--1976 TO CURRENT YEAR. EXTREMES FOR PERIOD OF RECORD.--HIGHEST WATER LEVEL, 30.56 FT(9.31 M) BELOW LAND-SURFACE DATUM, MAR 13, 1979; LOWEST WATER LEVEL, 32.61 FT(9.94 M) BELOW LAND-SURFACE DATUM, JAN 26, 1983.

WATER LEVELS IN FEET BELOW LAND SURFACE DATUM, CLIMATIC YEARS APRIL 1981 TO MARCH 1983

$\begin{array}{cccc}\text { DATE } & \text { WATER } & & \text { WATER } \\ \text { LEVEL } & \text { DATE } & \text { LEVEL } \\ \text { MAR 09, 1982 } & 32.22 & \text { JAN 26, 1983 } & 32.61\end{array}$




\section{GROUND-WATER LEVELS}

\section{JACKSON COUNTY -- CONTINUED}

\section{LOCAL NUMBER;}

LOCATION,--LAT 34 27'44", LONG 099

01S-25W-13 CCC 1. AQUIFER.--BLAINE GYPSUM. PERIOD OF RECORD.--1976 TO CURRENT YEAR. EXTREMES FOR PERIOD OF RECORD.--HIGHEST WATER LEVEL, 75.20 FT(22.92 M) BELOW LAND-SURFACE DATUM,MAR 9, 1978; LOWEST WATER LEVEL,78.87 FT(24.04 M) BELOW LAND-SURFACE DATUM, DEC 28, 1976.

WATER LEVELS IN FEET BELOW LAND SURFACE DATUM, CLIMATIC YEARS APRIL 1981 TO MARCH 1983

\begin{tabular}{lccc}
\multicolumn{1}{c}{} & WATER & & WATER \\
DATE & LEVEL & DATE & LEVEL \\
L 09, 1982 & 76.78 & JAN 26, 1983 & 77.14
\end{tabular}


GROUND-WATER LEVELS

\section{JEFFERSON COUNTY}

340827097593301. LOCAL NUMBER;

05S-08W-12 BAD 1.

LOCATION,--LAT 34 08'27", LONG 097 59'33", HYDROLOGIC UNIT 11130208.

AQUIFER.--ALLUVIUM.

PERIOD OF RECORD. - 1979 TO CURRENT YEAR.

EXTREMES FOR PERIOD OF RECORD.--HIGHEST WATER LEVEL, $24.30 \mathrm{FT}(7.41 \mathrm{M})$ BELOW LAND-SURFACE DATUM,FEB 20, 1979; LOWEST WATER LEVEL,25.52 FT(7.78 M) BELOW LAND-SURFACE DATUM, MAR 25, 1982 .

WATER LEVELS IN FEET BELOW LAND SURFACE DATUM, CLIMATIC YEARS APRIL 1981 TO MARCH 1983

$\begin{array}{lccr}\text { DATE } & \text { WATER } & & \text { WATER } \\ \text { LEVEL } & \text { DATE } & \text { LEVEL } \\ \text { R 25, 1982 } & 25.45 & \text { FEB 08, 1983 } & 24.39\end{array}$




\section{GROUND-WATER LEVELS}

JOHNSTON COUNTY

342415096475101. LOCAL NUMBER;

LOCATION,--LAT 34 24'15", LONG 096

AQUIFER.--ARBUCKLE FORMATION.

PERIOD OF RECORD.-- 1980 TO CURRENT YEAR.

EXTREMES FOR PERIOD OF RECORD.--HIGHEST WATER LEVEL,43.50 FT(13.26 M) BELOW LAND-SURFACE DATUM,FEB 28, 1980; LOWEST WATER LEVEL,57.06 FT(17.39 M) BELOW LAND-SURFACE DATUM, MAR 2, 1981.

WATER LEVELS IN FEET BELOW LAND SURFACE DATUM, CLIMATIC YEARS APRIL 1981 T0 MARCH 1983

02S-05E-08 ABA 1.

47'51", HYDROLOGIC UNIT 11130201.
MAR 10,1982
Z
MAR 11, 1983
Z

WATER

DATE

WATER

LEVEL

LEVEL

GROUND-WATER LEVELS

342236096262701. LOCAL NUMBER; 02S-08E-14 CCC 1.

LOCATION,--LAT $34^{2} 22^{\prime} 36^{\prime \prime}$, LONG 096 26'27", HYDROLOGIC UNIT 11140104.

AQUIFER.--ALLUVIUM.

PERIOD OF RECORD.--1979 TO CURRENT YEAR.

EXTREMES FOR PERIOD OF RECORD.--HIGHEST WATER LEVEL, $5.75 \mathrm{FT}(1.75 \mathrm{M})$ BELOW LAND-SURFACE DATUM,MAR 9, 1982; LOWEST WATER LEVEL, 8.39 FT(2.56 M) BELOW LAND-SURFACE DATUM, FEB 28, 1980.

WATER LEVELS IN FEET BELOW LAND SURFACE DATUM, CLIMATIC YEARS APRIL 1981 TO MARCH 1983

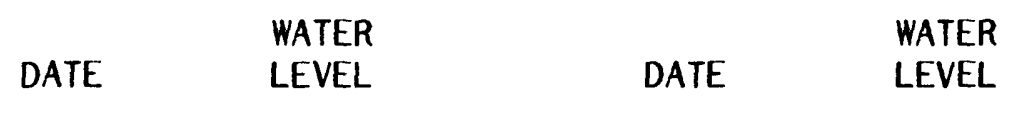

MAR 09, $1982 \quad 5.75 \quad$ MAR 08, $1983 \quad 5.90$ 
GROUND-WATER LEVELS

JOHNSTON COUNTY -- CONTINUED

341243096534501. LOCAL NUMBER; 04S-04E-06 BBC 1. LOCATION,--LAT 34 12'43", LONG 096 53'45", HYDROLOGIC UNIT 11130304. AQUIFER.--ALLUVIUM.

PERIOD OF RECORD.--1977 TO CURRENT YEAR. EXTREMES FOR PERIOD OF RECORD.--HIGHEST WATER LEVEL, $25.69 \mathrm{FT}(7.83 \mathrm{M})$ BELOW LAND-SURFACE DATUM, JAN 4, 1977; LOWEST WATER LEVEL, 31.05 FT(9.46 M) BELOW LAND-SURFACE DATUM, FEB 28, 1980.

WATER LEVELS IN FEET BELOW LAND SURFACE DATUM, CLIMATIC YEARS APRIL 1981 TO MARCH 1983

$\begin{array}{lccc}\text { DATE } & \begin{array}{l}\text { WATER } \\ \text { LEVEL }\end{array} & \text { DATE } & \text { WATER } \\ \text { LEVEL }\end{array}$


GROUND-WATER LEVELS

\section{KAY COUNTY}

364001097200001 . LOCAL NUMBER;

25N-01W-08 BBA 1.

LOCATION,--LAT 36 40'01", LONG 097 20'00", HYDROLOGIC UNIT 11060004.

AQUIFER .- -ALLUVIUM.

PERIOD OF RECORD.--1976 TO CURRENT YEAR.

EXTREMES FOR PERIOD OF RECORD.--HIGHEST WATER LEVEL,9.79 FT(2.98 M) BELOW LAND-SURFACE DATUM,FEB 1, 1980; LOWEST WATER LEVEL,11.79 FT(3.59 M) BELOW LAND-SURFACE DATUM, MAR 28, 1979.

WATER LEVELS IN FEET BELOW LAND SURFACE DATUM, CLIMATIC YEARS APRIL 1981 TO MARCH 1983

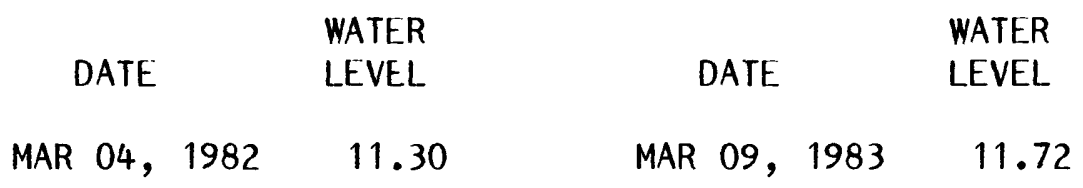

GROUND-WATER LEVELS

364222097025601. LOCAL NUMBER; 26N-02E-25 BBC 1. LOCATION,--LAT 36 42'22", LONG 097 02'56", HYDROLOGIC UNIT 11060004. AQUIFER.--TERRACE DEPOSITS. PERIOD OF RECORD.--1975 TO CURRENT YEAR. EXTREMES FOR PERIOD OF RECORD.--HIGHEST WATER LEVEL, 12.18 FT(3.71 M) BELOW LAND-SURFACE DATUM, MAR 6, 1975; LOWEST WATER LEVEL,29.90 FT(9.11 M) BELOW LAND-SURFACE DATUM, JAN 23, 1981 .

WATER LEVELS IN FEET BELOW LAND SURFACE DATUM, CLIMATIC YEARS APRIL 1981 TO MARCH 1983

\begin{tabular}{|c|c|c|}
\hline$I T$ & WATER & DATE: \\
\hline & & \\
\hline
\end{tabular}

MAR 04, $1982 \quad 0 \quad$ MAR 09, $1983 \quad 24.50$ 


\section{GROUND-WATER LEVELS}

\section{KAY COUNTY -- CONTINUED}

365824097272901. LOCAL NUMBER; LOCATION,--LAT $3658^{\prime} 24^{\prime \prime}$, LONG 097 AQUIFER.--TERRACE DEPOSITS. PERIOD OF RECORD. - 1975 TO CURRENT YEAR.

EXTREMES FOR PERIOD OF RECORD.--HIGHEST WATER LEVEL, $31.04 \mathrm{FT}(9.46 \mathrm{M})$ BELOW LAND-SURFACE DATUM, MAR 5, 1975; LOWEST WATER LEVEL, $34.00 \mathrm{FT}(10.36 \mathrm{M})$ BELOW LAND-SURFACE DATUM, JAN 23, 1981.

WATER LEVELS IN FEET BELOW LAND SURFACE DATUM, CLIMATIC YEARS APRIL 1981 TO MARCH 1983
29N-02W-19 CDC 1.

27'29", HYDROLOGIC UNIT 11060005.
WATER

DATE

LEVEL

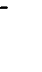




\section{GROUND-WATER LEVELS}

\section{KINGF ISHER COUNTY}

355302097561701. LOCAL NUMBER;

LOCATION,--LAT $3553^{\prime} 02^{\prime \prime}$, LONG 097

AQUIFER.--TERRACE DEPOSITS.

PERIOD OF RECORD.--1981 TO CURRENT

EXTREMES FOR PERIOD OF RECORD.--HIGHEST WATER LEVEL, $13.49 \mathrm{FT}(4.11 \mathrm{M})$ BELOW LAND-SURFACE DATUM,FEB 20, 1981; LOWEST WATER LEVEL, 13.49 FT(4.11 M) BELOW LAND-SURFACE DATUM, FEB 20, 1981.

WATER LEVELS IN FEET BELOW LAND SURFACE DATUM, CLIMATIC YEARS APRIL 1981 TO MARCH 1983

DATE

WATER

LEVEL

Z MAR 16,1983

WATER

LEVEL

MAR 16, 1982
$16 \mathrm{~N}-07 \mathrm{~W}-10$ BBB 1.

$56^{\prime} 17$ ', HYDROLOGIC UNIT 11050002.

$\begin{array}{ll} & \text { WATER } \\ \text { DATE } & \text { LEVEL }\end{array}$

GROUND-WATER LEVELS

355724097430301. LOCAL NUMBER; 17N-05W-10 DCC 1. LOCATION,--LAT 35 57'24", LONG 097 43'03", HYDROLOGIC UNIT 11050002. AQUIFER.--TERRACE DEPOSITS. PERIOD OF RECORD . - 1975 TO CURRENT YEAR. EXTREMES FOR PERIOD OF RECORD.--HIGHEST WATER LEVEL, 16.69 FT(5.09 M) BELOW LAND-SURFACE DATUM,JAN 12, 1976; LOWEST WATER LEVEL,20.04 FT(6.11 M) BELOW LAND-SURFACE DATUM, FEB 21, 1979.

WATER LEVELS IN FEET BELOW LAND SURFACE DATUM, CLIMATIC YEARS APRIL 1981 TO MARCH 1983

\begin{tabular}{|c|c|c|}
\hline & WATER & \\
\hline DATE & LEVEL & DATE \\
\hline
\end{tabular}

MAR 17, $1982 \quad 18.34 \quad$ MAR 16, $1983 \quad 18.60$


GROUND-WATER LEVELS

KINGF ISHER COUNTY -- CONTINUED

355632097464801. LOCAL NUMBER;

$17 \mathrm{~N}-05 \mathrm{~W}-18$ CCC 1 .

LOCATION,--LAT 35 56'32", LONG 097 46'48", HYDROLOGIC UNIT 11050002.

AQUIFER.--TERRACE DEPOSITS.

PERIOD OF RECORD.--1975 TO CURRENT YEAR.

EXTREMES FOR PERIOD OF RECORD.--HIGHEST WATER LEVEL,24.65 FT(7.51 M) BELOW LAND-SURFACE DATUM, JAN 20, 1975; LOWEST WATER LEVEL, $36.65 \mathrm{FT}(11.17 \mathrm{M})$ BELOW LAND-SURFACE DATUM, MAR 17, 1982 .

WATER LEVELS IN FEET BELOW LAND SURFACE DATUM, CLIMATIC YEARS APRIL 1981 TO MARCH 1983

\begin{tabular}{|c|c|}
\hline & WATER \\
\hline DATE & LEVEL \\
\hline
\end{tabular}

MAR $17,1982 \quad 36.65 \quad$ MAR $16,1983 \quad 26.59$

GROUND-WATER LEVELS

355447097423901. LOCAL NUMBER; 17N-05W-27 DDD 1.

LOCATION,--LAT 35 54'47", LONG 097 42'39", HYDROLOGIC UNIT 11050002.

AQUIFER.--TERRACE DEPOSITS.

PERIOD OF RECORD.--1975 TO CURRENT YEAR.

EXTREMES FOR PERIOD OF RECORD.--HIGHEST WATER LEVEL,4.11 FT(1.25 M) BELOW LAND-SURFACE DATUM, JAN 20, 1975; LOWEST WATER LEVEL,8.50 FT(2.59 M) BELOW LAND-SURFACE DATUM, JAN 3, 1978.

WATER LEVELS IN FEET BELOW LAND SURFACE DATUM, CLIMATIC YEARS APRIL 1981 TO MARCH 1983

$\begin{array}{lll} & \text { WATER } & \text { WATER } \\ \text { DATE } & \text { LEVEL } & \text { DATE }\end{array}$

MAR 17, 1982

$0 \quad$ MAR 16,1983

$N$ 
KINGFISHER COUNTY -- CONTINUED

355902097480001. LOCAL NUMBER; 17N-06W-02 AAA 1. LOCATION,--LAT 35 59'02", LONG 097 48'00", HYDROLOGIC UNIT 11050002.

AQUIFER.--TERRACE DEPOSITS.

PERIOD OF RECORD.--1975 TO CURRENT YEAR.

EXTREMES FOR PERIOD OF RECORD.--HIGHEST WATER LEVEL, 15.84 FT(4.83 M) BELOW LAND-SURFACE DATUM, JAN 12, 1976; LOWEST WATER LEVEL,20.20 FT(6.16 M) BELOW LAND-SURFACE DATUM, FEB 21, 1979.

WATER LEVELS IN FEET BELOW LAND SURFACE DATUM, CLIMATIC YEARS APRIL 1981 TO MARCH 1983

\begin{tabular}{|c|c|}
\hline & WATER \\
\hline DATE & LEVEL \\
\hline
\end{tabular}

MAR $17,1982 \quad 17.80 \quad$ MAR $16,1983 \quad 17.09$

GROUND-WATER LEVELS

355724097480001. LOCAL NUMBER; 17N-06W-11 DDD 1. LOCATION,--LAT $3557^{\prime} 24^{\prime \prime}$, LONG $09748^{\circ} 00^{\prime \prime}$, HYDROLOGIC UNIT 11050002.

AQUIFER.--TERRACE DEPOSITS.

PERIOD OF RECORD.--1975 TO CURRENT YEAR.

EXTREMES FOR PERIOD OF RECORD.--HIGHEST WATER LEVEL, $33.01 \mathrm{FT}(10.06 \mathrm{M})$ BELOW LAND-SURFACE DATUM, JAN 12, 1976; LOWEST WATER LEVEL, $35.05 \mathrm{FT}(10.68 \mathrm{M})$ BELOW LAND-SURFACE DATUM, JAN 3, 1978.

WATER LEVELS IN FEET BELOW LAND SURFACE DATUM, CLIMATIC YEARS APRIL 1981 TO MARCH 1983

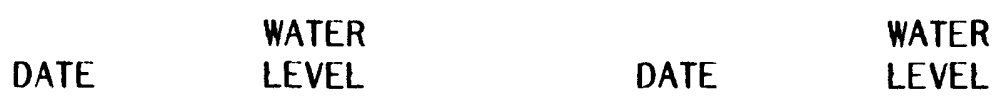

MAR 17, $1982 \quad 33.82$ MAR 16, $1983 \quad 33.12$ 
KINGFISHER COUNTY -- CONTINUED

355810097465601. LOCAL NUMBER; 17N-06W-12 AAA 1. LOCATION,--LAT 35 58'10", LONG 097 46'56", HYDROLOGIC UNIT 11050002. AQUIFER.--TERRACE DEPOSITS. PERIOD OF RECORD.--1975 TO CURRENT YEAR. EXTREMES FOR PERIOD OF RECORD.--HIGHEST WATER LEVEL, 34.08 FT (10.39 M) BELOW LAND-SURFACE DATUM, JAN 12, 1976; LOWEST WATER LEVEL, $37.98 \mathrm{FT}(11.58 \mathrm{M})$ BELOW LAND-SURFACE DATUM, JAN 21, 1975.

WATER LEVELS IN FEET BELOW LAND SURFACE DATUM, CLIMATIC YEARS APRIL 1981 TO MARCH 1983

$\begin{array}{cccc}\text { DATE } & \begin{array}{c}\text { WATER } \\ \text { LEVEL }\end{array} & \text { DATE } & \text { WATER } \\ \text { LEVEL }\end{array}$

\section{GROUND-WATER LEVELS}

355658097531201. LOCAL NUMBER;

$17 \mathrm{~N}-06 \mathrm{~W}-18 \mathrm{BCC} \uparrow$.

LOCATION,--LAT $3556^{\prime} 58^{\prime \prime}$, LONG 097 53'12", HYDROLOGIC UNIT 11050002.

AQUIFER.--TERRACE DEPOSITS.

PERIOD OF RECORD.--1975 TO CURRENT YEAR.

EXTREMES FOR PERIOD OF RECORD.--HIGHEST WATER LEVEL, .18 FT(.05 M) BELOW LAND-SURFACE DATUM, JAN 21, 1975; LOWEST WATER LEVEL,5.93 FT( $1.81 \mathrm{M})$ BELOW LAND-SURFACE DATUM, JAN 3, 1978.

WATER LEVELS IN FEET BELOW LAND SURFACE DATUM, CLIMATIC YEARS APRIL 1981 TO MARCH 1983

$\begin{array}{lll} & \text { WATER } & \text { WATER } \\ \text { DATE } & \text { LEVEL } & \text { DATE }\end{array}$

MAR 17, $1982 \quad 4.29 \quad$ MAR $16,1983 \quad 4.00$ 
GROUND-WATER LEVELS

KINGFISHER COUNTY -- CONTINUED

355829097544001. LOCAL NUMBER; 17N-07W-02 DBD 1.

LOCATION,--LAT 35 58'29", LONG 097 54'40", HYDROLOGIC UNIT 11050002.

AQUIFER.--TERRACE DEPOSITS.

PERIOD OF RECORD.--1975 TO CURRENT YEAR.

EXTREMES FOR PERIOD OF RECORD.--HIGHEST WATER LEVEL, 2.70 FT (.82 M) BELOW LAND-SURFACE DATUM,JAN 21, 1975; LOWEST WATER LEVEL, $10.80 \mathrm{FT}(3.29 \mathrm{M})$ BELOW LAND-SURFACE DATUM, JAN 3, 1978.

WATER LEVELS IN FEET BELOW LAND SURFACE DATUM, CLIMATIC YEARS APRIL 1981 TO MARCH 1983

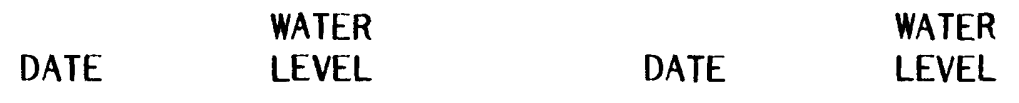

MAR 17, $198210.13 \quad$ MAR 16, $1983 \quad 7.96$

GROUND-WATER LEVELS

355743097544001. LOCAL NUMBER; 17N-07W-11 DBA 1.

LOCATION,--LAT 35 57'43", LONG 097 54'40", HYDROLOGIC UNIT 11050002.

AQUIFER.--TERRACE DEPOSITS.

PERIOD OF RECORD. - -1975 TO CURRENT YEAR.

EXTREMES FOR PERIOD OF RECORD.--HIGHEST WATER LEVEL,9.94 FT(3.03 M) BELOW LAND-SURFACE DATUM, JAN 21, 1975; LOWEST WATER LEVEL, 16.92 FT(5.16 M) BELOW LAND-SURFACE DATUM, MAR 19, 1979.

WATER LEVELS IN FEET BELOW LAND SURFACE DATUM, CLIMATIC YEARS APRIL 1981 TO MARCH 1983

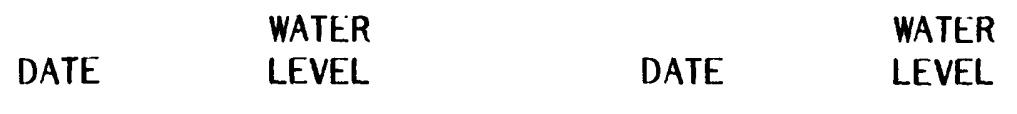

$\begin{array}{llll}\text { MAR } 17,1982 & 15.63 & \text { MAR 16, } 1983 \quad 14.90\end{array}$ 
GROUND-WATER LEVELS

KINGF ISHER COUNTY -- CONTINUED

355724097544001. LOCAL NUMBER; 17N-07W-11 DCD 2.

LOCATION,--LAT 35 57'24", LONG 097 54'40", HYDROLOGIC UNIT 11050002.

AQUIFER.--TERRACE DEPOSITS.

PERIOD OF RECORD.--1975 TO CURRENT YEAR.

EXTREMES FOR PERIOD OF RECORD.--HIGHEST WATER LEVEL, 10.80 FT(3.29 M) BELOW LAND-SURFACE DATUM, MAR 17, 1982; LOWEST WATER LEVEL, 13.06 FT(3.98 M) BELOW LAND-SURFACE DATUM, JAN 3, 1978.

WATER LEVELS IN FEET BELOW LAND SURFACE DATUM, CLIMATIC YEARS APRIL 1981 TO MARCH 1983

\begin{tabular}{|c|c|}
\hline & WATER \\
\hline DATE & LEVEL \\
\hline
\end{tabular}

MAR 17, $1982 \quad 10.80 \quad$ MAR $16,1983 \quad 11.47$

GROUND-WATER LEVELS

355743097532001. LOCAL NUMBER; 17N-07W-12 DAA 1. LOCATION,--LAT 35 57'43", LONG 097 53'20", HYDROLOGIC UNIT 11050002.

AQUIFER.--TERRACE DEPOSITS.

PERIOD OF RECORD.--1975 TO CURRENT YEAR.

EXTREMES FOR PERIOD OF RECORD.--HIGHEST WATER LEVEL,5.52 FT(1.68 M) BELOW LAND-SURFACE DATUM,JAN 21, 1975; LOWEST WATER LEVEL, 13.50 FT(4.11 M) BELOW LAND-SURFACE DATUM, JAN 3, 1978.

WATER LEVELS IN FEET BELOW LAND SURFACE DATUM, CLIMATIC YEARS APRIL 1981 TO MARCH 1983

\begin{tabular}{|c|c|c|}
\hline & WATER & \\
\hline DATE & LEVEL & DATE \\
\hline
\end{tabular}

MAR $17,1982 \quad 11.26 \quad$ MAR $16,1983 \quad 9.87$


KINGF ISHER COUNTY -- CONTINUED

355823097020901. LOCAL NUMBER;

$17 \mathrm{~N}-08 \mathrm{~W}-03$ DCA 1.

LOCATION,--LAT 35 58'23", LONG 097 02'09", HYDROLOGIC UNIT 11050002.

AQUIFER.--TERRACE DEPOSITS.

PERIOD OF RECORD.--1981 TO CURRENT YEAR.

EXTREMES FOR PERIOD OF RECORD.---HIGHEST WATER LEVEL, $1.23 \mathrm{FT}(.37 \mathrm{M})$ BELOW LAND-SURFACE DATUM,MAR 17, 1982; LOWEST WATER LEVEL,7.30 FT(2.23 M) BELOW LAND-SURFACE DATUM, JAN $28,1981$.

WATER LEVELS IN FEET BELOW LAND SURFACE DATUM, CLIMATIC YEARS APRIL 1981 TO MARCH 1983

\begin{tabular}{|c|c|c|}
\hline DATE & $\begin{array}{l}\text { WATER } \\
\text { LEVEL }\end{array}$ & DATE \\
\hline
\end{tabular}

MAR $17,1982 \quad 1.23 \quad$ MAR 16, $1983 \quad$ Z

GROUND-WATER LEVELS

360342097495201. LOCAL NUMBER; 18N-06W-03 CBD 1. LOCATION,--LAT 36 03'42", LONG 097 49'52", HYDROLOGIC UNIT 11050002. AQUIFER.--TERRACE DEPOSITS. PERIOD OF RECORD.--1975 TO CURRENT YEAR. LXTREMES FOR PERIOD OF RECORD.--HIGHEST WATER LEVEL,6.32 FT(1.93 M) BELOW LAND-SURFACE DATUM, MAR 16, 1982; LOWEST WATER LEVEL,9.28 FT(2.83 M) BELOW LAND-SURFACE DATUM, FEB 21, 1979.

WATER LEVELS IN FEET BELOW LAND SURFACE DATUM, CLIMATIC YEARS APRIL 1981 TO MARCH 1983

\begin{tabular}{|c|c|c|}
\hline & WATER & \\
\hline ATE & LEVEL & DATE \\
\hline
\end{tabular}

MAR 16, $1982 \quad 6.32 \quad$ MAR $15,1983 \quad 8.25$ 
GROUND-WATER LEVELS

KINGFISHER COUNTY -- CONTINUED

360316097522401. LOCAL NUMBER; 18N-06W-07 AAC 1.

LOCATION,--LAT 36 03'16", LONG 097 52'24", HYDROLOGIC UNIT 11050002.

AQUIFER.--TERRACE DEPOSITS.

PERIOD OF RECORD.--1975 TO CURRENT YEAR.

EXTREMES FOR PERIOD OF RECORD.--HIGHEST WATER LEVEL, 3.66 FT(1.12 M) BELOW LAND-SURFACE DATUM, JAN 21, 1975; LOWEST WATER LEVEL,8.37 FT(2.55 M) BELOW LAND-SURFACE DATUM, FEB 21, 1979.

WATER LEVELS IN FEET BELOW LAND SURFACE DATUM, CLIMATIC YEARS APRIL 1981 TO MARCH 1983

\begin{tabular}{|c|c|}
\hline & WATER \\
\hline DATE & LEVEL \\
\hline
\end{tabular}

MAR 16, $1982 \quad 6.40 \quad$ MAR $16,1983 \quad 5.95$

GROUND-WATER LEVELS

360237097490401. LOCAL NUMBER;

$18 \mathrm{~N}-06 \mathrm{~W}-10$ DDD 1

LOCATION,--LAT 36 02'37", LONG 097 49'04", HYDROLOGIC UNIT 11050002.

AQUIFER.--TERRACE DEPOSITS.

PERIOD OF RECORD.--1975 TO CURRENT YEAR.

EXTREMES FOR PERIOD OF RECORD.--HIGHEST WATER LEVEL,2.88 FT(.88 M) BELOW LAND-SURFACE DATUM, JAN 21, 1975; LOWEST WATER LEVEL,9.70 FT(2.96 M) BELOW LAND-SURFACE DATUM, FEB 21, 1979.

WATER LEVELS IN FEET BELOW LAND SURFACE DATUM, CLIMATIC YEARS APRIL 1981 TO MARCH 1983

\begin{tabular}{|c|c|c|}
\hline & WATER & \\
\hline DAIE. & LEVEL & DATE \\
\hline
\end{tabular}

MAR $16,1982 \quad 4.42 \quad$ MAR $15,1983 \quad 3.64$ 


\section{GROUND-WATER LEVELS}

KINGF ISHER COUNTY -- CONTINUED

360145097505601. LOCAL NUMBER; 18N-06W-16 CCD 1. LOCATION,--LAT 3601 '45", LONG 097 50'56", HYDROLOGIC UNIT 11050002. AQUIFER.--TERRACE DEPOSITS. PERIOD OF RECORD.--1975 TO CURRENT YEAR. EXTREMES FOR PERIOD OF RECORD.--HIGHEST WATER LEVEL, 19.05 FT $(5.81 \mathrm{M})$ BELOW LAND-SURFACE DATUM,JAN 13, 1976; LOWEST WATER LEVEL,26.02 FT(7.93 M) BELOW LAND-SURFACE DATUM, JAN 21, 1975.

WATER LEVELS IN FEET BELOW LAND SURFACE DATUM, CLIMATIC YEARS APRIL 1981 TO MARCH 1983

\begin{tabular}{|c|c|}
\hline & WATER \\
\hline DATE & LEVEL \\
\hline
\end{tabular}

MAR $16,1982 \quad 21.49 \quad$ MAR $15,1983 \quad 19.64$

GROUND-WATER LEVELS

360230097512001. LOCAL NUMBER; 18N-06W-17 AAB 1. LOCATION,--LAT 36 02'30", LONG 097 51'20", HYDROLOGIC UNIT 11050002. AQUIFER.--TERRACE DEPOSITS. PERIOD OF RECORD.--1975 TO CURRENT YEAR.

EXTREMES FOR PERIOD OF RECORD.--HIGHEST WATER LEVEL,27.97 FT(8.53 M) BELOW LAND-SURFACE DATUM, MAR 15, 1983; LOWEST WATER LEVEL,36.59 FT(11.15 M) BELOW LAND-SURFACE DATUM, JAN 21, 1975.

WATER LEVELS IN FEET BELOW LAND SURFACE DATUM, CLIMATIC YEARS APRIL 1981 TO MARCH 1983

$\begin{array}{lll} & \text { WATER } & \\ \text { DATE } & \text { LEVEL } & \text { WATER } \\ \text { LATE } & \text { LEVEL }\end{array}$

MAR $16,1982 \quad 31.36 \quad$ MAR $15,1983 \quad 27.97$ 
GROUND-WATER LEVELJ

KINGFISHER COUNTY -- CONTINUED

360059097520001 . LOCAL NUMBER; 18N-06W-20 CCA 1. LOCATION,--LAT 36 00'59", LONG 097 52'00", HYDROLOGIC UNIT 11050002.

AQUIFER.--TERRACE DEPOSITS.

PERIOD OF RECORD.--1975 TO CURRENT YEAR.

EXTREMES FOR PERIOD OF RECORD.--HIGHEST WATER LEVEL, $8.00 \mathrm{FT}(2.44 \mathrm{M})$ BELOW LAND-SURFACE DATUM, JAN 13, 1976; LOWEST WATER LEVEL, $14.70 \mathrm{FT}(4.48 \mathrm{M})$ BELOW LAND-SURFACE DATUM, JAN 27, 1981.

WATER LEVELS IN FEET BELOW LAND SURFACE DATUM, CLIMATIC YEARS APRIL 1981 TO MARCH 1983

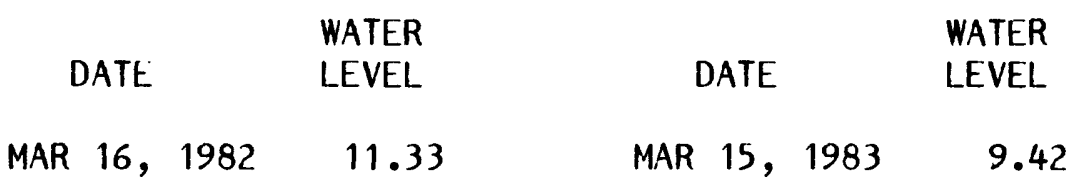

\section{GROUND-WATER LEVELS}

355954097524801. LOCAL NUMBER; 18N-06W-31 BAA 1. LOCATION,--LAT 35 59'54", LONG 097 52'48", HYDROLOGIC UNIT 11050002. AQUIFER.--TERRACE DEPOSITS. PERIOD OF RECORD.--1975 TO CURRENT YEAR. EXTREMES FOR PERIOD OF RECORD.--HIGHEST WATER LEVEL, 11.68 FT(3.56 M) BELOW LAND-SURFACE DATUM, JAN 13, 1976; LOWEST WATER LEVEL, $16.56 \mathrm{FT}(5.05 \mathrm{M})$ BELOW LAND-SURFACE DATUM, MAR 19, 1979.

WATER LEVELS IN FEET BELOW LAND SURFACE DATUM, CLIMATIC YEARS APRIL 1981 TO MARCH 1983

$\begin{array}{lll} & \text { WATER } & \\ \text { DATE } & \text { LEVEL } & \text { WATER } \\ & \text { DATE } & \text { LEVEL }\end{array}$

$\begin{array}{lllll}\text { MAR } 17,1982 & 14.29 & \text { MAR } 16,1983 & 12.44\end{array}$ 
GROUND-WATER LEVELS

KINGFISHER COUNTY -- CONTINUED

355908097521601. LOCAL NUMBER;

$18 \mathrm{~N}-06 \mathrm{~W}-31$ DDD 2.

LOCATION,--LAT 35 59'08", LONG 097 52'16", HYDROLOGIC UNIT 11050002.

AQUIFER.--TERRACE DEPOSITS.

PERIOD OF RECORD.--197S TO CURRENT YEAR.

EXTREMES FOR PERIOD OF RECORD.--HIGHEST WATER LEVEL,2.74 FT(.84 M) BELOW LAND-SURFACE DATUM, JAN 21, 1975; LOWEST WATER LEVEL,4.70 FT(1.43 M) BELOW LAND-SURFACE DATUM, JAN 4, 1978.

WATER LEVELS IN FEET BELOW LAND SURFACE DATUM, CLIMATIC YEARS APRIL 1981 TO MARCH 1983

$\begin{array}{lll} & \text { WATER } & \text { WATER } \\ \text { DATE } & \text { LEVEL } & \text { DATE }\end{array}$

MAR 17, $1982 \quad 3.03 \quad$ MAR $16,1983 \quad 2.84$

GROUND-WATER LEVELS

355954097514401. LOCAL NUMBER; 18N-06W-32 BAA 1.

LOCATION,--LAT 35 59'54", LONG 097 51'44", HYDROLOGIC UNIT 11050002.

AQUIFER.--TERRACE DEPOSITS.

PERIOD OF RECORD.--1975 TO CURRENT YEAR.

EXTREMES FOR PERIOD OF RECORD.--HIGHEST WATER LEVEL, $8.85 \mathrm{FT}(2.70 \mathrm{M})$ BELOW LAND-SURFACE DATUM, JAN 13, 1976; LOWEST WATER LEVEL, 13.55 FT(4.13 M) BELOW LAND-SURFACE DATUM, MAR 19, 1979.

WATER LEVELS IN FEET BELOW LAND SURFACE DATUM, CLIMATIC YEARS APRIL 1981 TO MARCH 1983

$\begin{array}{lll} & \text { WATER } & \text { WATER } \\ \text { DATE } & \text { LEVEL } & \text { DATE }\end{array}$
MAR 17, 1982
10.75
MAR 16, 1983
9.04 
GROUND-WATER LEVELS

KINGF ISHER COUNTY -- CONTINUED

360052097564801. LOCAL NUMBER; 18N-07W-21 DCD 1.

LOCATION,--LAT 3600 '52", LONG 097 56'48", HYDROLOGIC UNIT 11050002.

AQUIFER.--TERRACE DEPOSITS.

PERIOD OF RECORD.-- 1975 TO CURRENT YEAR.

EXTREMES FOR PERIOD OF RECORD.---HIGHEST WATER LEVEL, $20.92 \mathrm{FT}(6.38 \mathrm{M})$ BELOW LAND-SURFACE DATUM,JAN 13, 1976; LOWEST WATER LEVEL,27.00 FT(8.23 M) BELOW LAND-SURFACE DATUM, JAN 20, 1978.

WATER LEVELS IN FEET BELOW LAND SURFACE DATUM, CLIMATIC YEARS APRIL 1981 TO MARCH 1983

$\begin{array}{ccccc}\text { DATE } & \begin{array}{c}\text { WATER } \\ \text { LEVEL }\end{array} & \text { DATE } & \begin{array}{c}\text { WATER } \\ \text { LEVEL }\end{array} \\ \text { MAR 16, 1982 } & & \text { Z } & \text { MAR 16, 1983 } & 23.19\end{array}$

GROUND-WATER LEVELS

$360322098023301 . \quad L O C A L$ NUMBER; 18N-08W-10 BAB 1. LOCATION,--LAT 36 03'22", LONG 098 02'33", HYDROLOGIC UNIT 11050002.

AQUIFER.--TERRACE DEPOSITS.

PERIOD OF RECORD.--1975 TO CURRENT YEAR.

EXTREMES FOR PERIOD OF RECORD.--HIGHEST WATER LEVEL,7.13 FT(2.17 M) BELOW LAND-SURFACE DATUM,FEB 21, 1980; LOWEST WATER LEVEL, 14.85 FT(4.53 M) BELOW LAND-SURFACE DATUM, MAR 19, 1979.

WATER LEVELS IN FEET BELOW LAND SURFACE DATUM, CLIMATIC YEARS APRIL 1981 TO MARCH 1983

$\begin{array}{lll}\text { WATER } & & \text { WATER } \\ \text { DATE } & \text { LEVEL } & \text { DATE } \\ \text { LEVEL }\end{array}$

MAR 16, $1982 \quad 12.90 \quad$ MAR 15, $1983 \quad 9.93$ 
GROUND-WATER LEVELS

KINGFISHER COUNTY -- CONTINUED

360230098004101. LOCAL NUMBER;

$18 \mathrm{~N}-08 \mathrm{~W}-13$ BBB 1.

LOCATION,--LAT 36 02'30", LONG 098 00'41", HYDROLOGIC UNIT 11050002.

AQUIFER.--TERRACE DEPOSITS.

PERIOD OF RECORD.--1975 TO CURRENT YEAR.

EXTREMES FOR PERIOD OF RECORD.--HIGHEST WATER LEVEL, $1.19 \mathrm{FT}(.36 \mathrm{M})$ BELOW LAND-SURFACE DATUM,FEB 21, 1980; LOWEST WATER LEVEL, 11.20 FT(3.41 M) BELOW LAND-SURFACE DATUM, DEC 9, 1976.

WATER LEVELS IN FEET BELOW LAND SURFACE DATUM, CLIMATIC YEARS APRIL 1981 TO MARCH 1983

\begin{tabular}{crrrr}
\multicolumn{2}{c}{ WATER } & & \multicolumn{1}{c}{ WATER } \\
DATE & LEVEL & DATE & \multicolumn{1}{c}{ LEVEL } \\
MAR 16, 1982 & 2.87 & MAR 15, 1983 & 1.79
\end{tabular}

GROUND-WATER LEVELS

360651097553601. LOCAL NUMBER; 19N-07W-22 AAB 1. LOCATION,--LAT 36 06'51", LONG 097 55'36", HYDROLOGIC UNIT 11050002.

AQUIFER.--TERRACE DEPOSITS.

PERIOD OF RECORD.--1975 TO CURRENT YEAR.

EXTREMES FOR PERIOD OF RECORD.--HIGHEST WATER LEVEL, 13.18 FT(4.02 M) BELOW LAND-SURFACE DATUM, JAN 22, 1975; LOWEST WATER LEVEL,17.18 FT(5.24 M) BELOW LAND-SURFACE DATUM, DEC 9, 1976.

WATER LEVELS IN FEET BELOW LAND SURFACE DATUM, CLIMATIC YEARS APRIL 1981 TO MARCH 1983

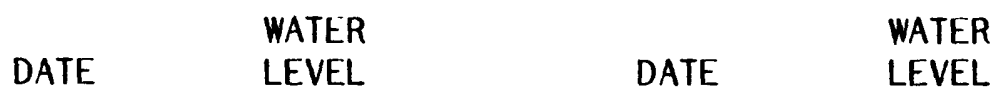

MAR $16,1982 \quad 0 \quad$ MAR 15, $1983 \quad \mathrm{~N}$ 


\section{GROUND-WATER LEVELS}

KINGF ISHER COUNTY -- CONTINUED

360651097560001. LOCAL NUMBER;

$19 N-07 W-22$ BAA 2.

LOCATION,--LAT 36 06'51", LONG 097

AQUIFER.--TERRACE DEPOSITS.

PERIOD OF RECORD.-- 1975 TO CURRENT YEAR.

EXTREMES FOR PERIOD OF RECORD.--HIGHEST WATER LEVEL,4.20 FT(1.28 M) BELOW LAND-SURFACE DATUM,JAN 22, 1975; LOWEST WATER LEVEL, 10.98 FT(3.35 M) BELOW LAND-SURFACE DATUM, MAR 20, 1979.

WATER LEVELS IN FEET BELOW LAND SURFACE DATUM, CLIMATIC YEARS APRIL 1981 TO MARCH 1983

$\begin{array}{lll} & \text { WATER } & \text { WATER } \\ \text { DATE } & \text { LEVEL } & \text { DATE }\end{array}$

MAR 16, $1982 \quad 7.40 \quad$ MAR $15,1983 \quad 6.68$

GROUND-WATER LEVELS

361045097590501. LOCAL NUMBER; 19N-07W-30 DCD 1.

LOCATION,--LAT 36 10'45", LONG 097 59'05", HYDROLOGIC UNIT 11050002.

AQUIFER.--TERRACE DEPOSITS.

PERIOD OF RECORD.--1976 TO CURRENT YEAR.

EXTREMES FOR PERIOD OF RECORD.--HIGHEST WATER LEVEL, $29.95 \mathrm{FT}(9.13 \mathrm{M})$ BELOW LAND-SURFACE DATUM, JAN 13, 1976; LOWEST WATER LEVEL,37.88 FT(11.55 M) BELOW LAND-SURFACE DATUM, FEB 21, 1980.

WATER LEVELS IN FEET BELOW LAND SURFACE DATUM, CLIMATIC YEARS APRIL 1981 TO MARCH 1983

$\begin{array}{lll} & \text { WATER } & \text { WATER } \\ \text { DATE } & \text { LEVEL } & \text { DATE }\end{array}$

MAR $16,1982 \quad 36.90 \quad$ MAR $15,1983 \quad 32.53$ 
360922098021401. LOCAL NUMBER; LOCATION,--LAT 36 09'22", LONG 098 AQUIFER.--TERRACE DEPOSITS. PERIOD OF RECORD.--1975 TO CURRENT YEAR. EXTREMES FOR PERIOD OF RECORD.--HIGHEST WATER LEVEL, $1.90 \mathrm{FT}(.58 \mathrm{M})$ BELOW LAND-SURFACE MAR 20, 1979.

WATER LEVELS IN FEET BELOW LAND SURFACE DATUM, CLIMATIC YEARS APRIL 1981 TO MARCH 1983
19N-08W-03 AAA 1.

02 '14", HYDROLOGIC UNIT 11050002.

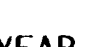
MAR 16, 1982
5.87

WATER

LEVEL

\begin{tabular}{crrrr}
\multicolumn{2}{c}{} & WATER & & \multicolumn{1}{c}{ WATER } \\
DATE & LEVEL & DATE & LEVEL \\
MAR 16, 1982 & 5.87 & MAR 16, 1983 & 2.08
\end{tabular}

\section{GROUND-WATER LEVELS}

360657098041701. LOCAL NUMBER; LOCATION, --LAT 3606 '57", LONG 098 AQUIFER.--TERRACE DEPOSITS. PERIOD OF RECORD.--1975 TO CURRENT EXTREMES FOR PERIOD OF RECORD.--HIGHEST WATER LEVEL,4.89 FT(1.49 M) BELOW LAND-SURFACE DATUM, JAN 13, 1976; LOWEST WATER LEVEL,11.27 FT(3.44 M) BELOW LAND-SURFACE DATUM, MAR 16, 1982.

WATER LEVELS IN FEET BELOW LAND SURFACE DATUM, CLIMATIC YEARS APRIL 1981 TO MARCH 1983

19N-08W-17 DCD 1

$04^{\prime} 17^{\prime \prime}$, HYDROLOGIC UNIT 11050002.

YEAR.
MAR 16, $1982 \quad 11.27$
MAR 15, 1983

WATER

LEVEL 


\section{GROUND-WATER LEVELS}

KINGF ISHER COUNTY -- CONTINUED

360526098020101. LOCAL NUMBER;

19N-08W-27 DAC 1

LOCATION,--LAT 36 05'26", LONG 098 02'01", HYDROLOGIC UNIT 11050002.

AQUIFER.--TERRACE DEPOSITS.

PERIOD OF RECORD .--1975 TO CURRENT YEAR.

EXTREMES FOR PERIOD OF RECORD .--HIGHEST WATER LEVEL,4.63 FT(1.41 M) BELOW LAND-SURFACE DATUM,JAN 13, 1976; LOWEST WATER LEVEL,77.84 FT(23.73 M) BELOW LAND-SURFACE DATUM, MAR 20, 1979.

WATER LEVELS IN FEET BELOW LAND SURFACE DATUM, CLIMATIC YEARS APRIL 1981 TO MARCH 1983

\begin{tabular}{|c|c|}
\hline & WATER \\
\hline DATE & LEVEL \\
\hline
\end{tabular}

MAR 16, $1982 \quad 10.64 \quad$ MAR 15, $1983 \quad 6.66$

GROUND-WATER LEVELS

360750098084901. LOCAL NUMBER; 19N-09W-10 CDD 1.

LOCATION,--LAT 36 07'50", LONG 098 08'49", HYDROLOGIC UNIT 11050002.

AQUIFER.--TERRACE DEPOSITS.

PERIOD OF RECORD.--1975 TO CURRENT YEAR.

EXTREMES FOR PERIOD OF RECORD.--HIGHEST WATER LEVEL, $3.29 \mathrm{FT}(1.00 \mathrm{M})$ BELOW LAND-SURFACE DATUM, JAN 22, 1975; LOWEST WATER LEVEL,9.25 FT(2.82 M) BELOW LAND-SURFACE DATUM, MAR 20, 1979.

WATER LEVELS IN FEET BELOW LAND SURFACE DATUM, CLIMATIC YEARS APRIL 1981 TO MARCH 1983

\begin{tabular}{|c|c|}
\hline & WATER \\
\hline DATE & LEVEL \\
\hline
\end{tabular}

MAR 16, $1982 \quad 7.82 \quad$ MAR 15, $1983 \quad 4.97$




\section{GROUND-WATER LEVELS}

KIOWA COUNTY

344005098591001. LOCAL NUMBER;

02N-17W-04 DDC 1.

LOCATION, --LAT 34 40'05", LONG 098

59'10", HYDROLOGIC UNIT 11120303.

AQUIFER.--TERRACE DEPOSITS.

PERIOD OF RECORD.--1978 TO CURRENT YEAR.

EXTREMES FOR PERIOD OF RECORD.--HIGHEST WATER LEVEL, 11.00 FT(3.35 M) BELOW LAND-SURFACE DATUM,FEB 09, 1983; LOWEST WATER LEVEL, 14.15 FT(4.31 M) BELOW LAND-SURFACE DATUM, MAR 27, 1979.

WATER LEVELS IN FEET BELOW LAND SURFACE DATUM, CLIMATIC YEARS APRIL 1981 TO MARCH 1983

\begin{tabular}{|c|c|c|}
\hline & WATER & \\
\hline DATE & LEVEL & DATE \\
\hline
\end{tabular}

MAR $11,1982 \quad 12.80 \quad$ FEB 09, $1983 \quad 11.00$

GROUND-WATER LEVELS

343913098592601. LOCAL NUMBER; 02N-17W-09 DCC 1. LOCATION,--LAT 34 39'13", LONG 098 59'26", HYDROLOGIC UNIT 11120303. AQUIFER.--TERRACE DEPOSITS.

PERIOD OF RECORD.--1979 TO CURRENT YEAR.

EXTREMES FOR PERIOD OF RECORD.--HIGHEST WATER LEVEL, 12.19 FT(3.72 M) BELOW LAND-SURFACE DATUM,FEB 09, 1983; LOWEST WATER LEVEL,20.95 FT(6.39 M) BELOW LAND-SURFACE DATUM, MAR 27, 1979.

WATER LEVELS IN FEET BELOW LAND SURFACE DATUM, CLIMATIC YEARS APRIL 1981 TO MARCH 1983

\begin{tabular}{|c|c|}
\hline & $\begin{array}{l}\text { WATER } \\
\text { LEVEL }\end{array}$ \\
\hline
\end{tabular}

$\begin{array}{llll}\text { MAR } 11,1982 & 18.12 & \text { FEB 09, } 1983 \quad 12.19\end{array}$ 
KIOWA COUNTY -- CONTINUED

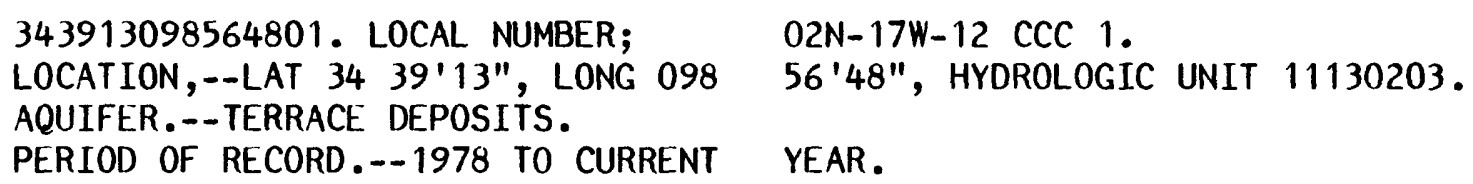

WATER LEVELS IN FEET BELOW LAND SURFACE DATUM, CLIMATIC YEARS APRIL 1981 TO MARCH 1983

\begin{tabular}{|c|c|c|}
\hline & WATER & \\
\hline DATE & LEVEL & DATE \\
\hline
\end{tabular}

MAR $11 ; 1982 \quad 20.27 \quad$ FEB 09, $1983 \quad 19.75$

GROUND-WATER LEVELS

363903099580001. LOCAL NUMBER; 02N-17W-15 AAA 1.

LOCATION,--LAT 36 39'03", LONG 099 58'00", HYDROLOGIC UNIT 11120303.

AQUIFER.--TERRACE DEPOSITS.

PERIOD OF RECORD.--1979 TO CURRENT YEAR.

EXTREMES FOR PERIOD OF RECORD.--HIGHEST WATER LEVEL,20.18 FT(6.15 M) BELOW LAND-SURFACE DATUM,MAR 4, 1980; LOWEST WATER LEVEL,21.70 FT(6.61 M) BELOW LAND-SURFACE DATUM, FEB 9, 1983.

WATER LEVELS IN FEET BELOW LAND SURFACE DATUM, CLIMATIC YEARS APRIL 1981 TO MARCH 1983

$\begin{array}{lll} & \text { WATER } & \text { WATER } \\ \text { DATE } & \text { LEVEL } & \text { DATE }\end{array}$

MAR 11, $1982 \quad 21.63 \quad$ FEB 09, $1983 \quad 21.70$ 


\section{GROUND-WATER LEVELS}

KIOWA COUNTY -- CONTINUED

343820098592501. LOCAL NUMBER; 02N-17W-16 DCC 1. LOCATION,--LAT 34 38'20", LONG 098 59'25", HYDROLOGIC UNIT 11120303.

AQUIFER.--TERRACE DEPOSITS.

PERIOD OF RECORD.--1974 TO CURRENT YEAR.

EXTREMES FOR PERIOD OF RECORD.--HIGHEST WATER LEVEL, $25.19 \mathrm{FT}(7.68 \mathrm{M})$ BELOW LAND-SURFACE DATUM,MAR 4, 1980; LOWEST WATER LEVEL, $27.62 \mathrm{FT}(8.42 \mathrm{M})$ BELOW LAND-SURFACE DATUM, MAR 11, 1982.

WATER LEVELS IN FEET BELOW LAND SURFACE DATUM, CLIMATIC YEARS APRIL 1981 TO MARCH 1983

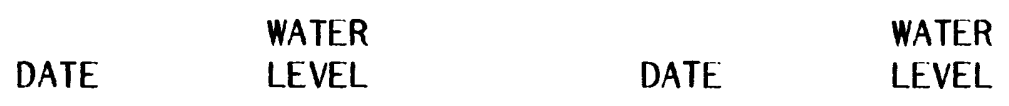

$\begin{array}{lll}\text { MAR } 11,1982 \quad 27.62 & \text { FEB 04, } 1983 \quad 27.53\end{array}$

GROUND-WATER LEVELS

343846099000501. LOCAL NUMBER; 02N-17W-17 ADD 1. LOCATION,--LAT 34 38'46", LONG 099 00'05", HYDROLOGIC UNIT 11120303.

AQUIFER.--TERRACE DEPOSITS.

PERIOD OF RECORD.--1979 TO CURRENT YEAR.

EXTREMES FOR PERIOD OF RECORD.--HIGHEST WATER LEVEL, $15.93 \mathrm{FT}(4.86 \mathrm{M})$ BELOW LAND-SURFACE DATUM, MAR 22, 1983; LOWEST WATER LEVEL, $17.93 \mathrm{FT}(5.47 \mathrm{M})$ BELOW LAND-SURFACE DATUM, MAR 24, 1981.

WATER LEVELS IN FEET BELOW LAND SURFACE DATUM, CLIMATIC YEARS APRIL 1981 TO MARCH 1983

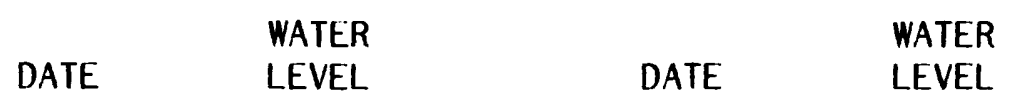

MAR 11, $1982 \quad 17.40 \quad$ MAR 22, $1983 \quad 15.93$ 
GROUND-WATER LEVELS

KIOWA COUNTY -- CONTINUED

344235098541801. LOCAL NUMBER;

$03 N-16 W-29$ BAA 1.

LOCATION,--LAT 34 42'35", LONG 098 54'18", HYDROLOGIC UNIT 11120303.

AQUIFER.--TERRACE DEPOSITS.

PERIOD OF RECORD.--1978 TO CURRENT YEAR.

EXTREMES FOR PERIOD OF RECORD.--HIGHEST WATER LEVEL, $17.12 \mathrm{FT}(5.22 \mathrm{M})$ BELOW LAND-SURFACE DATUM, MAR 22, 1983; LOWEST WATER LEVEL,21.74 FT(6.63 M) BELOW LAND-SURFACE DATUM, MAR 11, 1982 .

WATER LEVELS IN FEET BELOW LAND SURFACE DATUM, CLIMATIC YEARS APRIL 1981 TO MARCH 1983

$\begin{array}{lll} & \text { WATER } & \text { WATER } \\ \text { DATE } & \text { LEVEL } & \text { DATE }\end{array}$

MAR $11,1982 \quad 21.74 \quad$ MAR 22, $1983 \quad 17.12$

GROUND-WATER LEVELS

344149098582301. LOCAL NUMBER;

$03 \mathrm{~N}-17 \mathrm{~W}-27$ DCC 1

LOCATION,--LAT 34 41'49", LONG 098 58'23", HYDROLOGIC UNIT 11120303.

AQUIFER.--TERRACE DEPOSITS.

PERIOD OF RECORD.--1978 TO CURRENT YEAR.

EXTREMES FOR PERIOD OF RECORD.--HIGHEST WATER LEVEL, $32.80 \mathrm{FT}(10.00 \mathrm{M})$ BELOW LAND-SURFACE DATUM,FEB 9, 1983; LOWEST WATER LEVEL, 35.30 FT(10.76 M) BELOW LAND-SURFACE DATUM, AUG $30,1978$.

WATER LEVELS IN FEET BELOW LAND SURFACE DATUM, CLIMATIC YEARS APRIL 1981 TO MARCH 1983

$\begin{array}{lll} & \text { WATER } & \\ \text { DATE } & \text { LEVEL } & \text { WATER } \\ & \text { DATE } & \text { LEVEL }\end{array}$

$\begin{array}{llll}\text { MAR } 11,1982 & 33.42 & \text { FEB 09, } 1983 \quad 32.80\end{array}$ 


\section{GROUND-WATER LEVELS}

KIOWA COUNTY -- CONTINUED

344210098593501. LOCAL NUMBER;

LOCATION, --LAT 34 42'10", LONG 098

AQUIFER.--TERRACE DEPOSITS.

PERIOD OF RECORD.--1974 TO CURRENT YEAR.

EXTREMES FOR PERIOD OF RECORD.--HIGHEST WATER LEVEL, 20.57 FT (6.27 M) BELOW LAND-SURFACE

DATUM, MAR 27, 1979; LOWEST WATER LEVEL,21.33 FT(6.50 M) BELOW LAND-SURFACE DATUM, FEB 9, 1983.

WATER LEVELS IN FEET BELOW LAND SURFACE DATUM, CLIMATIC YEARS APRIL 1981 TO MARCH 1983
$03 N-17 W-28$ CAD 1.

59'35", HYDROLOGIC UNIT 11120303. 
GROUND-WATER LEVELS

KIOWA COUNTY -- CONTINUED

344745099083001. LOCAL NUMBER;

LOCATION,--LAT 34 47'45", LONG 099 AQUIFER.--TERRACE DEPOSITS.

PERIOD OF RECORD.--1978 TO CURRENT YEAR.

EXTREMES FOR PERIOD OF RECORD.--HIGHEST WATER LEVEL, 10.94 FT(3.33 M) BELOW LAND-SURFACE DATUM, AUG 29, 1978; LOWEST WATER LEVEL, 13.16 FT(4.01 M) BELOW LAND-SURFACE DATUM, MAR 11, 1982.

WATER LEVELS IN FEET BELOW LAND SURFACE DATUM, CLIMATIC YEARS APRIL 1981 TO MARCH 1983
$04 \mathrm{~N}-19 \mathrm{~W}-25$ AAA 1

$08^{\prime} 30^{\prime \prime}$, HYDROLOGIC UNIT 11120303.

\begin{tabular}{|c|c|}
\hline & WATER \\
\hline ATE & LEVEL \\
\hline
\end{tabular}

MAR $11,1982 \quad 13.16 \quad$ MAR $11,1983 \quad 13.10$

GROUND-WATER LEVELS

345844099193001. LOCAL NUMBER; 06N-20W-20 DAA 1.

LOCATION,--LAT 34 58'44", LONG 099 19'30", HYDROLOGIC UNIT 11120302.

AQUIFER.--TERRACE DEPOSITS.

PERIOD OF RECORD.--1976 TO CURRENT YEAR.

EXTREMES FOR PERIOD OF RECORD.--HIGHEST WATER LEVEL, 24.10 FT(7.35 M) BELOW LAND-SURFACE DATUM, MAR 27, 1979; LOWEST WATER LEVEL,28.02 FT(8.54 M) BELOW LAND-SURFACE DATUM, DEC 29, 1976.

WATER LEVELS IN FEET BELOW LAND SURFACE DATUM, CLIMATIC YEARS APRIL 1981 TO MARCH 1983

$\begin{array}{lll} & \text { WATER } & \text { WATER } \\ \text { DATE } & \text { LEVEL } & \text { DATE }\end{array}$

MAR $12,1982 \quad 26.24 \quad$ MAR $11,1983 \quad 27.86$ 
KIOWA COUNTY -- CONTINUED

345857099193001. LOCAL NUMBER; LOCATION,--LAT $3458^{\prime} 57^{\prime \prime}$, LONG 099 AQUIFER.--TERRACE DEPOSITS.

PERIOD OF RECORD.--1976 TO CURRENT

EXTREMES FOR PERIOD OF RECORD.--HIGHEST WATER LEVEL, 27.28 FT(8.31 M) BELOW LAND-SURFACE DATUM, MAR 6, 1979; LOWEST WATER LEVEL, 31.10 FT(9.48 M) BELOW LAND-SURFACE DATUM, DEC 29, 1976.

WATER LEVELS IN FEET BELOW LAND SURFACE DATUM, CLIMATIC YEARS APRIL 1981 TO MARCH 1983
GROUND-WATER LEVELS

345831099191401. LOCAL NUMBER;

$06 \mathrm{~N}-20 \mathrm{~W}-21$ CCA 1 .

LOCATION,--LAT $3458^{\prime} 31$ ", LONG 099 19'14", HYDROLOGIC UNIT 11120302.

AQUIFER.--TERRACE DEPOSITS.

PERIOD OF RECORD.--1976 TO CURRENT YEAR.

EXTREMES FOR PERIOD OF RECORD.--HIGHEST WATER LEVEL, 27.74 FT(8.46 M) BELOW LAND-SURFACE DATUM,MAR 6, 1979; LOWEST WATER LEVEL,33.73 FT(10.28 M) BELOW LAND-SURFACE DATUM, DEC 29, 1976.

WATER LEVELS IN FEET BELOW LAND SURFACE DATUM, CLIMATIC YEARS APRIL 1981 TO MARCH 1983
MAR 12, $1982 \quad 30.82 \quad$ MAR $11,1983 \quad 31.38$
MAR 12, $1982 \quad 30.82 \quad$ MAR $11,1983 \quad 31.38$

WATER

LEVEL 
KIOWA COUNTY -- CONTINUED

345824099185001. LOCAL NUMBER; 06N-20W-21 DCC 2. LOCATION,--LAT 34 58'24", LONG 099 18'50", HYDROLOGIC UNIT 11120302.

AQUIFER.--TERRACE DEPOSITS.

PERIOD OF RECORD .--1976 TO CURRENT YEAR.

EXTREMES FOR PERIOD OF RECORD.--HIGHEST WATER LEVEL, $24.43 \mathrm{FT}(7.45 \mathrm{M})$ BELOW LAND-SURFACE DATUM,MAR 6, 1979; LOWEST WATER LEVEL, 31.05 FT(9.46 M) BELOW LAND-SURFACE DATUM, DEC 29, 1976.

WATER LEVELS IN FEET BELOW LAND SURFACE DATUM, CLIMATIC YEARS APRIL 1981 TO MARCH 1983

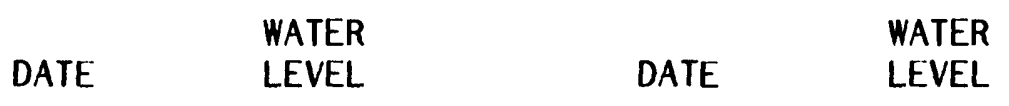

MAR 12, $1982 \quad 28.28 \quad$ MAR 21, $1983 \quad 30.34$

GROUND-WATER LEVELS

345824099165901. LOCAL NUMBER; 06N-20W-23 CDC 1. LOCATION,--LAT 34 58'24", LONG 099 16'59", HYDROLOGIC UNIT 11120302. AQUIFER .--TERRACE DEPOSITS.

PERIOD OF RECORD.--1976 TO CURRENT YEAR.

EXTREMES FOR PERIOD OF RECORD.--HIGHEST WATER LEVEL, $14.82 \mathrm{FT}(4.52 \mathrm{M})$ BELOW LAND-SURFACE DATUM, MAR 8, 1978; LOWEST WATER LEVEL, $17.65 \mathrm{FT}(5.38 \mathrm{M})$ BELOW LAND-SURFACE DATUM, MAR 12, 1982.

WATER LEVELS IN FEET BELOW LAND SURFACE DATUM, CLIMATIC YEARS APRIL 1981 TO MARCH 1983

\begin{tabular}{|c|c|c|}
\hline $\mathbf{A T}$ & WATER & DATE \\
\hline AT & & DAIE \\
\hline
\end{tabular}

MAR 12, $1982 \quad 17.65 \quad$ MAR $11,1983 \quad 17.41$ 
GROUND-WATER LEVELS

KIOWA COUNTY -- CONTINUED

345732099181801. LOCAL NUMBER; $06 \mathrm{~N}-20 \mathrm{~W}-27$ CCC 1 . LOCATION,--LAT 34 57'32", LONG 099 18'18", HYDROLOGIC UNIT 11120302. AQUIFER.--TERRACE DEPOSITS.

PERIOD OF RECORD.--1976 TO CURRENT YEAR.

EXTREMES FOR PERIOD OF RECORD.--HIGHEST WATER LEVEL, 18.63 FT(5.68 M) BELOW LAND-SURFACE DATUM,MAR 6, 1979; LOWEST WATER LEVEL,23.32 FT(7.11 M) BELOW LAND-SURFACE DATUM, MAR 11, 1983.

WATER LEVELS IN FEET BELOW LAND SURFACE DATUM, CLIMATIC YEARS APRIL 1981 TO MARCH 1983

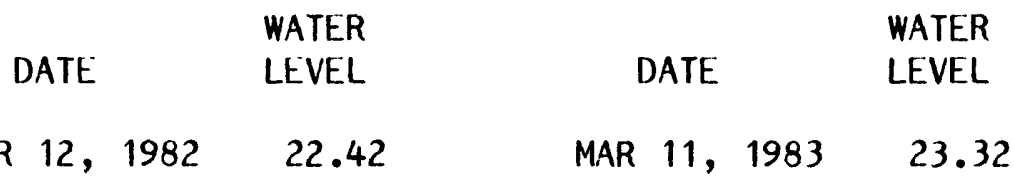

GROUND-WATER LEVELS

345751099185801. LOCAL NUMBER; 06N-20W-28 CAA 1. LOCATION,--LAT 34 57'51", LONG 099 18'58", HYDROLOGIC UNIT 11120302.

AQUIFER.--TERRACE DEPOSITS.

PERIOD OF RECORD.--1979 TO CURRENT YEAR.

EXTREMES FOR PERIOD OF RECORD.--HIGHEST WATER LEVEL, 12.75 FT(3.89 M) BELOW LAND-SURFACE DATUM,MAR 8, 1979; LOWEST WATER LEVEL,20.39 FT(6.21 M) BELOW LAND-SURFACE DATUM, MAR 21, 1983.

WATER LEVELS IN FEET BELOW LAND SURFACE DATUM, CLIMATIC YEARS APRIL 1981 TO MARCH 1983

\begin{tabular}{|c|c|c|}
\hline & WATER & \\
\hline DATE & LEVEL & DATE \\
\hline
\end{tabular}

MAR 12, $1982 \quad P \quad$ MAR 21, $1983 \quad 20.39$ 
KIOWA COUNTY -- CONTINUED

345818099193001. LOCAL NUMBER; LOCATION,--LAT 34 58'18", LONG 099 AQUIFER.--TERRACE DEPOSITS. PERIOD OF RECORD.--1979 TO CURRENT YEAR. EXTREMES FOR PERIOD OF RECORD.--HIGHEST WATER LEVEL,29.69 FT(9.05 M) BELOW LAND-SURFACE DATUM, MAR 27, 1979; LOWEST WATER LEVEL,33.42 FT(10.19 M) BELOW LAND-SURFACE DATUM, MAR 21, 1983.

WATER LEVELS IN FEET BELOW LAND SURFACE DATUM, CLIMATIC YEARS APRIL 1981 TO MARCH 1983
06N-20W-29 AAA 1.

19'30", HYDROLOGIC UNIT 11120302.

\section{WATER}

DATE

LEVEL
33.41
MAR 12, 1982
MAR $21,1983 \quad 33.42$

DATE

WATER

LEVEL.

\section{GROUND-WATER LEVELS}

345659099162001. LOCAL NUMBER; 06N-20W-35 DAA 1. AQUIFER.--TERRACE DEPOSITS. PERIOD OF RECORD.--1976 TO CURRENT YEAR. EXTREMES FOR PERIOD OF RECORD.--HIGHEST WATER LEVEL,7.53 FT(2.30 M) BELOW LAND-SURFACE DATUM,DEC 29, 1976; LOWEST WATER LEVEL, 10.83 FT(3.30 M) BELOW LAND-SURFACE DATUM, MAR 12,1982 .

WATER LEVELS IN FEET BELOW LAND SURFACE DATUM, CLIMATIC YEARS APRIL 1981 TO MARCH 1983

\begin{tabular}{|c|c|}
\hline & WATER \\
\hline ATE & LEVEL \\
\hline
\end{tabular}

MAR 12, $1982 \quad 10.83 \quad$ MAR 21, $1983 \quad P$


GROUND-WATER LEVELS

KIOWA COUNTY -- CONTINUED

350457098453401. LOCAL NUMBER; 07N-15W-14 BCC 1.

LOCATION,--LAT 35 04'57", LONG 098 45'34", HYDROLOGIC UNIT 11130302.

AQUIFER.--TERRACE DEPOSITS.

PERIOD OF RECORD .--1976 TO CURRENT YEAR.

EXTREMES FOR PERIOD OF RECORD.--HIGHEST WATER LEVEL, $16.51 \mathrm{FT}(5.03 \mathrm{M})$ BELOW LAND-SURFACE DATUM, MAR 17, 1983; LOWEST WATER LEVEL,20.21 FT(6.16 M) BELOW LAND-SURFACE DATUM, MAR 28, 1979.

WATER LEVELS IN FEET BELOW LAND SURFACE DATUM, CLIMATIC YEARS APRIL 1981 TO MARCH 1983

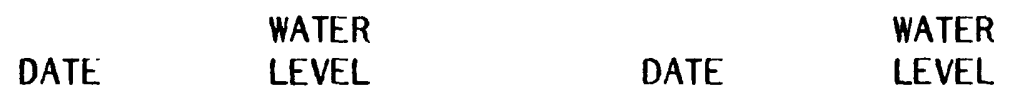

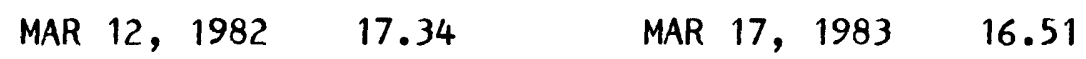

GROUND-WATER LEVELS

350226099074501. LOCAL NUMBER; 07N-18W-32 BCB 1. LOCATION,--LAT 35 02'26", LONG 099 07'45", HYDROLOGIC UNIT 11120303. AQUIFER.--TERRACE DEPOSITS.

PERIOD OF RECORD.--1976 TO CURRENT YEAR.

EXTREMES FOR PERIOD OF RECORD.--HIGHEST WATER LEVEL, 18.26 FT(5.57 M) BELOW LAND-SURFACE DATUM, MAR 23, 1981; LOWEST WATER LEVEL,20.05 FT(6.11 M) BELOW LAND-SURFACE DATUM, MAR 6, 1979.

WATER LEVELS IN FEET BELOW LAND SURFACE DATUM, CLIMATIC YEARS APRIL 1981 TO MARCH 1983

\begin{tabular}{|c|c|c|}
\hline & WATER & \\
\hline DATE & LEVEL & DATE \\
\hline
\end{tabular}

$\begin{array}{llll}\text { MAR } 12,1982 & 20.02 & \text { FEB } 09,1983 \quad 18.84\end{array}$ 


\section{GROUND-WATER LEVELS}

\section{LATIMER COUNTY}

\section{LOCAL NUMBER;}

05N-19E-09 CBB 1.

LOCATION,--LAT 34 55'09", LONG 095 18'49", HYDROLOGIC UNIT 11110105.

AQUIFER.--ANTLERS SAND.

PERIOD OF RECORD.--1976 TO CURRENT YEAR.

EXTREMES FOR PERIOD OF RECORD.--HIGHEST WATER LEVEL, $1.59 \mathrm{FT}(.48 \mathrm{M})$ BELOW LAND-SURFACE

DATUM, MAR 10, 1981; LOWEST WATER LEVEL, $3.38 \mathrm{FT}(1.03 \mathrm{M})$ BELOW LAND-SURFACE DATUM, JUNE $16,1976$.

WATER LEVELS IN FEET BELOW LAND SURFACE DATUM, CLIMATIC YEARS APRIL 1981 TO MARCH 1983

\begin{tabular}{lrrrr} 
DATE & $\begin{array}{l}\text { WATER } \\
\text { LEVEL }\end{array}$ & DATE & \multicolumn{1}{l}{ WATER } \\
LEVEL
\end{tabular}


GROUND-WATER LEVELS

LE FLORE COUNTY

345021094320801. LOCAL NUMBER; 04N-26E-11 BBD 1. LOCATION,--LAT 34 50'21", LONG 094 32'08", HYDROLOGIC UNIT 11110105. AQUIFER.--ALLUVIUM.

PERIOD OF RECORD.--1980 TO CURRENT YEAR. EXTREMES FOR PERIOD OF RECORD.--HIGHEST WATER LEVEL,45.79 FT(13.96 M) BELOW LAND-SURFACE DATUM,FEB 26, 1980; LOWEST WATER LEVEL,70.18 FT(21.39 M) BELOW LAND-SURFACE DATUM, MAR 22, 1983.

WATER LEVELS IN FEET BELOW LAND SURFACE DATUM, CLIMATIC YEARS APRIL 1981 TO MARCH 1983

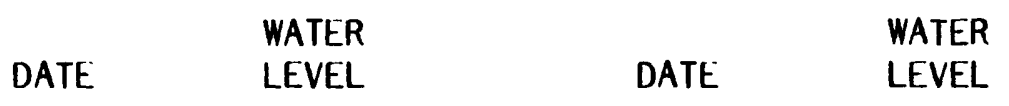

MAR 24, $1982 \quad 57.34 \quad$ MAR 22, $1983 \quad 70.18$

GROUND-WATER LEVELS

352005094432801. LOCAL NUMBER; 10N-24E-13 CCC 1. LOCATION,--LAT 35 20'05", LONG 094 43'28", HYDROLOGIC UNIT 11110104. AQUIFER.--TERRACE DEPOSITS. PERIOD OF RECORD.--1976 TO CURRENT YEAR. EXTREMES FOR PERIOD OF RECORD.--HIGHEST WATER LEVEL,20.12 FT(6.13 M) BELOW LAND-SURFACE DATUM,APR 22, 1976; LOWEST WATER LEVEL,24.92 FT(7.60 M) BELOW LAND-SURFACE DATUM, MAR 10, 1981.

WATER LEVELS IN FEET BELOW LAND SURFACE DATUM, CLIMATIC YEARS APRIL 1981 TO MARCH 1983

$\begin{array}{lll} & \text { WATER } & \\ \text { DATE } & \text { LEVEL } & \text { WATER } \\ \text { LEVEL }\end{array}$
MAR 24, 1982
0
MAR 22, 1983
N 
GROUND-WATER LEVELS

\section{LINCOLN COUNTY}

352907096411001. LOCAL NUMBER;

12N-06E-29 DAB 1. LOCATION,--LAT 35 29'07", LONG 096 41'10", HYDROLOGIC UNIT 11100302. AQUIFER.--GARBER SANDSTONE.

PERIOD OF RECORD.--1980 TO CURRENT YEAR.

EXTREMES FOR PERIOD OF RECORD.--HIGHEST WATER LEVEL, $65.21 \mathrm{FT}(19.88 \mathrm{M})$ BELOW LAND-SURFACE DATUM, MAR 8, 1983; LOWEST WATER LEVEL,71.12 FT(21.68 M) BELOW LAND-SURFACE DATUM, JAN 25, 1980 .

WATER LEVELS IN FEET BELOW LAND SURFACE DATUM, CLIMATIC YEARS APRIL 1981 TO MARCH 1983

\begin{tabular}{|c|c|}
\hline & WATER \\
\hline DATE & LEVEL \\
\hline
\end{tabular}

MAR 04, $1982 \quad 67.05 \quad$ MAR 08, $1983 \quad 65.21$

GROUND-WATER LEVELS

354205096530801. LOCAL NUMBER; 14N-04E-09 CAB 1. LOCATION,--LAT 35 42'05", LONG 096 53'08", HYDROLOGIC UNIT 11100303. AQUIFER .--GARBER SANDSTONE.

PERIOD OF RECORD.--1980 TO CURRENT YEAR.

EXTREMES FOR PERIOD OF RECORD.--HIGHEST WATER LEVEL,2.22 FT(.68 M) BELOW LAND-SURFACE DATUM,MAR 8, 1983; LOWEST WATER LEVEL,25.72 FT(7.84 M) BELOW LAND-SURFACE DATUM, MAR 4, 1982.

WATER LEVELS IN FEET BELOW LAND SURFACE DATUM, CLIMATIC YEARS APRIL 1981 TO MARCH 1983

\begin{tabular}{|c|c|c|}
\hline & WATER & \\
\hline DATE & LEVEL & DATE \\
\hline
\end{tabular}

MAR $04,1982 \quad 25.72 \quad$ MAR 08, $1983 \quad 2.22$


GROUND-WATER LEVELS

\section{LINCOLN COUNTY -- CONTINUED}

355540097054901. LOCAL NUMBER;

LOCATION,--LAT $3555^{\prime} 40 "$ ", LONG 097

AQUIFER.--VAMOOSA.

PERIOD OF RECORD.--1977 TO CURRENT YEAR.

EXTREMES FOR PERIOD OF RECORD.--HIGHEST WATER LEVEL,23.24 FT(7.08 M) BELOW LAND-SURFACE DATUM, MAR 8, 1983; LOWEST WATER LEVEL,27.18 FT(8.28 M) BELOW LAND-SURFACE DATUM, MAR 20, 1978.

WATER LEVELS IN FEET BELOW LAND SURFACE DATUM, CLIMATIC YEARS APRIL 1981 TO MARCH 1983

$\begin{array}{lccc}\text { DATE } & \begin{array}{l}\text { WATER } \\ \text { LEVEL }\end{array} & \text { DATE } & \text { WATER } \\ \text { LEVEL }\end{array}$


GROUND-WATER LEVELS

LOGAN COUNTY

355632097365501. LOCAL NUMBER;

$17 N-04 W-15 \operatorname{CDC} 1$.

LOCAT1ON,--LAT $3556^{\prime} 32^{\prime \prime}$, LONG 097

36 '55", HYDROLOGIC UNIT 11050002.

AQUIFER. - -TERRACE DEPOSITS.

PERIOD OF RECORD.--1975 TO CURRENT YEAR.

EXTREMES FOR PERIOD OF RECORD.--HIGHEST WATER LEVEL, $11.12 \mathrm{FT}(3.39 \mathrm{M})$ BELOW LAND-SURFACE DATUM, JAN 12, 1976; LOWEST WATER LEVEL, 14.10 FT(4.30 M) BELOW LAND-SURFACE DATUM, JAN $5,1978$.

WATER LEVELS IN FEET BELOW LAND SURFACE DATUM, CLIMATIC YEARS APRIL 1981 TO MARCH 1983

$\begin{array}{lccc} & \text { WATER } & & \text { WATER } \\ \text { DATE } & \text { LEVEL } & \text { DATE } & \text { LEVEL } \\ & & & \\ \text { R 03, 1982 } & 12.85 & \text { MAR 16, 1983 } & 11.56\end{array}$

GROUND-WATER LEVELS

355632097395901. LOCAL NUMBER; 17N-04W-18 CDD 1.

LOCATION,--LAT 35 56'32", LONG 097 39'59", HYDROLOGIC UNIT 11050002.

AQUIFER.--TERRACE DEPOSITS.

PERIOD OF RECORD.--1980 TO CURRENT YEAR.

EXTREMES FOR PERIOD OF RECORD.--HIGHEST WATER LEVEL, $25.42 \mathrm{FT}(7.75 \mathrm{M})$ BELOW LAND-SURFACE DATUM,MAR 16, 1983; LOWEST WATER LEVEL,26.54 FT(8.09 M) BELOW LAND-SURFACE DATUM, JAN 23, 1980.

WATER LEVELS IN FEET BELOW LAND SURFACE DATUM, CLIMATIC YEARS APRIL 1981 TO MARCH 1983

$\begin{array}{lll} & \text { WATER } & \text { WATER } \\ \text { DATE } & \text { LEVEL } & \text { DATE }\end{array}$

MAR 03, $1982 \quad 25.65 \quad$ MAR 16, $1983 \quad 25.42$




\section{GROUND-WATER LEVELS}

\section{LOGAN COUNTY -- CONTINUED}

355606097392701. LOCAL NUMBER; 17N-04W-19 ADD 1. LOCATION,--LAT 35 56'06", LONG 097 39'27", HYDROLOGIC UNIT 11050002. AQUIFER.--TERRACE DEPOSITS. PERIOD OF RECORD.--1975 TO CURRENT YEAR. EXTREMES FOR PERIOD OF RECORD.--HIGHEST WATER LEVEL, $16.49 \mathrm{FT}(5.03 \mathrm{M})$ BELOW LAND-SURFACE DATUM,MAR 17, 1983; LOWEST WATER LEVEL,22.42 FT(6.83 M) BELOW LAND-SURFACE DATUM, JAN 20, 1975.

WATER LEVELS IN FEET BELOW LAND SURFACE DATUM, CLIMATIC YEARS APRIL 1981 TO MARCH 1983

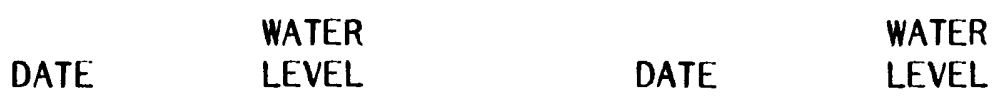

MAR 03, $1982 \quad 20.30 \quad$ MAR 17, $1983 \quad 16.49$

GROUND-WATER LEVELS

355507097371101. LOCAL NUMBER; 17N-04W-27 CBB 1. LOCATION,--LAT 35 55'07", LONG 097 37'11", HYDROLOGIC UNIT 11050002. AQUIFER.--TERRACE DEPOSITS. PERIOD OF RECORD.--1975 TO CURRENT YEAR. EXTREMES FOR PERIOD OF RECORD.--HIGHEST WATER LEVEL, 3.65 FT(1.11 M) BELOW LAND-SURFACE DATUM,JAN 12, 1976; LOWEST WATER LEVEL,7.30 FT(2.23 M) BELOW LAND-SURFACE DATUM, JAN $5,1978$.

WATER LEVELS IN FEET BELOW LAND SURFACE DATUM, CLIMATIC YEARS APRIL 1981 T0 MARCH 1983

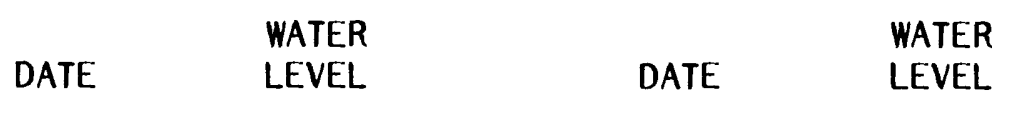

MAR 03, $1982 \quad Z \quad$ MAR 18, $1983 \quad$ Z


LOGAN COUNTY -- CONTINUED

355447097365501. LOCAL NUMBER; 17N-04W-27 CDC 2. LOCATION,--LAT 35 54'47", LONG 097 36'55", HYDROLOGIC UNIT 11050002. AQUIFER.--TERRACE DEPOSITS.

PERIOD OF RECORD.--1975 TO CURRENT YEAR.

EXTREMES FOR PERIOD OF RECORD.--HIGHEST WATER LEVEL, $5.58 \mathrm{FT}(1.70 \mathrm{M})$ BELOW LAND-SURFACE DATUM,JAN 12, 1976; LOWEST WATER LEVEL,9.03 FT(2.75 M) BELOW LAND-SURFACE DATUM, JAN 5, 1978.

WATER LEVELS IN FEET BELOW LAND SURFACE DATUM, CLIMATIC YEARS APRIL 1981 TO MARCH 1983

\begin{tabular}{|c|c|c|}
\hline & WATER & \\
\hline DATE & LEVEL & DATE \\
\hline
\end{tabular}
MAR 03, 1982
6.65
MAR 16, 1983
8.83

GROUND-WATER LEVELS

355355097402301. LOCAL NUMBER; 17N-04W-31 CCC 1. LOCATION,--LAT 35 53'55", LONG 097 40'23", HYDROLOGIC UNIT 11050002. AQUIFER.--TERRACE DEPOSITS.

PERIOD OF RECORD.--1975 TO CURRENT YEAR.

EXTREMES FOR PERIOD OF RECORD.--HIGHEST WATER LEVEL, 18.68 FT(5.69 M) BELOW LAND-SURFACE DATUM, JAN 12, 1976; LOWEST WATER LEVEL,21.65 FT(6.60 M) BELOW LAND-SURFACE DATUM, FEB 6, 1979.

WATER LEVELS IN FEET BELOW LAND SURFACE DATUM, CLIMATIC YEARS APRIL 1981 TO MARCH 1983

$\begin{array}{lll} & \text { WATER } & \\ \text { DATE } & \text { WEVEL } & \text { WATER } \\ & \text { LEVEL }\end{array}$

MAR 03, $1982 \quad 20.24 \quad$ MAR $16,1983 \quad 20.13$ 
GROUND-WATER LEVELS

LOGAN COUNTY -- CONTINUED

355441097363901. LOCAL NUMBER;

$17 \mathrm{~N}-04 \mathrm{~W}-34$ ABB 1.

LOCATION,--LAT $3554^{\prime} 41$ ", LONG 097

AQUIFER.--TERRACE DEPOSITS.

PERIOD OF RECORD.--1975 TO CURRENT YEAR.

EXTREMES FOR PERIOD OF RECORD.--HIGHEST WATER LEVEL,27.77 FT(8.46 M) BELOW LAND-SURFACE DATUM,JAN 12, 1976; LOWEST WATER LEVEL,31.45 FT(9.59 M) BELOW LAND-SURFACE DATUM, DEC 6, 1976.

WATER LEVELS IN FEET BELOW LAND SURFACE DATUM, CLIMATIC YEARS APRIL 1981 TO MARCH 1983

\begin{tabular}{|c|c|}
\hline & WATER \\
\hline DATE & LEVEL \\
\hline
\end{tabular}

MAR 03, $1982 \quad 29.72 \quad$ MAR 16, $1983 \quad$ Z

GROUND-WATER LEVELS

355402097371101. LOCAL NUMBER; 17N-04W-34 CCB 2.

LOCATION,--LAT $3554^{\prime} 02 "$ ", LONG 097 37'11", HYDROLOGIC UNIT 11050002.

AQUIFER.--TERRACE DEPOSITS.

PERIOD OF RECORD.--1975 TO CURRENT YEAR.

EXTREMES FOR PERIOD OF RECORD.--HIGHEST WATER LEVEL, 47.37 FT ( $14.44 \mathrm{M})$ BELOW LAND-SURFACE DATUM,JAN 21, 1976; LOWEST WATER LEVEL,49.51 FT(15.09 M) BELOW LAND-SURFACE DATUM, MAR $16,1983$.

WATER LEVELS IN FEET BELOW LAND SURFACE DATUM, CLIMATIC YEARS APRIL 1981 TO MARCH 1983

$\begin{array}{lll} & \text { WATER } & \text { WATER } \\ \text { DATE } & \text { LEVEL } & \text { DATE }\end{array}$
MAR 03, 1982
Z
MAR 06, 1983
49.51 
335106097244901. LOCAL NUMBER; 08S-02W-21 BAA 1. LOCATION,--LAT 33 51'06", LONG 097 24'49", HYDROLOGIC UNIT 11130201.

AQUIFER.--TERRACE DEPOSITS.

PERIOD OF RECORD.--1980 TO CURRENT YEAR.

EXTREMES FOR PERIOD OF RECORD.--HIGHEST WATER LEVEL, $39.84 \mathrm{FT}(12.14 \mathrm{M})$ BELOW LAND-SURFACE DATUM,MAR 2, 1983; LOWEST WATER LEVEL,45.56 FT(13.89 M) BELOW LAND-SURFACE DATUM, MAR 3, 1981.

WATER LEVELS IN FEET BELOW LAND SURFACE DATUM, CLIMATIC YEARS APRIL 1981 TO MARCH 1983

\begin{tabular}{|c|c|c|}
\hline DATE & $\begin{array}{l}\text { WATER } \\
\text { IFVFI }\end{array}$ & DATF \\
\hline & & DAIE \\
\hline
\end{tabular}

MAR 08, $1982 \quad 43.26 \quad$ MAR 02, $1983 \quad 39.84$ 
MCCLAIN COUNTY

345646097160401. LOCAL NUMBER; 06N-01W-35 DDB 1. LOCATION,--LAT 34 56'46", LONG 097 16'04", HYDROLOGIC UNIT 11090202. AQUIFER.--ALLUVIUM.

PERIOD OF RECORD.--1976 TO CURRENT YEAR.

EXTREMES FOR PERIOD OF RECORD.--HIGHEST WATER LEVEL,9.01 FT(2.75 M) BELOW LAND-SURFACE DATUM,MAR 1, 1983; LOWEST WATER LEVEL, 13.34 FT(4.07 M) BELOW LAND-SURFACE DATUM, MAR 2, 1982.

WATER LEVELS IN FEET BELOW LAND SURFACE DATUM, CLIMATIC YEARS APRIL 1981 TO MARCH 1983

\begin{tabular}{|c|c|}
\hline & WATER \\
\hline DATE & LEVEL \\
\hline
\end{tabular}

MAR $02,1982 \quad 13.34 \quad$ MAR 01, $1983 \quad 9.01$

GROUND-WATER LEVELS

351043097294001. LOCAL NUMBER; O8N-03W-10 DDA 1.

LOCATION,--LAT 35 10'43", LONG 097 29'40", HYDROLOGIC UNIT 11090202.

AQUIFER.--ALLUVIUM.

PERIOD OF RECORD.--1976 TO CURRENT YEAR.

EXTREMES FOR PERIOD OF RECORD.--HIGHEST WATER LEVEL, 8.83 FT(2.69 M) BELOW LAND-SURFACE DATUM, MAR 10, 1983; LOWEST WATER LEVEL, $12.53 \mathrm{FT}(3.82 \mathrm{M})$ BELOW LAND-SURFACE DATUM, JAN 23, 1981.

WATER LEVELS IN FEET BELOW LAND SURFACE DATUM, CLIMATIC YEARS APRIL 1981 TO MARCH 1983

$\begin{array}{lll} & \text { WATER } & \text { WATER } \\ \text { DATE } & \text { LEVEL } & \text { DATE }\end{array}$

MAR 02, $1982 \quad 11.83 \quad$ MAR $10,1983 \quad 8.83$ 


\section{MCCLAIN COUNTY -- CONTINUED}

350957097274901. LOCAL NUMBER; 08N-03W-13 CBC 1. LOCATION,--LAT 35 09'57", LONG 097 27'49", HYDROLOGIC UNIT 11090202. AQUIFER.--TERRACE DEPOSITS. PERIOD OF RECORD.--1980 TO CURRENT YEAR. EXTREMES FOR PERIOD OF RECORD.--HIGHEST WATER LEVEL,9.99 FT(3.04 M) BELOW LAND-SURFACE DATUM,MAR 10, 1983; LOWEST WATER LEVEL,13.12 FT(4.00 M) BELOW LAND-SURFACE DATUM, MAR 2, 1982.

WATER LEVELS IN FEET BELOW LAND SURFACE DATUM, CLIMATIC YEARS APRIL 1981 TO MARCH 1983

$\begin{array}{cccr} & \text { WATER } & & \\ \text { DATE } & \text { LEVEL } & \text { WATE } & \text { WATER } \\ \text { LEVEL }\end{array}$


MCCURTAIN COUNTY

340042095051801. LOCAL NUMBER; 06S-21E-27 AAA 1. LOCATION,--LAT 34 00'42", LONG 095 05'18", HYDROLOGIC UNIT 11140107. AQUIFER.--ANTLERS SAND.

PERIOD OF RECORD.-- 1976 TO CURRENT YEAR.

EXTREMES FOR PERIOD OF RECORD.--HIGHEST WATER LEVEL,69.92 FT(21.31 M) BELOW LAND-SURFACE DATUM, APR 22, 1976; LOWEST WATER LEVEL,73.67 FT(22.45 M) BELOW LAND-SURFACE DATUM, MAR 10, 1982 .

WATER LEVELS IN FEET BELOW LAND SURFACE DATUM, CLIMATIC YEARS APRIL 1981 TO MARCH 1983

\begin{tabular}{|c|c|}
\hline & WATER \\
\hline DATE & LEVEL \\
\hline
\end{tabular}

MAR $10,1982 \quad 73.67 \quad$ MAR 09, $1983 \quad 72.42$ 
GROUND-WATER LEVELS

MCINTOSH COUNTY

351206095504201. LOCAL NUMBER; 08N-14E-05 AAB 1. LOCATION,--LAT 35 12'06", LONG 095 50'42", HYDROLOGIC UNIT 11090204. AQUIFER.--TERRACE DEPOSITS. PERIOD OF RECORD.--1977 TO CURRENT YEAR. EXTREMES FOR PERIOD OF RECORD.--HIGHEST WATER LEVEL,2.25 FT(.69 M) BELOW LAND-SURFACE DATUM,MAR 14, 1978; LOWEST WATER LEVEL,8.00 FT(2.44 M) BELOW LAND-SURFACE DATUM, MAR 9, 1981.

WATER LEVELS IN FEET BELOW LAND SURFACE DATUM, CLIMATIC YEARS APRIL 1981 TO MARCH 1983

$\begin{array}{lll} & \text { WATER } & \text { WATER } \\ \text { DATE } & \text { LEVEL } & \text { DATE }\end{array}$

MAR 23, $1982 \quad 3.82 \quad$ MAR 22, $1983 \quad 3.66$

GROUND-WATER LEVELS

352512095285701. LOCAL NUMBER; 11N-17E-23 BBB 1. LOCATION,--LAT 35 25'12", LONG 095 28'57", HYDROLOGIC UNIT 11090204.

AQUIFER . - BOGGY FORMATION.

PERIOD OF RECORD.--1980 TO CURRENT YEAR.

EXTREMES FOR PERIOD OF RECORD.--HIGHEST WATER LEVEL,73.59 FT(22.43 M) BELOW LAND-SURFACE DATUM,FEB 25, 1980; LOWEST WATER LEVEL, $102.50 \mathrm{FT}(31.24 \mathrm{M})$ BELOW LAND-SURFACE DATUM, MAR 22, 1983.

WATER LEVELS IN FEET BELOW LAND SURFACE DATUM, CLIMATIC YEARS APRIL 1981 TO MARCH 1983

$\begin{array}{lll} & \text { WATER } & \text { WATER } \\ \text { DATE } & \text { LEVEL } & \text { DATE }\end{array}$

MAR 25, $1982 \quad \mathrm{P} \quad$ MAR 22, $1983 \quad 102.50 \mathrm{R}$ 
GROUND-WATER LEVELS

MAJOR COUNTY

361440098092101. LOCAL NUMBER;

20N-09W-04 AAA 1.

LOCATION,--LAT 36 14'40", LONG 098 09'21", HYDROLOGIC UNIT 11050002.

AQUIFER.--TERRACE DEPOSITS.

PERIOD OF RECORD.--1978 TO CURRENT YEAR.

EXTREMES FOR PERIOD OF RECORD.--HIGHEST WATER LEVEL, $14.47 \mathrm{FT}(4.41 \mathrm{M})$ BELOW LAND-SURFACE DATUM,FEB 28, 1983; LOWEST WATER LEVEL, $16.92 \mathrm{FT}(5.16 \mathrm{M})$ BELOW LAND-SURFACE DATUM, MAR 20, 1981.

WATER LEVELS IN FEET BELOW LAND SURFACE DATUM, CLIMATIC YEARS APRIL 1981 TO MARCH 1983

$\begin{array}{lll} & \text { WATER } & \text { WATER } \\ \text { DATE } & \text { LEVEL } & \text { DATE }\end{array}$

MAR 23, $1982 \quad 16.77 \quad$ FEB 28, $1983 \quad 14.47$

GROUND-WATER LEVELS

361355098112101. LOCAL NUMBER; 20N-09W-05 CCC 1.

LOCATION,--LAT 36 13'55", LONG 098 11'21", HYDROLOGIC UNIT 11050002.

AQUIFER.--TERRACE DEPOSITS.

PERIOD OF RECORD.--1976 TO CURRENT YEAR.

EXTREMES FOR PERIOD OF RECORD.--HIGHEST WATER LEVEL,6.99 FT(2.13 M) BELOW LAND-SURFACE DATUM,JAN 13, 1976; LOWEST WATER LEVEL, $14.00 \mathrm{FT}(4.27 \mathrm{M})$ BELOW LAND-SURFACE DATUM, MAR 11, 1980.

WATER LEVELS IN FEET BELOW LAND SURFACE DATUM, CLIMATIC YEARS APRIL 1981 TO MARCH 1983

$\begin{array}{lll} & \text { WATER } & \text { WATER } \\ \text { DATE } & \text { LEVEL } & \text { DATE }\end{array}$

MAR 23, $1982 \quad 10.14 \quad$ FEB 28, $1983 \quad 7.33$ 
MAJOR COUNTY -- CONTINUED

361302098130601. LOCAL NUMBER; LOCATION,--LAT 36 13'02", LONG 098 AQUIFER.--TERRACE DEPOSITS.

PERIOD OF RECORD.--1976 TO CURRENT YEAR .

EXTREMES FOR PERIOD OF RECORD.--HIGHEST WATER LEVEL, 16.63 FT(5.07 M) BELOW LAND-SURFACE DATUM,JAN 13, 1976; LOWEST WATER LEVEL,21.02 FT(6.41 M) BELOW LAND-SURFACE DATUM, MAR 23, 1982.

WATER LEVELS IN FEET BELOW LAND SURFACE DATUM, CLIMATIC YEARS APRIL 1981 TO MARCH 1983
20N-10W-12 CDD 1.

13'06", HYDROLOGIC UNIT 11050002.

$\begin{array}{lll} & & \\ & & \\ \text { DATE } & \text { WATER } & \text { WATER } \\ \text { LEVEL } & \text { DATE } & \text { LEVEL }\end{array}$

MAR 23, $1982 \quad 21.02 \quad$ FEB 28, $1983 \quad 18.29$

GROUND-WATER LEVELS

361155098360801. LOCAL NUMBER; 20N-13W-20 ADA 1.

LOCATION,--LAT 36 11'55", LONG 098 36'08", HYDROLOGIC UNIT 11100301.

AQUIFER.--TERRACE DEPOSITS.

PERIOD OF RECORD.--1978 TO CURRENT YEAR.

EXTREMES FOR PLRIOD OF RECORD.--HIGHEST WATER LEVEL, 19.87 FT(6.06 M) BELOW LAND-SURFACE DATUM,MAR 1, 1983; LOWEST WATER LEVEL,21.42 FT(6.53 M) BELOW LAND-SURFACE DATUM, NOV $20,1978$.

WATER LEVELS IN FEET BELOW LAND SURFACE DATUM, CLIMATIC YEARS APRIL 1981 TO MARCH 1983

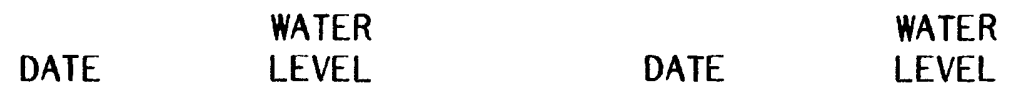

MAR 23, $1982 \quad 20.39 \quad$ MAR $01,1983 \quad 19.87$ 
MAJOR COUNTY -- CONTINUED

361245098421001. LOCAL NUMBER; 20N-14W-16 BDB 1. LOCATION,--LAT 36 12'45", LONG 098 42'10", HYDROLOGIC UNIT 11100301. AQUIFER.--TERRACE DEPOSITS.

PERIOD OF RECORD.--1978 TO CURRENT YEAR.

EXTREMES FOR PERIOD OF RECORD.--HIGHEST WATER LEVEL, $52.03 \mathrm{FT}(15.86 \mathrm{M})$ BELOW LAND-SURFACE DATUM, MAR 23, 1982; LOWEST WATER LEVEL,53.39 FT(16.27 M) BELOW LAND-SURFACE DATUM, MAR 14, 1979.

WATER LEVELS IN FEET BELOW LAND SURFACE DATUM, CLIMATIC YEARS APRIL 1981 TO MARCH 1983

\begin{tabular}{|c|c|c|}
\hline & WATER & \\
\hline DATE & LEVEL & DATE \\
\hline
\end{tabular}

MAR 23, $1982 \quad 52.03 \quad$ MAR 01, $1983 \quad 53.05$

GROUND-WATER LEVELS

361035098490701. LOCAL NUMBER; 20N-15W-29 DCA 1.

LOCATION,--LAT 36 10'35", LONG 098 49'07", HYDROLOGIC UNIT 11100301.

AQUIFER.--TERRACE DEPOSITS.

PERIOD OF RECORD ..-1978 TO CURRENT YEAR.

EXTREMES FOR PERIOD OF RECORD.--HIGHEST WATER LEVEL, 33.08 FT(10.08 M) BELOW LAND-SURFACE DATUM,MAR 20, 1981; LOWEST WATER LEVEL, 35.15 FT(10.71 M) BELOW LAND-SURFACE DATUM, OCT 19, 1978.

WATER LEVELS IN FEET BELOW LAND SURFACE DATUM, CLIMATIC YEARS APRIL 1981 TO MARCH 1983

\begin{tabular}{|c|c|c|}
\hline DATE & $\begin{array}{l}\text { WATER } \\
\text { LEVEL }\end{array}$ & DATE \\
\hline
\end{tabular}

MAR 22, $1982 \quad$ Z MAR 01, $1983 \quad$ W 


\section{GROUND-WATER LEVELS}

\section{MAJOR COUNTY -- CONTINUED}

361249098515501. LOCAL NUMBER; LOCATION,--LAT 3612 '49", LONG 098 AQUIFER.--TERRACE DEPOSITS.

PERIOD OF RECORD.--1978 TO CURRENT YEAR.

EXTREMES FOR PERIOD OF RECORD.--HIGHEST WATER LEVEL,28.90 FT(8.81 M) BELOW LAND-SURFACE DATUM,MAR 16, 1981; LOWEST WATER LEVEL,31.03 FT(9.46 M) BELOW LAND-SURFACE DATUM, MAY 16, 1979.

WATER LEVELS IN FEET BELOW LAND SURFACE DATUM, CLIMATIC YEARS APRIL 1981 TO MARCH 1983

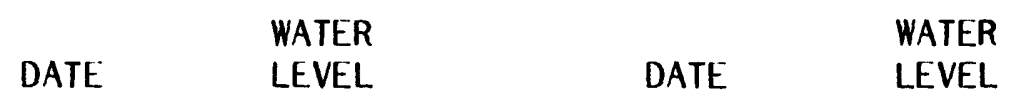
MAR 22, $1982 \quad 29.63$
MAR 01, 1983
29.31

\section{GROUND-WATER LEVELS}

361117098543101. LOCAL NUMBER; LOCATION,--LAT 3611 '17", LONG 098 AQUIFER.--TERRACE DEPOSITS.

PERIOD OF RECORD.--1978 TO CURRENT YEAR.

EXTREMES FOR PERIOD OF RECORD.--HIGHEST WATER LEVEL, 10.40 FT(3.17 M) BELOW LAND-SURFACE DATUM,MAR 1, 1983; LOWEST WATER LEVEL, 12.20 FT(3.72 M) BELOW LAND-SURFACE DATUM, SEP 20, 1978.

WATER LEVELS IN FEET BELOW LAND SURFACE DATUM, CLIMATIC YEARS APRIL 1981 TO MARCH 1983
$20 \mathrm{~N}-16 \mathrm{~W}-21 \mathrm{DCD} 1$

54'31", HYDROLOGIC UNIT 11100301.

$\begin{array}{cccc}\text { WATER } & & \\ \text { LEVEL } & \text { WATER } \\ \text { MAR 22, } 1982 & 10.94 & \text { MAR 01, 1983 } & \text { LEVEL } \\ \text { MATE } & 10.40\end{array}$




\section{GROUND-WATER LEVELS}

MAJOR COUNTY -- CONTINUED

361940098062601. LOCAL NUMBER;

$21 \mathrm{~N}-09 \mathrm{~W}-01 \mathrm{ADB} 1$.

LOCATION,--LAT 36 19'40", LONG 098 06'26", HYDROLOGIC UNIT 11150002.

AQUIFER.--TERRACE DEPOSITS.

PERIOD OF RECORD.--1978 TO CURRENT YEAR.

EXTREMES FOR PERIOD OF RECORD.--HIGHEST WATER LEVEL, $5.90 \mathrm{FT}(1.80 \mathrm{M})$ BELOW LAND-SURFACE DATUM,MAR 23, 1982; LOWEST WATER LEVEL,8.30 FT(2.53 M) BELOW LAND-SURFACE DATUM, MAR $30,1979$.

WATER LEVELS IN FEET BELOW LAND SURFACE DATUM, CLIMATIC YEARS APRIL 1981 TO MARCH 1983

$\begin{array}{lll} & \text { WATER } & \text { WATER } \\ \text { DATE } & \text { LEVEL } & \text { DATE }\end{array}$
MAR 23, 1982
5.90
FEB 28, 1983
6.21

GROUND-WATER LEVELS

361907098123501. LOCAL NUMBER; 21N-09W-06 CCC 1.

LOCATION,--LAT 36 19'07', LONG 098 12'35", HYDROLOGIC UNIT 11050002.

AQUIFER.--TERRACE DEPOSITS.

PERIOD OF RECORD.--1976 TO CURRENT YEAR.

EXTREMES FOR PERIOD OF RECORD.--HIGHEST WATER LEVEL, 22.77 FT(6.94 M) BELOW LAND-SURFACE DATUM,FEB 28, 1983; LOWEST WATER LEVEL,27.34 FT(8.33 M) BELOW LAND-SURFACE DATUM, MAR 11, 1980.

WATER LEVELS IN FEET BELOW LAND SURFACE DATUM, CLIMATIC YEARS APRIL 1981 TO MARCH 1983

$\begin{array}{lll} & \text { WATER } & \\ \text { DATE } & \text { LEVEL } & \text { WATER } \\ \text { DATE } & \text { LEVEL }\end{array}$

$\begin{array}{llll}\text { MAR 23, } 1982 & 25.08 & \text { FEB 28, } 1983 \quad 22.77\end{array}$ 
MAJOR COUNTY -- CONTINUED

361631098103501. LOCAL NUMBER; 21N-09W-20 DDD 1. LOCATION,--LAT 36 16'31", LONG 098 10'35", HYDROLOGIC UNIT 11050002.

AQUIFER. - -TERRACE DEPOSITS.

PERIOD OF RECORD.--1976 TO CURRENT YEAR.

EXTREMES FOR PERIOD OF RECORD.--HIGHEST WATER LEVEL,8.62 FT(2.63 M) BELOW LAND-SURFACE DATUM, JAN 15, 1976; LOWEST WATER LEVEL, $17.20 \mathrm{FT}(5.24 \mathrm{M})$ BELOW LAND-SURFACE DATUM, MAR 11, 1980.

WATER LEVELS IN FEET BELOW LAND SURFACE DATUM, CLIMATIC YEARS APRIL 1981 TO MARCH 1983

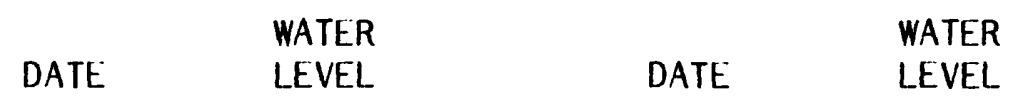

MAR 23, $1982 \quad 16.86 \quad$ FEB 28, $1983 \quad 14.80$

GROUND-WATER LEVELS

361539098093001. LOCAL NUMBER; 21N-09W-28 DDD 1.

LOCATION,--LAT 36 15'39", LONG 098 09'30", HYDROLOGIC UNIT 11050002.

AQUIFER.--TERRACE DEPOSITS.

PERIOD OF RECORD.--1976 TO CURRENT YEAR.

EXTREMES FOR PERIOD OF RECORD.--HIGHEST WATER LEVEL, 15.02 FT(4.58 M) BELOW LAND-SURFACE DATUM, JAN 13, 1976; LOWEST WATER LEVEL,27.73 FT(8.45 M) BELOW LAND-SURFACE DATUM, MAR 23, 1982.

WATER LEVELS IN FEET BELOW LAND SURFACE DATUM, CLIMATIC YEARS APRIL 1981 TO MARCH 1983

\begin{tabular}{|c|c|c|c|c|c|}
\hline & WATER & & WATER & & WATER \\
\hline DATE & LEVEL & DATE & LEVEL & DATE & LEVEL \\
\hline 23,1982 & 27.73 & FEB 28, 1983 & 21.81 & MAR 23, 1983 & 27.73 \\
\hline
\end{tabular}




\title{
GROUND-WATER LEVELS
}

\author{
MAJOR COUNTY -- CONTINUED
}

361624098120301 . LOCAL NUMBER; LOCATION,--LAT $3616^{\prime} 24^{\prime \prime}$, LONG 098 AQUIFER.--TERRACE DEPOSITS. PERIOD OF RECORD.--1976 TO CURRENT YEAR. EXTREMES FOR PERIOD OF RECORD.--HIGHEST WATER LEVEL, $7.39 \mathrm{FT}(2.25 \mathrm{M})$ BELOW LAND-SURFACE DATUM, JAN 15, 1976; LOWEST WATER LEVEL,8.58 FT(2.62 M) BELOW LAND-SURFACE DATUM, MAR 29, 1979.

WATER LEVELS IN FEET BELOW LAND SURFACE DATUM, CLIMATIC YEARS APRIL 1981 TO MARCH 1983
$21 \mathrm{~N}-09 \mathrm{~W}-30$ ABB 1. 12 '03", HYDROLOGIC UNIT 11050002.

WATER LEVELS IN

$\begin{array}{lll} & \text { WATER } & \\ \text { DATE } & \text { LEVEL } & \text { WATER } \\ & \text { DATE } & \text { LEVEL }\end{array}$
MAR 23, 1982
8.53
FEB 28, 1983
7.96

GROUND-WATER LEVELS

361723098164401. LOCAL NUMBER; $21 \mathrm{~N}-10 \mathrm{~W}-16$ CCD 1 LOCATION,--LAT 36 17'23", LONG 098 16'44", HYDROLOGIC UNIT 11050002. AQUIFER.--TERRACE DEPOSITS. PERIOD OF RECORD.--1976 TO CURRENT YEAR. EXTREMES FOR PERIOD OF RECORD.--HIGHEST WATER LEVEL, 16.21 FT(4.94 M) BELOW LAND-SURFACE DATUM, MAR 1, 1983; LOWEST WATER LEVEL,21.69 FT(6.61 M) BELOW LAND-SURFACE DATUM, MAR 29, 1979.

WATER LEVELS IN FEET BELOW LAND SURFACE DATUM, CLIMATIC YEARS APRIL 1981 TO MARCH 1983

\begin{tabular}{|c|c|c|}
\hline $\mathrm{D} A \mathrm{TC}$ & WATER & \\
\hline D & LEVEL & DATE \\
\hline
\end{tabular}
MAR 23, 1982
21.10
MAR 01, 1983
16.21 
WATER LEVELS IN FEET BELOW LAND SURFACE DATUM, CLIMATIC YEARS APRIL 1981 TO MARCH 1983

\begin{abstract}
MAJOR COUNTY -- CONTINUED
361631098155601. LOCAL NUMBER; LOCATION,--LAT $3616^{\prime} 31 "$, LONG 098 AQUIFER.--TERRACE DEPOSITS.

PERIOD OF RECORD.--1976 TO CURRENT YEAR.

EXTREMES FOR PERIOD OF RECORD.--HIGHEST WATER LEVEL, $15.92 \mathrm{FT}(4.85 \mathrm{M})$ BELOW LAND-SURFACE DATUM, MAR 1, 1983; LOWEST WATER LEVEL,23.50 FT(7.16 M) BELOW LAND-SURFACE DATUM, JAN S, 1978.

$21 \mathrm{~N}-10 \mathrm{~W}-21$ DDD 1.

15'56", HYDROLOGIC UNIT 11050002.
\end{abstract}
WATER WATER
DATE LEVEL DATE LEVEL
MAR 23, $1982 \quad 17.24 \quad$ MAR 01, $1983 \quad 15.92$
GROUND-WATER LEVELS

362045098183101. LOCAL NUMBER; 22N-10W-31 BAA 1. LOCATION,--LAT 36 20'45", LONG 098 18'31", HYDROLOGIC UNIT 11050002. AQUIFER.--TERRACE DEPOSITS.

PERIOD OF RECORD.--1979 TO CURRENT YEAR.

EXTREMES FOR PERIOD OF RECORD.--HIGHEST WATER LEVEL,21.08 FT(6.43 M) BELOW LAND-SURFACE DATUM,MAR 2, 1983; LOWEST WATER LEVEL,24.90 FT(7.59 M) BELOW LAND-SURFACE DATUM, MAR 29, 1979.

WATER LEVELS IN FEET BELOW LAND SURFACE DATUM, CLIMATIC YEARS APRIL 1981 TO MARCH 1983

\begin{tabular}{|c|c|c|}
\hline & WATER & \\
\hline DATE & LEVEL & DATE \\
\hline
\end{tabular}

MAR 23, $1982 \quad 21.89 \quad$ MAR 02, $1983 \quad 21.08$ 
MAJOR COUNTY -- CONTINUED

362210098201301. LOCAL NUMBER; 22N-11W-23 ADD 1. LOCATION,--LAT 36 22'10", LONG 098 20'13", HYDROLOGIC UNIT 11050002.

AQUIFER.--TERRACE DEPOSITS.

PERIOD OF RECORD.--1976 TO CURRENT YEAR.

EXTREMES FOR PERIOD OF RECORD.--HIGHEST WATER LEVEL, $24.50 \mathrm{FT}(7.47 \mathrm{M})$ BELOW LAND-SURFACE DATUM, JAN 5, 1978; LOWEST WATER LEVEL,28.01 FT(8.54 M) BELOW LAND-SURFACE DATUM, MAR 23, 1982.

WATER LEVELS IN FEET BELOW LAND SURFACE DATUM, CLIMATIC YEARS APRIL 1981 TO MARCH 1983

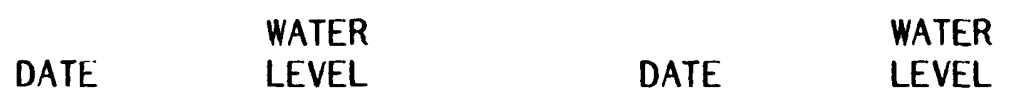

MAR 23, $1982 \quad 28.01 \quad$ MAR 02, $1983 \quad 27.58$

GROUND-WATER LEVELS

362045098235801. LOCAL NUMBER; 22N-11W-32 BAA 1. LOCATION,--LAT 36 20'45", LONG 098 23'58", HYDROLOGIC UNIT 11050002.

AQUIFER.--TERRACE DEPOSITS.

PERIOD OF RECORD.--1976 TO CURRENT YEAR.

EXTREMES FOR PERIOD OF RECORD.--HIGHEST WATER LEVEL, 16.68 FT(5.08 M) BELOW LAND-SURFACE DATUM, JAN 15, 1976; LOWEST WATER LEVEL,20.64 FT(6.29 M) BELOW LAND-SURFACE DATUM, MAR 23, 1982.

WATER LEVELS IN FEET BELOW LAND SURFACE DATUM, CLIMATIC YEARS APRIL 1981 TO MARCH 1983

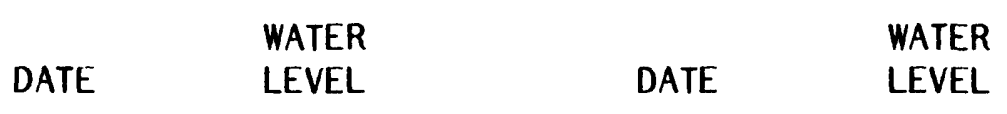

MAR 23, $1982 \quad 20.64 \quad$ MAR 02, $1983 \quad 20.52$ 
MAJOR COUNTY -- CONTINUED

362716098243001. LOCAL NUMBER;

LOCATION,--LAT 36 27'16", LONG 098 24'30", HYDROLOGIC UNIT 11050001. AQUIFER.--TERRACE DEPOSITS. PERIOD OF RECORD.--1976 TO CURRENT YEAR.

EXTREMES FOR PERIOD OF RECORD.--HIGHEST WATER LEVEL, $5.00 \mathrm{FT}(1.52 \mathrm{M})$ BELOW LAND-SURFACE DATUM,MAR 23, 1982; LOWEST WATER LEVEL,9.47 FT(2.89 M) BELOW LAND-SURFACE DATUM, JAN 5, 1978.

WATER LEVELS IN FEET BELOW LAND SURFACE DATUM, CLIMATIC YEARS APRIL 1981 TO MARCH 1983
$23 N-11 W-19$ DAA 1
DATE WATER

MAR 23, $1982 \quad 5.00$ LEVEL

DATE

WATER

LEVEL

MAR 02, $1983 \quad 5.59$ 
GROUND-WATER LEVELS

MARSHALL COUNTY

335621096380301. LOCAL NUMBER; 07S-06E-24 BBA 1. LOCATION,--LAT $3356^{\prime} 21^{\prime \prime}$, LONG $09638^{\prime} 03^{\prime \prime}$, HYDROLOGIC UNIT 11130210. AQUIFER.--TERRACE DEPOSITS.

PERIOD OF RECORD.-- 1978 TO CURRENT YEAR.

EXTREMES FOR PERIOD OF RECORD.--HIGHEST WATER LEVEL, 111.35 FT(33.94 M) BELOW LAND-SURFACE DATUM,FEB 26, 1980; LOWEST WATER LEVEL,118.61 FT(36.15 M) BELOW LAND-SURFACE DATUM, MAR 7, 1983.

WATER LEVELS IN FEET BELOW LAND SURFACE DATUM, CLIMATIC YEARS APRIL 1981 TO MARCH 1983

\begin{tabular}{|c|c|c|}
\hline & WATER & \\
\hline DATE & LEVEL & DATE \\
\hline
\end{tabular}

MAR 09, $1982 \quad 116.84 \quad$ MAR 07, $1983 \quad 118.61$ 


\section{GRDUND-WATER LEVELS}

MAYES COUNTY

361132095130901. LDCAL NUMBER;

$20 \mathrm{~N}-20 \mathrm{E}-19$ CCD 1.

LOCATION,--LAT 3611 '32", LONG 095

$13^{\prime}$ '09", HYDROLOGIC UNIT 11070209.

AQUIFER.--BOONE FORMATION.

PERIOD OF RECORD.--1979 TO CURRENT YEAR.

EXTREMES FOR PERIOD OF RECORD.--HIGHEST WATER LEVEL, $34.25 \mathrm{FT}(10.44 \mathrm{M})$ BELOW LAND-SURFACE DATUM, MAR 29, 1982; LOWEST WATER LEVEL, $37.47 \mathrm{FT}(11.42 \mathrm{M})$ BELOW LAND-SURFACE DATUM, MAR 10, 1981.

WATER LEVELS IN FEET BELOW LAND SURFACE DATUM, CLIMATIC YEARS APRIL 1981, TO MARCH 1983

\begin{tabular}{|c|c|}
\hline & WATER \\
\hline ATE & LEVEL \\
\hline
\end{tabular}

MAR 29, $1982 \quad 34.55 \quad$ MAR $11,1983 \quad 34.25$

GROUND-WATER LEVELS

361040095104601. LOCAL NUMBER; 2ON-20E-28 CDD 1.

LOCATION,--LAT 36 10'40", LONG 095 10'46", HYDROLOGIC UNIT 11070209.

AQUIFER .--BOONE FORMATION.

PERIOD OF RECORD.--1979 TO CURRENT YEAR.

EXTREMES FOR PERIOD OF RECORD.--HIGHEST WATER LEVEL, $16.60 \mathrm{FT}(5.06 \mathrm{M})$ BELOW LAND-SURFACE DATUM,FEB 26, 1980; LOWEST WATER LEVEL,25.40 FT(7.74 M) BELOW LAND-SURFACE DATUM, MAR 8, 1979.

WATER LEVELS IN FEET BELOW LAND SURFACE DATUM, CLIMATIC YEARS APRIL 1981 TO MARCH 1983

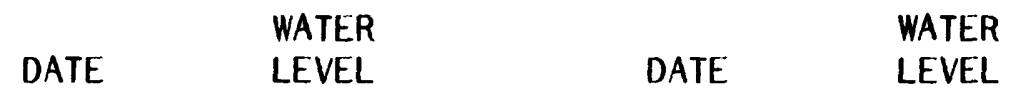

MAR 29, $1982 \quad 20.94 \quad$ MAR 11, $1983 \quad 0$




\section{GROUND-WATER LEVELS}

MAYES COUNTY -- CONTINUED

361547095151901. LOCAL NUMBER;

$21 \mathrm{~N}-19 \mathrm{E}-35$ BAB 1.

LOCATION,--LAT 36 15'47", LONG 095 15'19", HYDROLOGIC UNIT 11070209.

AQUIFER.--MOOREF IELD FORMATION.

PERIOD OF RECORD.-- 1977 TO CURRENT YEAR.

EXTREMES FOR PERIOD OF RECORD.--HIGHEST WATER LEVEL, 15.46 FT(4.71 M) BELOW LAND-SURFACE DATUM, MAR 29, 1982; LOWEST WATER LEVEL,27.86 FT(8.49 M) BELOW LAND-SURFACE DATUM, MAR. 22, 1977.

WATER LEVELS IN FEET BELOW LAND SURFACE DATUM, CLIMATIC YEARS APRIL 1981 TO MARCH 1983

$\begin{array}{lll} & \text { WATER } & \text { WATER } \\ \text { DATE } & \text { LEVEL } & \text { DATE }\end{array}$

MAR 29, $1982 \quad 15.46 \quad$ MAR $11,1983 \quad 17.50$ 


\section{GROUND-WATER LEVELS}

MURRAY COUNTY

343208096494101. LOCAL NUMBER; 01N-04E-25 AAA 1. LOCATION,--LAT 34 32'08", LONG 096 49'41", HYDROLOGIC UNIT 11130304. AQUIFER.--ARBUCKLE FORMATION. PERIOD OF RECORD.--1976 TO CURRENT YEAR. EXTREMES FOR PERIOD OF RECORD.--HIGHEST WATER LEVEL, $80.47 \mathrm{FT}(24.53 \mathrm{M})$ BELOW LAND-SURFACE DATUM,MAR 8, 1982; LOWEST WATER LEVEL,133.48 FT(40.68 M) BELOW LAND-SURFACE DATUM, MAR 3, 1981.

WATER LEVELS IN FEET BELOW LAND SURFACE DATUM, CLIMATIC YEARS APRIL 1981 TO MARCH 1983

$\begin{array}{lll} & \text { WATER } & \\ \text { DATE } & \text { LEVEL } & \text { WATER } \\ \text { LEVEL }\end{array}$

MAR 08, $1982 \quad 80.47 \quad$ MAR 07, $1983 \quad 94.59$




\section{GROUND-WATER LEVELS}

\section{MUSKOGEE COUNTY}

352143095131301. LOCAL NUMBER;

10N-20E-07 AAA 1.

LOCATION,--LAT $3521^{\prime} 43^{\prime \prime}$, LONG 095 13'13", HYDROLOGIC UNIT 11090204. AQUIFER.--ALLUVIUM.

PERIOD OF RECORD.--1981 TO CURRENT YEAR.

EXTREMES FOR PERIOD OF RECORD.--HIGHEST WATER LEVEL, $3.00 \mathrm{FT}(.91 \mathrm{M})$ BELOW LAND-SURFACE DATUM,MAR 1, 1983; LOWEST WATER LEVEL,14.68 FT(4.47 M) BELOW LAND-SURFACE DATUM, MAR 10, 1981.

WATER LEVELS IN FEET BELOW LAND SURFACE DATUM, CLIMATIC YEARS APRIL 1981 TO MARCH 1983

$\begin{array}{lll} & \text { WATER } & \text { WATER } \\ \text { DATE } & \text { LEVEL } & \text { DATE }\end{array}$

MAR 23, $1982 \quad 6.22 \quad$ MAR 01, $1983 \quad 3.00$


GROUND-WATER LEVELS

NOBLE COUNTY

361651097165401. LOCAL NUMBER; 21N-01W-23 CBB 1.

LOCATION,--LAT 36 16'51", LONG 097 16'54", HYDROLOGIC UNIT 11060006.

AQUIFER.--GARBER SANDSTONE.

PERIOD OF RECORD.-- 1977 TO CURRENT YEAR.

EXTREMES FOR PERIOD OF RECORD.--HIGHEST WATER LEVEL, 15.95 FT(4.86 M) BELOW LAND-SURFACE DATUM, APR 22, 1977; LOWEST WATER LEVEL, $18.82 \mathrm{FT}(5.74 \mathrm{M})$ BELOW LAND-SURFACE DATUM, JAN $31,1980$.

WATER LEVELS IN FEET BELOW LAND SURFACE DATUM, CLIMATIC YEARS APRIL 1981 TO MARCH 1983

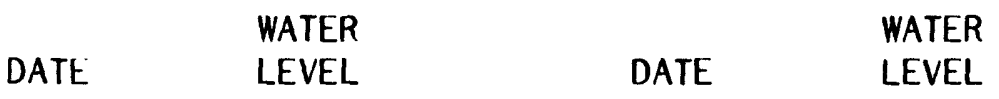

MAR 04, $198218.03 \quad$ MAR 09, $1983 \quad 17.08$




\section{GROUND-WATER LEVELS}

NOWATA COUNTY

365439095270101. LOCAL NUMBER; 28N-17E-14 ADD 1.

LOCATION,--LAT 36 54'39", LONG 095 27'01", HYDROLOGIC UNIT 11070103.

AQUIFER.--OGALLALA.

PERIOD OF RECORD.--1979 TO CURRENT YEAR.

EXTREMES FOR PERIOD OF RECORD.--HIGHEST WATER LEVEL, 3.97 FT(1.21 M) BELOW LAND-SURFACE DATUM, MAR 30, 1982; LOWEST WATER LEVEL,6.95 FT(2.12 M) BELOW LAND-SURFACE DATUM, MAR 14, 1979.

WATER LEVELS IN FEET BELOW LAND SURFACE DATUM, CLIMATIC YEARS APRIL 1981 TO MARCH 1983

\begin{tabular}{|c|c|}
\hline & $\begin{array}{l}\text { WATER } \\
\text { LEVEL }\end{array}$ \\
\hline
\end{tabular}

MAR $30,1982 \quad 3.97 \quad$ MAR 04, $1983 \quad 4.64$

GROUND-WATER LEVELS

365321095282901. LOCAL NUMBER; 28N-17E-22 DCC 1. LOCATION,--LAT 36 53'21", LONG 095 28'29", HYDROLOGIC UNIT 11070103. AQUIFER.--OGALLALA.

PERIOD OF RECORD.--1979 TO CURRENT YEAR.

EXTREMES FOR PERIOD OF RECORD.--HIGHEST WATER LEVEL,..17 FT(.05 M) BELOW LAND-SURFACE DATUM, MAR 14, 1979; LOWEST WATER LEVEL,3.76 FT(1.15 M) BELOW LAND-SURFACE DATUM, MAR 12, 1981.

WATER LEVELS IN FEET BELOW LAND SURFACE DATUM, CLIMATIC YEARS APRIL 1981 TO MARCH 1983

$\begin{array}{lll} & \text { WATER } & \text { WATER } \\ \text { DATE } & \text { LEVEL } & \text { DATE }\end{array}$

$\begin{array}{llll}\text { MAR } 30,1982 & 1.12 & \text { MAR 04, } 1983 & 1.09\end{array}$


NOWATA COUNTY -- CONTINUED

365903095390001. LOCAL NUMBER; LOCATION,--LAT 36 59'03", LONG 095 39'00", HYDROLOGIC UNIT 11070103. AQUIFER.--FRANCIS FORMATION. PERIOD OF RECORD.--1979 TO CURRENT YEAR. EXTREMES FOR PERIOD OF RECORD.--HIGHEST WATER LEVEL,2.66 FT(.81 M) BELOW LAND-SURFACE DATUM,MAR 4, 1983; LOWEST WATER LEVEL, 11.83 FT(3.61 M) BELOW LAND-SURFACE DATUM, MAR 12, 1981.

WATER LEVELS IN FEET BELOW LAND SURFACE DATUM, CLIMATIC YEARS APRIL 1981 TO MARCH 1983

\begin{tabular}{crrrr} 
DATE & WATER & & \multicolumn{1}{c}{ WATER } \\
LEVEL & DATE & \multicolumn{1}{c}{ LEVEL } \\
MAR 30, 1982 & 4.61 & MAR 04, 1983 & 2.66
\end{tabular}


OKFUSKEE COUNTY

353532096353601. LOCAL NUMBER;

13N-07E-20 BBC 1.

LOCATION,--LAT 35 35'32", LONG 096

AQUIFER.--VAMOOSA.

PERIOD OF RECORD.--1977 TO CURRENT YEAR.

EXTREMES FOR PERIOD OF RECORD.--HIGHEST WATER LEVEL, 26.96 FT (8.22 M) BELOW LAND-SURFACE DATUM,FEB 21, 1980; LOWEST WATER LEVEL,46.50 FT(14.17 M) BELOW LAND-SURFACE DATUM, JAN 29, 1981.

WATER LEVELS IN FEET BELOW LAND SURFACE DATUM, CLIMATIC YEARS APRIL 1981 T0 MARCH 1983

$\begin{array}{ll} & \text { WATER } \\ \text { DATE } & \text { LEVEL }\end{array}$

MAR 02, 1982

W

GROUND-WATER LEVELS

353354096352901. L.OCAL NUMBER; 13N-07E-32 BBA 1.

LOCATION,--LAT 35 33'54", LONG 096 35'29", HYDROLOGIC UNIT 11100303.

AQUIFER .--VAMOOSA.

PERIOD OF RECORD.--1982 TO CURRENT YEAR.

EXTREMES FOR PERIOD OF RECORD.--HIGHEST WATER LEVEL, $12.20 \mathrm{FT}(3.72 \mathrm{M})$ BELOW LAND-SURFACE DATUM,MAR 24, 1983; LOWEST WATER LEVEL, 12.78 FT(3.90 M) BELOW LAND-SURFACE DATUM, MAR 2, 1982.

WATER LEVELS IN FEET BELOW LAND SURFACE DATUM, CLIMATIC YEARS APRIL 1981 TO MARCH 1983

\begin{tabular}{|c|c|c|}
\hline & WATER & \\
\hline DATE & LEVEL & DATE \\
\hline
\end{tabular}

MAR $02,1982 \quad 12.78 \quad$ MAR $24,1983 \quad 12.20$ 
GROUND-WATER LEVELS

OKLAHOMA COUNTY

352749097260101. LOCAL NUMBER; LOCATION, --LAT $3527 \cdot 49 "$ ", LONG 097 AQUIFER.--GARBER SANDSTONE.

PERIOD OF RECORD.--1983 TO CURRENT YEAR.

EXTREMES FOR PERIOD OF RECORD.--HIGHEST WATER LEVEL,244.61 FT(74.56 M) BELOW LAND-SURFACE DATUM,FEB 25, 1983; LOWEST WATER LEVEL,244.61 FT(74.56 M) BELOW LAND-SURFACE DATUM, FEB 25, 1983.

WATER LEVELS IN FEET BELOW LAND SURFACE DATUM, CLIMATIC YEARS APRIL 1981 TO MARCH 1983

$\begin{array}{ll}\text { WATER } \\ \text { DATE } & \text { LEVEL }\end{array}$

FEB 25, $1983 \quad 244.61$

GROUND-WATER LEVELS

352749097192301. LOCAL NUMBER; 11N-01W-05 ABA 1. LOCATION,--LAT 35 27'49", LONG 097 19'23", HYDROLOGIC UNIT 11100302. AQUIFER .--GARBER SANDSTONE.

PERIOD OF RECORD.--1978 TO CURRENT YEAR.

EXTREMES FOR PERIOD OF RECORD.--HIGHEST WATER LEVEL, $156.67 \mathrm{FT}(47.75 \mathrm{M})$ BELOW LAND-SURFACE DATUM,MAR 20, 1978; LOWEST WATER LEVEL,200.00 FT(60.96 M) BELOW LAND-SURFACE DATUM, FEB 7, 1981.

WATER LEVELS IN FEET BELOW LAND SURFACE DATUM, CLIMATIC YEARS APRIL 1981 TO MARCH 1983

\begin{tabular}{|c|c|c|}
\hline & WATER & \\
\hline DATE & LEVEL & DATE \\
\hline
\end{tabular}
MAR 11, 1982
Z FEB 25,1983
0 


\section{GROUND-WATER LEVELS}

OKLAHOMA COUNTY -- CONTINUED

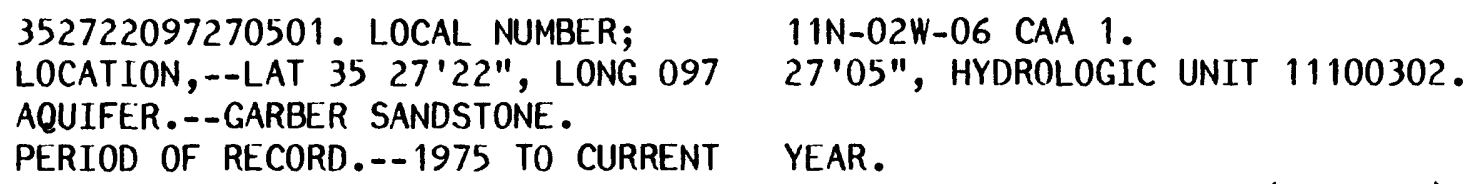

WATER LEVELS IN FEET BELOW LAND SURFACE DATUM, CLIMATIC YEARS APRIL 1981 TO MARCH 1983

$\begin{array}{lll} & \text { WATER } & \text { WATER } \\ \text { DATE } & \text { LEVEL } & \text { DATE }\end{array}$

MAR 11, $1982 \quad Z \quad$ FEB 25, $1983 \quad 234.84$

GROUND-WATER LEVELS

352637097253701. LOCAL NUMBER; 11N-02W-08 ADC 1. LOCATION,--LAT 35 26'37", LONG 097 25'37", HYDROLOGIC UNIT 11100302. AQUIFER.--GARBER SANDSTONE. PERIOD OF RECORD.--1975 TO CURRENT YEAR. EXTREMES FOR PERIOD OF RECORD.--HIGHEST WATER LEVEL, $156.00 \mathrm{FT}(47.55 \mathrm{M})$ BELOW LAND-SURFACE DATUM,FEB 3, 1976; LOWEST WATER LEVEL, $330.00 \mathrm{FT}(100.58 \mathrm{M})$ BELOW LAND-SURFACE DATUM, OCT 10, 1975.

WATER LEVELS IN FEET BELOW LAND SURFACE DATUM, CLIMATIC YEARS APRIL 1981 TO MARCH 1983

$\begin{array}{lll} & \text { WATER } & \\ \text { DATE } & \text { LEVEL } & \text { WATER } \\ \text { LEVEL }\end{array}$

MAR 11, $1982195.00 \quad$ FEB 25, $1983 \quad 240.72$ 
OKLAHOMA COUNTY -- CONTINUED

352637097234601. LOCAL NUMBER; 11N-02W-10 ACC 1.

LOCATION,--LAT 35 26'37", LONG 097 23'46", HYDROLOGIC UNIT 11100302.

AQUIFER.--GARBER SANDSTONE.

PERIOD OF RECORD.--1979 TO CURRENT YEAR.

EXTREMES FOR PERIOD OF RECORD.--HIGHEST WATER LEVEL,235.00 FT(71.63 M) BELOW LAND-SURFACE DATUM,APR 3, 1980; LOWEST WATER LEVEL, 301.00 FT(91.74 M) BELOW LAND-SURFACE DATUM, FEB 25, 1983.

WATER LEVELS IN FEET BELOW LAND SURFACE DATUM, CLIMATIC YEARS APRIL 1981 TO MARCH 1983

\begin{tabular}{|c|c|c|}
\hline & WATER & \\
\hline TF & LEVEL & DATE \\
\hline
\end{tabular}

MAR 11, $1982 \quad Z \quad$ FEB 25, $1983 \quad 301.00$

GROUND-WATER LEVELS

352630097230601. LOCAL NUMBER; 11N-02W-11 CBA 1.

LOCATION,--LAT 35 26'30", LONG 097 23'06", HYDROLOGIC UNIT 11100302.

AQUIFER.--GARBER SANDSTONE.

PERIOD OF RECORD.--1975 TO CURRENT YEAR.

EXTREMES FOR PERIOD OF RECORD.--HIGHEST WATER LEVEL,202.90 FT(61.84 M) BELOW LAND-SURFACE DATUM, JAN 4, 1977; LOWEST WATER LEVEL, 323.50 FT(98.60 M) BELOW LAND-SURFACE DATUM, AUG 19, 1975.

WATER LEVELS IN FEET BELOW LAND SURFACE DATUM, CLIMATIC YEARS APRIL 1981 TO MARCH 1983

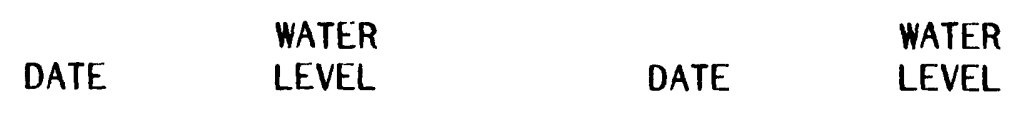

MAR 11, $1982 \quad Z \quad$ MAR 09, $1983 \quad 317.30$ 
GROUND-WATER LEVELS

OKLAHOMA COUNTY -- CONTINUED

352604097241801. LOCAL NUMBER; 11N-02W-15 BBB 1.

LOCATION,--LAT 35 26'04", LONG 097 24'18", HYDROLOGIC UNIT 11100302.

AQUIFER.--GARBER SANDSTONE.

PERIOD OF RECORD.--1975 TO CURRENT YEAR.

EXTREMES FOR PERIOD OF RECORD.--HIGHEST WATER LEVEL,213.00 FT(64.92 M) BELOW LAND-SURFACE DATUM,JAN 4, 1977; LOWEST WATER LEVEL,298.50 FT(90.98 M) BELOW LAND-SURFACE DATUM, MAR 9, 1983.

WATER LEVELS IN FEET BELOW LAND SURFACE DATUM, CLIMATIC YEARS APRIL 1981 TO MARCH 1983

\begin{tabular}{cccc}
\multicolumn{1}{c}{ WATER } & & WATER \\
DATE & LEVEL & DATE & LEVEL \\
MAR 11, 1982 & 250.99 & MAR 09, 1983 & 298.50
\end{tabular}

GROUND-WATER LEVELS

352452097222601. LOCAL NUMBER; 11N-02W-23 ADC 1.

LOCATION,--LAT 35 24'52", LONG 097 22'26", HYDROLOGIC UNIT 11100302.

AQUIFER.--GARBER SANDSTONE.

PERIOD OF RECORD.--1975 TO CURRENT YEAR.

EXTREMES FOR PERIOD OF RECORD.--HIGHEST WATER LEVEL, 251.02 FT(76.51 M) BELOW LAND-SURFACE DATUM, MAR 11, 1982; LOWEST WATER LEVEL,273.56 FT(83.38 M) BELOW LAND-SURFACE DATUM, MAR 9, 1983.

WATER LEVELS IN FEET BELOW LAND SURFACE DATUM, CLIMATIC YEARS APRIL 1981 TO MARCH 1983

$\begin{array}{lll} & \text { WATER } & \\ \text { DATE } & \text { LEVEL } & \text { WATER } \\ \text { LATE } & \text { LEVEL }\end{array}$

MAR 11, $1982 \quad 251.02 \quad$ MAR 09, $1983 \quad 273.56$ 
352426097231401. LOCAL NUMBER; LOCATION,--LAT 35 24'26", LONG 097 AQUIFER.--GARBER SANDSTONE.

PERIOD OF RECORD.--1976 TO CURRENT

EXTREMES FOR PERIOD OF RECORD.--HIGHEST WATER LEVEL,246.00 FT(74.98 M) BELOW LAND-SURFACE DATUM,FEB 3, 1976; LOWEST WATER LEVEL,293.50 FT(89.46 M) BELOW LAND-SURFACE DATUM, MAR 13, 1979.

WATER LEVELS IN FEET BELOW LAND SURFACE DATUM, CLIMATIC YEARS APRIL 1981 TO MARCH 1983

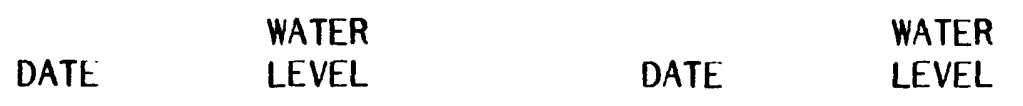

MAR 11, $1982 \quad 273.30 \quad$ MAR 09, $1983 \quad P$
GROUND-WATER LEVELS

352630097323801. LOCAL NUMBER; 11N-03W-08 CBA 1. LOCATION,--LAT 35 26'30", LONG 097 32'38", HYDROLOGIC UNIT 11100302.

AQUIFER .--GARBER SANDSTONE.

PERIOD OF RECORD.--1975 TO CURRENT YEAR.

EXTREMES FOR PERIOD OF RECORD.--HIGHEST WATER LEVEL,260.00 FT(79.25 M) BELOW LAND-SURFACE DATUM,DEC 16, 1976; LOWEST WATER LEVEL,317.75 FT(96.85 M) BELOW LAND-SURFACE DATUM, FEB 16, 1983.

WATER LEVELS IN FEET BELOW LAND SURFACE DATUM, CLIMATIC YEARS APRIL 1981 TO MARCH 1983
MAR 10, $1982 \quad 290.00$
FEB $16,1983 \quad 317.75$ 
OKLAHOMA COUNTY -- CONTINUED

352519097351701. LOCAL NUMBER; 11N-04W-14 DCD 1. LOCATION,--LAT 35 25'19", LONG 097 35'17", HYDROLOGIC UNIT 11100302. AQUIFER.--GARBER SANDSTONE.

PERIOD OF RECORD.--1979 TO CURRENT YEAR.

EXTREMES FOR PERIOD OF RECORD.--HIGHEST WATER LEVEL,289.00 FT(88.09 M) BELOW LAND-SURFACE DATUM, MAR 15, 1979; LOWEST WATER LEVEL, $447.00 \mathrm{FT}(136.25 \mathrm{M})$ BELOW LAND-SURFACE DATUM, FEB 24, 1983.

WATER LEVELS IN FEET BELOW LAND SURFACE DATUM, CLIMATIC YEARS APRIL 1981 TO MARCH 1983

\begin{tabular}{|c|c|}
\hline & WATER \\
\hline DATE & LEVEL \\
\hline
\end{tabular}

MAR 09, $1982 \quad 420.00 \quad$ FEB 24, $1983 \quad 447.00$

GROUND-WATER LEVELS

352604097370901. LOCAL NUMBER; 11N-04W-16 AAA 1.

LOCATION,--LAT 35 26'04", LONG 097 37'09", HYDROLOGIC UNIT 11100302.

AQUIFER.--GARBER SANDSTONE.

PERIOD OF RECORD.--1980 TO CURRENT YEAR.

EXTREMES FOR PERIOD OF RECORD.--HIGHEST WATER LEVEL, $319.00 \mathrm{FT}(97.23 \mathrm{M})$ BELOW LAND-SURFACE DATUM,APR 3, 1980; LOWEST WATER LEVEL, $460.00 \mathrm{FT}(140.21 \mathrm{M})$ BELOW LAND-SURFACE DATUM, MAR 25, 1981.

WATER LEVELS IN FEET BELOW LAND SURFACE DATUM, CLIMATIC YEARS APRIL 1981 TO MARCH 1983

\begin{tabular}{|c|c|}
\hline & WATER \\
\hline DATE & LEVEL \\
\hline
\end{tabular}

MAR 09, $1982 \quad 445.00 \mathrm{R} \quad$ FEB 22, $1983 \quad 436.00$ 


\section{GROUND-WATER LEVELS}

OKLAHOMA COUNTY -- CONTINUED

352420097344601. LOCAL NUMBER; 11N-04W-25 BBA 1. LOCATION,--LAT 35 24'20", LONG 097 34'46", HYDROLOGIC UNIT 11100302. AQUIFER .--GARBER SANDSTONE.

PERIOD OF RECORD.--1979 TO CURRENT YEAR.

EXTREMES FOR PERIOD OF RECORD.--HIGHEST WATER LEVEL, $315.00 \mathrm{FT}(96.01 \mathrm{M})$ BELOW LAND-SURFACE DATUM,MAR 9, 1982; LOWEST WATER LEVEL,440.00 FT(134.11 M) BELOW LAND-SURFACE DATUM, FEBB 23, 1983.

WATER LEVELS IN FEET BELOW LAND SURFACE DATUM, CLIMATIC YEARS APRIL 1981 TO MARCH 1983

$\begin{array}{lll} & \text { WATER } & \text { WATER } \\ \text { DATE } & \text { LEVEL } & \text { DATE }\end{array}$

MAR 09, $1982 \quad 315.00 \mathrm{R} \quad$ FEB 23, $1983 \quad 440.00$

GROUND-WATER LEVELS

353045097181201. LOCAL NUMBER; 12N-01W-16 DAC 1.

LOCATION,--LAT 35 30'45", LONG 097 18'12", HYDROLOGIC UNIT 11100302.

AQUIFER.--GARBER SANDSTONE.

PERIOD OF RECORD.--1975 TO CURRENT YEAR.

EXTREMES FOR PERIOD OF RECORD.--HIGHEST WATER LEVEL, $126.90 \mathrm{FT}(38.68 \mathrm{M})$ BELOW LAND-SURFACE DATUM,MAR 21, 1978; LOWEST WATER LEVEL,222.73 FT(67.89 M) BELOW LAND-SURFACE DATUM, FEB 16, 1983.

WATER LEVELS IN FEET BELOW LAND SURFACE DATUM, CLIMATIC YEARS APRIL 1981 TO MARCH 1983

$\begin{array}{lll} & \text { WATER } & \text { WATER } \\ \text { DATE } & \text { LEVEL } & \text { DATE }\end{array}$

MAR 09, $1982 \quad \mathrm{Z} \quad$ FEB $16,1983 \quad 222.73 \mathrm{R}$ 
OKLAHOMA COUNTY -- CONTINUED

352907097194701. LOCAL NUMBER; 12N-01W-29 CAB 1. LOCATION,--LAT 35 29'07", LONG 097 19'47", HYDROLOGIC UNIT 11100302.

AQUIFER.--GARBER SANDSTONE.

PERIOD OF RECORD.--1975 TO CURRENT YEAR.

EXTREMES FOR PERIOD OF RECORD.--HIGHEST WATER LEVEL, 10.31 FT(3.14 M) BELOW LAND-SURFACE DATUM,FEB 28, 1983; LOWEST WATER LEVEL, $165.00 \mathrm{FT}(50.29 \mathrm{M})$ BELOW LAND-SURFACE DATUM, JULY 18, 1975.

WATER LEVELS IN FEET BELOW LAND SURFACE DATUM, CLIMATIC YEARS APRIL 1981 TO MARCH 1983

$\begin{array}{lll} & \text { WATER } & \text { WATER } \\ \text { DATE } & \text { LEVEL } & \text { DATE }\end{array}$

$\begin{array}{llll}\text { MAR } 31,1982 & Z & \text { FEB } 28,1983 & 10.31\end{array}$

GROUND-WATER LEVELS

353117097221001. LOCAL NUMBER; 12N-02W-13 BBB 1. LOCATION,--LAT $3533^{\prime} 17^{\prime \prime}$, LONG 097 22'10", HYDROLOGIC UNIT 11100302. AQUIFER.--GARBER SANDSTONE. PERIOD OF RECORD.--1975 TO CURRENT YEAR. EXTREMES FOR PERIOD OF RECORD.--HIGHEST WATER LEVEL, 31.80 FT(9.69 M) BELOW LAND-SURFACE DATUM,MAR 23, 1978; LOWEST WATER LEVEL,69.80 FT(21.28 M) BELOW LAND-SURFACE DATUM, JULY 30, 1975.

WATER LEVELS IN FEET BELOW LAND SURFACE DATUM, CLIMATIC YEARS APRIL 1981 TO MARCH 1983

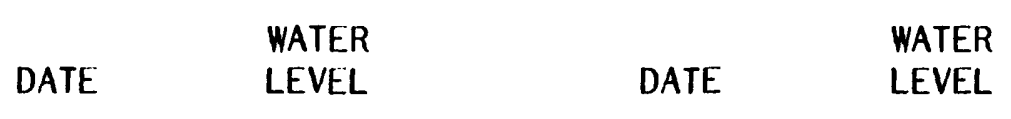
MAR 31, 1982
Z
FEB 18, $1983 \quad 47.55$ 
OKLAHOMA COUNTY -- CONTINUED

352940097225001. LOCAL NUMBER; 12N-02W-23 CDD 1.

LOCATION,--LAT 35 29'40", LONG 097 22'50", HYDROLOGIC UNIT 11100302.

AQUIFER.--GARBER SANDSTONE.

PERIOD OF RECORD..-1979 TO CURRENT YEAR.

EXTREMES FOR PERIOD OF RECORD.--HIGHEST WATER LEVEL,35.72 FT(10.89 M) BELOW LAND-SURFACE DATUM,APR 4, 1980; LOWEST WATER LEVEL,42.80 FT(13.05 M) BELOW LAND-SURFACE DATUM, MAR 30, 1979.

WATER LEVELS IN FEET BELOW LAND SURFACE DATUM, CLIMATIC YEARS APRIL 1981 TO MARCH 1983

\begin{tabular}{|c|c|c|}
\hline & WATER & \\
\hline DATE & LEVEL & DATE \\
\hline
\end{tabular}

MAR 31, $1982 \quad Z \quad$ FEB $18,1983 \quad 37.50$

GROUND-WATER LEVELS

352926097261701. LOCAL NUMBER; 12N-02W-29 BBD 1.

LOCATION,--LAT 35 29'26", LONG 097 26'17", HYDROLOGIC UNIT 11100302.

AQUIFER.--GARBER SANDSTONE.

PERIOD OF RECORD.--1975 TO CURRENT YEAR.

EXTREMES FOR PERIOD OF RECORD.--HIGHEST WATER LEVEL, 119.73 FT(36.49 M) BELOW LAND-SURFACE DATUM,FEB 16, 1983; LOWEST WATER LEVEL, 135.50 FT(41.30 M) BELOW LAND-SURFACE DATUM, JAN 22, 1976.

WATER LEVELS IN FEET BELOW LAND SURFACE DATUM, CLIMATIC YEARS APRIL 1981 TO MARCH 1983

\begin{tabular}{|c|c|c|}
\hline DATE & $\begin{array}{l}\text { WATER } \\
\text { LEVEL }\end{array}$ & DATE \\
\hline
\end{tabular}

$\begin{array}{llll}\text { MAR 31, } 1982 & Z & \text { FEB } 16,1983 & 119.73\end{array}$ 
OKLAHOMA COUNTY -- CONTINUED

353236097301501. LOCAL NUMBER; 12N-03W-03 CAA 1. LOCATION,--LAT $3532^{\prime} 36^{\prime \prime}$, LONG 097 30'15", HYDROLOGIC UNIT 11100302. AQUIFER.--GARBER SANDSTONE. PERIOD OF RECORD..- 1979 TO CURRENT YEAR. EXTREMES FOR PERIOD OF RECORD.--HIGHEST WATER LEVEL,216.98 FT(66.14 M) BELOW LAND-SURFACE DATUM,FEB 22, 1983; LOWEST WATER LEVEL, $350.00 \mathrm{FT}(106.68 \mathrm{M})$ BELOW LAND-SURFACE DATUM, MAR 5, 1979.

WATER LEVELS IN FEET BELOW LAND SURFACE DATUM, CLIMATIC YEARS APRIL 1981 TO MARCH 1983

$\begin{array}{lll} & \text { WATER } & \text { WATER } \\ \text { DATE } & \text { LEVEL } & \text { DATE }\end{array}$

MAR 09, $1982 \quad 222.00 \quad$ FEB 22, $1983 \quad 216.98$

\section{GROUND-WATER LEVELS}

353236097332601. LOCAL NUMBER; 12N-03W-06 CAA 1. LOCATION,--LAT 35 32'36", LONG 097 33'26", HYDROLOGIC UNIT 11100303. AQUIFER.--GARBER SANDSTONE.

PERIOD OF RECORD.--1976 TO CURRENT YEAR.

EXTREMES FOR PERIOD OF RECORD.--HIGHEST WATER LEVEL,221.60 FT(67.54 M) BELOW LAND-SURFACE DATUM,MAR 5, 1979; LOWEST WATER LEVEL,256.26 FT(78.11 M) BELOW LAND-SURFACE DATUM, APR 1, 1980.

WATER LEVELS IN FEET BELOW LAND SURFACE DATUM, CLIMATIC YEARS APRIL 1981 TO MARCH 1983

$\begin{array}{lll} & \text { WATER } & \\ \text { DATE } & \text { WEVEL } & \text { WATER } \\ \text { LEVEL }\end{array}$

MAR 11, $1982 \quad 232.89 \quad$ MAR $01,1983 \quad 236.40$ 
OKLAHOMA COUNTY -- CONTINUED

352940097300701. LOCAL NUMBER; 12N-03W-22 DCC 1.

LOCATION,--LAT 35 29'40", LONG 097 30'07", HYDROLOGIC UNIT 11100303.

AQUIFER.--GARBER SANDSTONE.

PERIOD OF RECORD.--1976 TO CURRENT YEAR.

EXTREMES FOR PERIOD OF RECORD.--HIGHEST WATER LEVEL, $250.00 \mathrm{FT}(76.20 \mathrm{M})$ BELOW LAND-SURFACE DATUM,NOV 4, 1976; LOWEST WATER LEVEL, 365.00 FT(111.25 M) BELOW LAND-SURFACE DATUM, MAR 30, 1979.

WATER LEVELS IN FEET BELOW LAND SURFACE DATUM, CLIMATIC YEARS APRIL 1981 TO MARCH 1983

$\begin{array}{lll} & \text { WATER } & \text { WATER } \\ \text { DATE } & \text { LEVEL } & \text { DATE }\end{array}$

MAR 31, $1982 \quad \mathrm{Z} \quad$ MAR 01, $1983 \quad 363.30$

GROUND-WATER LEVELS

353006097345401. LOCAL NUMBER; 12N-04W-24 BCC 1.

LOCATION,--LAT 35 30'06", LONG 097 34'54", HYDROLOGIC UNIT 11100303.

AQUIFER.--GARBER SANDSTONE.

PERIOD OF RECORD.--1976 TO CURRENT YEAR.

EXTREMLS FOR PERIOD OF RECORD.--HIGHEST WATER LEVEL, $247.00 \mathrm{FT}(75.29 \mathrm{M})$ BELOW LAND-SURFACE DATUM, MAR 21, 1978; LOWEST WATER LEVEL,304.00 FT(92.66 M) BELOW LAND-SURFACE DATUM, FEB 16, 1983.

WATER LEVELS IN FEET BELOW LAND SURFACE DATUM, CLIMATIC YEARS APRIL 1981 TO MARCH 1983

\begin{tabular}{|c|c|c|}
\hline DATE & $\begin{array}{l}\text { WATER } \\
\text { LEVEL }\end{array}$ & DATE \\
\hline
\end{tabular}

MAR 31, $1982 \quad Z \quad$ FEB $16,1983 \quad 304.00$ 


\section{GROUND-WATER LEVELS}

- OKLAHOMA COUNTY -- CONTINUED

353558097182101. LOCAL NUMBER; LOCATION,--LAT 35 35'58', LONG 097 AQUIFER.--GARBER SANDSTONE.

PERIOD OF RECORD.--1979 TO CURRENT YEAR.

EXTREMES FOR PERIOD OF RECORD.--HIGHEST WATER LEVEL, 120.10 FT(36.61 M) BELOW LAND-SURFACE DATUM,MAR 7, 1979; LOWEST WATER LEVEL, 122.27 FT(37.27 M) BELOW LAND-SURFACE DATUM, APR 1, 1980.

WATER LEVELS IN FEET BELOW LAND SURFACE DATUM, CLIMATIC YEARS APRIL 1981 TO MARCH 1983
$13 \mathrm{~N}-01 \mathrm{~W}-16$ DBD 1.

$18^{\prime} 21^{\prime \prime}$, HYDROLOGIC UNIT 11100302.

$\begin{array}{lll} & \text { WATER } & \text { WATER } \\ \text { DATE } & \text { LEVEL } & \text { DATE }\end{array}$
JAN 01, 1982
Z MAR 16,1983
$\mathbf{P}$

GROUND-WATER LEVELS

353315097173301. LOCAL NUMBER; 13N-01W-34 CDA 1. LOCATION,--LAT 35 33'15", LONG 097 17'33", HYDROLOGIC UNIT 11100302. AQUIFER - - GARBER SANDSTONE. PERIOD OF RECORD..-1975 TO CURRENT YEAR. EXTREMES FOR PERIOD OF RECORD.--HIGHEST WATER LEVEL,93.50 FT(28.50 M) BELOW LAND-SURFACE DATUM,MAR 23, 1978; LOWEST WATER LEVEL,200.00 FT(60.96 M) BELOW LAND-SURFACE DATUM, MAR 23, 1981.

WATER LEVELS IN FEET BELOW LAND SURFACE DATUM, CLIMATIC YEARS APRIL 1981 TO MARCH 1983

\begin{tabular}{|c|c|c|}
\hline & WATER & \\
\hline DATE & LEVEL & DATE \\
\hline
\end{tabular}
MAR 31, 1982
Z MAR 18,1983
$\mathbf{P}$ 
OKLAHOMA COUNTY -- CONTINUED

353749097243601. LOCAL NUMBER;

13N-02W-04 DAB 1.

LOCATION, --LAT 35 37'49", LONG 097

24'36", HYDROLOGIC UNIT 11100303.

AQUIFER.--GARBER SANDSTONE.

PERIOD OF RECORD.--1977 TO CURRENT YEAR.

EXTREMES FOR PERIOD OF RECORD.--HIGHEST WATER LEVEL,65.23 FT(19.88 M) BELOW LAND-SURFACE DATUM, APR 1, 1977; LOWEST WATER LEVEL,68.00 FT(20.73 M) BELOW LAND-SURFACE DATUM, MAR 7, 1979.

WATER LEVELS IN FEET BELOW LAND SURFACE DATUM, CLIMATIC YEARS APRIL 1981 TO MARCH 1983

$\begin{array}{lll} & \text { WATER } & \\ \text { DATE } & \text { LEVEL } & \text { WATER } \\ \text { DATE } & \text { LEVEL }\end{array}$

MAR 09, $198266.20 \quad$ FEB $17,1983 \quad 0$

GROUND-WATER LEVELS

353815097280401. LOCAL NUMBER;

$13 \mathrm{~N}-03 \mathrm{~W}-01$ ABB 1.

LOCATION,--LAT 35 38'15", LONG 097 28'04", HYDROLOGIC UNIT 11100303.

AQUIFER .--GARBER SANDSTONE.

PERIOD OF RECORD.--1976 TO CURRENT YEAR.

EXTREMES FOR PERIOD OF RECORD.--HIGHEST WATER LEVEL, 239.00 FT(72.85 M) BELOW LAND-SURFACE DATUM, MAR 21, 1983; LOWEST WATER LEVEL,376.00 FT(114.60 M) BELOW LAND-SURFACE DATUM, JAN 15, 1976.

WATER LEVELS IN FEET BELOW LAND SURFACE DATUM, CLIMATIC YEARS APRIL 1981 TO MARCH 1983

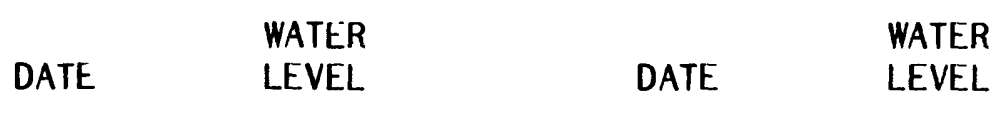

MAR $12,1982 \quad 366.00 R \quad$ MAR $21,1983 \quad 239.00$ 


\section{GROUND-WATER LEVELS}

OKLAHOMA COUNTY -- CONTINUED

353749097294001. LOCAL NUMBER; _OCATION,--LAT 35 37'49", LONG 097 AQUIFER.--GARBER SANDSTONE.

DERIOD OF RECORD.--1976 TO CURRENT YEAR.

EXTREMES FOR PERIOD OF RECORD.--HIGHEST WATER LEVEL,242.00 FT(73.76 M) BELOW LAND-SURFACE DATUM,JAN 15, 1976; LOWEST WATER LEVEL, 302.00 FT(92.05 M) BELOW LAND-SURFACE DATUM, APR 3, 1980.

WATER LEVELS IN FEET BELOW LAND SURFACE DATUM, CLIMATIC YEARS APRIL 1981 TO MARCH 1983

WATER

DATE LEVEL

MAR 08, $1982 \quad 260.00$
$13 \mathrm{~N}-03 \mathrm{~W}-02$ CBB 1. $29^{\prime} 40^{\prime \prime}$, HYDROLOGIC UNIT 11100303.

GROUND-WATER LEVELS

353723097294001. LOCAL NUMBER;

$13 \mathrm{~N}-03 \mathrm{~W}-11$ BBB 1. LOCATION,--LAT 35 37'23", LONG 097 29'40", HYDROLOGIC UNIT 11100303. AQUIFER .--GARBER SANDSTONE. PERIOD OF RECORD. - -1972 TO CURRENT YEAR.

EXTREMES FOR PERIOD OF RECORD.--HIGHEST WATER LEVEL, $107.00 \mathrm{FT}(32.61 \mathrm{M})$ BELOW LAND-SURFACE DATUM, JAN 1, 1972; LOWEST WATER LEVEL,282.00 FT(85.95 M) BELOW LAND-SURFACE DATUM, JAN 15, 1976.

WATER LEVELS IN FEET BELOW LAND SURFACE DATUM, CLIMATIC YEARS APRIL 1981 TO MARCH 1983

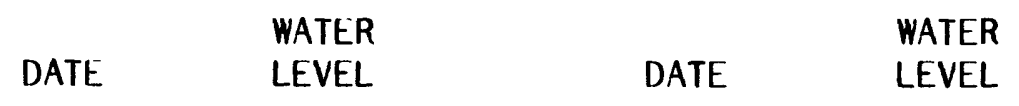

MAR 08, $1982 \quad 263.00 \quad$ MAR 21, $1983 \quad 262.00$ 
GROUND-WATER LEVELS

OKLAHOMA COUNTY -- CONTINUED

353401097282801. LOCAL NUMBER;

$13 \mathrm{~N}-03 \mathrm{~W}-25$ CCD 1.

LOCATION,--LAT 35 34'01", LONG 097 28'28", HYDROLOGIC UNIT 11100303.

AQUIFER.--GARBER SANDSTONE.

PERIOD OF RECORD.--1981 TO CURRENT YEAR.

EXTREMES FOR PERIOD OF RECORD.--HIGHEST WATER LEVEL,79.50 FT(24.23 M) BELOW LAND-SURFACE DATUM, MAR 11, 1981; LOWEST WATER LEVEL,79.50 FT(24.23 M) BELOW LAND-SURFACE DATUM, MAR 11, 1981.

WATER LEVELS IN FEET BELOW LAND SURFACE DATUM, CLIMATIC YEARS APRIL 1981 TO MARCH 1983

\begin{tabular}{|c|c|}
\hline & WATER \\
\hline
\end{tabular}
MAR 31, 1982
Z
FEB 16, 1983
Z

GROUND-WATER LEVELS

353440097314001. LOCAL NUMBER; 13N-03W-28 BBD 1. LOCATION,--LAT 35 34'40", LONG 097 31'40", HYDROLOGIC UNIT 11100303. AQUIFER.--GARBER SANDSTONE.

PERIOD OF RECORD.--1973 TO CURRENT YEAR.

EXTREMES FOR PERIOD OF RECORD.--HIGHEST WATER LEVEL,207.30 FT(63.19 M) BELOW LAND-SURFACE DATUM,MAR 21, 1978; LOWEST WATER LEVEL,275.30 FT(83.91 M) BELOW LAND-SURFACE DATUM, APR 9, 1980.

WATER LEVELS IN FEET BELOW LAND SURFACE DATUM, CLIMATIC YEARS APRIL 1981 TO MARCH 1983

$\begin{array}{lcccc} & \text { WATER } & & \text { WATER } \\ \text { DATE } & \text { LEVEL } & \text { DATE } & \text { LEVEL } \\ \text { 09, 1982 } & & \mathrm{Z} & \text { FEB 16, 1983 } & 224.30\end{array}$


GROUND-WATER LEVELS

OKLAHOMA COUNTY -- CONTINUED

353321097332301. LOCAL NUMBER; 13N-03W-31 DBC 1.

LOCATION,--LAT 35 33'21", LONG 097 33'23", HYDROLOGIC UNIT 11050002.

AQUIFER.--GARBER SANDSTONE.

PERIOD OF RECORD.--1973 TO CURRENT YEAR.

EXTREMES FOR PERIOD OF RECORD.--HIGHEST WATER LEVEL, 240.00 FT(73.15 M) BELOW LAND-SURFACE DATUM,DEC 11, 1976; LOWEST WATER LEVEL, $350.00 \mathrm{FT}(106.68 \mathrm{M})$ BELOW LAND-SURFACE DATUM, FEB 25, 1981.

WATER LEVELS IN FEET BELOW LAND SURFACE DATUM, CLIMATIC YEARS APRIL 1981 TO MARCH 1983

$\begin{array}{lll} & \text { WATER } & \text { WATER } \\ \text { DATE } & \text { LEVEL } & \text { DATE }\end{array}$

MAR 09, $1982 \quad 345.00 \quad$ FEB 22, $1983 \quad 308.50$

GROUND-WATER LEVELS

353308097294001. LOCAL NUMBER; 13N-03W-35 CCC 1.

LOCATION,--LAT 35 33'08", LONG 097 29'40", HYDROLOGIC UNIT 11100303.

AQUIFER .--GARBER SANDSTONE.

PERIOD OF RECORD.--1973 TO CURRENT YEAR.

EXTREMES FOR PERIOD OF RECORD.--HIGHEST WATER LEVEL, 193.04 FT(58.84 M) BELOW LAND-SURFACE

DATUM,APR 3, 1980; LOWEST WATER LEVEL,246.00 FT(74.98 M) BELOW LAND-SURFACE DATUM,

JUNE $1,1973$.

WATER LEVELS IN FEET BELOW LAND SURFACE DATUM, CLIMATIC YEARS APRIL 1981 TO MARCH 1983

$\begin{array}{lll} & \text { WATER } & \text { WATER } \\ \text { DATE } & \text { LEVEL } & \text { DATE }\end{array}$

MAR 11, $1982 \quad 219.00 \quad$ FEB 22, $1983 \quad 226.22$ 
GROUND-WATER LEVELS

OKLAHOMA COUNTY -- CONTINUED

353334097274801. LOCAL NUMBER; 13N-03W-36 CDA 1.

LOCATION,--LAT 35 33'34", LONG 097 27'48", HYDROLOGIC UNIT 11100303.

AQUIFER .--GARBER SANDSTONE.

PERIOD OF RECORD.--1980 TO CURRENT YEAR.

EXTREMES FOR PERIOD OF RECORD.--HIGHEST WATER LEVEL, $73.85 \mathrm{FT}(22.51 \mathrm{M})$ BELOW LAND-SURFACE

DATUM, MAR 1, 1983; LOWEST WATER LEVEL,78.52 FT(23.93 M) BELOW LAND-SURFACE DATUM, MAR 31, 1981.

WATER LEVELS IN FEET BELOW LAND SURFACE DATUM, CLIMATIC YEARS APRIL 1981 TO MARCH 1983

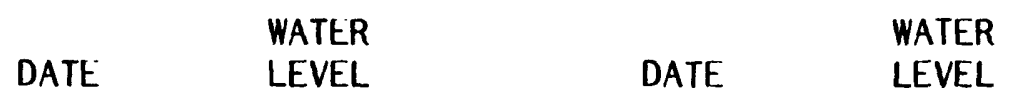

MAR 31, $1982 \quad$ Z MAR 01, $1983 \quad 73.85$

GROUND-WATER LEVELS

353354097282801. LOCAL NUMBER; 13N-03W-36 BBA 1.

LOCATION,--LAT 35 33'54", LONG 097 28'28", HYDROLOGIC UNIT 11100303.

AQUIFER .--GARBER SANDSTONE.

PERIOD OF RECORD.--1981 TO CURRENT YEAR.

EXTREMES FOR PERIOD OF RECORD.--HIGHEST WATER LEVEL, 100.92 FT(30.76 M) BELOW LAND-SURFACE DATUM,FEB 22, 1983; LOWEST WATER LEVEL, 102.77 FT(31.32 M) BELOW LAND-SURFACE DATUM, MAR 11, 1981 .

WATER LEVELS IN FEET BELOW LAND SURFACE DATUM, CLIMATIC YEARS APRIL 1981 TO MARCH 1983

\begin{tabular}{|c|c|}
\hline & WATER \\
\hline DATE & LEVEL \\
\hline
\end{tabular}

MAR 31, $1982 \quad \mathrm{Z} \quad$ FEB 22, $1983 \quad 100.92 \mathrm{R}$ 


\section{GROUND-WATER LEVELS}

OKLAHOMA COUNTY -- CONTINUED

354231097140501. LOCAL NUMBER; 14N-01E-07 ABA 1.

LOCATION,--LAT 35 42'31", LONG 097 14'05", HYDROLOGIC UNIT 11100303.

AQUIFER.--GARBER SANDSTONE.

PERIOD OF RECORD.--1977 TO CURRENT YEAR.

EXTREMES FOR PERIOD OF RECORD.--HIGHEST WATER LEVEL,61.50 FT(18.75 M) BELOW LAND-SURFACE DATUM,JAN 7, 1977; LOWEST WATER LEVEL,76.20 FT(23.23 M) BELOW LAND-SURFACE DATUM, MAR 8, 1979.

WATER LEVELS IN FEET BELOW LAND SURFACE DATUM, CLIMATIC YEARS APRIL 1981 TO MARCH 1983

\begin{tabular}{|c|c|}
\hline & WATER \\
\hline DATE & LEVEL \\
\hline
\end{tabular}

MAR 31, $1982 \quad Z \quad$ FEB 28, $1983 \quad 65.95$

GROUND-WATER LEVELS

353948097112501. LOCAL NUMBER; 14N-01E-27 BBD 1. LOCATION,--LAT 35 39'48", LONG 097 11'25", HYDROLOGIC UNIT 11100303. AQUIFER.--GARBER SANDSTONE. PERIOD OF RECORD.--1973 TO CURRENT YEAR. EXTREMES FOR PERIOD OF RECORD.--HIGHEST WATER LEVEL,41.20 FT(12.56 M) BELOW LAND-SURFACE DATUM, MAY 15, 1973; LOWEST WATER LEVEL,75.50 FT(23.01 M) BELOW LAND-SURFACE DATUM, MAR 31, 1981.

WATER LEVELS IN FEET BELOW LAND SURFACE DATUM, CLIMATIC YEARS APRIL 1981 TO MARCH 1983

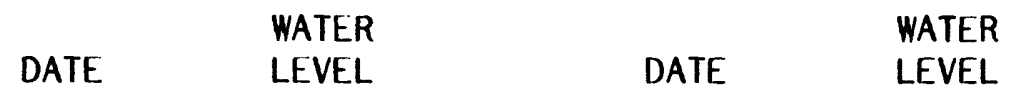

$\begin{array}{llll}\text { MAR } 31,1982 & Z & \text { FEB } 18,1983 & Z\end{array}$


GROUND-WATER LEVELS

OKLAHOMA COUNTY -- CONTINUED

354025097200501. LOCAL NUMBER;

$14 \mathrm{~N}-01 \mathrm{~W}-20$ CBB 1.

LOCATION, --LAT 35 40'25", LONG 097

AQUIFER.--GARBER SANDSTONE.

PERIOD OF RECORD.--1956 TO CURRENT YEAR.

EXTREMES FOR PERIOD OF RECORD.--HIGHEST WATER LEVEL, $20.35 \mathrm{FT}(6.20 \mathrm{M})$ BELOW LAND-SURFACE DATUM,MAR 7, 1979; LOWEST WATER LEVEL, $31.00 \mathrm{FT}(9.45 \mathrm{M})$ BELOW LAND-SURFACE DATUM, NOV 1, 1956.

WATER LEVELS IN FEET BELOW LAND SURFACE DATUM, CLIMATIC YEARS APRIL 1981 TO MARCH 1983

$\begin{array}{lll} & \text { WATER } & \text { WATER } \\ \text { DATE } & \text { LEVEL } & \text { DATE } \\ \end{array}$

MAR 09, $1982 \quad 28.38 \quad$ FEB 16, $1983 \quad 27.72$

GROUND-WATER LEVELS

354328097234801. LOCAL NUMBER; 14N-02W-03 ABB 1.

LOCATION,--LAT 35 43'28", LONG 097 23'48", HYDROLOGIC UNIT 11100303.

AQUIFER .--GARBER SANDSTONE.

PERIOD OF RECORD.--1976 TO CURRENT YEAR.

EXTREMES FOR PERIOD OF RECORD.--HIGHEST WATER LEVEL, 66.30 FT(20.21 M) BELOW LAND-SURFACE DATUM,DEC 27, 1976; LOWEST WATER LEVEL,67.60 FT(20.60 M) BELOW LAND-SURFACE DATUM, MAR 7, 1979.

WATER LEVELS IN FEET BELOW LAND SURFACE DATUM, CLIMATIC YEARS APRIL 1981 TO MARCH 1983

\begin{tabular}{|c|c|c|}
\hline & WATER & \\
\hline DATE & LEVEL & DATE \\
\hline
\end{tabular}

MAR 09, $1982 \quad Z \quad$ FEB 28, $1983 \quad 67.25$ 
EXTREMES FOR PERIOD OF RECORD.--HIGHEST WATER LEVEL,69.98 FT(21.33 M) BELOW LAND-SURFACE DATUM, FEB 17, 1983; LOWEST WATER LEVEL,98.05 FT(29.89 M) BELOW LAND-SURFACE DATUM, MAR 7, 1979.

NATER LEVELS IN FEET BELOW LAND SURFACE DATUM, CLIMATIC YEARS APRIL 1981 TO MARCH 1983

\begin{tabular}{ccccc}
\multicolumn{1}{c}{} & WATER & & & WATER \\
DATE & LEVEL & DATE & LEVEL \\
R 31, 1982 & & Z & FEB 17, 1983 & 69.98
\end{tabular}

GROUND-WATER LEVELS

354150097304401. LOCAL NUMBER; 14N-03W-10 CCC 1.

LOCATION,--LAT 35 41'50", LONG 097 30'44", HYDROLOGIC UNIT 11050002.

AQUIFER . --GARBER SANDSTONE.

PERIOD OF RECORD.--1979 TO CURRENT YEAR.

EXTREMES FOR PERIOD OF RECORD.--HIGHEST WATER LEVEL,71.00 FT(21.64 M) BELOW LAND-SURFACE DATUM, MAR 2, 1981; LOWEST WATER LEVEL, 121.00 FT(36.88 M) BELOW LAND-SURFACE DATUM, APR 3,1980 .

WATER LEVELS IN FEET BELOW LAND SURFACE DATUM, CLIMATIC YEARS APRIL 1981 TO MARCH 1983

$\begin{array}{lll} & \text { WATER } & \text { WATER } \\ \text { DATE } & \text { LEVEL } & \text { DATE }\end{array}$

MAR 18, $1982 \quad 83.00 \quad$ MAR 21, $1983 \quad 71.00$ 
GROUND-WATER LEVELS

OKLAHOMA COUNTY -- CONTINUED

354111097325201. LOCAL NUMBER;

$14 \mathrm{~N}-03 \mathrm{~W}-17 \mathrm{CBC} 1$.

LOCATION,--LAT 35 41'11", LONG 097 32'52", HYDROLOGIC UNIT 11050002.

AQUIFER.--GARBER SANDSTONE.

PERIOD OF RECORD.--1973 TO CURRENT YEAR.

EXTREMES FOR PERIOD OF RECORD.--HIGHEST WATER LEVEL, $147.50 \mathrm{FT}(44.96 \mathrm{M})$ BELOW LAND-SURFACE DATUM,FEB 28, 1983; LOWEST WATER LEVEL,210.19 FT(64.07 M) BELOW LAND-SURFACE DATUM, APR $10,1980$.

WATER LEVELS IN FEET BELOW LAND SURFACE DATUM, CLIMATIC YEARS APRIL 1981 TO MARCH 1983

\begin{tabular}{|c|c|}
\hline & WATER \\
\hline DATE & LEVEL \\
\hline
\end{tabular}

MAR 31, $1982 \quad Z \quad$ FEB 28, $1983 \quad 147.50$

GROUND-WATER LEVELS

354052097274001. LOCAL NUMBER; 14N-03W-24 AAA 1.

LOCATION,--LAT 35 40'52", LONG 097 27'40", HYDROLOGIC UNIT 11100303.

AQUIFER.--GARBER SANDSTONE.

PERIOD OF RECORD.--1979 TO CURRENT YEAR.

EXTREMES FOR PERIOD OF RECORD.--HIGHEST WATER LEVEL, 130.00 FT(39.62 M) BELOW LAND-SURFACE DATUM,FEB 6, 1979; LOWEST WATER LEVEL,170.00 FT(51.82 M) BELOW LAND-SURFACE DATUM, MAR 21, 1983.

WATER LEVELS IN FEET BELOW LAND SURFACE DATUM, CLIMATIC YEARS APRIL 1981 TO MARCH 1983

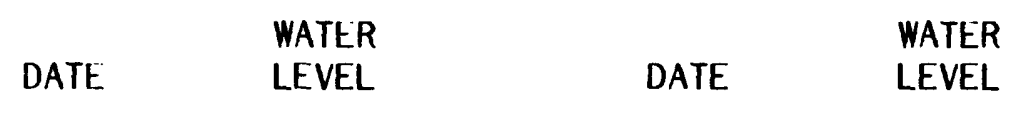

MAR 12, $1982 \quad 160.00 R \quad$ MAR 21, $1983 \quad 170.00$ 
OKLAHOMA COUNTY -- CONTINUED

353914097280401. LOCAL NUMBER; LOCATION,--LAT 35 39'14", LONG 097 AQUIFER.--GARBER SANDSTONE.

PERIOD OF RECORD.--1973 TO CURRENT YEAR.

EXTREMES FOR PERIOD OF RECORD.--HIGHEST WATER LEVEL,244.00 FT(74.37 M) BELOW LAND-SURFACE DATUM,DEC 27, 1976; LOWEST WATER LEVEL,417.00 FT(127.10 M) BELOW LAND-SURFACE DATUM, MAR 2, 1981.

WATER LEVELS IN FEET BELOW LAND SURFACE DATUM, CLIMATIC YEARS APRIL 1981 TO MARCH 1983

GROUND-WATER LEVELS

353914097274001. LOCAL NUMBER; LOCATION, --LAT 35 39'14", LONG 097 AQUIFER.--GARBER SANDSTONE.

PERIOD OF RECORD.--1967 TO CURRENT YEAR. EXTREMES FOR PERIOD OF RECORD.--HIGHEST WATER LEVEL, $170.00 \mathrm{FT}(51.82 \mathrm{M})$ BELOW LAND-SURFACE DATUM, JAN 1, 1967; LOWEST WATER APR 1, 1980.
$14 \mathrm{~N}-03 \mathrm{~W}-25$ DDD 1 .

$27^{\prime} 40^{\prime \prime}$, HYDROLOGIC UNIT 11100303.

LEVEL, $324.00 \mathrm{FT}(98.76 \mathrm{M})$ BELOW LAND-SURFACE DATUM,

WATER LEVELS IN FEET BELOW LAND SURFACE DATUM, CLIMATIC YEARS APRIL 1981 TO MARCH 1983
DATE
WATER
LEVEL
DATE
WATER
LEVEL

MAR 18, 1982

P $\quad$ MAR $21,1983 \quad 263.00$ 


\author{
OKLAHOMA COUNTY -- CONTINUED
}

353927097285201. LOCAL NUMBER; 14N-03W-26 DAC 1. LOCATION,--LAT 35 39'27", LONG 097 28'52", HYDROLOGIC UNIT 11100303. AQUIFER.--GARBER SANDSTONE. PERIOD OF RECORD.--1949 TO CURRENT YEAR. EXTREMES FOR PERIOD OF RECORD.--HIGHEST WATER LEVEL, $117.00 \mathrm{FT}(35.66 \mathrm{M})$ BELOW LAND-SURFACE DATUM,MAR 18, 1982; LOWEST WATER LEVEL,245.00 FT(74.68 M) BELOW LAND-SURFACE DATUM, DEC 27, 1976.

WATER LEVELS IN FEET BELOW LAND SURFACE DATUM, CLIMATIC YEARS APRIL 1981 TO MARCH 1983

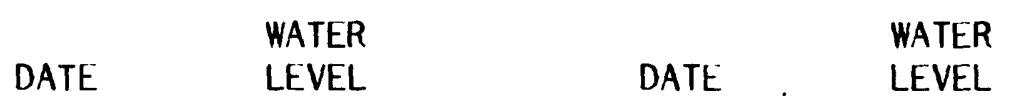

MAR $18,1982 \quad 117.00 \quad$ MAR 21, $1983 \quad 223.00$

GROUND-WATER LEVELS

353914097325901. LOCAL NUMBER; 14N-03W-30 DDD 1.

LOCATION,--LAT 35 39'14", LONG 097 32'59", HYDROLOGIC UNIT 11050002.

AQUIFER .--GARBER SANDSTONE.

PERIOD OF RECORD.--1976 TO CURRENT YEAR.

EXTREMES FOR PERIOD OF RECORD.--HIGHEST WATER LEVEL, 87.92 FT(26.80 M) BELOW LAND-SURFACE DATUM,FEB 28, 1983; LOWEST WATER LEVEL,92.91 FT(28.32 M) BELOW LAND-SURFACE DATUM, MAR 9, 1982 .

WATER LEVELS IN FEET BELOW LAND SURFACE DATUM, CLIMATIC YEARS APRIL 1981 TO MARCH 1983

\begin{tabular}{cccc}
\multicolumn{1}{c}{ WATER } & & WATER \\
DATE & LEVEL & DATE & LEVEL \\
MAR 09, 1982 & 92.91 & FEB 28, 1983 & 87.92
\end{tabular}


GROUND-WATER LEVELS

OKLAHOMA COUNTY -- CONTINUED

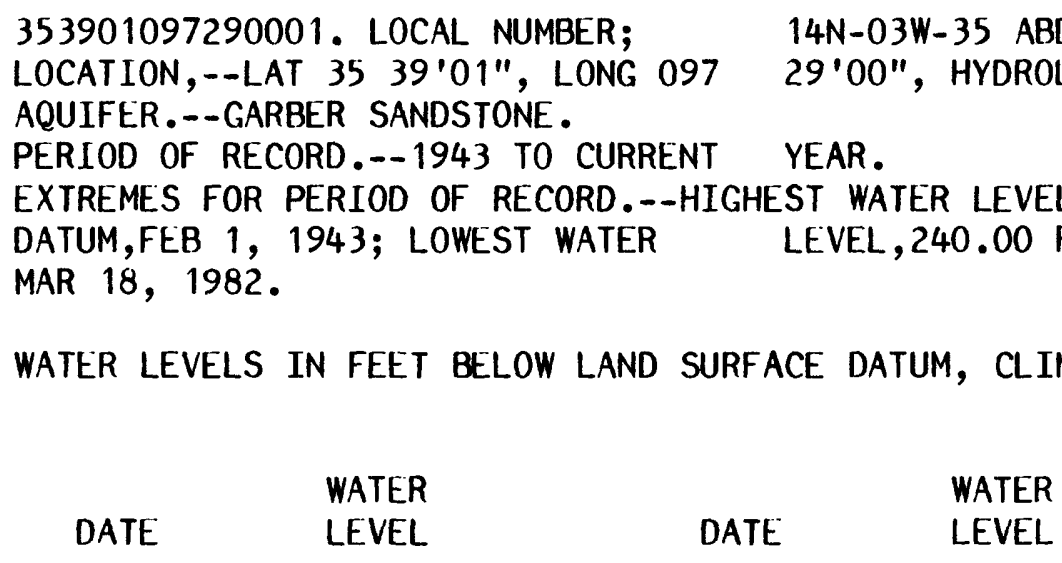

MAR $18,1982 \quad 240.00 \quad$ MAR 21, $1983 \quad 223.70$ 


\section{GROUND-WATER LEVELS}

OKMULGEE COUNTY

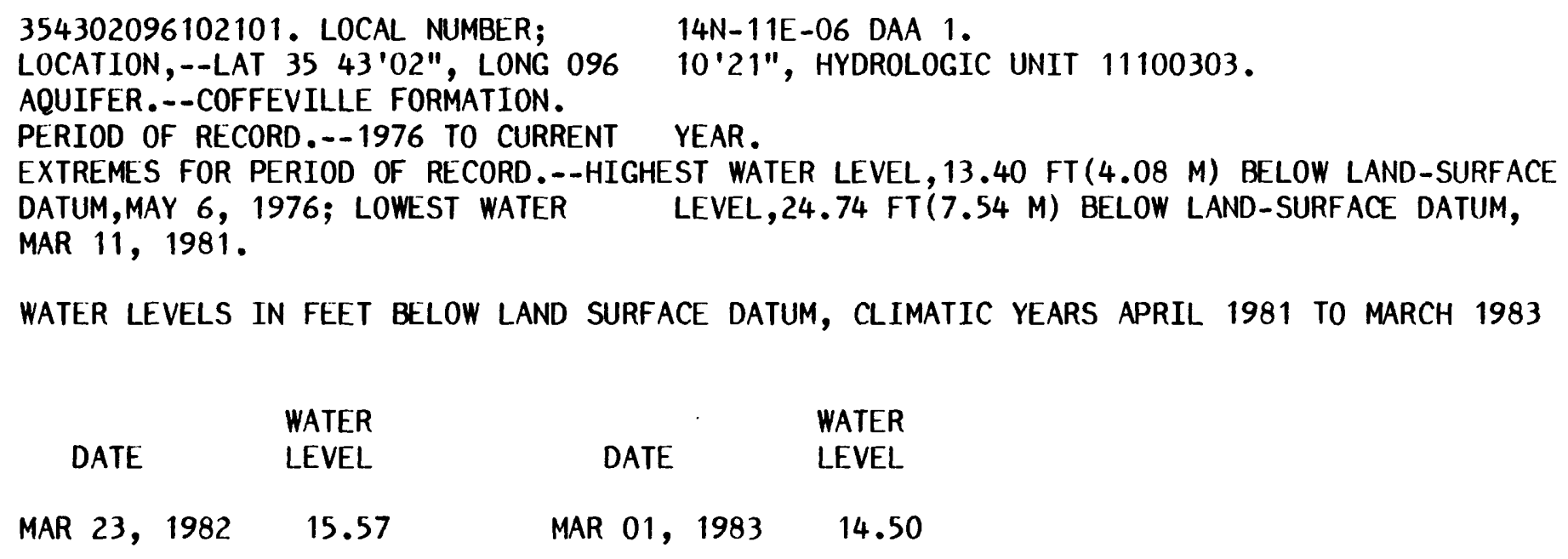

WATER LEVELS IN FEET BELOW LAND SURFACE DATUM, CLIMATIC YEARS APRIL 1981 TO MARCH 1983

$\begin{array}{lll} & \text { WATER } & \\ \text { DATE } & \text { WEVEL } & \text { WATER } \\ \text { LEVEL } & \text { LETE }\end{array}$
MAR 23, 1982
15.57
MAR 01, $1983 \quad 14.50$ 
GROUND-WATER LEVELS

OSAGE COUNTY

361526096084201. LOCAL NUMBER; 21N-11E-32 ABC 1. LOCATION,--LAT 36 15'26", LONG 096 08'42", HYDROLOGIC UNIT 11070107. AQUIFER.--CHANUTE FORMATION.

PERIOD OF RECORD.--1979 TO CURRENT YEAR.

EXTREMES FOR PERIOD OF RECORD.--HIGHEST WATER LEVEL, 22.12 FT(6.74 M) BELOW LAND-SURFACE DATUM,FEB 25, 1983; LOWEST WATER LEVEL, $36.25 \mathrm{FT}(11.05 \mathrm{M})$ BELOW LAND-SURFACE DATUM, MAR 11, 1980.

WATER LEVELS IN FEET BELOW LAND SURFACE DATUM, CLIMATIC YEARS APRIL 1981 TO MARCH 1983

$\begin{array}{lll} & \text { WATER } & \\ \text { DATE } & \text { WEVEL } & \text { WATER } \\ \text { LEVEL }\end{array}$

FEB 23, $1982 \quad 24.12 \quad$ FEB 25, $1983 \quad 22.12$ 
GROUND-WATER LEVELS

OTTAWA COUNTY

365745094580101. LOCAL NUMBER;

$29 \mathrm{~N}-22 \mathrm{E}-30$ CAA 1.

LOCATION,--LAT 36 57'45", LONG 094 AQUIFER.--BOONE FORMATION.

PERIOD OF RECORD. - 1979 TO CURRENT YEAR.

EXTREMES FOR PERIOD OF RECORD.--HIGHEST WATER LEVEL,2.23 FT(.68 M) BELOW LAND-SURFACE DATUM, MAR 16, 1983; LOWEST WATER LEVEL, $17.50 \mathrm{FT}(5.33 \mathrm{M})$ BELOW LAND-SURFACE DATUM, MAR 11, 1981.

WATER LEVELS IN FEET BELOW LAND SURFACE DATUM, CLIMATIC YEARS APRIL 1981 TO MARCH 1983

\begin{tabular}{|c|c|}
\hline & WATER \\
\hline DATE & LEVEL \\
\hline
\end{tabular}

MAR $30,1982 \quad 2.42$ MAR 16, $1983 \quad 2.23$ 
GROUND-WATER LEVELS

PAWNEE COUNTY

361631096301901 . LOCAL NUMBER;

$21 \mathrm{~N}-07 \mathrm{E}-24 \mathrm{CDD} 1$.

LOCATION,--LAT 36 16'31', LONG 096

30'19", HYDROLOGIC UNIT 11160006.

AQUIFER.--IOLA L.S.

PERIOD OF RECORD.-- 1979 TO CURRENT YEAR.

EXTREMES FOR PERIOD OF RECORD.--HIGHEST WATER LEVEL, 39.98 FT(12.19 M) BELOW LAND-SURFACE DATUM,FEB 19, 1980; LOWEST WATER LEVEL,43.10 FT(13.14 M) BELOW LAND-SURFACE DATUM, MAR 28, 1979.

WATER LEVELS IN FEET BELOW LAND SURFACE DATUM, CLIMATIC YEARS APRIL 1981 TO MARCH 1983

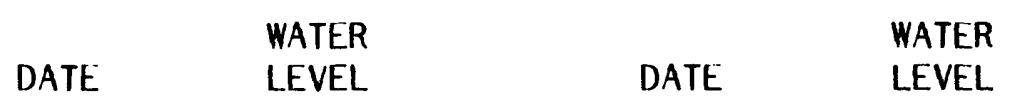

FEB 23, $1982 \quad 41.19 \quad$ FEB 25, $1983 \quad 41.47 \mathrm{R}$

GROUND-WATER LEVELS

363058096440101. LOCAL NUMBER;

$24 \mathrm{~N}-05 \mathrm{E}-35$ ACA 1.

LOCATION,--LAT 36 30'58", LONG 096 44'01", HYDROLOGIC UNIT 11160006.

AQUIFER.--TERRACE DEPOSITS.

PERIOD OF RECORD.--1978 TO CURRENT YEAR.

EXTREMES FOR PERIOD OF RECORD.--HIGHEST WATER LEVEL,8.94 FT(2.72 M) BELOW LAND-SURFACE DATUM,MAR 28, 1979; LOWEST WATER LEVEL, $12.66 \mathrm{FT}(3.86 \mathrm{M})$ BELOW LAND-SURFACE DATUM, MAR 24, 1981.

WATER LEVELS IN FEET BELOW LAND SURFACE DATUM, CLIMATIC YEARS APRIL 1981 TO MARCH 1983

$\begin{array}{lll} & \text { WATER } & \\ \text { DATE } & \text { LEVEL } & \text { WATER } \\ & \text { DATE } & \text { LEVEL }\end{array}$
FEB 23, $1982 \quad 10.83$
FEB 25, 1983
$10.87 \mathrm{~S}$ 
PAYNE COUNTY

355810097134101. LOCAL NUMBER; 17N-01E-08 BBB 1. LOCATION,--LAT 35 58'10", LONG 097 13'41", HYDROLOGIC UNIT 11050003. AQUIFER.--ALLUVIUM. PERIOD OF RECORD.--1983 TO CURRENT YEAR. EXTREMES FOR PERIOD OF RECORD.--HIGHEST WATER LEVEL, $15.00 \mathrm{FT}(4.57 \mathrm{M})$ BELOW LAND-SURFACE DATUM, MAR 17, 1983; LOWEST WATER LEVEL, 15.00 FT(4.57 M) BELOW LAND-SURFACE DATUM, MAR 17, 1983.

WATER LEVELS IN FEET BELOW LAND SURFACE DATUM, CLIMATIC YEARS APRIL 1981 T0 MARCH 1983

$\begin{array}{ll} & \text { WATER } \\ \text { DATE } & \text { LEVEL }\end{array}$

MAR $17,1983 \quad 15.00$

\section{GROUND-WATER LEVELS}

355908097030101. LOCAL NUMBER;

$18 \mathrm{~N}-02 \mathrm{E}-36$ CCC 1. LOCATION,--LAT 35 59'08", LONG 097 AQUIFER.--TERRACE DEPOSITS. PERIOD OF RECORD.--1979 TO CURRENT YEAR. EXTREMES FOR PERIOD OF RECORD.--HIGHEST WATER LEVEL, 30.55 FT(9.31 M) BELOW LAND-SURFACE DATUM,MAR 28, 1979; LOWEST WATER LEVEL,33.20 FT(10.12 M) BELOW LAND-SURFACE DATUM, MAR 5, 1982.

WATER LEVELS IN FEET BELOW LAND SURFACE DATUM, CLIMATIC YEARS APRIL 1981 TO MARCH 1983

$\begin{array}{lll} & \text { WATER } & \\ \text { DATE } & \text { LEVEL } & \text { WATER } \\ & \text { DATE } & \text { LEVEL }\end{array}$

$\begin{array}{llll}\text { MAR } 05,1982 \quad 33.20 & \text { MAR 15, } 1983 \quad 30.58\end{array}$ 
GROUND-WATER LEVELS

PAYNE COUNTY -- CONTINUED

355908096554101. LOCAL NUMBER; 18N-03E-36 DDD 1. LOCATION,--LAT 35 59'08", LONG 096 55'41", HYDROLOGIC UNIT 11050003. AQUIFER.--ALLUVIUM.

PERIOD OF RECORD.--1983 TO CURRENT YEAR.

EXTREMES FOR PERIOD OF RECORD.--HIGHEST WATER LEVEL, $25.00 \mathrm{FT}(7.62 \mathrm{M})$ BELOW LAND-SURFACE DATUM, MAR 15, 1983; LOWEST WATER LEVEL,25.00 FT(7.62 M) BELOW LAND-SURFACE DATUM, MAR 15, 1983.

WATER LEVELS IN FEET BELOW LAND SURFACE DATUM, CLIMATIC YEARS APRIL 1981 TO MARCH 1983

$\begin{array}{ll}\text { WATER } \\ \text { DATE } & \text { LEVEL }\end{array}$

MAR 15, $1983 \quad 25.00$

GROUND-WATER LEVELS

360329096450101. LOCAL NUMBER; 18N-05E-03 DDD 1.

LOCATION,--LAT 36 03'29", LONG 096 45'01", HYDROLOGIC UNIT 11050003. AQUIFER .--ALLUVIUM.

PERIOD OF RECORD.--1983 TO CURRENT YEAR.

EXTREMES FOR PERIOD OF RECORD.--HIGHEST WATER LEVEL, 16.32 FT(4.97 M) BELOW LAND-SURFACE DATUM,MAR 15, 1983; LOWEST WATER LEVEL, 16.32 FT(4.97 M) BELOW LAND-SURFACE DATUM, MAR 15, 1983.

WATER LEVELS IN FEET BELOW LAND SURFACE DATUM, CLIMATIC YEARS APRIL 1981 TO MARCH 1983

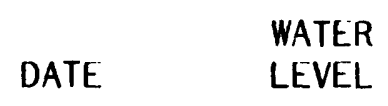

MAR $15,1983 \quad 16.32$ 
PAYNE COUNTY -- CONTINUED

355908096442101. LOCAL NUMBER; LOCATION,--LAT $3555^{\prime} 08^{\prime \prime}$, LONG 096 AQUIFER.--VAMOOSA.

PERIOD OF RECORD.--1977 TO CURRENT

EXTREMES FOR PERIOD OF RECORD.--HIGHEST WATER LEVEL, 124.32 FT(37.89 M) BELOW LAND-SURFACE DATUM, MAR 5, 1982; LOWEST WATER MAR 29, 1977.

WATER LEVELS IN FEET BELOW LAND SURFACE DATUM, CLIMATIC YEARS APRIL 1981 TO MARCH 1983
$18 \mathrm{~N}-05 \mathrm{E}-35$ DCC 1

$44^{\prime} 21$ ', HYDROLOGIC UNIT 11050003.

YEAR.

\begin{tabular}{|c|c|}
\hline & WATER \\
\hline DATE & \\
\hline
\end{tabular}
MAR 05, 1982
124.32
MAR 18, 1983
Z

GROUND-WATER LEVELS

360927097211001. LOCAL NUMBER; 19N-01W-06 BBB 1.

LOCATION,--LAT 36 09'27", LONG 097 21'10", HYDROLOGIC UNIT 11050003.

AQUIFER .--GARBER SANDSTONE.

PERIOD OF RECORD.--1977 TO CURRENT YEAR.

EXTREMES FOR PERIOD OF RECORD.--HIGHEST WATER LEVEL,6.59 FT(2.01 M) BELOW LAND-SURFACE

DATUM, JAN 24, 1980; LOWEST WATER LEVEL, 12.15 FT(3.70 M) BELOW LAND-SURFACE DATUM, JAN 22, 1981.

WATER LEVELS IN FEET BELOW LAND SURFACE DATUM, CLIMATIC YEARS APRIL 1981 TO MARCH 1983
DATE
WATER
LEVEL DATE LEVEL
WATER

MAR 05, $1982 \quad 7.74 \quad$ MAR 16, $1983 \quad 6.92$ 


\section{GROUND-WATER LEVELS}

\section{PITTSBURG COUNTY}

345128095441501. LOCAL NUMBER; LOCATION,--LAT 3451 '28", LONG 095 AQUIFER.--SAVANNA FORMATION.

PERIOD OF RECORD.--1976 TO CURRENT YEAR.

EXTREMES FOR PERIOD OF RECORD.--HIGHEST WATER LEVEL, 7.05 FT(2.15 M) BELOW LAND-SURFACE DATUM, MAY 3, 1976; LOWEST WATER LEVEL, 11.09 FT(3.38 M) BELOW LAND-SURFACE DATUM, FEB 27, 1980.

WATER LEVELS IN FEET BELOW LAND SURFACE DATUM, CLIMATIC YEARS APRIL 1981 TO MARCH 1983
05N-15E-32 DDA 1.

44'15", HYDROLOGIC UNIT 11090204.

WATER LEVELS IN

DATE

MAR 22, 1982

\section{WATER}

LEVEL

$$
9.54
$$

DATE

MAR 22, 1983
WATER

LEVEL 
PONTOTOC COUNTY

343523096410901. LOCAL NUMBER; 01N-06E-04 BCB 1.

LOCATION,--LAT 34 35'23", LONG 096 41'09", HYDROLOGIC UNIT 11140102.

AQUIFER.--ARBUCKLE FORMATION.

PLRIOD OF RECORD.--1977 TO CURRENT YEAR.

EXTREMES FOR PERIOD OF RECORD.--HIGHEST WATER LEVEL,63.76 FT(19.43 M) BELOW LAND-SURFACE DATUM,JAN 3, 1977; LOWEST WATER LEVEL,87.18 FT(26.57 M) BELOW LAND-SURFACE DATUM, FEB 21, 1980.

WATER LEVELS IN FEET BELOW LAND SURFACE DATUM, CLIMATIC YEARS APRIL 1981 TO MARCH 1983

\begin{tabular}{|c|c|c|}
\hline & WATER & \\
\hline DATE & LEVEL & DATE \\
\hline
\end{tabular}

MAR 22, $1982 \quad 64.27 \quad$ MAR 21, $1983 \quad 71.18$ 
POTTANATOMIE COUNTY

350035097031801. LOCAL NUMBER;

06N-02E-11 ADA 1.

LOCATION,--LAT 35 00'35", LONG 097 03'18", HYDROLOGIC UNIT 11090203.

AQUIFER. - - TERRACE DEPOSITS.

PERIOD OF RECORD. - 1978 TO CURRENT YEAR.

EXTREMES FOR PERIOD OF RECORD.--HIGHEST WATER LEVEL, 40.48 FT( $12.34 \mathrm{M})$ BELOW LAND-SURFACE DATUM,FEB 22, 1980; LOWEST WATER LEVEL,43.77 FT(13.34 M) BELOW LAND-SURFACE DATUM, FEB 22, 1982.

WATER LEVELS IN FEET BELOW LAND SURFACE DATUM, CLIMATIC YEARS APRIL 1981 TO MARCH 1983

$\begin{array}{lll} & \text { WATER } & \text { WATER } \\ \text { DATE } & \text { LEVEL } & \text { DATE }\end{array}$

FEB 22, $1982 \quad 43.77 \quad$ MAR 31, $1983 \quad$ Z

GROUND-WATER LEVELS

351604096475401. LOCAL NUMBER; 09N-05E-08 CAA 1. LOCATION,--LAT 35 16'04", LONG 096 47'54", HYDROLOGIC UNIT 11100302. AQUIFER.--TERRACE DEPOSITS. PERIOD OF RECORD.--1978 TO CURRENT YEAR. EXTREMES FOR PERIOD OF RECORD.--HIGHEST WATER LEVEL, 37.18 FT(11.33 M) BELOW LAND-SURFACE DATUM, MAR 4, 1983; LOWEST WATER LEVEL,44.65 FT(13.61 M) BELOW LAND-SURFACE DATUM, MAR 16, 1978.

WATER LEVELS IN FEET BELOW LAND SURFACE DATUM, CLIMATIC YEARS APRIL 1981 TO MARCH 1983

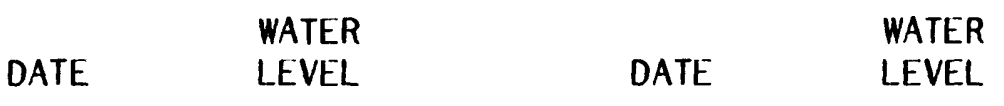

FEB 22, $1982 \quad 38.97$ MAR 04, $1983 \quad 37.18$ 
GROUND-WATER LEVELS

POTTAWATOMIE COUNTY -- CONTINUED

352617097055401. LOCAL NUMBER;

LOCATION,--LAT 35 26'17", LONG 097 05'54", HYDROLOGIC UNIT 11100302.

AQUIFER.--TERRACE DEPOSITS.

PERIOD OF RECORD.--1982 TO CURRENT YEAR.

EXTREMES FOR PERIOD OF RECORD.--HIGHEST WATER LEVEL, $142.00 \mathrm{FT}(43.28 \mathrm{M})$ BELOW LAND-SURFACE DATUM,MAR 8, 1983; LOWEST WATER LEVEL, 145.00 FT(44.20 M) BELOW LAND-SURFACE DATUM, MAR 4, 1982 .

WATER LEVELS IN FEET BELOW LAND SURFACE DATUM, CLIMATIC YEARS APRIL 1981 TO MARCH 1983

\begin{tabular}{|c|c|c|}
\hline & WATER & \\
\hline DATE & LEVEL & DATE \\
\hline
\end{tabular}

MAR 04, $1982 \quad 145.00 \quad$ MAR 08, $1983 \quad 142.00$

GROUND-WATER LEVELS

352558097053001. LOCAL NUMBER; 11N-02E-16 AAC 1.

LOCATION,--LAT 35 25'58", LONG 097 05'30", HYDROLOGIC UNIT 11100302.

AQUIFER .--TERRACE DEPOSITS.

PERIOD OF RECORD.--1979 TO CURRENT YEAR.

EXTREMES FOR PERIOD OF RECORD.--HIGHEST WATER LEVEL, 69.00 FT(21.03 M) BELOW LAND-SURFACE

DATUM, MAR 8, 1983; LOWEST WATER LEVEL, 120.00 FT(36.58 M) BELOW LAND-SURFACE DATUM, MAR $30,1979$.

WATER LEVELS IN FEET BELOW LAND SURFACE DATUM, CLIMATIC YEARS APRIL 1981 TO MARCH 1983

$\begin{array}{lll} & \text { WATER } & \text { WATER } \\ \text { DATE } & \text { LEVEL } & \text { DATE }\end{array}$

$\begin{array}{llll}\text { MAR 31, } 1982 & \text { M } & \text { MAR 08, } 1983 & 69.00\end{array}$ 
GROUND-WATER LEVELS

POTTANATOMIE COUNTY -- CONTINUED

352604097060901. LOCAL NUMBER; 11N-02E-16 BBA 1.

LOCATION,--LAT 35 26'04", LONG 097 06'09", HYDROLOGIC UNIT 11100302.

AQUIFER.--TERRACE DEPOSITS.

PERIOD OF RECORD.--1979 TO CURRENT YEAR.

EXTREMES FOR PERIOD OF RECORD.--HIGHEST WATER LEVEL, 160.00 FT(48.77 M) BELOW LAND-SURFACE DATUM, MAR 4, 1982; LOWEST WATER LEVEL, 170.00 FT(51.82 M) BELOW LAND-SURFACE DATUM, MAR 30, 1979.

WATER LEVELS IN FEET BELOW LAND SURFACE DATUM, CLIMATIC YEARS APRIL 1981 TO MARCH 1983

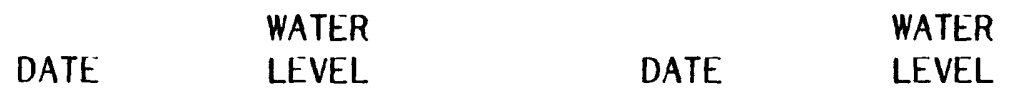

MAR 31, $1982 \quad$ Z $\quad$ MAR 08, $1983 \quad 170.00$

GROUND-WATER LEVELS

352551097060901. LOCAL NUMBER; 11N-02E-16 BCA 1.

LOCATION,--LAT 35 25'51", LONG 097 06'09", HYDROLOGIC UNIT 11100302.

AQUIFER.--TERRACE DEPOSITS.

PERIOD OF RECORD.--1982 TO CURRENT YEAR.

EXTREMES FOR PERIOD OF RECORD.--HIGHEST WATER LEVEL, $145.00 \mathrm{FT}(44.20 \mathrm{M})$ BELOW LAND-SURFACE DATUM,MAR 8, 1983; LOWEST WATER LEVEL, 160.00 FT(48.77 M) BELOW LAND-SURFACE DATUM, MAR 4, 1982 .

WATER LEVELS IN FEET BELOW LAND SURFACE DATUM, CLIMATIC YEARS APRIL 1981 TO MARCH 1983

\begin{tabular}{|c|c|c|}
\hline DATE & $\begin{array}{l}\text { WATER } \\
\text { LEVEL }\end{array}$ & DATE \\
\hline
\end{tabular}

MAR 04, $1982 \quad 160.00 \quad$ MAR 08, $1983 \quad 145.00$ 
POTTAWATOMIE COUNTY -- CONTINUED

352519096544701. LOCAL NUMBER; 11N-04E-18 DDD 1.

LOCATION,--LAT 35 25'19", LONG 096 54'47", HYDROLOGIC UNIT 11100302.

AQUIFER.--TERRACE DEPOSITS.

PERIOD OF RECORD.--1981 TO CURRENT YEAR.

EXTREMES FOR PERIOD OF RECORD.--HIGHEST WATER LEVEL, $54.65 \mathrm{FT}(16.66 \mathrm{M})$ BELOW LAND-SURFACE DATUM, MAY 21, 1981; LOWEST WATER LEVEL,74.69 FT(22.77 M) BELOW LAND-SURFACE DATUM, MAR $4,1983$.

WATER LEVELS IN FEET BELOW LAND SURFACE DATUM, CLIMATIC YEARS APRIL 1981 TO MARCH 1983

\begin{tabular}{|c|c|}
\hline & WATER \\
\hline DATE & LEVEL \\
\hline
\end{tabular}

MAR 09, $1982 \quad 71.60 \quad$ MAR 04, $1983 \quad 74.69 \mathrm{R}$

GROUND-WATER LEVELS

352519096545501. LOCAL NUMBER; 11N-04E-18 CDD 1.

LOCATION,--LAT 35 25'19", LONG 096 54'55", HYDROLOGIC UNIT 11100302.

AQUIFER.--TERRACE DEPOSITS.

PERIOD OF RECORD.--1981 TO CURRENT YEAR.

EXTREMES FOR PERIOD OF RECORD.--HIGHEST WATER LEVEL, $69.95 \mathrm{FT}(21.32 \mathrm{M})$ BELOW LAND-SURFACE DATUM, MAY 29, 1981; LOWEST WATER LEVEL,97.60 FT(29.75 M) BELOW LAND-SURFACE DATUM, MAR 9, 1982.

WATER LEVELS IN FEET BELOW LAND SURFACE DATUM, CLIMATIC YEARS APRIL 1981 TO MARCH 1983

\begin{tabular}{|c|c|c|}
\hline DATE & $\begin{array}{l}\text { WATER } \\
\text { LEVEL }\end{array}$ & DATE \\
\hline
\end{tabular}

MAR 09, $1982 \quad 97.60 \mathrm{P} \quad$ MAR 04, $1983 \quad 97.59$ 


\section{GROUND-WATER LEVELS}

PUSHMATAHA COUNTY

341435095350801. LOCAL NUMBER; LOCATION,--LAT 34 14'35", LONG 095 35'08", HYDROLOGIC UNIT 11140105. AQUIFER.--ANTLERS SAND. PERIOD OF RECORD.--1976 TO CURRENT YEAR. EXTREMES FOR PERIOD OF RECORD.--HIGHEST WATER LEVEL,17.13 FT(5.22 M) BELOW LAND-SURFACE DATUM, APR 21, 1976; LOWEST WATER LEVEL, 19.90 FT(6.07 M) BELOW LAND-SURFACE DATUM, MAR 4, 1981.

WATER LEVELS IN FEET BELOW LAND SURFACE DATUM, CLIMATIC YEARS APRIL 1981 TO MARCH 1983

$\begin{array}{lll} & \text { WATER } & \\ \text { DATE } & \text { LEVEL } & \text { WATER } \\ & \text { DATE } & \text { LEVEL }\end{array}$

MAR 11, $198219.79 \quad$ MAR 08, $1983 \quad 18.04$

\section{GROUND-WATER LEVELS}

341344095373801. LOCAL NUMBER; LOCATION,--LAT 34 13'44", LONG 095 AQUIFER . - PALUXY SAND. PERIOD OF RECORD.--1980 TO CURRENT YEAR.

EXTREMES FOR PERIOD OF RECORD.--HIGHEST WATER LEVEL,8.50 FT(2.59 M) BELOW LAND-SURFACE DATUM,MAR 8, 1983; LOWEST WATER LEVEL, $12.50 \mathrm{FT}(3.81 \mathrm{M})$ BELOW LAND-SURFACE DATUM, MAR 3, 1981.

WATER LEVELS IN FEET BELOW LAND SURFACE DATUM, CLIMATIC YEARS APRIL 1981 TO MARCH 1983

$$
\text { 04S-16E-09 ABB } 1 .
$$$$
\text { 37'38", HYDROLOGIC UNIT } 11130102 .
$$ 


\section{GROUND-WATER LEVELS}

ROGER MILLS COUNTY

352750099530001. LOCAL NUMBER;

$11 \mathrm{~N}-25 \mathrm{~W}-06$ ABB 1.

LOCATION,--LAT 35 27'50", LONG 099 53'00", HYDROLOGIC UNIT 11120302.

AQUIFER.--OGALLALA.

PERIOD OF RECORD.--1979 TO CURRENT YEAR.

EXTREMES FOR PERIOD OF RECORD.--HIGHEST WATER LEVEL,9.84 FT(3.00 M) BELOW LAND-SURFACE DATUM,FEB 18, 1983; LOWEST WATER LEVEL,43.40 FT(13.23 M) BELOW LAND-SURFACE DATUM, APR 10, 1979.

WATER LEVELS IN FEET BELOW LAND SURFACE DATUM, CLIMATIC YEARS APRIL 1981 TO MARCH 1983

\begin{tabular}{|c|c|}
\hline & WATER \\
\hline DATE & LEVEL \\
\hline
\end{tabular}

JAN 21, $1982 \quad 41.15 \quad$ FEB 18, $1983 \quad 9.84$

\section{GROUND-WATER LEVELS}

353206099422201. LOCAL NUMBER; 12N-24W-11 ABC 1.

LOCATION,--LAT 35 32'06", LONG 099 42'22", HYDROLOGIC UNIT 11130301.

AQUIFER .--OGALLALA.

PERIOD OF RECORD.--1980 TO CURRENT YEAR.

EXTREMES FOR PERIOD OF RECORD.--HIGHEST WATER LEVEL,9.84 FT(3.00 M) BELOW LAND-SURFACE DATUM, FEB 18, 1983; LOWEST WATER LEVEL, 13.88 FT(4.23 M) BELOW LAND-SURFACE DATUM, JAN 21,1982 .

WATER LEVELS IN FEET BELOW LAND SURFACE DATUM, CLIMATIC YEARS APRIL 1981 TO MARCH 1983

\begin{tabular}{|c|c|}
\hline 47 & WATER \\
\hline & \\
\hline
\end{tabular}

JAN 21, $1982 \quad 13.88 \quad$ FEB $18,1983 \quad 9.84$ 
GROUND-WATER LEVELS

ROGER MILLS COUNTY -- CONTINUED

353100099460801. LOCAL NUMBER;

LOCATION,--LAT 35 31'00", LONG 099

AQUIFER . - OGALLALA.

PERIOD OF RECORD.--1980 TO CURRENT YEAR.

EXTREMES FOR PERIOD OF RECORD.--HIGHEST WATER LEVEL, $38.56 \mathrm{FT}(11.75 \mathrm{M})$ BELOW LAND-SURFACE DATUM,FEB 18, 1983; LOWEST WATER LEVEL,39.25 FT(11.96 M) BELOW LAND-SURFACE DATUM, MAR 26, 1980.

WATER LEVELS IN FEET BELOW LAND SURFACE DATUM, CLIMATIC YEARS APRIL 1981 TO MARCH 1983

\begin{tabular}{|c|c|c|}
\hline & WATER & \\
\hline DATE & LEVEL & DATE \\
\hline
\end{tabular}

JAN 21, $1982 \quad 38.77 \quad$ FEB $18,1983 \quad 38.56$

GROUND-WATER LEVELS

353242099471801. LOCAL NUMBER; 12N-25W-01 ADD 1.

LOCATION,--LAT 35 32'42", LONG 099 47'18", HYDROLOGIC UNIT 11130301.

AQUIFER .--OGALLALA.

PERIOD OF RECORD.--1980 TO CURRENT YEAR.

EXTREMES FOR PERIOD OF RECORD.--HIGHEST WATER LEVEL, 58.77 FT(17.91 M) BELOW LAND-SURFACE DATUM,MAR 26, 1980; LOWEST WATER LEVEL,59.23 FT(18.05 M) BELOW LAND-SURFACE DATUM, FEB 18, 1983.

WATER LEVELS IN FEET BELOW LAND SURFACE DATUM, CLIMATIC YEARS APRIL 1981 TO MARCH 1983

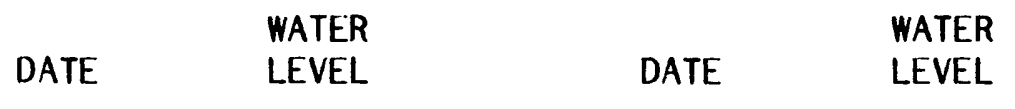

JAN 21, $1982 \quad 58.90 \quad$ FEB 18, $1983 \quad 59.23$ 
353044099492601 . LOCAL NUMBER; LOCATION,--LAT 35 30'44", LONG 099 AQUIFER . - OGALLALA.

EXTREMES FOR PERIOD OF RECORD.--HIGHEST WATER LEVEL, 18.69 FT(5.70 M) BELOW LAND-SURFACE DATUM,MAR 26, 1980; LOWEST WATER LEVEL,20.98 FT(6.39 M) BELOW LAND-SURFACE DATUM, JAN 21, 1982.

WATER LEVELS IN FEET BELOW LAND SURFACE DATUM, CLIMATIC YEARS APRIL 1981 TO MARCH 1983

\section{DATE}

WATER

LEVEL

JAN 21, $1982 \quad 20.98 \quad$ FEB 18, $1983 \quad 19.03$

12N-25W-15 DDA 1.

49'26", HYDROLOGIC UNIT 11120302.
352834099533701. LOCAL NUMBER; LOCATION,--LAT $3528^{\prime} 34^{\prime \prime}$, LONG 099 AQUIFER.--OGALLALA.

PERIOD OF RECORD. -1979 TO CURRENT YEAR.

EXTREMES FOR PERIOD OF RECORD.--HIGHEST WATER LEVEL,48.18 FT(14.69 M) BELOW LAND-SURFACE DATUM,MAR 26, 1981; LOWEST WATER LEVEL,64.78 FT(19.74 M) BELOW LAND-SURFACE DATUM, FEB 15, 1982 .

WATER LEVELS IN FEET BELOW LAND SURFACE DATUM, CLIMATIC YEARS APRIL 1981 TO MARCH 1983

\section{DATE}

WATER

LEVEL

DATE

WATER

LEVEL

FEB 15, $1982 \quad 64.78 \quad$ FEB 18, $1983 \quad 54.47$ 
ROGER MILLS COUNTY -- CONTINUED

353722099400801. LOCAL NUMBER;

$13 N-23 W-08$ ABB 1.

LOCATION,--LAT 35 37'22", LONG 099 40'08", HYDROLOGIC UNIT 11130301.

AQUIFER.--OGALLALA.

PERIOD OF RECORD.--1976 TO CURRENT YEAR.

EXTREMES FOR PERIOD OF RECORD.--HIGHEST WATER LEVEL,7.99 FT(2.44 M) BELOW LAND-SURFACE DATUM, MAR 26, 1980; LOWEST WATER LEVEL, 17.59 FT(5.36 M) BELOW LAND-SURFACE DATUM, DEC 22, 1976.

WATER LEVELS IN FEET BELOW LAND SURFACE DATUM, CLIMATIC YEARS APRIL 1981 TO MARCH 1983

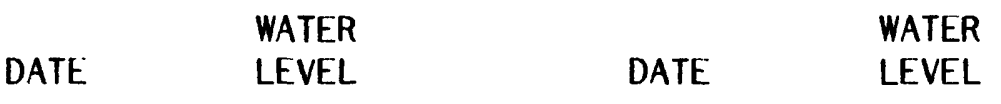

JAN 22, $1982 \quad 12.64 \quad$ FEB $18,1983 \quad 8.72$

GROUND-WATER LEVELS

353648099521601. LOCAL NUMBER; 13N-25W-09 CBC 1.

LOCATION,--LAT 35 36'48", LONG 099 52'16", HYDROLOGIC UNIT 11130301.

AQUIFER .--OGALLALA.

PERIOD OF RECORD.--1981 TO CURRENT YEAR.

EXTREMES FOR PERIOD OF RECORD.--HIGHEST WATER LEVEL, $35.92 \mathrm{FT}(10.95 \mathrm{M})$ BELOW LAND-SURFACE DATUM, MAR 27, 1981; LOWEST WATER LEVEL, 35.92 FT(10.95 M) BELOW LAND-SURFACE DATUM, MAR 27, 1981.

WATER LEVELS IN FEET BELOW LAND SURFACE DATUM, CLIMATIC YEARS APRIL 1981 TO MARCH 1983

\begin{tabular}{|c|c|}
\hline DATE & $\begin{array}{l}\text { WATER } \\
\text { LEVEL }\end{array}$ \\
\hline
\end{tabular}

JAN 22, $1982 \quad P \quad$ FEB 18, $1983 \quad P$


ROGER MILLS COUNTY -- CONTINUED

353440099534001. LOCAL NUMBER; 13N-25W-30 AAC 1.

LOCATION,--LAT 35 34'40", LONG 099 53'40", HYDROLOGIC UNIT 11130301.

AQUIFER. - -OGALLALA.

PERIOD OF RECORD.--1980 TO CURRENT YEAR.

EXTREMES FOR PERIOD OF RECORD.--HIGHEST WATER LEVEL,50.42 FT(15.37 M) BELOW LAND-SURFACE DATUM,FEB 18, 1983; LOWEST WATER LEVEL,51.07 FT(15.57 M) BELOW LAND-SURFACE DATUM, MAR 27, 1980.

WATER LEVELS IN FEET BELOW LAND SURFACE DATUM, CLIMATIC YEARS APRIL 1981 TO MARCH 1983

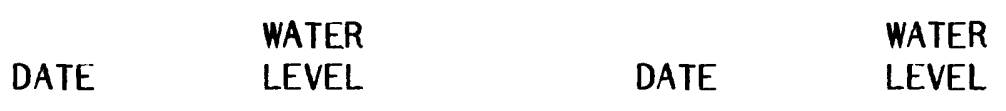

FEB $15,1982 \quad 50.70 \quad$ FEB $18,1983 \quad 50.42$

GROUND-WATER LEVELS

353338099533401. LOCAL NUMBER; 13N-25W-31 ADB 1.

LOCATION,--LAT 35 33'38", LONG 099 53'34", HYDROLOGIC UNIT 11130301.

AQUIFER .--ALLUVIUM.

PERIOD OF RECORD.--1979 TO CURRENT YEAR.

EXTREMES FOR PERIOD OF RECORD.--HIGHEST WATER LEVEL, $73 \mathrm{FT}(.22 \mathrm{M})$ BELOW LAND-SURFACE DATUM, APR 10, 1979; LOWEST WATER LEVEL, $1.84 \mathrm{FT}(.56 \mathrm{M})$ BELOW LAND-SURFACE DATUM, MAR 26, 1981.

WATER LEVELS IN FEET BELOW LAND SURFACE DATUM, CLIMATIC YEARS APRIL 1981 TO MARCH 1983

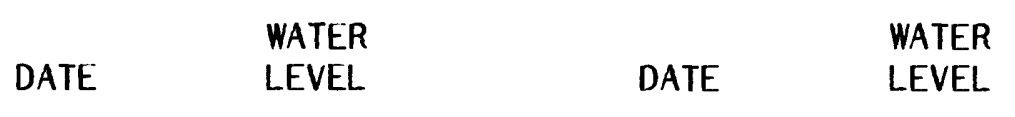

FEB 15, $1982 \quad 1.26 \quad$ FEB 18, $1983 \quad 1.54$ 


\section{GROUND-WATER LEVELS}

ROGER MILLS COUNTY -- CONTINUED

353712099582001. LOCAL NUMBER; 13N-26W-09 BDB 1. LOCATION,--LAT 35 37'12", LONG 099 58'20", HYDROLOGIC UNIT 11130301. AQUIFER.--OGALLALA. PERIOD OF RECORD._- 1980 TO CURRENT YEAR. EXTREMES FOR PERIOD OF RECORD.--HIGHEST WATER LEVEL,70.09 FT(21.36 M) BELOW LAND-SURFACE DATUM,MAR 26, 1981; LOWEST WATER LEVEL,71.94 FT(21.93 M) BELOW LAND-SURFACE DATUM, FEB 15, 1982 .

WATER LEVELS IN FEET BELOW LAND SURFACE DATUM, CLIMATIC YEARS APRIL 1981 TO MARCH 1983

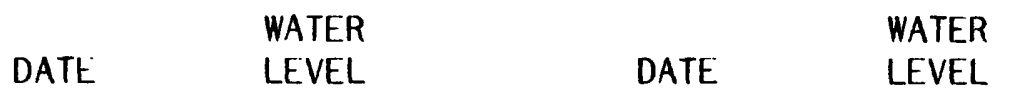

FEB $15,1982 \quad 71.94 \quad$ FEB $18,1983 \quad 71.49$

GROUND-WATER LEVELS

353440099375601. LOCAL NUMBER; 13N-26W-28 AAC 1. LOCATION,--LAT 35 34'40", LONG 099 57'56", HYDROLOGIC UNIT 11130301. AQUIFER.--OGALLALA. PERIOD OF RECORD.--1980 TO CURRENT YEAR. EXTREMES FOR PERIOD OF RECORD.--HIGHEST WATER LEVEL, 109.43 FT(33.35 M) BELOW LAND-SURFACE DATUM,FEB 18, 1983; LOWEST WATER LEVEL,115.72 FT(35.27 M) BELOW LAND-SURFACE DATUM, FEB 15, 1982 .

WATER LEVELS IN FEET BELOW LAND SURFACE DATUM, CLIMATIC YEARS APRIL 1981 TO MARCH 1983

\begin{tabular}{|c|c|}
\hline & WATER \\
\hline DATE & LEVEL \\
\hline
\end{tabular}

FEB 15, $1982 \quad 115.72 \quad$ FEB 18, $1983 \quad 109.43$ 
ROGER MILLS COUNTY -- CONTINUED

353854099402401. LOCAL NUMBER; LOCATION,--LAT 3538 '54", LONG 099 AQUIFER.--OGALLALA. PERIOD OF RECORD.--1979 TO CURRENT YEAR.

EXTREMES FOR PERIOD OF RECORD.--HIGHEST WATER LEVEL, 25.02 FT(7.63 M) BELOW LAND-SURFACE DATUM,MAR 27, 1981; LOWEST WATER LEVEL,27.52 FT(8.39 M) BELOW LAND-SURFACE DATUM, MAR 29, 1979.

WATER LEVELS IN FEET BELOW LAND SURFACE DATUM, CLIMATIC YEARS APRIL 1981 TO MARCH 1983
$14 \mathrm{~N}-23 \mathrm{~W}-32$ BDD 1.

40'24", HYDROLOGIC UNIT 11130301.

WATER LEVELS IN FEET BELOW LAND SURFACE DATUM, CLIMATIC YEARS APRIL 1981 TO MARCH 1983

$\begin{array}{lll} & \text { WATER } & \\ \text { DATE } & \text { WEVEL } & \text { DATER } \\ & & \text { LEVEL }\end{array}$

FEB 17, $1982 \quad 26.84 \quad$ FEB $18,1983 \quad 26.67$

GROUND-WATER LEVELS

353924099492801. LOCAL NUMBER; 14N-25W-26 DCA 1. LOCATION,--LAT 35 39'24", LONG 099 49'28", HYDROLOGIC UNIT 11130301. AQUIFER.--OGALLALA.

PERIOD OF RECORD.--1980 TO CURRENT YEAR.

EXTREMES FOR PERIOD OF RECORD.--HIGHEST WATER LEVEL,59.36 FT(18.09 M) BELOW LAND-SURFACE DATUM,FEB 16, 1983; LOWEST WATER LEVEL,61.83 FT(18.85 M) BELOW LAND-SURFACE DATUM, MAR 27, 1980.

WATER LEVELS IN FEET BELOW LAND SURFACE DATUM, CLIMATIC YEARS APRIL 1981 TO MARCH 1983

\begin{tabular}{|c|c|c|}
\hline DATE & $\begin{array}{l}\text { WATER } \\
\text { LEVEL }\end{array}$ & DATE \\
\hline
\end{tabular}

JAN 22, $1982 \quad 60.00 \quad$ FEB $16,1983 \quad 59.36$ 
ROGER MILLS COUNTY -- CONTINUED

353904099542201. LOCAL NUMBER; LOCATION,--LAT 35 39'04", LONG 099 54'22", HYDROLOGIC UNIT 11130301. AQUIFER.--OGALLALA. PERIOD OF RECORD.--1980 TO CURRENT YEAR.

EXTREMES FOR PERIOD OF RECORD.--HIGHEST WATER LEVEL, 32.62 FT(9.94 M) BELOW LAND-SURFACE DATUM, MAR 27, 1980; LOWEST WATER LEVEL,42.58 FT(12.98 M) BELOW LAND-SURFACE DATUM, MAR 26, 1981.

WATER LEVELS IN FEET BELOW LAND SURFACE DATUM, CLIMATIC YEARS APRIL 1981 TO MARCH 1983

WATER

DATE

JAN 22, 1982

\author{
LEVEL
}

42.40
DATE

FEB 16, 1983

WATER

LEVEL

41.94

\section{GROUND-WATER LEVELS}

354702099465501. LOCAL NUMBER; LOCATION,--LAT 35 47'02", LONG 099 AQUIFER .--OGALLALA.

PERIOD OF RECORD. --1980 TO CURRENT YEAR.

EXTREMES FOR PERIOD OF RECORD.--HIGHEST WATER LEVEL, $100.90 \mathrm{FT}(30.75 \mathrm{M})$ BELOW LAND-SURFACE DATUM, FEB 16, 1983; LOWEST WATER LEVEL,118.90 FT(36.24 M) BELOW LAND-SURFACE DATUM, FEB 17, 1982.

WATER LEVELS IN FEET BELOW LAND SURFACE DATUM, CLIMATIC YEARS APRIL 1981 TO MARCH 1983
$15 \mathrm{~N}-24 \mathrm{~W}-08$ CCC 1 46'55", HYDROLOGIC UNIT 11130301.
DATE
WATER
$\begin{array}{ll} & \text { WATER } \\ \text { DATE } & \text { LEVEL }\end{array}$

FEB 17, $1982 \quad 118.90$

FEB $16,1983 \quad 100.90$ 


\author{
ROGER MILLS COUNTY -- CONTINUED
}

354651099434101. LOCAL NUMBER;

$15 \mathrm{~N}-24 \mathrm{~W}-14 \mathrm{BBC} 1$. LOCATION,--LAT 3546 '51', LONG 099 AQUIFER.--OGALLALA. PERIOD OF RECORD.--1980 TO CURRENT YEAR. EXTREMES FOR PERIOD OF RECORD.--HIGHEST WATER LEVEL,66.50 FT(20.27 M) BELOW LAND-SURFACE DATUM,FEB 17, 1982; LOWEST WATER LEVEL,68.88 FT(20.99 M) BELOW LAND-SURFACE DATUM, MAR 27, 1981.

WATER LEVELS IN FEET BELOW LAND SURFACE DATUM, CLIMATIC YEARS APRIL 1981 TO MARCH 1983

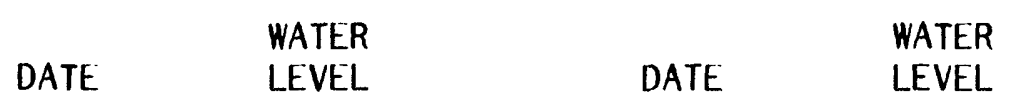

FEB $17,1982 \quad 66.50 \quad$ FEB $16,1983 \quad 67.58$

GROUND-WATER LEVELS

334519099465901. LOCAL NUMBER;

$15 \mathrm{~N}-24 \mathrm{~W}-19$ DDD 1.

LOCATION,--LAT 35 45'19', LONG 099

AQUIFER.--OGALLALA.

PERIOD OF RECORD.--1980 TO CURRENT YEAR.

EXTREMES FOR PERIOD OF RECORD.--HIGHEST WATER LEVEL, $57.94 \mathrm{FT}(17.66 \mathrm{M})$ BELOW LAND-SURFACE DATUM,MAR 27, 1980; LOWEST WATER LEVEL,58.38 FT(17.79 M) BELOW LAND-SURFACE DATUM, FEB 17, 1982.

WATER LEVELS IN FEET BELOW LAND SURFACE DATUM, CLIMATIC YEARS APRIL 1981 TO MARCH 1983

$\begin{array}{lll} & \text { WATER } & \text { WATER } \\ \text { DATE } & \text { LEVEL } & \text { DATE }\end{array}$

FEB 17, $1982 \quad 58.38 \quad$ FEB $16,1983 \quad$ Z 
GROUND-WATER LEVELS

ROGER MILLS COUNTY -- CONTINUED

354340099422501. LOCAL NUMBER;

$15 \mathrm{~N}-24 \mathrm{~W}-36$ CAC 1.

LOCATION,--LAT 35 43'40", LONG 099

AQUIFER.--OGALLALA.

PERIOD OF RECORD.--1976 TO CURRENT YEAR.

EXTREMES FOR PERIOD OF RECORD.--HIGHEST WATER LEVEL,4.10 FT(1.25 M) BELOW LAND-SURFACE DATUM,FEB 17, 1982; LOWEST WATER LEVEL,7.99 FT(2.44 M) BELOW LAND-SURFACE DATUM, DEC 22, 1976.

WATER LEVELS IN FEET BELOW LAND SURFACE DATUM, CLIMATIC YEARS APRIL 1981 TO MARCH 1983

\begin{tabular}{|c|c|c|}
\hline $\mathrm{DATC}$ & WATER & \\
\hline DAIE & & . \\
\hline
\end{tabular}

FEB 17, $1982 \quad 4.10 \quad$ FEB 16, $1983 \quad 6.85$

GROUND-WATER LEVELS

354725099511501. LOCAL NUMBER; 15N-25W-09 DAA 1.

LOCATION,--LAT 35 47'25", LONG 099 51'15", HYDROLOGIC UNIT 11130301.

AQUIFER .--OGALLALA.

PERIOD OF RLCORD.--1980 TO CURRENT YEAR.

EXTREMES FOR PERIOD OF RECORD.--HIGHEST WATER LEVEL, 113.04 FT(34.45 M) BELOW LAND-SURFACE DATUM,MAR 27, 1981; LOWEST WATER LEVEL,119.68 FT(36.48 M) BELOW LAND-SURFACE DATUM, MAR 27, 1980 .

WATER LEVELS IN FEET BELOW LAND SURFACE DATUM, CLIMATIC YEARS APRIL 1981 TO MARCH 1983

\begin{tabular}{|c|c|}
\hline & WATER \\
\hline DATE & LEVEL \\
\hline
\end{tabular}
FEB 17, 1982
0
FEB 16, 1983
0 
354845099581801. LOCAL NUMBER; LOCATION,--LAT 35 48'45", LONG 099 AQUIFER.--OGALLALA. PERIOD OF RECORD. - 1980 TO CURRENT YEAR. EXTREMES FOR PERIOD OF RECORD.--HIGHEST WATER LEVEL, $146.08 \mathrm{FT}(44.53 \mathrm{M})$ BELOW LAND-SURFACE DATUM, MAR 27, 1980; LOWEST WATER LEVEL, 146.47 FT(44.64 M) BELOW LAND-SURFACE DATUM, MAR 27, 1980.

WATER LEVELS IN FEET BELOW LAND SURFACE DATUM, CLIMATIC YEARS APRIL 1981 TO MARCH 1983
FEB 15, 1982
$Z \quad$ FEB 16,1983
Z

GROUND-WATER LEVELS

354704099563201. LOCAL NUMBER; 15N-26W-11 CCC 1. LOCATION,--LAT 35 47'04", LONG 099 56'32", HYDROLOGIC UNIT 11130301. AQUIFER .--OGALLALA. PERIOD OF RECORD.--1980 TO CURRENT YEAR. EXTREMES FOR PERIOD OF RECORD.--HIGHEST WATER LEVEL, $36.50 \mathrm{FT}(11.13 \mathrm{M})$ BELOW LAND-SURFACE DATUM,FEB 16, 1983; LOWEST WATER LEVEL,40.34 FT(12.30 M) BELOW LAND-SURFACE DATUM, MAR 27, 1980.

WATER LEVELS IN FEET BELOW LAND SURFACE DATUM, CLIMATIC YEARS APRIL 1981 TO MARCH 1983

$\begin{array}{lll} & \text { WATER } & \\ \text { DATE } & \text { WEVEL } & \text { WATER } \\ \text { LEVEL }\end{array}$

FEB $17,1982 \quad Z \quad$ FEB $16,1983 \quad 36.50$ 
ROGER MILLS COUNTY -- CONTINUED

354700099552901. LOCAL NUMBER; 15N-26W-13 BBB 1. LOCATION,--LAT 35 47'00", LONG 099 55'29", HYDROLOGIC UNIT 11130301. AQUIFER. - OGALLALA.

PERIOD OF RECORD.--1980 TO CURRENT YEAR.

EXTREMES FOR PERIOD OF RECORD.--HIGHEST WATER LEVEL,53.89 FT(16.43 M) BELOW LAND-SURFACE DATUM, FEB 17, 1982; LOWEST WATER LEVEL,55.00 FT(16.76 M) BELOW LAND-SURFACE DATUM, MAR 27, 1980.

WATER LEVELS IN FEET BELOW LAND SURFACE DATUM, CLIMATIC YEARS APRIL 1981 TO MARCH 1983

\begin{tabular}{|c|c|}
\hline & WATER \\
\hline DATE & LEVEL \\
\hline
\end{tabular}

FEB $17,1982 \quad 53.89 \quad$ FEB $16,1983 \quad Z$

GROUND-WATER LEVELS

354344099593601. LOCAL NUMBER; 15N-26W-32 CCA 1.

LOCATION,--LAT 35 43'44", LONG 099 59'36", HYDROLOGIC UNIT 11130301.

AQUIFER .--OGALLALA.

PERIOD OF RECORD.--1981 TO CURRENT YEAR.

EXTREMES FOR PERIOD OF RECORD.--HIGHEST WATER LEVEL, $76.94 \mathrm{FT}(23.45 \mathrm{M})$ BELOW LAND-SURFACE DATUM,FEB 16, 1983; LOWEST WATER LEVEL,78.66 FT(23.98 M) BELOW LAND-SURFACE DATUM, MAR 27, 1981.

WATER LEVELS IN FEET BELOW LAND SURFACE DATUM, CLIMATIC YEARS APRIL 1981 TO MARCH 1983

$\begin{array}{lll} & \text { WATER } & \text { WATER } \\ \text { DATE } & \text { LEVEL } & \text { DATE }\end{array}$

FEB 15, $1982 \quad 77.80 P \quad$ FEB $16,1983 \quad 76.94$ 
ROGER MILLS COUNTY -- CONTINUED

354935099460501. LOCAL NUMBER; 16N-24W-32 AAB 1. LOCATION,--LAT 3S 49'35", LONG 099 46'05", HYDROLOGIC UNIT 11090201. AQUIFER .--OGALLALA.

PERIOD OF RECORD.--1980 TO CURRENT YEAR.

EXTREMES FOR PERIOD OF RECORD.--HIGHEST WATER LEVEL, $42.29 \mathrm{FT}(12.89 \mathrm{M})$ BELOW LAND-SURFACE DATUM,MAR 27, 1980; LOWEST WATER LEVEL,42.81 FT(13.05 M) BELOW LAND-SURFACE DATUM, MAR 27, 1981.

WATER LEVELS IN FEET BELOW LAND SURFACE DATUM, CLIMATIC YEARS APRIL 1981 TO MARCH 1983

\begin{tabular}{|c|c|c|}
\hline & WATER & \\
\hline DATE & LEVEL & DATE \\
\hline
\end{tabular}

FEB 17, $1982 \quad Z \quad$ FEB 16, $1983 \quad Z$

GROUND-WATER LEVELS

355047099540501. LOCAL NUMBER; 16N-25W-19 CAC 1.

LOCATION,--LAT $3550^{\prime} 47^{\prime \prime}$, LONG $09954^{\prime} 05^{\prime \prime}$, HYDROLOGIC UNIT 11130301.

AQUIFER.--OGALLALA.

PERIOD OF RECORD.--1980 TO CURRENT YEAR.

EXTREMES FOR PERIOD OF RECORD.--HIGHEST WATER LEVEL, 123.95 FT(37.78 M) BELOW LAND-SURFACE DATUM,MAR 26, 1981; LOWEST WATER LEVEL, 124.21 FT(37.86 M) BELOW LAND-SURFACE DATUM, JAN 21, 1982 .

WATER LEVELS IN FEET BELOW LAND SURFACE DATUM, CLIMATIC YEARS APRIL 1981 TO MARCH 1983

$\begin{array}{lll} & \text { WATER } & \text { WATER } \\ \text { DATE } & \text { LEVEL } & \text { DATE }\end{array}$

JAN 21, $1982 \quad 124.21 \quad$ FEB 16, $1983 \quad 123.95$ 
GROUND-WATER LEVELS

ROGER MILLS COUNTY -- CONTINUED

355047099540601. LOCAL NUMBER; 16N-25W-31 CAC 1.

LOCATION,--LAT 35 50'47", LONG 099 54'06", HYDROLOGIC UNIT 11130301.

AQUIFER.--OGALLALA.

PERIOD OF RECORD.--1980 TO CURRENT YEAR.

EXTREMES FOR PERIOD OF RECORD.--HIGHEST WATER LEVEL,9.24 FT(2.82 M) BELOW LAND-SURFACE DATUM,FEB 16, 1983; LOWEST WATER LEVEL, 10.00 FT(3.05 M) BELOW LAND-SURFACE DATUM, MAR 27, 1981.

WATER LEVELS IN FEET BELOW LAND SURFACE DATUM, CLIMATIC YEARS APRIL 1981 TO MARCH 1983

$\begin{array}{lll} & \text { WATER } & \text { WATER } \\ \text { DATE } & \text { LEVEL } & \text { DATE }\end{array}$

FEB $17,1982 \quad 10.00 \quad$ FEB $16,1983 \quad 9.24$

GROUND-WATER LEVELS

355310099574301. LOCAL NUMBER; 16N-26W-04 DCD 1.

LOCATION,--LAT 35 53'10", LONG 099 57'43", HYDROLOGIC UNIT 11090201.

AQUIFER .--OGALLALA.

PERIOD OF RECORD.--1980 TO CURRENT YEAR.

EXTREMES FOR PERIOD OF RECORD.--HIGHEST WATER LEVEL,20.62 FT(6.28 M) BELOW LAND-SURFACE DATUM,JAN 14, 1983; LOWEST WATER LEVEL,21.08 FT(6.43 M) BELOW LAND-SURFACE DATUM, JAN 21, 1982.

WATER LEVELS IN FEET BELOW LAND SURFACE DATUM, CLIMATIC YEARS APRIL 1981 TO MARCH 1983

$\begin{array}{lll} & \text { WATER } & \text { WATER } \\ \text { DATE } & \text { LEVEL } & \text { DATE }\end{array}$

JAN 21, $1982 \quad 21.08 \quad$ JAN 14, $1983 \quad 20.62$ 
ROGER MILLS COUNTY -- CONTINUED

355219099574501. LOCAL NUMBER; 16N-26W-09 DDD 1. LOCATION,--LAT 35 52'19", LONG 099 57'45", HYDROLOGIC UNIT 11090201. AQUIFER . - OGALLALA. PERIOD OF RECORD.--1980 TO CURRENT YEAR. EXTREMES FOR PERIOD OF RECORD.--HIGHEST WATER LEVEL,5.10 FT(1.55 M) BELOW LAND-SURFACE DATUM, JAN 14, 1983; LOWEST WATER LEVEL,8.77 FT(2.67 M) BELOW LAND-SURFACE DATUM, MAR 26, 1980 .

WATER LEVELS IN FEET BELOW LAND SURFACE DATUM, CLIMATIC YEARS APRIL 1981 TO MARCH 1983

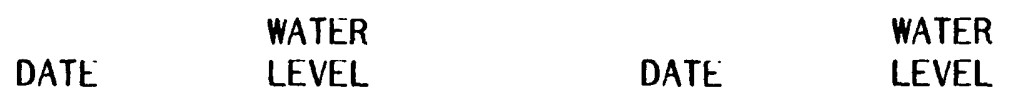

JAN 21, $1982 \quad 5.47 \quad$ JAN 14, $1983 \quad 5.10$

\section{GROUND-WATER LEVELS}

355214099581101. LOCAL NUMBER; 16N-26W-16 BAA 1.

LOCATION,--LAT 35 52'14", LONG 099 58'11", HYDROLOGIC UNIT 11090201. AQUIFER . - OGALLALA.

PERIOD OF RECORD.--1981 TO CURRENT YEAR.

EXTREMES FOR PERIOD OF RECORD.--HIGHEST WATER LEVEL, $41.00 \mathrm{FT}(12.50 \mathrm{M})$ BELOW LAND-SURFACE DATUM,MAR 26, 1981; LOWEST WATER LEVEL,41.12 FT(12.53 M) BELOW LAND-SURFACE DATUM, JAN 21, 1982.

WATER LEVELS IN FEET BELOW LAND SURFACE DATUM, CLIMATIC YEARS APRIL 1981 TO MARCH 1983

$\begin{array}{lll} & \text { WATER } & \text { WATER } \\ \text { DATE } & \text { LEVEL } & \text { DATE } \\ \end{array}$

JAN 21, $1982 \quad 41.12 \quad$ JAN 14, $1983 \quad 0$




\section{GROUND-WATER LEVELS}

ROGER MILLS COUNTY -- CONTINUED

355409099535201. LOCAL NUMBER;

$17 \mathrm{~N}-25 \mathrm{~W}-32$ BBC 1. LOCATION, --LAT 35 54'09", LONG 099 AQUIFER.--OGALLALA.

PERIOD OF RECORD.-- 1980 TO CURRENT YEAR.

EXTREMES FOR PERIOD OF RECORD.--HIGHEST WATER LEVEL, $35.80 \mathrm{FT}(10.91 \mathrm{M})$ BELOW LAND-SURFACE DATUM,MAR 26, 1980; LOWEST WATER LEVEL,37.14 FT(11.32 M) BELOW LAND-SURFACE DATUM, MAR 26, 1981.

WATER LEVELS IN FEET BELOW LAND SURFACE DATUM, CLIMATIC YEARS APRIL 1981 TO MARCH 1983

\begin{tabular}{|c|c|}
\hline & WATER \\
\hline DATE & LEVEL \\
\hline
\end{tabular}
FEB 17,1982
Z
FEB 16,1983
Z

\section{GROUND-WATER LEVELS}

355429099502801. LOCAL NUMBER; LOCATION, --LAT 35 54'29", LONG 099 AQUIFER.--OGALLALA. PERIOD OF RECORD.--1980 TO CURRENT YEAR. EXTREMES FOR PERIOD OF RECORD.--HIGHEST WATER LEVEL, 19.89 FT(6.06 M) BELOW LAND-SURFACE DATUM,FEB 16, 1983; LOWEST WATER LEVEL,22.83 FT(6.96 M) BELOW LAND-SURFACE DATUM, MAR 26, 1981.

WATER LEVELS IN FEET BELOW LAND SURFACE DATUM, CLIMATIC YEARS APRIL 1981 TO MARCH 1983

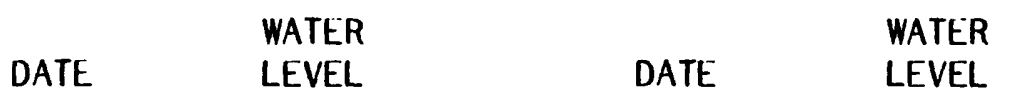

FEB $17,1982 \quad 20.32$ FEB 16, $1983 \quad 19.89$ 
GROUND-WATER LEVELS

ROGERS COUNTY

362305095420301. LOCAL NUMBER; 22N-15E-15 CBD 1. LOCATION,--LAT 36 23'05", LONG 095 42'03", HYDROLOGIC UNIT 11070106. AQUIFER ---OGALLALA. PERIOD OF RECORD.--1979 TO CURRENT YEAR. EXTREMES FOR PERIOD OF RECORD.--HIGHEST WATER LEVEL,9.63 FT (2.94 M) BELOW LAND-SURFACE DATUM,MAR 7, 1983; LOWEST WATER LEVEL, 12.80 FT(3.90 M) BELOW LAND-SURFACE DATUM, MAR 11, 1981.

WATER LEVELS IN FEET BELOW LAND SURFACE DATUM, CLIMATIC YEARS APRIL 1981 TO MARCH 1983

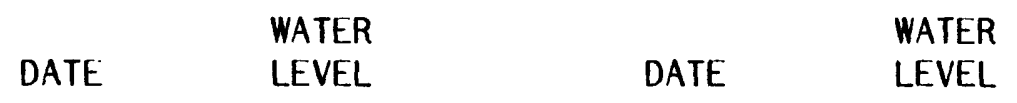

MAR $30,1982 \quad 11.48 \quad$ MAR $07,1983 \quad 9.63$

GROUND-WATER LEVELS

363306095242201. LOCAL NUMBER; 24N-18E-20 AAC 1. LOCATION,--LAT 36 33'06", LONG 095 24'22", HYDROLOGIC UNIT 11070209. AQUIFER .--SENORA FORMATION. PERIOD OF RECORD.-- 1979 TO CURRENT YEAR.

EXTREMES FOR PERIOD OF RECORD.--HIGHEST WATER LEVEL, $18.70 \mathrm{FT}(5.70 \mathrm{M})$ BELOW LAND-SURFACE DATUM, MAR 7, 1983; LOWEST WATER LEVEL,31.00 FT(9.45 M) BELOW LAND-SURFACE DATUM, MAR 15, 1979.

WATER LEVELS IN FEET BELOW LAND SURFACE DATUM, CLIMATIC YEARS APRIL 1981 TO MARCH 1983

$\begin{array}{lll} & \text { WATER } & \\ \text { DATE } & \text { WEVEL } & \text { WATER } \\ & \text { DATE } & \text { LEVEL }\end{array}$

$\begin{array}{llll}\text { MAR } 30,1982 & 19.10 \quad \text { MAR 07, } 1983 & 18.70\end{array}$ 


\section{GROUND-WATER LEVELS}

\section{SEMINOLE COUNTY}

350653096441901. LOCAL NUMBER;

LOCATION, --LAT $3506^{\prime} 53^{\prime \prime}$, LONG 096 AQUIFER.--ALLUVIUM.

PERIOD OF RECORD.--1980 TO CURRENT YEAR.

EXTREMES FOR PERIOD OF RECORD.--HIGHEST WATER LEVEL,45.65 FT(13.91 M) BELOW LAND-SURFACE

DATUM,FEB 2, 1980; LOWEST WATER LEVEL,123.90 FT(37.76 M) BELOW LAND-SURFACE DATUM, MAR 21, 1983.

WATER LEVELS IN FEET BELOW LAND SURFACE DATUM, CLIMATIC YEARS APRIL 1981 T0 MARCH 1983

WATER

DATE LEVEL

FEB 22, $1982 \quad 101.84 \mathrm{R}$
07N-05E-02 AAA 1.

44'19", HYDROLOGIC UNIT 11090203.
WATER

DATE LEVEL

MAR $21,1983 \quad 123.90$

GROUND-WATER LEVELS

352558096271701. LOCAL NUMBER; 11N-08E-16 AAD 1.

LOCATION,--LAT 35 25'58", LONG 096 27'17", HYDROLOGIC UNIT 11100302.

AQUIFER .--VAMOOSA.

PERIOD OF RECORD.--1976 TO CURRENT YEAR.

EXTREMES FOR PERIOD OF RECORD.--HIGHEST WATER LEVEL,5.50 FT(1.68 M) BELOW LAND-SURFACE DATUM, MAR 14, 1978; LOWEST WATER LEVEL,21.71 FT(6.62 M) BELOW LAND-SURFACE DATUM, MAR 24, 1981.

WATER LEVELS IN FEET BELOW LAND SURFACE DATUM, CLIMATIC YEARS APRIL 1981 TO MARCH 1983

$\begin{array}{lll} & \text { WATER } & \text { WATER } \\ \text { DATE } & \text { LEVEL } & \text { DATE }\end{array}$

FEB 22, $1982 \quad 13.34 \quad$ MAR 21, $1983 \quad 11.16$ 
GROUND-WATER LEVELS

SEQUOYAH COUNTY

353117095065201. LOCAL NUMBER;

$12 \mathrm{~N}-21 \mathrm{E}-18$ AAA 1.

LOCATION,--LAT 35 31'17", LONG 095 06'52", HYDROLOGIC UNIT 11110103.

AQUIFER.--ALLUVIUM.

PERIOD OF RECORD. - -1977 TO CURRENT YEAR.

EXTREMES FOR PERIOD OF RECORD.--HIGHEST WATER LEVEL, $13.59 \mathrm{FT}(4.14 \mathrm{M})$ BELOW LAND-SURFACE DATUM,MAR 8, 1983; LOWEST WATER LEVEL,16.80 FT(5.12 M) BELOW LAND-SURFACE DATUM, FEB 26, 1980.

WATER LEVELS IN FEET BELOW LAND SURFACE DATUM, CLIMATIC YEARS APRIL 1981 TO MARCH 1983

$\begin{array}{lll} & \text { WATER } & \text { WATER } \\ \text { DATE } & \text { LEVEL } & \text { DATE }\end{array}$

MAR 29, $1982 \quad 15.86 \quad$ MAR 08, $1983 \quad 13.59$

GROUND-WATER LEVELS

353624094585601. LOCAL NUMBER; 13N-22E-16 BAD 1. LOCATION,--LAT 35 36'24", LONG 094 58'56", HYDROLOGIC UNIT 11110103.

AQUIFER .--ATOKA FORMATION.

PERIOD OF RECORD.--1979 TO CURRENT YEAR.

EXTREMES FOR PERIOD OF RECORD.--HIGHEST WATER LEVEL,.20 FT(.06 M) BELOW LAND-SURFACE DATUM,MAR 8, 1983; LOWEST WATER LEVEL,3.55 FT(1.08 M) BELOW LAND-SURFACE DATUM, FEB 26, 1980.

WATER LEVELS IN FEET BELOW LAND SURFACE DATUM, CLIMATIC YEARS APRIL 1981 TO MARCH 1983

$\begin{array}{lll} & \text { WATER } & \text { WATER } \\ \text { DATE } & \text { LEVEL } & \text { DATE }\end{array}$

MAR 29, $1982 \quad 1.95 \quad$ MAR 08, $1983 \quad 0.20$ 


\section{GROUND-WATER LEVELS}

\section{STEPHENS COUNTY}

344050097553101. LOCAL NUMBER;

02N-07W-03 BAB 1.

LOCATION,--LAT 34 40'50", LONG 097 55'31", HYDROLOGIC UNIT 11130303. AQUIFER.--RUSH SPRINGS FORMATION.

PERIOD OF RECORD.--1976 TO CURRENT

YEAR.

EXTREMES FOR PERIOD OF RECORD.--HIGHEST WATER LEVEL, $59.51 \mathrm{FT}(18.14 \mathrm{M})$ BELOW LAND-SURFACE DATUM,DEC 10, 1976; LOWEST WATER LEVEL,69.21 FT(21.10 M) BELOW LAND-SURFACE DATUM, FEB 11, 1983.

WATER LEVELS IN FEET BELOW LAND SURFACE DATUM, CLIMATIC YEARS APRIL 1981 TO MARCH 1983

\begin{tabular}{|c|c|c|}
\hline & $\begin{array}{l}\text { WATER } \\
\text { LEVEL }\end{array}$ & DATE \\
\hline
\end{tabular}

$\begin{array}{llll}\text { MAR 25, } 1982 & P & \text { FEB 11, } 1983 & 69.21\end{array}$ 
363346101594001. LOCAL NUMBER; 01N-10E-09 CBC 1. LOCATION,--LAT 36 33'46", LONG 101 59'40", HYDROLOGIC UNIT 11100101. AQUIFER.--OGALLALA.

PERIOD OF RECORD.--1966 TO CURRENT YEAR.

EXTREMES FOR PERIOD OF RECORD.--HIGHEST WATER LEVEL, $179.90 \mathrm{FT}(54.83 \mathrm{M})$ BELOW LAND-SURFACE DATUM, MAR 1, 1966; LOWEST WATER LEVEL,221.80 FT(67.60 M) BELOW LAND-SURFACE DATUM, JAN 7, 1981.

WATER LEVELS IN FEET BELOW LAND SURFACE DATUM, CLIMATIC YEARS APRIL 1981 TO MARCH 1983

\begin{tabular}{|c|c|c|}
\hline & WATER & \\
\hline DATE & & תוL \\
\hline
\end{tabular}

JAN $18,1982 \quad 218.69 \quad$ JAN $11,1983 \quad 219.20$

GROUND-WATER LEVELS

363336101554701. LOCAL NUMBER; 01N-10E-12 DCD 1.

LOCATION,--LAT 36 33'36", LONG 101 55'47", HYDROLOGIC UNIT 11100101. AQUIFER .- OGALLALA.

PERIOD OF RECORD.--1966 TO CURRENT YEAR.

EXTREMES FOR PERIOD OF RECORD.--HIGHEST WATER LEVEL, 144.60 FT(44.07 M) BELOW LAND-SURFACE DATUM, MAR 1, 1966; LOWEST WATER LEVEL, 172.05 FT(52.44 M) BELOW LAND-SURFACE DATUM, JAN 11, 1983.

WATER LEVELS IN FEET BELOW LAND SURFACE. DATUM, CLIMATIC YEARS APRIL 1981 TO MARCH 1983

$\begin{array}{cccc}\text { DATE } & \begin{array}{l}\text { WATER } \\ \text { LEVEL }\end{array} & \text { DATE } & \text { WATER } \\ \text { LEVEL }\end{array}$




\section{GROUND-WATER LEVELS}

TEXAS COUNTY -- CONTINUED

363318101583801. LOCAL NUMBER; LOCATION,--LAT 36 33'18", LONG 101 AQUIFER .--OGALLALA.

PERIOD OF RECORD.--1966 TO CURRENT YEAR. EXTREMES FOR PERIOD OF RECORD.--HIGHEST WATER LEVEL, $170.40 \mathrm{FT}(51.94 \mathrm{M})$ BELOW LAND-SURFACE DATUM,MAR 1, 1966; LOWEST WATER LEVEL, 199.44 FT(60.79 M) BELOW LAND-SURFACE DATUM, JAN $15,1979$.

WATER LEVELS IN FEET BELOW LAND SURFACE DATUM, CLIMATIC YEARS APRIL 1981 TO MARCH 1983
JAN 18,1982
183.97
JAN 11, $1983 \quad 186.00$

\section{GROUND-WATER LEVELS}

363030102003701. LOCAL NUMBER; 01N-10E-32 CBA 1. LOCATION,--LAT 36 30'30", LONG 102 00'37", HYDROLOGIC UNIT 11100101. AQUIFER .--OGALLALA.

PERIOD OF RECORD.--1966 TO CURRENT YEAR. EXTREMES FOR PERIOD OF RECORD.--HIGHEST WATER LEVEL,221.76 FT(67.59 M) BELOW LAND-SURFACE DATUM, JAN 14, 1969; LOWEST WATER LEVEL,294.40 FT(89.73 M) BELOW LAND-SURFACE DATUM, JAN 11, 1983.

WATER LEVELS IN FEET BELOW LAND SURFACE DATUM, CLIMATIC YEARS APRIL 1981 TO MARCH 1983

\begin{tabular}{|c|c|}
\hline & WATER \\
\hline DATE & LEVEL \\
\hline
\end{tabular}
JAN $18,1982 \quad 275.16$
JAN $11,1983 \quad 294.40$ 


\section{GROUND-WATER LEVELS}

TEXAS COUNTY -- CONTINUED

363451101542001. LOCAL NUMBER; 01N-11E-05 CBB 1. LOCATION,--LAT 36 34'51", LONG 101 54'20", HYDROLOGIC UNIT 11100101. AQUIFER .--OGALLALA.

PERIOD OF RECORD.--1966 TO CURRENT YEAR.

EXTREMES FOR PERIOD OF RECORD.--HIGHEST WATER LEVEL, $144.50 \mathrm{FT}(44.04 \mathrm{M})$ BELOW LAND-SURFACE DATUM,MAR 1, 1966; LOWEST WATER LEVEL, 173.50 FT(52.88 M) BELOW LAND-SURFACE DATUM, JAN 11, 1983.

WATER LEVELS IN FEET BELOW LAND SURFACE DATUM, CLIMATIC YEARS APRIL 1981 TO MARCH 1983

$\begin{array}{llcl} & \text { WATER } & & \\ \text { DATE } & \text { LEVEL } & \text { WATE } & \text { WATER } \\ \text { LEVEL }\end{array}$

GROUND-WATER LEVELS

363118101511001. LOCAL NUMBER; 01N-11E-22 DDC 1. LOCATION,--LAT 36 31'18", LONG 101 51'10", HYDROLOGIC UNIT 11100101. AQUIFER . - OGALLALA.

PERIOD OF RECORD.--1966 TO CURRENT YEAR.

EXTREMES FOR PERIOD OF RECORD.--HIGHEST WATER LEVEL, $116.29 \mathrm{FT}(35.45 \mathrm{M})$ BELOW LAND-SURFACE DATUM,JAN 12, 1971; LOWEST WATER LEVEL,119.60 FT(36.45 M) BELOW LAND-SURFACE DATUM, JAN 7, 1981.

WATER LEVELS IN FEET BELOW LAND SURFACE DATUM, CLIMATIC YEARS APRIL 1981 TO MARCH 1983

\begin{tabular}{|c|c|c|}
\hline & WATER & \\
\hline DATE & LEVEL & DATE \\
\hline
\end{tabular}

$\begin{array}{llll}\text { JAN 18, } 1982 & P & \text { JAN 11, } 1983 & P\end{array}$ 
GROUND-WATER LEVELS

TEXAS COUNTY -- CONTINUED

363118101510001 . LOCAL NUMBER; 01N-11E-26 CBB 1.

LOCATION,--LAT 36 31'18", LONG 101 51'00", HYDROLOGIC UNIT 11100101.

AQUIFER.--OGALLALA.

PERIOD OF RECORD.--1966 TO CURRENT YEAR.

EXTREMES FOR PERIOD OF RECORD.--HIGHEST WATER LEVEL, $119.50 \mathrm{FT}(36.42 \mathrm{M})$ BELOW LAND-SURFACE DATUM, MAR 2, 1966; LOWEST WATER LEVEL, 133.21 FT(40.60 M) BELOW LAND-SURFACE DATUM, JAN 11, 1983.

WATER LEVELS IN FEET BELOW LAND SURFACE DATUM, CLIMATIC YEARS APRIL 1981 TO MARCH 1983

\begin{tabular}{|c|c|c|}
\hline & WATER & \\
\hline DATE & LEVEL & DATE \\
\hline
\end{tabular}

JAN 18, 1982 131.27 JAN 11, $1983 \quad 133.21$

GROUND-WATER LEVELS

363336101470901. LOCAL NUMBER; 01N-12E-08 DCD 1.

LOCATION,--LAT 36 33'36", LONG 101 47'09", HYDROLOGIC UNIT 11100101.

AQUIFER.--OGALLALA.

PERIOD OF RECORD.--1966 TO CURRENT YEAR.

EXTREMES FOR PERIOD OF RECORD.--HIGHEST WATER LEVEL, 80.49 FT(24.53 M) BELOW LAND-SURFACE DATUM,JAN 27, 1967; LOWEST WATER LEVEL,89.32 FT(27.22 M) BELOW LAND-SURFACE DATUM, JAN $11,1983$.

WATER LEVELS IN FEET BELOW LAND SURFACE DATUM, CLIMATIC YEARS APRIL 1981 TO MARCH 1983

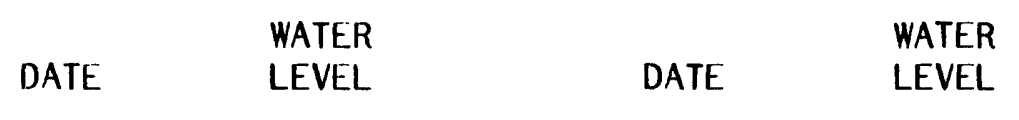

JAN 18, 198287.39 JAN 11, $1983 \quad 89.32$ 
GROUND-WATER LEVELS

TEXAS COUNTY -- CONTINUED

363335101440001. LOCAL NUMBER; $01 \mathrm{~N}-12 \mathrm{E}-11$ DBB 1.

LOCATION,--LAT 36 33'35", LONG 101 44'00", HYDROLOGIC UNIT 11100103.

AQUIFER .--OGALLALA.

PERIOD OF RECORD.--1966 TO CURRENT YEAR.

EXTREMES FOR PERIOD OF RECORD.--HIGHEST WATER LEVEL,223.20 FT (68.03 M) BELOW LAND-SURFACE

DATUM,MAR 1, 1966; LOWEST WATER LEVEL,261.17 FT(79.60 M) BELOW LAND-SURFACE DATUM, JAN $18,1982$.

WATER LEVELS IN FEET BELOW LAND SURFACE DATUM, CLIMATIC YEARS APRIL 1981 TO MARCH 1983

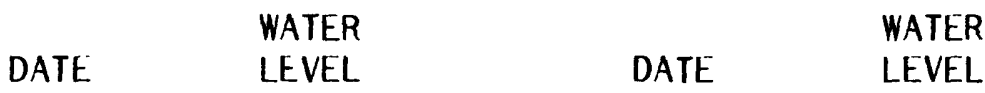

JAN 18, $1982 \quad 261.17 \quad$ JAN $11,1983 \quad$ Z

GROUND-WATER LEVELS

363459101370001. LOCAL NUMBER; 01N-13E-01 BCB 2.

LOCATION,--LAT 36 34'59", LONG 101 37'00", HYDROLOGIC UNIT 11100103.

AQUIFER .--OGALLALA.

PERIOD OF RECORD.--1966 TO CURRENT YEAR.

EXTREMES FOR PERIOD OF RECORD.--HIGHEST WATER LEVEL, $146.00 \mathrm{FT}(44.50 \mathrm{M})$ BELOW LAND-SURFACE DATUM,FEB 27, 1966; LOWEST WATER LEVEL, $189.20 \mathrm{FT}(57.67 \mathrm{M})$ BELOW LAND-SURFACE DATUM, JAN 18, 1982.

WATER LEVELS IN FEET BELOW LAND SURFACE DATUM, CLIMATIC YEARS APRIL 1981 TO MARCH 1983

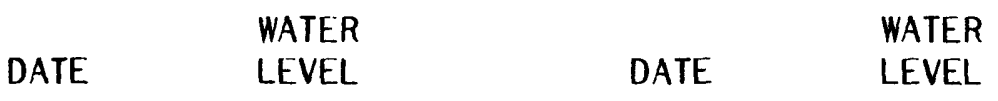

JAN $18,1982189.20 \quad$ JAN $12,1983 \quad 0$ 
TEXAS COUNTY -- CONTINUED

363405101390401. LOCAL NUMBER; LOCATION,--LAT $3634^{\prime} 05^{\prime \prime}$, LONG 101 AQUIFER.--OGALLALA.

PERIOD OF RECORD.--1966 TO CURRENT YEAR.

EXTREMES FOR PERIOD OF RECORD.--HIGHEST WATER LEVEL, $153.50 \mathrm{FT}(46.79 \mathrm{M})$ BELOW LAND-SURFACE DATUM,MAR 1, 1966; LOWEST WATER LEVEL, 191.50 FT(58.37 M) BELOW LAND-SURFACE DATUM, JAN 12, 1983.

WATER LEVELS IN FEET BELOW LAND SURFACE DATUM, CLIMATIC YEARS APRIL 1981 TO MARCH 1983

GROUND-WATER LEVELS

363118101373101. LOCAL NUMBER; $\quad 01 \mathrm{~N}-13 \mathrm{E}-26$ DBB 1. LOCATION,--LAT 36 31'18", LONG 101 37'31", HYDROLOGIC UNIT 11100103. AQUIFER.--OGALLALA.

PERIOD OF RECORD.--1966 TO CURRENT YEAR.

EXTREMES FOR PERIOD OF RECORD. --HIGHEST WATER LEVEL, $150.00 \mathrm{FT}(45.72 \mathrm{M})$ BELOW LAND-SURFACE DATUM,MAR 1, 1966; LOWEST WATER LEVEL,177.09 FT(53.98 M) BELOW LAND-SURFACE DATUM, JAN 12, 1983.

WATER LEVELS IN FEET BELOW LAND SURFACE DATUM, CLIMATIC YEARS APRIL 1981 TO MARCH 1983

WATER

DATE LEVEL

JAN 18, $1982 \quad 175.66$ $\begin{array}{ll} & \text { WATER } \\ \text { DATE } & \text { LEVEL }\end{array}$

JAN 12, $1983 \quad 177.09$ 


\section{GROUND-WATER LEVELS}

TEXAS COUNTY -- CONTINUED

363359101321201. LOCAL NUMBER; 01N-14E-10 BDD 1. LOCATION,--LAT 36 33'59", LONG 101 32'12", HYDROLOGIC UNIT 11100103. AQUIFER.--OGALLALA.

PERIOD OF RECORD.--1966 TO CURRENT YEAR.

EXTREMES FOR PERIOD OF RECORD.--HIGHEST WATER LEVEL, 67.20 FT (20.48 M) BELOW LAND-SURFACE DATUM,JAN 28, 1967; LOWEST WATER LEVEL,92.57 FT(28.22 M) BELOW LAND-SURFACE DATUM, JAN 10,1980 .

WATER LEVELS IN FEET BELOW LAND SURFACE DATUM, CLIMATIC YEARS APRIL 1981 TO MARCH 1983

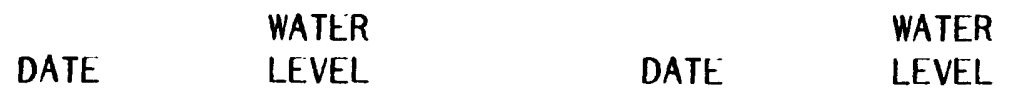

JAN 18, $1982 \quad 88.57 \quad$ JAN 12, $1983 \quad P$

\section{GROUND-WATER LEVELS}

363032101355101. LOCAL NUMBER; 01N-14E-31 CBB 1. LOCATION,--LAT 36 30'32", LONG 101 35'51", HYDROLOGIC UNIT 11100103. AQUIFER .--OGALLALA.

PERIOD OF RECORD.--1966 TO CURRENT YEAR.

EXTREMES FOR PERIOD OF RECORD.--HIGHEST WATER LEVEL, 189.70 FT(57.82 M) BELOW LAND-SURFACE DATUM,FEB 27, 1966; LOWEST WATER LEVEL,225.30 FT(68.67 M) BELOW LAND-SURFACE DATUM, JAN 10, 1977.

WATER LEVELS IN FEET BELOW LAND SURFACE DATUM, CLIMATIC YEARS APRIL 1981 TO MARCH 1983

\begin{tabular}{|c|c|}
\hline & WATER \\
\hline DATE & LEVEL \\
\hline
\end{tabular}

JAN 18, $1982 \quad 218.80$ JAN 12, $1983 \quad 216.67$ 
TEXAS COUNTY -- CONTINUED

363314101242901. LOCAL NUMBER; 01N-15E-14 ACA 1.

LOCATION,--LAT 36 33'14", LONG 101 24'29", HYDROLOGIC UNIT 11100103.

AQUIFER.--OGALLALA.

PERIOD OF RECORD.--1980 TO CURRENT YEAR.

EXTREMES FOR PERIOD OF RECORD.--HIGHEST WATER LEVEL,79.20 FT(24.14 M) BELOW LAND-SURFACE DATUM, MAY 6, 1980; LOWEST WATER LEVEL,82.86 FT(25.26 M) BELOW LAND-SURFACE DATUM, JAN 13, 1983.

WATER LEVELS IN FEET BELOW LAND SURFACE DATUM, CLIMATIC YEARS APRIL 1981 TO MARCH 1983

\begin{tabular}{|c|c|}
\hline & WATER \\
\hline DATE & LEVEL \\
\hline
\end{tabular}

JAN 19, $1982 \quad 82.44 \quad$ JAN 13, $1983 \quad 82.86$

GROUND-WATER LEVELS

363236101292701. LOCAL NUMBER; $\quad 01 \mathrm{~N}-15 \mathrm{E}-19$ BBB 1.

LOCATION,--LAT 36 32'36", LONG 101 29'27", HYDROLOGIC UNIT 11100103.

AQUIFER .--OGALLALA.

PERIOD OF RECORD.--1966 TO CURRENT YEAR.

EXTREMES FOR PERIOD OF RECORD.--HIGHEST WATER LEVEL, $166.70 \mathrm{FT}(50.81 \mathrm{M})$ BELOW LAND-SURFACE DATUM, JAN 18, 1966; LOWEST WATER LEVEL, $194.58 \mathrm{FT}(59.31 \mathrm{M})$ BELOW LAND-SURFACE DATUM, JAN 12, 1983.

WATER LEVELS IN FEET BELOW LAND SURFACE DATUM, CLIMATIC YEARS APRIL 1981 TO MARCH 1983

$\begin{array}{lll} & \text { WATER } & \\ \text { DATE } & \text { LEVEL } & \text { WATER } \\ & \text { DATE } & \text { LEVEL }\end{array}$

JAN 19, $1982187.67 \quad$ JAN 12, $1983 \quad 194.58$ 
GROUND-WATER LEVELS

TEXAS COUNTY -- CONTINUED

363209101283201. LOCAL NUMBER; 01N-15E-19 DAA 1.

LOCATION,--LAT 36 32'09", LONG 101 28'32", HYDROLOGIC UNIT 11100103.

AQUIFER.--OGALLALA.

PERIOD OF RECORD.--1966 TO CURRENT YEAR.

EXTREMES FOR PERIOD OF RECORD.--HIGHEST WATER LEVEL, $168.70 \mathrm{FT}(51.42 \mathrm{M})$ BELOW LAND-SURFACE

DATUM,JAN 18, 1966; LOWEST WATER LEVEL, 183.70 FT(55.99 M) BELOW LAND-SURFACE DATUM,

JAN 12, 1983.

WATER LEVELS IN FEET BELOW LAND SURFACE DATUM, CLIMATIC YEARS APRIL 1981 T0 MARCH 1983

\begin{tabular}{ccccc}
\multicolumn{1}{c}{ WATER } & WATE & LEVEL & DATE & WATER \\
LEVEL
\end{tabular}

GROUND-WATER LEVELS

363107101261501. LOCAL NUMBER; 01N-15E-27 CCB 1. LOCATION,--LAT 36 31'07", LONG 101 26'15", HYDROLOGIC UNIT 11100103.

AQUIFER .--OGALLALA.

PERIOD OF RECORD.--1966 TO CURRENT YEAR.

EXTREMES FOR PERIOD OF RECORD.--HIGHEST WATER LEVEL, 72.22 FT(22.01 M) BELOW LAND-SURFACE DATUM, JAN 17, 1969; LOWEST WATER LEVEL,92.25 FT(28.12 M) BELOW LAND-SURFACE DATUM, JAN 10, 1983.

WATER LEVELS IN FEET BELOW LAND SURFACE DATUM, CLIMATIC YEARS APRIL 1981 TO MARCH 1983

\begin{tabular}{|c|c|}
\hline & WATER \\
\hline DATE & LEVEL \\
\hline
\end{tabular}

JAN 19, $1982 \quad 91.71 \quad$ JAN 10, $1983 \quad 92.25$


TEXAS COUNTY -- CONTINUED

363027101282301. LOCAL NUMBER; 01N-15E-32 CBB 1.

LOCATION,--LAT 36 30'27", LONG 101 28'23", HYDROLOGIC UNIT 11100103.

AQUIFER .--OGALLALA.

PERIOD OF RECORD.--1966 TO CURRENT YEAR.

EXTREMES FOR PERIOD OF RECORD.--HIGHEST WATER LEVEL,201.47 FT(61.41 M) BELOW LAND-SURFACE DATUM,JAN 18, 1968; LOWEST WATER LEVEL,217.80 FT(66.39 M) BELOW LAND-SURFACE DATUM, JAN $12,1983$.

WATER LEVELS IN FEET BELOW LAND SURFACE DATUM, CLIMATIC YEARS APRIL 1981 TO MARCH 1983

$\begin{array}{lll} & \text { WATER } & \text { WATER } \\ \text { DATE } & \text { LEVEL } & \text { DATE } \\ \text { LEVEL }\end{array}$

JAN 19, $1982 \quad 216.63 \quad$ JAN $12,1983 \quad 217.80$

GROUND-WATER LEVELS

363512101203801. LOCAL NUMBER; 01N-16E-04 BAB 1.

LOCATION,--LAT 36 35'12", LONG 101 20'38", HYDROLOGIC UNIT 11100103.

AQUIFER.--OGALLALA.

PERIOD OF RECORD.--1980 TO CURRENT YEAR.

EXTREMES FOR PERIOD OF RECORD.--HIGHEST WATER LEVEL,21.18 FT(6.46 M) BELOW LAND-SURFACE DATUM,MAY 7, 1980; LOWEST WATER LEVEL,59.62 FT(18.17 M) BELOW LAND-SURFACE DATUM, JAN 10, 1983.

WATER LEVELS IN FEET BELOW LAND SURFACE DATUM, CLIMATIC YEARS APRIL 1981 TO MARCH 1983

$\begin{array}{lll} & \text { WATER } & \\ \text { DATE } & \text { WEVEL } & \text { WATER } \\ \text { LEVEL }\end{array}$

JAN 19, $1982 \quad 59.06 \quad$ JAN 10, $1983 \quad 59.62$ 
TEXAS COUNTY -- CONTINUED

363202101213301. LOCAL NUMBER; 01N-16E-20 CAD 1. LOCATION,--LAT 36 32'02", LONG 101 21'33", HYDROLOGIC UNIT 11100103. AQUIFER.--OGALLALA. PERIOD OF RECORD.--1980 TO CURRENT YEAR. EXTREMES FOR PERIOD OF RECORD.--HIGHEST WATER LEVEL, 163.79 FT(49.92 M) BELOW LAND-SURFACE DATUM, MAY 6, 1980; LOWEST WATER LEVEL,254.43 FT(77.55 M) BELOW LAND-SURFACE DATUM, JAN 10, 1983.

WATER LEVELS IN FEET BELOW LAND SURFACE DATUM, CLIMATIC YEARS APRIL 1981 TO MARCH 1983

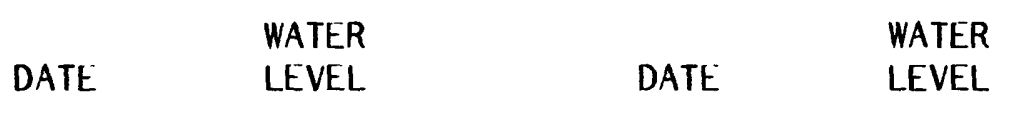

JAN 19, $1982 \quad 236.80 \quad$ JAN $10,1983 \quad 254.43$

GROUND-WATER LEVELS

363439101110701. LOCAL NUMBER; 01N-17E-01 CBC 1.

LOCATION,--LAT 36 34'39", LONG 101 11'07", HYDROLOGIC UNIT 11100104.

AQUIFER .--OGALLALA.

PERIOD OF RECORD.--1966 TO CURRENT YEAR.

EXTREMES FOR PERIOD OF RECORD.--HIGHEST WATER LEVEL, 124.34 FT(37.90 M) BELOW LAND-SURFACE DATUM, JAN 28, 1967; LOWEST WATER LEVEL, 139.60 FT (42.55 M) BELOW LAND-SURFACE DATUM, JAN 16, 1979.

WATER LEVELS IN FEET BELOW LAND SURFACE DATUM, CLIMATIC YEARS APRIL 1981 TO MARCH 1983

$\begin{array}{lll} & \text { WATER } & \\ \text { DATE } & \text { LEVEL } & \text { WATER } \\ & \text { DATE } & \text { LEVEL }\end{array}$

JAN 19, $1982138.05 \quad$ JAN $10,1983 \quad 135.26$ 
TEXAS COUNTY -- CONTINUED

363338101131801. LOCAL NUMBER; $01 \mathrm{~N}-17 \mathrm{E}-10$ CCC 1. LOCATION,--LAT 36 33'38", LONG 101 13'18", HYDROLOGIC UNIT 11100102. AQUIFER.--OGALLALA.

PERIOD OF RECORD.--1966 TO CURRENT YEAR.

EXTREMES FOR PERIOD OF RECORD.--HIGHEST WATER LEVEL, $143.90 \mathrm{FT}(43.86 \mathrm{M})$ BELOW LAND-SURFACE DATUM, JAN 16, 1969; LOWEST WATER LEVEL, 148.06 FT(45.13 M) BELOW LAND-SURFACE DATUM, JAN 22, 1975.

WATER LEVELS IN FEET BELOW LAND SURFACE DATUM, CLIMATIC YEARS APRIL 1981 TO MARCH 1983

$\begin{array}{lll} & \text { WATER } & \\ \text { DATE } & \text { LEVEL } & \text { WATER } \\ & \text { DATE } & \text { LEVEL }\end{array}$

JAN 19, $1982147.07 \quad$ JAN $10,1983 \quad 147.76$

GROUND-WATER LEVELS

363211101161101. LOCAL NUMBER; 01N-17E-19 CCC 1. LOCATION,--LAT 36 32'11", LONG 101 16'11", HYDROLOGIC UNIT 11100102. AQUIFER .--OGALLALA.

PERIOD OF RECORD.--1966 TO CURRENT YEAR.

EXTREMES FOR PERIOD OF RECORD.--HIGHEST WATER LEVEL, $166.60 \mathrm{FT}(50.78 \mathrm{M})$ BELOW LAND-SURFACE DATUM,FEB 25, 1966; LOWEST WATER LEVEL, $191.00 \mathrm{FT}(58.22 \mathrm{M})$ BELOW LAND-SURFACE DATUM, JAN 10, 1983.

WATER LEVELS IN FEET BELOW LAND SURFACE DATUM, CLIMATIC YEARS APRIL 1981 TO MARCH 1983

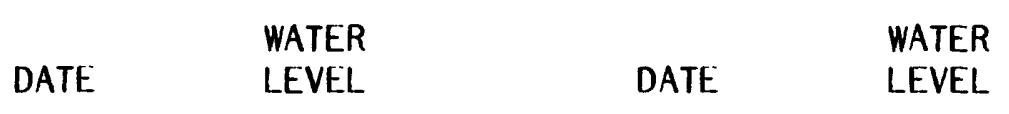

JAN 19, $1982190.14 \quad$ JAN 10, $1983 \quad 191.00$ 
TEXAS COUNTY -- CONTINUED

363115101160001. LOCAL NUMBER; 01N-17E-30 DBB 1. LOCATION,--LAT 36 31'15", LONG 101 16'00", HYDROLOGIC UNIT 11100104. AQUIFER. - OGALLALA.

PERIOD OF RECORD.--1970 TO CURRENT YEAR. EXTREMES FOR PERIOD OF RECORD.--HIGHEST WATER LEVEL, $165.00 \mathrm{FT}(50.29 \mathrm{M})$ BELOW LAND-SURFACE DATUM, JAN 20, 1972; LOWEST WATER LEVEL, 179.14 FT(54.60 M) BELOW LAND-SURFACE DATUM, JAN 10, 1983.

WATER LEVELS IN FEET BELOW LAND SURFACE DATUM, CLIMATIC YEARS APRIL 1981 TO MARCH 1983

$\begin{array}{lll} & \text { WATER } & \text { WATER } \\ \text { DATE } & \text { LEVEL } & \text { DATE }\end{array}$

JAN 19, $1982178.53 \quad$ JAN 10, $1983 \quad 179.14$

GROUND-WATER LEVELS

363242101042301. LOCAL NUMBER; $01 \mathrm{~N}-18 \mathrm{E}-13$ CDC 1. LOCATION,--LAT 36 32'42", LONG 101 04'23", HYDROLOGIC UNIT 11100104. AQUIFER .--OGALLALA.

PERIOD OF RECORD.--1966 TO CURRENT YEAR.

EXTREMES FOR PERIOD OF RECORD.--HIGHEST WATER LEVEL,9.15 FT(2.79 M) BELOW LAND-SURFACE DATUM, JAN 11, 1983; LOWEST WATER LEVEL, 14.15 FT(4.31 M) BELOW LAND-SURFACE DATUM, JAN $10,1980$.

WATER LEVELS IN FEET BELOW LAND SURFACE DATUM, CLIMATIC YEARS APRIL 1981 TO MARCH 1983

$\begin{array}{lll} & \text { WATER } & \text { WATER } \\ \text { DATE } & \text { LEVEL } & \text { DATE }\end{array}$

JAN 19, $1982 \quad 11.13 \quad$ JAN 11, $1983 \quad 9.15$ 


\section{GROUND-WATER LEVELS}

TEXAS COUNTY -- CONTINUED

363048101064601. LOCAL NUMBER; LOCATION,--LAT 36 30'48", LONG 101 AQUIFER. - -OGALLALA.

PERIOD OF RECORD.--1966 TO CURRENT YEAR.

EXTREMES FOR PERIOD OF RECORD.--HIGHEST WATER LEVEL,70.40 FT(21.46 M) BELOW LAND-SURFACE DATUM,FEB 25, 1966; LOWEST WATER LEVEL,77.34 FT(23.57 M) BELOW LAND-SURFACE DATUM, JAN 15, 1969.

WATER LEVELS IN FEET BELOW LAND SURFACE DATUM, CLIMATIC YEARS APRIL 1981 TO MARCH 1983

\begin{tabular}{lccc} 
& WATER & & \\
DATE & LEVEL & WATER \\
LATE & LEVEL \\
\hline 19,1982 & 74.04 & JAN 11, 1983 & 74.60
\end{tabular}

GROUND-WATER LEVELS

363742102002301. LOCAL NUMBER; 02N-10E-20 BBD 1. LOCATION,--LAT 36 37'42", LONG 102 00'23", HYDROLOGIC UNIT 11100101. AQUIFER .--OGALLALA.

PERIOD OF RECORD.--1967 TO CURRENT YEAR.

EXTREMES FOR PERIOD OF RECORD.--HIGHEST WATER LEVEL, 183.35 FT(55:89 M) BELOW LAND-SURFACE DATUM, JAN 28, 1967; LOWEST WATER LEVEL,222.60 FT(67.85 M) BELOW LAND-SURFACE DATUM, JAN 10, 1980.

WATER LEVELS IN FEET BELOW LAND SURFACE DATUM, CLIMATIC YEARS APRIL 1981 TO MARCH 1983

\begin{tabular}{|c|c|}
\hline DATE & $\begin{array}{l}\text { WATER } \\
\text { LEVEL }\end{array}$ \\
\hline
\end{tabular}

JAN 18, $1982208.03 \quad$ JAN 11, $1983 \quad 207.90$ 


\section{GROUND-WATER LEVELS}

TEXAS COUNTY -- CONTINUED

363648101584901 . LOCAL NUMBER;

$02 \mathrm{~N}-10 \mathrm{E}-28 \mathrm{BCB} 1$.

LOCATION,--LAT 36 36'48", LONG 101 58'49", HYDROLOGIC UNIT 11100101.

AQUIFER.--OGALLALA.

PERIOD OF RECORD.--1966 TO CURRENT YEAR.

EXTREMES FOR PERIOD OF RECORD.--HIGHEST WATER LEVEL, $170.30 \mathrm{FT}(51.91 \mathrm{M})$ BELOW LAND-SURFACE DATUM, MAR 1, 1966; LOWEST WATER LEVEL, $185.58 \mathrm{FT}(56.56 \mathrm{M})$ BELOW LAND-SURFACE DATUM, JAN $10,1980$.

WATER LEVELS IN FEET BELOW LAND SURFACE DATUM, CLIMATIC YEARS APRIL 1981 TO MARCH 1983

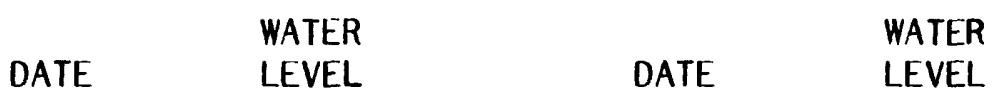

JAN $18,1982182.36 \quad$ JAN $11,1983 \quad 182.70$

GROUND-WATER LEVELS

363534101521001. LOCAL NUMBER; 02N-11E-34 CCB 1.

LOCATION,--LAT 36 35'34", LONG 101 52'10", HYDROLOGIC UNIT 11100101.

AQUIFER .--OGALLALA.

PERIOD OF RECORD.--1966 TO CURRENT YEAR.

EXTREMES FOR PERIOD OF RECORD.--HIGHEST WATER LEVEL, 147.70 FT(45.02 M) BELOW LAND-SURFACE DATUM,MAR 2, 1966; LOWEST WATER LEVEL, 174.87 FT(53.30 M) BELOW LAND-SURFACE DATUM, JAN 11, 1983.

WATER LEVELS IN FEET BELOW LAND SURFACE DATUM, CLIMATIC YEARS APRIL 1981 TO MARCH 1983

\begin{tabular}{cccc}
\multicolumn{1}{c}{ WATER } & & WATER \\
DATE & LEVEL & DATE & LEVEL \\
JAN 18, 1982 & 171.61 & JAN 11, 1983 & 174.87
\end{tabular}


TEXAS COUNTY -- CONTINUED

363632101423901. LOCAL NUMBER; 02N-12E-25 DBB 1. LOCATION,--LAT 36 36'32", LONG 101 42'39", HYDROLOGIC UNIT 11100101. AQUIFER .--OGALLALA. PERIOD OF RECORD.--1966 TO CURKENT YEAR. EXTREMES FOR PERIOD OF RECORD.--HIGHEST WATER LEVEL,220.80 FT(67.30 M) BELOW LAND-SURFACE DATUM,MAR 1, 1966; LOWEST WATER LEVEL,280.64 FT(85.54 M) BELOW LAND-SURFACE DATUM, JAN 12, 1983.

WATER LEVELS IN FEET BELOW LAND SURFACE DATUM, CLIMATIC YEARS APRIL 1981 TO MARCH 1983

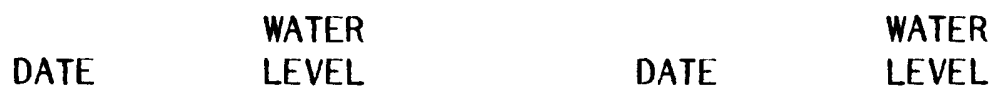

JAN 18, $1982 \quad 278.89 \quad$ JAN $12,1983 \quad 280.64$

\section{GROUND-WATER LEVELS}

363604101462601. LOCAL NUMBER; 02N-12E-33 BAB 1. LOCATION,--LAT 36 36'04", LONG 101 46'26", HYDROLOGIC UNIT 11100101. AQUIFER .--OGALLALA.

PERIOD OF RECORD.--1966 TO CURRENT YEAR.

EXTREMES FOR PERIOD OF RECORD.--HIGHEST WATER LEVEL, 15.74 FT(4.80 M) BELOW LAND-SURFACE DATUM, JAN 26, 1967; LOWEST WATER LEVEL,23.00 FT(7.01 M) BELOW LAND-SURFACE DATUM, JAN 11, 1983.

WATER LEVELS IN FEET BELOW LAND SURFACE DATUM, CLIMATIC YEARS APRIL 1981 TO MARCH 1983

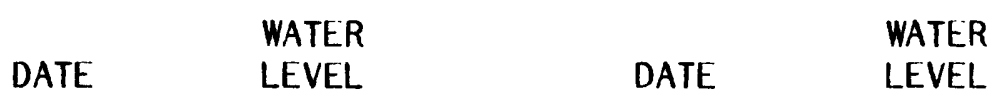

JAN 18, $1982 \quad 22.59$ JAN 11, $1983 \quad 23.00$ 
TEXAS COUNTY -- CONTINUED

363819101363001. LOCAL NUMBER; 02N-13E-13 CAA 1. LOCATION,--LAT 36 38'19", LONG 101 36'30", HYDROLOGIC UNIT 11100101. AQUIFER .- OGALLALA.

PERIOD OF RECORD.--1966 TO CURRENT YEAR.

EXTREMES FOR PERIOD OF RECORD..--HIGHEST WATER LEVEL, $155.50 \mathrm{FT}(47.40 \mathrm{M})$ BELOW LAND-SURFACE DATUM,FEB 28, 1966; LOWEST WATER LEVEL, $192.98 \mathrm{FT}(58.82 \mathrm{M})$ BELOW LAND-SURFACE DATUM, JAN 12, 1983.

WATER LEVELS IN FEET BELOW LAND SURFACE DATUM, CLIMATIC YEARS APRIL 1981 TO MARCH 1983

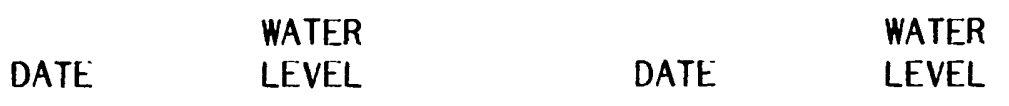

JAN 18, $1982 \quad 191.88 \quad$ JAN $12,1983 \quad 192.98$

\section{GROUND-WATER LEVELS}

363843101401201. LOCAL NUMBER; 02N-13E-16 BBB 1. LOCATION,--LAT 36 38'43", LONG 101 40'12", HYDROLOGIC UNIT 11100101. AQUIFER. - -OGALLALA.

PERIOD OF RECORD.--1966 TO CURRENT YEAR.

EXTREMES FOR PERIOD OF RECORD.--HIGHEST WATER LEVEL, $199.00 \mathrm{FT}(60.66 \mathrm{M})$ BELOW LAND-SURFACE DATUM, MAR 1, 1966; LOWEST WATER LEVEL,245.53 FT(74.84 M) BELOW LAND-SURFACE DATUM, JAN 16, 1978.

WATER LEVELS IN FEET BELOW LAND SURFACE DATUM, CLIMATIC YEARS APRIL 1981 TO MARCH 1983

$\begin{array}{lll} & \text { WATER } & \text { WATER } \\ \text { DATE } & \text { LEVEL } & \text { DATE }\end{array}$

JAN 18, $1982 \quad 234.08 \quad$ JAN 12, $1983 \quad 238.40$ 
TEXAS COUNTY -- CONTINUED

363520101411701. LOCAL NUMBER; 02N-13E-32 CCC 1.

LOCATION,--LAT $3635^{\prime} 20^{\prime \prime}$, LONG $101 \quad 41^{\prime 17} "$, HYDROLOGIC UNIT 11100101.

AQUIFER.--OGALLALA.

PERIOD OF RECORD.--1966 TO CURRENT YEAR.

EXTREMES FOR PERIOD OF RECORD.--HIGHEST WATER LEVEL, 178.40 FT(54.38 M) BELOW LAND-SURFACE DATUM, MAR 1, 1966; LOWEST WATER LEVEL,230.19 FT(70.16 M) BELOW LAND-SURFACE DATUM, JAN $18,1982$.

WATER LEVELS IN FEET BELOW LAND SURFACE DATUM, CLIMATIC YEARS APRIL 1981 TO MARCH 1983

$\begin{array}{lll} & \text { WATER } & \text { WATER } \\ \text { DATE } & \text { LEVEL } & \text { DATE } \\ \text { LEVEL }\end{array}$

JAN 18, $1982 \quad 230.19 \quad$ JAN 12, $1983 \quad 227.30$

GROUND-WATER LEVELS

363909101312301. LOCAL NUMBER; 02N-14E-11 CBA 1. LOCATION,--LAT 36 39'09", LONG 101 31'23", HYDROLOGIC UNIT 11100101. AQUIFER --OGALLALA.

PERIOD OF RECORD.--1966 TO CURRENT YEAR.

EXTREMES FOR PERIOD OF RECORD.---HIGHEST WATER LEVEL, 132.20 FT(40.29 M) BELOW LAND-SURFACE DATUM, JAN 29, 1967; LOWEST WATER LEVEL, 159.34 FT(48.57 M) BELOW LAND-SURFACE DATUM, JAN $12,1983$.

WATER LEVELS IN FEET BELOW LAND SURFACE DATUM, CLIMATIC YEARS APRIL 1981 TO MARCH 1983

$\begin{array}{lll} & \text { WATER } & \text { WATER } \\ \text { DATE } & \text { LEVEL } & \text { DATE } \\ \text { LEVEL }\end{array}$

JAN 18, $1982 \quad 156.36 \quad$ JAN 12, $1983 \quad 159.34$ 
TEXAS COUNTY -- CONTINUED

363718101313001 . LOCAL NUMBER; LOCATION,--LAT 36 37'18", LONG 101 AQUIFER.--OGALLALA.

PERIOD OF RECORD..-1970 TO CURRENT YEAR.

EXTREMES FOR PERIOD OF RECORD.--HIGHEST WATER LEVEL, 138.38 FT(42.18 M) BELOW LAND-SURFACE DATUM,JAN 13, 1970; LOWEST WATER LEVEL,167.97 FT(51.20 M) BELOW LAND-SURFACE DATUM, JAN 12, 1983.

WATER LEVELS IN FEET BELOW LAND SURFACE DATUM, CLIMATIC YEARS APRIL 1981 TO MARCH 1983
GROUND-WATER LEVELS

363716101303101. LOCAL NUMBER; LOCATION,--LAT 36 37'16", LONG 101 AQUIFER.--OGALLALA.

PERIOD OF RECORD .-1980 TO CURRENT YEAR.

EXTREMES FOR PERIOD OF RECORD.--HIGHEST WATER LEVEL, $162.56 \mathrm{FT}(49.55 \mathrm{M})$ BELOW LAND-SURFACE DATUM, APR 3, 1980; LOWEST WATER LEVEL, $172.69 \mathrm{FT}(52.64 \mathrm{M})$ BELOW LAND-SURFACE DATUM, JAN 12, 1983.

WATER LEVELS IN FEET BELOW LAND SURFACE DATUM, CLIMATIC YEARS APRIL 1981 TO MARCH 1983 
TEXAS COUNTY -- CONTINUED

363911101271401. LOCAL NUMBER; 02N-15E-09 BCB 1. LOCATION,--LAT 36 39'11", LONG 101 27'14", HYDROLOGIC UNIT 11100102. AQUIFER. - OGALLALA.

PERIOD OF RECORD.--1966 TO CURRENT YEAR.

EXTREMES FOR PERIOD OF RECORD.--HIGHEST WATER LEVEL,207.40 FT(63.22 M) BELOW LAND-SURFACE DATUM,FEB 24, 1966; LOWEST WATER LEVEL,289.50 FT(88.24 M) BELOW LAND-SURFACE DATUM, JAN 18,1982 .

WATER LEVELS IN FEET BELOW LAND SURFACE DATUM, CLIMATIC YEARS APRIL 1981 TO MARCH 1983

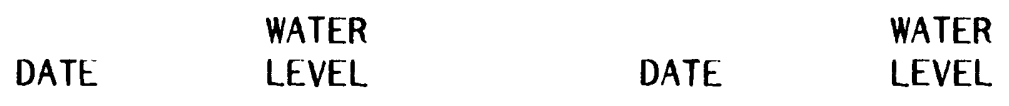

JAN $18,1982 \quad 289.50 \quad$ JAN 12, $1983 \quad P$

GROUND-WATER LEVELS

363843101254501. LOCAL NUMBER; 02N-15E-15 ABB 1. LOCATION,--LAT 36 38'43", LONG 101 25'45", HYDROLOGIC UNIT 11100102. AQUIFER.--OGALLALA.

PERIOD OF RECORD.--1970 TO CURRENT YEAR.

EXTREMES FOR PERIOD OF RECORD.--HIGHEST WATER LEVEL,208.29 FT(63.49 M) BELOW LAND-SURFACE DATUM, JAN 15, 1970; LOWEST WATER LEVEL,284.37 FT(86.68 M) BELOW LAND-SURFACE DATUM, JAN 12, 1983.

WATER LEVELS IN FEET BELOW LAND SURFACE DATUM, CLIMATIC YEARS APRIL 1981 TO MARCH 1983

$\begin{array}{lll} & \text { WATER } & \text { WATER } \\ \text { DATE } & \text { LEVEL } & \text { DATE }\end{array}$

JAN $19,1982 \quad 270.84 \quad$ JAN $12,1983 \quad 284.37$ 


\section{GROUND-WATER LEVELS}

TEXAS COUNTY -- CONTINUED

364003101201901. LOCAL NUMBER; 02N-16E-04 DBA 1. LOCATION,--LAT 36 40'03", LONG 101 20'19", HYDROLOGIC UNIT 11100102. AQUIFER .--OGALLALA.

PERIOD OF RECORD.--1966 TO CURRENT YEAR.

EXTREMES FOR PERIOD OF RECORD.--HIGHEST WATER LEVEL, 157.90 FT(48.13 M) BELOW LAND-SURFACE DATUM,FEB 24, 1966; LOWEST WATER LEVEL,238.39 FT(72.66 M) BELOW LAND-SURFACE DATUM, JAN 7, 1981.

WATER LEVELS IN FEET BELOW LAND SURFACE DATUM, CLIMATIC YEARS APRIL 1981 TO MARCH 1983

\begin{tabular}{|c|c|}
\hline & WATER \\
\hline ATE & LEVEL \\
\hline
\end{tabular}
JAN 19, $1982 \quad 237.72$
JAN 12, 1983
0

GROUND-WATER LEVELS

363927101225701. LOCAL NUMBER; 02N-16E-07 BBB 1.

LOCATION,--LAT 36 39'27", LONG 101 22'57", HYDROLOGIC UNIT 11100102.

AQUIFER .--OGALLALA.

PERIOD OF RECORD.--1966 TO CURRENT YEAR.

EXTREMES FOR PERIOD OF RECORD.--HIGHEST WATER LEVEL, 204.90 FT (62.45 M) BELOW LAND-SURFACE DATUM,FEB 24, 1966; LOWEST WATER LEVEL, $305.46 \mathrm{FT}(93.10 \mathrm{M})$ BELOW LAND-SURFACE DATUM, JAN 12, 1983.

WATER LEVELS IN FEET BELOW LAND SURFACE DATUM, CLIMATIC YEARS APRIL 1981 TO MARCH 1983

$\begin{array}{lll} & \text { WATER } & \text { WATER } \\ \text { DATE } & \text { LEVEL } & \text { DATE }\end{array}$

JAN 19, $1982 \quad 302.93 \quad$ JAN 12, $1983 \quad 305.46$ 


\title{
GROUND-WATER LEVELS
}

\author{
TEXAS COUNTY -- CONTINUED
}

363929101210701. LOCAL NUMBER; 02N-16E-08 AAA 1. LOCATION,--LAT 36 39'29", LONG 101 21'07", HYDROLOGIC UNIT 11100102. AQUIFER.--OGALLALA.

PERIOD OF RECORD.--1966 TO CURRENT YEAR.

EXTREMES FOR PERIOD OF RECORD.--HIGHEST WATER LEVEL, $163.00 \mathrm{FT}(49.68 \mathrm{M})$ BELOW LAND-SURFACE DATUM,FEB 24, 1966; LOWEST WATER LEVEL,264.38 FT(80.58 M) BELOW LAND-SURFACE DATUM, JAN 12, 1983.

WATER LEVELS IN FEET BELOW LAND SURFACE DATUM, CLIMATIC YEARS APRIL 1981 TO MARCH 1983

$\begin{array}{lll} & \text { WATER } & \\ \text { DATE } & \text { WEVEL } & \text { WATER } \\ & \text { LEVEL }\end{array}$

JAN 19, $1982 \quad P \quad$ JAN 12, $1983 \quad 264.38$

GROUND-WATER LEVELS

363816101184201. LOCAL NUMBER; O2N-16E-14 CBB 1.

LOCATION,--LAT 36 38'16", LONG 101 18'42", HYDROLOGIC UNIT 11100103.

AQUIFER . - OGALLALA.

PERIOD OF RECORD.--1966 TO CURRENT YEAR.

EXTREMES FOR PERIOD OF RECORD.--HIGHEST WATER LEVEL, $165.40 \mathrm{FT}(50.41 \mathrm{M})$ BELOW LAND-SURFACE DATUM,FEB 24, 1966; LOWEST WATER LEVEL,218.95 FT(66.74 M) BELOW LAND-SURFACE DATUM, JAN 12, 1983.

WATER LEVELS IN FEET BELOW LAND SURFACE DATUM, CLIMATIC YEARS APRIL 1981 TO MARCH 1983

$\begin{array}{cccc}\text { DATE } & \begin{array}{c}\text { WATER } \\ \text { LEVEL }\end{array} & \text { DATE } & \begin{array}{l}\text { WATER } \\ \text { LEVEL }\end{array} \\ \text { JAN 19, 1982 } & 216.01 & \text { JAN 12, 1983 } & 218.95\end{array}$


TEXAS COUNTY -- CONTINUED

363848101163101. LOCAL NUMBER; 02N-17E-07 CCC 1. LOCATION,--LAT 36 38'48", LONG 101 16'31", HYDROLOGIC UNIT 11100102. AQUIFER .--OGALLALA. PERIOD OF RECORD.--1966 TO CURRENT YEAR. EXTREMES FOR PERIOD OF RECORD.--HIGHEST WATER LEVEL, $171.00 \mathrm{FT}(52.12 \mathrm{M})$ BELOW LAND-SURFACE DATUM,FEB 24, 1966; LOWEST WATER LEVEL,202.30 FT(61.66 M) BELOW LAND-SURFACE DATUM, JAN $12,1983$.

WATER LEVELS IN FEET BELOW LAND SURFACE DATUM, CLIMATIC YEARS APRIL 1981 TO MARCH 1983

\begin{tabular}{cccc}
\multicolumn{1}{c}{ WATER } & & WATER \\
DATE & LEVEL & DATE & LEVEL \\
JAN 19, 1982 & 199.28 & JAN 12, 1983 & 202.30
\end{tabular}

GROUND-WATER LEVELS

363837101125201. LOCAL NUMBER

$2 \mathrm{~N}-17 \mathrm{E}-15$ ABB 1 . LOCATION,--LAT 36 38'37", LONG 101 12'52", HYDROLOGIC UNIT 11100102 AQUIFER.--ALLUVIUM.

PERIOD OF RECORD.--1966 TO CURRENT YEAR.

EXTREMES FOR PERIOD OF RECORD.--HICHEST WATER LEVEL, 6.33 FT(1.93 M) BELOW LAND-SURFACE DATUM,FEB 15, 1966; LOWEST WATER LEVEL,8.44 FT(2.57 M) BELOW LAND-SURFACE DATUM, JAN 23, 1975

WATER LEVELS IN FEET BELOW LAND SURFACE DATUM, CLIMATIC YEARS APRIL 1981 TO MARCH 1983

$\begin{array}{lll} & \text { WATER } & \\ \text { DATE } & \text { WEVEL } & \text { WATER } \\ \text { LATE } & \text { LEVEL }\end{array}$

JAN 19, $1982 \quad 8.20 \quad$ JAN 13, 1983 DRY 
TEXAS COUNTY -- CONTINUED

363932101064401. LOCAL NUMBER;

$02 \mathrm{~N}-18 \mathrm{E}-03$ BBA 1. LOCATION,--LAT 36 39'32", LONG 101 06'44", HYDROLOGIC UNIT 11100102. AQUIFER.--OGALLALA. PERIOD OF RECORD.--1967 TO CURRENT YEAR. EXTREMES FOR PERIOD OF RECORD.--HIGHEST WATER LEVEL,2.16 FT(.66 M) BELOW LAND-SURFACE DATUM, JAN 15, 1969; LOWEST WATER LEVEL,7.35 FT(2.24 M) BELOW LAND-SURFACE DATUM, SEP $20,1968$.

WATER LEVELS IN FEET BELOW LAND SURFACE DATUM, CLIMATIC YEARS APRIL 1981 TO MARCH 1983

\begin{tabular}{lrrrr} 
& WATER & & \multicolumn{1}{c}{ WATER } \\
DATE & LEVEL & DATE & \multicolumn{1}{l}{ LEVEL } \\
LAN 19, 1982 & 3.61 & JAN 11, 1983 & 3.63
\end{tabular}

GROUND-WATER LEVELS

363938101090101. LOCAL NUMBER; 02N-18E-08 BAD 1. LOCATION,--LAT 36 39'38", LONG 101 09'01", HYOROLOGIC UNIT 11100103. AQUIFER . - OGALLALA. PERIOD OF RECORD.--1968 TO CURRENT YEAR. EXTREMES FOR PERIOD OF RECORD.--HIGHEST WATER LEVEL, 86.04 FT(26.22 M) BELOW LAND-SURFACE DATUM, JAN 13, 1983; LOWEST WATER LEVEL,97.92 FT(29.85 M) BELOW LAND-SURFACE DATUM, JAN 15, 1969.

WATER LEVELS IN FEET BELOW LAND SURFACE DATUM, CLIMATIC YEARS APRIL 1981 TO MARCH 1983

$\begin{array}{lll} & \text { WATER } & \text { WATER } \\ \text { DATE } & \text { LEVEL } & \text { DATE } \\ \end{array}$

JAN 19, $1982 \quad 86.29 \quad$ JAN 13, $1983 \quad 86.04$ 
363907101051001. LOCAL NUMBER;

$02 \mathrm{~N}-18 \mathrm{E}-11$ DBB 1.

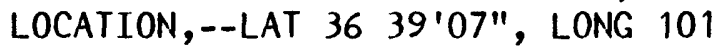
AQUIFER. - -OGALLALA. PERIOD OF RECORD . - 1966 TO CURRENT YEAR. EXTREMES FOR PERIOD OF RECORD.--HIGHEST WATER LEVEL, $56.60 \mathrm{FT}(17.25 \mathrm{M})$ BELOW LAND-SURFACE DATUM,FEB 26, 1966; LOWEST WATER LEVEL,79.18 FT(24.13 M) BELOW LAND-SURFACE DATUM, JAN 12, 1977.

WATER LEVELS IN FEET BELOW LAND SURFACE DATUM, CLIMATIC YEARS APRIL 1981 TO MARCH 1983

$\begin{array}{lccc} & \text { WATER } & & \text { WATER } \\ \text { DATE } & \text { LEVEL } & \text { DATE } & \text { LEVEL } \\ \text { LAN 19, 1982 } & 68.07 & \text { JAN 11, 1983 } & 68.30\end{array}$

363758101051301. LOCAL NUMBER; 02N-18E-14 DCD 1. LOCATION,--LAT 36 37'58", LONG 101 05'13", HYDROLOGIC UNIT 11100102. AQUIFER .--OGALLALA. PERIOD OF RECORD.--1966 TO CURRENT YEAR. EXTREMES FOR PERIOD OF RECORD.--HIGHEST WATER LEVEL, $126.90 \mathrm{FT}(38.68 \mathrm{M})$ BELOW LAND-SURFACE DATUM,FEB 26, 1966; LOWEST WATER LEVEL, 162.89 FT(49.65 M) BELOW LAND-SURFACE DATUM, JAN 22, 1975.

WATER LEVELS IN FEET BELOW LAND SURFACE DATUM, CLIMATIC YEARS APRIL 1981 TO MARCH 1983

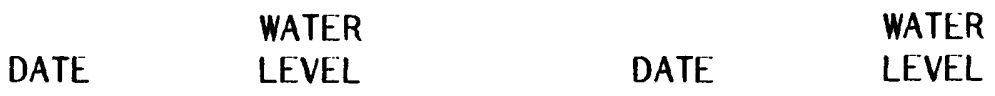

JAN 19, $1982 \quad 142.72 \quad$ JAN $11,1983 \quad 138.70$ 


\section{GROUND-WATER LEVELS}

TEXAS COUNTY -- CONTINUED

363727101044101. LOCAL NUMBER; 02N-18E-24 BCC 1. LOCATION,--LAT 36 37'27", LONG 101 04'41", HYDROLOGIC UNIT 11100104. AQUIFER.--OGALLALA.

PERIOD OF RECORD.--1970 TO CURRENT YEAR.

EXTREMES FOR PERIOD OF RECORD.--HIGHEST WATER LEVEL, 134.05 FT(40.86 M) BELOW LAND-SURFACE DATUM, JAN 16, 1970; LOWEST WATER LEVEL, 144.70 FT(44.10 M) BELOW LAND-SURFACE DATUM, JAN 16, 1979.

WATER LEVELS IN FEET BELOW LAND SURFACE DATUM, CLIMATIC YEARS APRIL 1981 TO MARCH 1983

\begin{tabular}{|c|c|c|}
\hline & WATER & \\
\hline DATE & LEVEL & DATE \\
\hline
\end{tabular}

JAN $17,1982140.08 \quad$ JAN 13, $1983 \quad 138.91$

GROUND-WATER LEVELS

363636101064801. LOCAL NUMBER; 02N-18E-27 BCC 1.

LOCATION,--LAT 36 36'36", LONG 101 06'48", HYDROLOGIC UNIT 11100104.

AQUIFER .--OGALLALA.

PERIOD OF RECORD.--1966 TO CURRENT YEAR.

EXTREMES FOR PERIOD OF RECORD.--HIGHEST WATER LEVEL, 122.00 FT(37.19 M) BELOW LAND-SURFACE DATUM,FEB 26, 1966; LOWEST WATER LEVEL, 153.98 FT(46.93 M) BELOW LAND-SURFACE DATUM, JAN 12, 1977.

WATER LEVELS IN FEET BELOW LAND SURFACE DATUM, CLIMATIC YEARS APRIL 1981 TO MARCH 1983

$\begin{array}{lll} & \text { WATER } & \text { WATER } \\ \text { DATE } & \text { LEVEL } & \text { DATE }\end{array}$

JAN 19, 1982141.97 JAN $11,1983 \quad 137.50$ 
TEXAS COUNTY -- CONTINUED

363629101100401. LOCAL NUMBER; 02N-18E-30 CBB 1. LOCATION,--LAT 36 36'29", LONG 101 10'04", HYDROLOGIC UNIT 11100102. AQUIFER.--OGALLALA.

PERIOD OF RECORD.--1966 TO CURRENT YEAR.

EXTREMES FOR PERIOD OF RECORD.--HIGHEST WATER LEVEL, $157.90 \mathrm{FT}(48.13 \mathrm{M})$ BELOW LAND-SURFACE DATUM,FEB 26, 1966; LOWEST WATER LEVEL, 186.13 FT(56.73 M) BELOW LAND-SURFACE DATUM, JAN 7, 1981.

WATER LEVELS IN FEET BELOW LAND SURFACE DATUM, CLIMATIC YEARS APRIL 1981 TO MARCH 1983

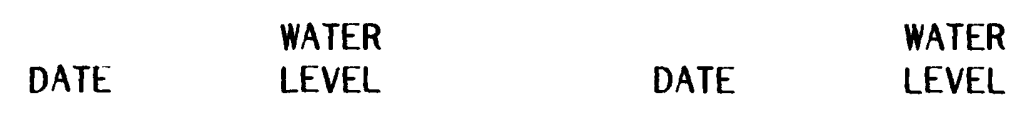

JAN 19, $1982170.80 \quad$ JAN 10, $1983 \quad 170.89$

GROUND-WATER LEVELS

363539101064901. LOCAL NUMBER;

02N-18E-34 CBB 1. LOCATION,--LAT 36 35'39", LONG 101 06'49", HYDROLOGIC UNIT 11100104. AQUIFER .--OGALLALA. PERIOD OF RECORD.--1966 TO CURRENT YEAR. EXTREMES FOR PERIOD OF RECORD.--HIGHEST WATER LEVEL, $107.52 \mathrm{FT}(32.77 \mathrm{M})$ BELOW LAND-SURFACE DATUM, JAN 18, 1968; LOWEST WATER LEVEL,116.52 FT(35.52 M) BELOW LAND-SURFACE DATUM, JAN 12, 1977.

WATER LEVELS IN FEET BELOW LAND SURFACE DATUM, CLIMATIC YEARS APRIL 1981 TO MARCH 1983

\begin{tabular}{|c|c|c|}
\hline DATE & $\begin{array}{l}\text { WATER } \\
\text { LEVEL }\end{array}$ & DATE \\
\hline
\end{tabular}

JAN 19, $1982 \quad 109.27 \mathrm{R} \quad$ JAN 11, $1983 \quad 109.20$ 
TEXAS COUNTY -- CONTINUED

363904101031501 . LOCAL NUMBER; LOCATION,--LAT 36 39'04", LONG 101 03'15", HYDROLOGIC UNIT 11100102. AQUIFER .--OGALLALA. PERIOD OF RECORD.--1966 TO CURRENT YEAR. EXTREMES FOR PERIOD OF RECORD.--HIGHEST WATER LEVEL, $52.25 \mathrm{FT}(15.93 \mathrm{M})$ BELOW LAND-SURFACE DATUM, JAN 22, 1975; LOWEST WATER LEVEL, 58.59 FT(17.86 M) BELOW LAND-SURFACE DATUM, JAN 15, 1969.

WATER LEVELS IN FEET BELOW LAND SURFACE DATUM, CLIMATIC YEARS APRIL 1981 TO MARCH 1983

\begin{tabular}{|c|c|}
\hline & WATER \\
\hline DATE & LEVEL \\
\hline
\end{tabular}

JAN 19, $1982 \quad 56.77 \quad$ JAN $11,1983 \quad 54.00$

\section{GROUND-WATER LEVELS}

364447101573301. LOCAL NUMBER; 03N-10E-11 BBB 2 . LOCATION,--LAT 36 44'47", LONG 101 57'33", HYDROLOGIC UNIT 11100101. AQUIFER .--OGALLALA.

PERIOD OF RECORD.--1966 TO CURRENT YEAR.

EXTREMES FOR PERIOD OF RECORD.--HIGHEST WATER LEVEL, $118.91 \mathrm{FT}(36.24 \mathrm{M})$ BELOW LAND-SURFACE DATUM, JAN 14, 1970; LOWEST WATER LEVEL, 128.25 FT(39.09 M) BELOW LAND-SURFACE DATUM, JAN 10, 1983.

WATER LEVELS IN FEET BELOW LAND SURFACE DATUM, CLIMATIC YEARS APRIL 1981 TO MARCH 1983

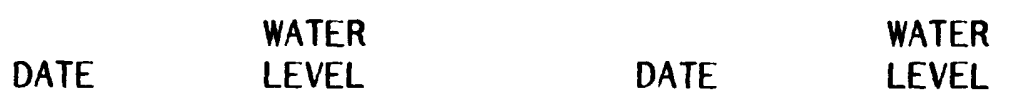

JAN 18, $1982127.06 \quad$ JAN $10,1983 \quad 128.25$


GROUND-WATER LEVELS

TEXAS COUNTY -- CONTINUED

364220102014101. LOCAL NUMBER; 03N-10E-19 CDC 1.

LOCATION,--LAT 36 42'20", LONG 102 01'41", HYDROLOGIC UNIT 11100101.

AQUIFER.--OGALLALA.

PERIOD OF RECORD.--1966 TO CURRENT YEAR.

EXTREMES FOR PERIOD OF RECORD.--HIGHEST WATER LEVEL,203.90 FT(62.15 M) BELOW LAND-SURFACE DATUM, MAR 1, 1966; LOWEST WATER LEVEL,230.62 FT(70.29 M) BELOW LAND-SURFACE DATUM,

JAN 14, 1969.

WATER LEVELS IN FEET BELOW LAND SURFACE DATUM, CLIMATIC YEARS APRIL 1981 TO MARCH 1983

\begin{tabular}{|c|c|}
\hline & WATER \\
\hline DATE & LEVEL \\
\hline
\end{tabular}

JAN 18, $1982 \quad 220.39 \quad$ JAN $10,1983 \quad 221.90$

GROUND-WATER LEVELS

364305101562401. LOCAL NUMBER; 03N-10E-24 BBB 1.

LOCATION,--LAT 36 43'05", LONG 101 56'24", HYDROLOGIC UNIT 11100101.

AQUIFER . - OGALLALA.

PERIOD OF RECORD.--1966 TO CURRENT YEAR.

EXTREMES FOR PERIOD OF RECORD.--HIGHEST WATER LEVEL, $173.90 \mathrm{FT}(53.00 \mathrm{M})$ BELOW LAND-SURFACE DATUM, MAR 1, 1966; LOWEST WATER LEVEL,186.60 FT(56.88 M) BELOW LAND-SURFACE DATUM, JAN 10, 1983.

WATER LEVELS IN FEET BELOW LAND SURFACE DATUM, CLIMATIC YEARS APRIL 1981 TO MARCH 1983

$\begin{array}{lll} & \text { WATER } & \text { WATER } \\ \text { DATE } & \text { LEVEL } & \text { DATE }\end{array}$

JAN 18, $1982 \quad 186.50 \quad$ JAN $10,1983 \quad 186.60$ 
GROUND-WATER LEVELS

TEXAS COUNTY -- CONTINUED

364325101552501. LOCAL NUMBER; 03N-11E-18 CBC 1.

LOCATION,--LAT 36 43'25", LONG 101 55'25", HYDROLOGIC UNIT 11100101.

AQUIFER .--OGALLALA.

PERIOD OF RECORD.--1966 TO CURRENT YEAR.

EXTREMES FOR PERIOD OF RECORD.--HIGHEST WATER LEVEL, 147.64 FT (45.00 M) BELOW LAND-SURFACE DATUM, JAN 27, 1967; LOWEST WATER LEVEL, 161.39 FT(49.19 M) BELOW LAND-SURFACE DATUM, JAN 18, 1979.

WATER LEVELS IN FEET BELOW LAND SURFACE DATUM, CLIMATIC YEARS APRIL 1981 TO MARCH 1983

\begin{tabular}{ccccc} 
DATE & WATER & & \multicolumn{2}{c}{ WATER } \\
LEVEL & DATE & LEVEL \\
JAN 18, 1982 & 159.47 & JAN 10, 1983 &
\end{tabular}

GROUND-WATER LEVELS

364331101482301. LOCAL NUMBER; 03N-12E-18 DBB 1. LOCATION,--LAT 36 43'31", LONG 101 48'23", HYDROLOGIC UNIT 11100101. AQUIFER.--OGALLALA.

PERIOD OF RECORD.--1980 TO CURRENT YEAR.

EXTREMES FOR PERIOD OF RECORD.--HIGHEST WATER LEVEL,60.83 FT(18.54 M) BELOW LAND-SURFACE DATUM,APR 16, 1980; LOWEST WATER LEVEL,62.97 FT(19.19 M) BELOW LAND-SURFACE DATUM, JAN 18, 1982.

WATER LEVELS IN FEET BELOW LAND SURFACE DATUM, CLIMATIC YEARS APRIL 1981 TO MARCH 1983

$\begin{array}{lll} & \text { WATER } & \\ \text { DATE } & \text { LEVEL } & \text { WATER } \\ & \text { DATE } & \text { LEVEL }\end{array}$

JAN 18, $1982 \quad 62.97 \quad$ JAN 10, $1983 \quad \mathrm{P}$


TEXAS COUNTY -- CONTINUED

364459101373601. LOCAL NUMBER; 03N-13E-02 CDD 1. LOCATION,--LAT 36 44'59", LONG 101 37'36", HYDROLOGIC UNIT 11100101. AQUIFER.--OGALLALA.

PERIOD OF RECORD.--1966 TO CURRENT YEAR.

EXTREMES FOR PERIOD OF RECORD.--HIGHEST WATER LEVEL,61.36 FT(18.70 M) BELOW LAND-SURFACE DATUM,JAN 15, 1980; LOWEST WATER LEVEL,62.89 FT(19.17 M) BELOW LAND-SURFACE DATUM, JAN 11, 1977.

WATER LEVELS IN FEET BELOW LAND SURFACE DATUM, CLIMATIC YEARS APRIL 1981 TO MARCH 1983

\begin{tabular}{|c|c|c|}
\hline DATF & $\begin{array}{l}\text { WATER } \\
\text { I FVEI }\end{array}$ & DATF \\
\hline & & oAl \\
\hline
\end{tabular}

JAN 18, $198262.58 \quad$ JAN $10,1983 \quad 62.60$

GROUND-WATER LEVELS

364301101175401. LOCAL NUMBER; 03N-16E-23 AAB 1.

LOCATION,--LAT 36 43'01", LONG 101 17'54", HYDROLOGIC UNIT 11100102. AQUIFER . - OĜALLALA.

PERIOD OF RECORD.--1966 TO CURRENT YEAR.

EXTREMES FOR PERIOD OF RECORD.--HIGHEST WATER LEVEL, 113.42 FT(34.57 M) BELOW LAND-SURFACE DATUM, JAN 15, 1971; LOWEST WATER LEVEL, 123.95 FT(37.78 M) BELOW LAND-SURFACE DATUM, JAN 11, 1983.

WATER LEVELS IN FEET BELOW LAND SURFACE DATUM, CLIMATIC YEARS APRIL 1981 TO MARCH 1983

$\begin{array}{lll} & \text { WATER } & \text { WATER } \\ \text { DATE } & \text { LEVEL } & \text { DATE }\end{array}$

JAN 19, $1982 \quad 118.18 \quad$ JAN $11,1983 \quad 123.95$ 
GROUND-WATER LEVELS

TEXAS COUNTY -- CONTINUED

364202101195501. LOCAL NUMBER; 03N-16E-28 AAD 1. LOCATION,--LAT 36 42'02", LONG 101 19'55", HYDROLOGIC UNIT 11100102.

AQUIFER.--OGALLALA.

PERIOD OF RECORD.--1966 TO CURRENT YEAR.

EXTREMES FOR PERIOD OF RECORD.--HIGHEST WATER LEVEL, $116.74 \mathrm{FT}(35.58 \mathrm{M})$ BELOW LAND-SURFACE DATUM, JAN 19, 1982; LOWEST WATER LEVEL, $124.79 \mathrm{FT}(38.04 \mathrm{M})$ BELOW LAND-SURFACE DATUM, JAN 16, 1970.

WATER LEVELS IN FEET BELOW LAND SURFACE DATUM, CLIMATIC YEARS APRIL 1981 TO MARCH 1983

\begin{tabular}{|c|c|c|}
\hline DA 1 & $\begin{array}{l}\text { WATER } \\
\text { LEVEL }\end{array}$ & DATE \\
\hline
\end{tabular}

JAN 19, $1982 \quad 116.74 \quad$ JAN 12, $1983 \quad 116.85$

GROUND-WATER LEVELS

364334101111101. LOCAL NUMBER; 03N-17E-13 CBB 1. LOCATION,--LAT 36 43'34", LONG 101 11'11", HYDROLOGIC UNIT 11100102. AQUIFER .--OGALLALA.

PERIOD OF RECORD.--1966 TO CURRENT YEAR.

EXTREMLS FOR PERIOD OF RECORD.--HIGHEST WATER LEVEL, 82.10 FT(25.02 M) BELOW LAND-SURFACE DATUM,FEB 2, 1966; LOWEST WATER LEVEL,90.30 FT(27.52 M) BELOW LAND-SURFACE DATUM, JAN 12, 1983.

WATER LEVELS IN FEET BELOW LAND SURFACE DATUM, CLIMATIC YEARS APRIL 1981 TO MARCH 1983

$\begin{array}{lll} & \text { WATER } & \text { WATER } \\ \text { DATE } & \text { LEVEL } & \text { DATE }\end{array}$

JAN 19, $198290.02 \quad$ JAN 12, $1983 \quad 90.30$ 
TEXAS COUNTY -- CONTINUED

365033101524702. LOCAL NUMBER; LOCATION,--LAT $3650^{\prime 3} 3$ ", LONG 101 AQUIFER.--OGALLALA. PERIOD OF RECORD.--1966 TO CURRENT YEAR. EXTREMES FOR PERIOD OF RECORD.--HIGHEST WATER LEVEL, $162.90 \mathrm{FT}(49.65 \mathrm{M})$ BELOW LAND-SURFACE DATUM, JAN 18, 1966; LOWEST WATER LEVEL, $179.98 \mathrm{FT}(54.86 \mathrm{M})$ BELOW LAND-SURFACE DATUM, JAN 13, 1977.

WATER LEVELS IN FEET BELOW LAND SURFACE DATUM, CLIMATIC YEARS APRIL 1981 TO MARCH 1983
04N-11E-04 DBD 1.

52'47", HYDROLOGIC UNIT 11100101.

$\begin{array}{cccc}\text { DATE } & \begin{array}{c}\text { WATER } \\ \text { LEVEL }\end{array} & \text { DATE } & \text { WATER } \\ \text { LEVEL }\end{array}$

365006101520801. LOCAL NUMBER;
LOCATION,--LAT 36 50'06", LONG $101 \quad 52^{\prime} 08^{\prime \prime}$, HYDROLOGI

UNIT 11100101.

AQUIFER.--OGALLALA.

PERIOD OF RECORD.--1966 TO CURRENT YEAR.

EXTREMES FOR PERIOD OF RECORD.--HIGHEST WATER LEVEL, 150.10 FT(45.75 M) BELOW LAND-SURFACE DATUM, JAN 18, 1966; LOWEST WATER LEVEL, 159.81 FT(48.71 M) BELOW LAND-SURFACE DATUM, JAN 13, 1977.

WATER LEVELS IN FEET BELOW LAND SURFACE DATUM, CLIMATIC YEARS APRIL 1981 TO MARCH 1983

$\begin{array}{lll} & \text { WATER } & \\ \text { DATE } & \text { LEVEL } & \text { WATER } \\ & \text { LEVEL }\end{array}$

JAN 21, $1982 \quad 157.05 \quad$ JAN 10, $1983 \quad 15.79$ 


\section{GROUND-WATER LEVELS}

TEXAS COUNTY -- CONTINUED

365009101444101. LOCAL NUMBER; 04N-12E-03 DDD 1. LOCATION,--LAT 36 50'09", LONG 101 44'41", HYDROLOGIC UNIT 11100101. AQUIFER.--OGALLALA.

PERIOD OF RECORD.--1966 TO CURRENT YEAR.

EXTREMES FOR PERIOD OF RECORD.--HIGHEST WATER LEVEL, 134.30 FT(40.93 M) BELOW LAND-SURFACE DATUM, JAN 10, 1980; LOWEST WATER LEVEL, 137.99 FT(42.06 M) BELOW LAND-SURFACE DATUM, JAN 13, 1977.

WATER LEVELS IN FEET BELOW LAND SURFACE DATUM, CLIMATIC YEARS APRIL 1981 TO MARCH 1983

\begin{tabular}{|c|c|}
\hline & $\begin{array}{l}\text { WATER } \\
\text { LEVEL }\end{array}$ \\
\hline
\end{tabular}

JAN 21, $1982 \quad 135.87 \quad$ JAN $10,1983 \quad 137.47$

GROUND-WATER LEVELS

364705101382801. LOCAL NUMBER; 04N-13E-27 ACD 1. LOCATION,--LAT 36 47'05", LONG 101 38'28", HYDROLOGIC UNIT 11100101. AQUIFER .--OGALLALA.

PERIOD OF RECORD.--1970 TO CURRENT YEAR.

EXTREMES FOR PERIOD OF RECORD.--HIGHEST WATER LEVEL, 114.52 FT(34.91 M) BELOW LAND-SURFACE DATUM, JAN 24, 1973; LOWEST WATER LEVEL, 116.62 FT(35.55 M) BELOW LAND-SURFACE DATUM, JAN 7, 1981.

WATER LEVELS IN FEET BELOW LAND SURFACE DATUM, CLIMATIC YEARS APRIL 1981 TO MARCH 1983

$\begin{array}{ll}\text { WATER } \\ \text { DATE } & \text { LEVEL }\end{array}$

JAN 21, 1982 
TEXAS COUNTY -- CONTINUED

365032101321201. LOCAL NUMBER; 04N-14E-03 BCC 2. LOCATION,--LAT 36 50'32", LONG 101 32'12", HYDROLOGIC UNIT 11100102. AQUIFER . - OGALLALA. PERIOD OF RECORD.--1966 TO CURRENT YEAR. EXTREMES FOR PERIOD OF RECORD.--HIGHEST WATER LEVEL, 180.40 FT(54.99 M) BELOW LAND-SURFACE DATUM,MAR 2, 1966; LOWEST WATER LEVEL,225.64 FT(68.78 M) BELOW LAND-SURFACE DATUM, JAN 12, 1983.

WATER LEVELS IN FEET BELOW LAND SURFACE DATUM, CLIMATIC YEARS APRIL 1981 TO MARCH 1983

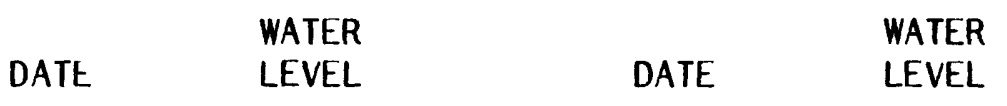

JAN $20,1982 \quad 222.35 \quad$ JAN $12,1983 \quad 225.64$

GROUND-WATER LEVELS

365046101345101. LOCAL NUMBER; $\quad 04 \mathrm{~N}-14 \mathrm{E}-05 \mathrm{BBC} 1$. LOCATION,--LAT 36 50'46", LONG 101 34'51", HYDROLOGIC UNIT 11100101. AQUIFER.--OGALLALA.

PERIOD OF RECORD.--1966 TO CURRENT YEAR.

EXTREMES FOR PERIOD OF RECORD.--HIGHEST WATER LEVEL, 156.34 FT(47.65 M) BELOW LAND-SURFACE DATUM, JAN 15, 1979; LOWEST WATER LEVEL, 178.69 FT(54.46 M) BELOW LAND-SURFACE DATUM, JAN $10,1977$.

WATER LEVELS IN FEET BELOW LAND SURFACE DATUM, CLIMATIC YEARS APRIL 1981 TO MARCH 1983

$\begin{array}{lll} & \text { WATER } & \text { WATER } \\ \text { DATE } & \text { LEVEL } & \text { DATE }\end{array}$

JAN 20, $1982159.22 \quad$ JAN $12,1983 \quad 159.80$ 


\section{GROUND-WATER LEVELS}

TEXAS COUNTY -- CONTINUED

364851101303901. LOCAL NUMBER;
LOCATION,--LAT 36 48'51", LONG $101 \quad 30^{\prime} 39 "$ ", HYDROLOGIC UNIT 11100101.

AQUIFER.--OGALLALA.

PERIOD OF RECORD.--1966 TO CURRENT YEAR.

EXTREMES FOR PERIOD OF RECORD.--HIGHEST WATER LEVEL, 180.10 FT(54.89 M) BELOW LAND-SURFACE DATUM,JAN 13, 1977; LOWEST WATER LEVEL, 196.49 FT(59.89 M) BELOW LAND-SURFACE DATUM, JAN 24, 1973.

WATER LEVELS IN FEET BELOW LAND SURFACE DATUM, CLIMATIC YEARS APRIL 1981 TO MARCH 1983

\begin{tabular}{cccc}
\multicolumn{1}{c}{ WATER } & & WATER \\
DATE & LEVEL & DATE & LEVEL \\
JAN 20, 1982 & 183.89 & JAN 12, 1983 & 184.45
\end{tabular}

GROUND-WATER LEVELS

364939101262101. LOCAL NUMBER; 04N-15E-09 ADD 1. LOCATION,--LAT 36 49'39", LONG 101 26'21", HYDROLOGIC UNIT 11100102.

AQUIFER.--OGALLALA.

PERIOD OF RECORD.--1966 TO CURRENT YEAR.

EXTREMES FOR PERIOD OF RECORD.--HIGHEST WATER LEVEL, $152.90 \mathrm{FT}(46.60 \mathrm{M})$ BELOW LAND-SURFACE DATUM,MAR 2, 1966; LOWEST WATER LEVEL, 198.87 FT(60.62 M) BELOW LAND-SURFACE DATUM, JAN 12, 1983.

WATER LEVELS IN FEET BELOW LAND SURFACE DATUM, CLIMATIC YEARS APRIL 1981 TO MARCH 1983

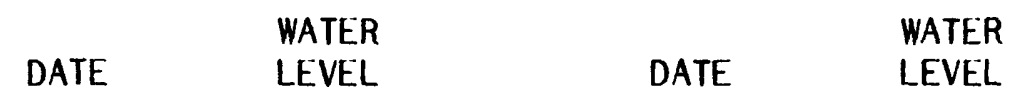

JAN 20, $1982196.72 \quad$ JAN $12,1983 \quad 198.87$ 
TEXAS COUNTY -- CONTINUED

364947101244101. LOCAL NUMBER; 04N-15E-11 BDA 1.

LOCATION,--LAT 36 49'47", LONG 101 24'41", HYDROLOGIC UNIT 11100102.

AQUIFER _--OGALLALA.

PERIOD OF RECORD.--1966 TO CURRENT YEAR.

EXTREMES FOR PERIOD OF RECORD.--HIGHEST WATER LEVEL, 142.15 FT(43.33 M) BELOW LAND-SURFACE DATUM,MAR 2, 1966; LOWEST WATER LEVEL, 182.35 FT(55.58 M) BELOW LAND-SURFACE DATUM, JAN 10, 1983.

WATER LEVELS IN FEET BELOW LAND SURFACE DATUM, CLIMATIC YEARS APRIL 1981 TO MARCH 1983

$\begin{array}{cccc}\text { DATE } & \begin{array}{l}\text { WATER } \\ \text { LEVEL }\end{array} & \text { DATE } & \text { WATER } \\ \text { LEVEL }\end{array}$

GROUND-WATER LEVELS

364844101255801. LOCAL NUMBER; $\quad 04 \mathrm{~N}-15 \mathrm{E}-15$ CAB 1. LOCATION,--LAT 36 48'44", LONG 101 25'58", HYDROLOGIC UNIT 11100102. AQUIFER .--OGALLALA.

PERIOD OF RECORD.--1966 TO CURRENT YEAR.

EXTREMES FOR PERIOD OF RECORD.--HIGHEST WATER LEVEL, 146.10 FT(44.53 M) BELOW LAND-SURFACE DATUM,MAR 2, 1966; LOWEST WATER LEVEL,202.97 FT(61.87 M) BELOW LAND-SURFACE DATUM, JAN $9,1981$.

WATER LEVELS IN FEET BELOW LAND SURFACE DATUM, CLIMATIC YEARS APRIL 1981 TO MARCH 1983

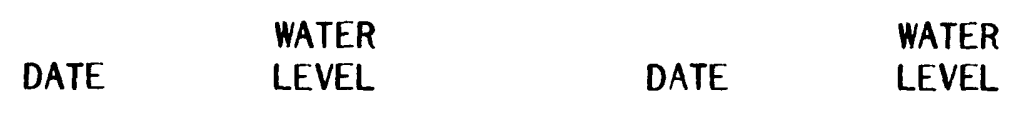

JAN. 20, $1982 \quad 202.71 \quad$ JAN 12, $1983 \quad 202.73$ 
GROUND-WATER LEVELS

TEXAS COUNTY -- CONTINUED

364703101233501. LOCAL NUMBER; .

04N-15E-25 DBB 1. LOCATION,--LAT 36 47'03", LONG 101 AQUIFER.--OGALLALA. PERIOD OF RECORD.--1966 TO CURRENT YEAR. EXTREMES FOR PERIOD OF RECORD.--HIGHEST WATER LEVEL, 137.49 FT(41.91 M) BELOW LAND-SURFACE DATUM, JAN 26, 1967; LOWEST WATER LEVEL, $172.06 \mathrm{FT}(52.44 \mathrm{M})$ BELOW LAND-SURFACE DATUM, JAN 10, 1983.

WATER LEVELS IN FEET BELOW LAND SURFACE DATUM, CLIMATIC YEARS APRIL 1981 TO MARCH 1983

\begin{tabular}{|c|c|}
\hline & WATER \\
\hline DATE & LEVEL \\
\hline
\end{tabular}

JAN 19, 1982170.57 JAN 10, $1983 \quad 172.06$

GROUND-WATER LEVELS

364705101264701. LOCAL NUMBER; 04N-15E-28 ACC 1. LOCATION,--LAT 36 47'05", LONG 101 26'47", HYDROLOGIC UNIT 11100102. AQUIFER .--OGALLALA.

PERIOD OF RECORD.--1966 TO CURRENT YEAR.

EXTREMES FOR PERIOD OF RECORD.--HIGHEST WATER LEVEL, $151.20 \mathrm{FT}(46.09 \mathrm{M})$ BELOW LAND-SURFACE DATUM,MAR 2, 1966; LOWEST WATER LEVEL, 188.94 FT(57.59 M) BELOW LAND-SURFACE DATUM, JAN 12, 1983.

WATER LEVELS IN FEET BELOW LAND SURFACE DATUM, CLIMATIC YEARS APRIL 1981 TO MARCH 1983

$\begin{array}{lll} & \text { WATER } & \text { WATER } \\ \text { DATE } & \text { LEVEL } & \text { DATE }\end{array}$

JAN 20, $1982 \quad 186.95 \quad$ JAN $12,1983 \quad 188.94$ 


\section{GROUND-WATER LEVELS}

TEXAS COUNTY -- CONTINUED

365028101215901. LOCAL NUMBER; 04N-16E-05 CCB 1.

LOCATION,--LAT 3650 '28", LONG 101 21'59", HYDROLOGIC UNIT 11100102.

AQUIFER .--OGALLALA.

PERIOD OF RECORD.--1966 TO CURRENT YEAR.

EXTREMES FOR PERIOD OF RECORD.--HIGHEST WATER LEVEL, $113.95 \mathrm{FT}(34.73 \mathrm{M})$ BELOW LAND-SURFACE DATUM,MAR 2, 1966; LOWEST WATER LEVEL,166.63 FT(50.79 M) BELOW LAND-SURFACE DATUM, JAN $10,1983$.

WATER LEVELS IN FEET BELOW LAND SURFACE DATUM, CLIMATIC YEARS APRIL 1981 TO MARCH 1983

$\begin{array}{lll} & \text { WATER } & \text { WATER } \\ \text { DATE } & \text { LEVEL } & \text { DATE }\end{array}$

JAN 19, $1982160.19 \quad$ JAN $10,1983 \quad 166.63$

GROUND-WATER LEVELS

364858101213301. LOCAL NUMBER; $\quad 04 \mathrm{~N}-16 \mathrm{E}-17$ BAD 1. LOCATION,--LAT 36 48'58", LONG 101 21'33", HYDROLOGIC UNIT 11100102. AQUIFER .--OGALLALA.

PERIOD OF RECORD.--1966 TO CURRENT YEAR. EXTREMES FOR PERIOD OF RECORD.--HIGHEST WATER LEVEL, $99.69 \mathrm{FT}(30.39 \mathrm{M})$ BELOW LAND-SURFACE DATUM, JAN 26, 1967; LOWEST WATER LEVEL, 133.97 FT(40.83 M) BELOW LAND-SURFACE DATUM, JAN 10, 1983.

WATER LEVELS IN FEET BELOW LAND SURFACE DATUM, CLIMATIC YEARS APRIL 1981 TO MARCH 1983

\begin{tabular}{|c|c|}
\hline & WATER \\
\hline DATE & LEVEL \\
\hline
\end{tabular}

JAN 19, $1982129.89 \quad$ JAN 10, $1983 \quad 133.97$ 
TEXAS COUNTY -- CONTINUED

364724101215601. LOCAL NUMBER;

$04 \mathrm{~N}-16 \mathrm{E}-29$ BBB 1.

LOCATION,--LAT 36 47'24", LONG 101 21'56", HYDROLOGIC UNIT 11100102.

AQUIFER .--OGALLALA.

PERIOD OF RECORD.--1966 TO CURRENT YEAR.

EXTREMES FOR PERIOD OF RECORD.--HIGHEST WATER LEVEL, $113.90 \mathrm{FT}(34.72 \mathrm{M})$ BELOW LAND-SURFACE DATUM,MAR 2, 1966; LOWEST WATER LEVEL, 140.78 FT(42.91 M) BELOW LAND-SURFACE DATUM, JAN 10, 1983.

WATER LEVELS IN FEET BELOW LAND SURFACE DATUM, CLIMATIC YEARS APRIL 1981 TO MARCH 1983

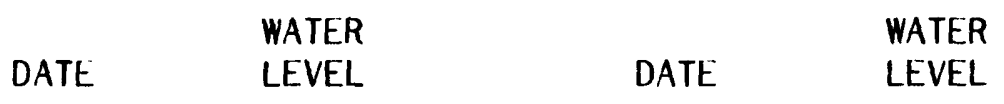

JAN 19, $1982139.64 \quad$ JAN $10,1983 \quad 140.78$

GROUND-WATER LEVELS

364740101190501. LOCAL NUMBER; LOCATION,--LAT 36 47'40", LONG 101 AQUIFER.--OGALLALA.

PERIOD OF RECORD.--1966 TO CURRENT YEAR.

EXTREMES FOR PERIOD OF RECORD.--HIGHEST WATER LEVEL, 100.20 FT(30.54 M) BELOW LAND-SURFACE DATUM,MAR 2, 1966; LOWEST WATER LEVEL,126.36 FT(38.51 M) BELOW LAND-SURFACE DATUM, JAN $17,1979$.

WATER LEVELS IN FEET BELOW LAND SURFACE DATUM, CLIMATIC YEARS APRIL 1981 TO MARCH 1983
JAN 19, 1982121.32
JAN 11, 1983
0 
GROUND-WATER LEVELS

TEXAS COUNTY -- CONTINUED

365015101132301. LOCAL NUMBER; 04N-17E-03 CBC 1.

LOCATION,--LAT 36 50'15", LONG 101 13'23", HYDROLOGIC UNIT 11100102.

AQUIFER.--OGALLALA.

PERIOD OF RECORD.--1966 TO CURRENT YEAR.

EXTREMES FOR PERIOD OF RECORD.--HIGHEST WATER LEVEL, $113.30 \mathrm{FT}(34.53 \mathrm{M})$ BELOW LAND-SURFACE DATUM, MAR 2, 1966; LOWEST WATER LEVEL, 134.71 FT(41.06 M) BELOW LAND-SURFACE DATUM, JAN 10, 1983.

WATER LEVELS IN FEET BELOW LAND SURFACE DATUM, CLIMATIC YEARS APRIL 1981 TO MARCH 1983

$\begin{array}{lll} & \text { WATER } & \text { WATER } \\ \text { DATE } & \text { LEVEL } & \text { DATE }\end{array}$

JAN 20, $1982 \quad 131.59 \quad$ JAN $10,1983 \quad 134.71$

GROUND-WATER LEVELS

364822101121101. LOCAL NUMBER; 04N-17E-14 CCD 1.

LOCATION,--LAT 36 48'22", LONG 101 12'11", HYDROLOGIC UNIT 11100102.

AQUIFER .--OGALLALA.

PERIOD OF RECORD.--1966 TO CURRENT YEAR.

EXTREMES FOR PERIOD OF RECORD.--HIGHEST WATER LEVEL, $118.10 \mathrm{FT}(36.00 \mathrm{M})$ BELOW LAND-SURFACE DATUM,MAR 2, 1966; LOWEST WATER LEVEL, $166.65 \mathrm{FT}(50.79 \mathrm{M})$ BELOW LAND-SURFACE DATUM, JAN 10, 1983.

WATER LEVELS IN FEET BELOW LAND SURFACE DATUM, CLIMATIC YEARS APRIL 1981 TO MARCH 1983

$\begin{array}{lll} & \text { WATER } & \\ \text { DATE } & \text { LEVEL } & \text { WATER } \\ \text { DATE } & \text { LEVEL }\end{array}$

JAN 20, 1982160.59 JAN 10, 1983166.65 
TEXAS COUNTY -- CONTINUED

364817101133101. LOCAL NUMBER; LOCATION,--LAT 36 48'17", LONG 101 AQUIFER.--OGALLALA.

PERIOD OF RECORD.--1966 TO CURRENT YEAR.

EXTREMES FOR PERIOD OF RECORD.--HIGHEST WATER LEVEL, 124.30 FT(37.89 M) BELOW LAND-SURFACE DATUM,MAR 2, 1966; LOWEST WATER LEVEL, 177.73 FT(54.17 M) BELOW LAND-SURFACE DATUM, JAN 10, 1983.

WATER LEVELS IN FEET BELOW LAND SURFACE DATUM, CLIMATIC YEARS APRIL 1981 TO MARCH 1983
04N-17E-21 AAA 1.

13'3\}', HYDROLOGIC UNIT 11100102.

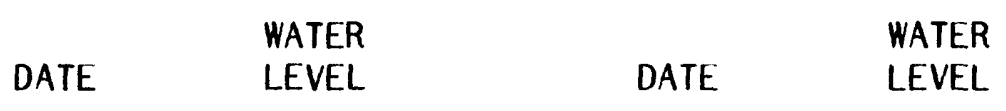

JAN $20,1982 \quad 174.39$

GROUND-WATER LEVELS

364810101112501 . LOCAL NUMBER;

LOCATION,--LAT 36 48'10", LONG 101 11'25", HYDROLOGIC UNIT 11100102.

AQUIFER .--OGALLALA.

PERIOD OF RECORD.-- 1966 TO CURRENT YEAR.

EXTREMES FOR PERIOD OF RECORD.--HIGHEST WATER LEVEL, 108.50 FT(33.07 M) BELOW LAND-SURFACE DATUM, MAR 3, 1966; LOWEST WATER LEVEL, $157.93 \mathrm{FT}(48.14 \mathrm{M})$ BELOW LAND-SURFACE DATUM, JAN 10, 1983.

WATER LEVELS IN FEET BELOW LAND SURFACE DATUM, CLIMATIC YEARS APRIL 1981 TO MARCH 1983 
TEXAS COUNTY -- CONTINUED

364614101133001. LOCAL NUMBER; 04N-17E-33 ADD 1.

LOCATION,--LAT 36 46'14", LONG 101 13'30", HYDROLOGIC UNIT 11100102.

AQUIFER. - OGALLALA.

PERIOD OF RECORD.--1966 TO CURRENT YEAR.

EXTREMES FOR PERIOD OF RECORD.--HIGHEST WATER LEVEL, $100.55 \mathrm{FT}(30.65 \mathrm{M})$ BELOW LAND-SURFACE DATUM, JAN 10, 1983; LOWEST WATER LEVEL, $155.36 \mathrm{FT}(47.35 \mathrm{M})$ BELOW LAND-SURFACE DATUM, JAN 13, 1977.

WATER LEVELS IN FEET BELOW LAND SURFACE DATUM, CLIMATIC YEARS APRIL 1981, T0 MARCH 1983

$\begin{array}{lll} & \text { WATER } & \text { WATER } \\ \text { DATE } & \text { LEVEL } & \text { DATE }\end{array}$

JAN 20, $1982 \quad 155.01 \quad$ JAN $10,1983 \quad 100.55$

GROUND-WATER LEVELS

364658101044301. LOCAL NUMBER; 04N-18E-25 CBB 1.

LOCATION,--LAT 36 46'58", LONG 101 04'43", HYDROLOGIC UNIT 11100102.

AQUIFER.--OGALLALA.

PERIOD OF RECORD.--1966 TO CURRENT YEAR.

EXTREMES FOR PERIOD OF RECORD.--HIGHEST WATER LEVEL, $94.20 \mathrm{FT}(28.71 \mathrm{M})$ BELOW LAND-SURFACE DATUM,MAR 2, 1966; LOWEST WATER LEVEL, 123.25 FT(37.57 M) BELOW LAND-SURFACE DATUM, JAN 23, 1975.

WATER LEVELS IN FEET BELOW LAND SURFACE DATUM, CLIMATIC YEARS APRIL 1981 TO MARCH 1983

\begin{tabular}{|c|c|c|}
\hline & WATER & \\
\hline DATE & LEVEL & DATE \\
\hline
\end{tabular}

JAN 20, 1982109.25 JAN 10, $1983 \quad 109.15$ 
AQUIFER.--OGALLALA.

PERIOD OF RECORD.--1966 TO CURRENT YEAR.

EXTREMES FOR PERIOD OF RECORD.--HIGHEST WATER LEVEL,97.20 FT(29.63 M) BELOW LAND-SURFACE DATUM, JAN 26, 1967; LOWEST WATER LEVEL, 117.45 FT(35.80 M) BELOW LAND-SURFACE DATUM, JAN 17, 1979.

WATER LEVELS IN FEET BELOW LAND SURFACE DATUM, CLIMATIC YEARS APRIL 1981 TO MARCH 1983

\begin{tabular}{cccc} 
& WATER & & WATER \\
DATE & LEVEL & DATE & LEVEL \\
\hline JAN 20, 1982 & 115.23 & JAN 10, 1983 & 116.36
\end{tabular}

WATER LEVELS IN FEET BELOW LAND SURFACE DATUM, CLIMATIC YEARS APRIL 1981 TO MARCH 1983

$\begin{array}{lll} & \text { WATER } & \text { WATER } \\ \text { DATE } & \text { LEVEL } & \text { DATE }\end{array}$

JAN 20, $1982 \quad 99.24 \quad$ JAN $10,1983 \quad$ W


TEXAS COUNTY -- CONTINUED

365407101530001. LOCAL NUMBER; 05N-11E-16 BCB 1. LOCATION,--LAT 36 54'07", LONG 101 53'00", HYDROLOGIC UNIT 11100101. AQUIFER.--OGALLALA.

PERIOD OF RECORD.--1966 TO CURRENT YEAR. EXTREMES FOR PERIOD OF RECORD.-- HIGHEST WATER LEVEL,201.55 FT(61.43 M) BELOW LAND-SURFACE DATUM,FEB 27, 1966; LOWEST WATER LEVEL,245.04 FT(74.69 M) BELOW LAND-SURFACE DATUM, JAN 21, 1982 .

WATER LEVELS IN FEET BELOW LAND SURFACE DATUM, CLIMATIC YEARS APRIL 1981 TO MARCH 1983

\begin{tabular}{|c|c|c|}
\hline & WATER & \\
\hline DATE & LEVEL & DATE \\
\hline
\end{tabular}

JAN 21, $1982 \quad 245.04 \quad$ JAN 10, $1983 \quad 239.42$

GROUND-WATER LEVELS

365607101481101. LOCAL NUMBER; 05N-12E-06 BAA 1. LOCATION,--LAT 36 56'07", LONG 101 48'11", HYDROLOGIC UNIT 11100101. AQUIFER .--OGALLALA.

PERIOD OF RECORD.--1968 TO CURRENT YEAR. EXTREMES FOR PERIOD OF RECORD.--HIGHEST WATER LEVEL,210.38 FT(64.12 M) BELOW LAND-SURFACE DATUM,JAN 14, 1969; LOWEST WATER LEVEL,263.77 FT(80.40 M) BELOW LAND-SURFACE DATUM, JAN 7, 1981.

WATER LEVELS IN FEET BELOW LAND SURFACE DATUM, CLIMATIC YEARS APRIL 1981 TO MARCH 1983

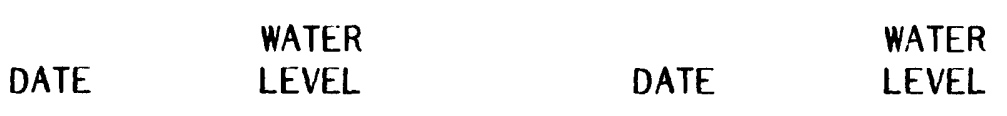

JAN 21, $1982 \quad 259.58 \quad$ JAN 10, $1983 \quad 260.22$ 
TEXAS COUNTY -- CONTINUED

365422101462601. LOCAL NUMBER;

05N-12E-16 BBB 1.

LOCAT10N,-..LAT 36 54'22", LONG 101

46'26", HYDROLOGIC UNIT 11100101.

AQUIFER .--OGALLALA.

PERIOD OF RECORD.--1966 TO CURRENT YEAR.

EXTREMES FOR PERIOD OF RECORD.--HIGHEST WATER LEVEL,201.30 FT(61.36 M) BELOW LAND-SURFACE DATUM,FEB 26, 1966; LOWEST WATER LEVEL,249.37 FT(76.01 M) BELOW LAND-SURFACE DATUM, JAN 15, 1979.

WATER LEVELS IN FEET BELOW LAND SURFACE DATUM, CLIMATIC YEARS APRIL 1981 TO MARCH 1983

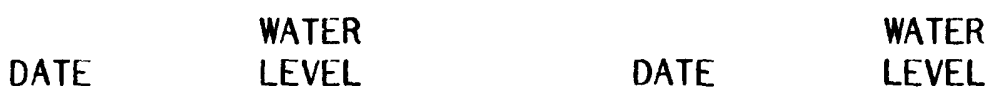

JAN 21, $1982 \quad 245.72 \quad$ JAN $10,1983 \quad 247.43$

GROUND-WATER LEVELS

365325101454001. LOCAL NUMBER; 05N-12E-21 AAB 1.

LOCATION,--LAT 36 53'25", LONG 101 45'40", HYDROLOGIC UNIT 11100101.

AQUIFER .--OGALLALA.

PERIOD OF RECORD.--1981 TO CURRENT YEAR.

EXTREMES FOR PERIOD OF RECORD.--HIGHEST WATER LEVEL, 199.22 FT(60.72 M) BELOW LAND-SURFACE DATUM,MAR 4, 1981; LOWEST WATER LEVEL,201.75 FT(61.49 M) BELOW LAND-SURFACE DATUM, JAN 10, 1983.

WATER LEVELS IN FEET BELOW LAND SURFACE DATUM, CLIMATIC YEARS APRIL 1981 TO MARCH 1983

\begin{tabular}{|c|c|}
\hline & WATER \\
\hline DATE & LEVEL \\
\hline
\end{tabular}

JAN 21, $1982199.64 \quad$ JAN $10,1983 \quad 201.75$ 
TEXAS COUNTY -- CONTINUED

365313101451801. LOCAL NUMBER; O5N-12E-22 CBB 1. LOCATION,--LAT 36 53'13", LONG 101 45'18", HYDROLOGIC UNIT 11100101.

AQUIFER .--OGALLALA.

PERIOD OF RECORD.--1981 TO CURRENT YEAR.

EXTREMES FOR PERIOD OF RECORD.--HIGHEST WATER LEVEL, 178.22 FT(54.32 M) BELOW LAND-SURFACE DATUM, JAN 21, 1982; LOWEST WATER LEVEL, 179.84 FT(54.82 M) BELOW LAND-SURFACE DATUM, JAN 10, 1983.

WATER LEVELS IN FEET BELOW LAND SURFACE DATUM, CLIMATIC YEARS APRIL 1981 TO MARCH 1983

\begin{tabular}{cccc}
\multicolumn{1}{c}{ WATER } & & WATER \\
DATE & LEVEL & DATE & LEVEL \\
JAN 21, 1982 & 178.22 & JAN 10, 1983 & 179.84
\end{tabular}

\section{GROUND-WATER LEVELS}

365546101363301. LOCAL NUMBER; 05N-13E-01 CBA 1. LOCATION,--LAT 36 55'46", LONG 101 36'33", HYDROLOGIC UNIT 11100101. AQUIFER .--OGALLALA. PERIOD OF RECORD.--1966 TO CURRENT YEAR. EXTREMES FOR PERIOD OF RECORD.--HIGHEST WATER LEVEL,219.90 FT(67.03 M) BELOW LAND-SURFACE DATUM,FEB 26, 1966; LOWEST WATER LEVEL, 310.04 FT(94.50 M) BELOW LAND-SURFACE DATUM, JAN 17, 1978.

WATER LEVELS IN FEET BELOW LAND SURFACE DATUM, CLIMATIC YEARS APRIL 1981 TO MARCH 1983

$\begin{array}{lll} & \text { WATER } & \text { WATER } \\ \text { DATE } & \text { LEVEL } & \text { DATE }\end{array}$

JAN 20, $1982 \quad 296.46 \quad$ JAN 11, $1983 \quad P$ 
TEXAS COUNTY -- CONTINUED

365545101395501. LOCAL NUMBER; LOCATION,--LAT $3655^{\prime} 45^{\prime \prime}$, LONG 101 AQUIFER.--OGALLALA.

PERIOD OF RECORD.--1966 TO CURRENT YEAR.

EXTREMES FOR PERIOD OF RECORD.--HIGHEST WATER LEVEL,227.40 FT(69.31 M) BELOW LAND-SURFACE DATUM,FEB 26, 1966; LOWEST WATER LEVEL,290.58 FT(88.57 M) BELOW LAND-SURFACE DATUM, JAN 11, 1983.

WATER LEVELS IN FEET BELOW LAND SURFACE DATUM, CLIMATIC YEARS APRIL 1981 TO MARCH 1983
05N-13E-04 CBB 1.

39'55", HYDROLOGIC UNIT 11100101.
ST WATER LEVEL, $227.40 \mathrm{FT}(69.31 \mathrm{M})$ BELOW LAND-SURFACE
LEVEL, $290.58 \mathrm{FT}(88.57 \mathrm{M})$ BELOW LAND-SURFACE DATUM, WATER LEVELS IN

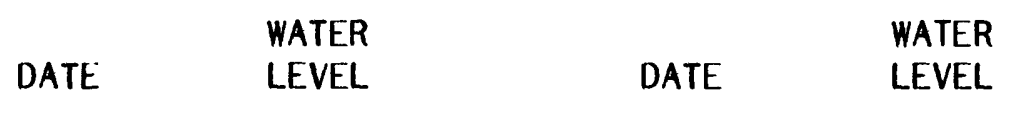

JAN 20, $1982 \quad 285.94 \quad$ JAN 11, $1983 \quad 290.58$

GROUND-WATER LEVELS

365517101405901. LOCAL NUMBER;

$05 \mathrm{~N}-13 \mathrm{E}-08$ BBB 1.

LOCATION,--LAT 36 55'17", LONG 101 40'59", HYDROLOGIC UNIT 11100101.

AQUIFER.--OGALLALA.

PERIOD OF RECORD.--1966 TO CURRENT YEAR.

EXTREMES FOR PERIOD OF RECORD.--HIGHEST WATER LEVEL,212.60 FT(64.80 M) BELOW LAND-SURFACE DATUM,FEB 26, 1966; LOWEST WATER LEVEL,266.42 FT(81.20 M) BELOW LAND-SURFACE DATUM, JAN 12, 1983.

WATER LEVELS IN FEET BELOW LAND SURFACE DATUM, CLIMATIC YEARS APRIL 1981 TO MARCH 1983

$\begin{array}{cccc}\text { DATE } & \begin{array}{l}\text { WATER } \\ \text { LEVEL }\end{array} & \text { DATE } & \begin{array}{l}\text { WATER } \\ \text { LEVEL }\end{array} \\ \text { DAN 20, 1982 } & 262.91 & \text { JAN 12, 1983 } & 266.42\end{array}$


TEXAS COUNTY -- CONTINUED

365358101371201. LOCAL NUMBER;

$05 \mathrm{~N}-13 \mathrm{E}-14$ DBB 1.

LOCATION,--LAT 36 53'58", LONG 101 37'12", HYDROLOGIC UNIT 11100102.

AQUIFER.--OGALLALA.

PERIOD OF RECORD. - 1966 TO CURRENT YEAR.

EXTREMES FOR PERIOD OF RECORD.--HIGHEST WATER LEVEL,203.70 FT(62.09 M) BELOW LAND-SURFACE DATUM,FEB 26, 1966; LOWEST WATER LEVEL,254.12 FT(77.46 M) BELOW LAND-SURFACE DATUM, JAN 20, 1982.

WATER LEVELS IN FEET BELOW LAND SURFACE DATUM, CLIMATIC YEARS APRIL 1981 TO MARCH 1983

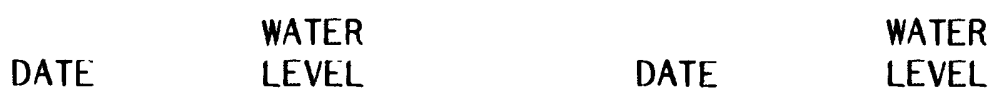

JAN 20, $1982 \quad 254.12 \quad$ JAN 12, $1983 \quad 240.00$

GROUND-WATER LEVELS

365335101294001. LOCAL NUMBER;

$05 \mathrm{~N}-14 \mathrm{E}-01$ ABB 1.

LOCATION,--LAT 36 53'35", LONG 101 29'40", HYDROLOGIC UNIT 11100102.

AQUIFER.--OGALLALA.

PERIOD OF RECORD.--1966 TO CURRENT YEAR.

EXTREMES FOR PERIOD OF RECORD.--HIGHEST WATER LEVEL, $202.40 \mathrm{FT}(61.69 \mathrm{M})$ BELOW LAND-SURFACE DATUM,FEB 25, 1966; LOWEST WATER LEVEL,266.85 FT(81.34 M) BELOW LAND-SURFACE DATUM, JAN 11, 1983.

WATER LEVELS IN FEET BELOW LAND SURFACE DATUM, CLIMATIC YEARS APRIL 1981 TO MARCH 1983

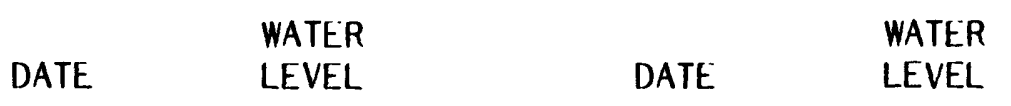

JAN 20, $1982 \quad 261.91 \quad$ JAN $11,1983 \quad 266.85$ 
TEXAS COUNTY -- CONTINUED

365451101321901. LOCAL NUMBER; 05N-14E-10 CBB 1.

LOCATION,--LAT 36 54'51", LONG 101 32'19", HYDROLOGIC UNIT 11100102.

AQUIFER .--OGALLALA.

PERIOD OF RECORD.--1966 TO CURRENT YEAR.

EXTREMES FOR PERIOD OF RECORD.--HIGHEST WATER LEVEL,201.20 FT(61.33 M) BELOW LAND-SURFACE DATUM,FEB 25, 1966; LOWEST WATER LEVEL,241.01 FT(73.46 M) BELOW LAND-SURFACE DATUM,

JAN 14, 1976.

WATER LEVELS IN FEET BELOW LAND SURFACE DATUM, CLIMATIC YEARS APRIL 1981 TO MARCH 1983

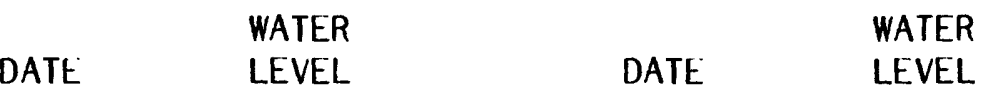

JAN 20, $1982 \quad$ JAN 11, $1983 \quad 0$

GROUND-WATER LEVELS

365130101350001 . LOCAL NUMBER; 05N-14E-31 ACC 1. LOCATION,--LAT 36 51'30", LONG 101 35'00", HYDROLOGIC UNIT 11100102. AQUIFER .--OGALLALA. PERIOD OF RECORD.--1966 TO CURRENT YEAR.

EXTREMES FOR PERIOD OF RECORD.--HIGHEST WATER LEVEL, $172.80 \mathrm{FT}(52.67 \mathrm{M})$ BELOW LAND-SURFACE DATUM,FEB 25, 1966; LOWEST WATER LEVEL,202.89 FT(61.84 M) BELOW LAND-SURFACE DATUM, JAN 12, 1983.

WATER LEVELS IN FEET BELOW LAND SURFACE DATUM, CLIMATIC YEARS APRIL 1981 T0 MARCH 1983

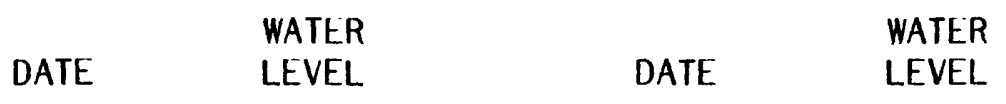

JAN $20,1982 \quad 201.50 \quad$ JAN $12,1983 \quad 202.89$ 


\section{GROUND-WATER LEVELS}

TEXAS COUNTY -- CONTINUED

365428101273901. LOCAL NUMBER; 05N-15E-08 CDD 1.

LOCATION,--LAT 36 54'28", LONG 101 27'39", HYDROLOGIC UNIT 11100102.

AQUIFER.--OGALLALA.

PERIOD OF RECORD.--1966 TO CURRENT YEAR.

EXTREMES FOR PERIOD OF RECORD.--HIGHEST WATER LEVEL, $141.00 \mathrm{FT}(42.98 \mathrm{M})$ BELOW LAND-SURFACE DATUM,FEB 25, 1966; LOWEST WATER LEVEL, 195.02 FT(59.44 M) BELOW LAND-SURFACE DATUM, JAN 12, 1983.

WATER LEVELS IN FEET BELOW LAND SURFACE DATUM, CLIMATIC YEARS APRIL 1981 T0 MARCH 1983

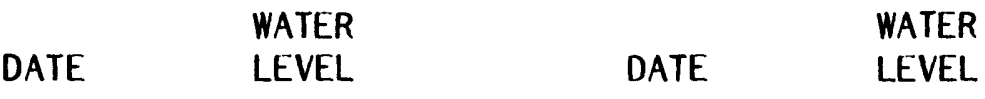

JAN 20, $1982191.59 \quad$ JAN $12,1983 \quad 195.02$

GROUND-WATER LEVELS

365354101244401. LOCAL NUMBER; 05N-15E-14 CBA 1. LOCATION,--LAT 36 53'54", LONG 101 24'44", HYDROLOGIC UNIT 11100102.

AQUIFER .--OGALLALA.

PERIOD OF RECORD.--1966 TO CURRENT YEAR.

EXTREMES FOR PERIOD OF RECORD.--HIGHEST WATER LEVEL, 147.00 FT(44.81 M) BELOW LAND-SURFACE DATUM,FEB 25, 1966; LOWEST WATER LEVEL, 192.35 FT(58.63 M) BELOW LAND-SURFACE DATUM, JAN 11, 1983.

WATER LEVELS IN FEET BELOW LAND SURFACE DATUM, CLIMATIC YEARS APRIL 1981 TO MARCH 1983

\begin{tabular}{|c|c|c|}
\hline & $\begin{array}{l}\text { WATER } \\
\text { LEVEL }\end{array}$ & DATE \\
\hline
\end{tabular}

JAN 19, $1982 \quad 187.64 \quad$ JAN 11, $1983 \quad 192.35$ 
TEXAS COUNTY -- CONTINUED

365300101271001. LOCAL NUMBER; LOCATION, --LAT 36 53'00", LONG 101 AQUIFER.--OGALLALA. PERIOD OF RECORD.--1966 TO CURRENT YEAR. EXTREMES FOR PERIOD OF RECORD.--HIGHEST WATER LEVEL, 138.20 FT(42.12 M) BELOW LAND-SURFACE DATUM,FEB 25, 1966; LOWEST WATER LEVEL, 182.78 FT(55.71 M) BELOW LAND-SURFACE DATUM, JAN 20, 1982 .

WATER LEVELS IN FEET BELOW LAND SURFACE DATUM, CLIMATIC YEARS APRIL 1981 TO MARCH 1983
05N-15E-20 DAB 1. $27^{\prime} 10^{\prime \prime}$, HYDROLOGIC UNIT 11100102.

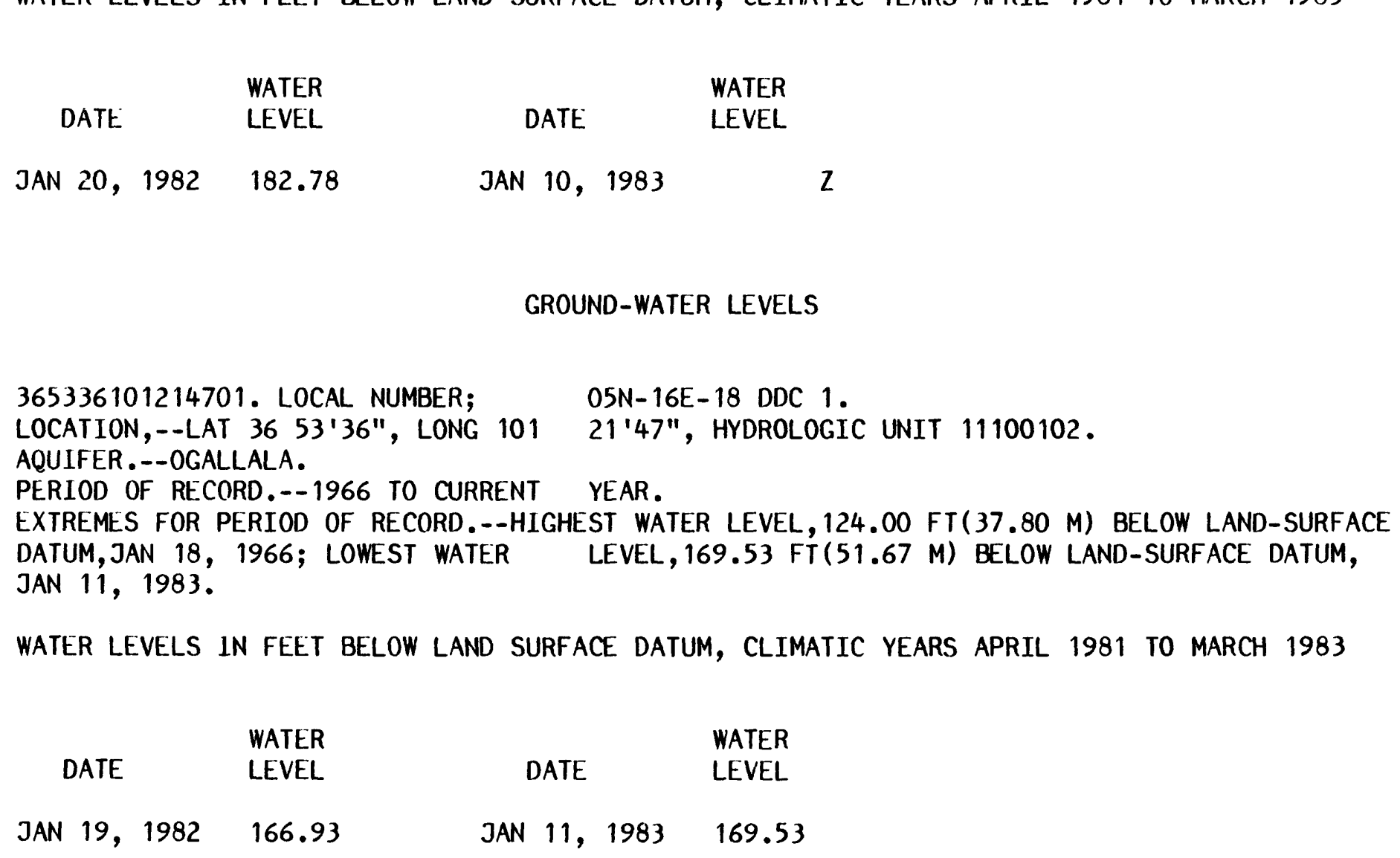




\section{GROUND-WATER LEVELS}

TEXAS COUNTY -- CONTINUED

365552101094901. LOCAL NUMBER; LOCATION,--LAT 36 55'52", LONG 101 AQUIFER. - OGALLALA.

PERIOD OF RECORD.--1966 TO CURRENT YEAR.

EXTREMES FOR PERIOD OF RECORD.--HIGHEST WATER LEVEL, $78.75 \mathrm{FT}(24.00 \mathrm{M})$ BELOW LAND-SURFACE DATUM, JAN 13, 1977; LOWEST WATER LEVEL, 85.15 FT(25.95 M) BELOW LAND-SURFACE DATUM, JAN 15, 1969.

WATER LEVELS IN FEET BELOW LAND SURFACE DATUM, CLIMATIC YEARS APRIL 1981 TO MARCH 1983
05N-17E-01 ADA 1.

09'49", HYDROLOGIC UNIT 11100102.
WATER LEVELS IN FEET BELON LAND SURFACE DATUR

$\begin{array}{lll} & \text { WATER } & \text { WATER } \\ \text { DATE } & \text { LEVEL } & \text { DATE }\end{array}$
JAN 19, $1982 \quad 82.00 \quad$ JAN $11,1983 \quad 83.27$

GROUND- WATER LEVELS

365437101130301. LOCAL NUMBER; 05N-17E-09 DDA 1.

LOCATION,--LAT 36 54'37", LONG 101 13'03", HYDROLOGIC UNIT 11100102.

AQUIFER .--OGALLALA.

PERIOD OF RECORD.--1966 TO CURRENT YEAR.

EXTREMES FOR PERIOD OF RECORD.--HIGHEST WATER LEVEL, 107.13 FT(32.65 M) BELOW LAND-SURFACE DATUM, JAN 16, 1976; LOWEST WATER LEVEL,117.10 FT(35.69 M) BELOW LAND-SURFACE DATUM, JAN 19, 1972 .

WATER LEVELS IN FEET BELOW LAND SURFACE DATUM, CLIMATIC YEARS APRIL 1981 TO MARCH 1983

$\begin{array}{lll} & \text { WATER } & \text { WATER } \\ \text { DATE } & \text { LEVEL } & \text { DATE }\end{array}$

JAN 20, $1982 \quad 113.83 \quad$ JAN 11, $1983 \quad 115.04$ 
TEXAS COUNTY -- CONTINUED

365427101111501. LOCAL NUMBER; LOCATION,--LAT 36 54'27", LONG 101 AQUIFER . - OGALLALA.

PERIOD OF RECORD.--1966 TO CURRENT YEAR.

EXTREMES FOR PERIOD OF RECORD.--HIGHEST WATER LEVEL, 102.26 FT(31.17 M) BELOW LAND-SURFACE DATUM, JAN 23, 1975; LOWEST WATER LEVEL,112.54 FT(34.30 M) BELOW LAND-SURFACE DATUM, JAN $19,1982$.

WATER LEVELS IN FEET BELOW LAND SURFACE DATUM, CLIMATIC YEARS APRIL 1981 TO MARCH 1983

$\begin{array}{cccr}\text { WATE } & \begin{array}{c}\text { WATER } \\ \text { LEVEL }\end{array} & \text { DATE } & \text { WATER } \\ \text { LEVEL }\end{array}$

\section{GROUND-WATER LEVELS}

365358101145301. LOCAL NUMBER;

05N-17E-17 CBA 1. LOCATION,--LAT 36 53'58", LONG 101 14'53", HYDROLOGIC UNIT 11100102. AQUIFER .--OGALLALA.

PERIOD OF RECORD.--1966 TO CURRENT YEAR.

EXTREMES FOR PERIOD OF RECORD.--HIGHEST WATER LEVEL, 124.92 FT(38.08 M) BELOW LAND-SURFACE DATUM,JAN 23, 1975; LOWEST WATER LEVEL,136.10 FT(41.48 M) BELOW LAND-SURFACE DATUM, JAN 14, 1971.

WATER LEVELS IN FEET BELOW LAND SURFACE DATUM, CLIMATIC YEARS APRIL 1981 TO MARCH 1983

\begin{tabular}{llcc}
\multicolumn{1}{c}{} & WATER & & WATER \\
DATE & LEVEL & DATE & LEVEL \\
LAN 19, 1982 & 126.39 & JAN 11, 1983 & 127.51
\end{tabular}


TEXAS COUNTY -- CONTINUED

365234101122001 . LOCAL NUMBER;

$05 \mathrm{~N}-17 \mathrm{E}-27$ ABC 1.

LOCATION, --LAT 36 52'34", LONG 101

AQUIFER.--OGALLALA.

PERIOD OF RECORD.--1966 TO CURRENT YEAR.

EXTREMES FOR PERIOD OF RECORD.--HIGHEST WATER LEVEL, $112.85 \mathrm{FT}(34.40 \mathrm{M})$ BELOW LAND-SURFACE DATUM,FEB 24, 1966; LOWEST WATER LEVEL, 126.79 FT(38.65 M) BELOW LAND-SURFACE DATUM, JAN 11, 1983.

WATER LEVELS IN FEET BELOW LAND SURFACE DATUM, CLIMATIC YEARS APRIL 1981 TO MARCH 1983

$\begin{array}{lll} & \text { WATER } & \text { WATER } \\ \text { DATE } & \text { LEVEL } & \text { DATE }\end{array}$

JAN $19,1982 \quad 124.70 \quad$ JAN $11,1983 \quad 126.79$

GROUND-WATER LEVELS

365541101044901. LOCAL NUMBER; 05N-18E-02 DBB 1.

LOCATION,--LAT 36 55'41", LONG 101 04'49", HYDROLOGIC UNIT 11100102.

AQUIFER .--OGALLALA.

PERIOD OF RECORD.--1966 TO CURRENT YEAR.

EXTREMES FOR PERIOD OF RECORD.--HIGHEST WATER LEVEL, $53.00 \mathrm{FT}(16.15 \mathrm{M})$ BELOW LAND-SURFACE DATUM,FEB 24, 1966; LOWEST WATER LEVEL, 149.65 FT (45.61 M) BELOW LAND-SURFACE DATUM, JAN 12, 1983.

WATER LEVELS IN FEET BELOW LAND SURFACE DATUM, CLIMATIC YEARS APRIL 1981 TO MARCH 1983

$\begin{array}{ccccc} & \text { WATER } & & & \text { WATER } \\ \text { DATE } & \text { LEVEL } & \text { DATE } & \text { LEVEL } \\ \text { LAN 19, 1982 } & & \text { Z } & \text { JAN 12, 1983 } & 149.65\end{array}$


TEXAS COUNTY -- CONTINUED

365539101002301. LOCAL NUMBER; LOCATION,--LAT 36 55'39", LONG 101 AQUIFER ._-OGALLALA.

PERIOD OF RECORD.--1966 TO CURRENT YEAR.

EXTREMES FOR PERIOD OF RECORD.--HIGHEST WATER LEVEL, 86.20 FT(26.27 M) BELOW LAND-SURFACE DATUM,FEB 25, 1966; LOWEST WATER LEVEL, $116.81 \mathrm{FT}(35.60 \mathrm{M})$ BELOW LAND-SURFACE DATUM, JAN 15, 1969.

WATER LEVELS IN FEET BELOW LAND SURFACE DATUM, CLIMATIC YEARS APRIL 1981 TO MARCH 1983

$\begin{array}{crcc} & \text { WATER } & & \text { WATER } \\ \text { DATE } & \text { LEVEL } & \text { DATE } & \text { LEVEL } \\ \text { LN 19, 1982 } & 92.35 & \text { JAN 12, 1983 } & 100.10\end{array}$

GROUND-WATER LEVELS

365513101023601. LOCAL NUMBER; OSN-19E-07 ABB 1.

LOCATION,--LAT 36 55'13", LONG 101 02'36", HYDROLOGIC UNIT 11100102.

AQUIFER.--OGALLALA.

PERIOD OF RECORD .--1980 TO CURRENT YEAR.

EXTREMES FOR PERIOD OF RECORD.--HIGHEST WATER LEVEL, 140.57 FT(42.85 M) BELOW LAND-SURFACE DATUM, MAY 7, 1980; LOWEST WATER LEVEL, $185.50 \mathrm{FT}(56.54 \mathrm{M})$ BELOW LAND-SURFACE DATUM, JAN 12, 1983.

WATER LEVELS IN FEET BELOW LAND SURFACE DATUM, CLIMATIC YEARS APRIL 1981 TO MARCH 1983

\begin{tabular}{|c|c|}
\hline DATE & $\begin{array}{l}\text { WATER } \\
\text { LEVEL }\end{array}$ \\
\hline
\end{tabular}

JAN 19, $1982148.89 \quad$ JAN $12,1983 \quad 185.50$ 
GROUND-WATER LEVELS

TEXAS COUNTY -- CONTINUED

365421100571201. LOCAL NUMBER; $05 \mathrm{~N}-19 \mathrm{E}-13$ ABB 1.

LOCATION,--LAT 36 54'21", LONG 100 57'12", HYDROLOGIC UNIT 11100102.

AQUIFER.--OGALLALA.

PERIOD OF RECORD.--1980 TO CURRENT YEAR.

EXTREMES FOR PERIOD OF RECORD.--HIGHEST WATER LEVEL, 100.10 FT(30.51 M) BELOW LAND-SURFACE DATUM, MAY 6, 1980; LOWEST WATER LEVEL, 111.80 FT(34.08 M) BELOW LAND-SURFACE DATUM, JAN 12, 1983.

WATER LEVELS IN FEET BELOW LAND SURFACE DATUM, CLIMATIC YEARS APRIL 1981 TO MARCH 1983

\begin{tabular}{|c|c|}
\hline & WATER \\
\hline DATE & LEVEL \\
\hline
\end{tabular}

JAN 20, $1982104.50 \quad$ JAN $12,1983 \quad 111.80$

GROUND-WATER LEVELS

365208101010601. LOCAL NUMBER; O5N-19E-29 DAA 1. LOCATION,--LAT $3652^{\prime} 08^{\prime \prime}$, LONG 101 01'06", HYDROLOGIC UNIT 11100102. AQUIFER.--OGALLALA.

PERIOD OF RECORD.--1970 TO CURRENT YEAR.

EXTREMES FOR PERIOD OF RECORD.--HIGHEST WATER LEVEL, 81.96 FT(24.98 M) BELOW LAND-SURFACE DATUM, JAN 17, 1974; LOWEST WATER LEVEL, 146.37 FT(44.61 M) BELOW LAND-SURFACE DATUM, JAN 13, 1977.

WATER LEVELS IN FEET BELOW LAND SURFACE DATUM, CLIMATIC YEARS APRIL 1981 TO MARCH 1983

$\begin{array}{lll} & \text { WATER } & \text { WATER } \\ \text { DATE } & \text { LEVEL } & \text { DATE }\end{array}$

JAN 20, $198296.07 \quad$ JAN 12, $1983 \quad 100.86$ 
TEXAS COUNTY -- CONTINUED

365934102014401. LOCAL NUMBER; O6N-10E-18 BBB 1. LOCATION,--LAT 36 59'34", LONG 102 01'44", HYDROLOGIC UNIT 11040002.

AQUIFER.--OGALLALA.

PLRIOD OF RECORD.--1970 TO CURRENT YEAR.

EXTREMES FOR PERIOD OF RECORD.--HIGHEST WATER LEVEL, 191.86 FT(58.48 M) BELOW LAND-SURFACE DATUM, NOV 24, 1970; LOWEST WATER LEVEL,205.28 FT(62.57 M) BELOW LAND-SURFACE DATUM, JAN 13, 1977.

WATER LEVELS IN FEET BELOW LAND SURFACE DATUM, CLIMATIC YEARS APRIL 1981 TO MARCH 1983

$\begin{array}{lll} & \text { WATER } & \text { WATER } \\ \text { DATE } & \text { LEVEL } & \text { DATE }\end{array}$

JAN 21, $1982 \quad 200.15 \quad$ JAN $10,1983 \quad 201.10$

GROUND-WATER LEVELS

365710102013901. LOCAL NUMBER; 06N-10E-30 CCC 1. LOCATION,--LAT 36 57'10", LONG 102 01'39", HYDROLOGIC UNIT 11100101. AQUIFER.--OGALLALA.

PERIOD OF RECORD.--1966 TO CURRENT YEAR.

EXTREMES FOR PERIOD OF RECORD.--HIGHEST WATER LEVEL,222.30 FT(67.76 M) BELOW LAND-SURFACE DATUM,FEB 27, 1966; LOWEST WATER LEVEL,239.51 FT(73.00 M) BELOW LAND-SURFACE DATUM, JAN 13, 1977.

WATER LEVELS IN FEET BELOW LAND SURFACE DATUM, CLIMATIC YEARS APRIL 1981 TO MARCH 1983

$\begin{array}{lll} & \text { WATER } & \text { WATER } \\ \text { DATE } & \text { LEVEL } & \text { DATE }\end{array}$

JAN 21, $1982 \quad P \quad$ JAN $10,1983 \quad 226.91$ 
GROUND-WATER LEVELS

TEXAS COUNTY -- CONTINUED

$365636101554301 . \quad$ LOCAL NUMBER; 06N-10E-36 DBB 1. LOCATION,--LAT 36 56'36", LONG 101 55'43", HYDROLOGIC UNIT 11100101. AQUIFER.--OGALLALA. PERIOD OF RECORD.--1966 TO CURRENT YEAR. EXTREMES FOR PERIOD OF RECORD.--HIGHEST WATER LEVEL,260.10 FT(79.28 M) BELOW LAND-SURFACE DATUM,FEB 27, 1966; LOWEST WATER LEVEL,316.95 FT(96.61 M) BELOW LAND-SURFACE DATUM, JAN 21, 1982 .

WATER LEVELS IN FEET BELOW LAND SURFACE DATUM, CLIMATIC YEARS APRIL 1981 TO MARCH 1983

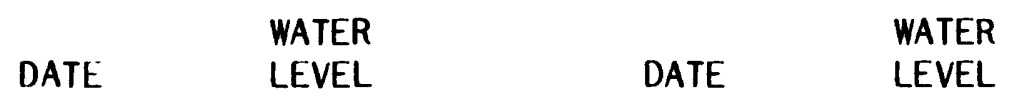

JAN 21, $1982 \quad 316.95 \quad$ JAN $10,1983 \quad 307.84$

GROUND-WATER LEVELS

365903101540201. LOCAL NUMBER; 06N-11E-17 CBC 1.

LOCATION,--LAT 36 59'03", LONG 101 54'02", HYDROLOGIC UNIT 11040006.

AQUIFER .--OGALLALA.

PERIOD OF RECORD.--1967 TO CURRENT YEAR.

EXTREMES FOR PERIOD OF RECORD.--HIGHEST WATER LEVEL,249.65 FT(76.09 M) BELOW LAND-SURFACE DATUM,JAN 29, 1967; LOWEST WATER LEVEL,299.07 FT(91.16 M) BELOW LAND-SURFACE DATUM, JAN 10, 1983.

WATER LEVELS IN FEET BELOW LAND SURFACE DATUM, CLIMATIC YEARS APRIL 1981 TO MARCH 1983

\begin{tabular}{|c|c|c|}
\hline DATE & $\begin{array}{l}\text { WATER } \\
\text { LEVEL }\end{array}$ & DATE \\
\hline
\end{tabular}

JAN 21, $1982 \quad 291.54 \quad$ JAN $10,1983 \quad 299.07$ 
TEXAS COUNTY -- CONTINUED

365731101493901. LOCAL NUMBER; LOCATION,--LAT 36 57'31", LONG 101 AQUIFER.--OGALLALA.

PERIOD OF RECORD.--1966 TO CURRENT YEAR.

EXTREMES FOR PERIOD OF RECORD.--HIGHEST WATER LEVEL,209.00 FT(63.70 M) BELOW LAND-SURFACE DATUM,FEB 27, 1966; LOWEST WATER LEVEL,306.90 FT(93.54 M) BELOW LAND-SURFACE DATUM, JAN $21,1982$.

WATER LEVELS IN FEET BELOW LAND SURFACE DATUM, CLIMATIC YEARS APRIL 1981 TO MARCH 1983
06N-11E-25 BCC 1.

49'39", HYDROLOGIC UNIT 11100101.

$\begin{array}{lll} & & \\ \text { DATE } & \text { WATER } & \text { WATER } \\ \text { LEVEL } & \text { DATE } & \text { LEVEL }\end{array}$

JAN 21, $1982 \quad 306.90 \quad$ JAN 10, $1983 \quad 285.20$

GROUND-WATER LEVELS

365739101504801. LOCAL NUMBER; O6N-11E-26 BCB 2.

LOCATION,--LAT 36 57'39", LONG 101 50'48", HYDROLOGIC UNIT 11100101.

AQUIFER .--OGALLALA.

PERIOD OF RECORD.--1966 TO CURRENT YEAR.

EXTREMES FOR PERIOD OF RECORD.--HIGHEST WATER LEVEL, 210.40 FT(64.13 M) BELOW LAND-SURFACE DATUM,FEB 27, 1966; LOWEST WATER LEVEL,286.02 FT(87.18 M) BELOW LAND-SURFACE DATUM, JAN $16,1979$.

WATER LEVELS IN FEET BELOW LAND SURFACE DATUM, CLIMATIC YEARS APRIL 1981 TO MARCH 1983

$\begin{array}{lll} & \text { WATER } & \text { WATER } \\ \text { DATE } & \text { LEVEL } & \text { DATE } \\ \text { LEVEL }\end{array}$

JAN 21, $1982 \quad 282.98 \quad$ JAN 10, $1983 \quad 282.98$ 
TEXAS COUNTY -- CONTINUED

365802101462501. LOCAL NUMBER; LOCATION,--LAT $3658^{\prime} 02^{\prime \prime}$, LONG 101 AQUIFER . - OGALLALA.

PERIOD OF RECORD.--1966 TO CURRENT YEAR.

EXTREMES FOR PERIOD OF RECORD.--HIGHEST WATER LEVEL,217.00 FT(66.14 M) BELOW LAND-SURFACE DATUM,FEB 26, 1966; LOWEST WATER LEVEL,272.20 FT(82.97 M) BELOW LAND-SURFACE DATUM, JAN $13,1977$.

WATER LEVELS IN FEET BELOW LAND SURFACE DATUM, CLIMATIC YEARS APRIL 1981 TO MARCH 1983

$06 \mathrm{~N}-12 \mathrm{E}-21 \mathrm{CCC} 1$

46'25", HYDROLOGIC UNIT 11100102.
DATE
WATER
DATE
WATER
JAN $21,1982 \quad 252.49$
JAN 10,1983
0

\section{GROUND-WATER LEVELS}

365614101431001. LOCAL NUMBER; 06N-12E-36 CCC 1. LOCATION,--LAT $3656^{\prime} 14^{\prime \prime}$, LONG 101 43'10", HYDROLOGIC UNIT 11100101. AQUIFER.--OGALLALA. PERIOD OF RECORD.--1966 TO CURRENT YEAR. EXTREMES FOR PERIOD OF RECORD.--HIGHEST WATER LEVEL, 204.70 FT(62.39 M) BELOW LAND-SURFACE DATUM, FEB 26, 1966; LOWEST WATER LEVEL,276.03 FT(84.13 M) BELOW LAND-SURFACE DATUM, JAN $8,1981$.

WATER LEVELS IN FEET BELOW LAND SURFACE DATUM, CLIMATIC YEARS APRIL 1981 TO MARCH 1983

\begin{tabular}{|c|c|c|}
\hline DATE & $\begin{array}{l}\text { WATER } \\
\text { LEVEL }\end{array}$ & DATE \\
\hline
\end{tabular}

JAN 20, $1982 \quad 0 \quad$ JAN $10,1983 \quad 0$


TEXAS COUNTY -- CONTINUED

365918101392301. LOCAL NUMBER; 06N-13E-16 ACC 1. LOCATION,--LAT 36 59'18", LONG 101 39'23", HYDROLOGIC UNIT 11040006. AQUIFER .--OGALLALA.

PERIOD OF RECORD.--1966 TO CURRENT YEAR. EXTREMES FOR PERIOD OF RECORD.--HIGHEST WATER LEVEL,235.10 FT(71.66 M) BELOW LAND-SURFACE DATUM,FEB 26, 1966; LOWEST WATER LEVEL, 309.02 FT(94.19 M) BELOW LAND-SURFACE DATUM, JAN $10,1980$.

WATER LEVELS IN FEET BELOW LAND SURFACE DATUM, CLIMATIC YEARS APRIL 1981 TO MARCH 1983

\begin{tabular}{|c|c|c|}
\hline DATE & $\begin{array}{l}\text { WATER } \\
\text { LEVEL }\end{array}$ & DATE \\
\hline
\end{tabular}

JAN 20, $1982 \quad 281.56 \quad$ JAN 11, $1983 \quad 0$

GROUND-WATER LEVELS

365810101400201. LOCAL NUMBER; 06N-13E-20 DDA 1. LOCATION,--LAT 36 58'10", LONG 101 40'02", HYDROLOGIC UNIT 11100102. AQUIFER . - OGALLALA. PERIOD OF RECORD.--1966 TO CURRENT YEAR. EXTREMES FOR PERIOD OF RECORD.--HIGHEST WATER LEVEL, $238.00 \mathrm{FT}(72.54 \mathrm{M})$ BELOW LAND-SURFACE DATUM,FEB 26, 1966; LOWEST WATER' LEVEL,336.65 FT(102.61 M) BELOW LAND-SURFACE DATUM, JAN 11, 1983.

WATER LEVELS IN FEET BELOW LAND SURFACE DATUM, CLIMATIC YEARS APRIL 1981 TO MARCH 1983

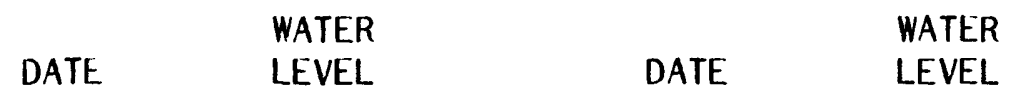

JAN 20, $1982 \quad 334.33 \quad$ JAN 11, $1983 \quad 336.65$ 
GROUND-WATER LEVELS

TEXAS COUNTY -- CONTINUED

365730101420601. LOCAL NUMBER; 06N-13E-30 CBB 1.

LOCATION,--LAT $3657^{\prime} 30^{\prime \prime}$, LONG 101 42'06", HYDROLOGIC UNIT 11100102.

AQUIFER.--OGALLALA.

PERIOD OF RECORD.--1966 TO CURRENT YEAR.

EXTREMES FOR PERIOD OF RECORD.--HIGHEST WATER LEVEL, $221.50 \mathrm{FT}(67.51 \mathrm{M})$ BELOW LAND-SURFACE DATUM,FEB 26, 1966; LOWEST WATER LEVEL,295.47 FT(90.06 M) BELOW LAND-SURFACE DATUM, JAN 20, 1982.

WATER LEVELS IN FEET BELOW LAND SURFACE DATUM, CLIMATIC YEARS APRIL 1981 TO MARCH 1983

$\begin{array}{lll} & \text { WATER } & \text { WATER } \\ \text { DATE } & \text { LEVEL } & \text { DATE }\end{array}$

JAN 20, $1982 \quad 295.47 \quad$ JAN 11, $1983 \quad 291.54$

GROUND-WATER LEVELS

365825101330001. LOCAL NUMBER; 06N-14E-21 ACB 1. LOCATION,--LAT $3658^{\prime 25 ", ~ L O N G ~} 101$ 33'00", HYDROLOGIC UNIT 11100102.

AQUIFER .--OGALLALA.

PERIOD OF RECORD.--1966 TO CURRENT YEAR.

EXTREMES FOR PERIOD OF RECORD.--HIGHEST WATER LEVEL,213.70 FT(65.14 M) BELOW LAND-SURFACE DATUM,FEB 25, 1966; LOWEST WATER LEVEL,241.36 FT(73.57 M) BELOW LAND-SURFACE DATUM, JAN 24, 1973.

WATER LEVELS IN FEET BELOW LAND SURFACE DATUM, CLIMATIC YEARS APRIL 1981 TO MARCH 1983

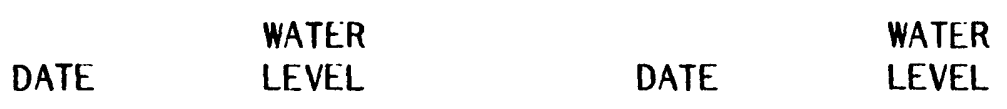

JAN 20, $1982 \quad 239.88 \quad$ JAN $11,1983 \quad 237.35$ 
GROUND-WATER LEVELS

TEXAS COUNTY -- CONTINUED

365630101323601. LOCAL NUMBER; 06N-14E-33 DAB 1. LOCATION,--LAT 36 56'30", LONG 101 32'36", HYDROLOGIC UNIT 11100102.

AQUIFER .- -OGALLALA.

PERIOD OF RECORD.--1966 TO CURRENT YEAR.

EXTREMES FOR PERIOD OF RECORD.--HIGHEST WATER LEVEL,226.00 FT(68.88 M) BELOW LAND-SURFACE DATUM,FEB 25, 1966; LOWEST WATER LEVEL, 322.18 FT(98.20 M) BELOW LAND-SURFACE DATUM, JAN 20, 1982.

WATER LEVELS IN FEET BELOW LAND SURFACE DATUM, CLIMATIC YEARS APRIL 1981 TO MARCH 1983

$\begin{array}{lll} & \text { WATER } & \\ \text { DATE } & \text { WEVEL } & \text { DATER } \\ \text { LEVEL }\end{array}$

JAN 20, $1982 \quad 322.18 \quad$ JAN 11, $1983 \quad 0$

\section{GROUND-WATER LEVELS}

365633101311501. LOCAL NUMBER; 06N-14E-35 CBB 1. LOCATION,--LAT 36 56'33", LONG 101 31'15", HYDROLOGIC UNIT 11100102. AQUIFER . - OGALLALA.

PERIOD OF RECORD.--1966 TO CURRENT YEAR.

EXTREMES FOR PERIOD OF RECORD.--HIGHEST WATER LEVEL, 192.80 FT(58.77 M) BELOW LAND-SURFACE DATUM,FEB 25, 1966; LOWEST WATER LEVEL,250.51 FT(76.36 M) BELOW LAND-SURFACE DATUM, JAN $11,1977$.

WATER LEVELS IN FEET BELOW LAND SURFACE DATUM, CLIMATIC YEARS APRIL 1981 TO MARCH 1983

$\begin{array}{lll} & \text { WATER } & \\ \text { DATE } & \text { WEVEL } & \text { WATER } \\ \text { LEVEL }\end{array}$

JAN 20, 1982

$0 \quad$ JAN 11,1983

0 
GROUND-WATER LEVELS

TEXAS COUNTY -- CONTINUED

365735101283501. LOCAL NUMBER;

$06 \mathrm{~N}-15 \mathrm{E}-30$ ACB 1.

LOCATION,--LAT 36 57'35", LONG 101 28'35", HYDROLOGIC UNIT 11100102.

AQUIFER.--OGALLALA.

PERIOD OF RECORD.--1966 TO CURRENT YEAR.

EXTREMES FOR PERIOD OF RECORD.--HIGHEST WATER LEVEL, $173.13 \mathrm{FT}(52.77 \mathrm{M})$ BELOW LAND-SURFACE DATUM,FEB 24, 1966; LOWEST WATER LEVEL,223.13 FT(68.01 M) BELOW LAND-SURFACE DATUM, JAN 11, 1983.

WATER LEVELS IN FEET BELOW LAND SURFACE DATUM, CLIMATIC YEARS APRIL 1981 TO MARCH 1983

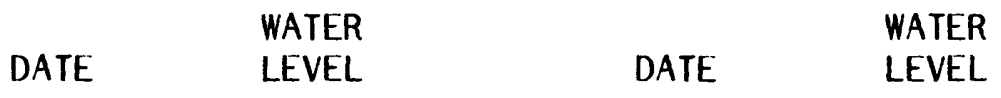

JAN 20, $1982 \quad 219.56 \quad$ JAN 11, $1983 \quad 223.13$

GROUND-WATER LEVELS

365649101270001. LOCAL NUMBER; 06N-15E-33 BBC 1. LOCATION,--LAT 36 56'49", LONG 101 27'00", HYDROLOGIC UNIT 11100102.

AQUIFER.--OGALLALA.

PERIOD OF RECORD.--1980 TO CURRENT YEAR.

EXTREMES FOR PERIOD OF RECORD.--HIGHEST WATER LEVEL, 176.10 FT(53.68 M) BELOW LAND-SURFACE DATUM, APR 23, 1980; LOWEST WATER LEVEL, 183.63 FT(55.97 M) BELOW LAND-SURFACE DATUM, JAN 20, 1982 .

WATER LEVELS IN FEET BELOW LAND SURFACE DATUM, CLIMATIC YEARS APRIL 1981 TO MARCH 1983

$\begin{array}{lll} & \text { WATER } & \text { WATER } \\ \text { DATE } & \text { LEVEL } & \text { DATE }\end{array}$

JAN 20, $1982183.63 \quad$ JAN 11, 1983 
TEXAS COUNTY -- CONTINUED

365750101195501 . LOCAL NUMBER; LOCATION, --LAT 36 57'50", LONG 101 AQUIFER.--OGALLALA. PERIOD OF RECORD.--1970 TO CURRENT YEAR. EXTREMES FOR PERIOD OF RECORD.---HIGHEST WATER LEVEL, 82.55 FT(25.16 M) BELOW LAND-SURFACE DATUM, JAN 17, 1974; LOWEST WATER LEVEL, 102.51 FT(31.25 M) BELOW LAND-SURFACE DATUM, JAN 11, 1983.

WATER LEVELS IN FEET BELOW LAND SURFACE DATUM, CLIMATIC YEARS APRIL 1981 TO MARCH 1983

$06 \mathrm{~N}-16 \mathrm{E}-27$ ACC 1.

19'55", HYDROLOGIC UNIT 11100102 .
JAN 21, 1982100.67

$\begin{array}{cccc}\text { DATE } & \begin{array}{c}\text { WATER } \\ \text { LEVEL }\end{array} & \text { DATE } & \begin{array}{c}\text { WATER } \\ \text { LEVEL }\end{array} \\ \text { JAN 21, 1982 } & 100.67 & \text { JAN 11, 1983 } & 102.51\end{array}$

GROUND-WATER LEVELS

365606101112001. LOCAL NUMBER; O6N-17E-14 DBB 1. LOCATION,--LAT 36 56'06", LONG 101 11'20", HYDROLOGIC UNIT 11100102. AQUIFER.--OGALLALA. PERIOD OF RECORD.--1966 TO CURRENT YEAR. EXTREMES FOR PERIOD OF RECORD.--HIGHEST WATER LEVEL, 116.44 FT(35.49 M) BELOW LAND-SURFACE DATUM, JAN 13, 1977; LOWEST WATER LEVEL,133.87 FT(40.80 M) BELOW LAND-SURFACE DATUM, JAN 17, 1979.

WATER LEVELS IN FEET BELOW LAND SURFACE DATUM, CLIMATIC YEARS APRIL 1981 TO MARCH 1983

$\begin{array}{cccc}\text { DATE } & \begin{array}{c}\text { WATER } \\ \text { LEVEL }\end{array} & \text { DATE } & \begin{array}{c}\text { WATER } \\ \text { LEVEL }\end{array} \\ \text { JAN 19, 1982 } & 128.57 & \text { JAN 11, 1983 } & 127.81\end{array}$




\section{GROUND-WATER LEVELS}

TEXAS COUNTY -- CONTINUED

365809101114701 . LOCAL NUMBER;

$06 \mathrm{~N}-17 \mathrm{E}-23 \mathrm{CBC} 1$.

LOCATION,--LAT $3658^{\prime} 09^{\prime \prime}$, LONG 101

$11^{\prime} 47$ ", HYDROLOGIC UNIT 11100102.

AQUIFER .--OGALLALA.

PERIOD OF RECORD.--1966 TO CURRENT YEAR.

EXTREMES FOR PERIOD OF RECORD.--HIGHEST WATER LEVEL,98.02 FT(29.88 M) BELOW LAND-SURFACE DATUM,JAN 16, 1976; LOWEST WATER LEVEL, $108.94 \mathrm{FT}(33.20 \mathrm{M})$ BELOW LAND-SURFACE DATUM, JAN 19, 1982.

WATER LEVELS IN FEET BELOW LAND SURFACE DATUM, CLIMATIC YEARS APRIL 1981 T0. MARCH 1983

$\begin{array}{lll} & \text { WATER } & \text { WATER } \\ \text { DATE } & \text { LEVEL } & \text { DATE }\end{array}$

JAN 19, $1982108.94 \quad$ JAN $11,1983 \quad 107.11$

GROUND-WATER LEVELS

365706101134001. LOCAL NUMBER; 06N-17E-28 CCD 1.

LOCATION,--LAT 36 57'06", LONG 101 13'40", HYDROLOGIC UNIT 11100102.

AQUIFER .--OGALLALA.

PERIOD OF RECORD.--1966 TO CURRENT YEAR.

EXTREMES FOR PERIOD OF RECORD.--HIGHEST WATER LEVEL, 102.37 FT(31.20 M) BELOW LAND-SURFACE DATUM, JAN 17, 1974; LOWEST WATER LEVEL, 119.69 FT(36.48 M) BELOW LAND-SURFACE DATUM, JAN 11, 1983.

WATER LEVELS IN FEET BELOW LAND SURFACE DATUM, CLIMATIC YEARS APRIL 1981 TO MARCH 1983

$\begin{array}{lll} & \text { WATER } & \text { WATER } \\ \text { DATE } & \text { LEVEL } & \text { DATE }\end{array}$

JAN 19, 1982118.39 JAN 11, $1983 \quad 119.69$ 
TEXAS COUNTY -- CONTINUED

365616101115701. LOCAL NUMBER;

06N-17E-34 DDD 1. LOCATION,--LAT 36 56'16", LONG 101 11 '57", HYDROLOGIC UNIT 11100102. AQUIFER.--OGALLALA. PERIOD OF RECORD.--1966 TO CURRENT YEAR. EXTREMES FOR PERIOD OF RECORD.--HIGHEST WATER LEVEL,89.76 FT(27.36 M) BELOW LAND-SURFACE DATUM,JAN 23, 1975; LOWEST WATER LEVEL,97.55 FT(29.73 M) BELOW LAND-SURFACE DATUM, JAN 11, 1983.

WATER LEVELS IN FEET BELOW LAND SURFACE DATUM, CLIMATIC YEARS APRIL 1981 T0 MARCH 1983

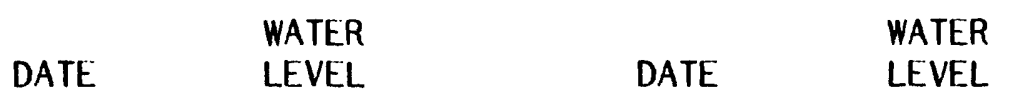

JAN 19, $1982 \quad P \quad$ JAN $11,1983 \quad 97.55$

GROUND-WATER LEVELS

365704101070301. LOCAL NUMBER; 06N-18E-28 CDD 1.

LOCATION,--LAT 36 57'04", LONG 101 07'03", HYDROLOGIC UNIT 11100102.

AQUIFER .--OGALLLALA.

PERIOD OF RECORD.--1966 TO CURRENT YEAR.

EXTREMES FOR PERIOD OF RECORD.--HIGHEST WATER LEVEL,73.18 FT(22.31 M) BELOW LAND-SURFACE DATUM, JAN 17, 1974; LOWEST WATER LEVEL,83.78 FT(25.54 M) BELOW LAND-SURFACE DATUM, JAN 11, 1983.

WATER LEVELS IN FEET BELOW LAND SURFACE DATUM, CLIMATIC YEARS APRIL 1981 TO MARCH 1983

\begin{tabular}{|c|c|}
\hline \\
\hline DATE & LEVEL \\
\hline
\end{tabular}

JAN 19, $1982 \quad 82.00 \quad$ JAN 11, $1983 \quad 83.78$ 
TEXAS COUNTY -- CONTINUED

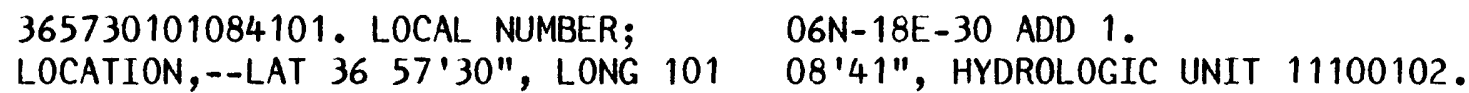

AQUIFER.--OGALLALA.

PERIOD OF RECORD.--1966 TO CURRENT YEAR.

EXTREMES FOR PERIOD OF RECORD.--HIGHEST WATER LEVEL, $84.90 \mathrm{FT}(25.88 \mathrm{M})$ BELOW LAND-SURFACE DATUM,FEB 24, 1966; LOWEST WATER LEVEL,95.51 FT(29.11 M) BELOW LAND-SURFACE DATUM, JAN 11, 1983.

WATER LEVELS IN FEET BELOW LAND SURFACE DATUM, CLIMATIC YEARS APRIL 1981 TO MARCH 1983

$\begin{array}{lll} & \text { WATER } & \text { WATER } \\ \text { DATE } & \text { LEVEL } & \text { DATE }\end{array}$

JAN 19, $1982 \quad 91.79 \quad$ JAN 11, $1983 \quad 95.51$

GROUND-WATER LEVELS

365857100595601. LOCAL NUMBER; O6N-19E-15 CCC 1.

LOCATION,--LAT 36 58'57", LONG 100 59'56", HYDROLOGIC UNIT 11100102.

AQUIFER.--OGALLALA.

PERIOD OF RECORD.--1966 TO CURRENT YEAR.

EXTREMES FOR PERIOD OF RECORD.--HIGHEST WATER LEVEL, 83.72 FT(25.52 M) BELOW LAND-SURFACE DATUM, JAN 23, 1975; LOWEST WATER LEVEL,97.70 FT(29.78 M) BELOW LAND-SURFACE DATUM, JAN 5, 1981.

WATER LEVELS IN FEET BELOW LAND SURFACE DATUM, CLIMATIC YEARS APRIL 1981 TO MARCH 1983

$\begin{array}{lll} & \text { WATER } & \text { WATER } \\ \text { DATE } & \text { LEVEL } & \text { DATE }\end{array}$

JAN 19, $198295.22 \quad$ JAN 12, $1983 \quad 96.58$ 
GROUND-WATER LEVELS

TEXAS COUNTY -- CONTINUED

363002101573401 . LOCAL NUMBER; LOCATION,--LAT $3630^{\prime} 02^{\prime \prime}$, LONG 101 AQUIFER.--OGALLALA.

PERIOD OF RECORD.--1966 TO CURRENT YEAR.

EXTREMES FOR PERIOD OF RECORD.--HIGHEST WATER LEVEL, 133.10 FT(40.57 M) BELOW LAND-SURFACE DATUM, JAN 27, 1967; LOWEST WATER LEVEL, 182.00 FT(55.47 M) BELOW LAND-SURFACE DATUM, JAN 18, 1982.

WATER LEVELS IN FEET BELOW LAND SURFACE DATUM, CLIMATIC YEARS APRIL 1981 TO MARCH 1983

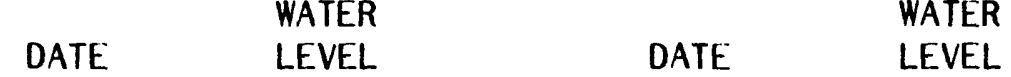

JAN $18,1982 \quad 182.00 \quad$ JAN 11, $1983 \quad$ Z

GROUND-WATER LEVELS

363003101220301. LOCAL NUMBER; 01S-16E-06 AAA 1. LOCATION,--LAT 36 30'03", LONG 101 22'03", HYDROLOGIC UNIT 11100103. AQUIFER.--OGALLALA.

PERIOD OF RECORD.--1966 TO CURRENT YEAR.

EXTREMES FOR PERIOD OF RECORD.--HIGHEST WATER LEVEL,214.80 FT(65.47 M) BELOW LAND-SURFACE DATUM,FEB 25, 1966; LOWEST WATER LEVEL,252.28 FT(76.89 M) BELOW LAND-SURFACE DATUM, JAN 7, 1981.

WATER LEVELS IN FEET BELOW LAND SURFACE DATUM, CLIMATIC YEARS APRIL 1981 TO MARCH 1983

$\begin{array}{lll} & \text { WATER } & \text { WATER } \\ \text { DATE } & \text { LEVEL } & \text { DATE }\end{array}$

JAN 19, $1982 \quad 251.16 \quad$ JAN 13, $1983 \quad 249.85$ 
GROUND-WATER LEVELS

TILLMAN COUNTY

343122099013201. LOCAL NUMBER; LOCATION,--LAT 3431 '22", LONG 099 AQUIFER.--TERRACE DEPOSITS.

PERIOD OF RECORD.--1969 TO CURRENT YEAR.

EXTREMES FOR PERIOD OF RECORD.--HIGHEST WATER LEVEL, $15.05 \mathrm{FT}(4.59 \mathrm{M})$ BELOW LAND-SURFACE DATUM,MAR 8, 1977; LOWEST WATER LEVEL,20.11 FT(6.13 M) BELOW LAND-SURFACE DATUM, FEB 18, 1969.

WATER LEVELS IN FEET BELOW LAND SURFACE DATUM, CLIMATIC YEARS APRIL 1981 TO MARCH 1983

\begin{tabular}{cccc} 
DATE & $\begin{array}{c}\text { WATER } \\
\text { LEVEL }\end{array}$ & DATE & $\begin{array}{c}\text { WATER } \\
\text { LEVEL }\end{array}$ \\
\hline R 11, 1982 & 18.54 & MAR 03, 1983 & 17.99
\end{tabular}

GROUND-WATER LEVELS

343511099025101. LOCAL NUMBER; 01N-18W-01 CAB 1. LOCATION,--LAT 34 35'11", LONG 099 02'51", HYDROLOGIC UNIT 11120303. AQUIFER.--TERRACE DEPOSITS. PERIOD OF RECORD.--1969 TO CURRENT YEAR. EXTREMES FOR PERIOD OF RECORD.--HIGHEST WATER LEVEL, $12.33 \mathrm{FT}(3.76 \mathrm{M})$ BELOW LAND-SURFACE DATUM,MAR 28, 1978; LOWEST WATER LEVEL, 15.46 FT(4.71 M) BELOW LAND-SURFACE DATUM, MAR 9, 1983.

WATER LEVELS IN FEET BELOW LAND SURFACE DATUM, CLIMATIC YEARS APRIL 1981 TO MARCH 1983

$\begin{array}{lll} & \text { WATER } & \text { WATER } \\ \text { DATE } & \text { LEVEL } & \text { DATE }\end{array}$
MAR 11, 1982
0
MAR 09, $1983 \quad 15.46$ 


\section{GROUND-WATER LEVELS}

TILLMAN COUNTY -- CONTINUED

343419099041001. LOCAL NUMBER;

$01 \mathrm{~N}-18 \mathrm{~W}-11 \mathrm{CBB} 1$.

LOCATION,--LAT 34 34'19", LONG 099 AQUIFER.--TERRACE DEPOSITS.

PERIOD OF RECORD.--1977 TO CURRENT YEAR.

EXTREMES FOR PERIOD OF RECORD.--HIGHEST WATER LEVEL, 14.11 FT(4.30 M) BELOW LAND-SURFACE DATUM, MAR 9, 1977; LOWEST WATER LEVEL, 17.18 FT(5.24 M) BELOW LAND-SURFACE DATUM, MAR 3, 1983.

WATER LEVELS IN FEET BELOW LAND SURFACE DATUM, CLIMATIC YEARS APRIL 1981 TO MARCH 1983

$\begin{array}{ccccc} & \text { WATER } & & \text { WATER } \\ \text { DATE } & \text { LEVEL } & \text { DATE } & \text { LEVEL } \\ \text { L 11, } 1982 & & 0 & \text { MAR 03, 1983 } & 17.18\end{array}$

GROUND-WATER LEVELS

343313099052901. LOCAL NUMBER; 01N-18W-16 DDB 1.

LOCATION,--LAT 34 33'13", LONG 099 05'29", HYDROLOGIC UNIT 11120303.

AQUIFER.--TERRACE DEPOSITS.

PERIOD OF RECORD.--1969 TO CURRENT YEAR.

EXTREMES FOR PERIOD OF RECORD.--HIGHEST WATER LEVEL, 19.79 FT(6.03 M) BELOW LAND-SURFACE DATUM,MAR 9, 1977; LOWEST WATER LEVEL,22.95 FT(7.00 M) BELOW LAND-SURFACE DATUM, MAR 9, 1983.

WATER LEVELS IN FEET BELOW LAND SURFACE DATUM, CLIMATIC YEARS APRIL 1981 TO MARCH 1983

$\begin{array}{lll} & \text { WATER } & \text { WATER } \\ \text { DATE } & \text { LEVEL } & \text { DATE } \\ \text { LEVEL }\end{array}$

MAR $10,1982 \quad 22.28 \quad$ MAR 09, $1983 \quad 22.95$ 
GROUND-WATER LEVELS

TILLMAN COUNTY -- CONTINUED

343122099062401. LOCAL NUMBER; 01N-18W-29 DDD 1.

LOCATION,--LAT 34 31'22", LONG 099 06'24", HYDROLOGIC UNIT 11120303.

AQUIFER.--TERRACE DEPOSITS.

PERIOD OF RECORD.--1977 TO CURRENT YEAR.

EXTREMES FOR PERIOD OF RECORD.--HIGHEST WATER LEVEL, 24.22 FT(7.38 M) BELOW LAND-SURFACE DATUM, MAR 28, 1978; LOWEST WATER LEVEL,27.45 FT(8.37 M) BELOW LAND-SURFACE DATUM, MAR 10, 1982 .

WATER LEVELS IN FEET BELOW LAND SURFACE DATUM, CLIMATIC YEARS APRIL 1981 TO MARCH 1983

$\begin{array}{lll} & \text { WATER } & \text { WATER } \\ \text { DATE } & \text { LEVEL } & \text { DATE }\end{array}$

MAR $10,1982 \quad 27.45 \quad$ MAR 09, $1983 \quad 26.70$

GROUND-WATER LEVELS

343300099083801. LOCAL NUMBER; 01N-19W-24 AAB 1. LOCATION,--LAT 34 33'00", LONG 099 08'38", HYDROLOGIC UNIT 11120303.

AQUIFER.--TERRACE DEPOSITS.

PERIOD OF RECORD.--1969 TO CURRENT YEAR.

EXTREMES FOR PERIOD OF RECORD.--HIGHEST WATER LEVEL, $26.73 \mathrm{FT}(8.15 \mathrm{M})$ BELOW LAND-SURFACE DATUM, MAR 9, 1977; LOWEST WATER LEVEL,31.50 FT(9.60 M) BELOW LAND-SURFACE DATUM, MAR 28, 1978.

WATER LEVELS IN FEET BELOW LAND SURFACE DATUM, CLIMATIC YEARS APRIL 1981 TO MARCH 1983

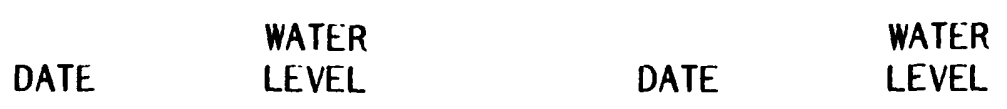

MAR $10,1982 \quad 28.18 \quad$ MAR 09, $1983 \quad 28.55$ 
342938099013401. LOCAL NUMBER; 01S-17W-06 CDD 1. LOCATION,--LAT 34 29'38", LONG 099 01'34", HYDROLOGIC UNIT 11130203. AQUIFER.--TERRACE DEPOSITS. PERIOD OF RECORD.--1969 TO CURRENT YEAR. EXTREMES FOR PERIOD OF RECORD.--HIGHEST WATER LEVEL, $15.28 \mathrm{FT}(4.66 \mathrm{M})$ BELOW LAND-SURFACE DATUM, MAR 28, 1978; LOWEST WATER LEVEL, $16.60 \mathrm{FT}(5.06 \mathrm{M})$ BELOW LAND-SURFACE DATUM, FEB 18, 1969.

WATER LEVELS IN FEET BELOW LAND SURFACE DATUM, CLIMATIC YEARS APRIL 1981 TO MARCH 1983

\begin{tabular}{|c|c|c|}
\hline & WATER & \\
\hline DATE & LEVEL & DATE \\
\hline
\end{tabular}

MAR 11, $1982 \quad 16.30 \quad$ MAR 09, $1983 \quad 16.06$

GROUND-WATER LEVELS

342938099053001. LOCAL NUMBER; 01S-18W-04 DCD 1.

LOCATION,--LAT 34 29'38", LONG 099 05'30", HYDROLOGIC UNIT 11120303.

AQUIFER.--TERRACE DEPOSITS.

PERIOD OF RECORD.--1970 TO CURRENT YEAR.

EXTREMES FOR PERIOD OF RECORD.--HIGHEST WATER LEVEL, $9.27 \mathrm{FT}(2.83 \mathrm{M})$ BELOW LAND-SURFACE DATUM,MAR 28, 1978; LOWEST WATER LEVEL, $15.63 \mathrm{FT}(4.76 \mathrm{M})$ BELOW LAND-SURFACE DATUM, JAN 21, 1970.

WATER LEVELS IN FEET BELOW LAND SURFACE DATUM, CLIMATIC YEARS APRIL 1981 TO MARCH 1983

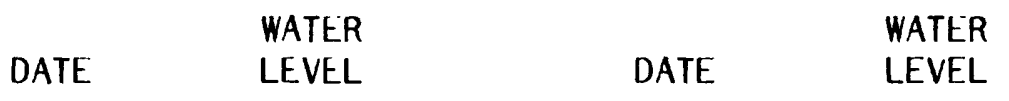

MAR $11,1982 \quad 14.87 \quad$ MAR 03, $1983 \quad 13.14$ 
GROUND-WATER LEVELS

TILLMAN COUNTY -- CONTINUED

342845099043501. LOCAL NUMBER; 01S-18W-10 DCC 1. LOCATION,--LAT 34 28'45", LONG 099 04'35", HYDROLOGIC UNIT 11120303. AQUIFER.--TERRACE DEPOSITS. PERIOD OF RECORD.-- 1970 TO CURRENT YEAR. EXTREMES FOR PERIOD OF RECORD.--HIGHEST WATER LEVEL,22.98 FT(7.00 M) BELOW LAND-SURFACE DATUM,MAR 28, 1978; LOWEST WATER LEVEL,26.87 FT(8.19 M) BELOW LAND-SURFACE DATUM, MAR 25, 1981.

WATER LEVELS IN FEET BELOW LAND SURFACE DATUM, CLIMATIC YEARS APRIL 1981 TO MARCH 1983

$\begin{array}{lll} & \text { WATER } & \text { WATER } \\ \text { DATE } & \text { LEVEL } & \text { DATE }\end{array}$

MAR $11,1982 \quad 26.40 \quad$ MAR 09, $1983 \quad 26.82$

GROUND-WATER LEVELS

342727099072101. LOCAL NUMBER; 01S-18W-19 ADD 1. LOCATION,--LAT 34 27'27", LONG 099 07'21", HYDROLOGIC UNIT 11120303. AQUIFER.--TERRACE DEPOSITS. PERIOD OF RECORD.--1970 TO CURRENT YEAR. EXTREMES FOR PERIOD OF RECORD.--HIGHEST WATER LEVEL, $12.50 \mathrm{FT}(3.81 \mathrm{M})$ BELOW LAND-SURFACE DATUM, MAR 8, 1977; LOWEST WATER LEVEL, 17.80 FT(5.43 M) BELOW LAND-SURFACE DATUM, JAN 21, 1970.

WATER LEVELS IN FEET BELOW LAND SURFACE DATUM, CLIMATIC YEARS APRIL 1981 TO MARCH 1983

$\begin{array}{lll} & \text { WATER } & \text { WATER } \\ \text { DATE } & \text { LEVEL } & \text { DATE }\end{array}$

MAR $11,1982 \quad 17.30 \quad$ MAR 09, $1983 \quad 16.28$ 
TILLMAN COUNTY -- CONTINUED

342700099040301. LOCAL NUMBER; LOCATION,--LAT 34 27'00", LONG 099 AQUIFER.--TERRACE DEPOSITS.

PERIOD OF RECORD.--1969 TO CURRENT YEAR.

EXTREMES FOR PERIOD OF RECORD.--HIGHEST WATER LEVEL,23.20 FT(7.07 M) BELOW LAND-SURFACE DATUM,MAR 28, 1978; LOWEST WATER LEVEL,25.24 FT(7.69 M) BELOW LAND-SURFACE DATUM, MAR 11, 1982.

WATER LEVELS IN FEET BELOW LAND SURFACE DATUM, CLIMATIC YEARS APRIL 1981 TO MARCH 1983
DATE
WATER
LEVEL
DATE
WATER
LEVEL

MAR $11,1982 \quad 25.24 \quad$ MAR 09, $1983 \quad 24.27$

GROUND-WATER LEVELS

342654099054601. LOCAL NUMBER; 01S-18W-28 BAA 1. LOCATION,--LAT 34 26'54", LONG 099 05'46", HYDROLOGIC UNIT 11120303. AQUIFER.--TERRACE DEPOSITS. PERIOD OF RECORD.--1977 TO CURRENT YEAR. EXTREMES FOR PERIOD OF RECORD.--HIGHEST WATER LEVEL, 15.22 FT(4.64 M) BELOW LAND-SURFACE DATUM,FEB 26, 1980; LOWEST WATER LEVEL, 17.24 FT(5.25 M) BELOW LAND-SURFACE DATUM, MAR 25, 1981 .

WATER LEVELS IN FEET BELOW LAND SURFACE DATUM, CLIMATIC YEARS APRIL 1981 TO MARCH 1983

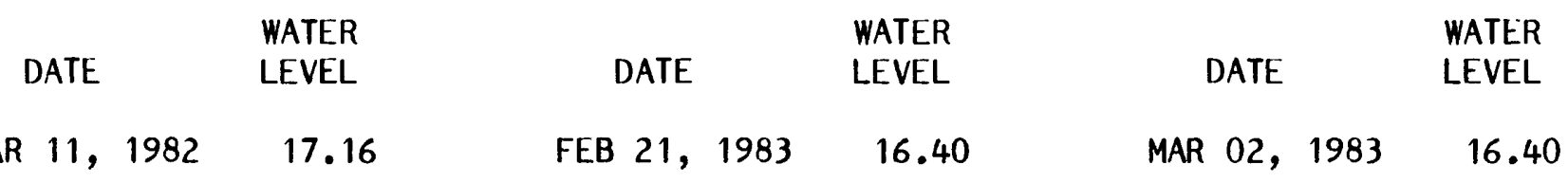




\section{GROUND-WATER LEVELS}

TILLMAN COUNTY -- CONTINUED

342615099072101. LOCAL NUMBER; LOCATION, --LAT 34 26'15", LONG 099 AQUIFER.--TERRACE DEPOSITS.

PERIOD OF RECORD.--1981 TO CURRENT YEAR.

EXTREMES FOR PERIOD OF RECORD.--HIGHEST WATER LEVEL,20.35 FT(6.20 M) BELOW LAND-SURFACE DATUM, MAR 25, 1981; LOWEST WATER LEVEL,20.82 FT(6.35 M) BELOW LAND-SURFACE DATUM, MAR 11, 1982 .

WATER LEVELS IN FEET BELOW LAND SURFACE DATUM, CLIMATIC YEARS APRIL 1981 TO MARCH 1983
015-18W-30 DDA 1.

$07 ' 21$ ', HYDROLOGIC UNIT 11120303.
342601099051401. LOCAL NUMBER; LOCATION, --LAT 34 26'01', LONG 099 AQUIFER.--TERRACE DEPOSITS. PERIOD OF RECORD.--1969 TO CURRENT YEAR. EXTREMES FOR PERIOD OF RECORD.--HIGHEST WATER LEVEL, $22.87 \mathrm{FT}(6.97 \mathrm{M})$ BELOW LAND-SURFACE DATUM, MAR 6, 1978; LOWEST WATER LEVEL,26.97 FT(8.22 M) BELOW LAND-SURFACE DATUM, JAN $6,1969$.

WATER LEVELS IN FEET BELOW LAND SURFACE DATUM, CLIMATIC YEARS APRIL 1981 TO MARCH 1983
$015-18 W-33$ AAA 1

05'14", HYDROLOGIC UNIT 11120303.
WATER

DATE

MAR $11,1982 \quad 24.50$
MAR $11,1982 \quad 20.82$
MAR 09, $1983 \quad 20.62$ 
TILLMAN COUNTY -- CONTINUED

342542099030001 . LOCAL NUMBER; LOCATION,--LAT 34 25'42", LONG 099 AQUIFER.--TERRACE DEPOSITS. PERIOD OF RECORD.-- 1977 TO CURRENT YEAR. EXTREMES FOR PERIOD OF RECORD.--HIGHEST WATER LEVEL, 27.39 FT(8.35 M) BELOW LAND-SURFACE DATUM,MAR 6, 1978; LOWEST WATER LEVEL,28.34 FT(8.64 M) BELOW LAND-SURFACE DATUM, MAR 8, 1977.

WATER LEVELS IN FEET BELOW LAND SURFACE DATUM, CLIMATIC YEARS APRIL 1981 TO MARCH 1983
$015-18 W-36$ BCC 1.

$03^{\prime}$ '00", HYDROLOGIC UNIT 11130102. WA

\begin{tabular}{|c|c|c|}
\hline & WATER & \\
\hline DATE & LEVEL & DATE \\
\hline
\end{tabular}

MAR 11, $1982 \quad 28.22 \quad$ MAR 09, $1983 \quad 27.90$

\section{GROUND-WATER LEVELS}

343017099103001. LOCAL NUMBER; 01S-19W-03 AAD 1. LOCATION,--LAT 34 30'17", LONG 099 10'30", HYDROLOGIC UNIT 11120303. AQUIFER.--TERRACE DEPOSITS. PERIOD OF RECORD.-- 1969 TO CURRENT YEAR. EXTREMES FOR PERIOD OF RECORD.--HIGHEST WATER LEVEL, 29.55 FT(9.01 M) BELOW LAND-SURFACE DATUM, MAR 09, 1983; LOWEST WATER LEVEL, $32.36 \mathrm{FT}(9.86 \mathrm{M})$ BELOW LAND-SURFACE DATUM, FEB 19, 1969.

WATER LEVELS IN FEET BELOW LAND SURFACE DATUM, CLIMATIC YEARS APRIL 1981 TO MARCH 1983

\begin{tabular}{cccc}
\multicolumn{1}{c}{} & WATER & & WATER \\
DATE & LEVEL & DATE & LEVEL \\
MAR 10, 1982 & 29.83 & MAR 09, 1983 & 32.36
\end{tabular}


342931099122001. LOCAL NUMBER; 01S-19W-09 BBA 1. LOCATION,--LAT 34 29'31", LONG 099 12'20", HYDROLOGIC UNIT 11120303. AQUIFER.--TERRACE DEPOSITS. PERIOD OF RECORD.--1970 TO CURRENT YEAR. EXTREMES FOR PERIOD OF RECORD.--HIGHEST WATER LEVEL,9.15 FT (2.79 M) BELOW LAND-SURFACE DATUM,MAR 28, 1978; LOWEST WATER LEVEL, 15.24 FT(4.65 M) BELOW LAND-SURFACE DATUM, MAR 9, 1983.

WATER LEVELS IN FEET BELOW LAND SURFACE DATUM, CLIMATIC YEARS APRIL 1981 TO MARCH 1983

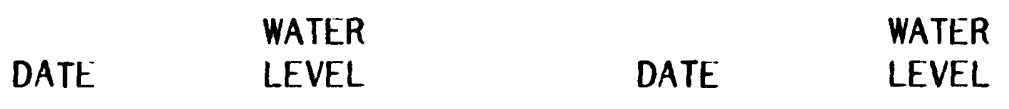

MAR $10,1982 \quad 14.64 \quad$ MAR 09, $1983 \quad 15.24$

GROUND-WATER LEVELS

342806099092701. LOCAL NUMBER; 01S-19W-14 DAD 1. LOCATION,--LAT 34 28'06", LONG 099 09'27", HYDROLOGIC UNIT 11120303. AQUIFER.--TERRACE DEPOSITS. PERIOD OF RECORD.--1970 TO CURRENT YEAR.

EXTREMES FOR PERIOD OF RECORD.--HIGHEST WATER LEVEL, $22.96 \mathrm{FT}(7.00 \mathrm{M})$ BELOW LAND-SURFACE DATUM, MAR 25, 1981; LOWEST WATER LEVEL,27.20 FT(8.29 M) BELOW LAND-SURFACE DATUM, JAN 21, 1970 .

WATER LEVELS IN FEET BELOW LAND SURFACE DATUM, CLIMATIC YEARS APRIL 1981 TO MARCH 1983

\begin{tabular}{|c|c|c|}
\hline & WATER & \\
\hline DATE & LEVEL & DATE \\
\hline
\end{tabular}

MAR $10,1982 \quad 24.03 \quad$ MAR 09, $1983 \quad 23.07$ 


\section{TILLMAN COUNTY -- CONTINUED}

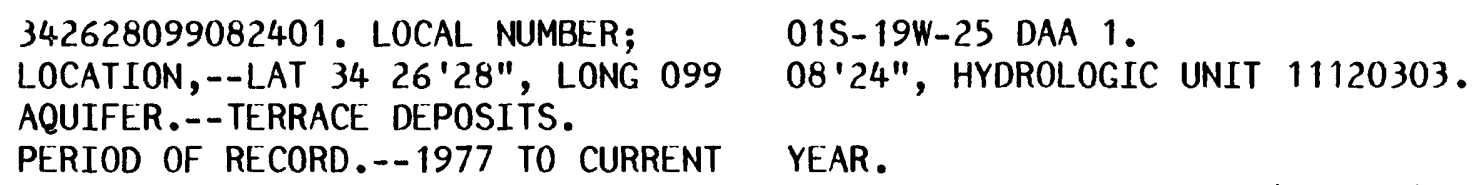

GROUND-WATER LEVELS

342654099105301. LOCAL NUMBER; 01S-19W-27 ABB 1.

LOCATION,--LAT 34 26'54", LONG 099 10'53", HYDROLOGIC UNIT 11120303.

AQUIFER.--TERRACE DEPOSITS.

PERIOD OF RECORD.--1969 TO CURRENT YEAR.

EXTREMES FOR PERIOD OF RECORD.--HIGHEST WATER LEVEL, 22.04 FT(6.72 M) BELOW LAND-SURFACE DATUM, MAR 28, 1978; LOWEST WATER LEVEL,25.57 FT(7.79 M) BELOW LAND-SURFACE DATUM, MAR 3, 1983.

WATER LEVELS IN FEET BELOW LAND SURFACE DATUM, CLIMATIC YEARS APRIL 1981 TO MARCH 1983

$\begin{array}{lll} & \text { WATER } & \text { WATER } \\ \text { DATE } & \text { LE.VEL } & \text { DATE }\end{array}$

MAR $10,1982 \quad 25.00 \quad$ MAR $03,1983 \quad 25.57$ 
TILLMAN COUNTY -- CONTINUED

342535099091901. LOCAL NUMBER; LOCATION,--LAT 34 25'35", LONG 099 AQUIFER.--TERRACE DEPOSITS. PERIOD OF RECORD.--1969 TO CURRENT YEAR. EXTREMES FOR PERIOD OF RECORD.--HIGHEST WATER LEVEL,26.78 FT(8.16 M) BELOW LAND-SURFACE DATUM,FEB 26, 1980; LOWEST WATER LEVEL,31.27 FT(9.53 M) BELOW LAND-SURFACE DATUM, FEB 19, 1969.

WATER LEVELS IN FEET BELOW LAND SURFACE DATUM, CLIMATIC YEARS APRIL 1981 TO MARCH 1983
01S-19W-36 CBB 1 .

09'19", HYDROLOGIC UNIT 11120303.

$\begin{array}{lll} & & \\ & \text { WATER } & \\ \text { DATE } & \text { LEVEL } & \text { WATER } \\ & \text { DATE } & \text { LEVEL }\end{array}$
MAR 10, 1982
28.83
MAR 09, $1983 \quad 28.35$

GROUND-WATER LEVELS

343735099010801. LOCAL NUMBER; $\quad 025-17 W-19$ DDA 1. LOCATION,--LAT 34 37'35", LONG 099 01'08", HYDROLOGIC UNIT 11120303. AQUIFER.--TERRACE DEPOSITS. PERIOD OF RECORD.--1969 TO CURRENT YEAR. EXTREMES FOR PERIOD OF RECORD.--HIGHEST WATER LEVEL,23.90 FT(7.28 M) BELOW LAND-SURFACE DATUM,FEB 21, 1979; LOWEST WATER LEVEL,25.68 FT(7.83 M) BELOW LAND-SURFACE DATUM, MAR 9, 1983.

WATER LEVELS IN FEET BELOW LAND SURFACE DATUM, CLIMATIC YEARS APRIL 1981 TO MARCH 1983

\begin{tabular}{|c|c|c|}
\hline & WATER & \\
\hline DATE & LEVEL & DATE \\
\hline
\end{tabular}

MAR $11,1982 \quad 25.18 \quad$ MAR 09, $1983 \quad 25.68$ 
TILLMAN COUNTY -- CONTINUED

342430099053801. LOCAL NUMBER; 02S-18W-04 DCB 2.

LOCATION,--LAT 34 24'30", LONG 099 05'38", HYDROLOGIC UNIT 11130102.

AQUIFER.--TERRACE DEPOSITS.

PERIOD OF RECORD.-- 1970 TO CURRENT YEAR.

EXTREMES FOR PERIOD OF RECORD.--HIGHEST WATER LEVEL, 47.46 FT(14.47 M) BELOW LAND-SURFACE DATUM, MAR 9, 1983; LOWEST WATER LEVEL,53.97 FT(16.45 M) BELOW LAND-SURFACE DATUM, JAN 21, 1970.

WATER LEVELS IN FEET BELOW LAND SURFACE DATUM, CLIMATIC YEARS APRIL 1981 TO MARCH 1983

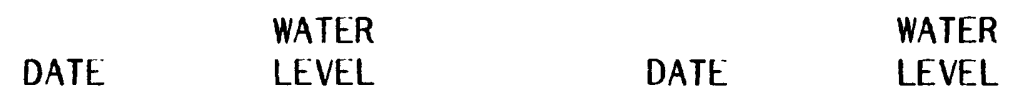

MAR $11,1982 \quad 48.17 \quad$ MAR 09, $1983 \quad 47.46$

GROUND-WATER LEVELS

342245099050701. LOCAL NUMBER; 02S-18W-15 CCB 1.

LOCATION,--LAT 34 22'45", LONG 099 05'07", HYDROLOGIC UNIT 11130102.

AQUIFER.--TERRACE DEPOSITS.

PERIOD OF RECORD.--1970 TO CURRENT YEAR.

EXTREMES FOR PERIOD OF RECORD.--HIGHEST WATER LEVEL, 38.94 FT(11.87 M) BELOW LAND-SURFACE DATUM,FEB 11, 1983; LOWEST WATER LEVEL,47.09 FT(14.35 M) BELOW LAND-SURFACE DATUM, JAN 21, 1970.

WATER LEVELS IN FEET BELOW LAND SURFACE DATUM, CLIMATIC YEARS APRIL 1981. T0 MARCH 1983

$\begin{array}{lll} & \text { WATER } & \text { WATER } \\ \text { DATE } & \text { LEVEL } & \text { DATE }\end{array}$

MAR $11,1982 \quad 39.67 \quad$ FEB $11,1983 \quad 38.94$ 


\section{TILLMAN COUNTY -- CONTINUED}

342443099134701. LOCAL NUMBER;

LOCATION,--LAT $3424^{\prime} 43^{\prime \prime}$, LONG 099

AQUIFER.--TERRACE DEPOSITS.

PERIOD OF RECORD.--1970 TO CURRENT YEAR.

EXTREMES FOR PERIOD OF RECORD.--HIGHEST WATER LEVEL, $5.29 \mathrm{FT}(1.61 \mathrm{M})$ BELOW LAND-SURFACE DATUM,MAR 8, 1977; LOWEST WATER LEVEL,7.59 FT(2.31 M) BELOW LAND-SURFACE DATUM, MAR 10, 1982.

WATER LEVELS IN FEET BELOW LAND SURFACE DATUM, CLIMATIC YEARS APRIL 1981 TO MARCH 1983
02S-19W-06 DAB 1.

13 '47", HYDROLOGIC UNIT 11120303. 
TILLMAN COUNTY -- CONTINUED

341559099003901. LOCAL NUMBER; 035-17W-29 CAB 1.

LOCATION,--LAT 34 15'59", LONG 099 00'39", HYDROLOGIC UNIT 11130102.

AQUIFER.--TERRACE DEPOSITS.

PERIOD OF RECORD.--1970 TO CURRENT YEAR.

EXTREMES FOR PERIOD OF RECORD.--HIGHEST WATER LEVEL,6.96 FT(2.12 M) BELOW LAND-SURFACE DATUM, JAN 20, 1970; LOWEST WATER LEVEL, 12.76 FT(3.89 M) BELOW LAND-SURFACE DATUM, MAR 25, 1981.

WATER LEVELS IN FEET BELOW LAND SURFACE DATUM, CLIMATIC YEARS APRIL 1981 TO MARCH 1983

\begin{tabular}{|c|c|c|}
\hline & WATER & \\
\hline DATE & LEVEL & DATE \\
\hline
\end{tabular}

MAR 10, $1982 \quad 10.95 \quad$ MAR $03,1983 \quad 8.69$

GROUND-WATER LEVELS

341539099015701. LOCAL NUMBER; 03S-17W-30 CCC 1.

LOCATION,--LAT 34 15'39", LONG 099 01'57", HYDROLOGIC UNIT 11130102.

AQUIFER.--CEDAR HILL SANDSTONE.

PERIOD OF RECORD.--1983 TO CURRENT YEAR.

EXTREMES FOR PERIOD OF RECORD.--HIGHEST WATER LEVEL, $27.59 \mathrm{FT}(8.41 \mathrm{M})$ BELOW LAND-SURFACE DATUM,FEB 2, 1983; LOWEST WATER LEVEL,27.59 FT(8.41 M) BELOW LAND-SURFACE DATUM, FEB 2, 1983.

WATER LEVELS IN FEET BELOW LAND SURFACE DATUM, CLIMATIC YEARS APRIL 1981 TO MARCH 1983

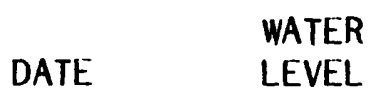

FEB $02,1983 \quad 27.59$ 


\section{GROUND-WATER LEVELS}

\section{TILLMAN COUNTY -- CONTINUED}

341955099051401 . LOCAL NUMBER;

LOCATION,--LAT 34 19'55", LONG 099 AQUIFER.--TERRACE DEPOSITS.

PERIOD OF RECORD.--1977 TO CURRENT

EXTREMES FOR PERIOD OF RECORD.--HIGHEST WATER LEVEL, 16.12 FT (4.91 M) BELOW LAND-SURFACE DATUM,MAR 8, 1977; LOWEST WATER LEVEL, $17.43 \mathrm{FT}(5.31 \mathrm{M})$ BELOW LAND-SURFACE DATUM, FEB 20, 1979.

WATER LEVELS IN FEET BELOW LAND SURFACE DATUM, CLIMATIC YEARS APRIL 1981 TO MARCH 1983
03S-18W-04 AAA 1.

05'14", HYDROLOGIC UNIT 11130102.

YEAR.
WATER LEVELS IN FEET BELON LAND SURFACE DATUM, CLIMATIC YEARS APRIL 1981 TO MARCH 1983

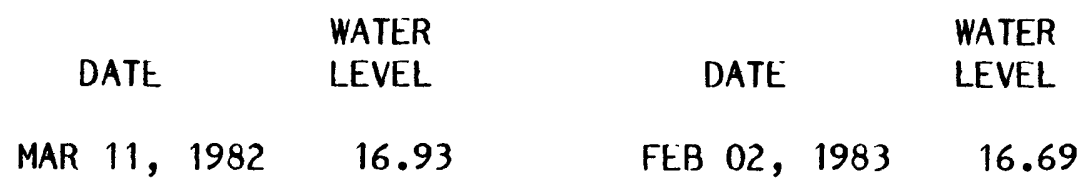

GROUND-WATER LEVELS

341723099052401. LOCAL NUMBER; 03S-18W-21 BAA 1. LOCATION,--LAT 34 17'23", LONG 099 05'24", HYDROLOGIC UNIT 11130102. AQUIFER.--TERRACE DEPOSITS. PERIOD OF RECORD.--1974 TO CURRENT YEAR. EXTREMES FOR PERIOD OF RECORD.--HIGHEST WATER LEVEL, 19.70 FT(6.00 M) BELOW LAND-SURFACE DATUM,FEB 2, 1983; LOWEST WATER LEVEL,26.96 FT(8.22 M) BELOW LAND-SURFACE DATUM, DEC 12, 1974.

WATER LEVELS IN FEET BELOW LAND SURFACE DATUM, CLIMATIC YEARS APRIL 1981 TO MARCH 1983

\begin{tabular}{cccc}
\multicolumn{1}{c}{ WATER } & WATER & & WATER \\
LEVEL & DATE & LEVEL \\
MAR 11, 1982 & 20.03 & FEB 02, 1983 & 19.70
\end{tabular}


341447099062501 . LOCAL NUMBER; LOCATION,--LAT 34 14'47", LONG 099 AQUIFER.--TERRACE DEPOSITS. PERIOD OF RECORD.--1969 TO CURRENT YEAR. EXTREMES FOR PERIOD OF RECORD.--HIGHEST WATER LEVEL,28.25 FT(8.61 M) BELOW LAND-SURFACE DATUM,FEB 26, 1980; LOWEST WATER LEVEL,33.14 FT(10.10 M) BELOW LAND-SURFACE DATUM, FEB $18,1969$.

WATER LEVELS IN FEET BELOW LAND SURFACE DATUM, CLIMATIC YEARS APRIL 1981 TO MARCH 1983

$\begin{array}{cccc}\text { DATE } & \begin{array}{c}\text { WATER } \\ \text { LEVEL }\end{array} & \text { DATE } & \text { WATER } \\ \text { LEVEL } \\ \text { D 10, 1982 } & 30.64 & \text { FEB 02, 1983 } & 30.08\end{array}$

GROUND-WATER LEVELS

341951099091801. LOCAL NUMBER; 03S-19W-02 ABB 1. LOCATION,--LAT 34 19'51", LONG 099 09'18", HYDROLOGIC UNIT 11130102. AQUIFER. - -TERRACE DEPOSITS.

PERIOD OF RECORD. --1974 TO CURRENT YEAR.

EXTREMES FOR PERIOD OF RECORD.--HIGHEST WATER LEVEL, 25.86 FT(7.88 M) BELOW LAND-SURFACE DATUM,FEB 26, 1980; LOWEST WATER LEVEL,29.18 FT(8.89 M) BELOW LAND-SURFACE DATUM, DEC $10,1974$.

WATER LEVELS IN FEET BELOW LAND SURFACE DATUM, CLIMATIC YEARS APRIL 1981 TO MARCH 1983

\begin{tabular}{|c|c|}
\hline & WATER \\
\hline DATE & LEVEL \\
\hline
\end{tabular}

MAR $10,1982 \quad 27.30 \quad$ MAR 09, $1983 \quad 0$ 


\section{TILLMAN COUNTY -- CONTINUED}

341718099103001. LOCAL NUMBER; 03S-19W-22 AAA 1. LOCATION,--LAT 34 17'18", LONG 099 10'30", HYDROLOGIC UNIT 11130102. AQUIFER.--TERRACE DEPOSITS. PERIOD OF RECORD.--1977 TO CURRENT YEAR. EXTREMES FOR PERIOD OF RECORD.--HIGHEST WATER LEVEL, $16.70 \mathrm{FT}(5.09 \mathrm{M})$ BELOW LAND-SURFACE DATUM,MAR 3, 1983; LOWEST WATER LEVEL,28.30 FT(8.63 M) BELOW LAND-SURFACE DATUM, MAR 10, 1982.

WATER LEVELS IN FEET BELOW LAND SURFACE DATUM, CLIMATIC YEARS APRIL 1981 TO MARCH 1983

\begin{tabular}{|c|c|c|}
\hline & WATER & \\
\hline DATE & LEVEL & DATE \\
\hline
\end{tabular}

MAR $10,1982 \quad 28.30 \quad$ MAR 03, $1983 \quad 16.70$

GROUND-WATER LEVELS

341256099013401. LOCAL NUMBER; 04S-17W-18 BAA 1.

LOCATION,--LAT 34 12'56", LONG 099 01'34", HYDROLOGIC UNIT 11130102.

AQUIFER.--ALLUVIUM.

PERIOD OF RECORD.--1981 TO CURRENT YEAR.

EXTREMES FOR PERIOD OF RECORD.--HIGHEST WATER LEVEL,7.26 FT(2.21 M) BELOW LAND-SURFACE DATUM,MAR 25, 1981; LOWEST WATER LEVEL,7.26 FT(2.21 M) BELOW LAND-SURFACE DATUM, MAR 25, 1981 .

WATER LEVELS IN FEET BELOW LAND SURFACE DATUM, CLIMATIC YEARS APRIL 1981 TO MARCH 1983

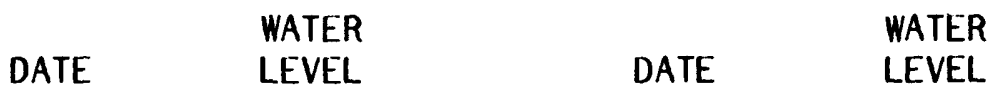

MAR 10, $1982 \quad \mathrm{Z} \quad$ MAR 03, $1983 \quad \mathrm{~N}$ 
341342099030001. LOCAL NUMBER; LOCATION,--LAT 34 13'42", LONG 099 AQUIFER.--TERRACE DEPOSITS. PERIOD OF RECORD.--1981 TO CURRENT YEAR. EXTREMES FOR PERIOD OF RECORD.--HIGHEST WATER LEVEL, $32.35 \mathrm{FT}(9.86 \mathrm{M})$ BELOW LAND-SURFACE DATUM,MAR 25, 1981; LOWEST WATER LEVEL, 34.58 FT(10.54 M) BELOW LAND-SURFACE DATUM, MAR 10, 1982 .

WATER LEVELS IN FEET BELOW LAND SURFACE DATUM, CLIMATIC YEARS APRIL 1981 TO MARCH 1983

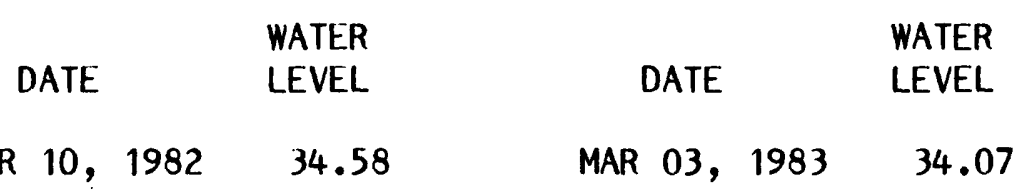

\section{GROUND-WATER LEVELS}

341441099084701. LOCAL NUMBER; LOCATION,--LAT 34 14'41", LONG 099 . 08'47", HYDROLOGIC UNIT 11130102. AQUIFER.--TERRACE DEPOSITS. PERIOD OF RECORD.--1981 TO CURRENT YEAR. EXTREMES FOR PERIOD OF RECORD.--HIGHEST WATER LEVEL, 26.32 FT (8.02 M) BELOW LAND-SURFACE DATUM, MAR 25, 1981; LOWEST WATER LEVEL, 30.45 FT(9.28 M) BELOW LAND-SURFACE DATUM, FEB 2, 1983.

WATER LEVELS IN FEET BELOW LAND SURFACE DATUM, CLIMATIC YEARS APRIL 1981 TO MARCH 1983

\begin{tabular}{cccc} 
& WATER & & WATER \\
DATE & LEVEL & DATE & \multicolumn{1}{l}{ LEVEL } \\
R 10, 1982 & 27.60 & FEB 02, 1983 & 30.45
\end{tabular}




\section{GROUND-WATER LEVELS}

\section{TULSA COUNTY}

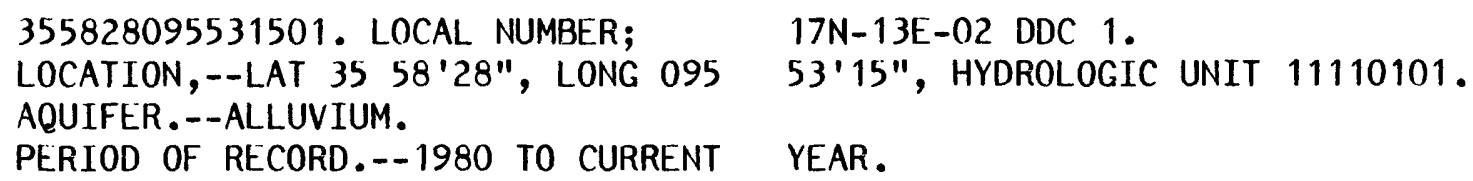

WATER LEVELS IN FEET BELOW LAND SURFACE DATUM, CLIMATIC YEARS APRIL 1981 T0 MARCH 1983

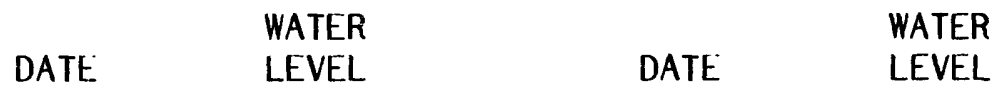

MAR 23, $1982 \quad 9.05 \quad$ FEB 08, $1983 \quad 9.20$




\section{GROUND-WATER LEVELS}

WAGONER COUNTY

355210095224401. LOCAL NUMBER; LOCATION,--LAT 3552 '10", LONG 095 AQUIFER .--ALLUVIUM.

PERIOD OF RECORD.--1976 TO CURRENT EXTREMES FOR PERIOD OF RECORD.--HIGHEST WATER LEVEL,6.39 FT(1.95 M) BELOW LAND-SURFACE

DATUM, MAY 4, 1976; LOWEST WATER LEVEL,8.19 FT(2.50 M) BELOW LAND-SURFACE DATUM, MAR 22, 1977. WATER LEVELS IN FEET BELOW LAND SURFACE DATUM, CLIMATIC YEARS APRIL 1981 TO MARCH 1983
$16 \mathrm{~N}-18 \mathrm{E}-15$ AAA 1

22 '44", HYDROLOGIC UNIT 11090201.
YEAR 。

$$
\text { WATER }
$$

DATE

MAR 23, 1982

$$
\text { LEVEL }
$$

$$
6.40
$$

$\begin{array}{lll} & \text { WATER } & \\ \text { DATE } & \text { LEVEL } & \text { WATER } \\ & \text { DATE } & \text { LEVEL }\end{array}$

MAR $01,1983 \quad 6.60$

MAR 10, 1983

6.60 
WASHINGTON COUNTY

363819095523901 . LOCAL NUMBER; 25N-13E-23 AAD 1. LOCATION,--LAT 36 38'19", LONG 095 52'39", HYDROLOGIC UNIT 11070106. AQUIFER.--ALLUVIUM.

PERIOD OF RECORD.--1979 TO CURRENT YEAR.

EXTREMES FOR PERIOD OF RECORD.--HIGHEST WATER LEVEL,22.67 FT(6.91 M) BELOW LAND-SURFACE DATUM, MAR 15, 1979; LOWEST WATER LEVEL,26.36 FT(8.03 M) BELOW LAND-SURFACE DATUM, MAR 11, 1981.

WATER LEVELS IN FEET BELOW LAND SURFACE DATUM, CLIMATIC YEARS APRIL 1981 TO MARCH 1983

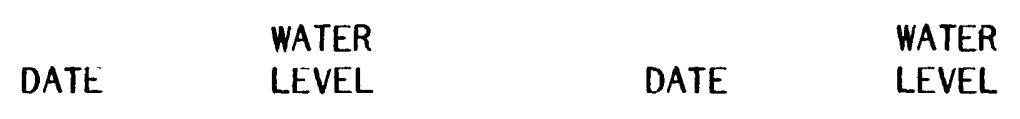

MAR $30,1982 \quad 24.49 \quad$ MAR $04,1983 \quad 23.41$

GROUND-WATER LEVELS

364919095523101. LOCAL NUMBER; 27N-13E-13 CBB 1.

LOCATION,--LAT 36 49'19", LONG 095 52'31", HYDROLOGIC UNIT 11070106.

AQUIFER.--DEWEY FORMATION.

PERIOD OF RECORD.--1977 TO CURRENT YEAR.

EXTREMES FOR PERIOD OF RECORD.--HIGHEST WATER LEVEL, 11.88 FT(3.62 M) BELOW LAND-SURFACE DATUM, MAR 21, 1978; LOWEST WATER LEVEL, 14.68 FT(4.47 M) BELOW LAND-SURFACE DATUM, MAR 11, 1981.

WATER LEVELS IN FEET BELOW LAND SURFACE DATUM, CLIMATIC YEARS APRIL 1981 TO MARCH 1983

$\begin{array}{lll} & \text { WATER } & \text { WATER } \\ \text { DATE } & \text { LEVEL } & \text { DATE }\end{array}$

MAR $30,1982 \quad 13.91 \quad$ MAR 04, $1983 \quad 12.40$ 


\section{GROUND-WATER LEVELS}

\section{WASHINGTON COUNTY -- CONTINUED}

$\begin{array}{ll}365557095521501 . \text { LOCAL NUMBER; } & \text { 28N-13E-01 CDC } 1 . \\ \text { LOCATION,--LAT 36 55'57", LONG } 095 & 52 \cdot 15^{\prime \prime}, \text { HYDROLOGIC UNIT } 11070106 .\end{array}$

AQUIFER.--WANN FORMATION.

PERIOD OF RECORD.--1979 TO CURRENT YEAR.

EXTREMES FOR PERIOD OF RECORD.--HIGHEST WATER LEVEL,9.04 FT(2.76 M) BELOW LAND-SURFACE DATUM,MAR 30, 1982; LOWEST WATER LEVEL, $19.50 \mathrm{FT}(5.94 \mathrm{M})$ BELOW LAND-SURFACE DATUM, FEB 27, 1980.

WATER LEVELS IN FEET BELOW LAND SURFACE DATUM, CLIMATIC YEARS APRIL 1981 TO MARCH 1983

\begin{tabular}{|c|c|c|}
\hline & WATER & \\
\hline DATE & LEVEL & DATE \\
\hline
\end{tabular}

MAR 30, $1982 \quad 9.04 \quad$ MAR 04, $1983 \quad 9.70$ 
WASHITA COUNTY

351630098432401. LOCAL NUMBER; LOCATION,--LAT $3516^{\prime} 30^{\prime \prime}$, LONG 098 AQUIFER.--RUSH SPRINGS FORMATION. PERIOD OF RECORD.--1979 TO CURRENT YEAR. EXTREMES FOR PERIOD OF RECORD.--HIGHEST WATER LEVEL, 18.87 FT(5.75 M) BELOW LAND-SURFACE DATUM,MAR 12, 1982; LOWEST WATER LEVEL,26.40 FT(8.05 M) BELOW LAND-SURFACE DATUM, MAR 10, 1981.

WATER LEVELS IN FEET BELOW LAND SURFACE DATUM, CLIMATIC YEARS APRIL 1981 TO MARCH 1983

\begin{tabular}{|c|c|c|}
\hline$T$ & WATER & DAT \\
\hline DAIE & & InIL \\
\hline
\end{tabular}

MAR $12,1982 \quad 18.87 \quad$ MAR 04, $1983 \quad 22.72$

GROUND-WATER LEVELS

351308098395701. LOCAL NUMBER; 09N-14W-27 DCD 1. LOCATION,--LAT 35 13'08", LONG 098 39'57", HYDROLOGIC UNIT 11130302. AQUIFER.--TERRACE DEPOSITS. PERIOD OF RECORD.--1979 TO CURRENT YEAR. EXTREMES FOR PERIOD OF RECORD.--HIGHEST WATER LEVEL, 78.55 FT (23.94 M) BELOW LAND-SURFACE DATUM,FEB 24, 1983; LOWEST WATER LEVEL,79.20 FT(24.14 M) BELOW LAND-SURFACE DATUM, MAR 3, 1980.

WATER LEVELS IN FEET BELOW LAND SURFACE DATUM, CLIMATIC YEARS APRIL 1981 TO MARCH 1983

\begin{tabular}{|c|c|c|}
\hline & WATER & \\
\hline DATE & LEVEL & DATE \\
\hline
\end{tabular}

MAR 11, $1982 \quad 79.08 \quad$ FEB 24, $1983 \quad 78.55$ 
WASHITA COUNTY -- CONTINUED

352229098384601. LOCAL NUMBER; 10N-14W-02 AAC 1.

LOCATION,--LAT 35 22'29", LONG 098 38'46", HYDROLOGIC UNIT 11130302.

AQUIFER.--RUSH SPRINGS FORMATION.

PERIOD OF RECORD.--1979 TO CURRENT YEAR.

EXTREMES FOR PERIOD OF RECORD.--HIGHEST WATER LEVEL,59.76 FT(18.21 M) BELOW LAND-SURFACE DATUM,MAR 28, 1979; LOWEST WATER LEVEL,62.86 FT(19.16 M) BELOW LAND-SURFACE DATUM, MAR 10, 1981.

WATER LEVELS IN FEET BELOW LAND SURFACE DATUM, CLIMATIC YEARS APRIL 1981 TO MARCH 1983

$\begin{array}{cccc}\text { DATE } & \text { WATER } & & \text { WATER } \\ \text { LEVEL } & \text { DATE } & \text { LEVEL } \\ \text { MAR 08, 1982 } & 62.38 & \text { MAR 04, 1983 } & 61.80\end{array}$

GROUND-WATER LEVELS

351939098394101. LOCAL NUMBER; 10N-14W-22 ADD 1.

LOCATION,--LAT 35 19'39", LONG 098 39'41", HYDROLOGIC UNIT 11130302.

AQUIFER.--RUSH SPRINGS FORMATION.

PERIOD OF RECORD.--1979 TO CURRENT YEAR.

EXTREMES FOR PERIOD OF RECORD.--HIGHEST WATER LEVEL, $3.55 \mathrm{FT}(1.08 \mathrm{M})$ BELOW LAND-SURFACE DATUM,MAR 8, 1982; LOWEST WATER LEVEL,4.68 FT(1.43 M) BELOW LAND-SURFACE DATUM, MAR 3, 1980 .

WATER LEVELS IN FEET BELOW LAND SURFACE DATUM, CLIMATIC YEARS APRIL 1981 TO MARCH 1983

$\begin{array}{lll} & \text { WATER } & \text { WATER } \\ \text { DATE } & \text { LEVEL } & \text { DATE }\end{array}$

MAR 08, $1982 \quad 3.56 \quad$ MAR 04, $1983 \quad 4.65$




\section{GROUND-WATER LEVELS}

WASHITA COUNTY -- CONTINUED

352749098422001. LOCAL NUMBER; LOCATION,--LAT 35 27'49", LONG 098 AQUIFER.--RUSH SPRINGS FORMATION. PERIOD OF RECORD.--1979 TO CURRENT EXTREMES FOR PERIOD OF RECORD.--HIGHEST WATER LEVEL, $38.45 \mathrm{FT}(11.72 \mathrm{M})$ BELOW LAND-SURFACE

EXTREMES FOR PERIOD OF RECORD.--HIGHEST WATER LEVEL, 38.45 FT(11.72 M) BELOW LAND-SURFACE
DATUM,MAR 4, 1983; LOWEST WATER LEVEL,43.80 FT(13.35 M) BELOW LAND-SURFACE DATUM, MAR 8, 1982 . WATER LEVELS IN FEET BELOW LAND SURFACE DATUM, CLIMATIC YEARS APRIL 1981 TO MARCH 1983

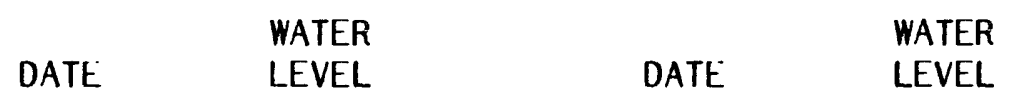
MAR 08, 1982
43.80
MAR 04, 1983
38.45

$11 \mathrm{~N}-14 \mathrm{~W}-05$ BAA 1.

$42^{\prime} 20^{\prime}$ ", HYDROLOGIC UNIT 11130302.

YEAR. 


\section{GROUND-WATER LEVELS}

WOODS COUNTY

362933098350501. LOCAL NUMBER; 23N-13W-03 CCC 1. LOCATION,--LAT 36 29'33", LONG 098 35'05", HYDROLOGIC UNIT 11050001. AQUIFER .--ALLUVIUM.

PERIOD OF RECORD.--1983 TO CURRENT YEAR.

EXTREMES FOR PERIOD OF RECORD.--HIGHEST WATER LEVEL, $12.50 \mathrm{FT}(3.81 \mathrm{M})$ BELOW LAND-SURFACE DATUM, MAR 24, 1983; LOWEST WATER LEVEL, $12.50 \mathrm{FT}(3.81 \mathrm{M})$ BELOW LAND-SURFACE DATUM, MAR 24, 1983.

WATER LEVELS IN FEET BELOW LAND SURFACE DATUM, CLIMATIC YEARS APRIL 1981 TO MARCH 1983

$\begin{array}{ll}\text { WATER } \\ \text { DATE } & \text { LEVEL }\end{array}$

MAR 24, $1983 \quad 12.50$

GROUND-WATER LEVELS

362755098371301. LOCAL NUMBER; LOCATION,--LAT 36 27'55", LONG 098 AQUIFER.--TERRACE DEPOSITS.

PERIOD OF RECORD.--1977 TO CURRENT YEAR.

EXTREMES FOR PERIOD OF RECORD.--HIGHEST WATER LEVEL,4.20 FT(1.28 M) BELOW LAND-SURFACE DATUM,MAR 23, 1982; LOWEST WATER LEVEL,7.10 FT(2.16 M) BELOW LAND-SURFACE DATUM, MAR $10,1977$.

WATER LEVELS IN FEET BELOW LAND SUAFACE DATUM, CLIMATIC YEARS APRIL 1981 TO MARCH 1983
$23 \mathrm{~N}-13 \mathrm{~W}-17 \mathrm{CCB} 1$.

37'13", HYDROLOGIC UNIT 11050001.
DATE
WATER
LEVEL
DATE
WATER
LEVEL

MAR 23, 1982

$\begin{array}{lll}4.20 & \text { MAR } 01,1983 \quad 5.48\end{array}$




\section{GROUND-WATER LEVELS}

WOODS COUNTY -- CONTINUED

363446098334401. LOCAL NUMBER; LOCATION,--LAT 36 34'46", LONG 098 AQUIFER.--ALLUVIUM.

PERIOD OF RECORD.--1983 TO CURRENT YEAR. EXTREMES FOR PERIOD OF RECORD.--HIGHEST WATER LEVEL, 1.72 FT(.52 M) BELOW LAND-SURFACE DATUM, MAR 23, 1983; LOWEST WATER LEVEL, 1.72 FT(.52 M) BELOW LAND-SURFACE DATUM, MAR 23, 1983.

WATER LEVELS IN FEET BELOW LAND SURFACE DATUM, CLIMATIC YEARS APRIL 1981 TO MARCH 1983
$24 N-13 W-02$ CDC 1 33'44", HYOROLOGIC UNIT 11050001.

$\begin{array}{ll} & \\ \text { DATE } & \text { WATER } \\ \text { LEVEL }\end{array}$

MAR 23, $1983 \quad 1.72$

GROUND-WATER LEVELS

363446098352901. LOCAL NUMBER; 24N-13W-04 DCD 1. LOCATION,--LAT 36 34'46", LONG 098 35'29", HYDROLOGIC UNIT 11050001. AQUIFER.--ALLUVIUM.

PERIOD OF RECORD.--1983 TO CURRENT YEAR.

EXTREMES FOR PERIOD OF RECORD.--HIGHEST WATER LEVEL, 2.43 FT(.74 M) BELOW LAND-SURFACE DATUM,MAR 23, 1983; LOWEST WATER LEVEL,2.43 FT(.74 M) BELOW LAND-SURFACE DATUM, MAR 23, 1983.

WATER LEVELS IN FEET BELOW LAND SURFACE DATUM, CLIMATIC YEARS APRIL 1981 TO MARCH 1983

$$
\begin{array}{ll} 
& \text { WATER } \\
\text { DATE } & \text { LEVEL }
\end{array}
$$

MAR 23, $1983 \quad 2.43$ 
WOODS COUNTY -- CONTINUED

363209098402601. LOCAL NUMBER;

LOCATION,--LAT 36 32'09", LONG 098 AQUIFER.--TERRACE DEPOSITS.

PERIOD OF RECORD. - 1977 TO CURRENT YEAR.

EXTREMES FOR PERIOD OF RECORD.--HIGHEST WATER LEVEL, 30.14 FT(9.19 M) BELOW LAND-SURFACE DATUM, MAR 23, 1982; LOWEST WATER LEVEL, $31.80 \mathrm{FT}(9.69 \mathrm{M})$ BELOW LAND-SURFACE DATUM, MAR 6, 1978.

WATER LEVELS IN FEET BELOW LAND SURFACE DATUM, CLIMATIC YEARS APRIL 1981 TO MARCH 1983
$24 \mathrm{~N}-14 \mathrm{~W}-23$ CCC 1. 40'26", HYDROLOGIC UNIT 11050001.

$\begin{array}{cccc}\text { DATE } & \text { WATER } & & \text { WATER } \\ \text { LEVEL } & \text { DATE } & \text { LEVEL } \\ \text { MAR 23, } 1982 & 30.14 & \text { MAR 01, 1983 } & \\ & & & \\ & & & \\ & & \text { GROUND-WATER LEVELS }\end{array}$

363150098444301. LOCAL NUMBER;

$24 N-14 W-30$ BCB 1 .

LOCATION,--LAT 36 31'50", LONG 098 44'43", HYDROLOGIC UNIT 11050001.

AQUIFER .--ALLUVIUM.

PERIOD OF RECORD.--1983. TO CURRENT YEAR.

EXTREMES FOR PERIOD OF RECORD.--HIGHEST WATER LEVEL,21.84 FT(6.66 M) BELOW LAND-SURFACE DATUM,MAR 24, 1983; LOWEST WATER LEVEL,21.84 FT(6.66 M) BELOW LAND-SURFACE DATUM, MAR 24, 1983.

WATER LEVELS IN FEET BELOW LAND SURFACE DATUM, CLIMATIC YEARS APRIL 1981 TO MARCH 1983

$\begin{array}{ll}\text { WATER } \\ \text { DATE } & \text { LEVEL }\end{array}$

MAR $24,1983 \quad 21.84$ 
WOODS COUNTY -- CONTINUED

363045098403401 . LOCAL NUMBER; LOCATION,--LAT 36 30'45", LONG 098 AQUIFER.--ALLUVIUM.

PERIOD OF RECORD.--1983 TO CURRENT YEAR.

EXTREMES FOR PERIOD OF RECORD.--HICHEST WATER LEVEL, 24.79 FT(7.56 M) BELOW LAND-SURFACE DATUM,MAR 24, 1983; LOWEST WATER LEVEL, 24.79 FT(7.56 M) BELOW LAND-SURFACE DATUM, MAR 24, 1983.

WATER LEVELS IN FEET BELOW LAND SURFACE DATUM, CLIMATIC YEARS APRIL 1981 TO MARCH 1983
$24 N-14 W-34$ DAA 1.

40'34", HYDROLOGIC UNIT 11050001. WATER

DATE LEVEL

MAR 24, $1983 \quad 24.79$

\section{GROUND-WATER LEVELS}

363446098514101. LOCAL NUMBER;

$24 \mathrm{~N}-16 \mathrm{~W}-01$ DCC 1 . LOCATION,--LAT 36 34'46", LONG 098 51'41", HYDROLOGIC UNIT 11050001. AQUIFER.--ALLUVIUM.

PERIOD OF RECORD.--1983 TO CURRENT YEAR.

EXTREMES FOR PERIOD OF RECORD.--HIGHEST WATER LEVEL, 26.00 FT(7.92 M) BELOW LAND-SURFACE DATUM,MAR 24, 1983; LOWEST WATER LEVEL,26.00 FT(7.92 M) BELOW LAND-SURFACE DATUM, MAR 24, 1983.

WATER LEVELS IN FEET BELOW LAND SURFACE DATUM, CLIMATIC YEARS APRIL 1981 TO MARCH 1983

$$
\begin{array}{ll}
\text { WATER } \\
\text { DATE } & \text { LEVEL }
\end{array}
$$

MAR 24, $1983 \quad 26.00$ 
WOODS COUNTY -- CONTINUED

363916098353601. LOCAL NUMBER;

LOCATION,--LAT 36 39'16", LONG 098 AQUIFER.--ALLUVIUM.

PERIOD OF RECORD.--1983 TO CURRENT YEAR.

EXTREMES FOR PERIOD OF RECORD.--HIGHEST WATER LEVEL,3.89 FT(1.19 M) BELOW LAND-SURFACE DATUM, MAR 25, 1983; LOWEST WATER LEVEL,3.89 FT(1.19 M) BELOW LAND-SURFACE DATUM, MAR 25, 1983.

WATER LEVELS IN FEET BELOW LAND SURFACE DATUM, CLIMATIC YEARS APRIL 1981 TO MARCH 1983

DATE

WATER

LEVEL

MAR 25, $1983 \quad 3.89$
35'36", HYDROLOGIC UN1T 11050001.
$25 \mathrm{~N}-13 \mathrm{~W}-10$ CCC 1

GROUND-WATER LEVELS

363751098395501. LOCAL NUMBER; 25N-14W-24 CBB 1. LOCATION,--LAT 36 37'51", LONG 098 39'55", HYDROLOGIC UNIT 11050001. AQUIFER.--ALLUVIUM.

PERIOD OF RECORD.--1983 TO CURRENT YEAR.

EXTREMES FOR PERIOD OF RECORD.--HIGHEST WATER LEVEL,7.49 FT(2.28 M) BELOW LAND-SURFACE DATUM, MAR 22, 1983; LOWEST WATER LEVEL,7.49 FT(2.28 M) BELOW LAND-SURFACE DATUM, MAR 22, 1983.

WATER LEVELS IN FEET BELOW LAND SURFACE DATUM, CLIMATIC YEARS APRIL 1981 TO MARCH 1983 $\begin{array}{ll}\text { WATER } \\ \text { DATE } & \text { LEVEL }\end{array}$

MAR 22, $1983 \quad 7.49$ 
WOODS COUNTY -- CONTINUED

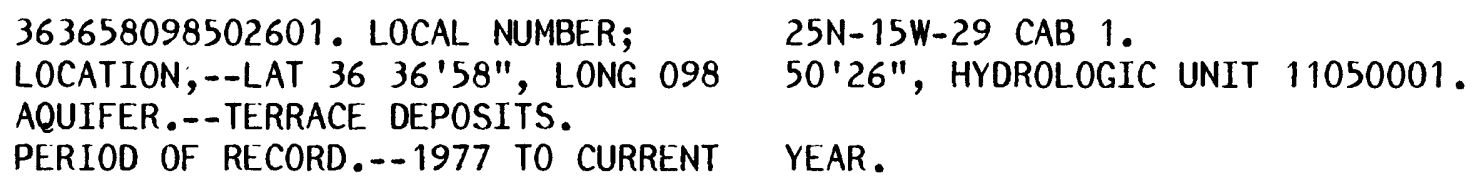

WATER LEVELS IN FEET BELOW LAND SURFACE DATUM, CLIMATIC YEARS APRIL 1981 TO MARCH 1983

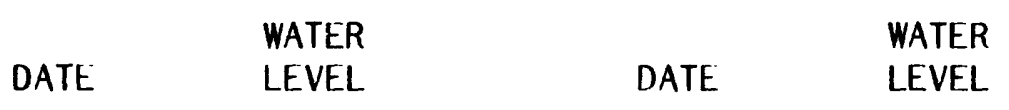

MAR 23, $1982 \quad 40.35 \quad$ MAR 01, $1983 \quad 39.18$

GROUND-WATER LEVELS

363547098505001. LOCAL NUMBER; 25N-15W-31 DDD 1.

LOCATION,--LAT 36 35'47", LONG 098 50'50", HYDROLOGIC UNIT 11050001.

AQUIFER.--ALLUVIUM.

PERIOD OF RECORD.--1983 TO CURRENT YEAR.

EXTREMES FOR PERIOD OF RECORD.--HIGHEST WATER LEVEL, $46.30 \mathrm{FT}(14.11 \mathrm{M})$ BELOW LAND-SURFACE DATUM, MAR 24, 1983; LOWEST WATER LEVEL,46.30 FT(14.11 M) BELOW LAND-SURFACE DATUM, MAR 24, 1983.

WATER LEVELS IN FEET BELOW LAND SURFACE DATUM, CLIMATIC YEARS APRIL 1981 TO MARCH 1983

$\begin{array}{ll} & \text { WATER } \\ \text { DATE } & \text { LEVEL }\end{array}$

MAR 24, $1983 \quad 46.30$ 
WOODS COUNTY -- CONTINUED

364422098374601. LOCAL NUMBER; 26N-13W-17 BBB 1.

LOCATION,--LAT 36 44'22", LONG 098 37'46", HYDROLOGIC UNIT 11050001.

AQUIFER.--ALLUVIUM.

PERIOD OF RECORD.--1983 TO CURRENT YEAR.

EXTREMES FOR PERIOD OF RECORD.--HIGHEST WATER LEVEL, $14.79 \mathrm{FT}(4.51 \mathrm{M})$ BELOW LAND-SURFACE DATUM,MAR 23, 1983; LOWEST WATER LEVEL, 14.79 FT(4.51 M) BELOW LAND-SURFACE DATUM, MAR 24, 1983.

WATER LEVELS IN FEET BELOW LAND SURFACE DATUM, CLIMATIC YEARS APRIL 1981 TO MARCH 1983

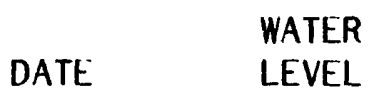

MAR 24, $1983 \quad 14.79$

GROUND-WATER LEVELS

364330098401901. LOCAL NUMBER; 26N-14W-23 ABA 1.

LOCATION,--LAT 36 43'30", LONG 098 40'19", HYDROLOGIC UNIT 11050001. AQUIFER.--ALLUVIUM.

PERIOD OF RECORD.--1983 TO CURRENT YEAR.

EXTREMES FOR PERIOD OF RECORD. --HIGHEST WATER LEVEL, $9.24 \mathrm{FT}(2.82 \mathrm{M})$ BELOW LAND-SURFACE DATUM, MAR 22, 1983; LOWEST WATER LEVEL,9.24 FT(2.82 M) BELOW LAND-SURFACE DATUM, MAR 22, 1983.

WATER LEVELS IN FEET BELOW LAND SURFACE DATUM, CLIMATIC YEARS APRIL 1981 TO MARCH 1983

$\begin{array}{ll} & \text { WATER } \\ \text { DATE } & \text { LEVEL }\end{array}$

MAR 22, $1983 \quad 9.24$ 


\section{GROUND-WATER LEVELS}

WOODS COUNTY -- CONTINUED

364613098391401. LOCAL NUMBER; 27N-14W-36 DCD 1.

LOCATION,--LAT 36 46'13", LONG 098 39'14", HYDROLOGIC UNIT 11050001. AQUIFER. - -ALLUVIUM.

PERIOD OF RECORD.--1983 TO CURRENT YEAR.

EXTREMES FOR PERIOD OF RECORD.--HIGHEST WATER LEVEL, 18.10 FT(5.52 M) BELOW LAND-SURFACE DATUM,MAR 22, 1983; LOWEST WATER LEVEL, 18.10 FT(5.52 M) BELOW LAND-SURFACE DATUM, MAR 22, 1983.

WATER LEVELS IN FEET BELOW LAND SURFACE DATUM, CLIMATIC YEARS APRIL 1981 TO MARCH 1983

$\begin{array}{ll} & \text { WATER } \\ \text { DATE } & \text { LEVEL }\end{array}$

MAR 22, $1983 \quad 18.10$ 


\section{GROUND-WATER LEVELS}

WOODWARD COUNTY

361447099105501. LOCAL NUMBER;

20N-19W-01 BAA 1.

LOCATION,--LAT 36 14'47", LONG 099 10'55", HYDROLOGIC UNIT 11100301.

AQUIFER.--RUSH SPRINGS FORMATION.

PERIOD OF RECORD.--1977 TO CURRENT YEAR.

EXTREMES FOR PERIOD OF RECORD.--HIGHEST WATER LEVEL, 195.65 FT(59.63 M) BELOW LAND-SURFACE DATUM, JAN 10, 1983; LOWEST WATER LEVEL, 198.61 FT(60.54 M) BELOW LAND-SURFACE DATUM, JAN $18,1982$.

WATER LEVELS IN FEET BELOW LAND SURFACE DATUM, CLIMATIC YEARS APRIL 1981 TO MARCH 1983

$\begin{array}{lll} & \text { WATER } & \text { WATER } \\ \text { DATE } & \text { LEVEL } & \text { DATE }\end{array}$

JAN 18, $1982 \quad 198.61 \quad$ JAN 10, $1983 \quad 195.65$

GROUND-WATER LEVELS

361221099182601. LOCAL NUMBER; 20N-20W-14 BBA 1. LOCATION,--LAT 36 12'21", LONG 099 18'26", HYDROLOGIC UNIT 11100301. AQUIFER .--OGALLALA.

PERIOD OF RECORD.--1980 TO CURRENT YEAR. EXTREMES FOR PERIOD OF RECORD.--HIGHEST WATER LEVEL, $46.13 \mathrm{FT}(14.06 \mathrm{M})$ BELOW LAND-SURFACE DATUM, JAN 10, 1983; LOWEST WATER LEVEL,47.45 FT(14.46 M) BELOW LAND-SURFACE DATUM, JAN 31, 1980.

WATER LEVELS IN FEET BELOW LAND SURFACE DATUM, CLIMATIC YEARS APRIL 1981 TO MARCH 1983

$\begin{array}{lll} & \text { WATER } & \text { WATER } \\ \text { DATE } & \text { LEVEL } & \text { DATE }\end{array}$

JAN $18,1982 \quad 47.14 \quad$ JAN $10,1983 \quad 46.13$




\section{GROUND-WATER LEVELS}

WOODWARD COUNTY -- CONTINUED

361128099210601. LOCAL NUMBER; 20N-20W-20 DCA 1. LOCATION,--LAT 36 11'28", LONG 099 21'06", HYDROLOGIC UNIT 11100301. AQUIFER.--OGALLALA. PERIOD OF RECORD.--1980 TO CURRENT YEAR. EXTREMES FOR PERIOD OF RECORD.--HIGHEST WATER LEVEL,43.64 FT(13.30 M) BELOW LAND-SURFACE DATUM, JAN 10, 1983; LOWEST WATER LEVEL,44.68 FT(13.62 M) BELOW LAND-SURFACE DATUM, JAN 31, 1980.

WATER LEVELS IN FEET BELOW LAND SURFACE DATUM, CLIMATIC YEARS APRIL 1981 TO MARCH 1983

\begin{tabular}{|c|c|c|}
\hline & WATER & \\
\hline DATE & & DATE \\
\hline
\end{tabular}

JAN 18, $1982 \quad 44.63 \quad$ JAN $10,1983 \quad 43.64$

\section{GROUND-WATER LEVELS}

361412099225701. LOCAL NUMBER; 20N-21W-01 DAD 1. LOCATION,--LAT 36 14'12", LONG 099 22'57", HYDROLOGIC UNIT 11100301. AQUIFER .--OGALLALA.

PERIOD OF RECORD.--1980 TO CURRENT YEAR.

EXTREMES FOR PERIOD OF RECORD.--HIGHEST WATER LEVEL, $120.60 \mathrm{FT}(36.76 \mathrm{M})$ BELOW LAND-SURFACE DATUM,FEB 3, 1981; LOWEST WATER LEVEL, 120.90 FT(36.85 M) BELOW LAND-SURFACE DATUM, JAN 18, 1982.

WATER LEVELS IN FEET BELOW LAND SURFACE DATUM, CLIMATIC YEARS APRIL 1981 TO MARCH 1983

$\begin{array}{lll} & \text { WATER } & \text { WATER } \\ \text { DATE } & \text { LEVEL } & \text { DATE }\end{array}$
JAN 18, $1982 \quad 120.90$
JAN 10,1983
$P$ 


\section{GROUND-WATER LEVELS}

WOODWARD COUNTY -- CONTINUED

361340099252201. LOCAL NUMBER;

$20 N-21 W-10$ ACA 1.

LOCATION,--LAT 36 13'40", LONG 099

25'22", HYDROLOGIC UNIT 11100301.

AQUIFER . - OGALLALA.

PERIOD OF RECORD.--1980 TO CURRENT YEAR.

EXTREMES FOR PERIOD OF RECORD.--HIGHEST WATER LEVEL, $113.20 \mathrm{FT}(34.50 \mathrm{M})$ BELOW LAND-SURFACE DATUM,JAN 19, 1982; LOWEST WATER LEVEL, $114.39 \mathrm{FT}(34.87 \mathrm{M})$ BELOW LAND-SURFACE DATUM, FLB 5, 1980.

WATER LEVELS IN FEET BELOW LAND SURFACE DATUM, CLIMATIC YEARS APRIL 1981 TO MARCH 1983

$\begin{array}{lll} & \text { WATER } & \text { WATER } \\ \text { DATE } & \text { LEVEL } & \text { DATE }\end{array}$

JAN 19, $1982 \quad 113.20 \quad$ JAN $10,1983 \quad 113.57$

GROUND-WATER LEVELS

361432099301001. LOCAL NUMBER; 20N-22W-01 BCA 1.

LOCATION,--LAT 36 14'32", LONG 099 30'10", HYDROLOGIC UNIT 11100301.

AQUIFER .--OGALLALA.

PERIOD OF RECORD.--1980 TO CURRENT YEAR.

EXTREMES FOR PERIOD OF RECORD.--HIGHEST WATER LEVEL, 153.44 FT(46.77 M) BELOW LAND-SURFACE DATUM,FEB 5, 1980; LOWEST WATER LEVEL, 158.78 FT(48.40 M) BELOW LAND-SURFACE DATUM, JAN 11, 1983.

WATER LEVELS IN FEET BELOW LAND SURFACE DATUM, CLIMATIC YEARS APRIL 1981 TO MARCH 1983

$\begin{array}{lll} & \text { WATER } & \text { WATER } \\ \text { DATE } & \text { LEVEL } & \text { DATE }\end{array}$
JAN 19, $1982 \quad 158.68$
JAN 11, $1983 \quad 158.78$ 


\section{GROUND-WATER LEVELS}

WOODWARD COUNTY -- CONTINUED

361146099395001. LOCAL NUMBER; 20N-22W-19 ADD 1.

LOCATION,--LAT 36 11'46", LONG 099 39'50", HYDROLOGIC UNIT 11100203.

AQUIFER.--OGALLALA.

PERIOD OF RECORD.--1980 TO CURRENT YEAR.

EXTREMES FOR PERIOD OF RECORD.--HIGHEST WATER LEVEL, 135.40 FT(41.27 M) BELOW LAND-SURFACE DATUM, JAN 19, 1982; LOWEST WATER LEVEL,136.10 FT(41.48 M) BELOW LAND-SURFACE DATUM, FLB 5, 1980.

WATER LEVELS IN FEET BELOW LAND SURFACE DATUM, CLIMATIC YEARS APRIL 1981 TO MARCH 1983

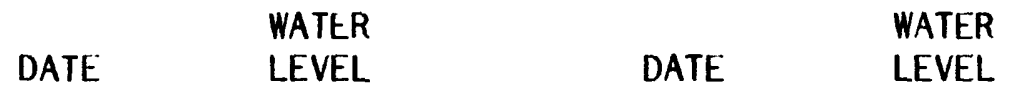

JAN 19, $1982135.40 \quad$ JAN $11,1983 \quad 135.54$

GROUND-WATER LEVELS

361006099293201. LOCAL NUMBER; 20N-22W-36 ADD 1. LOCATION,--LAT 36 10'06", LONG 099 29'32", HYDROLOGIC UNIT 11100301. AQUIFER .--OGALLALA.

PERIOD OF RECORD.--1957 TO CURRENT YEAR.

EXTREMES FOR PERIOD OF RECORD.--HIGHEST WATER LEVEL, 95.02 FT(28.96 M) BELOW LAND-SURFACE DATUM,SEP 27, 1961; LOWEST WATER LEVEL,99.91 FT(30.45 M) BELOW LAND-SURFACE DATUM, JAN 26, 1965 .

WATER LEVELS IN FEET BELOW LAND SURFACE DATUM, CLIMATIC YEARS APRIL 1981 TO MARCH 1983

\begin{tabular}{|c|c|c|}
\hline & WATER & \\
\hline DATE & LEVEL & DATE \\
\hline
\end{tabular}

JAN $19,1982 \quad 96.88 \quad$ JAN $11,1983 \quad 96.64$ 


\section{WOODWARD COUNTY -- CONTINUED}

361737099024301. LOCAL NUMBER; LOCATION, --LAT 36 17'37", LONG 099 AQUIFER. - - TERRACE DEPOSITS PERIOD OF RECORD..-1978 TO CURRENT YEAR. EXTREMES FOR PERIOD OF RECORD.--HIGHEST WATER LEVEL,77.38 FT(23.59 M) BELOW LAND-SURFACE DATUM, JAN 10, 1983; LOWEST WATER LEVEL,78.15 FT(23.82 M) BELOW LAND-SURFACE DATUM, MAR 14, 1979.

WATER LEVELS IN FEET BELOW LAND SURFACE DATUM, CLIMATIC YEARS APRIL 1981 TO MARCH 1983
$21 \mathrm{~N}-17 \mathrm{~W}-17 \mathrm{CDB} 1$. 02 '43", HYDROLOGIC UNIT 11050001. WATER LEVELS IN
DATE
WATER
LEVEL
DATE
WATER
JAN 18, $1982 \quad 77.65$
JAN $10,1983 \quad 77.38$

\section{GROUND-WATER LEVELS}

361610099070901. LOCAL NUMBER; LOCATION,--LAT 36 16'10", LONG 099 AQUIFER.--TERRACE DEPOSITS. PERIOD OF RECORD._-1977 TO CURRENT YEAR. EXTREMES FOR PERIOD OF RECORD.--HIGHEST WATER LEVEL, 33.16 FT(10.11 M) BELOW LAND-SURFACE DATUM, JAN 5, 1980; LOWEST WATER LEVEL, 36.95 FT(11.26 M) BELOW LAND-SURFACE DATUM, MAR 30, 1979.

$21 \mathrm{~N}-18 \mathrm{~W}-27 \mathrm{BCB} 1$. $07^{\prime} 09^{\prime \prime}$, HYOROLOGIC UNIT 11100301.

WATER LEVELS IN FEET BELOW LAND SURFACE DATUM, CLIMATIC YEARS APRIL 1981 TO MARCH 1983

$\begin{array}{lll} & \text { WATER } & \text { WATER } \\ \text { DATE } & \text { LEVEL } & \text { DATE } \\ \text { LEVEL }\end{array}$
JAN $18,1982 \quad 35.63$
JAN $10,1983 \quad 33.40$ 


\section{GROUND-WATER LEVELS}

WOODWARD COUNTY -- CONTINUED

361856099112401. LOCAL NUMBER; 21N-19W-12 BBD 1.

LOCATION,--LAT 36 18'56", LONG 099 11'24", HYDROLOGIC UNIT 11100301.

AQUIFER.--TERRACE DEPOSITS.

PERIOD OF RECORD.--1956 TO CURRENT YEAR.

EXTREMES FOR PERIOD OF RECORD.--HIGHEST WATER LEVEL,6.90 FT(2.10 M) BELOW LAND-SURFACE DATUM, JAN 10, 1983; LOWEST WATER LEVEL, 16.17 FT(4.93 M) BELOW LAND-SURFACE DATUM, AUG 15, 1968.

WATER LEVELS IN FEET BELOW LAND SURFACE DATUM, CLIMATIC YEARS APRIL 1981 TO. MARCH 1983

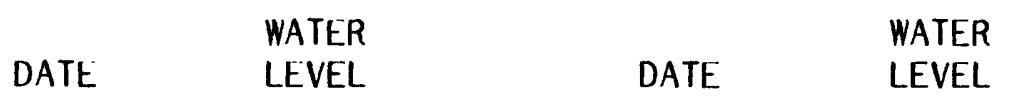

JAN $19,1982 \quad 9.20 \quad$ JAN $10,1983 \quad 6.90$

GROUND-WATER LEVELS

361949099192601. LOCAL NUMBER; 21N-20W-03 ABD 1.

LOCATION,--LAT 36 19'49", LONG 099 19'26", HYDROLOGIC UNIT 11100301.

AQUIFER .--OGALLALA.

PERIOD OF RECORD.--1980 TO CURRENT YEAR.

EXTREMES FOR PERIOD OF RECORD.--HIGHEST WATER LEVEL, $7.39 \mathrm{FT}(2.25 \mathrm{M})$ BELOW LAND-SURFACE DATUM, JAN 31, 1980; LOWEST WATER LEVEL,9.30 FT(2.83 M) BELOW LAND-SURFACE DATUM, FEB 3, 1981.

WATER LEVELS IN FEET BELOW LAND SURFACE DATUM, CLIMATIC YEARS APRIL 1981 TO MARCH 1983

$\begin{array}{lll} & \text { WATER } & \text { WATER } \\ \text { DATE } & \text { LEVEL } & \text { DATE }\end{array}$

JAN 19, $1982 \quad 9.08 \quad$ JAN 11, $1983 \quad 9.14$


GROUND-WATER LEVELS

WOODWARD COUNTY -- CONTINUED

361833099192201. LOCAL NUMBER; 21N-20W-10 DBD 1. LOCATION,--LAT 36 18'33", LONG 099 19'22", HYDROLOGIC UNIT 11100301. AQUIFER.--OGALLALA.

PERIOD OF RECORD.--1980 TO CURRENT YEAR.

EXTREMES FOR PERIOD OF RECORD.--HIGHEST WATER LEVEL,29.61 FT(9.03 M) BELOW LAND-SURFACE DATUM, JAN 11, 1983; LOWEST WATER LEVEL, 30.86 FT(9.41 M) BELOW LAND-SURFACE DATUM, JAN 31, 1980.

WATER LEVELS IN FEET BELOW LAND SURFACE DATUM, CLIMATIC YEARS APRIL 1981 TO MARCH 1983

\begin{tabular}{|c|c|c|}
\hline & WATER & \\
\hline DATE & LEVEL & DATE \\
\hline
\end{tabular}

JAN 19, $1982 \quad 30.62 \quad$ JAN 11, $1983 \quad 29.61$

GROUND-WATER LEVELS

361810099242201. LOCAL NUMBER; 21N-21W-12 CBB 1.

LOCATION,--LAT 36 18'10", LONG 099 24'22", HYDROLOGIC UNIT 11100301.

AQUIFER.--OGALLALA.

PERIOD OF RECORD.--1956 TO CURRENT YEAR.

EXTREMES FOR PERIOD OF RECORD.--HIGHEST WATER LEVEL, $13.01 \mathrm{FT}(3.97 \mathrm{M})$ BELOW LAND-SURFACE DATUM, JAN 10, 1983; LOWEST WATER LEVEL,28.93 FT(8.82 M) BELOW LAND-SURFACE DATUM, MAY 23, 1967.

WATER LEVELS IN FEET BELOW LAND SURFACE DATUM, CLIMATIC YEARS APRIL 1981 T0 MARCH 1983

$\begin{array}{lll} & \text { WATER } & \text { WATER } \\ \text { DATE } & \text { LEVEL } & \text { DATE }\end{array}$

JAN $19,1982 \quad 13.86 \quad$ JAN $10,1983 \quad 13.01$ 


\section{GROUND-WATER LEVELS}

WOODWARD COUNTY -- CONTINUED

361605099253201. LOCAL NUMBER;

LOCATION,--LAT 36 16'05", LONG 099 AQUIFER.--OGALLALA.

PERIOD OF RECORD.--1980 TO CURRENT YEAR.

EXTREMES FOR PERIOD OF RECORD.--HIGHEST WATER LEVEL,42.54 FT(12.97 M) BELOW LAND-SURFACE DATUM, JAN 10, 1983; LOWEST WATER LEVEL,43.94 FT(13.39 M) BELOW LAND-SURFACE DATUM, FEB 5, 1980.

WATER LEVELS IN FEET BELOW LAND SURFACE DATUM, CLIMATIC YEARS APRIL 1981 TO MARCH 1983

\begin{tabular}{|c|c|c|}
\hline & $\begin{array}{l}\text { WATER } \\
\text { LEVEL }\end{array}$ & DATE \\
\hline
\end{tabular}

JAN $19,1982 \quad 43.43 \quad$ JAN 10, $1983 \quad 42.54$

GROUND-WATER LEVELS

361748099334101. LOCAL NUMBER; $21 \mathrm{~N}-22 \mathrm{~W}-16$ CAA 1.

LOCATION,--LAT 36 17'48", LONG 099 33'41", HYDROLOGIC UNIT 11100203.

AQUIFER.--OGALLALA.

PERIOD OF RECORD.--1980 TO CURRENT YEAR.

EXTREMES FOR PERIOD OF RECORD.--HIGHEST WATER LEVEL, 129.30 FT(39.41 M) BELOW LAND-SURFACE DATUM, JAN 11, 1983; LOWEST WATER LEVEL, 129.81 FT(39.57 M) BELOW LAND-SURFACE DATUM, JAN 19, 1982.

WATER LEVELS IN FEET BELOW LAND SURFACE DATUM, CLIMATIC YEARS APRIL 1981 TO MARCH 1983

\begin{tabular}{llcl}
\multicolumn{1}{c}{} & WATER & & WATER \\
DATE & LEVEL & DATE & LEVEL \\
& & & \\
& 129.81 & JAN 11, 1983 & 129.30
\end{tabular}


WOODWARD COUNTY -- CONTINUED

362325099091901. LOCAL NUMBER; LOCATION,--LAT 36 23'25", LONG 099 AQUIFER.--TERRACE DEPOSITS.

PERIOD OF RECORD.--1978 TO CURRENT YEAR.

EXTREMES FOR PERIOD OF RECORD.--HIGHEST WATER LEVEL, $27.58 \mathrm{FT}(8.41 \mathrm{M})$ BELOW LAND-SURFACE DATUM,FEB 3, 1981; LOWEST WATER LEVEL, 30.28 FT(9.23 M) BELOW LAND-SURFACE DATUM, APR 20, 1978.

WATER LEVELS IN FEET BELOW LAND SURFACE DATUM, CLIMATIC YEARS APRIL 1981 TO MARCH 1983
$22 \mathrm{~N}-18 \mathrm{~W}-17$ BBB 1.

09'19", HYDROLOGIC UNIT 11100301.

$\begin{array}{ccccc}\text { DATE } & \text { WATER } & & \text { WATER } \\ \text { LEVEL } & \text { DATE } & \text { LEVEL } \\ \text { JAN 19, 1982 } & 28.97 & \text { JAN 10, 1983 } & 28.47\end{array}$

GROUND-WATER LEVELS

362002099081801. LOCAL NUMBER; 22N-18W-33 CCC 1.

LOCATION,--LAT 36 20'02", LONG 099 08'18", HYDROLOGIC UNIT 11100301.

AQUIFER.--TERRACE DEPOSITS.

PERIOD OF RECORD.--1977 TO CURRENT YEAR.

EXTREMES FOR PERIOD OF RECORD.--HIGHEST WATER LEVEL, 19.22 FT(5.86 M) BELOW LAND-SURFACE DATUM,JAN 10, 1983; LOWEST WATER LEVEL,23.45 FT(7.15 M) BELOW LAND-SURFACE DATUM, JAN 13, 1977.

WATER LEVELS IN FEET BELOW LAND SURFACE DATUM, CLIMATIC YEARS APRIL 1981 TO MARCH 1983

$\begin{array}{cccc}\text { DATE } & \text { WATER } & & \text { WATER } \\ \text { LEVEL } & \text { DATE } & \text { LEVEL } \\ \text { JAN 19, 1982 } & 20.80 & \text { JAN 10, 1983 } & 19.22\end{array}$




\section{GROUND-WATER LEVELS}

WOODWARD COUNTY -- CONTINUED

362144099174101. LOCAL NUMBER; 22N-20W-25 BBA 1. LOCATION,--LAT 36 21'44", LONG 099 17'41", HYDROLOGIC UNIT 11100301. AQUIFER.--TERRACE DEPOSITS. PERIOD OF RECORD.--1952 TO CURRENT YEAR. EXTREMES FOR PERIOD OF RECORD.--HIGHEST WATER LEVEL, $1.65 \mathrm{FT}(.50 \mathrm{M})$ BELOW LAND-SURFACE DATUM, JULY 1, 1957; LOWEST WATER LEVEL,7.65 FT(2.33 M) BELOW LAND-SURFACE DATUM, OCT 23, 1956.

WATER LEVELS IN FEET BELOW LAND SURFACE DATUM, CLIMATIC YEARS APRIL 1981 TO MARCH 1983

\begin{tabular}{|c|c|}
\hline & WATER \\
\hline DATE & LEVEL \\
\hline
\end{tabular}

JAN 19, $1982 \quad 3.59 \quad$ JAN $11,1983 \quad 4.16$

GROUND-WATER LEVELS

362147099224701. LOCAL NUMBER; 22N-21W-24 CCD 1. LOCATION,--LAT 36 21'47", LONG 099 22'47", HYDROLOGIC UNIT 11100301. AQUIFER.--OGALLALA.

PERIOD OF RECORD.--1980 TO CURRENT YEAR.

EXTREMES FOR PERIOD OF RECORD.--HIGHEST WATER LEVEL, 20.13 FT(6.14 M) BELOW LAND-SURFACE DATUM, JAN 11, 1983; LOWEST WATER LEVEL,21.31 FT(6.50 M) BELOW LAND-SURFACE DATUM, JAN 19, 1982 .

WATER LEVELS IN FEET BELOW LAND SURFACE DATUM, CLIMATIC YEARS APRIL 1981 TO MARCH 1983

$\begin{array}{lll} & \text { WATER } & \\ \text { DATE } & \text { WEVEL } & \text { WATER } \\ & \text { DATE } & \text { LEVEL }\end{array}$

JAN 19, $1982 \quad 21.31 \quad$ JAN 11, $1983 \quad 20.13$ 


\section{WOODWARD COUNTY -- CONTINUED}

362101099264701 . LOCAL NUMBER; 22N-21W-28 DDB 1. LOCATION,--LAT 36 21'01", LONG 099 26'47", HYDROLOGIC UNIT 11100301. AQUIFER.--OGALLALA. PERIOD OF RECORD.--1980 TO CURRENT YEAR. EXTREMES FOR PERIOD OF RECORD.--HIGHEST WATER LEVEL, 101.44 FT(30.92 M) BELOW LAND-SURFACE DATUM,JAN 11, 1983; LOWEST WATER LEVEL, 101.87 FT(31.05 M) BELOW LAND-SURFACE DATUM, FEB 4, 1981.

WATER LEVELS IN FEET BELOW LAND SURFACE DATUM, CLIMATIC YEARS APRIL 1981 TO MARCH 1983

\begin{tabular}{|c|c|c|}
\hline & WATER & \\
\hline DATE & LEVEL & DATE \\
\hline
\end{tabular}

JAN 19, $1982 \quad 101.71 \quad$ JAN $11,1983 \quad 101.44$

GROUND-WATER LEVELS

362429099301101. LOCAL NUMBER; 22N-22W-01 DCA 1. LOCATION,--LAT 36 24'29", LONG 099 30'11", HYDROLOGIC UNIT 11100301. AQUIFER.--OGALLALA. PERIOD OF RECORD.--1983 TO CURRENT YEAR. EXTREMES FOR PERIOD OF RECORD.--HIGHEST WATER LEVEL, $55.05 \mathrm{FT}(16.78 \mathrm{M})$ BELOW LAND-SURFACE DATUM, JAN 11, 1983; LOWEST WATER LEVEL,55.05 FT(16.78 M) BELOW LAND-SURFACE DATUM, JAN 11, 1983.

WATER LEVELS IN FEET BELOW LAND SURFACE DATUM, CLIMATIC YEARS APRIL 1981 TO MARCH 1983

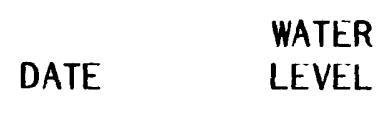

JAN 11, $1983 \quad 55.05$ 
362502099311401. LOCAL NUMBER; LOCATION,--LAT $3625^{\prime} 02^{\prime \prime}$, LONG 099 AQUIFER.--OGALLALA.

PERIOD OF RECORD.--1978 TO CURRENT YEAR. EXTREMES FOR PERIOD OF RECORD.--HIGHEST WATER LEVEL, $40.53 \mathrm{FT}(12.35 \mathrm{M})$ BELOW LAND-SURFACE DATUM,JAN 11, 1983; LOWEST WATER LEVEL,45.80 FT(13.96 M) BELOW LAND-SURFACE DATUM, JAN 29, 1980. WATER LEVELS IN FEET BELOW LAND SURFACE DATUM, CLIMATIC YEARS APRIL 1981 TO MARCH 1983
$22 \mathrm{~N}-22 \mathrm{~W}-02$ ABA 1

31 '14", HYDROLOGIC UNIT 11100301.
WATER

DATE

JAN 20, 1982
LEVEL

41.68
WATER

LEVEL

GROUND-WATER LEVELS

362350099341501. LOCAL NUMBER; 22N-22W-08 DAD 1. LOCATION,--LAT 36 23'50", LONG 099 34'15", HYDROLOGIC UNIT 11100203.

AQUIFER.--OGALLALA.

PERIOD OF RECORD.-- 1980 TO CURRENT YEAR. EXTREMES FOR PERIOD OF RECORD.--HIGHEST WATER LEVEL, $3.44 \mathrm{FT}(1.05 \mathrm{M})$ BELOW LAND-SURFACE DATUM, JAN 11, 1983; LOWEST WATER LEVEL, $11.56 \mathrm{FT}(3.52 \mathrm{M})$ BELOW LAND-SURFACE DATUM, MAR 25, 1980.

WATER LEVELS IN FEET BELOW LAND SURFACE DATUM, CLIMATIC YEARS APRIL 1981 TO MARCH 1983

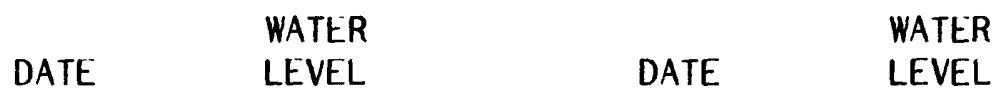

JAN 20, $1982 \quad 3.79 \quad$ JAN 11, $1983 \quad 3.44$ 
GROUND-WATER LEVELS

WOODWARD COUNTY -- CONTINUED

362149099361501. LOCAL NUMBER; LOCATION, --LAT 36 21'49", LONG 099 AQUIFER .--OGALLALA. PERIOD OF RECORD._-1955 TO CURRENT YEAR. EXTREMES FOR PERIOD OF RECORD.--HIGHEST WATER LEVEL, 13.08 FT(3.99 M) BELOW LAND-SURFACE DATUM, JAN 29, 1980; LOWEST WATER LEVEL,29.71 FT(9.06 M) BELOW LAND-SURFACE DATUM, OCT 17, 1956 .

WATER LEVELS IN FEET BELOW LAND SURFACE DATUM, CLIMATIC YEARS APRIL 1981 TO MARCH 1983

\begin{tabular}{|c|c|c|}
\hline & WATER & \\
\hline DATE & LEVEL & DATE \\
\hline
\end{tabular}

JAN 20, $1982 \quad 27.76 \quad$ JAN 11, $1983 \quad 28.12 \mathrm{R}$

GROUND-WATER LEVELS

362229099295801. LOCAL NUMBER; 22N-22W-24 AAB 1.

LOCATION,--LAT 36 22'29", LONG 099 29'58", HYDROLOGIC UNIT 11100203.

AQUIFER.--OGALLALA.

PERIOD OF RECORD.--1983 TO CURRENT YEAR.

EXTREMES FOR PERIOD OF RECORD.--HIGHEST WATER LEVEL, 122.20 FT(37.25 M) BELOW LAND-SURFACE DATUM, JAN 11, 1983; LOWEST WATER LEVEL, 122.20 FT(37.25 M) BELOW LAND-SURFACE DATUM, JAN 11, 1983.

WATER LEVELS IN FEET BELOW LAND SURFACE DATUM, CLIMATIC YEARS APRIL 1981 T0 MARCH 1983

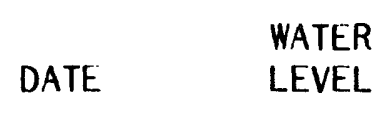

JAN $11,1983122.20$ 
362818099103001. LOCAL NUMBER; LOCATION,--LAT 36 28'18", LONG 099 AQUIFER.--TERRACE DEPOSITS. PERIOD OF RECORD.--1978 TO CURRENT YEAR. EXTREMES FOR PERIOD OF RECORD.--HIGHEST WATER LEVEL, $61.55 \mathrm{FT}(18.76 \mathrm{M})$ BELOW LAND-SURFACE DATUM, APR 20, 1978; LOWEST WATER LEVEL,64.59 FT(19.69 M) BELOW LAND-SURFACE DATUM, JAN 10, 1983.

WATER LEVELS IN FEET BELOW LAND SURFACE DATUM, CLIMATIC YEARS APRIL 1981 TO MARCH 1983
$23 \mathrm{~N}-19 \mathrm{~W}-13$ ADD 1.

10'30", HYDROLOGIC UNIT 11100301.
198264.26
JAN 10,1983
64.59

$\begin{array}{cll} & & \\ \text { DATE } & \text { WATER } & \text { WATER } \\ \text { LEVEL } & \text { DATE } & \text { LEVEL }\end{array}$

$\begin{array}{cll} & & \\ \text { DATE } & \text { WATER } & \text { WATER } \\ \text { LEVEL } & \text { DATE } & \text { LEVEL }\end{array}$

GROUND-WATER LEVELS

\begin{abstract}
362748099134401. LOCAL NUMBER; 23N-19E-21 AAA 1. LOCATION,--LAT 36 27'48", LONG 099 13'44", HYDROLOGIC UNIT 11100301. AQUIFER.--TERRACE DEPOSITS. PERIOD OF RECORD.--1978 TO CURRENT YEAR. EXTREMES FOR PERIOD OF RECORD.--HIGHEST WATER LEVEL, 41.17 FT(12.55 M) BELOW LAND-SURFACE DATUM, APR 20, 1978; LOWEST WATER LEVEL,43.32 FT(13.20 M) BELOW LAND-SURFACE DATUM, JAN 19, 1982 .
\end{abstract}

WATER LEVELS IN FEET BELOW LAND SURFACE DATUM, CLIMATIC YEARS APRIL 1981 TO MARCH 1983

$\begin{array}{lll} & \text { WATER } & \text { WATER } \\ \text { DATE } & \text { LEVEL } & \text { DATE }\end{array}$
JAN 19,1982
43.32
JAN 10, 1983
41.83 
GROUND-WATER LEVELS

WOODWARD COUNTY -- CONTINUED

362737099125101. LOCAL NUMBER; 23N-19W-22 AAC 1.

LOCATION,--LAT 36 27'37", LONG 099 12'51", HYDROLOGIC UNIT 11100301.

AQUIFER.--TERRACE DEPOSITS.

PERIOD OF RECORD.--1977 TO CURRENT YEAR.

EXTREMES FOR PERIOD OF RECORD.--HIGHEST WATER LEVEL,41.40 FT(12.62 M) BELOW LAND-SURFACE DATUM, JAN 10, 1983; LOWEST WATER LEVEL,43.86 FT(13.37 M) BELOW LAND-SURFACE DATUM, JAN 19, 1982.

WATER LEVELS IN FEET BELOW LAND SURFACE DATUM, CLIMATIC YEARS APRIL 1981 TO MARCH 1983

\begin{tabular}{ccccc}
\multicolumn{1}{c}{} & WATER & & & WATER \\
DATE & LEVEL & DATE & LEVEL \\
JAN 19, 1982 & 43.86 & JAN 10, 1983 & 41.40
\end{tabular}

GROUND-WATER LEVELS

363020099220201. LOCAL NUMBER; 23N-20W-05 BBA 1. LOCATION,--LAT 36 30'20", LONG 099 22'02", HYDROLOGIC UNIT 11100301. AQUIFER.-.TERRACE DEPOSITS.

PERIOD OF RECORD.-- 1977 TO CURRENT YEAR.

EXTREMES FOR PERIOD OF RECORD.--HIGHEST WATER LEVEL, 31.25 FT $(9.53 \mathrm{M})$ BELOW LAND-SURFACE DATUM, JAN 10, 1983; LOWEST WATER LEVEL, $32.96 \mathrm{FT}(10.05 \mathrm{M})$ BELOW LAND-SURFACE DATUM, JAN 13, 1977.

WATER LEVELS IN FEET BELOW LAND SURFACE DATUM, CLIMATIC YEARS APRIL 1981 TO MARCH 1983

$\begin{array}{lll} & \text { WATER } & \text { WATER } \\ \text { DATE } & \text { LEVEL } & \text { DATE }\end{array}$

JAN 21, $1982 \quad 32.11 \quad$ JAN $10,1983 \quad 31.25$ 
WOODWARD COUNTY -- CONTINUED

362717099231401. LOCAL NUMBER; 23N-20W-19 CBB 1.

LOCATION,--LAT 36 27'17", LONG 099 23'14", HYDROLOGIC UNIT 11100301.

AQUIFER.--TERRACE DEPOSITS.

PERIOD OF RECORD. - 1945 TO CURRENT YEAR.

EXTREMES FOR PERIOD OF RECORD.--HIGHEST WATER LEVEL, $1.02 \mathrm{FT}(.31 \mathrm{M})$ BELOW LAND-SURFACE DATUM, JULY 1, 1957; LOWEST WATER LEVEL,6.94 FT(2.12 M) BELOW LAND-SURFACE DATUM, OCT $9,1956$.

WATER LEVELS IN FEET BELOW LAND SURFACE DATUM, CLIMATIC YEARS APRIL 1981 TO MARCH 1983

\begin{tabular}{|c|c|}
\hline & WATER \\
\hline DATE & LEVEL \\
\hline
\end{tabular}
JAN 19, 1982
5.18
JAN 10,1983
W

\section{GROUND-WATER LEVELS}

362728099220201 . LOCAL NUMBER; LOCATION,--LAT 36 27'28", LONG 099 AQUIFER.--TERRACE DEPOSITS.

PERIOD OF RECORD.--1978 TO CURRENT YEAR. EXTREMES FOR PERIOD OF RECORD.--HIGHEST WATER LEVEL,6.02 FT(1.83 M) BELOW LAND-SURFACE DATUM,FEB 3, 1981; LOWEST WATER LEVEL, $12.51 \mathrm{FT}(3.81 \mathrm{M})$ BELOW LAND-SURFACE DATUM, APR $4,1978$. WATER. LEVELS IN FEET BELOW LAND SURFACE DATUM, CLIMATIC YEARS APRIL 1981 TO MARCH 1983
23N-20W-20 BCA 1.

22 '02", HYDROLOGIC UNIT 11100301.

$\begin{array}{lll} & \text { WATER } & \\ \text { DATE } & \text { WEVEL } & \text { DATER } \\ \text { LEVEL }\end{array}$

JAN 19, $1982 \quad 12.45 \quad$ JAN $10,1983 \quad 11.73$ 
GROUND-WATER LEVELS

WOODWARD COUNTY -- CONTINUED

362650099254401. LOCAL NUMBER; 23N-21W-27 AAB 1. LOCATION,--LAT 36 26'50", LONG 099 25'44", HYDROLOGIC UNIT 11100301. AQUIFER.--OGALLALA.

PERIOD OF RECORD.--1980 TO CURRENT YEAR.

EXTREMES FOR PERIOD OF RECORD.--HIGHEST WATER LEVEL, $15.37 \mathrm{FT}(4.68 \mathrm{M})$ BELOW LAND-SURFACE DATUM,JAN 31, 1980; LOWEST WATER LEVEL, $15.79 \mathrm{FT}(4.81 \mathrm{M})$ BELOW LAND-SURFACE DATUM, JAN 11, 1983.

WATER LEVELS IN FEET BELOW LAND SURFACE DATUM, CLIMATIC YEARS APRIL 1981 TO MARCH 1983

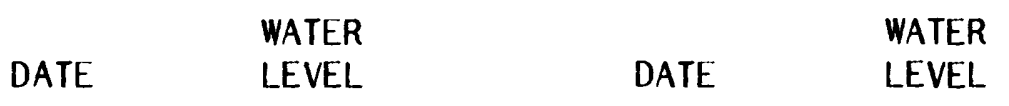

JAN 19, $1982 \quad 15.75 \quad$ JAN $11,1983 \quad 15.79$

GROUND-WATER LEVELS

362942099320301. LOCAL NUMBER; 23N-22W-03 DDA 1.

LOCATION,--LAT 36 29'42", LONG 099 32'03", HYDROLOGIC UNIT 11100203.

AQUIFER.--OGALLALA.

PERIOD OF RECORD.--1980 TO CURRENT YEAR.

EXTREMES FOR PERIOD OF RECORD.--HIGHEST WATER LEVEL, $32.87 \mathrm{FT}(10.02 \mathrm{M})$ BELOW LAND-SURFACE DATUM,FEB 4, 1981; LOWEST WATER LEVEL,36.23 FT(11.04 M) BELOW LAND-SURFACE DATUM, JAN 29, 1980.

WATER LEVELS IN FEET BELOW LAND SURFACE DATUM, CLIMATIC YEARS APRIL 1981 TO MARCH 1983

$\begin{array}{lll} & \text { WATER } & \text { WATER } \\ \text { DATE } & \text { LEVEL } & \text { DATE }\end{array}$

JAN 20, $1982 \quad 34.95 \quad$ JAN $11,1983 \quad 33.19$ 
WOODWARD COUNTY -- CONTINUED

362659099322201. LOCAL NUMBER; LOCATION,--LAT 36 26'59", LONG 099 AQUIFER.--OGALLALA. PERIOD OF RECORD.--1978 TO CURRENT YEAR. EXTREMES FOR PERIOD OF RECORD.--HIGHEST WATER LEVEL,26.67 FT(8.13 M) BELOW LAND-SURFACE DATUM, MAR 3, 1981; LOWEST WATER LEVEL,29.70 FT(9.05 M) BELOW LAND-SURFACE DATUM, APR 19, 1978.

WATER LEVELS IN FEET BELOW LAND SURFACE DATUM, CLIMATIC YEARS APRIL 1981 TO MARCH 1983
$23 N-22 W-22$ DCD 1 32 '22", HYDROLOGIC UNIT 11100203.

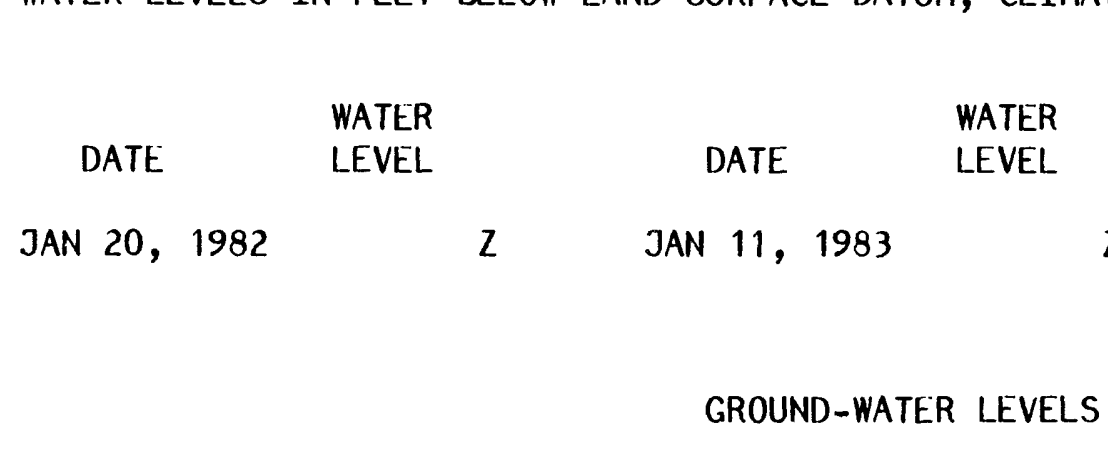

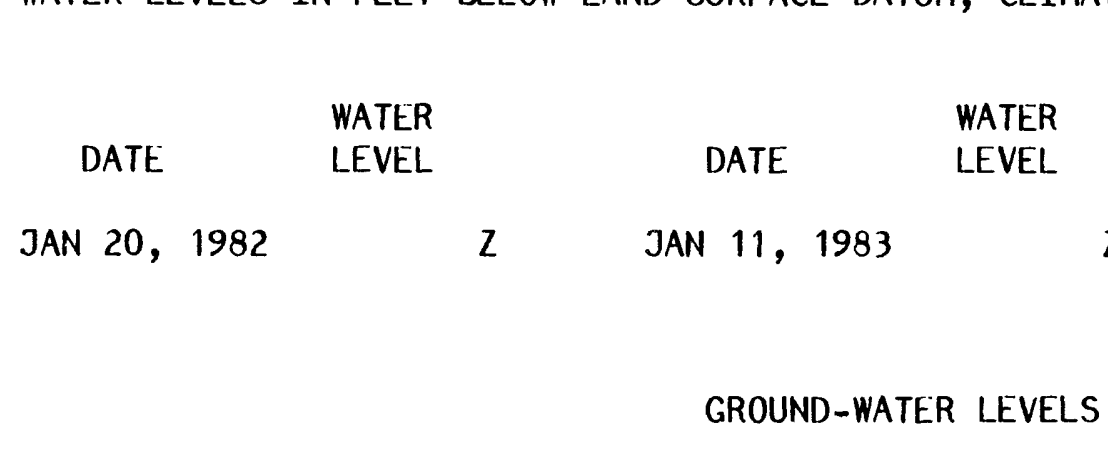

362658099321801. LOCAL NUMBER; 23N-22W-22 DDB 1. LOCATION,--LAT 36 26'58", LONG 099 32'18", HYDROLOGIC UNIT 11100203. AQUIFER.--OGALLALA. PERIOD OF RECORD.--1980 TO CURRENT YEAR. EXTREMES FOR PERIOD OF RECORD.--HIGHEST WATER LEVEL, 22.46 FT (6.85 M) BELOW LAND-SURFACE DATUM, JAN 11, 1983; LOWEST WATER LEVEL,24.90 FT(7.59 M) BELOW LAND-SURFACE DATUM, JAN 20, 1982.

WATER LEVELS IN FEET BELOW LAND SURFACE DATUM, CLIMATIC YEARS APRIL 1981 T0 MARCH 1983

\begin{tabular}{|c|c|c|}
\hline & WATER & \\
\hline DATE & & DATE \\
\hline
\end{tabular}

JAN 20, $1982 \quad 24.90 \quad$ JAN $11,1983 \quad 22.46$ 
WOODWARD COUNTY -- CONTINUED

362534099344501. LOCAL NUMBER; 23N-22W-32 DBD 1.

LOCATION,--LAT 36 25'34", LONG 099 34'45", HYDROLOGIC UNIT 11100203.

AQUIFER.--OGALLALA.

PERIOD OF RECORD.--1981 TO CURRENT YEAR.

EXTREMES FOR PERIOD OF RECORD.--HIGHEST WATER LEVEL,8.96 FT(2.73 M) BELOW LAND-SURFACE DATUM, JAN 11, 1983; LOWEST WATER LEVEL, 10.60 FT(3.23 M) BELOW LAND-SURFACE DATUM, JAN 20, 1982 .

WATER LEVELS IN FEET BELOW LAND SURFACE DATUM, CLIMATIC YEARS APRIL 1981 TO MARCH 1983

$\begin{array}{lll} & \text { WATER } & \text { WATER } \\ \text { DATE } & \text { LEVEL } & \text { DATE }\end{array}$

JAN 20, $1982 \quad 10.60 \quad$ JAN $11,1983 \quad 8.96$

GROUND-WATER LEVELS

363300099232001. LOCAL NUMBER; 24N-20W-18 CCC 1. LOCATION,--LAT 36 33'00", LONG 099 23'20", HYDROLOGIC UNIT 11100301. AQUIFER .--OGALLALA. PERIOD OF RECORD.--1978 TO CURRENT YEAR. EXTREMES FOR PERIOD OF RECORD.--HIGHEST WATER LEVEL,41.95 FT(12.79 M) BELOW LAND-SURFACE DATUM, JAN 10, 1983; LOWEST WATER LEVEL,44.50 FT(13.56 M) BELOW LAND-SURFACE DATUM, JAN 21, 1982.

WATER LEVELS IN FEET BELOW LAND SURFACE DATUM, CLIMATIC YEARS APRIL 1981 TO MARCH 1983

\begin{tabular}{|c|c|c|}
\hline & WATER & \\
\hline DATE & LEVEL & DATE \\
\hline
\end{tabular}

JAN 21, $1982 \quad 44.50 \quad$ JAN $10,1983 \quad 41.95$ 
WOODWARD COUNTY -- CONTINUED

363023099231901. LOCAL NUMBER; 24N-20W-31 CCC 1. LOCATION,--LAT 36 30'23", LONG 099 23'19", HYDROLOGIC UNIT 11100301. AQUIFER.--TERRACE DEPOSITS. PERIOD OF RECORD.--1978 TO CURRENT YEAR. EXTREMES FOR PERIOD OF RECORD.--HIGHEST WATER LEVEL, $49.05 \mathrm{FT}(14.95 \mathrm{M})$ BELOW LAND-SURFACE DATUM,JAN 10, 1983; LOWEST WATER LEVEL,54.82 FT(16.71 M) BELOW LAND-SURFACE DATUM, MAY 15, 1979.

WATER LEVELS IN FEET BELOW LAND SURFACE DATUM, CLIMATIC YEARS APRIL 1981 TO MARCH 1983

\begin{tabular}{|c|c|}
\hline & WATER \\
\hline DATE & LEVEL \\
\hline
\end{tabular}

JAN 21, $1982 \quad 49.25 \quad$ JAN 10, $1983 \quad 49.05$

\section{GROUND-WATER LEVELS}

363308099245801. LOCAL NUMBER; 24N-21W-14 CDA 1. LOCATION,--LAT 36 33'08", LONG 099 24'58", HYDROLOGIC UNIT 11100301.

AQUIFER.--TERRACE DEPOSITS.

PERIOD OF RECORD.--1978 TO CURRENT YEAR.

EXTREMES FOR PERIOD OF RECORD.--HIGHEST WATER LEVEL, $28.95 \mathrm{FT}(8.82 \mathrm{M})$ BELOW LAND-SURFACE DATUM, JAN 10, 1983; LOWEST WATER LEVEL, 31.75 FT(9.68 M) BELOW LAND-SURFACE DATUM, APR 12, 1978.

WATER LEVELS IN FEET BELOW LAND SURFACE DATUM, CLIMATIC YEARS APRIL 1981 TO MARCH 1983

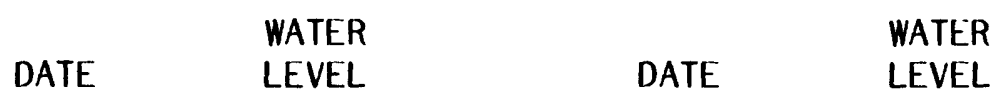

JAN 21, $1982 \quad 31.70 \mathrm{R} \quad$ JAN 10, $1983 \quad 28.95$ 
WOODWARD COUNTY -- CONTINUED

363534099331101. LOCAL NUMBER; 24N-22W-04 AAA 1. LOCATION,--LAT 36 35'34", LONG 099 33'11", HYDROLOGIC UNIT 11100203.

AQUIFER.--OGALLALA.

PERIOD OF RECORD. - 1980 TO CURRENT YEAR.

EXTREMES FOR PERIOD OF RECORD.--HIGHEST WATER LEVEL, $8.03 \mathrm{FT}(2.45 \mathrm{M})$ BELOW LAND-SURFACE DATUM,FEB 3, 1981; LOWEST WATER LEVEL,8.30 FT(2.53 M) BELOW LAND-SURFACE DATUM, JAN 20, 1982.

WATER LEVELS IN FEET BELOW LAND SURFACE DATUM, CLIMATIC YEARS APRIL 1981 TO MARCH 1983

\begin{tabular}{|c|c|}
\hline & WATER \\
\hline DATE & LEVEL \\
\hline
\end{tabular}

JAN 20, $1982 \quad 8.30 \quad$ JAN $11,1983 \quad 8.16$

GROUND-WATER LEVELS

363401099325601. LOCAL NUMBER;

LOCATION,--LAT 36 34'01', LONG 099

AQUIFER.--TERRACE DEPOSITS.

PERIOD OF RECORD.--1951 TO CURRENT YEAR.

EXTREMES FOR PERIOD OF RECORD.--HIGHEST WATER LEVEL, $8.93 \mathrm{FT}(2.72 \mathrm{M})$ BELOW LAND-SURFACE

DATUM, JULY 1, 1957; LOWEST WATER LEVEL, $17.00 \mathrm{FT}(5.18 \mathrm{M})$ BELOW LAND-SURFACE DATUM, JAN 27, 1960.

WATER LEVELS IN FEET BELOW LAND SURFACE DATUM, CLIMATIC YEARS APRIL 1981 TO MARCH 1983
$24 \mathrm{~N}-22 \mathrm{~W}-10$ CCC 1.

32 '56", HYDROLOGIC UNIT 11100203.

DATE

WATER

JAN 20, $1982 \quad 15.08$

DATE

WATER

LEVEL

JAN $11,1983 \quad 13.89$ 